\title{
Algebraic Approaches to Partial Differential Equations
}

\author{
Xiaoping $\mathrm{Xu}$ \\ Hua Loo-Keng Key Mathematical Laboratory \\ Institute of Mathematics \\ Academy of Mathematics and System Sciences \\ Chinese Academy of Sciences \\ 55 Zhongguancun Dong Lu \\ Beijing 100190, P. R. China \\ 2012
}




\section{Dedicated to My Wife Jing Jing}




\section{Contents}

Preface $\quad v$

Introduction vii

Notational Conventions . . . . . . . . . . . . . . . . . xviii

I Ordinary Differential Equations

1 First-Order Ordinary Differential Equations

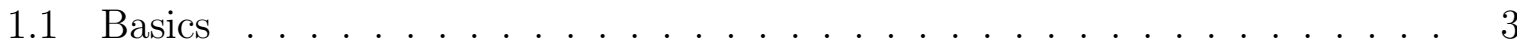

1.2 Special Equations . . . . . . . . . . . . . . . . . . 9

2 Higher-Order Ordinary Differential Equations $\quad 17$

2.1 Basics . . . . . . . . . . . . . . . . . . . . . . . . . . . .

2.2 Method of Undetermined Coefficients . . . . . . . . . . . . . . . 21

2.3 Method of Variation of Parameters . . . . . . . . . . . . . . 25

2.4 Series Method and Bessel Functions . . . . . . . . . . . . . . . . 28

3 Special Functions

3.1 Gamma and Beta Functions . . . . . . . . . . . . . . . . . 35

3.2 Gauss Hypergeometric Functions . . . . . . . . . . . . . . . . 40

3.3 Orthogonal Polynomials . . . . . . . . . . . . . . . . . 44

3.4 Weierstrass's Elliptic Functions . . . . . . . . . . . . . . 50

3.5 Jacobian Elliptic Functions . . . . . . . . . . . . . . . . . . 55

II Partial Differential Equations $\quad 59$

4 First-Order or Linear Equations $\quad 61$

4.1 Method of Characteristics . . . . . . . . . . . . . . 62

4.2 Characteristic Strip and Exact Equations . . . . . . . . . . . . . . 65

4.3 Polynomial Solutions of Flag Equations . . . . . . . . . . . . . . 67 
4.4 Use of Fourier Expansion I . . . . . . . . . . . . . . . . . . 84

4.5 Use of Fourier Expansion II . . . . . . . . . . . . . . . . . . . . . . 89

4.6 Calogero-Sutherland Model . . . . . . . . . . . . . . . . 102

4.7 Maxwell Equations . . . . . . . . . . . . . . . . . . . . . 111

4.8 Dirac Equation and Acoustic System . . . . . . . . . . . . . . . 116

5 Nonlinear Scalar Equations $\quad 123$

5.1 Kortweg and de Vries Equation . . . . . . . . . . . . . . . . . . . 124

5.2 Kadomtsev and Petviashvili Equation . . . . . . . . . . . . . . . . 130

5.3 Equation of Transonic Gas Flows . . . . . . . . . . . . . . . . . . 135

5.4 Short Wave Equation . . . . . . . . . . . . . . . . . . . . . . 141

5.5 Khokhlov and Zabolotskaya Equation . . . . . . . . . . . . . . . . 146

5.6 Equation of Geopotential Forecast . . . . . . . . . . . . . . . . . . . 150

6 Nonlinear Schrödinger and DS Equations $\quad 157$

6.1 Nonlinear Schrödinger Equation . . . . . . . . . . . . . . . . . . 157

6.2 Coupled Schrödinger Equations . . . . . . . . . . . . . . . . . 164

6.3 Davey and Stewartson Equations . . . . . . . . . . . . . 176

7 Dynamic Convection in a Sea $\quad 185$

7.1 Equations and Symmetries . . . . . . . . . . . . . . 185

7.2 Moving-Line Approach . . . . . . . . . . . . . . . . . . . 188

7.3 Approach of Cylindrical Product . . . . . . . . . . . . . . . . 191

7.4 Dimensional Reduction . . . . . . . . . . . . . . . . . . . 194

8 Boussinesq Equations in Geophysics $\quad 201$

8.1 Two-Dimensional Equations . . . . . . . . . . . . . . . . 201

8.2 Three-Dimensional Equations and Symmetry . . . . . . . . . . . . 215

8.3 Asymmetric Approach I . . . . . . . . . . . . . . . . . 217

8.4 Asymmetric Approach II . . . . . . . . . . . . . . . . . . . . . 222

8.5 Asymmetric Approach III . . . . . . . . . . . . . . . . . 227

9 Navier-Stokes Equations $\quad 231$

9.1 Background and Symmetry . . . . . . . . . . . . . . . 231

9.2 Asymmetric Approaches . . . . . . . . . . . . . . . . . 235

9.3 Moving-Frame Approach I . . . . . . . . . . . . . . . . . . . 245

9.4 Moving-Frame Approach II . . . . . . . . . . . . . . . . . . . 254

\begin{tabular}{l|l|} 
Bibliography & 271 \\
\hline
\end{tabular} 
Index

282 


\section{Preface}

Partial differential equations are fundamental tools in mathematics, sciences and engineering. For instance, the electrodynamics is governed by the Maxwell equations, the two-dimensional cubic nonlinear Schrödinger equation is used to describe the propagation of an intense laser beam through a medium with Kerr nonlinearity and the Navier-Stokes equations are the fundamental equations in fluid dynamics. There are three major ways of studying partial differential equations. The analytic way is to study the existence and uniqueness of certain solutions of partial differential equations and their mathematical properties. While the numerical way is to find certain numerical solutions of partial differential equations. In particular, physicists and engineers have developed their own computational methods of finding physical and practically useful numerical solutions, mostly motivated by experiments. The algebraic way is to study symmetries, conservation laws, exact solutions and complete integrability of partial differential equations.

This book belongs to the third category. It is mainly an exposition of the various algebraic techniques of solving partial differential equations for exact solutions developed by the author in recent years, with emphasis on physical equations such as: the CalogeroSutherland model of quantum many-body system in one-dimension, the Maxwell equations, the free Dirac equations, the generalized acoustic system, the Kortweg and de Vries $(\mathrm{KdV})$ equation, the Kadomtsev and Petviashvili (KP) equation, the equation of transonic gas flows, the short-wave equation, the Khokhlov and Zabolotskaya equation in nonlinear acoustics, the equation of geopotential forecast, the nonlinear Schrödinger equation and coupled nonlinear Schrödinger equations in optics, the Davey and Stewartson equations of three-dimensional packets of surface waves, the equation of the dynamic convection in a sea, the Boussinesq equations in geophysics, the incompressible Navier-Stokes equations and the classical boundary layer equations.

It is well known that most partial differential equations from geometry are treated as the equations of elliptic type and most partial differential equations from fluid dynamics are treated as the equations of hyperbolic type. Analytically, partial differential equations of elliptic type are easier than those of hyperbolic type. Most of the nonlinear partial differential equations in this book are from fluid dynamics. Our results show that algebraically, partial differential equations of hyperbolic type are easier than those of el- 
liptic type in terms of exact solutions. Algebraic approach and analytic approach have fundamental differences.

This book was written based on the author's lecture notes on partial differential equations taught at the Graduate University of Chinese Academy of Sciences. It turned out that the course with the same title as the book was welcome not only by mathematical graduate students but also by physical and engineering students. Some engineering faculty members had also showed their interests in the course. The book is self-contained with the minimal prerequisite of calculus and linear algebra. It progresses according to the complexity of equations and sophistication of the techniques involved. Indeed, it includes the basic algebraic techniques in ordinary differential equations and a brief introduction to special functions as the preparation for the main context.

In linear partial differential equations, we focus on finding all the polynomial solutions and solving the initial-value problems. Intuitive derivations of easily-using symmetry transformations of nonlinear partial differential equations are given. These transformations generate sophisticated solutions with more parameters from relatively simple ones. They are also used to simplify our process of finding exact solutions. We have extensively used moving frames, asymmetric conditions, stable ranges of nonlinear terms, special functions and linearizations in our approaches to nonlinear partial differential equations. The exact solutions we obtained usually contain multiple parameter functions and most of them are not of traveling-wave type.

The book can serve as a research reference book for mathematicians, scientists and engineers. It can also be treated as a text book after a proper selection of materials for training students' mathematical skills and enriching their knowledge.

Beijing, P. R. China

2012 


\section{Introduction}

In normal circumstances, the natural world operates according to physical laws. Many of these laws were formulated in terms of partial differential equations. For instance, the electromagnetic fields in physics are governed by the well-known Maxwell equations

$$
\partial_{t}(\mathbf{E})=\operatorname{curl} \mathbf{B}, \quad \partial_{t}(\mathbf{B})=-\operatorname{curl} \mathbf{E}
$$

with

$$
\operatorname{div} \mathbf{E}=f(x, y, z), \quad \operatorname{div} \mathbf{B}=g(x, y, z),
$$

where the vector function $\mathbf{E}$ stands for the electric field, the vector function $\mathbf{B}$ stands for the magnetic field, the scalar function $f$ is related to the charge density and the scalar function $g$ is related to the magnetic potential. The two-dimensional cubic nonlinear Schrödinger equation

$$
i \psi_{t}+\kappa\left(\psi_{x x}+\psi_{y y}\right)+\varepsilon|\psi|^{2} \psi=0
$$

is used to describe the propagation of an intense laser beam through a medium with Kerr nonlinearity, where $t$ is the distance in the direction of propagation, $x$ and $y$ are the transverse spacial coordinates, $\psi$ is a complex valued function in $t, x, y$ standing for electric field amplitude, and $\kappa, \varepsilon$ are nonzero real constants. Moreover, the coupled twodimensional cubic nonlinear Schrödinger equations

$$
\begin{aligned}
& i \psi_{t}+\kappa_{1}\left(\psi_{x x}+\psi_{y y}\right)+\left(\varepsilon_{1}|\psi|^{2}+\epsilon_{1}|\varphi|^{2}\right) \psi=0, \\
& i \varphi_{t}+\kappa_{2}\left(\varphi_{x x}+\varphi_{y y}\right)+\left(\varepsilon_{2}|\psi|^{2}+\epsilon_{2}|\varphi|^{2}\right) \varphi=0
\end{aligned}
$$

are used to describe the interaction of electromagnetic waves with different polarizations in nonlinear optics, where $\kappa_{1}, \kappa_{2}, \varepsilon_{1}, \varepsilon_{2}, \epsilon_{1}$ and $\epsilon_{2}$ are real constants.

The most fundamental differential equations in the motion of incompressible viscous fluids are the Navier-Stokes equations

$$
\begin{gathered}
u_{t}+u u_{x}+v u_{y}+w u_{z}+\frac{1}{\rho} p_{x}=\nu\left(u_{x x}+u_{y y}+u_{z z}\right), \\
v_{t}+u v_{x}+v v_{y}+w v_{z}+\frac{1}{\rho} p_{y}=\nu\left(v_{x x}+v_{y y}+v_{z z}\right),
\end{gathered}
$$




$$
\begin{aligned}
w_{t}+u w_{x}+v w_{y}+w w_{z}+\frac{1}{\rho} p_{z} & =\nu\left(w_{x x}+w_{y y}+w_{z z}\right), \\
u_{x}+v_{y}+w_{z} & =0
\end{aligned}
$$

where $(u, v, w)$ stands for the velocity vector of the fluid, $p$ stands for the pressure of the fluid, $\rho$ is the density constant and $\nu$ is the coefficient constant of the kinematic viscosity.

Algebraic study of partial differential equations traces back to Norwegian mathematician Sophus Lie [Lie], who invented the powerful tool of continuous groups (known as Lie groups) in 1874 in order to study symmetry of differential equations. Lie's idea has been carried on mainly by the mathematicians in the former states of Soviet Union, East Europe and some mathematicians in North America. Now it has become an important mathematical field known as "group analysis of differential equations," whose main objective is to find symmetry group of partial differential equations, related conservation laws and similarity solutions. The most influential modern books on the subject may be the book "Applications of Lie Groups to Differential Equations" by Olver [Op] and the book "Lie Group Analysis of Differential Equations" by Ibragimov (cf. [In2, In3]). In [X3], we found the complete set of functional generators for the differential invariants of classical groups.

Soliton phenomenon was first observed by J. Scott Russel in 1834 when he was riding on horseback beside the narrow Union Canal near Edinburgh, Scotland. The phenomenon had been theoretically studied by Russel, Airy (1845), Stokes (1847), Boussinesq (1871, 1872) and Rayleigh (1876). The problem was finally solved by Kortweg and de Vries (1895) in terms of the partial differential equation

$$
u_{t}+6 u u_{x}+u_{x x x}=0
$$

where $u$ is the surface elevation of the wave above the equilibrium level, $x$ is the distance from starting point and $t$ stands for time (later people also realized that the above equation and its one-soliton solution appeared in the Boussinesq's long paper $[\mathrm{Bj}]$ ). However, it was not until 1960 that any further application of the equation was discovered. Gardner and Morikawa [GM] (1960) rediscovered the KdV equation in the study of collision-free hydromagnetic waves. Subsequently, the KdV equation has arisen in a number of other physical contexts, such as, stratified internal waves, ion-acoustic waves, plasma physics and lattice dynamics etc. Later a group led by Kruskal [GGKM1, GGKM2, KMGZ, MGK] invented a special way of solving the KdV equation (known as "inverse scattering method") and discovered infinite number of conservation laws of the equation. Their works laid down the foundation for the field of integrable systems. We refer to the excellent book "Solitons, Nonlinear Evolution Equations and Inverse Scattering" by Ablowitz and Clarkson [AC] for the details. Galaktionov and Svirshschevskii [GS] gave an invariantsubspace approach to nonlinear partial differential equations. 
On the other hand, Gel'fand, Dikii and Dorfam [GDi1, GDi2, GDo1-GDo3] introduced in 1970s a theory of Hamiltonian operators in order to study the integrability of nonlinear evolution partial differential equations (also cf. [Mf]). Our first experience with partial differential equation was in the works [X1, X2, X4-X6] on the structure of Hamiltonian operators and their supersymmetric generalizations. In particular, we [X5] proved that vertex algebras are equivalent to linear Hamiltonian operators as mathematical structures. In this book, we are going to solve partial differential equations directly based on the algebraic characteristics of individual equations. The tools we have employed are: the grading technique from representation theory, the Campbell-Hausdorff-type factorization of exponential differential operators, Fourier expansions, matrix differential operators, stable-range of nonlinear terms, generalized power series method, moving frames, classical special functions in one variable and new multi-variable special functions found by us, asymmetric conditions, symmetry transformations and linearization techniques etc. The solved partial differential equations are: flag partial differential equations (including constant-coefficient linear equations), the Calogero-Sutherland model of quantum manybody system in one-dimension, the Maxwell equations, the free Dirac equations, the generalized acoustic system, the Kortweg and de Vries (KdV) equation, the Kadomtsev and Petviashvili (KP) equation, the equation of transonic gas flows, the short-wave equation, the Khokhlov and Zabolotskaya equation in nonlinear acoustics, the equation of geopotential forecast, the nonlinear Schrödinger equation and coupled nonlinear Schrödinger equations in optics, the Davey and Stewartson equations of three-dimensional packets of surface waves, the equation of the dynamic convection in a sea, the Boussinesq equations in geophysics, the Navier-Stokes equations and the classical boundary layer equations.

The book consists of two parts. The first part is about basic algebraic techniques of solving ordinary differential equations and a brief introduction to special functions, most of which are solutions of certain ordinary differential equations. This part serves as a preparation for later solving partial differential equations. It also makes the book accessible to the larger audience, who may even not know what differential equation is about but have the basic knowledge in calculus and linear algebra. The second part is our main context, which consists of linear partial differential equations, nonlinear scalar partial differential equations and systems of nonlinear partial differential equations. Below we give chapter-by-chapter detailed introductions.

In Chapter 1, we start with first-order linear ordinary differential equations, and then turn to first-order separable equations, homogenous equations and exact equations. Next we present the methods of solving more special first-order ordinary differential equations such as: the Bernoulli equations, the Darboux equations, the Riccati equations, the Abel equations and the Clairaut's equations.

Chapter 2 begins with solving homogeneous linear ordinary differential equations with 
constant coefficients by characteristic equations. Then we solve the Euler equations and exact equations. Moreover, the method of undetermined coefficients for solving nonhomogeneous linear ordinary differential equations is presented. Furthermore, we give the method of variation of parameters for solving second-order nonhomogeneous linear ordinary differential equations. In addition, we introduce the power series method to solve variable-coefficient linear ordinary differential equations and study the Bessel equation in detail.

Special functions are important objects both in mathematics and physics. The problem of finding a function of continuous variable $x$ that equals $n$ ! when $x=n$ is a positive integer, was suggested by Bernoulli and Goldbach, and was investigated by Euler in the late 1720s. In Chapter 3, we first introduce the gamma function $\Gamma(z)$, as a continuous generalization of $n$ !. Then we prove the following identities: (1) the beta function $B(x, y)=$ $\int_{0}^{1} t^{x-1}(1-t)^{y-1} d=\Gamma(x) \Gamma(y) / \Gamma(x+y) ;(2)$ Euler's reflection formula $\Gamma(z) \Gamma(1-z)=$ $\pi / \sin \pi z ;(3)$ the product formula

$$
\Gamma(z) \Gamma\left(z+\frac{1}{n}\right) \Gamma\left(z+\frac{2}{n}\right) \cdots \Gamma\left(z+\frac{n-1}{n}\right)=\frac{(2 \pi)^{(n-1) / 2}}{n^{n z-1 / 2}} \Gamma(n z) .
$$

In his thesis presented at Göttingen in 1812, Gauss discovered the one-variable function ${ }_{2} F_{1}(\alpha, \beta ; \gamma ; z)$. We introduce it in Chapter 3 as the power series solution of the Gauss hypergeometric equation

$$
z(1-z) y^{\prime \prime}+[\gamma-(\alpha+\beta+1) z] y^{\prime}-\alpha \beta y=0
$$

and prove the Euler's integral representation

$$
{ }_{2} F_{1}(\alpha, \beta ; \gamma ; z)=\frac{\Gamma(\gamma)}{\Gamma(\beta) \Gamma(\gamma-\beta)} \int_{0}^{1} t^{\beta-1}(1-t)^{\gamma-\beta-1}(1-z t)^{-\alpha} d t .
$$

Moreover, Jacobi polynomials are introduced from the finite-sum cases of the Gauss hypergeometric function and their orthogonality is proved. Legendre orthogonal polynomials are discussed in detail.

Weierstrass's elliptic function $\wp(z)$ is a double-periodic function with second-order poles, satisfying the nonlinear ordinary differential equation

$$
\wp^{\prime 2}(z)=4 \wp^{3}(z)-g_{2} \wp(z)-g_{3},
$$

whose consequence

$$
\wp^{\prime \prime}(z)=6 \wp^{2}(z)-\frac{g_{2}}{2}
$$

will be used later for solving nonlinear partial differential equations. Weierstrass's zeta function $\zeta(z)$ is an integral of $-\wp(z)$, that is, $\zeta^{\prime}(z)=-\wp(z)$. Moreover, Weierstrass's sigma function $\sigma(z)$ satisfies $\sigma^{\prime}(z) / \sigma(z)=\zeta(z)$. We discuss these functions and their properties in Chapter 3 to a certain depth. 
Finally in Chapter 3, we present Jacobi's elliptic functions sn $(z \mid m)$, cn $(z \mid m)$ and $\operatorname{dn}(z \mid m)$, and derive the nonlinear ordinary differential equations satisfied by them. These functions are also very useful in solving nonlinear partial differential equations.

Chapter 4 to Chapter 10 are the main contexts of this book. First in Chapter 4 . we derive the commonly used method of characteristic lines for solving first-order quasilinear partial differential equations, including boundary-value problems. Then we talk about more sophisticated method of characteristic strip for solving nonlinear first-order of partial differential equations. Exact first-order partial differential equations are also handled.

A partial differential equation of flag type is the linear differential equation of the form:

$$
\left(d_{1}+f_{1} d_{2}+f_{2} d_{3}+\cdots+f_{n-1} d_{n}\right)(u)=0
$$

where $d_{1}, d_{2}, \ldots, d_{n}$ are certain commuting locally nilpotent differential operators on the polynomial algebra $\mathbb{R}\left[x_{1}, x_{2}, \ldots, x_{n}\right]$ and $f_{1}, \ldots, f_{n-1}$ are polynomials satisfying

$$
d_{l}\left(f_{j}\right)=0 \quad \text { if } l>j
$$

Many variable-coefficient (generalized) Laplace equations, wave equations, Klein-Gordon equations, Helmholtz equations are equivalent to the equations of this type. A general equation of this type can not be solved by separation of variables. Flag partial differential equations also naturally appear in the representation theory of Lie algebras, in which the complete set of polynomial solutions is crucial in determining the structure of many natural representations. We use the grading technique from representation theory to solve flag partial differential equations and find the complete set of polynomial solutions. Our method also leads us to obtain the solution of initial-value problem of the following type of equations:

$$
\left(\partial_{x_{1}}^{m}-\sum_{r=1}^{m} \partial_{x_{1}}^{m-r} f_{r}\left(\partial_{x_{2}}, \ldots, \partial_{x_{n}}\right)\right)(u)=0,
$$

where $m$ and $n>1$ are positive integers, and

$$
f_{r}\left(\partial_{x_{2}}, \ldots, \partial_{x_{n}}\right) \in \mathbb{R}\left[\partial_{x_{2}}, \ldots, \partial_{x_{n}}\right] .
$$

It turns out that the following family of new special functions

$$
\mathcal{Y}_{\ell}\left(y_{1}, \ldots, y_{m}\right)=\sum_{\iota_{1}, \ldots, \iota_{m}=0}^{\infty}\left(\begin{array}{c}
\iota_{1}+\cdots+\iota_{m} \\
\iota_{1}, \ldots, \iota_{m}
\end{array}\right) \frac{y_{1}^{\iota_{1}} y_{2}^{\iota_{2}} \cdots y_{m}^{\iota_{m}}}{\left(\ell+\sum_{s=1}^{m} s \iota_{s}\right) !}
$$

play the key roles, where $\ell$ is a nonnegative integer. In the case when all $f_{r}=1$, we get that the functions

$$
\varphi_{r}(x)=x^{r} \mathcal{Y}_{r}\left(b_{1} x, b_{2} x^{2}, \ldots, b_{m} x^{m}\right) \text { with } r=0,1, \ldots, m-1
$$


form a fundamental set of solutions for the ordinary differential equation

$$
y^{(m)}-b_{1} y^{(m-1)}-\cdots-b_{m-1} y^{\prime}-b_{m}=0 .
$$

These results are taken from our work [X11].

Barros-Neto and Gel'fand [BG1,BG2] $(1998,2002)$ studied solutions of the equation

$$
u_{x x}+x u_{y y}=\delta\left(x-x_{0}, y-y_{0}\right)
$$

related to the Tricomi operator $\partial_{x}^{2}+x \partial_{y}^{2}$. A natural generalization of the Tricomi operator is $\partial_{x_{1}}^{2}+x_{1} \partial_{x_{2}}^{2}+\cdots+x_{n-1} \partial_{x_{n}}^{2}$. As pointed out in [BG1, BG2], the Tricomi operator is an analogue of the Laplace operator. So the equation

$$
u_{t}=u_{x_{1} x_{1}}+x_{1} u_{x_{2} x_{2}}+\cdots+x_{n-1} u_{x_{n} x_{n}}
$$

is a natural analogue of heat conduction equation. In Chapter 4, we use the method of characteristic lines to prove a Campbell-Hausdorff-type factorization of exponential differential operators and then solve the initial-value problem of the following more general evolution equation

$$
u_{t}=\left(\partial_{x_{1}}^{m_{1}}+x_{1} \partial_{x_{2}}^{m_{2}}+\cdots+x_{n-1} \partial_{x_{n}}^{m_{n}}\right)(u)
$$

by Fourier expansions. Indeed we have solved analogous more general equations related to tree diagrams. We also use the Campbell-Hausdorff-type factorization to solve the initial-value problem of analogous non-evolution flag partial partial differential equations. The results are due to our work [X7].

The Calogero-Sutherland model is an exactly solvable quantum many-body system in one-dimension (cf. [Cf], [Sb]), whose Hamiltonian is given by

$$
H_{C S}=\sum_{\iota=1}^{n} \partial_{x_{\iota}}^{2}+K \sum_{1 \leq p<q \leq n} \frac{1}{\sinh ^{2}\left(x_{p}-x_{q}\right)},
$$

where $K$ is a constant. The model was used to study long-range interactions of $n$ particles. Solving the model is equivalent to find eigenfunctions and their corresponding eigenvalues of the Hamiltonian $H_{C S}$ as a differential operator. We prove in Chapter 4 that the function

$$
e^{2 \mu_{1}\left(x_{1}+\cdots+x_{n}\right)}\left[\prod_{1 \leq p<q \leq n}\left(e^{2 x_{p}}-e^{2 x_{q}}\right)\right]^{\mu_{2}}
$$

is a solution of the Calogero-Sutherland model for any real numbers $\mu_{1}$ and $\mu_{2}$. If $n=2$, we find a connection between the Calogero-Sutherland model and the Gauss hypergeometric function. When $n>2$, a new class of multi-variable hypergeometric functions are found based on Etingof's work [Ep]. The results are taken from our work [X9]. Finally in Chapter 4, we use matrix differential operators and Fourier expansions to solve the 
Maxwell equations, the free Dirac equations and the generalized acoustic system. The results come from our work [X10].

Chapter 5 deals with nonlinear scalar (one dependent variable) partial differential equations. First we do symmetry analysis on the KdV equation (0.10), and obtain the Galilean boost $G_{c}(u(t, x))=u(t, x+c t)-c / 6$ for $c \in \mathbb{R}$. Solving the stationary equation $6 u u_{x}+u_{x x x}=0$ and using the Galilean boost $G_{c}$, we get the traveling-wave solutions of the $\mathrm{KdV}$ equation in terms of the functions $\wp(z), \tan ^{2} z, \operatorname{coth}^{2} z$ and $\mathrm{cn}^{2}(z \mid m)$, respectively. In particular, the soliton solution is obtained by taking $\lim _{m \rightarrow 1}$ of a special case of the last solution. Moreover, we derive the Hirota bilinear presentation of the KdV equation and use it to find the two-soliton solution.

The Kadomtsev and Petviashvili (KP) equation

$$
\left(u_{t}+6 u u_{x}+u_{x x x}\right)_{x}+3 \epsilon u_{y y}=0
$$

with $\epsilon= \pm 1$ is used to describe the evolution of long water waves of small amplitude if they are weakly two-dimensional (cf. [KP]). The choice of $\epsilon$ depends on the relevant magnitude of gravity and surface tension. The equation has also been proposed as a model for surface waves and internal waves in straits or channels of varying depth and width. The KP equation can be viewed as an extension of the KdV equation (0.10). In Chapter 5, we have done the symmetry analysis on the KP equation, and it possesses the following important symmetry transformation

$$
T_{3, \alpha}(u(t, x, y))=u\left(t, x-\epsilon \alpha^{\prime} y / 6, y+\alpha\right)+\epsilon\left(2 \alpha^{\prime \prime} y-\alpha^{\prime 2}\right) / 72,
$$

where $\alpha$ is any second-order differentiable equation in $t$. Any solution of the KdV equation is obviously a solution of the KP equation, and the above transformation $T_{3, \alpha}$ maps such a solution independent of $y$ to a more sophisticated solution of the KP equation that depends on $y$. However, not all the interesting solutions of the KP equation are obtained in this way. In fact, we solve the KP equation for solutions that are polynomial in $x$, and obtain many solutions that can not be obtained from the solutions of the KdV equation; for instance, we have the solution

$$
u=-\frac{\epsilon}{2}\left(x-\epsilon \alpha^{\prime} y / 6+\beta\right)^{2} \wp(y+\alpha)+\frac{2 \alpha^{\prime \prime} y-\alpha^{\prime 2}}{72 \epsilon}-\frac{\beta^{\prime}}{6},
$$

where $\alpha$ and $\beta$ are any functions in $t$ with the above indicated differentiability. Furthermore, we find the Hirota bilinear presentation of the KP equation and get the following lump solution of the KP equation:

$$
u=2 \partial_{x}^{2} \ln \left(\left(x-c y+3 \epsilon\left(b-c^{2}\right) t+a\right)^{2}+b(y+6 \epsilon c t)^{2}-\epsilon / b^{2}\right),
$$

where $a, b, c \in \mathbb{R}$ and $b \neq 0$. The above results in Chapter 5 are well-known (e.g., cf. $[\mathrm{AC}])$ and we reformulate them here just for pedagogic purpose. 
Lin, Reisner and Tsien [LRT] (1948) found the equation

$$
2 u_{t x}+u_{x} u_{x x}-u_{y y}=0
$$

for two-dimensional non-steady motion of a slender body in a compressible fluid, which was later called the "equation of transonic gas flows" (cf. [Me1]). We derive in Chapter 5 the symmetry transformations of the above equation. Using the stable range of the nonlinear term $u_{x} u_{x x}$ and generalized power series method, we find a family of singular solutions with seven arbitrary parameter functions in $t$ and a family of analytic solutions with six arbitrary parameter functions in $t$. For instance, we have the solution

$$
u=\frac{\left(x+\beta^{\prime} y+\alpha\right)^{3}}{3(y-\beta)^{2}}+\left({\beta^{\prime}}^{2}-2 \alpha^{\prime}\right) x+2\left(\beta^{\prime} \beta^{\prime \prime}-\alpha^{\prime \prime}\right) y^{2}-2 \beta^{\prime \prime} x y-\frac{2 \beta^{\prime \prime}}{3} y^{3}+\mu
$$

which blows up on a moving line $y=\beta$, where $\alpha, \beta$ and $\mu$ are any functions in $t$ with the above indicated differentiability. Such a solution may reflect the phenomenon of abrupt high-speed wind. The results are due to our work [X8].

Khristianovich and Rizhov [KR] (1958) discovered the equations of short waves:

$$
u_{y}-2 v_{t}-2(v-x) v_{x}-2 k v=0, \quad v_{y}+u_{x}=0
$$

in connection with the nonlinear reflection of weak shock waves, where $k$ is a real constant. Khokhlov and Zabolotskaya [KZ] (1969) found the equation

$$
2 u_{t x}+\left(u u_{x}\right)_{x}-u_{y y}=0
$$

for quasi-plane waves in nonlinear acoustics of bounded bundles. More specifically, the equation describes the propagation of a diffraction sound beam in a nonlinear medium. The solutions of the above equations similar to those of the equation (0.32) are derived in Chapter 5 based on our work [X13].

In a book on short term weather forecast [Kt], Kibel' (1954) used the partial differential equation

$$
\left(H_{x x}+H_{y y}\right)_{t}+H_{x}\left(H_{x x}+H_{y y}\right)_{y}-H_{y}\left(H_{x x}+H_{y y}\right)_{x}=k H_{x}
$$

for geopotential forecast on a middle level in earth sciences, where $k$ is a real constant. The symmetry transformations and two new families of exact solutions with multiple parameter functions of the above equation are derived in Chapter 5. The results are newly obtained by us.

In Chapter 6, we solve the two-dimensional cubic nonlinear Schrödinger equation (0.3) and the coupled two-dimensional cubic nonlinear Schrödinger equations (0.4) and (0.5) by imposing a quadratic condition on the related argument functions and using their symmetry transformations. More complete families of exact solutions of such type are 
obtained. The soliton solutions are included. Many of them are the periodic, quasiperiodic, aperiodic and singular solutions that may have practical significance. This was our work [14].

Davey and Stewartson [DS] (1974) used the method of multiple scales to derive the following system of nonlinear partial differential equations

$$
\begin{gathered}
2 i u_{t}+\epsilon_{1} u_{x x}+u_{y y}-2 \epsilon_{2}|u|^{2} u-2 u v=0, \\
v_{x x}-\epsilon_{1}\left(v_{y y}+2\left(|u|^{2}\right)_{x x}\right)=0
\end{gathered}
$$

that describe the long time evolution of three-dimensional packets of surface waves, where $u$ is a complex-valued function, $v$ is a real valued function and $\epsilon_{1}, \epsilon_{2}= \pm 1$. In Chapter 6 , we also apply the above quadratic-argument approach to the Davey-Stewartson equations and obtain four large families of solutions, including the soliton solution. This part is a revision of our earlier preprint [X18].

Both the atmospheric and oceanic flows are influenced by the rotation of the earth. In fact, the fast rotation and small aspect ratio are two main characteristics of the large scale atmospheric and oceanic flows. The small aspect ratio characteristic leads to the primitive equations, and the fast rotation leads to the quasi-geostropic equations. A main objective in climate dynamics and in geophysical fluid dynamics is to understand and predict the periodic, quasi-periodic, aperiodic, and fully turbulent characteristics of the large scale atmospheric and oceanic flows. The general model of atmospheric and oceanic flows is very complicated.

Ovsiannikov (1967) introduced the following equations in geophysics:

$$
\begin{gathered}
u_{x}+v_{y}+w_{z}=0, \quad \rho=p_{z}, \\
\rho_{t}+u \rho_{x}+v \rho_{y}+w \rho_{z}=0, \\
u_{t}+u u_{x}+v u_{y}+w u_{z}+v=-\frac{1}{\rho} p_{x}, \\
v_{t}+u v_{x}+v v_{y}+w v_{z}-u=-\frac{1}{\rho} p_{y}
\end{gathered}
$$

to describe the dynamic convection in a sea, where $u, v$ and $w$ are components of velocity vector of relative motion of fluid in Cartesian coordinates $(x, y, z), \rho=\rho(x, y, z, t)$ is the density of fluid and $p$ is the pressure (e.g., cf. Page 203 in [In3]). Moreover, he determined the Lie point symmetries of the above equations and found two very special solutions. In Chapter 7, we give intuitive derivation of the symmetry transformations of the above equations and solve them by the moving line, cylindrical product and dimension reduction. This chapter is a revision of our earlier preprint [X17]. 
The two-dimensional Boussinesq equations for the incompressible fluid in geophyics are

$$
\begin{gathered}
u_{t}+u u_{x}+v u_{y}-\nu \Delta u=-p_{x}, \quad v_{t}+u v_{x}+v v_{y}-\nu \Delta v-\theta=-p_{y}, \\
\theta_{t}+u \theta_{x}+v \theta_{y}-\kappa \Delta \theta=0, \quad u_{x}+v_{y}=0,
\end{gathered}
$$

where $(u, v)$ is the velocity vector field, $p$ is the scalar pressure, $\theta$ is the scalar temperature, $\nu \geq 0$ is the viscosity and $\kappa \geq 0$ is the thermal diffusivity. The above system is a simple model in atmospheric sciences (e.g., cf. [Ma], [Cd]). By imposing asymmetric conditions with respect to the spacial variables $x, y$ and using moving frame, we find four families of multi-parameter solutions of the above Boussinesq equations in Chapter 8.

Another slightly simplified version of the system of primitive equations in geophysics is the three-dimensional stratified rotating Boussinesq system (e.g., cf. [LTW1], [LTW2], [Ma], [HMW]):

$$
\begin{gathered}
u_{t}+u u_{x}+v u_{y}+w u_{z}-\frac{1}{R_{0}} v=\sigma\left(\Delta u-p_{x}\right), \\
v_{t}+u v_{x}+v v_{y}+w v_{z}+\frac{1}{R_{0}} u=\sigma\left(\Delta v-p_{y}\right), \\
w_{t}+u w_{x}+v w_{y}+w w_{z}-\sigma R T=\sigma\left(\Delta w-p_{z}\right), \\
T_{t}+u T_{x}+v T_{y}+w T_{z}=\Delta T+w, \\
u_{x}+v_{y}+w_{z}=0,
\end{gathered}
$$

where $(u, v, w)$ is the velocity vector filed, $T$ is the temperature function, $p$ is the pressure function, $\sigma$ is the Prandtle number, $R$ is the thermal Rayleigh number and $R_{0}$ is the Rossby number. Moreover, the vector $\left(1 / R_{0}\right)(-v, u, 0)$ represents the Coriolis force and the term $w$ in (0.48) is derived using stratification. By the similar method of solving the two-dimensional equations, we derive in Chapter 8 five classes of multi-parameter solutions of the equations (0.45)-(0.49). The results in Chapter 8 are reformulations of those in our work [X16].

In Chapter 9, we introduce a method of imposing asymmetric conditions on the velocity vector with respect to independent spacial variables and a method of moving frame for solving the three dimensional Navier-Stokes equations (0.6)-(0.9). Seven families of nonsteady rotating asymmetric solutions with various parameters are obtained. In particular, one family of solutions blow up on a moving plane, which may be used to study abrupt high-speed rotating flows. Using Fourier expansion and two families of our solutions, one can obtain discontinuous solutions that may be useful in study of shock waves. Another family of solutions are partially cylindrical invariant, containing two parameter functions in $t$, which may be used to describe incompressible fluid in a nozzle. Most of our solutions 
are globally analytic with respect to spacial variables. The results are due to our work $[\mathrm{X} 12]$.

In 1904, Prandtl observed that in the flow of slightly viscous fluid past bodies, the frictional effects are confined to a thin layer of fluid adjacent to the surface of the body. Moreover, he showed that the motion of a small amount of fluid in this boundary layer decides such important matters as the frictional drag, heat transfer, and transfer of momentum between the body and the fluid. The two-dimensional classical non-steady boundary layer equations

$$
\begin{gathered}
u_{t}+u u_{x}+v u_{y}+p_{x}=u_{y y}, \\
p_{y}=0, \quad u_{x}+v_{y}=0
\end{gathered}
$$

are used to describe the motion of a flat plate with the incident flow parallel to the plate and directed to along the $x$-axis in the Cartesian coordinates $(x, y)$, where $u$ and $v$ are the longitudinal and the transverse components of the velocity, and $p$ is the pressure (e.g., cf. [In3]). The three-dimensional classical non-steady boundary layer equations are:

$$
\begin{gathered}
u_{t}+u u_{x}+v u_{y}+w u_{z}=-\frac{1}{\rho} p_{x}+\nu u_{y y}, \\
w_{t}+u w_{x}+v w_{y}+w w_{z}=-\frac{1}{\rho} p_{z}+\nu w_{y y}, \\
p_{y}=0, \quad u_{x}+v_{y}+w_{z}=0,
\end{gathered}
$$

where $(u, v, w)$ denotes the velocity vector, $p$ stands for the pressure, $\rho$ is the density constant and $\nu$ is the coefficient constant of the kinematic viscosity (e.g., cf. [In3]).

In Chapter 10, we introduce various schemes with multiple parameter functions to solve these equations and obtain many families of new explicit exact solutions with multiple parameter functions. Moreover, symmetry transformations are used to simplify our arguments. The technique of moving frame is applied in the three-dimensional case in order to capture the rotational properties of the fluid. In particular, we obtain a family of solutions singular on any moving surface, which may be used to study abrupt high-speed rotating flows. Many other solutions are analytic related to trigonometric and hyperbolic functions, which reflect various wave characteristics of the fluid. Our solutions may also help engineers to develop more effective algorithms to find physical numeric solutions to practical models. The results are taken from our work [X15]. Note that most of the nonlinear partial differential equations in this book are from fluid dynamics. Our results show that algebraically, partial differential equations of hyperbolic type are easier than those of elliptic type in terms of exact solutions. The research in this book was partly supported by the National Natural Science Foundation of China (Grant No. 11171324). 


\section{Conventions}

$\mathbb{C}$ : the field of complex numbers.

$\overline{l, l+k}:\{l, l+1, i=l+2, \ldots, l+k\}$, an index set.

$\delta_{l, j}=1$ if $l=j$, and 0 if $l \neq j$.

$\mathbb{Z}$ : the ring of integers.

$\mathbb{N}:\{0,1,2,3, \ldots\}$, the set of nonnegative integers

$i=\sqrt{-1}$ : the imaginary number.

$\mathbb{R}$ : the field of real numbers.

$\partial_{x}$ : the operator of taking partial derivative with respect to $x$.

- We assume that all partial differential derivatives can change orders.

- We use prime' to denote the derivative of a one-variable function.

- When an expression appears, we always assume the conditions that it makes sense; e.g., $\sqrt{a-b} \Longrightarrow a \geq b$ if $a, b \in \mathbb{R}$. 


\section{Part I}

\section{Ordinary Differential Equations}





\section{Chapter 1}

\section{First-Order Ordinary Differential Equations}

In this chapter, we start with first-order linear ordinary differential equations, and then turn to first-order separable equations, homogenous equations and exact equations. Next we present the methods of solving more special first-order ordinary differential equations such as: the Bernoulli equations, the Darboux equations, the Riccati equations, the Abel equations and the Clairaut's equations.

\subsection{Basics}

In this section, we deal with first-order linear ordinary differential equations, separable equations, homogenous equations and exact equations.

Let $y$ be a function of $t$. We use $y^{\prime}=d y / d t$. A first-order linear ordinary differential equation is written as

$$
y^{\prime}+f(t) y=g(t)
$$

To solve the equation, we multiply the integrating factor $e^{\int f(t) d t}$ to the equation:

$$
y^{\prime} e^{\int f(t) d t}+f(t) y e^{\int f(t) d t}=g(t) e^{\int f(t) d t},
$$

which can be rewritten as

$$
\left(y e^{\int f(t) d t}\right)^{\prime}=g(t) e^{\int f(t) d t} .
$$

Thus

$$
y e^{\int f(t) d t}=\int g(t) e^{\int f(t) d t} d t+c,
$$

where $c$ is an arbitrary constant. So we obtain the general solution

$$
y=e^{-\int f(t) d t}\left[\int g(t) e^{\int f(t) d t} d t+c\right] .
$$


Example 1.1.1. Solve the following initial-value problem:

$$
t y^{\prime}+2 y=4 t^{2}, \quad y(1)=2
$$

Solution. Rewrite the equation in the standard form:

$$
y^{\prime}+\frac{2}{t} y=4 t
$$

Then $f(t)=2 / t$ and $g(t)=4 t$. We calculate

$$
e^{\int f(t) d t}=e^{\int(2 / t) d t} \stackrel{\text { choose }}{=} e^{2 \ln |t|}=e^{\ln t^{2}}=t^{2} .
$$

Thus the general solution is:

$$
y=\frac{\int 4 t \cdot t^{2} d t+c}{t^{2}}=\frac{t^{4}+c}{t^{2}}=t^{2}+c t^{-2}
$$

The initial condition $y(1)=2$ implies

$$
2=1+c \Longrightarrow c=1
$$

The final solution is:

$$
y=t^{2}+t^{-2}
$$

A first-order separable ordinary differential equation is written as $y^{\prime}=f(t) g(y)$. The general solution is given by

$$
\int \frac{1}{g(y)} d y=\int f(t) d t+c
$$

Example 1.1.2. Solve

$$
y^{\prime}=\frac{t y^{3}}{\sqrt{1+t^{2}}}, \quad y(0)=1 .
$$

Solution. We rewrite the equation as

$$
\frac{2 d y}{y^{3}}=\frac{2 t d t}{\sqrt{1+t^{2}}}
$$

So

$$
-\int \frac{2 d y}{y^{3}}=-\int \frac{2 t d t}{\sqrt{1+t^{2}}} \Longrightarrow \frac{1}{y^{2}}=c-2 \sqrt{1+t^{2}} \Longrightarrow y= \pm \frac{1}{\sqrt{c-2 \sqrt{1+t^{2}}}}
$$

Since $y(0)=1$, we choose positive sign and have

$$
1=\frac{1}{\sqrt{c-2}} \Longrightarrow c=3
$$


Thus the final solution is

$$
y=\frac{1}{\sqrt{3-2 \sqrt{1+t^{2}}}}
$$

A first-order homogeneous ordinary differential equation is written as $y^{\prime}=f(y / t)$. To solve it, we change variable $u(t)=y(t) / t$. Then

$$
y=t u \Longrightarrow y^{\prime}=u+t u^{\prime}
$$

Thus the equation $y^{\prime}=f(y / t)$ can be rewritten as

$$
u+t u^{\prime}=f(u) \Longrightarrow u^{\prime}=\frac{f(u)-u}{t},
$$

which is a separable equation.

Example 1.1.3. Find the general solution of the following homogeneous equation:

$$
y^{\prime}=\frac{2 y^{2}-3 t^{2}}{t y}
$$

Solution. Rewrite

$$
y^{\prime}=\frac{2(y / t)^{2}-3}{y / t}
$$

By changing variable $u(t)=y(t) / t$, we get

$$
u+t u^{\prime}=\frac{2 u^{2}-3}{u} \Longrightarrow t u^{\prime}=\frac{2 u^{2}-3}{u}-u=\frac{u^{2}-3}{u} .
$$

Thus

$$
\frac{u d u}{u^{2}-3}=\frac{d t}{t} \Longrightarrow \int \frac{2 u d u}{u^{2}-3}=\int \frac{2 d t}{t} \Longrightarrow \ln \left|u^{2}-3\right|=\ln t^{2}+c_{1}
$$

So

$$
u^{2}-3=c t^{2} \Longrightarrow u^{2}=3+c t^{2}
$$

Hence

$$
\left(\frac{y}{t}\right)^{2}=3+c t^{2} \Longrightarrow y^{2}=3 t^{2}+c t^{4}
$$

Example 1.1.4. Solve the following equation

$$
y^{\prime}=\frac{t+y-2}{t-y+4}
$$

Solution. In order to change the above equation to a homogeneous equation, we change variable

$$
\left\{\begin{array}{l}
T=t+k \\
Y=y+l
\end{array}\right.
$$


where $k$ and $l$ are constants to be determined. Since

$$
\frac{t+y-2}{t-y+4}=\frac{T+Y-k-l-2}{T-Y-k+l+4}
$$

we let

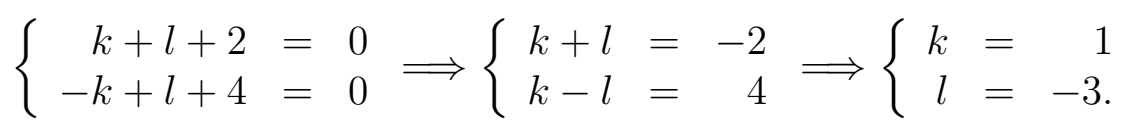

Hence

$$
\left\{\begin{array}{l}
T=t+1 \\
Y=y-3
\end{array}\right.
$$

The original equation changes to

$$
\frac{d Y}{d T}=\frac{T+Y}{T-Y}=\frac{1+\frac{Y}{T}}{1-\frac{Y}{T}}
$$

Let

So

$$
u=\frac{Y}{T} \Longrightarrow \frac{d Y}{d T}=u+u^{\prime} T \text {. }
$$

$$
\begin{gathered}
u+T u^{\prime}=\frac{1+u}{1-u} \Longrightarrow u^{\prime} T=\frac{1+u}{1-u}-u=\frac{1+u^{2}}{1-u} \\
\Longrightarrow \frac{1-u}{1+u^{2}} d u=\frac{d T}{T} \Longrightarrow \int \frac{1-u}{1+u^{2}} d u=\int \frac{d T}{T} \\
\Longrightarrow \arctan u-\frac{1}{2} \ln \left(1+u^{2}\right)=\ln |T|+c_{1} .
\end{gathered}
$$

Thus

$$
\frac{e^{\arctan u}}{\sqrt{1+u^{2}}}=c_{2} T
$$

equivalently,

$$
\begin{gathered}
e^{\arctan u}=c_{2} T \sqrt{1+u^{2}} \Longrightarrow e^{\arctan \frac{Y}{T}}=c_{2} T \sqrt{1+\frac{Y^{2}}{T^{2}}} \\
\Longrightarrow e^{\arctan \frac{Y}{T}}= \pm c_{2} \sqrt{T^{2}+Y^{2}}=c \sqrt{T^{2}+Y^{2}}
\end{gathered}
$$

The final solution is

$$
e^{\arctan \frac{y-3}{t+1}}=c \sqrt{(t+1)^{2}+(y-3)^{2}} .
$$

A first-order exact ordinary differential equation has the form

$$
f(t, y) d t+g(t, y) d y=0, \quad \text { where } \frac{\partial f}{\partial y}=\frac{\partial g}{\partial t} .
$$

In this case, the general solution is $U(t, y)=c$, where $U$ is a function determined from

$$
\frac{\partial U}{\partial t}=f, \quad \frac{\partial U}{\partial y}=g .
$$


Integrating the first equation yields $U=\int f(t, y) d t+\psi(y)$, where $\psi(y)$ is a function to be determined. In fact,

$$
\psi^{\prime}(y)=\frac{\partial U}{\partial y}-\frac{\partial \int f(t, y) d t}{\partial y}=g-\frac{\partial \int f(t, y) d t}{\partial y} .
$$

Example 1.1.5. Solve the following exact equation:

$$
\left(9 t^{2}+y-1\right) d t-(4 y-t) d y=0, \quad y(1)=0 .
$$

Solution. Let

$$
U(t, y)=\int\left(9 t^{2}+y-1\right) d t+\psi(y)=3 t^{3}+(y-1) t+\psi(y)
$$

Taking partial derivative of the above equation with respect to $y$, we have

$$
U_{y}=t+\psi^{\prime}(y)=-(4 y-t)
$$

Thus

$$
\psi^{\prime}(y)=-4 y . \quad \text { Choose } \psi(y)=-2 y^{2} .
$$

So $U=3 t^{3}+(y-1) t-2 y^{2}$ and the general solution is:

$$
3 t^{3}+(y-1) t-2 y^{2}=c
$$

When $y(1)=0$,

$$
3-1=c \Longrightarrow c=2 \text {. }
$$

The final solution is

$$
3 t^{3}+(y-1) t-2 y^{2}=2 .
$$

An integrating factor for the equation $f(t, y) d t+g(t, y) d y=0$ is a function $\mu(t, y)$ such that

$$
\mu(t, y) f(t, y) d t+\mu(t, y) g(t, y) d y=0
$$

is an exact equation, that is,

$$
\frac{\partial(\mu f)}{\partial y}=\frac{\partial(\mu g)}{\partial t} \Longrightarrow g \frac{\partial \mu}{\partial t}-f \frac{\partial \mu}{\partial y}=\left(\frac{\partial f}{\partial y}-\frac{\partial g}{\partial t}\right) \mu \sim g \mu_{t}-f \mu_{y}=\left(f_{y}-g_{t}\right) \mu .
$$

The condition for $\mu$ to be a pure function in $t$ (i.e, $\partial \mu / \partial y=0)$ is $\mu_{t} / \mu=\left(f_{y}-g_{t}\right) / g$ is a pure function in $t$.

Example 1.1.6. Solve the following equation by the method of exact equations and integrating factors:

$$
t\left(t^{2}+y^{2}+1\right) d t+y d y=0, \quad y(0)=2 .
$$


Solution. Note

$$
f=t\left(t^{2}+y^{2}+t\right), \quad g=y
$$

Moreover,

$$
f_{y}=2 t y, \quad g_{t}=0
$$

Since

$$
\frac{f_{y}-g_{t}}{g}=2 t
$$

we look for an integrating factor $\mu(t)$. In this case,

$$
\frac{\mu^{\prime}}{\mu}=2 t \stackrel{\text { choose }}{\Longrightarrow} \mu=e^{t^{2}}
$$

Thus the original equation is equivalent to the following exact equation:

$$
e^{t^{2}} t\left(t^{2}+y^{2}+1\right) d t+e^{t^{2}} y d y=0
$$

Let

$$
\begin{aligned}
U(t, y) & =\int e^{t^{2}} t\left(t^{2}+y^{2}+1\right) d t+\psi(y)=\frac{1}{2} \int e^{t^{2}}\left(t^{2}+y^{2}+1\right) d t^{2}+\psi(y) \\
& =\frac{e^{t^{2}}\left(t^{2}+y^{2}\right)}{2}+\psi(y)
\end{aligned}
$$

Then

$$
U_{y}(t, y)=e^{t^{2}} y+\psi^{\prime}(y)=e^{t^{2}} y \Longrightarrow \psi^{\prime}(y)=0 \Longrightarrow \psi \stackrel{\text { choose }}{=} 0 .
$$

Thus the general solution is:

$$
\frac{e^{t^{2}}\left(t^{2}+y^{2}\right)}{2}=c
$$

Since $y(0)=2$, we have:

$$
c=\frac{2^{2}}{2}=2 .
$$

Therefore, the final solution is:

$$
e^{t^{2}}\left(t^{2}+y^{2}\right)=4
$$

If $\left(f_{y}-g_{t}\right) / f$ is a pure function in $y$, then we have the integrating factor

$$
\mu=\int \frac{g_{t}-f_{y}}{f} d y
$$

Let $\varphi(z)$ be any one-variable function.

$$
\text { If } f=y \varphi(t y), g=t \varphi(t y) \Longrightarrow \mu=\frac{1}{t f-y g} \text {. }
$$




$$
\begin{gathered}
\text { When } \frac{f_{y}-g_{t}}{g-f}=\varphi(t+y) \Longrightarrow \mu=e^{\int \varphi(z) d z}, \quad z=x+y \text {. } \\
\text { If } \frac{f_{y}-g_{t}}{y g-t f}=\varphi(t y) \Longrightarrow \mu=e^{\int \varphi(z) d z}, \quad z=t y \text {. } \\
\text { When } \frac{t^{2}\left(f_{y}-g_{t}\right)}{y g+t f}=\varphi(y / t) \Longrightarrow \mu=e^{-\int \varphi(z) d z}, \quad z=\frac{y}{t} . \\
\text { If } \frac{f_{y}-g_{t}}{t g-y f}=\varphi\left(t^{2}+y^{2}\right) \Longrightarrow \mu=e^{(1 / 2) \int \varphi(z) d z}, \quad z=t^{2}+y^{2} .
\end{gathered}
$$

\section{Excises 1.1.}

1. Solve the equation:

$$
y^{\prime}+y \tan t=t
$$

2. Find the general solution of the equation:

$$
y^{\prime}=\frac{3 t^{2}\left(1+e^{y^{2}}\right)}{2 y\left(1+t^{3}\right)} .
$$

3. Solve the following equation

$$
y^{\prime}=\frac{t+2 y-1}{2 t+3 y+2}
$$

4. Find the general solution of the equation:

$$
y^{\prime}=\frac{3 t^{2}-y^{2}-7}{e^{y}+2 t y+1} .
$$

5. Solve the equation:

$$
\left[3 t^{2} \sin t y+y\left(t^{3}+3 y+1\right) \cos t y\right] d t+\left[3 \sin t y+t\left(t^{3}+3 y+1\right) \cos t y\right] d y=0
$$

\section{$1.2 \quad$ Special Equations}

We present in this section the methods of solving the Bernoulli equations, the Darboux equations, the Riccati equations, the Abel equations and the Clairaut's equations.

A Bernoulli equation has the form

$$
y^{\prime}+f(t) y=g(t) y^{a}, \quad a \neq 0,1 .
$$

Changing variable $u(t)=y^{1-a}$, we get

$$
u^{\prime}=(1-a) y^{-a} y^{\prime} \sim(1-a) y^{\prime}=y^{a} u^{\prime}
$$


and (1.2.1) becomes

$$
y^{a} u^{\prime}+(1-a) f y^{a} u=(1-a) g y^{a} \sim u^{\prime}+(1-a) f u=(1-a) g
$$

Example 1.2.1. Solve the following Bernoulli equation :

$$
y^{\prime}-\frac{1}{t} y=y^{3} \sin t^{3}
$$

Solution. Note that $y \equiv 0$ is an obvious solution.

We assume that $y \not \equiv 0$. Rewrite the equation as:

$$
\frac{y^{\prime}}{y^{3}}-\frac{1}{t y^{2}}=\sin t^{3}
$$

Change variable:

$$
u=\frac{1}{y^{2}} ; \quad u^{\prime}=-\frac{2 y^{\prime}}{y^{3}} .
$$

Thus the original equation is equivalent to:

$$
-\frac{u^{\prime}}{2}-\frac{u}{t}=\sin t^{3}
$$

equivalently,

$$
u^{\prime}+\frac{2}{t} u=-2 \sin t^{3}
$$

We calculate

$$
e^{\int \frac{2}{t} d t \stackrel{\text { choose }}{=}} e^{2 \ln |t|}=e^{\ln t^{2}}=t^{2}
$$

Thus

$$
u=\frac{\int-2 t^{2} \sin t^{3} d t+c}{t^{2}}=\frac{\frac{2}{3} \cos t^{3}+c}{t^{2}}=\frac{2 \cos t^{3}+c_{1}}{3 t^{2}} .
$$

Therefore,

$$
\frac{1}{y^{2}}=\frac{2 \cos t^{3}+c_{1}}{3 t^{2}} \Longrightarrow y= \pm \frac{\sqrt{3} t}{\sqrt{2 \cos t^{3}+c_{1}}}
$$

A Darboux equation can be represented as

$$
\left(f(y / t)+t^{a} h(y / t)\right) y^{\prime}=g(y / t)+y t^{a-1} h(y / t) .
$$

Using the substitution $y(t)=t z(t)$ and taking $z$ to be independent variable, we have

$$
\frac{d y}{d z}=y^{\prime} \frac{d t}{d z}=t+z \frac{d t}{d z} .
$$

So (1.2.12) becomes

$$
\left(f(z)+t^{a} h(z)\right) y^{\prime} \frac{d t}{d z}=\left(g(z)+z h(z) t^{a}\right) \frac{d t}{d z}
$$


equivalently,

$$
\left(f(z)+t^{a} h(z)\right)\left(t+z \frac{d t}{d z}\right)=\left(g(z)+z h(z) t^{a}\right) \frac{d t}{d z} .
$$

Thus

$$
(z f(z)-g(z)) \frac{d t}{d z}+f(z) t=-h(z) t^{a+1},
$$

which is a Bernoulli equation.

A Riccati equation has the general form

$$
y^{\prime}=f_{2}(t) y^{2}+f_{1}(t) y+f_{0}(t)
$$

If $f_{2}=0$, the equation is a linear equation. When $f_{0}=0$, it is a Bernoulli equation. Changing variable

$$
y=-\frac{u^{\prime}(t)}{f_{2}(t) u(t)}
$$

we have

$$
y^{\prime}=\frac{f_{2} u^{\prime 2}+f_{2}^{\prime} u u^{\prime}-f_{2} u u^{\prime \prime}}{f_{2}^{2} u^{2}}
$$

and (1.2.17) becomes

$$
\frac{f_{2} u^{\prime 2}+f_{2}^{\prime} u u^{\prime}-f_{2} u u^{\prime \prime}}{f_{2}^{2} u^{2}}=\frac{u^{\prime 2}}{f_{2} u^{2}}-\frac{f_{1} u^{\prime}}{f_{2} u}+f_{0} \sim u^{\prime \prime}=\left(\frac{f_{2}^{\prime}}{f_{2}}+f_{1}\right) u^{\prime}-f_{0} f_{2} u=0,
$$

which is a second-order linear ordinary equation.

Example 1.2.2. Solve the Riccati equation:

$$
y^{\prime}=e^{t} y^{2}-y+e^{-t}
$$

Solution. Now $f_{2}=e^{t}, f_{1}=-1$ and $f_{0}=e^{-t}$. Changing variable

$$
y(t)=-\frac{e^{-t} u^{\prime}(t)}{u(t)}
$$

we get

$$
u^{\prime \prime}=-u
$$

by (1.2.20). By a later method, the general solution of (1.2.23) is $u=c_{1} \sin \left(t+c_{2}\right)$. Thus the general solution of (1.2.21) is

$$
y=-e^{-t} \cot \left(t+c_{2}\right) .
$$

Suppose that $y=\varphi(t)$ is a particular solution of (1.2.17). Changing variable $y(t)=$ $\varphi(t)+u(t)$, we reduce (1.2.17) to the Bernoulli equation

$$
u^{\prime}=f_{2} u^{2}+\left(f_{1}+2 f_{2} \varphi\right) y .
$$


Example 1.2.3. Solve the Riccati equation:

$$
y^{\prime}=y^{2}+\frac{t \tan t+2}{t} y+\frac{t \tan t+2}{t^{2}} .
$$

Solution. Observe that $y=-1 / t$ is a particular solution of (1.2.26). Changing variable $y(t)=u(t)-1 / t$, we get

$$
u^{\prime}=u^{2}+\tan t u
$$

Set $w=1 / u$. Then $(1.2 .27)$ becomes

$$
w^{\prime}=-1-\tan t w \Longrightarrow w=\left[\frac{1}{2} \ln \frac{1-\sin t}{1+\sin t}+c\right] \cos t .
$$

So

$$
u=\frac{\sec t}{\frac{1}{2} \ln \frac{1-\sin t}{1+\sin t}+c} \Longrightarrow y=\frac{\sec t}{\frac{1}{2} \ln \frac{1-\sin t}{1+\sin t}+c}-\frac{1}{t}
$$

An Abel equation of the first kind has the general form

$$
y^{\prime}=f_{3}(t) y^{3}+f_{2}(t) y^{2}+f_{1}(t) y+f_{0}(t), \quad f_{3}(t) \not \equiv 0 .
$$

The above equation is not integrable for arbitrary $f_{n}(t)$. We only list two interesting special cases:

1. The Abel equation is generalized homogeneous:

$$
y^{\prime}=a t^{2 n+1} y^{3}+b t^{n} y^{2}+c \frac{y}{t}+d t^{-n-2} .
$$

Changing variable $y(t)=u(t) / t^{n+1}$, we obtain

$$
y^{\prime}=\frac{t u^{\prime}-(n+1) u}{t^{n+2}}
$$

and

$$
\frac{t u^{\prime}-(n+1) u}{t^{n+2}}=a \frac{u^{3}}{t^{n+2}}+b \frac{u^{2}}{t^{n+2}}+c \frac{u}{t^{n+2}}+d \frac{1}{t^{n+2}}
$$

equivalently,

$$
t u^{\prime}-(n+1) u=a u^{3}+b u^{2}+c u+d \sim t u^{\prime}=a u^{3}+b u^{2}+(c+n+1) u+d,
$$

which is a separable equation.

2. The Abel equation has the form:

$$
y^{\prime}=a t^{3 n-m} y^{3}+b t^{2 n} y^{2}+\frac{m-n}{t} y+d t^{2 m} .
$$


Changing variable $y(t)=t^{m-n} u(t)$, we obtain

$$
y^{\prime}=t^{m-n} u^{\prime}+(m-n) t^{m-n-1} u
$$

and

$$
t^{m-n} u^{\prime}+(m-n) t^{m-n-1} u=a t^{2 m} u^{3}+b t^{2 m} u^{2}+(m-n) t^{m-n-1} u+d t^{2 m}
$$

equivalently,

$$
t^{-m-n} u^{\prime}=a u^{3}+b u^{2}+d
$$

which is a separable equation.

From the above examples, we can try changing variable $y=g_{1}(t) u(t)+g_{0}(t)$ to reduce the Abel equation to a separable equation, where $g_{0}$ and $g_{1}$ are the functions to be determined.

An Abel equation of the second kind has the general form

$$
(y+g(t)) y^{\prime}=f_{2}(t) y^{2}+f_{1}(t) y+f_{0}(t), \quad g(t) \not \equiv 0 .
$$

Again the above equation is not integrable for arbitrary $f_{n}(t)$. We only list two interesting special cases:

1. The Abel equation of second kind is generalized homogeneous:

$$
\left(y+k t^{n}\right) y^{\prime}=a \frac{y^{2}}{t}+b t^{n-1} y+c t^{2 n-1} .
$$

Changing variable $y(t)=t^{n} u(t)$, we obtain

$$
y^{\prime}=t^{n} u^{\prime}+n t^{n-1} u
$$

and

$$
(u+k) t^{n}\left(t^{n} u^{\prime}+n t^{n-1} u\right)=a t^{2 n-1} u^{2}+b t^{2 n-1} u+c t^{2 n-1}
$$

equivalently,

$$
(u+k)\left(t u^{\prime}+n u\right)=a u^{2}+b u+c \sim t(u+k) u^{\prime}=(a-n) u^{2}+(b-n k) u+c,
$$

which is a separable equation.

2. The Abel equation of second kind has the form:

$$
(y+g(t)) y^{\prime}=f_{2}(t) y^{2}+f_{1}(t) y+f_{1}(t) g(t)-f_{2}(t) g^{2}(t)
$$

Note that $y=-g(t)$ is a solution. Changing variable $y(t)=u(t)-g(t)$, we obtain

$$
u\left(u^{\prime}-g^{\prime}\right)=f_{2}(u-g)^{2}+f_{1}(u-g)+f_{1} g-f_{2} g^{2}
$$


equivalently,

$$
u u^{\prime}=f_{2} u^{2}+\left(f_{1}+g^{\prime}-2 g f_{2}\right) u \sim u^{\prime}=f_{2} u+f_{1}+g^{\prime}-2 g f_{2},
$$

which is a first-order linear equation.

From the above examples, we can again try changing variable $y=g_{1}(t) u(t)+g_{0}(t)$ to reduce the Abel equation of second kind to an integrable equation, where $g_{0}$ and $g_{1}$ are the functions to be determined.

A Clairaut's equation has the general form

$$
f\left(t y^{\prime}-y\right)=g\left(y^{\prime}\right)
$$

Note that the linear function $y=a t-b$ for which $f(b)=g(a)$ is a solution. But the equation has more solutions in general. Differentiating (1.2.47), we get

$$
y^{\prime \prime}\left(t f^{\prime}\left(t y^{\prime}-y\right)-g^{\prime}\left(y^{\prime}\right)\right)=0 \text {. }
$$

Solving the system

$$
f\left(t y^{\prime}-y\right)=g\left(y^{\prime}\right), \quad t f^{\prime}\left(t y^{\prime}-y\right)=g^{\prime}\left(y^{\prime}\right)
$$

by viewing $y$ and $y^{\prime}$ as variables, we get a singular solution of $y$.

Example 1.2.4. Solve the equation

$$
\left(t y^{\prime}-y\right)^{2}-y^{\prime 2}-1=0
$$

Solution. Rewrite the equation as $\left(t y^{\prime}-y\right)^{2}=y^{\prime 2}+1$. Note $f(z)=z^{2}$ and $g(z)=z^{2}+1$. Let

$$
f(b)=g(a) \sim b^{2}=a^{2}+1 \sim b= \pm \sqrt{a^{2}+1} .
$$

So we have the solution

$$
y=a t \pm \sqrt{a^{2}+1}
$$

Now the second equation in (1.2.49) becomes

$$
t\left(t y^{\prime}-y\right)=y^{\prime} \Longrightarrow y^{\prime}=\frac{t y}{t^{2}-1} .
$$

According to $(1.2 .50)$,

$$
\frac{y^{2}}{\left(t^{2}-1\right)^{2}}-\frac{t^{2} y^{2}}{\left(t^{2}-1\right)^{2}}-1=0 \sim y^{2}+t^{2}=1 .
$$

We refer to [PZ] for more exact solutions of ordinary differential equations. 


\section{Exercises 1.2:}

1. Solve the following Bernoulli equation

$$
y^{\prime}-\frac{1}{t} y=2 y^{2} \tan t^{2}
$$

2. Solve the Riccati equation

$$
y^{\prime}=y^{2}+\frac{t \cot t+2}{t} y+\frac{t \cot t+2}{t^{2}} .
$$

3. Solve the Abel equation of the first kind:

$$
y^{\prime}=t^{5} y^{3}+t^{2} y^{2}-2 \frac{y}{t}+\frac{1}{t^{4}} .
$$

4. Solve the Abel equation of the first kind:

$$
y^{\prime}=t^{3} y^{3}-2 t^{4} y^{2}+\frac{y}{t}+t^{6} .
$$

5. Solve the Abel equation of the second kind:

$$
\left(y+5 t^{2}\right) y^{\prime}=5 \frac{y^{2}}{t}+10 t y+t^{3} .
$$

6. Solve the Abel equation of the second kind:

$$
\left(y+e^{t}\right) y^{\prime}=-\frac{y^{2}}{t}+y \sin 2 t+e^{t} \sin 2 t+\frac{e^{2 t}}{t} .
$$




\section{Chapter 2}

\section{Higher-Order Ordinary Differential Equations}

In this chapter, we begin with solving homogeneous linear ordinary differential equations with constant coefficients by characteristic equations. Then we solve the Euler equations and exact equations. Moreover, the method of undetermined coefficients for solving nonhomogeneous linear ordinary differential equations is presented. Furthermore, we give the method of variation of parameters for solving second-order nonhomogeneous linear ordinary differential equations. In addition, we introduce the power series method to solve variable-coefficient linear ordinary differential equations and study the Bessel equation in detail.

\subsection{Basics}

This section deals with homogeneous linear ordinary differential equation with constant coefficients, the Euler equations and exact equations.

A second-order homogeneous linear ordinary differential equation with constant coefficients is of the form

$$
a y^{\prime \prime}+b y^{\prime}+c y=0, \quad a, b, c \in \mathbb{R} .
$$

To find the general solution, we assume that $y=e^{\lambda t}$ is a solution of (2.1.1), where $\lambda$ is a constant to be determined. Substituting it into (2.1.1), we get

$$
a \lambda^{2} e^{\lambda t}+b \lambda e^{\lambda t}+c e^{\lambda t} \sim a \lambda^{2}+b \lambda+c=0
$$

which is called the characteristic equation of (2.1.1). If the above equation has two distinct real roots $\lambda_{1}$ and $\lambda_{2}$, then the general solution of (2.1.1) is

$$
y=c_{1} e^{\lambda_{1} t}+c_{2} e^{\lambda_{2} t}
$$

where $c_{1}$ and $c_{2}$ are arbitrary constants. When (2.1.2) has two complex roots $r_{1} \pm r_{2} i$, then the real part and imaginary part of $e^{\left(r_{1}+r_{2} i\right) t}$ are solutions of (2.1.1). So the general 
solution of $(2.1 .1)$ is

$$
y=c_{1} e^{r_{1} t} \sin r_{2} t+c_{2} e^{r_{1} t} \cos r_{2} t
$$

In the case that (2.1.2) has repeated root $r$, the general solution of (2.1.1) is

$$
y=\left(c_{1}+c_{2} t\right) e^{r t}
$$

Example 2.1.1. The general solution of the equation

$$
y^{\prime \prime}-2 y^{\prime}-3 y=0
$$

is

$$
y=c_{1} e^{3 t}+c_{2} e^{-t}
$$

because $\lambda=3$ and $\lambda=-1$ are real roots of the characteristic equation $\lambda^{2}-2 \lambda-3=0$. Moreover, the general solution of the equation

$$
y^{\prime \prime}-4 y^{\prime}+13 y=0
$$

is

$$
y=c_{1} e^{2 t} \sin 3 t+c_{2} e^{2 t} \cos 3 t
$$

because $\lambda=2+3 i$ and $\lambda=2-3 i$ are roots of the characteristic equation $\lambda^{2}-4 \lambda+13=0$. Furthermore, the general solution of the equation

$$
y^{\prime \prime}+6 y^{\prime}+9 y=0
$$

is

$$
y=\left(c_{1}+c_{2} t\right) e^{-3 t}
$$

In general, the algebraic equation

$$
b_{n} \lambda^{n}+b_{n-1} \lambda^{n-1}+\cdots+b_{0}=0
$$

is called the characteristic equation of the differential equation

$$
b_{n} y^{(n)}+b_{n-1} y^{(n-1)}+\cdots+b_{0} y=0, \quad b_{r} \in \mathbb{R} .
$$

If (2.1.12) has a real root $r$ with multiplicity $m$, then

$$
\left(c_{m-1} t^{m-1}+\cdots+c_{1} t+c_{0}\right) e^{r t}
$$

is a solution of (2.1.13) for arbitrary $c_{0}, c_{1}, \ldots, c_{m-1} \in \mathbb{R}$. When $r_{1}+r_{2} i$ is a complex root of (2.1.12) with multiplicity $m$, then

$$
\left(c_{m-1} t^{m-1}+\cdots+c_{1} t+c_{0}\right) e^{r_{1} t} \sin r_{2} t
$$


and

$$
\left(a_{m-1} t^{m-1}+\cdots+a_{1} t+a_{0}\right) e^{r_{1} t} \cos r_{2} t
$$

are solutions of (2.1.13) for arbitrary $c_{r}, a_{r} \in \mathbb{R}$. For instance, if

$$
(\lambda-1)(\lambda+2)^{3}\left(\lambda^{2}-4 \lambda+13\right)^{2}=0
$$

is the characteristic equation of a differential equation of the form (2.1.13), then the general solution of the differential equation is

$$
y=c_{1} e^{t}+\left(c_{2} t^{2}+c_{3} t+c_{4}\right) e^{-2 t}+\left(c_{5} t+c_{6}\right) e^{2 t} \sin 3 t+\left(c_{7} t+c_{8}\right) e^{2 t} \cos 3 t .
$$

An Euler ordinary differential equation has the general form

$$
b_{n} t^{n} y^{(n)}+b_{n-1} t^{n-1} y^{(n-1)}+\cdots+b_{1} t y^{\prime}+b_{0} y=0, \quad b_{r} \in \mathbb{R} .
$$

We solve it by changing variable $x=\ln t$. In fact,

$$
y^{\prime}=\frac{y_{x}}{t}, \quad y^{\prime \prime}=\frac{y_{x x}-y_{x}}{t^{2}}, \quad y^{\prime \prime \prime}=\frac{y_{x x x}-3 y_{x x}+2 y_{x}}{t^{3}} .
$$

Example 2.1.2. Solve the equation

$$
t^{2} y^{\prime \prime}-3 t y^{\prime}+5 y=0
$$

Solution. Changing variable $x=\ln t$, we get

$$
y_{x x}-y_{x}-3 y_{x}+5 y=0 \sim y_{x x}-4 y_{x}+5 y=0,
$$

whose characteristic equation is $\lambda^{2}-4 \lambda+5=0$. The roots are $\lambda=2 \pm i$. So the general solution is

$$
y=c_{1} e^{2 x} \sin x+c_{2} e^{2 x} \cos x=t^{2}\left(c_{1} \sin \ln t+c_{2} \cos \ln t\right)
$$

Example 2.1.3. Solve the Euler equation

$$
t^{3} y^{\prime \prime \prime}-t^{2} y^{\prime \prime}-2 t y^{\prime}-4 y=0
$$

Solution. Changing variable $x=\ln t$, we get

$$
y_{x x x}-3 y_{x x}+2 y_{x}-\left(y_{x x}-y_{x}\right)-2 y_{x}-4 y=0 \sim y_{x x x}-4 y_{x x}+y_{x}-4 y=0,
$$


whose characteristic equation is

$$
\lambda^{3}-4 \lambda^{2}+\lambda-4=(\lambda-4)\left(\lambda^{2}+1\right)=0
$$

Thus the general solution is

$$
y=c_{1} e^{4 x}+c_{2} \sin x+c_{3} \cos x=c_{1} t^{4}+c_{2} \sin \ln t+c_{3} \cos \ln t
$$

An $n$ th-order ordinary differential equation is called an exact equation if the equation can be rewritten as

$$
\frac{d \Phi\left(t, y, y^{\prime}, \ldots, y^{(n-1)}\right)}{d t}=0 .
$$

We try to find $\Phi$ term by term.

Example 2.1.4. Solve the equation

$$
t y y^{\prime \prime}+t y^{\prime 2}+y y^{\prime}=0
$$

Solution. Note that $\Phi=t y y^{\prime}$. Thus (2.1.29) can rewritten as $\left(t y y^{\prime}\right)^{\prime}=0$. Thus

$$
2 t y y^{\prime}=c_{1} \sim t\left(y^{2}\right)^{\prime}=c_{1} \Longrightarrow y^{2}=c_{1} \ln t+c_{2}
$$

Example 2.1.5. Solve the equation

$$
\left(1+t+t^{2}\right) y^{\prime \prime \prime}+(3+6 t) y^{\prime \prime}+6 y^{\prime}=6 t
$$

Solution. We rewrite $(2.1 .31)$ as

$$
\begin{gathered}
\left(1+t+t^{2}\right) y^{\prime \prime \prime}+(1+2 t) y^{\prime \prime}+(2+4 t) y^{\prime \prime}+6 y^{\prime}-6 t=0 \\
\Longrightarrow\left[\left(1+t+t^{2}\right) y^{\prime \prime}\right]^{\prime}+(2+4 t) y^{\prime \prime}+4 y^{\prime}+2 y^{\prime}-6 t=0 \\
\Longrightarrow\left[\left(1+t+t^{2}\right) y^{\prime \prime}\right]^{\prime}+\left[(2+4 t) y^{\prime}\right]^{\prime}+2 y^{\prime}-6 t=0 \\
\Longrightarrow\left[\left(1+t+t^{2}\right) y^{\prime \prime}\right]^{\prime}+\left[(2+4 t) y^{\prime}\right]^{\prime}+(2 y)^{\prime}-\left(3 t^{2}\right)^{\prime}=0 \\
\Longrightarrow\left[\left(1+t+t^{2}\right) y^{\prime \prime}+(2+4 t) y^{\prime}+2 y-3 t^{2}\right]^{\prime}=0 \\
\Longrightarrow\left(1+t+t^{2}\right) y^{\prime \prime}+(2+4 t) y^{\prime}+2 y-3 t^{2}=2 c_{1} \\
\Longrightarrow\left(1+t+t^{2}\right) y^{\prime \prime}+(1+2 t) y^{\prime}+(1+2 t) y^{\prime}+2 y-3 t^{2}=2 c_{1} \\
\Longrightarrow\left[\left(1+t+t^{2}\right) y^{\prime}+(1+2 t) y-t^{3}\right]^{\prime}=2 c_{1} \\
\Longrightarrow\left(1+t+t^{2}\right) y^{\prime}+(1+2 t) y-t^{3}=2 c_{1} t+c_{2}
\end{gathered}
$$




$$
\begin{array}{r}
\Longrightarrow\left[\left(1+t+t^{2}\right) y\right]^{\prime}-t^{3}=2 c_{1} t+c_{2} \\
\Longrightarrow\left(1+t+t^{2}\right) y-\frac{t^{4}}{4}=c_{1} t^{2}+c_{2} t+c_{3} .
\end{array}
$$

\section{Exercises 2.1}

1. Find the general solution of the equation

$$
y^{\prime \prime}-y^{\prime}-6 y=0 .
$$

2. Find the general solution of the equation

$$
y^{\prime \prime}+6 y^{\prime}+13 y=0 .
$$

3. Find the general solution of the equation

$$
y^{(4)}+8 y^{\prime \prime}+16 y=0 .
$$

4. Solve the Euler equation

$$
t^{3} y^{\prime \prime \prime}+3 t^{2} y^{\prime \prime}-2 t y^{\prime}+2 y=0 .
$$

5. Solve the equation

$$
t y y^{\prime \prime}+3 t y^{\prime} y^{\prime \prime}+2 y y^{\prime \prime}+2 y^{\prime 2}=2 \cos t-t \sin t .
$$

\subsection{Method of Undetermined Coefficients}

In this section, we present the method of undetermined coefficients for solving nonhomogeneous linear ordinary differential equations.

In order to solve linear nonhomogeneous ordinary differential equation

$$
f_{n}(t) y^{(n)}+f_{n-1}(t) y^{(n-1)}+\cdots+f_{1}(t) y=g(t),
$$

we find the general solution $\phi\left(t, c_{1}, \ldots, c_{n}\right)$ of the homogeneous equation

$$
f_{n}(t) y^{(n)}+f_{n-1}(t) y^{(n-1)}+\cdots+f_{1}(t) y=0
$$

and a particular solution $y_{0}(t)$ of (2.2.1). Then the general solution of (2.2.1) is $y=$ $\phi\left(t, c_{1}, \ldots, c_{n}\right)+y_{0}(t)$. It is often that $y_{0}$ is obtained by guessing it of certain form with undetermined coefficients based on the form of $g(t)$.

Example 2.2.1. Find the general solution of the equation

$$
y^{\prime \prime}-\frac{2}{t^{2}} y=7 t^{4}+3 t^{3} .
$$


Solution. It is easy to see that $y=t^{2}$ and $y=1 / t$ are solutions of

$$
y^{\prime \prime}-\frac{2}{t^{2}} y=0
$$

So the general solution of $(2.2 .4)$ is

$$
y=c_{1} t^{2}+\frac{c_{2}}{t}
$$

Based on the form of (2.2.3), we guess a particular solution $y_{0}(t)=a t^{6}+b t^{5}$, where $a$ and $b$ are the constants to be determined. Note

$$
y_{0}^{\prime}=6 a t^{5}+5 b t^{4} \Longrightarrow y_{0}^{\prime \prime}=30 a t^{4}+20 t^{3} .
$$

By (2.2.3),

$$
30 a t^{4}+20 t^{3}-2\left(a t^{4}+b t^{3}\right)=7 t^{4}+3 t^{3} \sim 28 a=7,18 b=3 \Longrightarrow a=\frac{1}{4}, b=\frac{1}{6} .
$$

Thus $y_{0}=t^{6} / 4+t^{5} / 6$. The general solution (2.2.3) is

$$
y=c_{1} t^{2}+\frac{c_{2}}{t}+\frac{t^{6}}{4}+\frac{t^{5}}{6} .
$$

Example 2.2.2. Solve the equation

$$
y^{\prime \prime}+3 y^{\prime}+2 y=3 \sin 2 t
$$

Solution. The general solution of $y^{\prime \prime}+3 y^{\prime}+2 y=0$ is $y=c_{1} e^{-t}+c_{2} e^{-2 t}$. We guess a particular solution of (2.2.9):

$$
y_{0}=a \sin 2 t+b \cos 2 t
$$

Then

$$
y_{0}^{\prime}=2 a \cos 2 t-2 b \sin 2 t, \quad y_{0}^{\prime \prime}=-4 a \sin 2 t-4 b \cos 2 t .
$$

By (2.2.9),

$$
-4 a \sin 2 t-4 b \cos 2 t+3(2 a \cos 2 t-2 b \sin 2 t)+2(a \sin 2 t+b \cos 2 t)=3 \sin 2 t,
$$

equivalently,

$$
-(2 a+6 b) \sin 2 t+(6 a-2 b) \cos 2 t=3 \sin 2 t .
$$

Hence

$$
-(2 a+6 b)=3, \quad 6 a-2 b=0 \Longrightarrow a=-\frac{3}{20}, b=-\frac{9}{20} .
$$

So

$$
y_{0}=-\frac{3}{20} \sin 2 t-\frac{9}{20} \cos 2 t
$$


and the general solution of $(2.2 .9)$ is

$$
y=c_{1} e^{-t}+c_{2} e^{-2 t}-\frac{3}{20} \sin 2 t-\frac{9}{20} \cos 2 t .
$$

Example 2.2.3. Find the solution of the following problem:

$$
y^{\prime \prime}+y=2 \cos t, \quad y(0)=1, y^{\prime}(0)=3 \text {. }
$$

Solution. The general solution of the corresponding homogeneous equation $y^{\prime \prime}+y=0$ is:

$$
y=c_{1} \cos t+c_{2} \sin t .
$$

Thus we can not guess a particular solution $y_{0}=a \cos t+b \sin t$. Instead, we guess that

$$
y_{0}=a t \cos t+b t \sin t
$$

is a particular solution. Then

$$
\begin{gathered}
y_{0}^{\prime}=(a+b t) \cos t+(b-a t) \sin t, \\
y_{0}^{\prime \prime}=(2 b-a t) \cos t-(2 a+b t) \sin t .
\end{gathered}
$$

Substituting them into the equation in (2.2.17), we get

$$
2 b \cos t-2 a \sin t=2 \cos t
$$

So

$$
a=0, b=1 ; y_{0}=t \sin t .
$$

Thus the general solution is:

$$
y=c_{1} \cos t+\left(c_{2}+t\right) \sin t .
$$

Next

$$
y^{\prime}=\left(c_{2}+t\right) \cos t+\left(1-c_{1}\right) \sin t .
$$

Then

$$
\begin{aligned}
y(0) & =1 \Longrightarrow c_{1}=1, \\
y^{\prime}(0) & =3 \Longrightarrow c_{2}=3 .
\end{aligned}
$$

The final solution is:

$$
y=\cos t+(3+t) \sin t .
$$

Example 2.2.4. Find the solution of the following problem:

$$
y^{\prime \prime}-4 y^{\prime}+4 y=4\left(t^{2}+e^{2 t}\right) .
$$


Solution. The corresponding homogeneous equation is

$$
y^{\prime \prime}-4 y^{\prime}+4 y=0
$$

whose characteristic equation is:

$$
r^{2}-4 r+4=0 \Longrightarrow r=2 \text { is a repeated root. }
$$

Thus the general solution is

$$
y=\left(c_{1}+c_{2} t\right) e^{2 t}
$$

First we want to find a particular solution of the equation:

$$
y^{\prime \prime}-4 y^{\prime}+4 y=4 t^{2}
$$

Let

$$
y_{0}=A t^{2}+B t+C
$$

be a particular solution. Then

$$
y_{0}^{\prime}=2 A t+B, \quad y_{0}^{\prime \prime}=2 A
$$

Substitute them into the equation,

$$
\begin{gathered}
2 A-4(2 A t+B)+4\left(A t^{2}+B t+C\right)=4 t^{2} \\
\Longrightarrow 4 A t^{2}+(4 B-8 A) t+2 A-4 B+4 C=4 t^{2} . \\
4 A=4,4 B-8 A=0,2 A-4 B+4 C=0 \Longrightarrow A=1, B=2, C=\frac{3}{2} .
\end{gathered}
$$

So

$$
y_{0}=t^{2}+2 t+\frac{3}{2}
$$

Next we want to find a particular solution of the equation:

$$
y^{\prime \prime}-4 y^{\prime}+4 y=4 e^{2 t} .
$$

Let

$$
y_{0}=A t^{2} e^{2 t}
$$

be a particular solution. Then

$$
y_{0}^{\prime}=2 A\left(t+t^{2}\right) e^{2 t}, \quad y_{0}^{\prime \prime}=2 A\left(1+4 t+2 t^{2}\right) e^{2 t} .
$$

Substitute them into the equation,

$$
2 A\left(1+4 t+2 t^{2}\right) e^{2 t}-8 A\left(t+t^{2}\right) e^{2 t}+4 A t^{2} e^{2 t}=4 e^{2 t} \Longrightarrow 2 A e^{2 t}=4 e^{2 t} .
$$


So $A=2$ and

$$
y_{0}=2 t^{2} e^{2 t}
$$

The final solution is

$$
y=\left(c_{1}+c_{2} t+2 t^{2}\right) e^{2 t}+t^{2}+2 t+\frac{3}{2} .
$$

\section{Excises 2.2.}

1. Find the general solution of the following equation:

$$
y^{\prime \prime}+y^{\prime}-2 y=2 t .
$$

2. Solve the following initial value problem:

$$
y^{\prime \prime}+2 y^{\prime}+5 y=4 e^{-x} \cos 2 x, \quad y(0)=1, \quad y^{\prime}(0)=0 .
$$

3. Solve the following initial value problem:

$$
y^{\prime \prime}-2 y^{\prime}-3 y=\left\{\begin{array}{ll}
3 e^{-t} & \text { if } 0 \leq t \leq 1, \\
2 t^{2} & \text { if } t>1 ;
\end{array} \quad y(0)=0, \quad y^{\prime}(0)=1 .\right.
$$

\subsection{Method of Variation of Parameters}

In this section, we give the method of variation of parameters for solving second-order nonhomogeneous linear ordinary differential equations.

Suppose that we know the fundamental solutions $y_{1}(t)$ and $y_{2}(t)$ of the linear homogeneous equation

$$
y^{\prime \prime}+f_{1}(t) y^{\prime}+f_{0}(t) y=0,
$$

that is, the general solution of (2.3.1) is $y=c_{1} y_{1}(t)+c_{2} y_{2}(t)$. We want to solve the linear nonhomogeneous equation

$$
y^{\prime \prime}+f_{1}(t) y^{\prime}+f_{0}(t) y=g(t) .
$$

Let $y=u_{1}(t) y_{1}+u_{2}(t) y_{2}$ be a solution of (2.3.2), where $u_{1}(t)$ and $u_{2}(t)$ are functions to be determined. Note

$$
y^{\prime}=u_{1}^{\prime} y_{1}+u_{2}^{\prime} y_{2}+u_{1} y_{1}^{\prime}+u_{2} y_{2}^{\prime} .
$$

In order to simplify the problem, we impose a condition

$$
u_{1}^{\prime} y_{1}+u_{2}^{\prime} y_{2}=0
$$

Then

$$
y^{\prime}=u_{1} y_{1}^{\prime}+u_{2} y_{2}^{\prime} \Longrightarrow y^{\prime \prime}=u_{1} y_{1}^{\prime \prime}+u_{2} y_{2}^{\prime \prime}+u_{1}^{\prime} y_{1}^{\prime}+u_{2}^{\prime} y_{2}^{\prime} .
$$


According to (2.3.2),

$$
\begin{aligned}
& u_{1} y_{1}^{\prime \prime}+u_{2} y_{2}^{\prime \prime}+u_{1}^{\prime} y_{1}^{\prime}+u_{2}^{\prime} y_{2}^{\prime}+f_{1}\left(u_{1} y_{1}^{\prime}+u_{2} y_{2}^{\prime}\right)+f_{0}\left(u_{1} f_{1}+u_{2} f_{2}\right)=g(t) \\
& \Longrightarrow u_{1}\left(y_{1}^{\prime \prime}+f_{1} y_{1}+f_{0} y_{1}\right)+u_{2}\left(y_{2}^{\prime \prime}+f_{2} y_{1}+f_{0} y_{2}\right)+u_{1}^{\prime} y_{1}^{\prime}+u_{2}^{\prime} y_{2}^{\prime}=g(t),
\end{aligned}
$$

equivalently,

$$
u_{1}^{\prime} y_{1}^{\prime}+u_{2}^{\prime} y_{2}^{\prime}=g(t)
$$

because $y_{1}$ and $y_{2}$ are solutions of (2.3.1).

The Wronskian of the functions $\left\{h_{1}, h_{2}, \ldots, h_{m}\right\}$ is the determinant

$$
W\left(h_{1}, h_{2}, \ldots, h_{m}\right)=\left|\begin{array}{cccc}
h_{1} & h_{2} & \cdots & h_{m} \\
h_{1}^{\prime} & h_{2}^{\prime} & \cdots & h_{m}^{\prime} \\
\vdots & \vdots & \vdots & \vdots \\
h_{1}^{(m-1)} & h_{2}^{(m-1)} & \cdots & h_{m}^{(m-1)}
\end{array}\right| .
$$

Solving the system (2.3.4) and (2.3.8) for $u_{1}^{\prime}$ and $u_{2}^{\prime}$ by Crammer's rule, we get

$$
u_{1}^{\prime}=-\frac{g(t) y_{2}(t)}{W\left(y_{1}, y_{2}\right)}, \quad u_{2}^{\prime}=\frac{g(t) y_{1}(t)}{W\left(y_{1}, y_{2}\right)} .
$$

Thus

$$
u_{1}=-\int \frac{g(t) y_{2}(t)}{W\left(y_{1}, y_{2}\right)} d t, \quad u_{2}=\int \frac{g(t) y_{1}(t)}{W\left(y_{1}, y_{2}\right)} d t
$$

The final solution is

$$
y=-y_{1}(t) \int \frac{g(t) y_{2}(t)}{W\left(y_{1}, y_{2}\right)} d t+y_{2}(t) \int \frac{g(t) y_{1}(t)}{W\left(y_{1}, y_{2}\right)} d t .
$$

The above method is called the method of variation of parameters.

Example 2.3.1. Find the general solution of the following equation by the method of variation of parameters:

$$
y^{\prime \prime}+4 y=\frac{4}{\sin 2 t}, \quad 0<t<\frac{\pi}{4} .
$$

Solution. The corresponding homogeneous equation is $y^{\prime \prime}+4 y=0$, whose fundamental solutions are $y_{1}=\cos 2 t$ and $y_{2}=\sin 2 t$. So

$$
W\left(y_{1}, y_{2}\right)=\left|\begin{array}{cc}
\cos 2 t & \sin 2 t \\
-2 \sin 2 t & 2 \cos 2 t
\end{array}\right|=2
$$

Thus

$$
\begin{gathered}
u_{1}=-\int \frac{g(t) y_{2}(t)}{W\left(y_{1}, y_{2}\right)} d t=-2 \int d t=c_{1}-2 t \\
u_{2}=\int \frac{g(t) y_{1}(t)}{W\left(y_{1}, y_{2}\right)} d t=\int \frac{2 \cos 2 t}{\sin 2 t} d t=\ln \sin 2 t+c_{2} .
\end{gathered}
$$


The final solution is

$$
y=\left(c_{1}-2 t\right) \cos 2 t+\left(c_{2}+\ln \sin 2 t\right) \sin 2 t .
$$

Example 2.3.2. Solve the following initial value problem by the method of variation of parameters:

$$
y^{\prime \prime}-4 y=g(t), \quad y(0)=1, \quad y^{\prime}(0)=-1 .
$$

Solution. First we solve the following initial value problem:

$$
u^{\prime \prime}-4 u=0, \quad u(0)=1, \quad u^{\prime}(0)=-1 .
$$

The general solution of the above equation is:

$$
u=c_{1} e^{2 t}+c_{2} e^{-2 t} .
$$

So

$$
\begin{gathered}
u^{\prime}=2\left(c_{1} e^{2 t}-c_{2} e^{-2 t}\right) . \\
\left\{\begin{array} { l } 
{ u ( 0 ) = 1 } \\
{ u ^ { \prime } ( 0 ) = - 1 }
\end{array} \Longrightarrow \left\{\begin{array} { l } 
{ c _ { 1 } + c _ { 2 } = 1 } \\
{ 2 ( c _ { 1 } - c _ { 2 } ) = - 1 }
\end{array} \Longrightarrow \left\{\begin{array}{l}
c_{1}=1 / 4 \\
c_{2}=3 / 4
\end{array}\right.\right.\right.
\end{gathered}
$$

The solution is:

$$
u=\frac{1}{4}\left(e^{2 t}+3 e^{-2 t}\right) .
$$

Next we want to solve the following problem:

$$
\begin{gathered}
v^{\prime \prime}-4 v=g(t), \quad v(0)=0, \quad v^{\prime}(0)=0 . \\
W\left(e^{2 t}, e^{-2 t}\right)=-4 . \\
v=-e^{2 t} \int_{0}^{t} \frac{g(s) e^{-2 s}}{-4} d s+e^{-2 t} \int_{0}^{t} \frac{g(s) e^{2 s}}{-4} d s=\frac{1}{2} \int_{0}^{t} g(s) \sinh 2(t-s) d s .
\end{gathered}
$$

The final solution is:

$$
y=u+v=\frac{1}{4}\left(e^{2 t}+3 e^{-2 t}\right)+\frac{1}{2} \int_{0}^{t} g(s) \sinh 2(t-s) d s .
$$

If

$$
v(t)=-y_{1}(t) \int_{0}^{t} \frac{g(s) y_{2}(s)}{W\left(y_{1}, y_{2}\right)(s)} d s+y_{2}(t) \int_{0}^{t} \frac{g(s) y_{1}(s)}{W\left(y_{1}, y_{2}\right)(s)} d s
$$

then

$$
v^{\prime}(t)=-y_{1}^{\prime}(t) \int_{0}^{t} \frac{g(s) y_{2}(s)}{W\left(y_{1}, y_{2}\right)(s)} d s+y_{2}^{\prime}(t) \int_{0}^{t} \frac{g(s) y_{1}(s)}{W\left(y_{1}, y_{2}\right)(s)} d s .
$$

Thus we always have $v(0)=v^{\prime}(0)=0$. 


\section{Excises 2.3.}

1. Solve the equation

$$
y^{\prime \prime}+9 y=\frac{9}{\cos 3 t}, \quad 0<t<\frac{\pi}{6}
$$

2. Solve the equation

$$
y^{\prime \prime}-2 y^{\prime}+y=\frac{e^{t}}{1+t^{2}} .
$$

3. Let $g(t)$ be a given function. Find the solution of the following problem

$$
y^{\prime \prime}-3 y^{\prime}-4 y=g(t), \quad y(0)=1, \quad y^{\prime}(0)=-1 .
$$

\subsection{Series Method and Bessel Functions}

In this section, we use power series to solve certain second-order linear ordinary differential equations with variable coefficients:

$$
y^{\prime \prime}+f_{1}(t) y^{\prime}+f_{0}(t) y=0 .
$$

Suppose that $f_{1}$ and $f_{0}$ are analytic at $t=0$. Around $t=0$,

$$
f_{0}=\sum_{n=0}^{\infty} a_{n} t^{n}, \quad f_{1}=\sum_{n=0}^{\infty} b_{n} t^{n}, \quad a_{n}, b_{n} \in \mathbb{R} .
$$

We consider the solution of the form

$$
\begin{gathered}
y=\sum_{n=0}^{\infty} c_{n} t^{n}, \quad \text { where } c_{n} \text { are to be determined. } \\
y^{\prime}=\sum_{n=1}^{\infty} n c_{n} t^{n-1}, \quad y^{\prime \prime}=\sum_{n=2}^{\infty} n(n-1) c_{n} t^{n-2} .
\end{gathered}
$$

Now (2.4.1) becomes

$$
\begin{gathered}
\sum_{n=0}^{\infty}(n+1)(n+2) c_{n+2} t^{n}+\left(\sum_{n=0}^{\infty} b_{n} t^{n}\right)\left(\sum_{n=0}^{\infty}(n+1) c_{n+1} t^{n}\right)+\left(\sum_{n=0}^{\infty} a_{n} t^{n}\right)\left(\sum_{n=0}^{\infty} c_{n} t^{n}\right)=0 . \\
(n+1)(n+2) c_{n+2}=-\sum_{r=0}^{n}\left[(r+1) b_{n-r} c_{r+1}+a_{n-r} c_{r}\right] .
\end{gathered}
$$

Example 2.4.1. Solve the equation $y^{\prime \prime}-t y^{\prime}-y=0$.

Solution. Suppose that $y=\sum_{n=0}^{\infty} c_{n} t^{n}$ is a solution. Note $a_{r}=-\delta_{r, 0}$ and $b_{r}=-\delta_{r, 1}$. Thus (2.4.6) becomes

$$
(n+1)(n+2) c_{n+2}=(n+1) c_{n} \sim c_{n+2}=\frac{c_{n}}{n+2} .
$$


Hence

$$
y=c_{0} \sum_{n=0}^{\infty} \frac{t^{2 n}}{(2 n) ! !}+c_{1} \sum_{n=0}^{\infty} \frac{t^{2 n+1}}{(2 n+1) ! !}
$$

Suppose

$$
f_{0}=\sum_{n=-2}^{\infty} a_{n} t^{n}, \quad f_{1}=\sum_{n=-1}^{\infty} b_{n} t^{n}, \quad a_{n}, b_{n} \in \mathbb{R} .
$$

Assume that $y=\sum_{n=0}^{\infty} c_{n} t^{n+\mu}$ is a solution of the equation (2.4.1) with $c_{0} \neq 0$. Substituting it into (2.4.1), we find that the coefficients of $t^{\mu-2}$ give

$$
\mu(\mu-1)+\mu b_{-1}+a_{-2}=0 \sim \mu^{2}+\left(b_{-1}-1\right) \mu+a_{-2}=0,
$$

which is called the indicial equation of (2.4.1) with (2.4.9). If (2.4.10) has two distinct real roots $\mu_{1}$ and $\mu_{2}$ such that $\mu_{1}-\mu_{2}$ is not an integer, then the equation (2.4.1) has two linearly independent solutions of the forms:

$$
y_{1}=t^{\mu_{1}} \sum_{n=0}^{\infty} c_{n} t^{n}, \quad y_{2}=t^{\mu_{2}} \sum_{n=0}^{\infty} d_{n} t^{n} .
$$

When (2.4.10) has a repeated root $\mu$, then the equation (2.4.1) has two linearly independent solutions of the forms:

$$
y_{1}=t^{\mu} \sum_{n=0}^{\infty} c_{n} t^{n}, \quad y_{2}=y_{1} \ln t+t^{\mu} \sum_{n=0}^{\infty} d_{n} t^{n} .
$$

If (2.4.10) has two distinct real roots $\mu_{1}$ and $\mu_{2}$ such that $\mu_{2}-\mu_{1}$ is an integer, then the equation (2.4.1) has two linearly independent solutions of the forms:

$$
y_{1}=t^{\mu_{1}} \sum_{n=0}^{\infty} c_{n} t^{n}, \quad y_{2}=k y_{1} \ln t+t^{\mu_{2}} \sum_{n=0}^{\infty} d_{n} t^{n}
$$

where $k$ many be zero.

Example 2.4.2. Solve the following equation by power series:

$$
t^{2} y^{\prime \prime}+3 t y^{\prime}+(1+t) y=0, \quad t>0 .
$$

Solution. Note that $t=0$ is a regular singular point. Let $y=\sum_{n=0}^{\infty} c_{n} t^{n+\mu}$ be a solution with $c_{0} \neq 0$. Then

$$
y^{\prime}=\sum_{n=0}^{\infty}(n+\mu) c_{n} t^{n+\mu-1}, \quad y^{\prime \prime}=\sum_{n=0}^{\infty}(n+\mu)(n+\mu-1) c_{n} t^{n+\mu-2} .
$$

Substituting them into the equation, we have:

$$
\sum_{n=0}^{\infty}(n+\mu)(n+\mu-1) c_{n} t^{n+\mu}+3 \sum_{n=0}^{\infty}(n+\mu) c_{n} t^{n+\mu}+(1+t) \sum_{n=0}^{\infty} c_{n} t^{n+\mu}=0,
$$


equivalently,

$$
\sum_{n=0}^{\infty}(n+\mu)(n+\mu-1) c_{n} t^{n+\mu}+3 \sum_{n=0}^{\infty}(n+\mu) c_{n} t^{n+\mu}+\sum_{n=0}^{\infty} c_{n} t^{n+\mu}+\sum_{n=0}^{\infty} c_{n} t^{n+\mu+1}=0 .
$$

So we have

$$
\left[\mu(\mu-1) c_{0}+3 \mu c_{0}+c_{0}\right] t^{\mu}+\sum_{n=1}^{\infty}\left((n+\mu)(n+\mu-1) c_{n}+3(n+\mu) c_{n}+c_{n}+c_{n-1}\right) t^{n+\mu}=0 .
$$

Thus $\mu(\mu-1) c_{0}+3 \mu c_{0}+c_{0}=0$ and for $n \geq 1$,

$$
\begin{gathered}
(n+\mu)(n+\mu-1) c_{n}+3(n+\mu) c_{n}+c_{n}+c_{n-1}=0 \Longrightarrow(n+\mu+1)^{2} c_{n}=-c_{n-1} . \\
c_{n}=-\frac{c_{n-1}}{(n+\mu+1)^{2}}=\frac{(-1)^{n} c_{0}}{\prod_{j=1}^{n}(j+\mu+1)^{2}} .
\end{gathered}
$$

Denote

$$
b_{n}=\frac{(-1)^{n}}{\prod_{j=1}^{n}(j+\mu+1)^{2}}
$$

Set

$$
\varphi(\mu, t)=t^{\mu}\left(1+\sum_{n=1}^{\infty} b_{n} t^{n}\right)
$$

The indicial equation is

$$
\mu(\mu-1)+3 \mu+1=0 \sim(\mu+1)^{2}=0 \Longrightarrow \mu=-1
$$

is a double root. Then

$$
y_{1}=\varphi(-1, t)=t^{-1}\left(1+\sum_{n=1}^{\infty} \frac{(-1)^{n}}{\prod_{j=1}^{n} j^{2}} t^{n}\right)=t^{-1}\left(1+\sum_{n=1}^{\infty} \frac{(-1)^{n}}{(n !)^{2}} t^{n}\right)
$$

is a solution of (2.4.14).

Observe

$$
t^{2} \varphi_{t t}+3 t \varphi_{t}+(1+t) \varphi=t^{\mu}(\mu+1)^{2}
$$

(cf. the left hand side of (2.4.18) with $c_{0}=1$ ). Taking partial derivative of (2.4.25) with respect to $\mu$, we get

$$
t^{2} \varphi_{t t \mu}+3 t \varphi_{t \mu}+(1+t) \varphi_{\mu}=(\ln t) t^{\mu}(\mu+1)^{2}+2 t^{\mu}(\mu+1)
$$

equivalently,

$$
t^{2} \varphi_{\mu t t}+3 t \varphi_{\mu t}+(1+t) \varphi_{\mu}=(2+(\mu+1) \ln t) t^{\mu}(\mu+1) .
$$

Taking $\mu=-1$ in the above equation, we find

$$
t^{2}\left(\frac{d}{d t}\right)^{2} \varphi_{\mu}(-1, t)+3 t \frac{d}{d t} \varphi_{\mu}(-1, t)+(1+t) \varphi_{\mu}(-1, t)=0 .
$$


Thus $y_{2}=\varphi_{\mu}(-1, t)$ is another solution. Note that for $n \geq 1$,

$$
\begin{aligned}
\frac{d b_{n}}{d \mu}(-1) & =\left.\left(\frac{(-1)^{n}}{\prod_{j=1}^{n}(j+\mu+1)^{2}}\right)^{\prime}\right|_{\mu=-1} \\
& =\left.\left(\frac{2(-1)^{n+1}}{\prod_{j=1}^{n}(j+\mu+1)^{2}}\right)\left(\sum_{j=1}^{n} \frac{1}{j+\mu+1}\right)\right|_{\mu=-1} \\
& =\frac{2(-1)^{n+1}}{(n !)^{2}}\left(\sum_{j=1}^{n} \frac{1}{j}\right) .
\end{aligned}
$$

Thus

$$
y_{2}(t)=\left.\varphi_{\mu}(-1, t)\right|_{r=-1}=y_{1}(t) \ln t+\sum_{n=1}^{\infty} \frac{2(-1)^{n+1}}{(n !)^{2}}\left(\sum_{j=1}^{n} \frac{1}{j}\right) t^{n-1} .
$$

The general solution is: $y=c_{1} y_{1}(t)+c_{2} y_{2}(t)$.

The Bessel equation has the form

$$
y^{\prime \prime}+t^{-1} y^{\prime}+\left(1-\nu^{2} t^{-2}\right) y=0,
$$

where $\nu$ is a constant called order. The indicial equation is

$$
\mu^{2}-\nu^{2}=0 \sim \mu= \pm \nu .
$$

We rewrite (2.4.31) as

$$
t^{2} y^{\prime \prime}+t y^{\prime}+\left(t^{2}-\nu^{2}\right) y=0 .
$$

Let $y=\sum_{n=0}^{\infty} c_{n} t^{n+\mu}$ be a solution of (2.4.33) with $\mu= \pm \nu$ and $c_{0} \neq 0$. We have

$$
t y^{\prime}=\sum_{n=0}^{\infty}(n+\mu) c_{n} t^{n+\mu}, \quad t^{2} y^{\prime \prime}=\sum_{n=0}^{\infty}(n+\mu)(n+\mu-1) c_{n} t^{n+\mu} .
$$

Denote by $\mathbb{N}$ the set of nonnegative integers. So (2.4.33) is equivalent to

$$
c_{1}\left[(\mu+1)^{2}-\nu^{2}\right]=0, \quad\left[(\mu+n+2)^{2}-\nu^{2}\right] c_{n+2}+c_{n}=0, \quad n \in \mathbb{N} .
$$

Thus $c_{2 r+1}=0$ for $r \in \mathbb{N}$, and

$$
c_{2 n}=\frac{c_{0}}{\prod_{r=1}^{n}\left[\nu^{2}-(\mu+2 r)^{2}\right]}=\frac{(-1)^{n} c_{0}}{n ! 2^{2 n} \prod_{r=1}^{n}(\mu+r)} .
$$

The function

$$
J_{\mu}(t)=\left(\frac{t}{2}\right)^{\mu}+\sum_{n=1}^{\infty} \frac{(-1)^{n}}{n ! \prod_{r=1}^{n}(\mu+r)}\left(\frac{t}{2}\right)^{2 n+\mu}
$$

is called a Bessel function of first kind. If $\nu$ is not an integer, then the general solution of (2.4.31) is

$$
y=c_{1} J_{\nu}(t)+c_{2} J_{-\nu}(t) .
$$


Note

$$
\frac{d}{d t}\left(t^{\mu} J_{\mu}\right)=\mu t^{\mu}\left[\left(\frac{t}{2}\right)^{\mu-1}+\sum_{n=1}^{\infty} \frac{(-1)^{n}}{n ! \prod_{r=1}^{n}(\mu+r-1)}\left(\frac{t}{2}\right)^{2 n+\mu-1}\right]=\mu t^{\mu} J_{\mu-1}
$$

and

$$
\frac{d}{d t}\left(t^{-\mu} J_{\mu}\right)=\sum_{n=1}^{\infty} \frac{(-1)^{n} t^{-\mu}}{(\mu+1)(n-1) ! \prod_{r=1}^{n-1}(\mu+r+1)}\left(\frac{t}{2}\right)^{2 n+\mu-1}=-\frac{t^{-\mu} J_{\mu+1}}{\mu+1} .
$$

Thus

$$
\frac{d}{t d t}\left(t^{\mu} J_{\mu}\right)=\mu t^{\mu-1} J_{\mu-1}, \quad \frac{d}{t d t}\left(t^{-\mu} J_{\mu}\right)=-\frac{t^{-\mu-1} J_{\mu+1}}{\mu+1} .
$$

By induction,

$$
\left(\frac{d}{t d t}\right)^{m}\left(t^{\mu} J_{\mu}\right)=\left[\prod_{r=0}^{m-1}(\mu-r)\right] t^{\mu-m} J_{\mu-m}
$$

and

$$
\left(\frac{d}{t d t}\right)^{m}\left(t^{-\mu} J_{\mu}\right)=(-1)^{m} \frac{t^{-\mu-m} J_{\mu+m}}{\prod_{r=1}^{m}(\mu+r)} .
$$

On the other hand, (2.4.39) gives

$$
\mu t^{\mu-1} J_{\mu}+t^{\mu} J_{\mu}^{\prime}=\mu t^{\mu} J_{\mu-1} \sim \mu J_{\mu}+t J_{\mu}^{\prime}=\mu t J_{\mu-1}
$$

and (2.4.40) yields

$$
-\mu t^{-\mu-1} J_{\mu}+t^{-\mu} J_{\mu}^{\prime}=-\frac{t^{-\mu} J_{\mu+1}}{\mu+1} \sim-\mu J_{\mu}+t J_{\mu}^{\prime}=-\frac{t J_{\mu+1}}{\mu+1} .
$$

Thus

$$
\mu J_{\mu-1}+\frac{J_{\mu+1}}{\mu+1}=\frac{2 \mu}{t} J_{\mu}, \quad \mu J_{\mu-1}-\frac{J_{\mu+1}}{\mu+1}=2 \mu J_{\mu}^{\prime}
$$

Observe that

$$
\left(\frac{d}{d t}\right) \frac{t^{n}}{n !}=\frac{t^{n-1}}{(n-1) !}
$$

for a positive integer $n$. If we have a continuous analogue of $n$ !, then we can simplify (2.4.42) and (2.4.43) by rescalling $J_{\mu}$. Indeed, it is the spacial function $\Gamma(s)$.

When $\nu=n+1 / 2$ with $n \in \mathbb{N}$, the indicial equation has two roots $\mu_{1}=n+1 / 2$ and $\mu_{2}=-n-1 / 2$. Moreover, $\mu_{1}-\mu_{2}=2 n+1$ is an integer. However, both $J_{n+1 / 2}(t)$ and $J_{-n-1 / 2}(t)$ are well defined by (2.4.37). They form a set of fundamental solutions of the Bessel equation. Suppose that $\nu=m$ is a positive integer. the indicial equation has two roots $\mu_{1}=m$ and $\mu_{2}=-m$. The function $J_{m}(t)$ is still well defined, but $J_{-m}(t)$ is not defined. If $\mu=-m$, by the second equation in (2.4.35) with $n=2 m-2$, we get

$$
0=\left[(-m+2 m-2+2)^{2}-m^{2}\right] c_{2 m}=-c_{2 m-2}=-\frac{c_{0}}{(m !)^{2}} \Longrightarrow c_{0}=0
$$


which contradicts the assumption $c_{0} \neq 0$. Thus we do not have a solution of the form $y=\sum_{n=0}^{\infty} c_{n} t^{n-m}$. We look for another fundamental solution of the form

$$
y=J_{m}(t) \ln t+\sum_{n=0}^{\infty} c_{n} t^{n-m},
$$

which is related to Bessel functions of second kind.

\section{Exercise 2.4}

Solve the following equations by power series :

1. $\left(1-t^{2}\right) y^{\prime \prime}-t y^{\prime}+16 t y=0$.

2. $t^{2} y^{\prime \prime}+7 t y^{\prime}+(9-t) y=0, \quad t>0$. 
34 CHAPTER 2. HIGHER-ORDER ORDINARY DIFFERENTIAL EQUATIONS 


\section{Chapter 3}

\section{Special Functions}

Special functions are important objects both in mathematics and physics. This chapter is a brief introduction to them. The reader may refer to $[\mathrm{AAR}]$ and $[\mathrm{WG}]$ for more extensive knowledge. First we introduce the gamma function $\Gamma(z)$ as a continuous generalization of $n$ ! and prove the beta-function identity, the Euler's reflection formula and the product formula of the gamma function. Then we introduce Gauss hypergeometric function as the power series solution of the Gauss hypergeometric equation and prove the Euler's integral representation. Moreover, Jacobi polynomials are introduced from the finite-sum cases of the Gauss hypergeometric function and their orthogonality is proved. Legendre orthogonal polynomials are discussed in detail.

Weierstrass's elliptic function $\wp(z)$ is a double-periodic function with second-order poles, which will be used later in solving nonlinear partial differential equations. Weierstrass's zeta function $\zeta(z)$ is an integral of $-\wp(z)$, that is, $\zeta^{\prime}(z)=-\wp(z)$. Moreover, Weierstrass's sigma function $\sigma(z)$ satisfies $\sigma^{\prime}(z) / \sigma(z)=\zeta(z)$. We discuss these functions and their properties in this chapter to a certain depth.

Finally in this chapter, we present Jacobi's elliptic functions sn $(z \mid m)$, cn $(z \mid m)$ and $\operatorname{dn}(z \mid m)$, and derive the nonlinear ordinary differential equations satisfied by them. These functions are also very useful in solving nonlinear partial differential equations.

\subsection{Gamma and Beta Functions}

The problem of finding a function of continuous variable $x$ that equals $n$ ! when $x=n$ is a positive integer, was suggested by Bernoulli and Goldbach, and was investigated by Euler in the late 1720 s. For $a \in \mathbb{C}$ and $n \in \mathbb{N}+1$, we denote

$$
(a)_{n}=a(a+1) \cdots(a+n-1), \quad(a)_{0}=1 .
$$

If $x$ and $n$ are positive integers, then

$$
x !=\frac{(x+n) !}{(x+1)_{n}}=\frac{n !(n+1)_{x}}{(x+1)_{n}}=\frac{n ! n^{x}}{(x+1)_{n}} \frac{(n+1)_{x}}{n^{x}} .
$$


Since

$$
\lim _{n \rightarrow \infty} \frac{(n+1)_{x}}{n^{x}}=1
$$

we have

$$
x !=\lim _{n \rightarrow \infty} \frac{n ! n^{x}}{(x+1)_{n}} .
$$

Observe that for any $z \in \mathbb{C} \backslash\{-\mathbb{N}-1\}$,

$$
\begin{aligned}
& \left(\frac{n}{n+1}\right)^{z} \prod_{r=1}^{n}\left(1+\frac{z}{r}\right)^{-1}\left(1+\frac{1}{r}\right)^{z} \\
= & \left(\frac{n}{n+1}\right)^{z} \prod_{r=1}^{n}\left(\frac{z+r}{r}\right)^{-1}\left(\frac{r+1}{r}\right)^{z} \\
= & \left(\frac{n}{n+1}\right)^{z}\left(\frac{(z+1)_{n}}{n !}\right)^{-1}(n+1)^{z}=\frac{n ! n^{z}}{(z+1)_{n}} .
\end{aligned}
$$

Moreover,

$$
\begin{aligned}
& \left(1+\frac{z}{r}\right)^{-1}\left(1+\frac{1}{r}\right)^{z} \\
= & \left(1-\frac{z}{r}+\frac{z^{2}}{r^{2}}+O\left(\frac{1}{r^{3}}\right)\right)\left(1+\frac{z}{r}+\frac{z(z-1)}{2 r^{2}}+O\left(\frac{1}{r^{3}}\right)\right) \\
= & 1+\frac{z(z-1)}{2 r^{2}}+O\left(\frac{1}{r^{3}}\right) .
\end{aligned}
$$

This shows that

$$
\lim _{n \rightarrow \infty} \prod_{r=1}^{n}\left(1+\frac{z}{r}\right)^{-1}\left(1+\frac{1}{r}\right)^{z} \text { exists. }
$$

Thus we have a function

$$
\Pi(z)=\lim _{n \rightarrow \infty} \frac{n ! n^{z}}{(z+1)_{n}}=\prod_{r=1}^{\infty}\left(1+\frac{z}{r}\right)^{-1}\left(1+\frac{1}{r}\right)^{z}
$$

and $\Pi(m)=m$ ! for $m \in \mathbb{N}$ by (3.1.4). For notional convenience, we define the gamma function

$$
\Gamma(z)=\Pi(z-1)=\lim _{n \rightarrow \infty} \frac{n ! n^{z-1}}{(z)_{n}} \quad \text { for } \quad z \in \mathbb{C} \backslash\{-\mathbb{N}-1\}
$$

Then

$$
\begin{aligned}
\Gamma(z+1) & =\lim _{n \rightarrow \infty} \frac{n ! n^{z}}{(z+1)_{n}}=z \lim _{n \rightarrow \infty} \frac{n ! n^{z}}{(z)_{n+1}} \\
& =z \lim _{n \rightarrow \infty} \frac{n}{z+n} \frac{n ! n^{z-1}}{(z)_{n}}=z\left(\lim _{n \rightarrow \infty} \frac{n}{z+n}\right)\left(\lim _{n \rightarrow \infty} \frac{n ! n^{z-1}}{(z)_{n}}\right) \\
& =z \lim _{n \rightarrow \infty} \frac{n ! n^{z-1}}{(z)_{n}}=z \Gamma(z) .
\end{aligned}
$$

By $(3.1 .9), \Gamma(1)=1$. So $\Gamma(m+1)=m$ ! for $m \in \mathbb{N}$. 
For $x, y \in \mathbb{C}$ with $\operatorname{Re} x>0$ and $\operatorname{Re} y>0$, we define the beta function

$$
B(x, y)=\int_{0}^{1} t^{x-1}(1-t)^{y-1} d t .
$$

Theorem 3.1.1. We have $B(x, y)=\Gamma(x) \Gamma(y) / \Gamma(x+y)$.

Proof. Note

$$
B(x, y+1)=\int_{0}^{1} t^{x-1}(1-t)(1-t)^{y-1} d t=B(x, y)-B(x+1, y) .
$$

On the other hand, integration by parts gives

$$
\begin{aligned}
B(x, y+1) & =\int_{0}^{1} t^{x-1}(1-t)^{y} d t \\
& =\left.\frac{t^{x}(1-t)^{y}}{x}\right|_{0} ^{1}+\frac{y}{x} \int_{0}^{1} t^{x}(1-t)^{y-1} d t=\frac{y}{x} B(x+1, y) .
\end{aligned}
$$

Thanks to the above two expressions, we have

$$
B(x, y)-\frac{x}{y} B(x, y+1)=B(x, y+1) \Longrightarrow B(x, y)=\frac{x+y}{y} B(x, y+1) .
$$

By induction

$$
B(x, y)=\frac{(x+y)_{n}}{(y)_{n}} B(x, y+n) .
$$

Rewrite the above equation as

$$
\begin{aligned}
B(x, y) & =\frac{(x+y)_{n}}{n !} \frac{n !}{(y)_{n}} \int_{0}^{1} t^{x-1}(1-t)^{y+n-1} d t \\
& \stackrel{t=s / n}{=} \frac{(x+y)_{n}}{n !} \frac{n !}{(y)_{n}} \int_{0}^{n} n^{1-x} s^{x-1}\left(1-\frac{s}{n}\right)^{y+n-1} \frac{d s}{n} \\
& =\frac{(x+y)_{n}}{n ! n^{x+y-1}} \frac{n ! n^{y-1}}{(y)_{n}} \int_{0}^{n} s^{x-1}\left(1-\frac{s}{n}\right)^{y+n-1} d s \\
& =\lim _{n \rightarrow \infty} \frac{(x+y)_{n}}{n ! n^{x+y-1}} \frac{n ! n^{y-1}}{(y)_{n}} \int_{0}^{n} s^{x-1}\left(1-\frac{s}{n}\right)^{y+n-1} d s \\
& =\frac{\Gamma(y)}{\Gamma(x+y)} \int_{0}^{\infty} s^{x-1} e^{-s} d s .
\end{aligned}
$$

Taking $y=1$ in the above equation, we have

$$
B(x, 1) \Gamma(x+1)=\int_{0}^{\infty} s^{x-1} e^{-s} d s .
$$

Furthermore, (3.1.11) gives

$$
B(x, 1)=\int_{0}^{1} t^{x-1} d t=\frac{1}{x} .
$$


Thus

$$
\Gamma(x)=\frac{\Gamma(x+1)}{x}=B(x, 1) \Gamma(x+1)=\int_{0}^{\infty} s^{x-1} e^{-s} d s
$$

Therefore,

$$
B(x, y)=\frac{\Gamma(y)}{\Gamma(x+y)} \int_{0}^{\infty} s^{x-1} e^{-s} d s=\frac{\Gamma(x) \Gamma(y)}{\Gamma(x+y)} .
$$

Recall the Euler's constant

$$
\gamma=\lim _{n \rightarrow \infty}\left(\sum_{r=1}^{n} \frac{1}{r}-\ln n\right)
$$

Theorem 3.1.2. The following equation holds:

$$
\frac{1}{\Gamma(z)}=z e^{\gamma z} \prod_{n=1}^{\infty}\left(1+\frac{z}{n}\right) e^{-z / n}
$$

Proof. Note

$$
\left(1+\frac{z}{n}\right) e^{-z / n}=\left(1+\frac{z}{n}\right)\left(1-\frac{z}{n}+\frac{z^{2}}{2 n^{2}}+O\left(\frac{1}{n^{3}}\right)\right)=1-\frac{z^{2}}{2 n^{2}}+O\left(\frac{1}{n^{3}}\right) .
$$

Thus the product in (3.1.22) converges. Moreover,

$$
\begin{aligned}
\frac{1}{\Gamma(z)} & =\lim _{n \rightarrow \infty} \frac{(z)_{n}}{n ! n^{z-1}}=\lim _{n \rightarrow \infty} \frac{z(z+1) \cdots(z+n-1)}{(n-1) ! n^{z}} \\
& =z \lim _{n \rightarrow \infty}\left[\prod_{r=1}^{n-1}\left(1+\frac{z}{r}\right)\right] e^{-z \ln n} \\
& =z \lim _{n \rightarrow \infty} e^{z\left[\sum_{r=1}^{n} 1 / r-\ln n\right]} e^{-z / n} \prod_{r=1}^{n-1}\left(1+\frac{z}{r}\right) e^{-z / r} \\
& =z e^{\gamma z} \prod_{r=1}^{\infty}\left(1+\frac{z}{r}\right) e^{-z / r} .
\end{aligned}
$$

Theorem 3.1.3. Euler's reflection formula:

$$
\Gamma(z) \Gamma(1-z)=\frac{\pi}{\sin \pi z} .
$$

Proof. From complex analysis,

$$
\frac{\sin \pi z}{\pi z}=\prod_{n=1}^{\infty}\left(1-\frac{z^{2}}{n^{2}}\right)
$$


According to $(3.1 .22)$,

$$
\begin{aligned}
\Gamma(z) \Gamma(-z) & =\left[z e^{\gamma z} \prod_{n=1}^{\infty}\left(1+\frac{z}{n}\right) e^{-z / n}\right]^{-1}\left[-z e^{-\gamma z} \prod_{n=1}^{\infty}\left(1-\frac{z}{n}\right) e^{z / n}\right]^{-1} \\
& =-\frac{1}{z^{2}}\left[\prod_{n=1}^{\infty}\left(1+\frac{z}{n}\right)\left(1-\frac{z}{n}\right)\right]^{-1} \\
& =-\frac{1}{z^{2}}\left[\prod_{n=1}^{\infty}\left(1-\frac{z^{2}}{n^{2}}\right)\right]^{-1}=-\frac{\pi}{z \sin \pi z}
\end{aligned}
$$

Now (3.1.25) follows from the fact $\Gamma(1-z)=-z \Gamma(-z)$.

Letting $z=1 / 2$ in (3.1.25), we get $\Gamma(1 / 2)=\sqrt{\pi}$. Taking the logarithm of (3.1.22), we have

$$
-\ln \Gamma(z)=\gamma z+\ln z+\sum_{n=1}^{\infty}\left[\ln \left(1+\frac{z}{n}\right)-\frac{z}{n}\right] .
$$

Differentiating (3.1.28), we get

$$
\psi(z)=\frac{\Gamma^{\prime}(z)}{\Gamma(z)}=-\gamma-\frac{1}{z}+\sum_{n=1}^{\infty}\left(\frac{1}{n}-\frac{1}{z+n}\right) .
$$

In particular,

$$
\psi^{\prime}(z)=\sum_{n=0}^{\infty} \frac{1}{(z+n)^{2}}=\zeta(2, z),
$$

where the Riemman zeta function

$$
\zeta(s, a)=\sum_{n=0}^{\infty} \frac{1}{(n+a)^{s}}, \quad \operatorname{Re} s>1 .
$$

Theorem 3.1.3. The following product formula holds:

$$
\Gamma(z) \Gamma\left(z+\frac{1}{n}\right) \Gamma\left(z+\frac{2}{n}\right) \cdots \Gamma\left(z+\frac{n-1}{n}\right)=\frac{(2 \pi)^{(n-1) / 2}}{n^{n z-1 / 2}} \Gamma(n z) .
$$

Proof. Set

$$
\phi(z)=\frac{n^{n z}}{n \Gamma(n z)} \prod_{p=0}^{n-1} \Gamma\left(z+\frac{p}{n}\right) .
$$

Then (3.1.9) says

$$
\begin{aligned}
\phi(z) & =\lim _{r \rightarrow \infty} n^{n z-1} \frac{\prod_{p=0}^{n-1} \frac{r ! r^{z+(p-n) / n}}{(z+p / n)_{r}}}{\frac{(n r) !(n r)^{n z-1}}{(n z)_{n r}}} \\
& =\lim _{r \rightarrow \infty} \frac{\prod_{p=0}^{n-1} \frac{r ! r^{(p-n) / n}}{(z+p / n)_{r}}}{\frac{(n r) ! r^{-1}}{(n z)_{n r}}}=\lim _{r \rightarrow \infty} \frac{(r !)^{n} n^{r n}}{(n r) ! r^{(n+1) / 2}} .
\end{aligned}
$$


Thus $\phi$ is a constant. Hence

$$
\phi(z)=\phi(1 / n)=\prod_{j=1}^{n-1} \Gamma\left(\frac{j}{n}\right)=\prod_{j=1}^{n-1} \Gamma\left(1-\frac{j}{n}\right)
$$

So

$$
\phi^{2}=\prod_{j=1}^{n-1} \Gamma\left(\frac{j}{n}\right) \Gamma\left(1-\frac{j}{n}\right)=\prod_{j=1}^{n-1} \frac{\pi}{\sin j \pi / n} .
$$

Note

$$
\sum_{r=0}^{n-1} z^{r}=\frac{z^{n}-1}{z-1}=\prod_{j=1}^{n-1}\left(z-e^{2 j \pi i / n}\right) .
$$

Hence

$$
\begin{aligned}
n & =\prod_{j=1}^{n-1}\left(1-e^{2 j \pi i / n}\right)=\prod_{j=1}^{n-1} e^{j \pi i / n}\left(e^{-j \pi i / n}-e^{j \pi i / n}\right) \\
& =e^{(n-1) \pi i / 2} \prod_{j=1}^{n-1}(-2 i \sin j \pi / n)=2^{n-1} e^{(n-1) \pi i / 2}(-i)^{n-1} \prod_{j=1}^{n-1} \sin j \pi / n \\
& =2^{n-1} e^{(n-1) \pi i / 2} e^{3(n-1) \pi i / 2} \prod_{j=1}^{n-1} \sin j \pi / n=2^{n-1} \prod_{j=1}^{n-1} \sin j \pi / n .
\end{aligned}
$$

By (3.1.36) and (3.1.38),

$$
\phi^{2}=\frac{(2 \pi)^{n-1}}{n} \Longrightarrow \phi=\frac{(2 \pi)^{(n-1) / 2}}{\sqrt{n}} .
$$

Then (3.1.32) follows from (3.1.33) and (3.1.39).

\subsection{Gauss Hypergeometric Functions}

The term of "hypergeometric" was first used by Wallis in Oxford as early as 1655 in his work Arithmetrica Infinitorm when referring to any series which could be regarded as a generalization of the ordinary geometric series $\sum_{n=0}^{\infty} z^{n}$. Nowadays a power series $\sum_{n=0}^{\infty} c_{n} z^{n+\mu}$ is called a hypergeometric function if $c_{n+1} / c_{n}$ is a rational function of $n$. In this section, we use $z$ to denote independent variable instead of $t$. The classical hypergeometric equation is

$$
z(1-z) y^{\prime \prime}+[\gamma-(\alpha+\beta+1) z] y^{\prime}-\alpha \beta y=0
$$

We look for the solution of the form

$$
y=\sum_{n=0}^{\infty} c_{n} z^{n+\mu}
$$


where $c_{n}$ and $\mu$ are constants to be determined. We calculate

$$
y^{\prime}=\sum_{n=0}^{\infty}(n+\mu) c_{n} z^{n+\mu-1}, \quad y^{\prime \prime}=\sum_{n=0}^{\infty}(n+\mu)(n+\mu-1) c_{n} z^{n+\mu-2} .
$$

Substituting (3.2.3) into (3.2.1), we get

$$
z^{\mu} \sum_{n=0}^{\infty}\left\{(n+\mu)(n+\mu-1) c_{n} z^{n-1}(1-z)+(n+\mu) c_{n} z^{n-1}[\gamma-(\alpha+\beta+1) z]-\alpha \beta c_{n} z^{n}\right\}=0,
$$

equivalently,

$$
\begin{gathered}
\mu(\mu-1+\gamma)=0 \\
(n+1+\mu)(n+\mu+\gamma) c_{n+1}=[(n+\mu)(n+\mu+\alpha+\beta)+\alpha \beta] c_{n}
\end{gathered}
$$

for $n \in \mathbb{N}$. We rewrite (3.2.6) as

$$
(n+1+\mu)(n+\mu+\gamma) c_{n+1}=(n+\mu+\alpha)(n+\mu+\beta) c_{n} .
$$

By induction, we have

$$
c_{n}=\frac{(\mu+\alpha)_{n}(\mu+\beta)_{n}}{(\mu+1)_{n}(\mu+\gamma)_{n}} c_{0} \quad \text { for } n \in \mathbb{N}+1 .
$$

Hence

$$
y=c_{0} \sum_{n=0}^{\infty} \frac{(\mu+\alpha)_{n}(\mu+\beta)_{n}}{(\mu+1)_{n}(\mu+\gamma)_{n}} z^{n+\mu} .
$$

According to (3.2.5), $\mu=0$ or $\mu=1-\gamma$. Considering $\mu=0$, we denote

$$
{ }_{2} F_{1}(\alpha, \beta ; \gamma ; z)=\sum_{n=0}^{\infty} \frac{(\alpha)_{n}(\beta)_{n}}{n !(\gamma)_{n}} z^{n},
$$

which was introduced and studied by Gauss in his thesis presented at Göttingen in 1812. We call it classical Gauss hypergeometric function. Since

$$
\lim _{n \rightarrow \infty}\left[\frac{(\alpha)_{n+1}(\beta)_{n+1}}{(n+1) !(\gamma)_{n+1}} / \frac{(\alpha)_{n}(\beta)_{n}}{n !(\gamma)_{n}}\right]=\lim _{n \rightarrow \infty} \frac{(\alpha+n)(\beta+n)}{(n+1)(\gamma+n)}=1,
$$

the series in (3.2.10) converges absolutely when $|z|<1$. It can be proved that ${ }_{2} F_{1}(\alpha, \beta ; \gamma ; z)$ has analytic extension on the whole complex $z$ plane by complex analysis. Note that ${ }_{2} F_{1}(\alpha-\gamma+1, \beta-\gamma+1 ; 2-\gamma ; z) z^{1-\gamma}$ is another solution of (3.2.1) by (3.2.9).

Observe

$$
{ }_{2} F_{1}(\alpha, \beta ; \gamma ; 0)=1 .
$$

By $(3.2 .9),{ }_{2} F_{1}(\alpha, \beta ; \gamma ; z)$ is the unique power series solution of (3.2.1) satisfying (3.2.12). It has close relations with elementary functions:

$$
{ }_{2} F_{1}(-\alpha, \beta ; \beta ;-z)=\sum_{n=0}^{\infty} \frac{(-1)^{n}(-\alpha)_{n}}{n !} z^{n}=\sum_{n=0}^{\infty}\left(\begin{array}{l}
\alpha \\
n
\end{array}\right) z^{n}=(1+z)^{\alpha},
$$




$$
\begin{aligned}
{ }_{2} F_{1}(1,1 ; 2 ;-z) z & =\sum_{n=0}^{\infty} \frac{n ! n !}{n !(n+1) !}(-1)^{n} z^{n+1}=\ln (1+z), \\
\lim _{\beta \rightarrow \infty}{ }_{2} F_{1}(1, \beta ; 1 ; z / \beta) & =\lim _{\beta \rightarrow \infty} \sum_{n=0}^{\infty} \frac{n !(\beta)_{n}}{n ! n ! \beta^{n}} z^{n}=\sum_{n=0}^{\infty} \frac{1}{n !} z^{n}=e^{z}, \\
\lim _{\alpha, \beta \rightarrow \infty} F_{1}\left(\alpha, \beta ; 3 / 2 ;-z^{2} / 4 \alpha \beta\right) z & =\lim _{\alpha, \beta \rightarrow \infty} \sum_{n=0}^{\infty} \frac{(\alpha)_{n}(\beta)_{n}}{n !(3 / 2)_{n} \alpha^{n} \beta^{n} 4^{n}}(-1)^{n} z^{2 n+1} \\
& =\sum_{n=0}^{\infty} \frac{(-1)^{n}}{(2 n+1) !} z^{2 n+1}=\sin z \\
\lim _{\alpha, \beta \rightarrow \infty}{ }_{2} F_{1}\left(\alpha, \beta ; 1 / 2 ;-z^{2} / 4 \alpha \beta\right) & =\lim _{\alpha, \beta \rightarrow \infty} \frac{\sum_{n=0}^{\infty} \frac{(\alpha)_{n}(\beta)_{n}}{n !(1 / 2)_{n} \alpha^{n} \beta^{n} 4^{n}}(-1)^{n} z^{2 n}}{\infty}=\sum_{n=0}^{\infty} \frac{(-1)^{n}}{(2 n) !} z^{2 n}=\cos z .
\end{aligned}
$$

Less obviously,

$$
{ }_{2} F_{1}\left(1 / 2,1 / 2 ; 3 / 2 ; z^{2}\right) z=\arcsin z, \quad{ }_{2} F_{1}\left(1 / 2,1 ; 3 / 2 ;-z^{2}\right) z=\arctan z
$$

In addition,

$$
\begin{aligned}
\frac{d}{d z}{ }_{2} F_{1}(\alpha, \beta ; \gamma ; z) & =\sum_{n=0}^{\infty} \frac{(\alpha)_{n}(\beta)_{n}}{(n-1) !(\gamma)_{n}} z^{n-1} \\
& =\sum_{n=0}^{\infty} \frac{(\alpha)_{n+1}(\beta)_{n+1}}{n !(\gamma)_{n+1}} z^{n} \\
& =\frac{\alpha \beta}{\gamma} \sum_{n=0}^{\infty} \frac{(\alpha+1)_{n}(\beta+1)_{n}}{n !(\gamma+1)_{n}} z^{n} \\
& =\frac{\alpha \beta}{\gamma}{ }_{2} F_{1}(\alpha+1, \beta+1 ; \gamma+1 ; z)
\end{aligned}
$$

Furthermore, we have the following Euler's Integral Representation.

Theorem 3.2.1. If $\operatorname{Re} \gamma>\operatorname{Re} \beta>0$, then

$$
{ }_{2} F_{1}(\alpha, \beta ; \gamma ; z)=\frac{\Gamma(\gamma)}{\Gamma(\beta) \Gamma(\gamma-\beta)} \int_{0}^{1} t^{\beta-1}(1-t)^{\gamma-\beta-1}(1-z t)^{-\alpha} d t
$$

in the $z$ plane cut along the real axis from 1 to $\infty$. 
Proof. First we suppose $|z|<1$. We calculate

$$
\begin{aligned}
& \frac{\Gamma(\gamma)}{\Gamma(\beta) \Gamma(\gamma-\beta)} \int_{0}^{1} t^{\beta-1}(1-t)^{\gamma-\beta-1}(1-z t)^{-\alpha} d t \\
= & \frac{\Gamma(\gamma)}{\Gamma(\beta) \Gamma(\gamma-\beta)} \sum_{n=0}^{\infty}(-1)^{n}\left(\begin{array}{c}
-\alpha \\
n
\end{array}\right) z^{n} \int_{0}^{1} t^{\beta+n-1}(1-t)^{\gamma-\beta-1} d t \\
= & \frac{\Gamma(\gamma)}{\Gamma(\beta) \Gamma(\gamma-\beta)} \sum_{n=0}^{\infty} \frac{(\alpha)_{n}}{n !} z^{n} B(\beta+n, \gamma-\beta) \\
= & \frac{\Gamma(\gamma)}{\Gamma(\beta) \Gamma(\gamma-\beta)} \sum_{n=0}^{\infty} \frac{(\alpha)_{n}}{n !} \frac{\Gamma(\beta+n) \Gamma(\gamma-\beta)}{\Gamma(\gamma+n)} z^{n} \\
= & \sum_{n=0}^{\infty} \frac{(\alpha)_{n} \Gamma(\beta+n) \Gamma(\gamma)}{n ! \Gamma(\beta) \Gamma(\gamma+n)} z^{n} \\
= & \sum_{n=0}^{\infty} \frac{(\alpha)_{n}(\beta)_{n}}{n !(\gamma)_{n}} z^{n}={ }_{2} F_{1}(\alpha, \beta ; \gamma ; z)
\end{aligned}
$$

by (3.1.10), (3.1.11) and Theorem 3.1.1. So the theorem holds for $|z|<1$.

Since the integral in (3.2.20) is analytic in the cut plane, the theorem holds for $z$ in this region as well.

Theorem 3.2.2 (Gauss (1812)). If $\operatorname{Re}(\gamma-\alpha-\beta)>0$, then

$$
{ }_{2} F_{1}(\alpha, \beta ; \gamma ; 1)=\frac{\Gamma(\gamma) \Gamma(\gamma-\alpha-\beta)}{\Gamma(\gamma-\alpha) \Gamma(\gamma-\beta)} .
$$

Proof. By Abel's continuity theorem, (3.2.20) and Theorem 3.1.1,

$$
\begin{aligned}
{ }_{2} F_{1}(\alpha, \beta ; \gamma ; 1) & =\lim _{z \rightarrow 1-} \frac{\Gamma(\gamma)}{\Gamma(\beta) \Gamma(\gamma-\beta)} \int_{0}^{1} t^{\beta-1}(1-t)^{\gamma-\beta-1}(1-z t)^{-\alpha} d t \\
& =\frac{\Gamma(\gamma)}{\Gamma(\beta) \Gamma(\gamma-\beta)} \int_{0}^{1} t^{\beta-1}(1-t)^{\gamma-\beta-\alpha-1} d t \\
& =\frac{\Gamma(\gamma)}{\Gamma(\beta) \Gamma(\gamma-\beta)} B(\beta, \gamma-\beta-\alpha) \\
& =\frac{\Gamma(\gamma) \Gamma(\gamma-\alpha-\beta)}{\Gamma(\gamma-\alpha) \Gamma(\gamma-\beta)}
\end{aligned}
$$

when $\operatorname{Re} \gamma>\operatorname{Re} \beta>0$ and $\operatorname{Re}(\gamma-\alpha-\beta)>0$. The condition $\operatorname{Re} \gamma>\operatorname{Re} \beta>0$ can be removed in (3.2.22) by the continuity in $\beta$ and $\gamma$.

By (3.1.10), we have:

Corollary 3.2.3 (Chu-Vandermonde). For $n \in \mathbb{N}$,

$$
{ }_{2} F_{1}(-n, \beta ; \gamma ; 1)=\frac{(\gamma-\beta)_{n}}{(\gamma)_{n}} .
$$




\subsection{Orthogonal Polynomials}

Let $k \in \mathbb{N}$,

$$
{ }_{2} F_{1}(-k, \beta ; \gamma ; z)=\sum_{n=0}^{k} \frac{(-k)_{n}(\beta)_{n}}{n !(\gamma)_{n}} z^{n}=\sum_{n=0}^{k}\left(\begin{array}{l}
k \\
n
\end{array}\right) \frac{(\beta)_{n}}{(\gamma)_{n}}(-z)^{n}
$$

is a polynomial. We calculate the generating function

$$
\begin{aligned}
& \sum_{k=0}^{\infty} \frac{(\gamma)_{k} x^{k}}{k !}{ }_{2} F_{1}(-k, \beta ; \gamma ; z)=\sum_{k=0}^{\infty} \sum_{n=0}^{k} \frac{(\gamma+n)_{k-n}(\beta)_{n}}{n !(k-n) !} x^{k}(-z)^{n} \\
= & \sum_{k=0}^{\infty} \sum_{n=0}^{k}\left(\begin{array}{c}
\gamma+k-1 \\
k-n
\end{array}\right)\left(\begin{array}{c}
-\beta \\
n
\end{array}\right) x^{k} z^{n}=\sum_{m, n=0}^{\infty}\left(\begin{array}{c}
\gamma+m+n-1 \\
m
\end{array}\right)\left(\begin{array}{c}
-\beta \\
n
\end{array}\right) x^{m+n} z^{n} \\
= & \sum_{n=0}^{\infty}(1-x)^{-\gamma-n}\left(\begin{array}{c}
-\beta \\
n
\end{array}\right) x^{n} z^{n}=(1-x)^{-\gamma}\left(1+\frac{x z}{1-x}\right)^{-\beta} \\
= & (1-x)^{\beta-\gamma}(1+(z-1) x)^{-\beta} .
\end{aligned}
$$

Set

$$
w_{k}(\vartheta, \gamma ; z)={ }_{2} F_{1}(-k, \vartheta+k ; \gamma ; z)
$$

According to (3.2.1),

$$
z(1-z) w_{k}^{\prime \prime}+[\gamma-(\vartheta+1) z] w_{k}^{\prime}+k(\vartheta+k) w_{k}=0
$$

Thus

$$
\frac{d}{d z}\left[z^{\gamma}(1-z)^{\vartheta-\gamma+1} w_{k}^{\prime}\right]+k(\vartheta+k) z^{\gamma-1}(1-z)^{\vartheta-\gamma} w_{k}=0 .
$$

Let $m, n \in \mathbb{N}$ such that $m \neq n$. Then

$$
w_{m} \frac{d}{d z}\left[z^{\gamma}(1-z)^{\vartheta-\gamma+1} w_{n}^{\prime}\right]+n(\vartheta+n) z^{\gamma-1}(1-z)^{\vartheta-\gamma} w_{m} w_{n}=0
$$

and

$$
w_{n} \frac{d}{d z}\left[z^{\gamma}(1-z)^{\vartheta-\gamma+1} w_{m}^{\prime}\right]+m(\vartheta+m) z^{\gamma-1}(1-z)^{\vartheta-\gamma} w_{m} w_{n}=0 .
$$

Assume that $\operatorname{Re} \gamma>0, \operatorname{Re}(\vartheta-\gamma)>-1$ and $\vartheta \notin-\mathbb{N}-1$. Then

$$
\begin{aligned}
& \int_{0}^{1} z^{\gamma-1}(1-z)^{\vartheta-\gamma} w_{m} w_{n} d z \\
= & \frac{1}{(m-n)(m+n+\vartheta)} \int_{0}^{1}[m(\vartheta+m)-n(\vartheta+n)] z^{\gamma-1}(1-z)^{\vartheta-\gamma} w_{m} w_{n} d z \\
= & \frac{1}{(m-n)(m+n+\vartheta)}\left[\int_{0}^{1} w_{m} \frac{d}{d z}\left[z^{\gamma}(1-z)^{\vartheta-\gamma+1} w_{n}^{\prime}\right] d z\right. \\
& \left.-\int_{0}^{1} w_{n} \frac{d}{d z}\left[z^{\gamma}(1-z)^{\vartheta-\gamma+1} w_{m}^{\prime}\right] d z\right] \\
= & \left.\frac{z^{\gamma}(1-z)^{\vartheta-\gamma+1}\left(w_{m} w_{n}^{\prime}-w_{m}^{\prime} w_{n}\right)}{(m-n)(m+n+\vartheta)}\right|_{0} ^{1}=0 .
\end{aligned}
$$


Let $\mathcal{C}_{z}$ be a loop around $z$. According to (3.3.2),

$$
\begin{aligned}
& \frac{(\gamma)_{k}}{k !}{ }_{2} F_{1}(-k, \beta ; \gamma ; z) \\
= & \frac{1}{2 \pi i} \int_{\mathcal{C}_{0}} \frac{(1-x)^{\beta-\gamma}(1+(z-1) x)^{-\beta}}{x^{k+1}} d x \\
x=\frac{s-z}{s(1-z)} & \frac{1}{2 \pi i} \int_{\mathcal{C}_{z}} \frac{[z(1-s) / s(1-z)]^{\beta-\gamma}(z / s)^{-\beta}}{[(s-z) / s(1-z)]^{k+1}} \frac{z}{s^{2}(1-z)} d s \\
= & \frac{z^{1-\gamma}(1-z)^{\gamma-\beta+k}}{2 \pi i} \int_{\mathcal{C}_{z}} \frac{s^{\gamma+k-1}(1-s)^{\beta-\gamma}}{(s-z)^{k+1}} d s \\
= & \frac{z^{1-\gamma}(1-z)^{\gamma-\beta+k}}{2 \pi i k !}\left(\frac{d}{d z}\right)^{k} \int_{\mathcal{C}_{z}} \frac{s^{\gamma+k-1}(1-s)^{\beta-\gamma}}{s-z} d s \\
= & \frac{z^{1-\gamma}(1-z)^{\gamma-\beta+k}}{k !}\left(\frac{d}{d z}\right)^{k}\left[z^{\gamma+k-1}(1-z)^{\beta-\gamma}\right] .
\end{aligned}
$$

Hence

$$
w_{k}(\vartheta, \gamma ; z)=\frac{z^{1-\gamma}(1-z)^{\gamma-\vartheta}}{(\gamma)_{k}}\left(\frac{d}{d z}\right)^{k}\left[z^{\gamma+k-1}(1-z)^{\vartheta-\gamma+k}\right] .
$$

By (3.3.1),

$$
\left(\frac{d}{d z}\right)^{k}\left(w_{k}\right)=\left(\frac{d}{d z}\right)^{k}\left(\sum_{n=0}^{k}\left(\begin{array}{l}
k \\
n
\end{array}\right) \frac{(\vartheta+k)_{n}}{(\gamma)_{n}}(-z)^{n}\right)=\frac{(-1)^{k} k !(\vartheta+k)_{k}}{(\gamma)_{k}} .
$$

Thus

$$
\begin{aligned}
& \int_{0}^{1} z^{\gamma-1}(1-z)^{\vartheta-\gamma} w_{k}^{2} d z \\
= & \frac{1}{(\gamma)_{k}} \int_{0}^{1} w_{k}\left(\frac{d}{d z}\right)^{k}\left[z^{\gamma+k-1}(1-z)^{\vartheta-\gamma+k}\right] d z \\
= & \frac{(-1)^{k}}{(\gamma)_{k}} \int_{0}^{1}\left(\frac{d}{d z}\right)^{k}\left(w_{k}\right) z^{\gamma+k-1}(1-z)^{\vartheta-\gamma+k} d z \\
= & \frac{k !(\vartheta+k)_{k}}{\left((\gamma)_{k}\right)^{2}} \int_{0}^{1} z^{\gamma+k-1}(1-z)^{\vartheta-\gamma+k} d z \\
= & \frac{k !(\vartheta+k)_{k} \Gamma(\gamma+k) \Gamma(\vartheta-\gamma+k+1)}{\left((\gamma)_{k}\right)^{2} \Gamma(\vartheta+2 k+1)} \\
= & \frac{k !(\vartheta+k)_{k} \Gamma(\gamma) \Gamma(\vartheta-\gamma+k+1)}{(\gamma)_{k} \Gamma(\vartheta+2 k+1)} .
\end{aligned}
$$

Therefore $\left\{w_{k}(\vartheta, \gamma ; z) \mid k \in \mathbb{N}\right\}$ forms a set of orthogonal polynomials with respect to the weight $z^{\gamma-1}(1-z)^{\vartheta-\gamma}$. The Jacobi polynomials

$$
P_{k}^{(\alpha, \beta)}(z)=\left(\begin{array}{c}
\alpha+k \\
k
\end{array}\right) w_{k}\left(\alpha+\beta+1, \alpha+1 ; \frac{1-z}{2}\right) .
$$


Indeed $\left\{P_{k}^{(\alpha, \beta)}(z) \mid k \in \mathbb{N}\right\}$ forms a complete set of orthogonal functions on $[-1,1]$ with respect to the weight $(1-z)^{\alpha}(1+z)^{\beta}$. According (3.3.10),

$$
P_{k}^{(\alpha, \beta)}(z)=\frac{(-2)^{-k}}{k !}(1-z)^{-\alpha}(1+z)^{-\beta}\left(\frac{d}{d z}\right)^{k}\left[(1-z)^{\alpha+k}(1+z)^{\beta+k}\right] .
$$

The well known Chebyshev polynomials of first kind

$$
T_{k}(z)=\frac{1}{\left(\begin{array}{c}
-1 / 2+k \\
k
\end{array}\right)} P_{k}^{(-1 / 2,-1 / 2)}(z)=\frac{(-1)^{k} \sqrt{1-z^{2}}}{(2 k-1) ! !}\left(\frac{d}{d z}\right)^{k}\left[\left(1-z^{2}\right)^{k-1 / 2}\right] .
$$

The well known Chebyshev polynomials of second kind

$$
U_{k}(z)=\frac{(k+1) !}{\left(\begin{array}{c}
1 / 2+k \\
k
\end{array}\right)} P_{k}^{(1 / 2,1 / 2)}(z)=\frac{(-1)^{k}(k+1) !}{(2 k+1) ! ! \sqrt{1-z^{2}}}\left(\frac{d}{d z}\right)^{k}\left[\left(1-z^{2}\right)^{k+1 / 2}\right] .
$$

Equation

$$
\left(1-z^{2}\right) y^{\prime \prime}-2 z y^{\prime}+\nu(\nu+1) y=0
$$

is called a Legendre equation, where $\nu$ is a constant. Suppose that $y=\sum_{n=0}^{\infty} c_{n} z^{n}$ is a solution of (3.3.17). Then

$$
\left(1-z^{2}\right)\left(\sum_{n=2}^{\infty} n(n-1) c_{n} z^{n-2}\right)-2 \sum_{n=1}^{\infty} n c_{n} z^{n}+\nu(\nu+1) \sum_{n=0}^{\infty} c_{n} z^{n}=0,
$$

equivalently,

$$
(n+2)(n+1) c_{n+2}+[\nu(\nu+1)-n(n+1)] c_{n}=0 .
$$

Thus

$$
c_{n+2}=\frac{(n-\nu)(n+1+\nu)}{(n+2)(n+1)} c_{n}
$$

By induction,

$$
\begin{gathered}
c_{2 n}=\frac{\prod_{i=0}^{n-1}(2 i-\nu)(2 i+1+\nu)}{(2 n) !} c_{0}=\frac{(-\nu / 2)_{n}((1+\nu) / 2)_{n}}{n !(1 / 2)_{n}} c_{0}, \\
c_{2 n+1}=\frac{\prod_{i=0}^{n-1}(2 i+1-\nu)(2 i+2+\nu)}{(2 n+1) !} c_{1}=\frac{((1-\nu) / 2)_{n}((2+\nu) / 2)_{n}}{n !(3 / 2)_{n}} c_{1} .
\end{gathered}
$$

Thus for generic $\nu$, we have the fundamental solutions

$$
\sum_{n=0}^{\infty} \frac{(-\nu / 2)_{n}((1+\nu) / 2)_{n}}{n !(1 / 2)_{n}} z^{2 n}={ }_{2} F_{1}\left(-\frac{\nu}{2}, \frac{1+\nu}{2} ; \frac{1}{2} ; z^{2}\right)
$$

and

$$
\sum_{n=0}^{\infty} \frac{((1-\nu) / 2)_{n}((2+\nu) / 2)_{n}}{n !(3 / 2)_{n}} z^{2 n+1}={ }_{2} F_{1}\left(\frac{1-\nu}{2}, \frac{2+\nu}{2} ; \frac{3}{2} ; z^{2}\right) z
$$


which are called Legendre functions. When $\nu=2 k$ is nonnegative even integer, the first solution is a polynomial and we denote the Legendre polynomial

$$
P_{2 k}(z)=\frac{(-1)^{k}(1 / 2)_{k}}{k !}{ }_{2} F_{1}\left(-k, \frac{1}{2}+k ; \frac{1}{2} ; z^{2}\right) .
$$

If $\nu=2 k+1$ is an odd integer, the second solution is a polynomial and we denote the Legendre polynomial

$$
P_{2 k+1}(z)=\frac{(-1)^{k} 2(1 / 2)_{k+1}}{k !}{ }_{2} F_{1}\left(-k, \frac{3}{2}+k ; \frac{3}{2} ; z^{2}\right) z .
$$

Theorem 3.3.1. For $n \in \mathbb{N}$,

$$
P_{n}(z)=\frac{1}{2^{n} n !}\left(\frac{d}{d z}\right)^{n}\left[\left(z^{2}-1\right)^{n}\right]
$$

Proof. For convenience, we set

$$
\psi_{n}=\left(\frac{d}{d z}\right)^{n}\left[\left(z^{2}-1\right)^{n}\right]
$$

We want to prove

$$
\left(1-z^{2}\right) \psi_{n}^{\prime \prime}-2 z \psi_{n}^{\prime}+n(n+1) \psi_{n}=0,
$$

which is equivalent to

$$
\left[\left(1-z^{2}\right) \psi_{n}^{\prime}\right]^{\prime}+n(n+1) \psi_{n}=0
$$

Explicitly, (3.3.30) is

$$
\left[\left(1-z^{2}\right)\left(\frac{d}{d z}\right)^{n+1}\left[\left(z^{2}-1\right)^{n}\right]+n(n+1)\left(\frac{d}{d z}\right)^{n-1}\left[\left(z^{2}-1\right)^{n}\right]\right]^{\prime}=0,
$$

equivalently,

$$
\left(1-z^{2}\right)\left(\frac{d}{d z}\right)^{n+1}\left[\left(z^{2}-1\right)^{n}\right]+n(n+1)\left(\frac{d}{d z}\right)^{n-1}\left[\left(z^{2}-1\right)^{n}\right]=0
$$


due to that both terms are equal to zero when $z=1$. Note

$$
\begin{aligned}
& \left(1-z^{2}\right)\left(\frac{d}{d z}\right)^{n+1}\left[\left(z^{2}-1\right)^{n}\right] \\
& =\left(1-z^{2}\right)\left(\frac{d}{d z}\right)^{n+1}\left[(z-1)^{n}(z+1)^{n}\right] \\
& =-\sum_{s=0}^{n-1}\left(\begin{array}{c}
n+1 \\
s+1
\end{array}\right)\left[\prod_{p=0}^{s}(n-p)\right]\left[\prod_{r=s+1}^{n} r\right](z-1)^{n-s}(z+1)^{s+1} \\
& =-\sum_{s=0}^{n-1} \frac{(n+1) !\left[\prod_{p=0}^{s}(n-p)\right]\left[\prod_{r=s+1}^{n} r\right]}{(s+1) !(n-s) !}(z-1)^{n-s}(z+1)^{s+1} \\
& =-\sum_{s=0}^{n-1} \frac{(n+1) !\left[\prod_{p=0}^{s-1}(n-p)\right]\left[\prod_{r=s+2}^{n} r\right]}{s !(n-s-1) !}(z-1)^{n-s}(z+1)^{s+1} \\
& =-n(n+1) \sum_{s=0}^{n-1} \frac{(n-1) !\left[\prod_{p=0}^{s-1}(n-p)\right]\left[\prod_{r=s+2}^{n} r\right]}{s !(n-s-1) !}(z-1)^{n-s}(z+1)^{s+1} \\
& =-n(n+1) \sum_{s=0}^{n-1}\left(\begin{array}{c}
n-1 \\
s
\end{array}\right)\left[\prod_{p=0}^{s-1}(n-p)\right]\left[\prod_{r=s+2}^{n} r\right](z-1)^{n-s}(z+1)^{s+1} \\
& =-n(n+1)\left(\frac{d}{d z}\right)^{n-1}\left[(z-1)^{n}(z+1)^{n}\right] \\
& =-n(n+1)\left(\frac{d}{d z}\right)^{n-1}\left[\left(z^{2}-1\right)^{n}\right] \text {, }
\end{aligned}
$$

that is (3.3.32) holds.

On the other hand,

$$
\frac{1}{2^{n} n !} \psi_{n}=\left(\frac{d}{d z}\right)^{n} \sum_{r=0}^{n} \frac{(-1)^{r} z^{2 n-2 r}}{r !(n-r) ! 2^{n}}
$$

Thus for $k \in \mathbb{N}$,

$$
\left.\frac{1}{2^{2 k}(2 k) !} \psi_{2 k}(z)\right|_{z=0}=\frac{(-1)^{k}(2 k) !}{(k !)^{2} 2^{2 k}}=\frac{(-1)^{k}(1 / 2)_{k}}{k !}
$$

and

$$
\left.\frac{1}{2^{2 k+1}(2 k+1) ! z} \psi_{2 k+1}(z)\right|_{z=0}=\frac{(-1)^{k}(2 k+2) !}{k !(k+1) ! 2^{2 k+1}}=\frac{(-1)^{k} 2(1 / 2)_{k+1}}{k !}
$$

This shows that both $\psi_{n}(z) /\left(2^{n} n !\right)$ and $P_{n}(z)$ are polynomial solutions of the equation

$$
\left(1-z^{2}\right) y^{\prime \prime}-2 z y^{\prime}+n(n+1) y=0
$$

with the same term of lowest degree. Observe that any power series solution $y=\sum_{r=0}^{\infty} c_{r} z^{r}$ of (3.3.37) must be a linear combination of (3.3.23) and (3.3.24), one of which is not polynomial. Thus any two polynomial solutions of (3.3.37) must be proportional. Hence $P_{n}(z)=\psi_{n}(z) /\left(2^{n} n !\right)$, that is, (3.3.27) holds. 
Let $m, n \in \mathbb{N}$ such that $m \neq n$. Then

$$
\begin{gathered}
{\left[\left(1-z^{2}\right) P_{m}^{\prime}(z)\right]^{\prime} P_{n}(z)+m(m+1) P_{m}(z) P_{n}(z)=0} \\
P_{m}(z)\left[\left(1-z^{2}\right) P_{n}^{\prime}(z)\right]^{\prime}+n(n+1) P_{m}(z) P_{n}(z)=0 .
\end{gathered}
$$

Thus

$$
\begin{aligned}
& \int_{-1}^{1} P_{m}(z) P_{n}(z) d z \\
= & \frac{1}{(m-n)(m+n+1)} \int_{-1}^{1}[m(m+1)-n(n+1)] P_{m}(z) P_{n}(z) d z \\
= & \frac{1}{(m-n)(m+n+1)}\left[\int_{-1}^{1} P_{m}(z)\left[\left(1-z^{2}\right) P_{n}^{\prime}(z)\right]^{\prime} d z-\int_{-1}^{1}\left[\left(1-z^{2}\right) P_{m}^{\prime}(z)\right]^{\prime} P_{n}(z) d z\right] \\
= & \left.\frac{1}{(m-n)(m+n+1)}\left(P_{m}(z) P_{n}^{\prime}(z)-P_{m}^{\prime}(z) P_{n}(z)\right)\left(1-z^{2}\right)\right|_{-1} ^{1}=0 .
\end{aligned}
$$

According to (3.3.34),

$$
\left(\frac{d}{d z}\right)^{n}\left(P_{n}(z)\right)=\frac{(2 n) !}{n ! 2^{n}}=(2 n-1) ! !
$$

Hence

$$
\begin{aligned}
\int_{-1}^{1}\left(P_{n}(z)\right)^{2} d z & =\frac{1}{n ! 2^{n}} \int_{-1}^{1}\left(\frac{d}{d z}\right)^{n}\left[\left(z^{2}-1\right)^{n}\right] P_{n}(z) d z \\
& =\frac{1}{n ! 2^{n}} \int_{-1}^{1}(-1)^{n}\left(z^{2}-1\right)^{n}\left(\frac{d}{d z}\right)^{n}\left(P_{n}(z)\right) d z \\
& =\frac{(2 n-1) ! !}{n ! 2^{n}} \int_{-1}^{1}\left(1-z^{2}\right)^{n} d z=\frac{2(2 n-1) ! !}{n ! 2^{n}} \int_{0}^{1}\left(1-z^{2}\right)^{n} d z \\
& =\frac{(2 n-1) ! !}{n ! 2^{n}} \int_{0}^{1} x^{-1 / 2}(1-x)^{n} d x=\frac{(2 n-1) ! ! \Gamma(1 / 2) \Gamma(n+1)}{n ! 2^{n} \Gamma(n+3 / 2)} \\
& =\frac{2(2 n-1) ! !}{(2 n+1) ! !}=\frac{2}{2 n+1} .
\end{aligned}
$$

Legendre polynomials $\left\{P_{k}(z) \mid k \in \mathbb{N}\right\}$ have been used to solve the quantum two-body system.

\section{Exercise 3.3}

Find the differential equations satisfied by Jacobi polynomials and prove that Chebyshev polynomials of each kind form a set of orthogonal polynomials. 


\subsection{Weierstrass's Elliptic Functions}

For two integers $m<n$, we denote

$$
\overline{m, n}=\{m, m+1, \ldots, n\}, \overline{m, m}=\{m\}, \overline{n, m}=\emptyset .
$$

Let $\omega_{1}$ and $\omega_{2}$ be two linearly independent elements in the complex $z$-plane. Denote the lattice

$$
L=\left\{m \omega_{1}+n \omega_{2} \mid m, n \in \mathbb{Z}\right\}, \quad L^{\prime}=L \backslash\{0\}
$$

Lemma 3.4.1. For any $2<a \in \mathbb{R}$, the series

$$
\sum_{\omega \in L^{\prime}} \frac{1}{\omega^{a}}
$$

converges absolutely.

Proof. For $k \in \mathbb{N}+1$, we denote

$$
P_{k}=\left\{ \pm k \omega_{1}+r \omega_{2}, r \omega_{1} \pm k \omega_{2} \mid r \in \overline{-k, k}\right\}
$$

the set of the elements in $L$ lying on the parallelogram with vertices $\left\{ \pm k \omega_{1} \pm k \omega_{2}\right\}$. Denote

$$
\delta=\min \left\{\left|\omega_{1}\right|,\left|\omega_{2}\right|\right\}
$$

Then

$$
k \delta \leq|\omega| \text { for any } \omega \in P_{k} .
$$

Moreover, the number of elements

$$
\left|P_{k}\right|=8 k \text {. }
$$

Now

$$
\sum_{\omega \in L^{\prime}} \frac{1}{|\omega|^{a}}=\sum_{k=1}^{\infty} \sum_{\omega \in P_{k}} \frac{1}{|\omega|^{a}}<\sum_{k=1}^{\infty} \frac{8 k}{(k \delta)^{a}}=8 \delta^{-a} \sum_{k=1}^{\infty} \frac{1}{k^{a-1}},
$$

where the last series converges by calculus.

The Weierstrass's Elliptic Function

$$
\wp(z)=\frac{1}{z^{2}}+\sum_{\omega \in L^{\prime}}\left[\frac{1}{(z-\omega)^{2}}-\frac{1}{\omega^{2}}\right] .
$$

For any $z \in \mathbb{C} \backslash L$,

$$
\lim _{|\omega| \rightarrow \infty} \frac{\left[\frac{1}{(z-\omega)^{2}}-\frac{1}{\omega^{2}}\right]}{\frac{1}{\omega^{3}}}=\lim _{|\omega| \rightarrow \infty} \frac{z \omega(2 \omega-z)}{(z-\omega)^{2}}=2 z .
$$


Since $\sum_{\omega \in L^{\prime}} \frac{1}{\omega^{3}}$ converges absolutely by Lemma 3.4.1, the series in (3.4.9) converges absolutely. As $L^{\prime}=-L^{\prime}$, we have

$$
\begin{aligned}
\wp(-z) & =\frac{1}{(-z)^{2}}+\sum_{\omega \in L^{\prime}}\left[\frac{1}{(-z-\omega)^{2}}-\frac{1}{\omega^{2}}\right] \\
& =\frac{1}{z^{2}}+\sum_{\omega \in L^{\prime}}\left[\frac{1}{(z+\omega)^{2}}-\frac{1}{\omega^{2}}\right] \\
& =\frac{1}{z^{2}}+\sum_{-\omega \in-L^{\prime}}\left[\frac{1}{(z-(-\omega))^{2}}-\frac{1}{(-\omega)^{2}}\right] \\
& =\frac{1}{z^{2}}+\sum_{\tilde{\omega} \in L^{\prime}}\left[\frac{1}{(z-\tilde{\omega})^{2}}-\frac{1}{\tilde{\omega}^{2}}\right]=\wp(z),
\end{aligned}
$$

that is, $\wp(z)$ is an even function.

We calculate

$$
\wp^{\prime}(z)=-\frac{2}{z^{3}}-2 \sum_{\omega \in L^{\prime}} \frac{1}{(z-\omega)^{3}}=-2 \sum_{\omega \in L} \frac{1}{(z-\omega)^{3}}
$$

which converges absolutely for any $z \in \mathbb{C} \backslash L$. Since $L=-L, \wp^{\prime}(z)$ is an odd function by the similar argument as (3.4.11). For any $\omega \in L$, we have $L-\omega=L$ and

$$
\begin{aligned}
\wp^{\prime}(z+\omega) & =-2 \sum_{\omega^{\prime} \in L} \frac{1}{\left(z+\omega-\omega^{\prime}\right)^{3}}=-2 \sum_{\omega^{\prime}-\omega \in L-\omega} \frac{1}{\left(z-\left(\omega^{\prime}-\omega\right)\right)^{3}} \\
& =-2 \sum_{\tilde{\omega} \in L} \frac{1}{(z-\tilde{\omega})^{3}}=\wp^{\prime}(z) .
\end{aligned}
$$

So the elements of $L$ are periods of $\wp^{\prime}(z)$. Thus

$$
\wp(z+\omega)=\wp(z)+C
$$

for some constant $C$. Letting $z=-\omega / 2$ in (3.4.14), we have

$$
\wp(\omega / 2)=\wp(-\omega / 2)+C \Longrightarrow C=0
$$

by (3.4.11). Thus

$$
\wp(z+\omega)=\wp(z) \quad \text { for } \omega \in L,
$$

that is, $\wp(z)$ is a doubly periodic function.

Note that the function

$$
\wp_{*}(z)=\wp(z)-\frac{1}{z^{2}}=\sum_{\omega \in L^{\prime}}\left[\frac{1}{(z-\omega)^{2}}-\frac{1}{\omega^{2}}\right] .
$$

is analytic at $z=0$. Moreover,

$$
\wp_{*}^{(n)}(z)=(-1)^{n}(n+1) ! \sum_{\omega \in L^{\prime}} \frac{1}{(z-\omega)^{n+2}} .
$$


In particular,

$$
\wp_{*}^{(n)}(0)=(n+1) ! \sum_{\omega \in L^{\prime}} \frac{1}{\omega^{n+2}}
$$

For $m \in \mathbb{N}$,

$$
\begin{aligned}
\wp_{*}^{(2 m+1)}(0) & =(2 m+2) ! \sum_{\omega \in L^{\prime}} \frac{1}{\omega^{2 m+3}}=-(2 m+2) ! \sum_{-\omega \in-L^{\prime}} \frac{1}{(-\omega)^{2 m+3}} \\
& =-(2 m+2) ! \sum_{\tilde{\omega} \in L^{\prime}} \frac{1}{\tilde{\omega}^{2 m+3}}=-\wp_{*}^{(2 m+1)}(0) .
\end{aligned}
$$

Thus $\wp_{*}^{(2 m+1)}(0)=0$. Thanks to $(3.4 .17), \wp_{*}(0)=0$. Hence

$$
\wp_{*}(z)=\sum_{m=1}^{\infty} c_{m+1} z^{2 m}
$$

with

$$
c_{m+1}=\frac{\wp_{*}^{(2 m)}(0)}{(2 m) !}=(2 m+1) \sum_{\omega \in L^{\prime}} \frac{1}{\omega^{2 m+2}}
$$

by (3.4.19).

Now

$$
\wp(z)=\frac{1}{z^{2}}+\sum_{m=1}^{\infty} c_{m+1} z^{2 m} \Longrightarrow \wp^{\prime}(z)=-\frac{2}{z^{3}}+\sum_{m=1}^{\infty} 2 m c_{m+1} z^{2 m-1} .
$$

Moreover,

$$
\begin{aligned}
& \wp^{3}(z)=\frac{1}{z^{6}}+\frac{3 c_{2}}{z^{2}}+3 c_{3}+O(z) \\
& \wp^{\prime 2}(z)=\frac{4}{z^{6}}-\frac{8 c_{2}}{z^{2}}-16 c_{3}+O(z)
\end{aligned}
$$

Thus

$$
\left.\wp^{\prime 2}(z)\right)-4 \wp^{3}(z)=-\frac{20 c_{2}}{z^{2}}-28 c_{3}+O(z)
$$

Hence

$$
\psi=\wp^{\prime 2}(z)-4 \wp^{3}(z)+20 c_{2} \wp(z)+28 c_{3}
$$

is a function with periods in $L$ and only possible singular points in $L$. Since $\psi(0)=0$, we have $\psi(\omega)=\psi(0)=0$ for any $\omega \in L$. Hence $\psi$ is a holomorphic doubly periodic function. So $\psi$ is bounded. Thus $\psi(z) \equiv \psi(0)=0$. This proves:

Theorem 3.4.2. For $z \in \mathbb{C} \backslash L$,

$$
\wp^{\prime 2}(z)=4 \wp^{3}(z)-g_{2} \wp(z)-g_{3}
$$

with

$$
g_{2}=20 c_{2}=60 \sum_{\omega \in L^{\prime}} \frac{1}{\omega^{4}}, \quad g_{3}=28 c_{3}=140 \sum_{\omega \in L^{\prime}} \frac{1}{\omega^{6}} .
$$


Differentiating (3.4.28), we get

$$
2 \wp^{\prime}(z) \wp^{\prime \prime}(z)=12 \wp^{2}(z) \wp^{\prime}(z)-g_{2} \wp^{\prime}(z) .
$$

Hence

$$
\wp^{\prime \prime}(z)=6 \wp^{2}(z)-\frac{g_{2}}{2}
$$

which is very important in solving nonlinear partial differential equation.

Remark 3.4.3. Suppose $\operatorname{Re} \omega_{1} \neq 0$ and $\operatorname{Im} \omega_{1} \neq 0$. Then $\omega_{1}$ and its complex conjugate $\overline{\omega_{1}}$ are linearly independent. So we can take $\omega_{2}=\overline{\omega_{1}}$. In this case, $\bar{L}=L$. If $z \in \mathbb{R}$, then

$$
\overline{\wp(z)}=\frac{1}{z^{2}}+\sum_{\omega \in L^{\prime}}\left[\frac{1}{(z-\bar{\omega})^{2}}-\frac{1}{\bar{\omega}^{2}}\right]=\frac{1}{z^{2}}+\sum_{\tilde{\omega} \in \overline{L^{\prime}}=L^{\prime}}\left[\frac{1}{(z-\tilde{\omega})^{2}}-\frac{1}{\tilde{\omega}^{2}}\right]=\wp(z) .
$$

So $\wp(z)$ is a real-valued function on $\mathbb{R}$. Similarly, $g_{2}$ and $g_{3}$ are real constants. Since $\omega_{1}$ has two real freedom, $g_{2}$ and $g_{3}$ can take any two real numbers such that $g_{2}^{3}-27 g_{3}^{2} \neq 0$ (the condition comes from ellipticity (cf. [ARR, WG])).

Observe

$$
\frac{1}{z-\omega}+\frac{1}{\omega}+\frac{z}{\omega^{2}}=\frac{1}{z-\omega}+\frac{z+\omega}{\omega^{2}}=\frac{z^{2}}{\omega^{2}(z-\omega)}
$$

Thus the series

$$
\sum_{\omega \in L^{\prime}}\left[\frac{1}{z-\omega}+\frac{1}{\omega}+\frac{z}{\omega^{2}}\right]
$$

converges absolutely for any $z \in \mathbb{C} \backslash L$. The Weierstrass's zeta function:

$$
\zeta(z)=\frac{1}{z}+\sum_{\omega \in L^{\prime}}\left[\frac{1}{z-\omega}+\frac{1}{\omega}+\frac{z}{\omega^{2}}\right] .
$$

It is not the Riemann's zeta function! Obviously,

$$
\zeta^{\prime}(z)=-\wp(z)
$$

As the argument (3.4.11), $\zeta(z)$ is an odd function. Moreover,

$$
\zeta^{\prime}(z+\omega)=-\wp(z+\omega)=-\wp(z+\omega)=\zeta^{\prime}(\omega) \quad \text { for } \omega \in L .
$$

In particular, this implies that

$$
\zeta\left(z+\omega_{1}\right)=\zeta(z)+2 \eta_{1}, \quad \zeta\left(z+\omega_{2}\right)=\zeta(z)+2 \eta_{2}
$$

for some constants $\eta_{1}, \eta_{2} \in \mathbb{C}$. Taking $z=-\omega_{r} / 2$, we get

$$
\zeta\left(\omega_{r} / 2\right)=\zeta\left(-\omega_{r} / 2\right)+2 \eta_{r}
$$


Hence

$$
\eta_{1}=\zeta\left(\omega_{1} / 2\right), \quad \eta_{2}=\zeta\left(\omega_{2} / 2\right)
$$

Now we assume

$$
\operatorname{Im} \frac{\omega_{2}}{\omega_{1}}>0
$$

Let

$$
A=-\frac{\omega_{1}}{2}+\frac{\omega_{2}}{2}, \quad B=\frac{\omega_{1}}{2}+\frac{\omega_{2}}{2}, C=\frac{\omega_{1}}{2}-\frac{\omega_{2}}{2}, \quad D=-\frac{\omega_{1}}{2}-\frac{\omega_{2}}{2} .
$$

Denote by $X Y$ the oriented segment from $X$ to $Y$ on the complex plane. Let $\mathcal{C}$ be the parallelogram $A B C D$ with counterclockwise orientation. Since $z=0$ is the only pole of $\zeta(z)$ enclosed by the parallelogram. We have

$$
\begin{aligned}
2 \pi i= & \int_{\mathcal{C}} \zeta(z) d z=\int_{D C}\left(\zeta(z)-\zeta\left(z+\omega_{2}\right)\right) d z \\
& +\int_{C B}\left(\zeta(z)-\zeta\left(z-\omega_{1}\right) d z=-2 \eta_{2} \omega_{1}+2 \eta_{1} \omega_{2} .\right.
\end{aligned}
$$

Thus

$$
\eta_{1} \omega_{2}-\eta_{2} \omega_{1}=\pi i
$$

Note

$$
\begin{aligned}
& \left(1-\frac{z}{\omega}\right) e^{\frac{z}{\omega}+\frac{z^{2}}{2 \omega^{2}}} \\
= & \left(1-\frac{z}{\omega}\right)\left(1+\frac{z}{\omega}+\frac{z^{2}}{\omega^{2}}+\frac{2 z^{3}}{3 \omega^{3}}+O\left(\frac{z^{4}}{\omega^{4}}\right)\right) \\
= & 1-\frac{z^{3}}{3 \omega^{3}}+O\left(\frac{z^{4}}{\omega^{4}}\right) .
\end{aligned}
$$

Since

$$
\sum_{\omega \in L^{\prime}}\left(\frac{C z^{4}}{\omega^{4}}-\frac{z^{3}}{3 \omega^{3}}\right)
$$

converges absolutely for any given $z$ and $C$, the product

$$
\prod_{\omega \in L^{\prime}}\left(1-\frac{z}{\omega}\right) e^{\frac{z}{\omega}+\frac{z^{2}}{2 \omega^{2}}} \text { converges absolutely for any } z \in \mathbb{C} \backslash L .
$$

We define the Weierstrass's sigma function:

$$
\sigma(z)=z \prod_{\omega \in L^{\prime}}\left(1-\frac{z}{\omega}\right) e^{\frac{z}{\omega}+\frac{z^{2}}{2 \omega^{2}}}
$$

Then

$$
\ln \sigma(z)=\ln z+\sum_{\omega \in L^{\prime}}\left[\ln \left(1-\frac{z}{\omega}\right)+\frac{z}{\omega}+\frac{z^{2}}{2 \omega^{2}}\right]
$$

Thus

$$
\frac{\sigma^{\prime}(z)}{\sigma(z)}=\frac{1}{z}+\sum_{\omega \in L^{\prime}}\left[\frac{1}{z-\omega}+\frac{1}{\omega}+\frac{z}{\omega^{2}}\right]=\zeta(z) .
$$


By a similar argument as that of (3.4.11), $\sigma(z)$ is an odd function. Moreover, (3.4.37) and (3.4.49) yield

$$
\frac{\sigma^{\prime}\left(z+\omega_{1}\right)}{\sigma\left(z+\omega_{1}\right)}-\frac{\sigma^{\prime}(z)}{\sigma(z)}=2 \eta_{1}, \frac{\sigma^{\prime}\left(z+\omega_{2}\right)}{\sigma\left(z+\omega_{2}\right)}-\frac{\sigma^{\prime}(z)}{\sigma(z)}=2 \eta_{2} .
$$

Thus

$$
\frac{d}{d z} \ln \frac{\sigma\left(z+\omega_{r}\right)}{\sigma(z)}=2 \eta_{r} \Longrightarrow \ln \frac{\sigma\left(z+\omega_{r}\right)}{\sigma(z)}=2 \eta_{r} z+C_{r} .
$$

So

$$
\sigma\left(z+\omega_{r}\right)=\sigma(z) e^{2 \eta_{r} z+C_{r}} .
$$

Taking $z=-\omega_{r} / 2$ in (3.4.51), we get

$$
\sigma\left(\omega_{r} / 2\right)=\sigma\left(-\omega_{r} / 2\right) e^{-\eta_{r} \omega_{r}+C_{r}} \Longrightarrow e^{C_{r}}=-e^{\eta_{r} \omega_{r}} .
$$

Therefore,

$$
\sigma\left(z+\omega_{1}\right)=-\sigma(z) e^{\left(2 z+\omega_{1}\right) \eta_{1}}, \quad \sigma\left(z+\omega_{2}\right)=-\sigma(z) e^{\left(2 z+\omega_{2}\right) \eta_{2}} .
$$

Suppose $\operatorname{Re} \omega_{1} \neq 0$ and $\operatorname{Im} \omega_{1}<0$. Taking $\omega_{2}=\overline{\omega_{1}}$, we get two real-valued functions $\zeta(z)$ and $\sigma(z)$ for $z \in \mathbb{R}$.

\subsection{Jacobian Elliptic Functions}

Let $0<m<1$ be a real constant. Jacobian elliptic function sn $(z \mid m)$ is the inverse function of the Legendre's elliptic integral of first kind

$$
z=\int_{0}^{x} \frac{d t}{\sqrt{\left(1-t^{2}\right)\left(1-m^{2} t^{2}\right)}}
$$

that is, $x=\operatorname{sn}(z \mid m)$. The number $m$ is the elliptic modulus of $\operatorname{sn}(z \mid m)$. Moreover, we define

$$
\operatorname{cn}(z \mid m)=\sqrt{1-\mathrm{sn}^{2}(z \mid m)}, \quad \operatorname{dn}(z \mid m)=\sqrt{1-m^{2} \mathrm{sn}^{2}(z \mid m)} .
$$

Note

$$
z=\lim _{m \rightarrow 0} \int_{0}^{x} \frac{d t}{\sqrt{\left(1-t^{2}\right)\left(1-m^{2} t^{2}\right)}}=\int_{0}^{x} \frac{d t}{\sqrt{\left(1-t^{2}\right)}}=\arcsin x .
$$

Thus

$$
\lim _{m \rightarrow 0} \operatorname{sn}(z \mid m)=\sin z, \quad \lim _{m \rightarrow 0} \operatorname{cn}(z \mid m)=\cos z, \quad \lim _{m \rightarrow 0} \operatorname{dn}(z \mid m)=1 .
$$

On the other hand,

$$
z=\lim _{m \rightarrow 1} \int_{0}^{x} \frac{d t}{\sqrt{\left(1-t^{2}\right)\left(1-m^{2} t^{2}\right)}}=\int_{0}^{x} \frac{d t}{1-t^{2}}=\frac{1}{2} \ln \frac{1+x}{1-x},
$$


equivalently,

$$
\begin{gathered}
\frac{1+x}{1-x}=e^{2 z} \sim \frac{2}{1-x}-1=e^{2 z} \sim 1-x=\frac{2}{e^{2 z}+1} \\
\Longrightarrow x=1-\frac{2}{e^{2 z}+1}=\frac{e^{2 z}-1}{e^{2 z}+1}=\frac{e^{z}-e^{-z}}{e^{z}+e^{-z}}=\tanh z .
\end{gathered}
$$

Hence

$$
\lim _{m \rightarrow 1} \operatorname{sn}(z \mid m)=\tanh z, \quad \lim _{m \rightarrow 1} \operatorname{cn}(z \mid m)=\lim _{m \rightarrow 1} \operatorname{dn}(z \mid m)=\operatorname{sech} z .
$$

Taking derivative with respect to $z$ in (3.5.1), we get

$$
1=\frac{1}{\sqrt{\left(1-x^{2}\right)\left(1-m^{2} x^{2}\right)}} \frac{d x}{d z} \sim \frac{d x}{d z}=\sqrt{\left(1-x^{2}\right)\left(1-m^{2} x^{2}\right)}
$$

So

$$
\frac{d}{d z} \operatorname{sn}(z \mid m)=\sqrt{\left(1-\operatorname{sn}^{2}(z \mid m)\right)\left(1-m^{2} \operatorname{sn}^{2}(z \mid m)\right)}=\operatorname{cn}(z \mid m) \operatorname{dn}(z \mid m)
$$

Moreover,

$$
\begin{gathered}
\frac{d}{d z} \operatorname{cn}(z \mid m)=-\frac{\operatorname{sn}(z \mid m)}{\sqrt{1-\mathrm{sn}^{2}(z \mid m)}} \frac{d}{d z} \operatorname{sn}(z \mid m)=-\operatorname{sn}(z \mid m) \operatorname{dn}(z \mid m), \\
\frac{d}{d z} \operatorname{dn}(z \mid m)=-\frac{m^{2} \operatorname{sn}(z \mid m)}{\sqrt{1-m^{2} \operatorname{sn}^{2}(z \mid m)}} \frac{d}{d z} \operatorname{sn}(z \mid m)=-m^{2} \operatorname{sn}(z \mid m) \operatorname{cn}(z \mid m) .
\end{gathered}
$$

Rewrite (3.5.2) as

$$
\operatorname{sn}^{2}(z \mid m)+\operatorname{cn}^{2}(z \mid m)=1, \quad \operatorname{dn}^{2}(z \mid m)+m^{2} \operatorname{sn}^{2}(z \mid m)=1 .
$$

Now

$$
\begin{aligned}
\left(\frac{d}{d z}\right)^{2} \operatorname{sn}(z \mid m) & =\left(\frac{d}{d z} \operatorname{cn}(z \mid m)\right) \operatorname{dn}(z \mid m)+\operatorname{cn}(z \mid m)\left(\frac{d}{d z} \operatorname{dn}(z \mid m)\right) \\
& =-\operatorname{sn}(z \mid m) \operatorname{dn}^{2}(z \mid m)-m^{2} \operatorname{sn}(z \mid m) \operatorname{cn}^{2}(z \mid m) \\
& =-\operatorname{sn}(z \mid m)\left(1-m^{2} \operatorname{sn}^{2}(z \mid m)\right)-m^{2} \operatorname{sn}(z \mid m)\left(1-\operatorname{sn}^{2}(z \mid m)\right) \\
& =2 m^{2} \operatorname{sn}^{3}(z \mid m)-\left(m^{2}+1\right) \operatorname{sn}(z \mid m) \\
\left(\frac{d}{d z}\right)^{2} \operatorname{cn}(z \mid m) & =-\left(\frac{d}{d z} \operatorname{sn}(z \mid m)\right) \operatorname{dn}(z \mid m)-\operatorname{sn}(z \mid m)\left(\frac{d}{d z} \operatorname{dn}(z \mid m)\right) \\
& =-\operatorname{cn}(z \mid m) \operatorname{dn}^{2}(z \mid m)+m^{2} \operatorname{cn}(z \mid m) \operatorname{sn}^{2}(z \mid m) \\
& =-\operatorname{cn}^{2}(z \mid m)\left(1-m^{2}+m^{2} \operatorname{cn}^{2}(z \mid m)\right)+m^{2} \operatorname{cn}(z \mid m)\left(1-\operatorname{cn}^{2}(z \mid m)\right) \\
& =-2 m^{2} \operatorname{cn}^{3}(z \mid m)+\left(2 m^{2}-1\right) \operatorname{cn}(z \mid m)
\end{aligned}
$$




$$
\begin{aligned}
\left(\frac{d}{d z}\right)^{2} \operatorname{dn}(z \mid m) & =-m^{2}\left(\frac{d}{d z} \operatorname{sn}(z \mid m)\right) \operatorname{cn}(z \mid m)-m^{2} \operatorname{sn}(z \mid m)\left(\frac{d}{d z} \operatorname{cn}(z \mid m)\right) \\
& =-m^{2} \operatorname{dn}(z \mid m) \operatorname{cn}^{2}(z \mid m)+m^{2} \operatorname{dn}(z \mid m) \operatorname{sn}^{2}(z \mid m) \\
& =\operatorname{dn}(z \mid m)\left(1-m^{2}-\operatorname{dn}^{2}(z \mid m)\right)+\operatorname{dn}(z \mid m)\left(1-\operatorname{dn}^{2}(z \mid m)\right) \\
& =-2 \operatorname{dn}^{3}(z \mid m)+\left(2-m^{2}\right) \operatorname{dn}(z \mid m) .
\end{aligned}
$$

The above three equations are very useful in solving nonlinear partial differential equations such as nonlinear Schrödinger equations.

It is quite often to use (3.5.14)-(3.5.16) with similar equations for trigonometric functions as follows:

$$
\begin{aligned}
\tan ^{\prime} z=\tan ^{2} z+1, \quad \tan ^{\prime \prime} z & =2 \tan ^{3} z+2 \tan z, \\
\sec ^{\prime} z=\sec z \tan z, \quad \sec ^{\prime \prime} z & =2 \sec ^{3} z-\sec z, \\
\operatorname{coth}^{\prime} z=1-\operatorname{coth}^{2} z, \quad \operatorname{coth}^{\prime \prime} z & =2 \operatorname{coth}^{3} z-2 \operatorname{coth} z, \\
\operatorname{csch}^{\prime} z=-\operatorname{csch} z \operatorname{coth} z, \quad \operatorname{csch}^{\prime \prime}(z) & =2 \operatorname{csch}^{3} z+\operatorname{csch} z .
\end{aligned}
$$




\section{Part II}

\section{Partial Differential Equations}





\section{Chapter 4}

\section{First-Order or Linear Equations}

First in this chapter, we derive the commonly used method of characteristic lines for solving first-order quasilinear partial differential equations, including boundary-value problems. Then we talk about more sophisticated method of characteristic strip for solving nonlinear first-order of partial differential equations. Exact first-order partial differential equations are also handled.

Linear partial differential equations of flag type, including linear equations with constant coefficients, appear in many areas of mathematics and physics. A general equation of this type can not be solved by separation of variables. We use the grading technique from representation theory to solve flag partial differential equations and find the complete set of polynomial solutions. Our method also leads us to find a family of new special functions by which we are able to solve the initial-value problem of a large class of linear equations with constant coefficients.

We use the method of characteristic lines to prove a Campbell-Hausdorff-type factorization of exponential differential operators and then solve the initial-value problem of flag evolution partial differential equations. We also use the Campbell-Hausdorff-type factorization to solve the initial-value problem of generalized wave equations of flag type.

The Calogero-Sutherland model is an exactly solvable quantum many-body system in one-dimension (cf. [Cf], [Sb]). The model was used to study long-range interactions of $n$ particles. We prove that a two-parameter generalization of the Weyl function of type $A$ in representation theory is a solution of the Calogero-Sutherland model. If $n=2$, we find a connection between the Calogero-Sutherland model and the Gauss hypergeometric function. When $n>2$, a new class of multi-variable hypergeometric functions are found based on Etingof's work [Ep]. Finally in Chapter 4, we use matrix differential operators and Fourier expansions to solve the Maxwell equations, the free Dirac equations and the generalized acoustic system. 


\subsection{Method of Characteristics}

Let $n$ be a positive integer and let $x_{1}, x_{2}, \ldots, x_{n}$ be $n$ independent variables. Denote

$$
\vec{x}=\left(x_{1}, x_{2}, \ldots, x_{n}\right) .
$$

Suppose that $u(\vec{x})=u\left(x_{1}, x_{2}, \ldots, x_{n}\right)$ is a function in $x_{1}, x_{2}, \ldots, x_{n}$ determined by the quasilinear partial differential equation

$$
f_{1}(\vec{x}, u) u_{x_{1}}+f_{2}(\vec{x}, u) u_{x_{2}}+\cdots+f_{n}(\vec{x}, u) u_{x_{n}}=g(\vec{x}, u)
$$

subject to the condition

$$
\psi(\vec{x}, u)=0 \quad \text { on the surface } h(\vec{x})=0 .
$$

Geometrically, the above problem is equivalent to find a hypersuface $u=u\left(x_{1}, x_{2}, \ldots, x_{n}\right)$ in the $(n+1)$-dimensional space of $\left\{x_{1}, \ldots, x_{n}, u\right\}$ passing through the codimension-2 boundary (4.1.3) satisfying the equation (4.1.2). The idea of the method of characteristics is to find all the lines on the hypersurface passing through any point on the boundary (called characteristic lines). Suppose that we have a line

$$
x_{1}=x_{1}(s), \quad x_{2}=x_{2}(s), \ldots, \quad x_{n}=x_{n}(s), u=u(s)
$$

passing through a point $\left(x_{1}, \ldots, x_{n}, u\right)=\left(t_{1}, \ldots, t_{n}, t_{n+1}\right)$ on the boundary (4.1.3). Since $u$ is a function of $x_{1}, \ldots, x_{n}$ determining the hypersurface, we have

$$
\frac{d u}{d s}=u_{x_{1}} \frac{d x_{1}}{d s}+u_{x_{2}} \frac{d x_{2}}{d s}+\cdots+u_{x_{n}} \frac{d x_{n}}{d s}
$$

equivalently,

$$
\left(u_{x_{1}}, \ldots, u_{x_{n}},-1\right) \cdot\left(\frac{d x_{1}}{d s}, \ldots, \frac{d x_{n}}{d s}, \frac{d u}{d s}\right)=0 .
$$

On the other hand, (4.1.2) can be rewritten as

$$
\left(u_{x_{1}}, \ldots, u_{x_{n}},-1\right) \cdot\left(f_{1}, \ldots, f_{n}, g\right)=0 .
$$

Comparing the above two equation, we find that original problem is equivalent to solve the system of ordinary differential equations:

$$
\frac{d u}{d s}=g(\vec{x}, u), \quad \frac{d x_{r}}{d s}=f_{r}(\vec{x}, u), \quad r \in \overline{1, n}
$$

subject to the initial conditions:

$$
\begin{gathered}
\left.u\right|_{s=0}=t_{n+1},\left.\quad x_{r}\right|_{s=0}=t_{r}, \quad r \in \overline{1, n}, \\
\psi\left(t_{1}, \ldots, t_{n}, t_{n+1}\right)=0, \quad h\left(t_{1}, \ldots, t_{n}\right)=0 .
\end{gathered}
$$


Solving (4.1.8) and (4.1.9), we find

$$
u=\phi_{n+1}\left(s, t_{1}, \ldots, t_{n+1}\right), \quad x_{r}=\phi_{r}\left(s, t_{1}, \ldots, t_{n+1}\right), \quad r \in \overline{1, n} .
$$

Eliminating possible variables in $\left\{s, t_{1}, \ldots, t_{n+1}\right\}$ by (4.1.10) and (4.1.11), we obtain the solution of the original problem.

Example 4.1.1. Solve the equation $u_{x_{1}}-c u_{x_{2}}=0$ subject to $\left.u\right|_{x_{1}=0}=f\left(x_{2}\right)$, where $c$ is a constant and $f$ is a given function.

Solution. The system of characteristic lines is:

$$
\frac{d u}{d s}=0, \frac{d x_{1}}{d s}=1, \frac{d x_{2}}{d s}=-c .
$$

Initial conditions are:

$$
\begin{gathered}
\left.x_{1}\right|_{s=0}=t_{1},\left.\quad x_{2}\right|_{s=0}=t_{2},\left.\quad u\right|_{s=0}=t_{3}, \\
t_{3}=f\left(t_{2}\right), \quad t_{1}=0 .
\end{gathered}
$$

The solution of (4.1.12) and (4.1.13) is

$$
x_{1}=s, \quad x_{2}=-c s+t_{2}, \quad u=t_{3} .
$$

Thus $t_{2}=c x_{1}+x_{2}$ and the final solution is

$$
u=f\left(c x_{1}+x_{2}\right)
$$

Example 4.1.2. Solve the equation

$$
u_{x}+x^{2} u_{y}=-y u \text { subject to } u=f(y) \text { on } x=0 \text {. }
$$

Solution. The system of characteristic lines is:

$$
\frac{d x}{d s}=1, \frac{d y}{d s}=x^{2}, \frac{d u}{d s}=-y u .
$$

Initial conditions are:

$$
\begin{gathered}
\left.x\right|_{s=0}=t_{1},\left.\quad y\right|_{s=0}=t_{2},\left.\quad u\right|_{s=0}=t_{3}, \\
t_{3}=f\left(t_{2}\right), \quad t_{1}=0 .
\end{gathered}
$$

The first equation in (4.1.18) gives $x=s$. Then the second equation becomes

$$
\frac{d y}{d s}=s^{2} \Longrightarrow y=\frac{s^{3}}{3}+t_{2}
$$

Now the third equation in (4.1.18) becomes

$$
\frac{d u}{d s}=-\left(\frac{s^{3}}{3}+t_{2}\right) u \sim \frac{d u}{u}=-\left(\frac{s^{3}}{3}+t_{2}\right) d s .
$$


Thus

$$
u=t_{3} e^{-s^{4} / 12-t_{2} s}=f\left(t_{2}\right) e^{-s^{4} / 12-t_{2} s} .
$$

Note $s=x$. So $t_{2}=y-x^{3} / 3$. Thus the final solution is

$$
u=f\left(y-x^{3} / 3\right) e^{x^{4} / 4-x y} .
$$

Example 4.1.3. Solve the the equation

$$
u_{x}+u_{y}+x y u_{z}=u^{2} \text { subject to } u=x^{2} \text { on } y=z .
$$

Solutions. The system of characteristic lines is:

$$
\frac{d x}{d s}=1, \quad \frac{d y}{d s}=1, \quad \frac{d z}{d s}=x y, \frac{d u}{d s}=u^{2} .
$$

Initial conditions are:

$$
\left.x\right|_{s=0}=t_{1},\left.\quad y\right|_{s=0}=t_{2},\left.\quad z\right|_{s=0}=t_{3},\left.\quad u\right|_{s=0}=t_{1}^{2}, \quad t_{2}=t_{3} .
$$

The first two equations in (4.1.26) gives $x=s+t_{1}$ and $y=s+t_{2}$. The third equation becomes

$$
\frac{d z}{d s}=\left(s+t_{1}\right)\left(s+t_{2}\right)=s^{2}+\left(t_{1}+t_{2}\right) s+t_{1} t_{2}
$$

Thus

$$
z=\frac{s^{3}}{3}+\frac{t_{1}+t_{2}}{2} s^{2}+t_{1} t_{2} s+t_{2}
$$

The last equation in (4.1.26) yields

$$
\frac{1}{u}=-s+\frac{1}{t_{1}^{2}} \Longrightarrow u=\frac{t_{1}^{2}}{1-s t_{1}^{2}} .
$$

Note $t_{1}=x-s$ and $t_{2}=y-s$. Thus we obtained the parametric solution

$$
u=\frac{(x-s)^{2}}{1-s(x-s)^{2}}, \quad z=\frac{s^{3}}{3}-\frac{x+y}{2} s^{2}+x y s+y-s .
$$

\section{Exercise 4.1}

1. Solve the following problem

$$
x^{2} u_{x}+2 y u_{y}+4 z^{3} u_{z}=0 \text { subject to } u=f(y, z) \text { on the plane } x=1 \text {. }
$$

2. Find the solution of the problem

$$
u_{x}+2 x u_{y}+3 y u_{z}=4 z u^{3}
$$

subject to

$$
u^{3}=x^{2}+y+3 \sin z \text { on the surface } x=y^{2}+z^{2} .
$$




\subsection{Characteristic Strip and Exact Equations}

Consider the partial differential equation

$$
F(x, y, u, p, q)=0, \quad p=u_{x}, q=u_{y}
$$

We search for solution by solving the following system of strip equations:

$$
\begin{array}{ll}
\frac{\partial x}{\partial s}=F_{p}, \quad \frac{\partial y}{\partial s}=F_{q}, & \frac{\partial u}{\partial s}=p F_{p}+q F_{q}, \\
\frac{\partial p}{\partial s}=-F_{x}-p F_{u}, & \frac{\partial q}{\partial s}=-F_{y}-q F_{u},
\end{array}
$$

where we view $\{x, y, u, p, q\}$ as functions of the two variables $\{s, t\}$, and $t$ is responsible for the initial condition. The third equation in (4.2.2) is derived from the first two via

$$
\frac{\partial u}{\partial s}=u_{x} \frac{\partial x}{\partial s}+u_{y} \frac{\partial y}{\partial s}=p F_{p}+q F_{q}
$$

Note $p_{y}=u_{x y}=u_{y x}=q_{x}$. Taking partial derivative of the first equation in (4.2.1) with respect to $x$, we have

$$
F_{x}+p F_{u}+p_{x} F_{p}+q_{x} F_{q}=0 \sim F_{x}+p F_{u}+p_{x} F_{p}+p_{y} F_{q}=0
$$

Under the assumption the first two equations in (4.2.2),

$$
\frac{\partial p}{\partial s}=p_{x} \frac{\partial x}{\partial s}+p_{y} \frac{\partial y}{\partial s}=p_{x} F_{p}+q_{x} F_{q}=-F_{x}-p F_{u}
$$

that is, the first equation in (4.2.3) holds. We can similarly derive the second equation in (4.2.3). A solution of the system (4.2.2) and (4.2.3) does give a characteristic line because

$$
\left(u_{x}, u_{y},-1\right) \cdot\left(\frac{\partial x}{\partial s}, \frac{\partial y}{\partial s}, \frac{\partial u}{\partial s}\right)=p F_{p}+q F_{q}-\left(p F_{p}+q F_{q}\right)=0
$$

Example 4.2.1. Solving the problem

$$
u_{x} u_{y}-2 u-x+2 y=0
$$

subject to $u=y^{2}$ on the line $x=0$.

Solution. Now $F=p q-2 u-x+2 y$. The strip equations are:

$$
\begin{gathered}
\frac{\partial x}{\partial s}=q, \quad \frac{\partial y}{\partial s}=p, \quad \frac{\partial u}{\partial s}=2 p q \\
\frac{\partial p}{\partial s}=1+2 p \quad \frac{\partial q}{\partial s}=-2+2 q .
\end{gathered}
$$

The initial conditions are given: when $s=0$,

$$
x=0, \quad y=t, \quad u=t^{2} .
$$


To find the condition for $p$ and $q$ when $s=0$, we calculate

$$
\frac{d u}{d t}=p \frac{d x}{d t}+q \frac{d y}{d t} \sim 2 t=p \cdot 0+q \cdot 1 \Longrightarrow q=2 t .
$$

On the other hand, when $s=0,(4.2 .8)$ becomes

$$
p q-2 t^{2}+2 t=0 \Longrightarrow p=t-1 \text {. }
$$

According to (4.2.10), (4.2.12) and (4.2.13), we have

$$
p=\frac{-1+(2 t-1) e^{2 s}}{2}, \quad q=1+(2 t-1) e^{2 s} .
$$

Next (4.2.9) becomes

$$
\begin{gathered}
\frac{\partial x}{\partial s}=1+(2 t-1) e^{2 s}, \quad \frac{\partial y}{\partial s}=\frac{-1+(2 t-1) e^{2 s}}{2}, \\
\frac{\partial u}{\partial s}=(2 t-1)^{2} e^{4 s}-1 .
\end{gathered}
$$

Thus

$$
\begin{gathered}
x=s+\frac{(2 t-1)\left(e^{2 s}-1\right)}{2}, \quad y=-\frac{s}{2}+t+\frac{(2 t-1)\left(e^{2 s}-1\right)}{4}, \\
u=t^{2}-s+\frac{(2 t-1)^{2}\left(e^{4 s}-1\right)}{4} .
\end{gathered}
$$

The equation

$$
f(x, y, u) u_{x}=g(x, y, u) u_{y}
$$

is called exact if $f_{x}=g_{y}$. For an exact equation, we look for a function $\Psi(x, y, u)$ such that $\Psi_{y}=f$ and $\Psi_{x}=g$. Then $\Psi(x, y, u)=0$ is a solution of (4.2.19). In fact, the equation $\Psi(x, y, u)=0$ gives

$$
\Psi_{x}+\Psi_{u} u_{x}=0, \quad \Psi_{y}+\Psi_{u} u_{y}=0
$$

Thus

$$
u_{x}=-\frac{\Psi_{x}}{\Psi_{u}}=-\frac{g}{\Psi_{u}}, u_{y}=-\frac{\Psi_{y}}{\Psi_{u}}=-\frac{f}{\Psi_{u}},
$$

which implies

$$
f u_{x}=-f \frac{g}{\Psi_{u}}=-g \frac{f}{\Psi_{u}}=g u_{y}
$$

Example 4.2.2. Solve the equation

$$
(x+\cos y+u) u_{x}=\left(y+e^{x}+u^{2}\right) u_{y} .
$$


Solution. Now $f=x+\cos y+u$ and $g=y+e^{x}+u^{2}$. Moreover, $f_{x}=1=g_{y}$. The equation is exact. Let

$$
\Psi=\int f(x, y, u) d y=\int(x+\cos y+u) d y=(x+u) y+\sin y+\phi(x, u) .
$$

Taking partial derivative of (4.2.24) with respect to $x$, we get

$$
y+\phi_{x}=\Psi_{x}=g=y+e^{x}+u^{2} \sim \phi_{x}=e^{x}+u^{2} .
$$

Hence

$$
\phi=\int\left(e^{x}+u^{2}\right) d x=e^{x}+x u^{2}+h(u),
$$

where $h(u)$ is any differentiable function. The final answer is

$$
(x+u) y+\sin y+e^{x}+x u^{2}+h(u)=0 .
$$

We refer to $[\mathrm{Z}]$ for more exact methods of solving differential equations.

\section{Exercise 4.2}

1. Find the solution of the following problem $u_{x} u_{y}-2 u+2 x=0$ subject to $u=x^{2} y$ on the line $x=y$.

2. Solve the equation $\left(2 x y+e^{y}\right) u_{x}=\left(y^{2}+x+\sin u\right) u_{y}$.

\subsection{Polynomial Solutions of Flag Equations}

A linear transformation $T$ on an infinite-dimensional vector space $U$ is called locally nilpotent if for any $u \in U$, there exists a positive integer $m$ (usually depends on $u$ ) such that $T^{m}(u)=0$.

A partial differential equation of flag type is the linear differential equation of the form:

$$
\left(d_{1}+f_{1} d_{2}+f_{2} d_{3}+\cdots+f_{n-1} d_{n}\right)(u)=0,
$$

where $d_{1}, d_{2}, \ldots, d_{n}$ are certain commuting locally nilpotent differential operators on the polynomial algebra $\mathbb{R}\left[x_{1}, x_{2}, \ldots, x_{n}\right]$ and $f_{1}, \ldots, f_{n-1}$ are polynomials satisfying

$$
d_{l}\left(f_{j}\right)=0 \quad \text { if } l>j .
$$

Examples of such equations are: (1) Laplace equation

$$
u_{x_{1} x_{1}}+u_{x_{2} x_{2}}+\cdots+u_{x_{n} x_{n}}=0
$$

(2) heat conduction equation

$$
u_{t}-u_{x_{1} x_{1}}-u_{x_{2} x_{2}}-\cdots-u_{x_{n} x_{n}}=0
$$


(3) generalized Laplace equation

$$
u_{x x}+x u_{y y}+y u_{z z}=0
$$

The aim of this section is to find all the polynomial solutions of the equation (4.3.1). The contents are taken from the author's work [X11].

Let $U$ be a vector space over $\mathbb{R}$ and let $U_{1}$ be a subspace of $U$. The quotient space

$$
U / U_{1}=\left\{u+U_{1} \mid u \in U\right\}
$$

with linear operation

$$
a\left(u_{1}+U_{1}\right)+b\left(u_{2}+U_{1}\right)=\left(a u_{1}+b u_{2}\right)+U_{1} \quad \text { for } u_{1}, u_{2} \in U, a, b \in \mathbb{R}
$$

where the zero vector in $U / U_{1}$ is $U_{1}$ and

$$
u+v+U_{1}=u+U_{1} \quad \text { for } u \in U, v \in U_{1} \text {. }
$$

For instance, $U=\mathbb{R} x+\mathbb{R} y+\mathbb{R} z$ and $U_{1}=\mathbb{R} x$. Then $U / U_{1}=\left\{b y+c z+U_{1}\right\} \cong \mathbb{R} y+\mathbb{R} z$ and $\left\{y+U_{1}, z+U_{1}\right\}$ forms a basis of $U / U_{1}$. Second example is $U=\mathbb{R}+\mathbb{R} x+\mathbb{R} x^{2}$ and $U_{1}=\mathbb{R}\left(1+x+x^{2}\right)$. In this case, $\left(1+U_{1}\right)+\left(x+U_{1}\right)+\left(x^{2}+U_{1}\right)=\left(1+x+x^{2}\right)+U_{1}=U_{1}$, the zero vector in $U / U_{1}$. Thus $U / U_{1}=\left\{a+b x+U_{1} \mid a, b \in \mathbb{R}\right\}=\left\{a x+b x^{2}+U_{1} \mid a, b \in \mathbb{R}\right\}$. Both $\left\{1+U_{1}, x+U_{1}\right\}$ and $\left\{x+U_{1}, x^{2}+U_{1}\right\}$ are bases of $U / U_{1}$. But we know $U / U_{1} \cong \mathbb{R}^{2}$.

Recall that $\mathbb{N}$ denotes the set of nonnegative integers. Let $1 \leq k<n$. Denote

$$
\mathcal{A}=\mathbb{R}\left[x_{1}, x_{2}, \ldots, x_{n}\right], \quad \mathcal{B}=\mathbb{R}\left[x_{1}, x_{2}, \ldots, x_{k}\right], \quad V=\mathbb{R}\left[x_{k+1}, x_{k+2}, \ldots, x_{n}\right] .
$$

Let $\left\{V_{m} \mid m \in \mathbb{N}\right\}$ be a set of subspaces of $V$ such that

$$
V_{r} \subset V_{r+1} \text { for } r \in \mathbb{N} \text { and } V=\bigcup_{r=0}^{\infty} V_{r} .
$$

For instance, we take $V_{r}=\{g \in V \mid \operatorname{deg} g \leq r\}$ in some special cases.

Lemma 4.3.1. Let $T_{1}$ be a differential operator on $\mathcal{A}$ with a right inverse $T_{1}^{-}$such that

$$
T_{1}(\mathcal{B}), T_{1}^{-}(\mathcal{B}) \subset \mathcal{B}, \quad T_{1}\left(\eta_{1} \eta_{2}\right)=T_{1}\left(\eta_{1}\right) \eta_{2}, \quad T_{1}^{-}\left(\eta_{1} \eta_{2}\right)=T_{1}^{-}\left(\eta_{1}\right) \eta_{2}
$$

for $\eta_{1} \in \mathcal{B}, \eta_{2} \in V$, and let $T_{2}$ be a differential operator on $\mathcal{A}$ such that

$$
T_{2}\left(V_{0}\right)=\{0\}, T_{2}\left(V_{r+1}\right) \subset \mathcal{B} V_{r}, T_{2}(f \zeta)=f T_{2}(\zeta) \text { for } r \in \mathbb{N}, \quad f \in \mathcal{B}, \zeta \in \mathcal{A} .
$$

Then we have

$$
\begin{aligned}
& \left\{f \in \mathcal{A} \mid\left(T_{1}+T_{2}\right)(f)=0\right\} \\
= & \operatorname{Span}\left\{\sum_{\iota=0}^{\infty}\left(-T_{1}^{-} T_{2}\right)^{\iota}(h g) \mid g \in V, h \in \mathcal{B} ; T_{1}(h)=0\right\}
\end{aligned}
$$


where the summation is finite. Moreover, the operator $\sum_{\iota=0}^{\infty}\left(-T_{1}^{-} T_{2}\right)^{\iota} T_{1}^{-}$is a right inverse of $T_{1}+T_{2}$.

Proof. For $h \in \mathcal{B}$ such that $T_{1}(h)=0$ and $g \in V$, we have

$$
\begin{aligned}
& \left(T_{1}+T_{2}\right)\left(\sum_{\iota=0}^{\infty}\left(-T_{1}^{-} T_{2}\right)^{\iota}(h g)\right) \\
= & T_{1}(h g)-\sum_{\iota=1}^{\infty} T_{1}\left[T_{1}^{-} T_{2}\left(-T_{1}^{-} T_{2}\right)^{\iota-1}(h g)\right]+\sum_{\iota=0}^{\infty} T_{2}\left[\left(-T_{1}^{-}\right)^{\iota}(h g)\right] \\
= & T_{1}(h) g-\sum_{\iota=1}^{\infty}\left(T_{1} T_{1}^{-}\right) T_{2}\left(-T_{1}^{-} T_{2}\right)^{\iota-1}(h g)+\sum_{\iota=0}^{\infty} T_{2}\left(-T_{1}^{-} T_{2}\right)^{\iota}(h g) \\
= & -\sum_{\iota=1}^{\infty} T_{2}\left(-T_{1}^{-} T_{2}\right)^{\iota-1}(h g)+\sum_{\iota=0}^{\infty} T_{2}\left(-T_{1}^{-} T_{2}\right)^{\iota}(h g)=0
\end{aligned}
$$

by (4.3.11). Set $V_{-1}=\{0\}$. For $j \in \mathbb{N}$, we take $\left\{\psi_{j, r} \mid r \in I_{j}\right\} \subset V_{j}$ such that

$$
\left\{\psi_{j, r}+V_{j-1} \mid r \in I_{j}\right\} \text { forms a basis of } V_{j} / V_{j-1},
$$

where $I_{j}$ is an index set. Let

$$
\mathcal{A}^{(m)}=\mathcal{B} V_{m}=\sum_{s=0}^{m} \sum_{r \in I_{s}} \mathcal{B} \psi_{s, r}
$$

Obviously,

$$
T_{1}\left(\mathcal{A}^{(m)}\right), T_{1}^{-}\left(\mathcal{A}^{(m)}\right), T_{2}\left(\mathcal{A}^{(m+1)}\right) \subset \mathcal{A}^{(m)} \quad \text { for } m \in \mathbb{N}
$$

by (4.3.11) and (4.3.12), and

$$
\mathcal{A}=\bigcup_{m=0}^{\infty} \mathcal{A}^{(m)}
$$

Suppose $\phi \in \mathcal{A}^{(m)}$ such that $\left(T_{1}+T_{2}\right)(\phi)=0$. If $m=0$, then

$$
\phi=\sum_{r \in I_{0}} h_{r} \psi_{0, r}, \quad h_{r} \in \mathcal{B}
$$

Now

$$
0=\left(T_{1}+T_{2}\right)(\phi)=\sum_{r \in I_{0}} T_{1}\left(h_{r}\right) \psi_{0, r}+\sum_{r \in I_{0}} h_{r} T_{2}\left(\psi_{0, r}\right)=\sum_{r \in I_{0}} T_{1}\left(h_{r}\right) \psi_{0, r},
$$

Since $T_{1}\left(h_{r}\right) \in \mathcal{B}$ by (4.3.11), (4.3.20) gives $T_{1}\left(h_{r}\right)=0$ for $r \in I_{0}$. Denote by $\mathcal{S}$ the right hand side of the equation (4.3.13). Then

$$
\phi=\sum_{r \in I_{0}} \sum_{m=0}^{\infty}\left(-T_{1}^{-} T_{2}\right)^{m}\left(h_{r} \psi_{0, r}\right) \in \mathcal{S} .
$$


Suppose $m>0$. We write

$$
\phi=\sum_{r \in I_{m}} h_{r} \psi_{m, r}+\phi^{\prime}, \quad h_{r} \in \mathcal{B}, \phi^{\prime} \in \mathcal{A}^{(m-1)}
$$

Then

$$
0=\left(T_{1}+T_{2}\right)(\phi)=\sum_{r \in I_{m}} T_{1}\left(h_{r}\right) \psi_{m, r}+T_{1}\left(\phi^{\prime}\right)+T_{2}(\phi) .
$$

Since $T_{1}\left(\phi^{\prime}\right)+T_{2}(\phi) \in \mathcal{A}^{(m-1)}$, we have $T_{1}\left(h_{r}\right)=0$ for $r \in I_{m}$. Now

$$
\phi-\sum_{r \in I_{m}} \sum_{j=0}^{\infty}\left(-T_{1}^{-} T_{2}\right)^{j}\left(h \psi_{m, r}\right)=\phi^{\prime}-\sum_{r \in I_{m}} \sum_{j=1}^{\infty}\left(-T_{1}^{-} T_{2}\right)^{j}\left(h_{r} \psi_{m, r}\right) \in \mathcal{A}^{(m-1)}
$$

and (4.3.14) implies

$$
\left(T_{1}+T_{2}\right)\left(\phi-\sum_{r \in I_{m}} \sum_{j=0}^{\infty}\left(-T_{1}^{-} T_{2}\right)^{j}\left(h_{r} \psi_{m, r}\right)\right)=0
$$

By induction on $m$,

$$
\phi-\sum_{r \in I_{m}} \sum_{j=0}^{\infty}\left(-T_{1}^{-} T_{2}\right)^{j}\left(h_{r} \psi_{m, r}\right) \in \mathcal{S}
$$

Therefore, $\phi \in \mathcal{S}$.

For any $f \in \mathcal{A}$, we have:

$$
\begin{aligned}
& \left(T_{1}+T_{2}\right)\left(\sum_{\iota=0}^{\infty}\left(-T_{1}^{-} T_{2}\right)^{\iota} T_{1}^{-}\right)(f) \\
= & f-\sum_{\iota=1}^{\infty} T_{2}\left(-T_{1}^{-} T_{2}\right)^{\iota-1} T_{1}^{-}(f)+\sum_{\iota=0}^{\infty} T_{2}\left(-T_{1}^{-} T_{2}\right)^{\iota} T_{1}^{-}(f)=f .
\end{aligned}
$$

Thus the operator $\sum_{\iota=0}^{\infty}\left(-T_{1}^{-} T_{2}\right)^{\iota} T_{1}^{-}$is a right inverse of $T_{1}+T_{2}$.

We remark that the above operators $T_{1}$ and $T_{2}$ may not commute. The assumption $T_{2}\left(V_{r+1}\right) \subset \mathcal{B} V_{r}$ instead of $T_{2}\left(V_{r+1}\right) \subset V_{r}$ because we want our lemma working for a special case like $T_{1}=\partial_{x_{1}}^{2}, T_{2}=x_{1} \partial_{x_{2}}^{2}, \mathcal{B}=\mathbb{R}\left[x_{1}\right]$ and $V=\mathbb{R}\left[x_{2}\right]$.

Define

$$
x^{\alpha}=x_{1}^{\alpha_{1}} x_{2}^{\alpha_{2}} \cdots x_{n}^{\alpha_{n}} \text { for } \alpha=\left(\alpha_{1}, \ldots, \alpha_{n}\right) \in \mathbb{N}^{n} .
$$

Moreover, we denote

$$
\epsilon_{\iota}=(0, \ldots, 0, \stackrel{\iota}{1}, 0, \ldots, 0) \in \mathbb{N}^{n} .
$$

For each $\iota \in \overline{1, n}$, we define the linear operator $\int_{\left(x_{\iota}\right)}$ on $\mathcal{A}$ by:

$$
\int_{\left(x_{\iota}\right)}\left(x^{\alpha}\right)=\frac{x^{\alpha+\epsilon_{\iota}}}{\alpha_{\iota}+1} \text { for } \alpha \in \mathbb{N}^{n} \text {. }
$$


Furthermore, we let

$$
\int_{\left(x_{\iota}\right)}^{(0)}=1, \quad \int_{\left(x_{\iota}\right)}^{(m)}=\overbrace{\int_{\left(x_{\iota}\right)} \cdots \int_{\left(x_{\iota}\right)}}^{m} \text { for } 0<m \in \mathbb{Z}
$$

and denote

$$
\partial^{\alpha}=\partial_{x_{1}}^{\alpha_{1}} \partial_{x_{2}}^{\alpha_{2}} \cdots \partial_{x_{n}}^{\alpha_{n}}, \quad \int^{(\alpha)}=\int_{\left(x_{1}\right)}^{\left(\alpha_{1}\right)} \int_{\left(x_{2}\right)}^{\left(\alpha_{2}\right)} \cdots \int_{\left(x_{n}\right)}^{\left(\alpha_{n}\right)} \quad \text { for } \alpha \in \mathbb{N}^{n} .
$$

Obviously, $\int^{(\alpha)}$ is a right inverse of $\partial^{\alpha}$ for $\alpha \in \mathbb{N}^{n}$. We remark that $\int^{(\alpha)} \partial^{\alpha} \neq 1$ if $\alpha \neq 0$ due to $\partial^{\alpha}(1)=0$.

Example 4.3.1. Find polynomial solutions of the heat conduction equation $u_{t}=u_{x x}$. Solution. In this case,

$$
\mathcal{A}=\mathbb{R}[t, x], \quad \mathcal{B}=\mathbb{R}[t], \quad V=\mathbb{R}[x], \quad V_{r}=\{g \in V \mid \operatorname{deg} g \leq r\} .
$$

The equation can be written as $\left(\partial_{t}-\partial_{x}^{2}\right)(u)=0$. So we take

$$
T_{1}=\partial_{t}, \quad T_{1}^{-}=\int_{(t)}, \quad T_{2}=-\partial_{x}^{2} .
$$

It can be verified that the conditions in Lemma 4.3.1 are satisfied. Note that

$$
\left\{f \in \mathcal{B} \mid T_{1}(f)=0\right\}=\left\{f \in \mathbb{R}[t] \mid \partial_{t}(f)=0\right\}=\mathbb{R} .
$$

We calculate

$$
\left(-T_{1} T_{2}\right)^{\iota}\left(x^{k}\right)=\left(\int_{(t)} \partial_{x}^{2}\right)^{\iota}\left(x^{k}\right)=\int_{(t)}^{\iota}(1) \partial_{x}^{2 \iota}\left(x^{k}\right)=\frac{\left[\prod_{s=0}^{2 \iota-1}(k-s)\right] t^{\iota} x^{k-2 \iota}}{\iota !} .
$$

Thus the space of the polynomial solutions is

$$
\operatorname{Span}\left\{\sum_{\iota=0}^{\llbracket k / 2 \rrbracket} \frac{\left[\prod_{s=0}^{2 \iota-1}(k-s)\right] t^{\iota} x^{k-2 \iota}}{\iota !} \mid k \in \mathbb{N}\right\} .
$$

Example 4.3.2. Find polynomial solutions of the Laplace equation $u_{x x}+u_{y y}=0$. Solution. In this case,

$$
\mathcal{A}=\mathbb{R}[x, y], \quad \mathcal{B}=\mathbb{R}[x], \quad V=\mathbb{R}[y], \quad V_{r}=\{g \in V \mid \operatorname{deg} g \leq r\} .
$$

Moreover, we take

$$
T_{1}=\partial_{x}^{2}, \quad T_{1}^{-}=\int_{(x)}^{2}, \quad T_{2}=\partial_{y}^{2} .
$$


It can be verified that the conditions in Lemma 4.3.1 are satisfied. Note that

$$
\left\{f \in \mathcal{B} \mid T_{1}(f)=0\right\}=\left\{f \in \mathbb{R}[x] \mid \partial_{x}^{2}(f)=0\right\}=\mathbb{R}+\mathbb{R} x
$$

We calculate

$$
\begin{aligned}
\left(-T_{1} T_{2}\right)^{\iota}\left(y^{k}\right) & =\left(-\int_{(x)}^{2} \partial_{y}^{2}\right)^{\iota}\left(y^{k}\right)=(-1)^{\iota} \int_{(x)}^{2 \iota}(1) \partial_{y}^{2 \iota}\left(y^{k}\right) \\
& =\frac{\left[\prod_{s=0}^{2 \iota-1}(k-s)\right]\left(-x^{2}\right)^{\iota} y^{k-2 \iota}}{(2 \iota) !} \\
\left(-T_{1} T_{2}\right)^{\iota}\left(x y^{k}\right) & =\left(-\int_{(x)}^{2} \partial_{y}^{2}\right)^{\iota}\left(x y^{k}\right)=(-1)^{\iota} \int_{(x)}^{2 \iota}(x) \partial_{y}^{2 \iota}\left(y^{k}\right) \\
& =\frac{(-1)^{\iota}\left[\prod_{s=0}^{2 \iota-1}(k-s)\right] x^{2 \iota+1} y^{k-2 \iota}}{(2 \iota+1) !} .
\end{aligned}
$$

Thus the space of the polynomial solutions is

$$
\begin{aligned}
& \operatorname{Span}\left\{\sum_{\iota=0}^{\llbracket k / 2 \rrbracket} \frac{\left[\prod_{s=0}^{2 \iota-1}(k-s)\right]\left(-x^{2}\right)^{\iota} y^{k-2 \iota}}{(2 \iota) !},\right. \\
& \left.\sum_{\iota=0}^{\llbracket k / 2 \rrbracket} \frac{(-1)^{\iota}\left[\prod_{s=0}^{2 \iota-1}(k-s)\right] x^{2 \iota+1} y^{k-2 \iota}}{(2 \iota+1) !} \mid k \in \mathbb{N}\right\} .
\end{aligned}
$$

Consider the wave equation in Riemannian space with a nontrivial conformal group:

$$
u_{t t}-u_{x_{1} x_{1}}-\sum_{\iota, j=2}^{n} g_{\iota, j}\left(x_{1}-t\right) u_{x_{\iota} x_{j}}=0
$$

where we assume that $g_{\iota, j}(z)$ are one-variable polynomials. Change variables:

$$
z_{0}=x_{1}+t, \quad z_{1}=x_{1}-t
$$

Then

$$
\partial_{t}^{2}=\left(\partial_{z_{0}}-\partial_{z_{1}}\right)^{2}, \quad \partial_{x_{1}}^{2}=\left(\partial_{z_{0}}+\partial_{z_{1}}\right)^{2} .
$$

So the equation (4.3.44) changes to:

$$
2 \partial_{z_{0}} \partial_{z_{1}}+\sum_{\iota, j=2}^{n} g_{\iota, j}\left(z_{1}\right) u_{x_{\iota} x_{j}}=0 .
$$

Denote

$$
T_{1}=2 \partial_{z_{0}} \partial_{z_{1}}, \quad T_{2}=\sum_{\iota, j=2}^{n} g_{\iota, j}\left(z_{1}\right) \partial_{x_{\iota}} \partial_{x_{j}}
$$


Take $T_{1}^{-}=\frac{1}{2} \int_{\left(z_{0}\right)} \int_{\left(z_{1}\right)}$, and

$$
\mathcal{B}=\mathbb{R}\left[z_{0}, z_{1}\right], \quad V=\mathbb{R}\left[x_{2}, \ldots, x_{n}\right], \quad V_{r}=\{f \in V \mid \operatorname{deg} f \leq r\} .
$$

Then the conditions in Lemma 4.3.1 hold. Thus we have:

Theorem 4.3.2. The space of all polynomial solutions for the equation (4.3.44) is:

$$
\begin{aligned}
& \operatorname{Span}\left\{\sum_{m=0}^{\infty}(-2)^{-m}\left(\sum_{\iota, j=2}^{n} \int_{\left(z_{0}\right)} \int_{\left(z_{1}\right)} g_{\iota, j}\left(z_{1}\right) \partial_{x_{\iota}} \partial_{x_{j}}\right)^{m}\left(f_{0} g_{0}+f_{1} g_{1}\right)\right. \\
& \left.\mid f_{0} \in \mathbb{R}\left[z_{0}\right], f_{1} \in \mathbb{R}\left[z_{1}\right], g_{0}, g_{1} \in \mathbb{R}\left[x_{2}, \ldots, x_{n}\right]\right\}
\end{aligned}
$$

with $z_{0}, z_{1}$ defined in (4.3.45).

Let $m_{1}, m_{2}, \ldots, m_{n}$ be positive integers. According to Lemma 4.3.1, the set

$$
\begin{aligned}
& \left\{\sum_{\substack{k_{2}, \ldots, k_{n}=0 \\
k_{2}}}^{\infty}(-1)^{k_{2}+\cdots+k_{n}}\left(\begin{array}{c}
k_{2}+\cdots+k_{k} \\
k_{2}, \ldots, k_{n}
\end{array}\right) \int_{\left(x_{1}\right)}^{\left(\left(k_{2}+\cdots+k_{n}\right) m_{1}\right)}\left(x_{1}^{\ell_{1}}\right)\right. \\
& \left.\times \partial_{x_{2}}^{k_{2} m_{2}}\left(x_{2}^{\ell_{2}}\right) \cdots \partial_{x_{n}}^{k_{n} m_{n}}\left(x_{n}^{\ell_{n}}\right) \mid \ell_{1} \in \overline{0, m_{1}-1}, \ell_{2}, \ldots, \ell_{n} \in \mathbb{N}\right\}
\end{aligned}
$$

forms a basis of the space of polynomial solutions for the equation

$$
\left(\partial_{x_{1}}^{m_{1}}+\partial_{x_{2}}^{m_{2}}+\cdots+\partial_{x_{n}}^{m_{n}}\right)(u)=0
$$

in $\mathcal{A}$.

The above results can theoretically generalized as follows. Let

$$
f_{\iota} \in \mathbb{R}\left[x_{1}, \ldots, x_{\iota}\right] \quad \text { for } \iota \in \overline{1, n-1} .
$$

Consider the equation:

$$
\left(\partial_{x_{1}}^{m_{1}}+f_{1} \partial_{x_{2}}^{m_{2}}+\cdots+f_{n-1} \partial_{x_{n}}^{m_{n}}\right)(u)=0
$$

Denote

$$
d_{1}=\partial_{x_{1}}^{m_{1}}, \quad d_{r}=\partial_{x_{1}}^{m_{1}}+f_{1} \partial_{x_{2}}^{m_{2}}+\cdots+f_{r-1} \partial_{x_{r}}^{m_{r}} \quad \text { for } r \in \overline{2, n} .
$$

We will apply Lemma 4.3.1 with $T_{1}=d_{r}, T_{2}=\sum_{\iota=r}^{n-1} f_{\iota} \partial_{x_{\iota+1}}^{m_{\iota+1}}$ and $\mathcal{B}=\mathbb{R}\left[x_{1}, \ldots, x_{r}\right], V=$ $\mathbb{R}\left[x_{r+1}, \ldots, x_{n}\right]$,

$$
V_{k}=\operatorname{Span}\left\{x_{r+1}^{\ell_{r+1}} \cdots x_{n}^{\ell_{n}} \mid \ell_{s} \in \mathbb{N}, \ell_{r+1}+\sum_{\iota=r+2}^{n} \ell_{\iota}\left(\operatorname{deg} f_{r+1}+1\right) \cdots\left(\operatorname{deg} f_{\iota-1}+1\right) \leq k\right\} .
$$

The motivation of the above definition can be shown by the spacial example $T_{2}=x_{1} \partial_{x_{2}}+$ $x_{2}^{3} \partial_{x_{3}}$ and $V=\mathbb{R}\left[x_{2}, x_{3}\right]$. In this example, $T_{2}$ does not reduce the usual degree of the 
polynomials in $V$. If we define new degree by $\operatorname{deg} x_{2}^{m}=m$ and $\operatorname{deg} x_{3}^{m}=4 m$, then $T_{2}$ does reduce the new degree of the polynomials in $V$. Since $T_{2}\left(V_{0}\right)=\{0\}$ and $T_{2}\left(V_{r+1}\right) \subset \mathcal{B} V_{r}$ for $r \in \mathbb{N}$, this gives a proof that $T_{2}$ is locally nilpotent.

Take a right inverse $d_{1}^{-}=\int_{\left(x_{1}\right)}^{\left(m_{1}\right)}$. Suppose that we have found a right inverse $d_{s}^{-}$of $d_{s}$ for some $s \in \overline{1, n-1}$ such that

$$
x_{\iota} d_{s}^{-}=d_{s}^{-} x_{\iota}, \quad \partial_{x_{\iota}} d_{s}^{-}=d_{s}^{-} \partial_{x_{\iota}} \quad \text { for } \iota \in \overline{s+1, n} .
$$

Lemma 4.3.1 enable us to take

$$
d_{s+1}^{-}=\sum_{\iota=0}^{\infty}\left(-d_{s}^{-} f_{s}\right)^{\iota} d_{s}^{-} \partial_{x_{s+1}}^{\iota m_{s+1}}
$$

as a right inverse of $d_{s+1}$. Obviously,

$$
x_{\iota} d_{s+1}^{-}=d_{s+1}^{-} x_{\iota}, \quad \partial_{x_{\iota}} d_{s+1}^{-}=d_{s+1}^{-} \partial_{x_{\iota}} \quad \text { for } \iota \in \overline{s+2, n}
$$

according to (4.3.55). By induction, we have found a right inverse $d_{s}^{-}$of $d_{s}$ such that (4.3.57) holds for each $s \in \overline{1, n}$.

We set

$$
\mathcal{S}_{r}=\left\{g \in \mathbb{R}\left[x_{1}, \ldots, x_{r}\right] \mid d_{r}(g)=0\right\} \quad \text { for } r \in \overline{1, k}
$$

By (4.3.55),

$$
\mathcal{S}_{1}=\sum_{i=0}^{m_{1}-1} \mathbb{R} x_{1}^{i} .
$$

Suppose that we have found $\mathcal{S}_{r}$ for some $r \in \overline{1, n-1}$. Given $h \in \mathcal{S}_{r}$ and $\ell \in \mathbb{N}$, we define

$$
\sigma_{r+1, \ell}(h)=\sum_{s=0}^{\infty}\left(-d_{r}^{-} f_{r}\right)^{s}(h) \partial_{x_{r+1}}^{s m_{r+1}}\left(x_{r+1}^{\ell}\right),
$$

which is actually a finite summation. Lemma 4.3.1 says

$$
\mathcal{S}_{r+1}=\sum_{\ell=0}^{\infty} \sigma_{r+1, \ell}\left(\mathcal{S}_{r}\right) \text {. }
$$

By induction, we obtain:

Theorem 4.3.3. The set

$$
\left\{\sigma_{n, \ell_{n}} \sigma_{n-1, \ell_{n-1}} \cdots \sigma_{2, \ell_{2}}\left(x_{1}^{\ell_{1}}\right) \mid \ell_{1} \in \overline{0, m_{1}-1}, \ell_{2}, \ldots, \ell_{n} \in \mathbb{N}\right\}
$$

forms a basis of the polynomial solution space $\mathcal{S}_{n}$ of the partial differential equation (4.3.54).

Example 4.3.3. Let $m_{1}, m_{2}, n$ be positive integers. Consider the following equations

$$
\partial_{x}^{m_{1}}(u)+x^{n} \partial_{y}^{m_{2}}(u)=0
$$


Now

$$
d_{1}=\partial_{x}^{m_{1}}, \quad d_{1}^{-}=\int_{(x)}^{\left(m_{1}\right)}
$$

Then

$$
\begin{aligned}
\sigma_{2, \ell_{2}}\left(x^{\ell_{1}}\right) & =\sum_{r=0}^{\infty}\left(-\int_{(x)}^{\left(m_{1}\right)} x^{n}\right)^{r}\left(x^{\ell_{1}}\right) \partial_{y}^{r m_{2}}\left(y^{\ell_{2}}\right) \\
& =x^{\ell_{1}} y^{\ell_{2}}+\sum_{r=1}^{\llbracket \ell_{2} / m_{2} \rrbracket} \frac{(-1)^{r}\left[\prod_{s=0}^{r m_{2}-1}\left(\ell_{2}-s\right)\right] x^{r\left(n+m_{1}\right)+\ell_{1}} y^{\ell_{2}-r m_{2}}}{\prod_{\iota=1}^{m_{1}} \prod_{j=1}^{r}\left(j n+(j-1) m_{1}+\iota+\ell_{1}\right)} .
\end{aligned}
$$

The polynomial solution space of (4.3.65) has a basis $\left\{\sigma_{2, \ell_{2}}\left(x^{\ell_{1}}\right) \mid \ell_{1} \in \overline{0, m_{1}-1}, \ell_{2} \in \mathbb{N}\right\}$.

In some practical problem, people found the linear wave equation with dissipation:

$$
u_{t t}+u_{t}-u_{x_{1} x_{1}}-u_{x_{2} x_{2}}-\cdots-u_{x_{n} x_{n}}=0
$$

In order to find the polynomial solutions for the equations of the above type pivoting at the variable $t$, we need the following lemma.

Lemma 4.3.4. Let $d=a \partial_{t}+\partial_{t}^{2}$ with $0 \neq a \in \mathbb{R}$. Take a right inverse

$$
d^{-}=\int_{(t)} \sum_{r=0}^{\infty} a^{-r-1}\left(-\partial_{t}\right)^{r}
$$

of $d$. Then

$$
\left(d^{-}\right)^{\iota}(1)=\frac{t^{\iota}}{\iota ! a^{\iota}}-\frac{t^{\iota-1}}{(\iota-2) ! a^{\iota+1}}+\sum_{r=2}^{\iota-1} \frac{(-1)^{r} \prod_{s=1}^{r-1}(\iota+s)}{(\iota-r-1) ! r ! a^{r+\iota}} t^{\iota-r} .
$$

Proof. For

$$
f(t)=\sum_{\iota=1}^{m} b_{\iota} t^{\iota} \in \mathbb{R}[t] t
$$

we have

$$
d(f(t))=a m b_{m} t^{m-1}+\sum_{\iota=1}^{m-1} \iota\left(a b_{\iota}+(\iota+1) b_{\iota+1}\right) t^{\iota-1} .
$$

Thus $d(f(t))=0$ if and only if $f(t) \equiv 0$. So for any given $g(t) \in \mathbb{R}[t]$, there exists a unique $f(t) \in \mathbb{R}[t] t$ such that $d(f(t))=g(t)$.

Set

$$
\xi_{a, \iota}(t)=\frac{t^{\iota}}{\iota ! a^{\iota}}-\frac{t^{\iota-1}}{(\iota-2) ! a^{\iota+1}}+\sum_{r=2}^{\iota-1} \frac{(-1)^{r} \prod_{s=1}^{r-1}(\iota+s)}{(\iota-r-1) ! r ! a^{r+\iota}} t^{\iota-r}
$$

where we treat

$$
\xi_{a, 0}(t)=1, \quad \xi_{a, 1}(t)=\frac{t}{a}, \quad \xi_{a, 2}(t)=\frac{t^{2}}{2 a^{2}}-\frac{t}{a^{3}}
$$


Easily verify $d\left(\xi_{a, \iota}(t)\right)=\xi_{a, \iota-1}(t)$ for $\iota=1,2$.

Assume $\iota>2$. We have

$$
\begin{aligned}
& d\left(\xi_{a, \iota}(t)\right) \\
& =\left(a \partial_{t}+\partial_{t}^{2}\right)\left(\frac{t^{\iota}}{\iota ! a^{\iota}}-\frac{t^{\iota-1}}{(\iota-2) ! a^{\iota+1}}+\sum_{r=2}^{\iota-1} \frac{(-1)^{r} \prod_{s=1}^{r-1}(\iota+s)}{(\iota-r-1) ! r ! a^{r+\iota}} t^{\iota-r}\right) \\
& =\frac{t^{\iota-1}}{(\iota-1) ! a^{\iota-1}}-\frac{(\iota-1) t^{\iota-2}}{(\iota-2) ! a^{\iota}}+\sum_{r=2}^{\iota-1} \frac{(-1)^{r}(\iota-r) \prod_{s=1}^{r-1}(\iota+s)}{(\iota-r-1) ! r ! a^{r+\iota-1}} t^{\iota-r-1} \\
& +\frac{t^{\iota-2}}{(\iota-2) ! a^{\iota}}-\frac{(\iota-1) t^{\iota-3}}{(\iota-3) ! a^{\iota+1}}+\sum_{r=2}^{\iota-1} \frac{(-1)^{r}(\iota-r) \prod_{s=1}^{r-1}(\iota+s)}{(\iota-r-2) ! r ! a^{r+\iota}} t^{\iota-r-2} \\
& =\frac{t^{\iota-1}}{(\iota-1) ! a^{\iota-1}}-\frac{t^{\iota-2}}{(\iota-3) ! a^{\iota}}+\frac{(\iota-2)(\iota+1)}{(\iota-3) ! 2 ! a^{\iota+1}} t^{\iota-3}-\frac{(\iota-1) t^{\iota-3}}{(\iota-3) ! a^{\iota+1}} \\
& +\sum_{r=3}^{\iota-1}(-1)^{r}\left[\frac{(\iota-r) \prod_{s=1}^{r-1}(\iota+s)}{r !}-\frac{(\iota-r+1) \prod_{s=1}^{r-2}(\iota+s)}{(r-1) !}\right] \frac{t^{\iota-r-1}}{(\iota-r-1) ! a^{r+\iota-1}} \\
& =\frac{t^{\iota-1}}{(\iota-1) ! a^{\iota-1}}-\frac{t^{\iota-2}}{(\iota-3) ! a^{\iota}}+\frac{(\iota-2)(\iota+1)-2(\iota-1)}{(\iota-3) ! 2 ! a^{\iota+1}} t^{\iota-3} \\
& +\sum_{r=3}^{\iota-1}(-1)^{r} \frac{[(\iota-r)(\iota+r-1)-r(\iota-r+1)] \prod_{s=1}^{r-2}(\iota+s)}{(\iota-r-1) ! r ! a^{r+\iota-1}} t^{\iota-r-1} \\
& =\frac{t^{\iota-1}}{(\iota-1) ! a^{\iota-1}}-\frac{t^{\iota-2}}{(\iota-3) ! a^{\iota}}+\frac{\iota(\iota-3)}{(\iota-3) ! 2 ! a^{\iota+1}} t^{\iota-3} \\
& +\sum_{r=3}^{\iota-1}(-1)^{r} \frac{\iota(\iota-1-r) \prod_{s=1}^{r-2}(\iota+s)}{(\iota-r-1) ! r ! a^{r+\iota-1}} t^{\iota-r-1} \\
& =\frac{t^{\iota-1}}{(\iota-1) ! a^{\iota-1}}-\frac{t^{\iota-2}}{(\iota-3) ! a^{\iota}}+\frac{\iota}{(\iota-4) ! 2 ! a^{\iota+1}} t^{\iota-3}+\sum_{r=3}^{\iota-2}(-1)^{r} \frac{\iota \prod_{s=1}^{r-2}(\iota+s)}{(\iota-r-2) ! r ! a^{r+\iota-1}} t^{\iota-r-1} \\
& =\frac{t^{\iota-1}}{(\iota-1) ! a^{\iota-1}}-\frac{t^{\iota-2}}{(\iota-3) ! a^{\iota}}+\sum_{r=2}^{\iota-2}(-1)^{r} \frac{\prod_{s=1}^{r-1}(\iota-1+s)}{(\iota-r-2) ! r ! a^{r+\iota-1}} t^{\iota-r-1} \\
& =\xi_{a, \iota-1}(t) \text {. }
\end{aligned}
$$

Since $\left(d^{-}\right)^{0}(1)=1,\left(d^{-}\right)^{\iota}(1) \in \mathbb{R}[t] t$ by (4.3.69) and $d\left[\left(d^{-}\right)^{\iota}(1)\right]=\left(d^{-}\right)^{\iota-1}(1)$ for $\iota \in \mathbb{N}+1$, we have $\left(d^{-}\right)^{r}(1)=\xi_{a, r}(t)$ for $r \in \mathbb{N}$ by the uniqueness, that is, (4.3.70) holds.

By Lemma 4.3.1 and the above lemma, we obtain:

Theorem 4.3.5. The set

$$
\begin{aligned}
& \left\{\sum_{r_{1}, \ldots, r_{n}=0}^{\infty}\left(\begin{array}{c}
r_{1}+\cdots+r_{n} \\
r_{1}, \ldots, r_{n}
\end{array}\right)\left[\prod_{\iota=1}^{n}\left(2 r_{\iota}\right) !\left(\begin{array}{c}
\ell_{\iota} \\
2 r_{\iota}
\end{array}\right)\right]\right. \\
& \left.\times \xi_{1, r_{1}+\cdots+r_{n}}(t) x_{1}^{\ell_{1}-2 r_{1}} \cdots x_{n}^{\ell_{n}-2 r_{n}} \mid \ell_{1}, \ldots, \ell_{n} \in \mathbb{N}\right\}
\end{aligned}
$$

forms a basis of the polynomial solution space of the equation (4.3.68). 
Consider the Klein-Gordan equation:

$$
u_{t t}-u_{x x}-u_{y y}-u_{z z}+a^{2} u=0,
$$

where $a$ is a nonzero real number. Changing variable $u=e^{a i t} v$, we get

$$
v_{t t}+2 a i v_{t}-v_{x x}-v_{y y}-v_{z z}=0 .
$$

We write

$$
\xi_{2 a i, \iota}=\zeta_{\iota, 0}(t)+\zeta_{\iota, 1}(t) i,
$$

where $\zeta_{\iota, 0}(t)$ and $\zeta_{\iota, 1}(t)$ are real functions. According to (4.3.73),

$$
\begin{aligned}
\zeta_{2 \iota, 0}(t)= & (-1)^{\iota}\left[\frac{t^{2 \iota}}{(2 \iota) !(2 a)^{2 \iota}}+\sum_{r=1}^{\iota-1} \frac{(-1)^{r} \prod_{s=1}^{2 r-1}(2 \iota+s)}{(2 r) !(2(\iota-r)-1) !(2 a)^{2(\iota+r)}} t^{2(\iota-r)}\right] \\
& \zeta_{2 \iota, 1}(t)=(-1)^{\iota}\left[\frac{t^{2 \iota-1}}{(2 \iota-2) !(2 a)^{2 \iota+1}}\right. \\
& \left.+\sum_{r=1}^{\iota-1} \frac{(-1)^{r} \prod_{s=1}^{2 r}(2 \iota+s)}{(2 r+1) ![2(\iota-r-1)] !(2 a)^{2 \iota+2 r+1}} t^{2 \iota-2 r-1}\right] \\
& \zeta_{2 \iota+1,0}(t)=(-1)^{\iota}\left[\frac{t^{2 \iota}}{(2 \iota-1) !(2 a)^{2(\iota+1)}}\right. \\
& \left.+\sum_{r=1}^{\iota-1} \frac{(-1)^{r} \prod_{s=1}^{2 r}(2 \iota+s+1)}{(2 r+1) !(2 \iota-2 r-1) !(2 a)^{2(\iota+r+1)}} t^{2(\iota-r)}\right], \\
\zeta_{2 \iota+1,1}(t)= & (-1)^{\iota+1}\left[\frac{t^{2 \iota+1}}{(2 \iota+1) !(2 a)^{2 \iota+1}}+\sum_{r=1}^{\iota} \frac{(-1)^{r} \prod_{s=1}^{2 r-1}(2 \iota+s) !(2 \iota-2 r) !(2 a)^{2 \imath+2 r+1}}{(2 r)} t^{2 \iota-2 r+1}\right] .
\end{aligned}
$$

Recall the three-dimensional Laplace operator $\Delta=\partial_{x}^{2}+\partial_{y}^{2}+\partial_{z}^{2}$. By Lemma 4.3 .1 and Lemma 4.3.4,

$$
\left\{e^{a i t} \sum_{r=0}^{\infty} \xi_{2 a i, r}(t) \Delta^{r}\left(x_{1}^{\ell} y^{\ell_{2}} z^{\ell_{3}}\right) \mid \ell_{1}, \ell_{2}, \ell_{3} \in \mathbb{N}\right\}
$$

are complex solutions of the Klein-Gordan equation (4.3.77). Taking real parts of (4.3.84), we get

Theorem 4.3.6. The Klein-Gordan equation (4.3.77) has the following set of linearly independent trigonometric-polynomial solution:

$$
\begin{aligned}
& \left\{\sum_{r_{1}, r_{2}, r_{3}=0}^{\infty}\left(\begin{array}{c}
r_{1}+r_{2}+r_{3} \\
r_{1}, r_{2}, r_{3}
\end{array}\right)\left[\prod_{s=1}^{3}\left(2 r_{s}\right) !\left(\begin{array}{c}
\ell_{s} \\
2 r_{s}
\end{array}\right)\right]\left(\zeta_{r_{1}+r_{2}+r_{3}, 0}(t) \cos a t-\zeta_{r_{1}+r_{2}+r_{3}, 1}(t) \sin a t\right)\right. \\
& \times x^{\ell_{1}-2 r_{1}} y^{\ell_{2}-2 r_{2}} z^{\ell_{3}-2 r_{3}}, \sum_{r_{1}, r_{2}, r_{3}=0}^{\infty}\left(\begin{array}{c}
r_{1}+r_{2}+r_{3} \\
r_{1}, r_{2}, r_{3}
\end{array}\right)\left[\prod_{s=1}^{3}\left(2 r_{s}\right) !\left(\begin{array}{c}
\ell_{s} \\
2 r_{s}
\end{array}\right)\right]\left(\zeta_{r_{1}+r_{2}+r_{3}, 0}(t) \sin a t\right. \\
& \left.\left.+\zeta_{r_{1}+r_{2}+r_{3}, 1}(t) \cos a t\right) x^{\ell_{1}-2 r_{1}} y^{\ell_{2}-2 r_{2}} z^{\ell_{3}-2 r_{3}}, \mid \ell_{1}, \ell_{2}, \ell_{3} \in \mathbb{N}\right\}
\end{aligned}
$$


The following lemmas will be used to handle some special cases when the operator $T_{1}$ in Lemma 4.3.1 does not have a right inverse. We again use the settings in (4.3.9) and (4.3.10).

Lemma 4.3.7. Let $T_{0}$ be a differential operator on $\mathcal{A}$ with right inverse $T_{0}^{-}$such that

$$
T_{0}(\mathcal{B}), T_{0}^{-}(\mathcal{B}) \subset \mathcal{B}, \quad T_{0}\left(\eta_{1} \eta_{2}\right)=T_{0}\left(\eta_{1}\right) \eta_{2} \quad \text { for } \eta_{1} \in \mathcal{B}, \eta_{2} \in V
$$

and let $T_{1}, \ldots, T_{m}$ be commuting differential operators on $\mathcal{A}$ such that $T_{\iota}(V) \subset V$,

$$
T_{0} T_{\iota}=T_{\iota} T_{0}, \quad T_{\iota}(f \zeta)=f T_{\iota}(\zeta) \quad \text { for } \iota \in \overline{1, m}, f \in \mathcal{B}, \zeta \in \mathcal{A} .
$$

If $T_{0}^{m}(h)=0$ with $h \in \mathcal{B}$ and $g \in V$, then

$$
\begin{aligned}
u= & \sum_{\iota=0}^{\infty}\left(\sum_{s=1}^{m}\left(T_{0}^{-}\right)^{s} T_{s}\right)^{\iota}(h g)=\sum_{\iota_{1}, \ldots, \iota_{m}=0}^{\infty}\left(\begin{array}{c}
\iota_{1}+\cdots+\iota_{m} \\
\iota_{1}, \ldots, \iota_{m}
\end{array}\right) \\
& \times\left(T_{0}^{-}\right)^{\sum_{s=1}^{m} s \iota_{s}}(h)\left(\prod_{r=1}^{m} T_{r}^{\iota_{r}}\right)(g)
\end{aligned}
$$

is a solution of the equation:

$$
\left(T_{0}^{m}-\sum_{r=1}^{m} T_{0}^{m-\iota} T_{\iota}\right)(u)=0 .
$$

Suppose

$$
T_{\iota}\left(V_{r}\right) \subset V_{r-1} \quad \text { for } \iota \in \overline{1, m}, r \in \mathbb{N},
$$

where $V_{-1}=\{0\}$. Then any polynomial solution of (4.3.89) is a linear combinations of the solutions of the form (4.3.88).

Proof. Note that

$$
T_{0}^{m-\iota}=T_{0}^{m}\left(T_{0}^{-}\right)^{\iota} \quad \text { for } \quad \iota \in \overline{1, m}
$$

and

$$
\begin{aligned}
& \sum_{\iota_{1}+\cdots+\iota_{m}=\iota+1}\left(\begin{array}{c}
\iota+1 \\
\iota_{1}, \ldots, \iota_{m}
\end{array}\right) y_{1}^{\iota_{1}} \cdots y_{m}^{\iota_{m}}=\left(y_{1}+\cdots+y_{m}\right)^{\iota+1} \\
= & \sum_{r=1}^{m} \sum_{\iota_{1}+\cdots+\iota_{m}=\iota}\left(\begin{array}{c}
\iota \\
\iota_{1}, \ldots, \iota_{m}
\end{array}\right) y_{r} y_{1}^{\iota_{1}} \cdots y_{m}^{\iota_{m}} .
\end{aligned}
$$

Thus

$$
\begin{aligned}
& \left(T_{0}^{m}-\sum_{p=1}^{m} T_{0}^{m-p} T_{p}\right)\left[\sum_{\iota_{1}, \ldots, \iota_{m}=0}^{\infty}\left(\begin{array}{c}
\iota_{1}+\cdots+\iota_{m} \\
\iota_{1}, \ldots, \iota_{m}
\end{array}\right)\left(T_{0}^{-}\right)^{\sum_{s=1}^{m} s \iota_{s}}(h)\left(\prod_{r=1}^{m} T_{r}^{\iota_{r}}\right)(g)\right] \\
= & \sum_{\iota_{1}, \ldots, \iota_{m} \in \mathbb{N} ; \iota_{1}+\cdots+\iota_{m}>0}\left(\begin{array}{c}
\iota_{1}+\cdots+\iota_{m} \\
\iota_{1}, \ldots, \iota_{m}
\end{array}\right) T_{0}^{m}\left(T_{0}^{-}\right)^{\sum_{s=1}^{m} s \iota_{s}}(h)\left(\prod_{r=1}^{m} T_{r}^{\iota_{r}}\right)(g) \\
& -\sum_{\iota_{1}, \ldots, \iota_{m}=0}^{\infty} \sum_{p=1}^{m}\left(\begin{array}{c}
\iota_{1}+\cdots+\iota_{m} \\
\iota_{1}, \ldots, \iota_{m}
\end{array}\right) T_{0}^{m}\left(T_{0}^{-}\right)^{\iota_{p}+\sum_{s=1}^{m} s \iota_{s}}(h)\left(T_{p} \prod_{r=1}^{m} T_{r}^{\iota_{r}}\right)(g)=0 .
\end{aligned}
$$


Suppose that (4.3.90) holds. Let $u \in \mathcal{B} V_{k} \backslash \mathcal{B} V_{k-1}$ be a solution of (4.3.89). Take a basis $\left\{\phi_{\iota}+V_{k-1} \mid \iota \in I\right\}$ of $V_{k} / V_{k-1}$. Write

$$
u=\sum_{\iota \in I} h_{\iota} \phi_{\iota}+u^{\prime}, \quad h_{\iota} \in \mathcal{B}, u^{\prime} \in \mathcal{B} V_{k-1} .
$$

Since

$$
T_{r}\left(\phi_{\iota}\right) \in V_{k-1} \quad \text { for } \iota \in I, r \in \overline{1, m}
$$

by (4.3.90), we have

$$
\left(T_{0}^{m}-\sum_{r=1}^{m} T_{0}^{m-\iota} T_{\iota}\right)(u) \equiv \sum_{\iota \in I} T_{0}^{m}\left(h_{\iota}\right) \phi_{\iota} \equiv 0\left(\bmod \mathcal{B} V_{k-1}\right) .
$$

Hence

$$
T_{0}^{m}\left(h_{\iota}\right)=0 \quad \text { for } \iota \in I \text {. }
$$

Now

$$
u-\sum_{j \in I} \sum_{\iota_{1}, \ldots, \iota_{m}=0}^{\infty}\left(\begin{array}{c}
\iota_{1}+\cdots+\iota_{m} \\
\iota_{1}, \ldots, \iota_{m}
\end{array}\right)\left(T_{0}^{-}\right)^{\sum_{s=1}^{m} s \iota_{s}}\left(h_{j}\right)\left(\prod_{r=1}^{m} T_{r}^{\iota_{r}}\right)\left(\phi_{j}\right) \in \mathcal{B} V_{k-1}
$$

is a solution of (4.3.89). By induction on $k, u$ is a linear combinations of the solutions of the form (4.3.88).

We remark that the above lemma does not imply Lemma 4.3 .1 because $T_{1}$ and $T_{2}$ in Lemma 4.3.1 may not commute.

Let $d_{1}$ be a differential operator on $\mathbb{R}\left[x_{1}, x_{2}, \ldots, x_{r}\right]$ and let $d_{2}$ be a locally nilpotent differential operator on $V=\mathbb{R}\left[x_{r+1}, \ldots, x_{n}\right]$. Set

$$
V_{m}=\left\{f \in V \mid d_{2}^{m+1}(f)=0\right\} \quad \text { for } m \in \mathbb{N} .
$$

Then $V=\bigcup_{m=0}^{\infty} V_{m}$ because $d_{2}$ is locally nilpotent. We treat $V_{-1}=\{0\}$. Take a subset $\left\{\psi_{m, j} \mid m \in \mathbb{N}, j \in I_{m}\right\}$ of $V$ such that $\left\{\psi_{m, j}+V_{m-1} \mid j \in I_{m}\right\}$ forms a basis of $V_{m} / V_{m-1}$ for $m \in \mathbb{N}$. In particular, $\left\{\psi_{m, j} \mid m \in \mathbb{N}, j \in I_{m}\right\}$ forms a basis of $V$. Fix $h \in \mathbb{R}\left[x_{1}, \ldots, x_{r}\right]$.

Lemma 4.3.8. Let $m$ be a positive integer. Suppose that

$$
u=\sum_{j \in I_{m}} f_{j} \psi_{m, j}+u^{\prime} \in \mathbb{R}\left[x_{1}, x_{2}, \ldots, x_{n}\right]
$$

with $f_{j} \in \mathbb{R}\left[x_{1}, \ldots, x_{r}\right]$ and $d_{2}^{m}\left(u^{\prime}\right)=0$ is a solution of the equation:

$$
\left(d_{1}-h d_{2}\right)(u)=0 .
$$

Then $d_{1}\left(f_{j}\right)=0$ for $j \in I_{m}$ and the system

$$
\xi_{0}=f_{j}, \quad d_{1}\left(\xi_{s+1}\right)=h \xi_{s} \quad \text { for } s \in \overline{0, m-1}
$$


has a solution $\xi_{1}, \ldots, \xi_{m} \in \mathbb{R}\left[x_{1}, \ldots, x_{r}\right]$ for each $j \in I_{m}$.

Proof. Observe that if $\left\{g_{j}+V_{p} \mid j \in J\right\}$ is a linearly independent subset of $V_{p+1} / V_{p}$, then $\left\{d_{2}^{s}\left(g_{j}\right)+V_{p-s} \mid j \in J\right\}$ is a linearly independent subset of $V_{p-s+1} / V_{p-s}$ for $s \in \overline{1, p+1}$ by (4.3.99). By induction, we take a subset $\left\{\phi_{m-s, j} \mid j \in J_{m-s}\right\}$ of $V_{m-s}$ for each $s \in \overline{1, m}$ such that

$$
\left\{d_{2}^{s}\left(\psi_{m, j_{1}}\right)+V_{m-s-1}, d_{2}^{s-p}\left(\phi_{m-p, j_{2}}\right)+V_{m-s-1} \mid p \in \overline{1, s}, j_{1} \in I_{m}, j_{2} \in J_{m-p}\right\}
$$

forms a basis of $V_{m-s} / V_{m-s-1}$ for $s \in \overline{1, m}$. Denote

$$
\mathcal{U}=\sum_{s=1}^{m} \sum_{p=0}^{m-s} \sum_{j \in J_{m-s}} \mathbb{R}\left[x_{1}, \ldots, x_{r}\right] d_{2}^{p}\left(\phi_{m-s, j}\right)
$$

Now we write

$$
u=\sum_{j \in I_{m}}\left[f_{j} \psi_{m, j}+\sum_{s=1}^{m} f_{s, j} d_{2}^{s}\left(\psi_{m, j}\right)\right]+v, \quad v \in \mathcal{U}, f_{s, j} \in \mathbb{R}\left[x_{1}, \ldots, x_{r}\right]
$$

Then (4.3.101) becomes

$$
\begin{aligned}
& \sum_{j \in I_{m}}\left[d_{1}\left(f_{j}\right) \psi_{m, j}+\left(d_{1}\left(f_{1, j}\right)-h f_{j}\right) d_{2}\left(\psi_{m, j}\right)+\sum_{s=2}^{m}\left(d_{1}\left(f_{s, j}\right)-h f_{s-1, j}\right) d^{s}\left(\psi_{m, j}\right)\right] \\
& +\left(d_{1}-h d_{2}\right)(v)=0 .
\end{aligned}
$$

Since $\left(d_{1}-h d_{2}\right)(v) \in \mathcal{U}$, we have:

$$
d_{1}\left(f_{j}\right)=0, \quad d_{1}\left(f_{1, j}\right)=h f_{j}, \quad d_{1}\left(f_{s, j}\right)=h f_{s-1, j}
$$

for $j \in I_{m}$ and $s \in \overline{2, m}$. So (4.3.102) has a solution $\xi_{1}, \ldots, \xi_{m} \in \mathbb{R}\left[x_{1}, \ldots, x_{r}\right]$ for each $j \in I_{m}$.

We remark that our above lemma implies that if (4.3.102) does not have a solution for some $j$, then the equation (4.3.101) does not have a solution of the form (4.3.100). Set

$$
\mathcal{S}_{0}=\left\{f \in \mathbb{R}\left[x_{1}, \ldots, x_{r}\right] \mid d_{1}(f)=0\right\}
$$

and

$$
\mathcal{S}_{m}=\left\{f_{0} \in \mathcal{S}_{0} \mid d_{1}\left(f_{s}\right)=h f_{s-1} \text { for some } f_{1}, \ldots, f_{m} \in \mathbb{R}\left[x_{1}, \ldots, x_{r}\right]\right\}
$$

for $m \in \mathbb{N}+1$. For each $m \in \mathbb{N}+1$ and $f \in \mathcal{S}_{m}$, we fix $\left\{\sigma_{1}(f), \ldots, \sigma_{m}(f)\right\} \subset \mathbb{R}\left[x_{1}, \ldots, x_{r}\right]$ such that

$$
d_{1}\left(\sigma_{1}(f)\right)=h f, \quad d_{1}\left(\sigma_{s}(f)\right)=h \sigma_{s-1}(f) \quad \text { for } s \in \overline{2, m} \text {. }
$$


Denote $\sigma_{0}(f)=f$.

Lemma 4.3.9. The set

$$
\mathcal{S}=\sum_{m=0}^{\infty} \sum_{j \in I_{m}} \sum_{f \in \mathcal{S}_{m}} \mathbb{R}\left(\sum_{s=0}^{m} \sigma_{s}(f) d_{2}^{s}\left(\psi_{m, j}\right)\right)
$$

is the solution space of the equation (4.3.101) in $\mathbb{R}\left[x_{1}, x_{2}, \ldots, x_{n}\right]$.

Proof. For $f \in \mathcal{S}_{m}$,

$$
\begin{aligned}
& \left(d_{1}-h d_{2}\right)\left(\sum_{s=0}^{m} \sigma_{s}(f) d_{2}^{s}\left(\psi_{m, j}\right)\right) \\
= & \sum_{s=1}^{m} h \sigma_{s-1}(f) d_{2}^{s}\left(\psi_{m, j}\right)-\sum_{s=0}^{m-1} h \sigma_{s}(f) d_{2}^{s+1}\left(\psi_{m, j}\right)=0 .
\end{aligned}
$$

Thus $\sum_{s=0}^{m} \sigma_{s}(f) d_{2}^{s}\left(\psi_{m, j}\right)$ is a solution of (4.3.101).

Suppose that $u$ is a solution (4.3.101). Then $u$ can be written as (4.3.100) such that $f_{j} \neq 0$ for some $j \in I_{m}$ due to $V=\bigcup_{m=0}^{\infty} V_{m}$. If $m=0$, then $u \in \mathcal{S}$ naturally. Assume that $u \in \mathcal{S}$ if $m<\ell$. Consider $m=\ell$. According to Lemma 4.3.8, $f_{j} \in \mathcal{S}_{m}$ for any $j \in I_{m}$ (cf. (4.3.109)). Thus $\sum_{j \in I_{m}} \sum_{s=0}^{m} \sigma_{s}\left(f_{j}\right) d_{2}^{s}\left(\psi_{m, j}\right)$ is a solution of the equation (4.3.101). Hence $u-\sum_{j \in I_{m}} \sum_{s=0}^{m} \sigma_{s}\left(f_{j}\right) d_{2}^{s}\left(\psi_{m, j}\right)$ is a solution of (4.3.101) and

$$
d_{2}^{m}\left(u-\sum_{j \in I_{m}} \sum_{s=0}^{m} \sigma_{s}\left(f_{j}\right) d_{2}^{s}\left(\psi_{m, j}\right)\right)=0 .
$$

So

$$
u-\sum_{j \in I_{m}} \sum_{s=0}^{m} \sigma_{s}\left(f_{j}\right) d_{2}^{s}\left(\psi_{m, j}\right) \in \mathbb{R}\left[x_{1}, \ldots, x_{r}\right] V_{m-1}
$$

By assumption,

$$
u-\sum_{j \in I_{m}} \sum_{s=0}^{m} \sigma_{s}\left(f_{j}\right) d_{2}^{s}\left(\psi_{m, j}\right) \in \mathcal{S} .
$$

Since $\sum_{j \in I_{m}} \sum_{s=0}^{m} \sigma_{s}\left(f_{j}\right) d_{2}^{s}\left(\psi_{m, j}\right) \in \mathcal{S}$, we have $u \in \mathcal{S}$. By induction, $u \in \mathcal{S}$ for any solution of (4.3.101).

Let $\epsilon \in\{1,-1\}$ and let $\lambda$ be a nonzero real number. Next we want to find all the polynomial solutions of the equation:

$$
u_{t t}+\frac{\lambda}{t} u_{t}-\epsilon\left(u_{x_{1} x_{1}}+u_{x_{2} x_{2}}+\cdots+u_{x_{n} x_{n}}\right)=0,
$$

which is the generalized anisymmetrical Laplace equation if $\epsilon=-1$. Rewrite the above equation as:

$$
t u_{t t}+\lambda u_{t}-\epsilon t\left(u_{x_{1} x_{1}}+u_{x_{2} x_{2}}+\cdots+u_{x_{n} x_{n}}\right)=0 .
$$


Set

$$
d_{1}=t \partial_{t}^{2}+\lambda \partial_{t}, \quad d_{2}=\Delta_{n}=\sum_{r=1}^{n} \partial_{x_{r}}^{n}, \quad h=\epsilon t
$$

Denote

$$
\mathcal{S}=\left\{f \in \mathbb{R}[t] \mid d_{1}(f)=0\right\}
$$

Note that

$$
d_{1}\left(t^{m}\right)=m(\lambda+m-1) t^{m-1} \quad \text { for } m \in \mathbb{N} \text {. }
$$

So

$$
\mathcal{S}= \begin{cases}\mathbb{R} & \text { if } \lambda \notin-(\mathbb{N}+1) \\ \mathbb{R}+\mathbb{R} t^{-\lambda+1} & \text { if } \lambda \in-(\mathbb{N}+1)\end{cases}
$$

In particular, $t^{-\lambda} \notin d_{1}(\mathbb{R}[t])$ and so $d_{1}$ does not have a right inverse when $\lambda$ is a negative integer. Otherwise $t^{-\lambda}=d_{1}\left(d_{1}^{-}\left(t^{-\lambda}\right)\right) \in d_{1}(\mathbb{R}[t])$.

Set

$$
\phi_{0}(t)=1, \quad \phi_{m}(t)=\frac{\epsilon^{m} t^{2 m}}{m ! 2^{m} \prod_{r=0}^{m-1}(\lambda+2 r+1)}
$$

for $m \in \mathbb{N}+1$ and $\lambda \neq-1,-3, \ldots,-(2 m-1)$. Then $d\left(\phi_{r+1}(t)\right)=\epsilon t \phi_{r}(t)$ for $r \in \overline{0, m-1}$. If $\lambda=-2 k-1$, there does not exist a function $\phi(t) \in \mathbb{R}[t]$ such that $d_{1}(\phi(t))=\epsilon t \phi_{k}(t)$ because $d_{1}\left(t^{2 k+2}\right)=(2 k+2)(2 k+1+\lambda) t^{2 k+1}=0$. When $\lambda \in-(\mathbb{N}+1)$, we set

$$
\psi_{0}=t^{1-\lambda}, \quad \psi_{m}=\frac{\epsilon^{m} t^{2 m+1-\lambda}}{2^{m} m ! \prod_{r=1}^{m}(2 r+1-\lambda)} \quad \text { for } m \in \mathbb{N}+1 .
$$

It can be verified that $d_{1}\left(\psi_{r+1}(t)\right)=\epsilon t \psi_{r}(t)$ for $r \in \mathbb{N}$. Define

$$
V=\mathbb{R}\left[x_{1}, x_{2}, \ldots, x_{n}\right], \quad \Delta_{2, n}=\sum_{s=2}^{n} \partial_{x_{s}}^{2}
$$

and

$$
V_{m}=\left\{f \in V \mid \Delta_{n}^{m+1}(f)=0\right\} \quad \text { for } m \in \mathbb{N} .
$$

Observe

$$
\begin{aligned}
& \sum_{j_{1}, \ldots, j_{\ell}=0}^{\infty}(-1)^{j_{1}+\cdots+j_{\ell}}\left(\begin{array}{c}
j_{1}+\cdots+j_{\ell} \\
j_{1}, \ldots, j_{\ell}
\end{array}\right) \prod_{r=1}^{\ell}\left[\left(\begin{array}{l}
\ell \\
r
\end{array}\right) t^{r}\right]^{j_{r}}=\sum_{p=0}^{\infty}\left(-\sum_{s=1}^{\ell}\left(\begin{array}{l}
\ell \\
s
\end{array}\right) t^{s}\right)^{p} \\
= & \frac{1}{(1+t)^{\ell}}=\sum_{r=0}^{\infty}(-1)^{r}\left(\begin{array}{c}
\ell+r-1 \\
r
\end{array}\right) t^{r}
\end{aligned}
$$

for $|t|<1$. Applying Lemma 4.3.7 to $\Delta_{n}^{m+1}=\sum_{r=0}^{m+1}\left(\begin{array}{c}m+1 \\ r\end{array}\right) \partial_{x_{1}}^{2(m+1-r)} \Delta_{2, n}^{r}, T_{0}=\partial_{x_{1}}^{2}$ and $T_{r}=-\left(\begin{array}{c}m+1 \\ r\end{array}\right) \Delta_{2, n}^{r}$ for $r \in \overline{1, m+1}$, we get a basis

$$
\left\{\sum_{r=0}^{\infty}(-1)^{r}\left(\begin{array}{c}
m+r \\
r
\end{array}\right) \frac{x_{1}^{\ell_{1}+2 r}}{\left(\ell_{1}+2 r\right) !} \Delta_{2, n}^{r}\left(x_{2}^{\ell_{2}} \cdots x_{n}^{\ell_{n}}\right) \mid \ell_{1} \in \overline{0,2 m+1}, \ell_{2}, \ldots, \ell_{n} \in \mathbb{N}\right\}
$$


of $V_{m}$. Hence we obtain:

Theorem 4.3.10. If $\lambda \notin-(\mathbb{N}+1)$, then the set

$$
\left\{\sum_{r=0}^{\infty} \phi_{r}(t) \Delta_{n}^{r}\left(x_{1}^{\ell_{1}} \cdots x_{n}^{\ell_{n}}\right) \mid \ell_{1}, \ldots, \ell_{n} \in \mathbb{N}\right\}
$$

forms a basis of the space of the polynomial solutions for the equation (4.3.117). When $\lambda$ is a negative even integer, the set

$$
\left\{\sum_{r=0}^{\infty} \phi_{r}(t) \Delta_{n}^{r}\left(x_{1}^{\ell_{1}} \cdots x_{n}^{\ell_{n}}\right), \sum_{r=0}^{\infty} \psi_{r}(t) \Delta_{n}^{r}\left(x_{1}^{\ell_{1}} \cdots x_{n}^{\ell_{n}}\right) \mid \ell_{1}, \ldots, \ell_{n} \in \mathbb{N}\right\}
$$

forms a basis of the space of the polynomial solutions for the equation (4.3.117). Assume that $\lambda=-2 k-1$ is a negative odd integer. The set

$$
\begin{aligned}
& \left\{\sum_{s=0}^{k} \sum_{r=0}^{\infty}(-1)^{r}\left(\begin{array}{c}
k+r \\
r
\end{array}\right) \phi_{s}(t) \Delta_{n}^{s}\left[\frac{x_{1}^{\ell_{1}+2 r}}{\left(\ell_{1}+2 r\right) !} \Delta_{2, n}^{r}\left(x_{2}^{\ell_{2}} \cdots x_{n}^{\ell_{n}}\right)\right],\right. \\
& \left.\sum_{r=0}^{\infty} \psi_{r}(t) \Delta_{n}^{r}\left(x_{1}^{\ell_{1}^{\prime}} x_{2}^{\ell_{2}} \cdots x_{n}^{\ell_{n}}\right) \mid \ell_{1} \in \overline{0,2 k+1}, \ell_{1}^{\prime}, \ell_{2}, \ldots, \ell_{n} \in \mathbb{N}\right\}
\end{aligned}
$$

is a basis of the space of the polynomial solutions for the equation (4.3.117).

Finally, we consider the special Euler-Poisson-Darboux equation:

$$
u_{t t}-u_{x_{1} x_{1}}-u_{x_{2} x_{2}}-\cdots-u_{x_{n} x_{n}}-\frac{m(m+1)}{t^{2}} u=0
$$

with $m \neq-1,0$. Change the equations to:

$$
t^{2} u_{t t}-t^{2}\left(u_{x_{1} x_{1}}+u_{x_{2} x_{2}}+\cdots+u_{x_{n} x_{n}}\right)-m(m+1) u=0 .
$$

Letting $u=t^{m+1} v$, we have:

$$
t^{2} u_{t t}=m(m+1) t^{m+1} v+2(m+1) t^{m+2} v_{t}+t^{m+3} v_{t t} .
$$

Substituting (4.3.133) into (4.2.132), we get

$$
t v_{t t}+2(m+1) v_{t}-t\left(v_{x_{1} x_{1}}+v_{x_{2} x_{2}}+\cdots+v_{x_{n} x_{n}}\right)=0 .
$$

If we change variable $u=t^{-m} v$, then the equation (4.3.132) becomes

$$
t v_{t t}-2 m v_{t}-t\left(v_{x_{1} x_{1}}+v_{x_{2} x_{2}}+\cdots+{ }_{x_{n} x_{n}}\right)=0 .
$$

Equations (4.3.134) and (4.3.135) are special cases of the equation (4.3.117) with $\epsilon=1$, and $\lambda=2(m+1)$ and $\lambda=-2 m$, respectively.

\section{Exercise 4.3}

Find a basis of the polynomial solution space of the generalized Laplace equation

$$
u_{x x}+x u_{y y}+y u_{z z}=0
$$




\subsection{Use of Fourier Expansion I}

In this section, we mainly use Fourier expansion to solve constant-coefficient linear partial differential equations. Let us first look at three simple examples which are commonly used in engineering mathematics. Kovalevskaya Theorem says that their solutions are unique.

Example 4.4.1. Solve the following heat conduction equation

$$
u_{t}=u_{x x} \quad \text { subject to } u(t,-\pi)=u(t, \pi) \text { and } u(0, x)=g(x) \text { for } x \in[-\pi, \pi] \text {, }
$$

where $g(x)$ is a given continuous function.

Solution. We assume the separation of variables $u=\eta(t) \xi(x)$. Then the equation becomes

$$
\eta^{\prime}(t) \xi(x)=\eta(t) \xi^{\prime \prime}(x) \Longrightarrow \frac{\eta^{\prime}(t)}{\eta(t)}=\frac{\xi^{\prime \prime}(x)}{\xi(x)}=\lambda
$$

is a constant. Solving the problem

$$
\xi^{\prime \prime}=\lambda \xi, \quad \xi(-\pi)=\xi(\pi)=0
$$

we take $\lambda=-n^{2}$ for some $n \in \mathbb{N}$ and $\xi=C_{1} \cos n x+C_{2} \sin n x$. Moreover, $\eta^{\prime}(t)=$ $-n^{2} \eta(t) \Longrightarrow \eta=C_{3} e^{-n^{2} t}$. Thus

$$
u=e^{-n^{2} t}(a \cos n x+b \sin n x)
$$

is a solution of the problem:

$$
u_{t}=u_{x x}, \quad u(t,-\pi)=u(t, \pi)
$$

By superposition principle (additivity of solutions for homogeneous linear equations), we have more general solutions of (4.4.5):

$$
u(t, x)=\sum_{n=0}^{\infty} e^{-n^{2} t}\left(a_{n} \cos n x+b_{n} \sin n x\right),
$$

where $a_{n}$ and $b_{n}$ are constants to be determined. To satisfy the last condition in (4.4.1), we require

$$
\sum_{n=0}^{\infty}\left(a_{n} \cos n x+b_{n} \sin n x\right)=u(0, x)=g(x) .
$$

According to the theory of Fourier expansion,

$$
a_{0}=\frac{1}{2 \pi} \int_{-\pi}^{\pi} g(s) d s, \quad a_{n}=\frac{1}{\pi} \int_{-\pi}^{\pi} g(s) \cos n s d s, \quad b_{n}=\frac{1}{\pi} \int_{-\pi}^{\pi} g(s) \sin n s d s
$$


for $n \geq 1$. So the final solution of (4.1) is

$$
\begin{aligned}
& u(t, x)=\frac{1}{2 \pi} \int_{-\pi}^{\pi} g(s) d s \\
+ & \frac{1}{\pi} \sum_{n=1}^{\infty} e^{-n^{2} t}\left[\cos n x \int_{-\pi}^{\pi} g(s) \cos n s d s+\sin n x \int_{-\pi}^{\pi} g(s) \sin n s d s\right] \\
= & \frac{1}{2 \pi} \int_{-\pi}^{\pi} g(s) d s+\frac{1}{\pi} \sum_{n=1}^{\infty} e^{-n^{2} t} \int_{-\pi}^{\pi} g(s)(\cos n x \cos +\sin n x \sin n s) d s \\
= & \frac{1}{2 \pi} \int_{-\pi}^{\pi} g(s) d s+\frac{1}{\pi} \sum_{n=1}^{\infty} e^{-n^{2} t} \int_{-\pi}^{\pi} g(s) \cos n(x-s) d s .
\end{aligned}
$$

Example 4.4.2. Solve the following wave equation

$$
u_{t t}=u_{x x} \quad \text { subject to } u(t,-\pi)=u(t, \pi)
$$

and

$$
u(0, x)=g_{1}(x), u_{t}(0, x)=g_{2}(x) \text { for } x \in[-\pi, \pi]
$$

where $g_{1}(x)$ and $g_{2}(x)$ are given continuous functions.

Solution. We assume the separation of variables $u=\eta(t) \xi(x)$. Then the equation becomes

$$
\eta^{\prime \prime}(t) \xi(x)=\eta(t) \xi^{\prime \prime}(x) \Longrightarrow \frac{\eta^{\prime \prime}(t)}{\eta(t)}=\frac{\xi^{\prime \prime}(x)}{\xi(x)}=\lambda
$$

is a constant. As the above example, we find the general solution of (4.4.10) is

$$
u(t, x)=\sum_{n=0}^{\infty} \cos n t\left(a_{n} \cos n x+b_{n} \sin n x\right)+\sum_{n=1}^{\infty} \sin n t\left(\hat{a}_{n} \cos n x+\hat{b}_{n} \sin n x\right)+\hat{a}_{0} t,
$$

where $a_{n}, b_{n}, \hat{a}_{n}, \hat{b}_{n} \in \mathbb{R}$. Note

$$
u(0, x)=\sum_{n=0}^{\infty}\left(a_{n} \cos n x+b_{n} \sin n x\right)=g_{1}(x) .
$$

Since

$$
u_{t}(t, x)=\sum_{n=1}^{\infty} n\left[\sin n t\left(a_{n} \cos n x+b_{n} \sin n x\right)+\cos n t\left(\hat{a}_{n} \cos n x+\hat{b}_{n} \sin n x\right)\right]+\hat{a}_{0},
$$

we have

$$
u_{t}(0, x)=\sum_{n=1}^{\infty} n\left(\hat{a}_{n} \cos n x+\hat{b}_{n} \sin n x\right)+\hat{a}_{0}=g_{2}(x) .
$$

As (4.4.6)-(4.4.9), the final solution is

$$
\begin{aligned}
u(t, x)= & \frac{1}{2 \pi} \int_{-\pi}^{\pi}\left(g_{1}(s)+t g_{2}(s)\right) d s+\frac{1}{\pi} \sum_{n=1}^{\infty} \cos n t \int_{-\pi}^{\pi} g_{1}(s) \cos n(x-s) d s \\
& +\frac{1}{\pi} \sum_{n=1}^{\infty} \frac{\sin n t}{n} \int_{-\pi}^{\pi} g_{2}(s) \cos n(x-s) d s .
\end{aligned}
$$


Example 4.4.3. Solve the following Laplace equation

$$
u_{x x}+u_{y y}=0 \quad \text { subject to } u(x,-\pi)=u(x, \pi)
$$

and

$$
u(0, y)=g_{1}(y), u_{x}(0, y)=g_{2}(y) \text { for } y \in[-\pi, \pi]
$$

where $g_{1}(y)$ and $g_{2}(y)$ are given continuous functions.

Solution. We assume the separation of variables $u=\eta(x) \xi(y)$. Then the equation becomes

$$
\eta^{\prime \prime}(x) \xi(y)=-\eta(x) \xi^{\prime \prime}(y) \Longrightarrow-\frac{\eta^{\prime \prime}(x)}{\eta(x)}=\frac{\xi^{\prime \prime}(y)}{\xi(y)}=\lambda
$$

is a constant. As Example 4.4.1, we find the general solution of (4.4.18) is

$$
\begin{aligned}
u(x, y)= & \sum_{n=0}^{\infty} \cosh n x\left(a_{n} \cos n y+b_{n} \sin n y\right)+\hat{a}_{0} x \\
& +\sum_{n=1}^{\infty} \sinh n x\left(\hat{a}_{n} \cos n y+\hat{b}_{n} \sin n y\right),
\end{aligned}
$$

where $a_{n}, b_{n}, \hat{a}_{n}, \hat{b}_{n} \in \mathbb{R}$. As (4.4.14)-(4.4.17), we get the final solution

$$
\begin{aligned}
u(x, y)= & \frac{1}{2 \pi} \int_{-\pi}^{\pi}\left(g_{1}(s)+x g_{2}(s)\right) d s+\frac{1}{\pi} \sum_{n=1}^{\infty} \cosh n x \int_{-\pi}^{\pi} g_{1}(s) \cos n(y-s) d s \\
& +\frac{1}{\pi} \sum_{n=1}^{\infty} \frac{\sinh n x}{n} \int_{-\pi}^{\pi} g_{2}(s) \cos n(y-s) d s .
\end{aligned}
$$

The rest of this section is taken from the author's work [X11].

Let $m$ and $n>1$ be positive integers and let

$$
f_{r}\left(\partial_{x_{2}}, \ldots, \partial_{x_{n}}\right) \in \mathbb{R}\left[\partial_{x_{2}}, \ldots, \partial_{x_{n}}\right] \quad \text { for } r \in \overline{1, m} \text {. }
$$

We want to solve the equation:

$$
\left(\partial_{x_{1}}^{m}-\sum_{r=1}^{m} \partial_{x_{1}}^{m-r} f_{r}\left(\partial_{x_{2}}, \ldots, \partial_{x_{n}}\right)\right)(u)=0
$$

with $x_{1} \in \mathbb{R}$ and $x_{r} \in\left[-a_{r}, a_{r}\right]$ for $r \in \overline{2, n}$, subject to the condition

$$
\partial_{x_{1}}^{s}(u)\left(0, x_{2}, \ldots, x_{n}\right)=g_{s}\left(x_{2}, \ldots, x_{n}\right) \quad \text { for } s \in \overline{0, m-1}
$$

where $a_{2}, \ldots, a_{n}$ are positive real numbers and $g_{0}, \ldots, g_{m-1}$ are continuous functions. For convenience, we denote

$$
k_{\iota}^{\dagger}=\frac{k_{\iota}}{a_{\iota}}, \quad \vec{k}^{\dagger}=\left(k_{2}^{\dagger}, \ldots, k_{n}^{\dagger}\right) \quad \text { for } \quad \vec{k}=\left(k_{2}, \ldots, k_{n}\right) \in \mathbb{Z}^{n-1} .
$$


Set

$$
e^{\pi\left(\vec{k}^{\dagger} \cdot \vec{x}\right) i}=e^{\sum_{r=2}^{n} \pi k_{r}^{\dagger} x_{r} i} .
$$

For $r \in \overline{0, m-1}$, Lemma 4.3.7 with $T_{0}=\partial_{x_{1}}$ and $T_{q}=f_{q}\left(\partial_{x_{2}}, \ldots, \partial_{x_{n}}\right)$ gives that

$$
\begin{aligned}
& \frac{1}{r !} \sum_{\iota_{1}, \ldots, \iota_{m}=0}^{\infty}\left(\begin{array}{c}
\iota_{1}+\cdots+\iota_{m} \\
\iota_{1}, \ldots, \iota_{m}
\end{array}\right) \int_{\left(x_{1}\right)}^{\left(\sum_{s=1}^{m} s \iota_{s}\right)}\left(x_{1}^{r}\right)\left(\prod_{p=1}^{m} f_{p}\left(\partial_{x_{2}}, \ldots, \partial_{x_{n}}\right)^{\iota_{p}}\right)\left(e^{\pi\left(\vec{k}^{\dagger} \cdot \vec{x}\right) i}\right) \\
= & \sum_{\iota_{1}, \ldots, \iota_{m}=0}^{\infty}\left(\begin{array}{c}
\iota_{1}+\cdots+\iota_{m} \\
\iota_{1}, \ldots, \iota_{m}
\end{array}\right) \frac{x_{1}^{r+\sum_{s=1}^{m} s \iota_{s}}}{\left(r+\sum_{s=1}^{m} s \iota_{s}\right) !} \\
& \times\left[\prod_{p=1}^{m} f_{p}\left(k_{2}^{\dagger} \pi i, \ldots, k_{n}^{\dagger} \pi i\right)^{\iota_{p}}\right] e^{\pi\left(\vec{k}^{\dagger} \cdot \vec{x}\right) i}
\end{aligned}
$$

is a complex solution of the equation (4.4.24) for any $\vec{k} \in \mathbb{Z}^{n-1}$. We write

$$
\begin{aligned}
& \sum_{\iota_{1}, \ldots, \iota_{m}=0}^{\infty}\left(\begin{array}{c}
\iota_{1}+\cdots+\iota_{m} \\
\iota_{1}, \ldots, \iota_{m}
\end{array}\right) \frac{x_{1}^{r} \prod_{p=1}^{m}\left(x_{1}^{p} f_{p}\left(k_{2}^{\dagger} \pi i, \ldots, k_{n}^{\dagger} \pi i\right)\right)^{\iota_{p}}}{\left(r+\sum_{s=1}^{m} s \iota_{s}\right) !} \\
= & \phi_{r}\left(x_{1}, \vec{k}\right)+\psi_{r}\left(x_{1}, \vec{k}\right) i,
\end{aligned}
$$

where $\phi_{r}\left(x_{1}, \vec{k}\right)$ and $\psi_{r}\left(x_{1}, \vec{k}\right)$ are real functions. Moreover,

$$
\partial_{x_{1}}^{s}\left(\phi_{r}\right)(0, \vec{k})=\delta_{r, s}, \quad \partial_{x_{1}}^{s}\left(\psi_{r}\right)(0, \vec{k})=0 \quad \text { for } s \in \overline{0, r}
$$

We define $\overrightarrow{0} \prec \vec{k}$ if its first nonzero coordinate is a positive integer. By superposition principle and Fourier expansions, we get:

Theorem 4.4.1. The solution of the equation (4.4.24) subject to the condition (4.4.25) is:

$$
\begin{aligned}
u= & \sum_{r=0}^{m-1} \sum_{\overrightarrow{0} \preceq \vec{k} \in \mathbb{Z}^{n-1}}\left[b_{r}(\vec{k})\left(\phi_{r}\left(x_{1}, \vec{k}^{\dagger}\right) \cos \pi\left(\vec{k}^{\dagger} \cdot \vec{x}\right)-\psi_{r}\left(x_{1}, \vec{k}^{\dagger}\right) \sin \pi\left(\vec{k}^{\dagger} \cdot \vec{x}\right)\right)\right. \\
& \left.+c_{r}(\vec{k})\left(\phi_{r}\left(x_{1}, \vec{k}^{\dagger}\right) \sin \pi\left(\vec{k}^{\dagger} \cdot \vec{x}\right)+\psi_{r}\left(x_{1}, \vec{k}^{\dagger}\right) \cos \pi\left(\vec{k}^{\dagger} \cdot \vec{x}\right)\right)\right],
\end{aligned}
$$

with

$$
\begin{aligned}
b_{r}(\vec{k})= & \frac{1}{2^{n-2+\delta_{\vec{k}, \overrightarrow{0}}} a_{2} \cdots a_{n}} \int_{-a_{2}}^{a_{2}} \cdots \int_{-a_{n}}^{a_{n}} g_{r}\left(x_{2}, \ldots, x_{n}\right) \cos \pi\left(\vec{k}^{\dagger} \cdot \vec{x}\right) d x_{n} \cdots d x_{2} \\
& -\sum_{s=0}^{r-1}\left(b_{s}(\vec{k}) \partial_{x_{1}}^{r}\left(\phi_{s}\right)(0, \vec{k})+c_{s}(\vec{k}) \partial_{x_{1}}^{r}\left(\psi_{s}\right)(0, \vec{k})\right) \\
c_{r}(\vec{k})= & \frac{1}{2^{n-2} a_{2} \cdots a_{n}} \int_{-a_{2}}^{a_{2}} \cdots \int_{-a_{n}}^{a_{n}} g_{r}\left(x_{2}, \ldots, x_{n}\right) \sin \pi\left(\vec{k}^{\dagger} \cdot \vec{x}\right) d x_{n} \cdots d x_{2} \\
& -\sum_{s=0}^{r-1}\left(c_{s}(\vec{k}) \partial_{x_{1}}^{r}\left(\phi_{s}\right)(0, \vec{k})-b_{s}(\vec{k}) \partial_{x_{1}}^{r}\left(\psi_{s}\right)(0, \vec{k})\right) .
\end{aligned}
$$


The convergence of the series (4.4.31) is guaranteed by the Kovalevskaya Theorem on the existence and uniqueness of the solution of linear partial differential equations when the functions in (4.4.25) are analytic.

Remark 4.4.2. (1) If we take $f_{\iota}=b_{\iota}$ with $\iota \in \overline{1, m}$ to be constant functions and $\vec{k}=\overrightarrow{0}$ in (4.4.29), we get $m$ fundamental solutions

$$
\varphi_{r}(x)=\sum_{\iota_{1}, \ldots, \iota_{m}=0}^{\infty}\left(\begin{array}{c}
\iota_{1}+\cdots+\iota_{m} \\
\iota_{1}, \ldots, \iota_{m}
\end{array}\right) \frac{x^{r} \prod_{p=1}^{m}\left(b_{p} x^{p}\right)^{\iota_{p}}}{\left(r+\sum_{s=1}^{m} s \iota_{s}\right) !}, \quad r \in \overline{0, m-1},
$$

of the constant-coefficient ordinary differential equation

$$
y^{(m)}-b_{1} y^{(m-1)}-\cdots-b_{m-1} y^{\prime}-b_{m}=0 .
$$

Given the initial conditions:

$$
y^{(r)}(0)=c_{r} \quad \text { for } r \in \overline{0, m-1}
$$

we define $a_{0}=c_{0}$ and

$$
a_{r}=c_{r}-\sum_{s=0}^{r-1} \sum_{\iota_{1}, \ldots, \iota_{r-s} \in \mathbb{N} ; \sum_{p=1}^{r} p \iota_{p}=r-s}\left(\begin{array}{c}
r-s \\
\iota_{1}, \ldots, \iota_{r-s}
\end{array}\right) a_{s} b_{1}^{\iota_{1}} \cdots b_{r-s}^{\iota_{r}-s}
$$

by induction on $r \in \overline{1, m-1}$. Now the solution of (4.4.35) subject to the condition (4.4.36) is exactly

$$
y=\sum_{r=0}^{m-1} a_{r} \varphi_{r}(x)
$$

From the above results, it seems that the following functions

$$
\mathcal{Y}_{r}\left(y_{1}, \ldots, y_{m}\right)=\sum_{\iota_{1}, \ldots, \iota_{m}=0}^{\infty}\left(\begin{array}{c}
\iota_{1}+\cdots+\iota_{m} \\
\iota_{1}, \ldots, \iota_{m}
\end{array}\right) \frac{y_{1}^{\iota_{1}} y_{2}^{\iota_{2}} \cdots y_{m}^{\iota_{m}}}{\left(r+\sum_{s=1}^{m} s \iota_{s}\right) !} \quad \text { for } r \in \mathbb{N}
$$

are important natural functions. Indeed,

$$
\begin{gathered}
\mathcal{Y}_{0}(x)=e^{x}, \mathcal{Y}_{0}\left(0,-x^{2}\right)=\cos x, \mathcal{Y}_{1}\left(0,-x^{2}\right)=\frac{\sin x}{x} \\
\varphi_{r}(x)=x^{r} \mathcal{Y}_{r}\left(b_{1} x, b_{2} x^{2}, \ldots, b_{m} x^{m}\right)
\end{gathered}
$$

and

$$
\left.\phi_{r}\left(x_{1}, \vec{x}\right)+\psi_{r}\left(x_{1}, \vec{x}\right) i=x_{1}^{r} \mathcal{Y}_{r}\left(x_{1} f_{1}\left(k_{2}^{\dagger} \pi i, \ldots, k_{n}^{\dagger} \pi i\right)\right), \ldots, x_{1}^{m} f_{m}\left(k_{2}^{\dagger} \pi i, \ldots, k_{n}^{\dagger} \pi i\right)\right)
$$

for $r \in \overline{0, m}$.

(2) We can solve the initial value problem (4.4.24) and (4.4.25) with the constantcoefficient differential operators $f_{\iota}\left(\partial_{2}, \ldots, \partial_{n}\right)$ replaced by variable-coefficient differential 
operators $\phi_{\iota}\left(\partial_{2}, \ldots, \partial_{n_{1}}\right) \psi_{\iota}\left(x_{n_{1}+1}, \ldots, x_{n}\right)$ for some $2<n_{1}<n$, where $\phi_{\iota}\left(\partial_{2}, \ldots, \partial_{n_{1}}\right)$ are polynomials in $\partial_{2}, \ldots, \partial_{n_{1}}$ and $\psi_{\iota}\left(x_{n_{1}+1}, \ldots, x_{n}\right)$ are polynomials in $x_{n_{1}+1}, \ldots, x_{n}$.

\section{Exercise 4.4}

1. Solve the following heat conduction problem: $u_{t}=2 u_{x x}$ subject to $u_{x}(t, 0)=$ $0, u_{x}(t, 3)=0$ and $u(0, x)=2 x-1$.

2. Find the solution of the wave equation $u_{t t}=3 u_{x x}$ subject to $u(t, 0)=u(t, 4)=0$ and $u(0, x)=2-x, u_{t}(0, x)=|x-2|$.

3. Find the solution of the equation

$$
u_{x x x}-u_{x x y}-u_{x z}-u_{z z}=0
$$

with $x \in \mathbb{R}$ and $y, z \in[-2,2]$ subject to

$$
u(0, y, z)=y+z, \quad u_{x}(0, y, z)=y-z, \quad u_{x x}(0, y, z)=y z
$$

\subsection{Use of Fourier Expansion II}

In this section, we mainly use Fourier expansion to solve the evolution equations and generalized wave equations of flag type subject to initial conditions. The results in this section are taken from the author's work [X7].

Barros-Neto and Gel'fand [BG1,BG2] $(1998,2002)$ studied solutions of the equation

$$
u_{x x}+x u_{y y}=\delta\left(x-x_{0}, y-y_{0}\right)
$$

related to the Tricomi operator $\partial_{x}^{2}+x \partial_{y}^{2}$. A natural generalization of the Tricomi operator is $\partial_{x_{1}}^{2}+x_{1} \partial_{x_{2}}^{2}+\cdots+x_{n-1} \partial_{x_{n}}^{2}$. The equation

$$
u_{t}=u_{x_{1} x_{1}}+u_{x_{2} x_{2}}+\cdots+u_{x_{n} x_{n}}
$$

is a well known classical heat conduction equation related to the Laplacian operator $\partial_{x_{1}}^{2}+\partial_{x_{2}}^{2}+\cdots+\partial_{x_{n}}^{2}$. As pointed out in [BG1, BG2], the Tricomi operator is an analogue of the Laplacian operator. An immediate analogue of heat conduction equation is

$$
u_{t}=u_{x_{1} x_{1}}+x_{1} u_{x_{2} x_{2}}+x_{2} u_{x_{3} x_{3}}+\cdots+x_{n-1} u_{x_{n} x_{n}} .
$$

Another related well-known equation is the wave equation

$$
u_{t t}=u_{x_{1} x_{1}}+u_{x_{2} x_{2}}+\cdots+u_{x_{n} x_{n}}
$$

Similarly, we have the following analogue of wave equation:

$$
u_{t t}=u_{x_{1} x_{1}}+x_{1} u_{x_{2} x_{2}}+x_{2} u_{x_{3} x_{3}}+\cdots+x_{n-1} u_{x_{n} x_{n}} .
$$


The purpose of this section is to give the methods of solving linear partial differential equations of the above types subject to initial conditions.

Graphically, the above equation are related to the Dynkin diagram of the special linear Lie algebra:

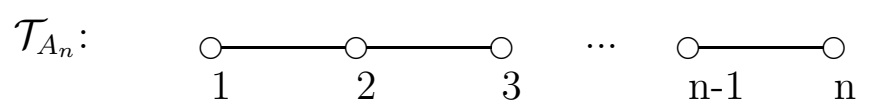

Naturally, we should also consider similar equations related to the graph:

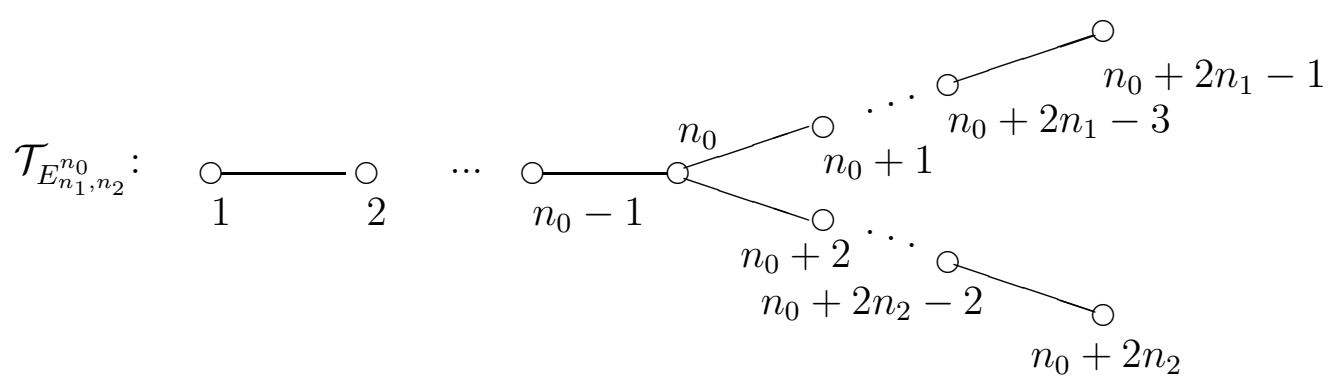

which is the Dynkin diagram of an orthogonal Lie algebra $o(2 n)$ when $n_{1}=n_{2}=1$, and the Dynkin diagram of the simple Lie algebra of types $E_{6}, E_{7}, E_{8}$ if $\left(n_{0}, n_{1}, n_{2}\right)=$ $(3,1,2),(3,1,3),(3,1,4)$, respectively. When $\left(n_{0}, n_{1}, n_{2}\right)=(3,2,2),(4,1,3),(6,1,2)$, it is also the Dynkin diagram of the affine Kac-Moody Lie algebra of types $E_{6}^{(1)}, E_{7}^{(1)}, E_{8}^{(1)}$, respectively (cf. $[\mathrm{Kv}])$. These diagrams are special examples of trees in graph theory.

A tree $\mathcal{T}$ consists of a finite set of nodes $\mathcal{N}=\left\{\iota_{1}, \iota_{2}, \ldots, \iota_{n}\right\}$ and a set of edges

$$
\mathcal{E} \subset\left\{\left(\iota_{p}, \iota_{q}\right) \mid 1 \leq p<q \leq n\right\}
$$

such that for each node $\iota_{q} \in \mathcal{N}$, there exists a unique sequence $\left\{\iota_{q_{1}}, \iota_{q_{2}}, \ldots, \iota_{q_{r}}\right\}$ of nodes with $1=q_{1}<q_{2}<\cdots<q_{r-1}<q_{r}=q$ for which

$$
\left(\iota_{q_{1}}, \iota_{q_{2}}\right),\left(\iota_{q_{2}}, \iota_{q_{3}}\right), \ldots,\left(\iota_{q_{r-2}}, \iota_{q_{r-1}}\right),\left(\iota_{q_{r-1}}, \iota_{q_{r}}\right) \in \mathcal{E} .
$$

We also denote the tree $\mathcal{T}=(\mathcal{N}, \mathcal{E})$. We identify a tree $\mathcal{T}=(\mathcal{N}, \mathcal{E})$ with a graph by depicting a small circle for each node in $\mathcal{N}$ and a segment connecting $r$ th circle to $j$ th circle for the edge $\left(\iota_{r}, \iota_{j}\right) \in \mathcal{E}$ (cf. the above dynkin diagrams of type $A$ and $E$ ).

For a tree $\mathcal{T}=(\mathcal{N}, \mathcal{E})$, we call the differential operator

$$
d_{\mathcal{T}}=\partial_{x_{1}}^{2}+\sum_{\left(\iota_{p}, \iota_{q}\right) \in \mathcal{E}} x_{p} \partial_{x_{q}}^{2}
$$

a generalized Tricomi operator of type $\mathcal{T}$. Moreover, we call the partial differential equation

$$
u_{t}=d_{\mathcal{T}}(u)
$$


a generalized heat conduction equation associated with the tree $\mathcal{T}$, where $u$ is a function in $t, x_{1}, x_{2}, \ldots, x_{n}$. For instance, the generalized heat equation of type $\mathcal{T}_{E_{n_{1}, n_{2}}^{n_{0}}}$ is:

$$
\begin{aligned}
u_{t}= & \left(\partial_{x_{1}}^{2}+\sum_{q=1}^{n_{0}-1} x_{q} \partial_{x_{q+1}}^{2}+\sum_{r=0}^{n_{2}-1} x_{n_{0}+2 r} \partial_{x_{n_{0}+2 r+2}}^{2}\right. \\
& \left.+x_{n_{0}} \partial_{x_{n_{0}+1}}^{2}+\sum_{p=1}^{n_{1}-1} x_{n_{0}+2 p-1} \partial_{x_{n_{0}+2 p+1}}^{2}\right)(u) .
\end{aligned}
$$

Similarly, we have the generalized wave equation associated with the tree $\mathcal{T}$ :

$$
u_{t t}=d_{\mathcal{T}}(u)
$$

Let $m_{0}, m_{1}, m_{2}, \ldots, m_{n}$ be $n+1$ positive integers. The difficulty of solving the equations (4.5.9) and (4.5.10) is the same as that of solving the following more general partial differential equation:

$$
\partial_{t}^{m_{0}}(u)=\left(\partial_{x_{1}}^{m_{1}}+\sum_{\left(\iota_{p}, \iota_{q}\right) \in \mathcal{E}} x_{p} \partial_{x_{q}}^{m_{q}}\right)(u) .
$$

Obviously, we want to use the operator $\sum_{\iota=0}^{\infty}\left(-T_{1}^{-} T_{2}\right)^{\iota}$ in Lemma 4.3.1. Then the main difficulty turns out to be how to calculate the powers of the operator $\partial_{x_{1}}^{m_{1}}+\sum_{\left(\iota_{p}, \iota_{q}\right) \in \mathcal{E}} x_{p} \partial_{x_{q}}^{m_{j}}$. This essentially involves the Campbell-Hausdorff formula, whose simplest nontrivial case $e^{t\left(\partial_{x_{1}}+x_{1} \partial_{x_{2}}\right)}=e^{t x_{1} \partial_{x_{2}}} e^{t \partial_{x_{1}}} e^{t \partial_{x_{2}} / 2}$ has been extensively used by physicists.

Lemma 4.5.1. Let $f(x)$ be a smooth function and let $b$ be a constant. Then

$$
e^{b \frac{d}{d x}}(f(x))=f(x+b) .
$$

Proof. Note

$$
e^{b \frac{d}{d x}}(f(x))=\sum_{n=0}^{\infty} \frac{f^{(n)}(x)}{n !} b^{n}=f(x+b)
$$

by Tayler's expansion.

For $n-1$ positive integers $m_{1}, m_{2}, \ldots, m_{n-1}$, we denote

$$
D=t\left(\partial_{x_{1}}+x_{1}^{m_{1}} \partial_{x_{2}}+x_{2}^{m_{2}} \partial_{x_{3}}+\cdots+x_{n-1}^{m_{n-1}} \partial_{x_{n}}\right)
$$

and set $\eta_{1}=t$,

$$
\eta_{\iota}=\int_{0}^{t}\left(x_{\iota-1}+\int_{0}^{y_{\iota-1}}\left(x_{\iota-2}+\ldots+\int_{0}^{y_{2}}\left(x_{1}+y_{1}\right)^{m_{1}} d y_{1} \ldots\right)^{m_{\iota-2}} d y_{\iota-2}\right)^{m_{\iota-1}} d y_{\iota-1}
$$

for $\iota \in \overline{2, n}$.

Lemma 4.5.2. We have the following Campbell-Hausdorff-type factorizaton:

$$
e^{D}=e^{\eta_{n} \partial_{x_{n}}} e^{\eta_{n-1} \partial_{x_{n-1}}} \cdots e^{\eta_{1} \partial_{x_{1}}} .
$$


Proof. Let $f\left(x_{1}, x_{2}, \ldots, x_{n}\right)$ be any given smooth function. We want to solve the equation

$$
u_{t}-u_{x_{1}}-x_{1}^{m_{1}} u_{x_{2}}-x_{2}^{m_{2}} u_{x_{3}}-\cdots-x_{n-1}^{m_{n-1}} u_{x_{n}}=0
$$

subject to $u\left(0, x_{1}, \ldots, x_{n}\right)=f\left(x_{1}, \ldots, x_{n}\right)$. According to the method of characteristic lines in Section 4.1, we solve the following problem:

$$
\frac{d t}{d s}=1, \quad \frac{d x_{1}}{d s}=-1, \quad \frac{d x_{r+1}}{d s}=-x_{r}^{m_{r}}, \quad r \in \overline{1, n-1}, \quad \frac{d u}{d s}=0,
$$

subject to

$$
\left.t\right|_{s=0}=0,\left.x_{p}\right|_{s=0}=t_{p}, \quad p \in \overline{1, n},\left.u\right|_{s=0}=t_{n+1}, \quad t_{n+1}=f\left(t_{1}, \ldots, t_{n}\right) .
$$

We find

$$
\begin{gathered}
u=t_{n+1}, \quad t=s, \quad x_{1}=-s+t_{1}, \quad x_{2}=t_{2}-\int_{0}^{s}\left(t_{1}-s_{1}\right)^{m_{1}} d s_{1}, \\
x_{3}=t_{3}-\int_{0}^{s}\left(t_{2}-\int_{0}^{s_{2}}\left(t_{1}-s_{1}\right)^{m_{1}} d s_{1}\right)^{m_{2}} d s_{2}, \ldots, \\
x_{r+1}=t_{r+1}-\int_{0}^{s}\left(t_{r}-\int_{0}^{s_{r}}\left(t_{r-1}-\cdots-\int_{0}^{s_{2}}\left(t_{1}-s_{1}\right)^{m_{1}} d s_{1} \cdots\right)^{m_{r-1}} d s_{r-1}\right)^{m_{r}} d s_{r} .
\end{gathered}
$$

Note that $t_{1}=x_{1}+t=x_{1}+\eta_{1}$,

$$
\begin{aligned}
& t_{2}=x_{2}+\int_{0}^{s}\left(t_{1}-s_{1}\right)^{m_{1}} d s_{1}=x_{2}+\int_{0}^{t}\left(x_{1}+t-s_{1}\right)^{m_{1}} d s_{1} \\
& \stackrel{y_{1}=\stackrel{t-s_{1}}{=}}{=} x_{2}-\int_{t}^{0}\left(x_{1}+y_{1}\right)^{m_{1}} d y_{1}=x_{2}+\int_{0}^{t}\left(x_{1}+y_{1}\right)^{m_{1}} d y_{1}=x_{2}+\eta_{2} \text {, } \\
& t_{3}=x_{3}+\int_{0}^{t}\left(t_{2}-\int_{0}^{s_{2}}\left(t_{1}-s_{1}\right)^{m_{1}} d s_{1}\right)^{m_{2}} d s_{2} \\
& =x_{3}+\int_{0}^{t}\left(x_{2}+\int_{0}^{t}\left(x_{1}+y_{1}\right)^{m_{1}} d y_{1}-\int_{0}^{s_{2}}\left(x_{1}+t-s_{1}\right)^{m_{1}} d s_{1}\right)^{m_{2}} d s_{2} \\
& =x_{3}+\int_{0}^{t}\left(x_{2}+\int_{0}^{t}\left(x_{1}+y_{1}\right)^{m_{1}} d y_{1}+\int_{t}^{t-s_{2}}\left(x_{1}+y_{1}\right)^{m_{1}} d y_{1}\right)^{m_{2}} d s_{2} \\
& =x_{3}+\int_{0}^{t}\left(x_{2}+\int_{0}^{t-s_{2}}\left(x_{1}+y_{1}\right)^{m_{1}} d y_{1}\right)^{m_{2}} d s_{2} \\
& \stackrel{y_{2}=\underline{t-s_{2}}}{=} x_{3}-\int_{t}^{0}\left(x_{2}+\int_{0}^{y_{2}}\left(x_{1}+y_{1}\right)^{m_{1}} d y_{1}\right)^{m_{2}} d y_{2} \\
& =x_{3}+\int_{0}^{t}\left(x_{2}+\int_{0}^{y_{2}}\left(x_{1}+y_{1}\right)^{m_{1}} d y_{1}\right)^{m_{2}} d y_{2}=x_{3}+\eta_{3} \text {. }
\end{aligned}
$$

This gives us a pattern of find general $t_{p}$. In the above, we have also proved

$$
t_{2}-\int_{0}^{s_{2}}\left(t_{1}-s_{1}\right)^{m_{1}} d s_{1}=x_{2}+\int_{0}^{t-s_{2}}\left(x_{1}+y_{1}\right)^{m_{1}} d y_{1}
$$


Suppose that $t_{r}=x_{r}+\eta_{r}$ and

$$
\begin{gathered}
t_{r-1}-\int_{0}^{s_{r-1}}\left(t_{r-2}-\cdots-\int_{0}^{s_{2}}\left(t_{1}-s_{1}\right)^{m_{1}} d s_{1} \cdots\right)^{m_{r-2}} d s_{r-2} \\
=x_{r-1}+\int_{0}^{y_{-s_{r-1}}}\left(x_{r-2}+\cdots+\int_{0}^{y_{2}}\left(x_{1}+y_{1}\right)^{m_{1}} d y_{1} \ldots\right)^{m_{r-2}} d y_{r-2} .
\end{gathered}
$$

Then we have

$$
\begin{aligned}
& t_{r}-\int_{0}^{s_{r}}\left(t_{r-1}-\cdots-\int_{0}^{s_{2}}\left(t_{1}-s_{1}\right)^{m_{1}} d s_{1} \cdots\right)^{m_{r-1}} d s_{r-1} \\
& =x_{r}+\int_{0}^{t}\left(x_{r-1}+\int_{0}^{y_{r-1}}\left(x_{r-2}+\ldots+\int_{0}^{y_{2}}\left(x_{1}+y_{1}\right)^{m_{1}} d y_{1} \ldots\right)^{m_{r-2}} d y_{r-2}\right)^{m_{r-1}} d y_{r-1} \\
& -\int_{0}^{s_{r}}\left(x_{r-1}+\int_{0}^{t-s_{r-1}}\left(x_{r-2}+\cdots+\int_{0}^{y_{2}}\left(x_{1}+y_{1}\right)^{m_{1}} d y_{1} \ldots\right)^{m_{r-2}} d y_{r-2}\right) d_{s_{r-1}} \\
& =x_{r}+\int_{0}^{t}\left(x_{r-1}+\int_{0}^{y_{r-1}}\left(x_{r-2}+\ldots+\int_{0}^{y_{2}}\left(x_{1}+y_{1}\right)^{m_{1}} d y_{1} \ldots\right)^{m_{r-2}} d y_{r-2}\right)^{m_{r-1}} d y_{r-1} \\
& +\int_{t}^{t-s_{r}}\left(x_{r-1}+\int_{0}^{y_{r-1}}\left(x_{r-2}+\cdots+\int_{0}^{y_{2}}\left(x_{1}+y_{1}\right)^{m_{1}} d y_{1} \ldots\right)^{m_{r-2}} d y_{r-2}\right) d y_{r-1} \\
& =x_{r}+\int_{0}^{t-s_{r}}\left(x_{r-1}+\ldots+\int_{0}^{y_{2}}\left(x_{1}+y_{1}\right)^{m_{1}} d y_{1} \ldots\right)^{m_{r-1}} d y_{r-1} \\
& t_{r+1}=x_{r+1}+\int_{0}^{t}\left(t_{r}-\int_{0}^{s_{r}}\left(t_{r-1}-\cdots-\int_{0}^{s_{2}}\left(t_{1}-s_{1}\right)^{m_{1}} d s_{1} \cdots\right)^{m_{r-1}} d s_{r-1}\right)^{m_{r}} d s_{r} \\
& =x_{r+1}+\int_{0}^{t}\left(x_{r}+\int_{0}^{t-s_{r}}\left(x_{r-1}+\ldots+\int_{0}^{y_{2}}\left(x_{1}+y_{1}\right)^{m_{1}} d y_{1} \ldots\right)^{m_{r-1}} d y_{r-1}\right)^{m_{r}} d s_{r} \\
& \stackrel{y=t-s_{r}}{=} x_{r+1}-\int_{t}^{0}\left(x_{r}+\int_{0}^{y_{r}}\left(x_{r-1}+\ldots+\int_{0}^{y_{2}}\left(x_{1}+y_{1}\right)^{m_{1}} d y_{1} \ldots\right)^{m_{r-1}} d y_{r-1}\right)^{m_{r}} d y_{r} \\
& =x_{r+1}+\int_{0}^{t}\left(x_{r}+\int_{0}^{y_{r}}\left(x_{r-1}+\ldots+\int_{0}^{y_{2}}\left(x_{1}+y_{1}\right)^{m_{1}} d y_{1} \ldots\right)^{m_{r-1}} d y_{r-1}\right)^{m_{r}} d y_{r} \\
& =x_{r+1}+\eta_{r+1} \text {. }
\end{aligned}
$$

By induction, we have

$$
t_{p}=x_{p}+\eta_{p} \quad \text { for } p \in \overline{1, n}
$$

Thus the solution

$$
\begin{aligned}
u & =t_{n+1}=f\left(t_{1}, t_{2}, \ldots, t_{n}\right) \\
& =f\left(x_{1}+\eta_{1}, x_{2}+\eta_{3}, \ldots, x_{n}+\eta_{n}\right) \\
& =e^{\eta_{n} \partial_{x_{n}}} e^{\eta_{n-1} \partial_{x_{n-1}}} \cdots e^{\eta_{1} \partial_{x_{1}}}\left(f\left(x_{1}, \ldots, x_{n-1}, x_{n}\right)\right) .
\end{aligned}
$$

According to (4.5.15),

$$
\partial_{t}\left(e^{D}(f)\right)=\left(\partial_{x_{1}}+x_{1}^{m_{1}} \partial_{x_{2}}+x_{2}^{m_{2}} \partial_{x_{3}}+\cdots+x_{n-1}^{m_{n-1}} \partial_{x_{n}}\right) e^{D}(f)
$$


and

$$
\left.e^{D}(f)\right|_{t=0}=e^{0}(f)=f
$$

Hence

$$
e^{D}(f)=u=e^{\eta_{n} \partial_{x_{n}}} e^{\eta_{n-1} \partial_{x_{n-1}}} \cdots e^{\eta_{1} \partial_{x_{1}}}(f) .
$$

Since $f$ is an arbitrary smooth function in $x_{1}, x_{2}, \ldots, x_{n},(4.5 .34)$ implies (4.5.17).

We remark that the above lemma was proved pure algebraically in [X7] by the CampbellHausdorff formula. The above result can be generalized as follows. Recall the definition of a tree given in the paragraph of (4.5.6) and (4.5.7). We define a tree diagram $\mathcal{T}^{d}$ to be a tree $\mathcal{T}=(\mathcal{N}, \mathcal{E})$ with a weight map $d: \mathcal{E} \rightarrow \mathbb{N}+1$, denoted as $\mathcal{T}^{d}=(\mathcal{N}, \mathcal{E}, d)$. Set

$$
D_{\mathcal{T}^{d}}=t\left(\partial_{x_{1}}+\sum_{\left(\iota_{p}, \iota_{q}\right) \in \mathcal{E}} x_{p}^{d\left[\left(\iota_{p}, \iota_{q}\right)\right]} \partial_{x_{q}}\right)
$$

In order to factorize $e^{D_{\mathcal{T}}}$, we need a new notion. For a node $\iota_{q}$ in a tree $\mathcal{T}$, the unique sequence

$$
\mathcal{C}_{q}=\left\{\iota_{q_{1}}, \iota_{q_{2}}, \ldots, \iota_{q_{r}}\right\}
$$

of nodes with $1=q_{1}<q_{2}<\cdots<q_{r-1}<q_{r}=q$ satisfying $\left(\iota_{q_{k}}, \iota_{q_{k+1}}\right) \in \mathcal{E}$ for $k \in \overline{1, r-1}$ is called the clan of the node $\iota_{q}$.

Again we define $\eta_{1}^{\mathcal{T}^{d}}=t$. For any $q \in \overline{2, n}$ with the clan (4.5.36), we define

$$
\begin{aligned}
\eta_{q}^{\mathcal{T}^{d}}= & \int_{0}^{t}\left(x_{q_{r-1}}+\int_{0}^{y_{q_{r-1}}}\left(x_{q_{r-2}}+\ldots+\int_{0}^{y_{q_{2}}}\left(x_{1}+y_{1}\right)^{d\left[\left(\iota_{q_{1}}, \iota_{q_{2}}\right)\right]}\right.\right. \\
& \left.\left.d y_{1} \ldots\right)^{d\left[\left(\iota_{q_{r-2}}, \iota_{q_{r-1}}\right)\right]} d y_{q_{r-2}}\right)^{d\left[\left(\iota_{q_{r-1}}, \iota_{q_{r}}\right)\right]} d y_{q_{r-1}} .
\end{aligned}
$$

Corollary 4.5.3. For a tree diagram $\mathcal{T}^{d}$ with $n$ nodes, we have

$$
e^{D_{\mathcal{T}^{d}}}=e^{\eta_{n}^{\mathcal{T}^{d}} \partial_{x_{n}}} e^{\eta_{n-1}^{\mathcal{T}^{d}} \partial_{x_{n-1}}} \cdots e^{\eta_{1}^{\mathcal{T}^{d}} \partial_{x_{1}}}
$$

In particular, $u=g\left(x_{1}+\eta_{1}^{\mathcal{T}^{d}}, x_{2}+\eta_{2}^{\mathcal{T}^{d}}, \ldots, x_{n}+\eta_{n}^{\mathcal{T}^{d}}\right)$ is the solution of the evolution equation

$$
u_{t}=\left(\partial_{x_{1}}+\sum_{\left(\iota_{p}, \iota_{q}\right) \in \mathcal{E}} x_{p}^{d\left[\left(\iota_{p}, \iota_{q}\right)\right]} \partial_{x_{q}}\right)(u)
$$

subject to $u\left(0, x_{1}, \ldots, x_{n}\right)=g\left(x_{1}, \ldots, x_{n}\right)$.

Since $d: \mathcal{E} \rightarrow \mathbb{N}+1$ is an arbitrary map, we can solve more general problem of replacing monomial functions by any first-order differentiable functions. Let $\vec{h}=\left\{h_{p, q}(x) \mid\left(\iota_{p}, \iota_{q}\right) \in\right.$ $\mathcal{E}\}$ be a set of first-order differentiable functions. Suppose $\mathcal{C}_{q}=\left\{\iota_{q_{1}}, \iota_{q_{2}}, \ldots, \iota_{q_{r}}\right\}$. We define

$$
\begin{aligned}
\eta_{q}^{\vec{h}}= & \int_{0}^{t} h_{q_{r-1}, q_{r}}\left(x_{q_{r-1}}+\int_{0}^{y_{q_{r-1}}} h_{q_{r-2}, q_{r-1}}\left(x_{q_{r-2}}\right.\right. \\
& \left.\left.+\ldots+\int_{0}^{y_{q_{2}}} h_{q_{1}, q_{2}}\left(x_{1}+y_{1}\right) d y_{q_{1}} \ldots\right) d y_{q_{r-2}}\right) d y_{q_{r-1}} .
\end{aligned}
$$


Set

$$
D_{\vec{h}}=t\left(\partial_{x_{1}}+\sum_{\left(\iota_{p}, \iota_{q}\right) \in \mathcal{E}} h_{p, q}\left(x_{p}\right) \partial_{x_{q}}\right) .
$$

Corollary 4.5.4. We have the factorization:

$$
e^{D_{\vec{h}}}=e^{\eta_{n}^{\vec{h}} \partial_{x_{n}}} e^{\eta_{n-1}^{\vec{h}} \partial_{x_{n-1}}} \cdots e^{\eta_{1}^{\vec{h}} \partial_{x_{1}}}
$$

In particular, $u=g\left(x_{1}+\eta_{1}^{\vec{h}}, x_{2}+\eta_{2}^{\vec{h}}, \ldots, x_{n}+\eta_{n}^{\vec{h}}\right)$ is the solution of the evolution equation

$$
u_{t}=\left(\partial_{x_{1}}+\sum_{\left(\iota_{p}, \iota_{q}\right) \in \mathcal{E}} h_{p, q}\left(x_{p}\right) \partial_{x_{q}}\right)(u)
$$

subject to the condition $u\left(0, x_{1}, \ldots, x_{n}\right)=g\left(x_{1}, \ldots, x_{n}\right)$.

Next we consider

$$
\hat{D}=t\left(\partial_{x_{1}}^{m_{1}}+x_{1} \partial_{x_{2}}^{m_{2}}+\cdots+x_{n-1} \partial_{x_{n}}^{m_{n}}\right) .
$$

To study the factorization of $e^{\hat{D}}$, we need the following preparations. Denote $\hat{\mathcal{A}}=$ $\mathbb{R}\left[x_{0}, x_{1}, \ldots, x_{n}\right]$. We denote

$$
x^{\alpha}=\prod_{r=0}^{n} x_{r}^{\alpha_{r}}, \quad \partial^{\alpha}=\prod_{r=0}^{n} \partial_{x_{r}}^{\alpha_{r}} \quad \text { for } \alpha=\left(\alpha_{0}, \alpha_{1}, \ldots, \alpha_{n}\right) \in \mathbb{N}^{n+1} .
$$

For $\alpha, \beta \in \mathbb{N}^{n+1}$, we define

$$
\beta \preceq \alpha \quad \text { if } \beta_{r} \leq \alpha_{r} \text { for } r \in \overline{0, n}
$$

and in this case,

$$
\left(\begin{array}{l}
\alpha \\
\beta
\end{array}\right)=\prod_{r=0}^{n}\left(\begin{array}{c}
\alpha_{r} \\
\beta_{r}
\end{array}\right), \quad \gamma !=\prod_{r=0}^{n} \gamma_{r} ! \quad \text { for } \beta \in \mathbb{N}^{n+1}
$$

Set

$$
\mathbb{A}=\operatorname{Span}\left\{x^{\alpha} \partial^{\beta} \mid \alpha, \beta \in \mathbb{N}^{n+1}\right\},
$$

the space of all algebraic differential operators on $\hat{\mathcal{A}}$. For $T_{1}, T_{2} \in \mathbb{A}$, the multiplication $T_{2} \cdot T_{2}$ is defined by

$$
\left(T_{1} \cdot T_{2}\right)(f)=T_{1}\left(T_{2}(f)\right) \quad \text { for } f \in \hat{\mathcal{A}} .
$$

Note that for $f, g_{1}, g_{2} \in \hat{\mathcal{A}}$ and $\alpha, \beta \in \mathbb{N}^{n+1}$,

$$
\left(g_{1} \partial^{\alpha} \cdot g_{2} \partial^{\beta}\right)(f)=\sum_{\alpha \succeq \gamma \in \mathbb{N}^{n+1}}\left(\begin{array}{l}
\alpha \\
\gamma
\end{array}\right) g_{1} \partial^{\gamma}\left(g_{2}\right) \partial^{\beta+\gamma}(f) .
$$

Thus

$$
g_{1} \partial^{\alpha} \cdot g_{2} \partial^{\beta}=\sum_{\alpha \succeq \gamma \in \mathbb{N}^{n+1}}\left(\begin{array}{l}
\alpha \\
\gamma
\end{array}\right) g_{1} \partial^{\gamma}\left(g_{2}\right) \partial^{\alpha+\beta-\gamma}
$$


So $(\mathbb{A}, \cdot)$ forms an associative algebra.

Define a linear transformation $\tau: \mathbb{A} \rightarrow \mathbb{A}$ by

$$
\tau\left(x^{\alpha} \partial^{\beta}\right)=x^{\beta} \partial^{\alpha} \quad \text { for } \alpha, \beta \in \mathbb{N}^{n+1} \text {. }
$$

Lemma 4.5.5. We have $\tau\left(T_{1} \cdot T_{2}\right)=\tau\left(T_{2}\right) \cdot \tau\left(T_{1}\right)$.

Proof. For $\alpha, \alpha^{\prime}, \beta, \beta^{\prime} \in \mathbb{N}^{n+1}$, we have

$$
\begin{aligned}
\tau\left(x^{\alpha} \partial^{\alpha^{\prime}} \cdot x^{\beta} \partial^{\beta^{\prime}}\right) & =\sum_{\alpha^{\prime} \succeq \gamma \in \mathbb{N}^{n+1}} \gamma !\left(\begin{array}{l}
\alpha^{\prime} \\
\gamma
\end{array}\right)\left(\begin{array}{l}
\beta \\
\gamma
\end{array}\right) \tau\left(x^{\alpha+\beta-\gamma} \partial^{\alpha^{\prime}+\beta^{\prime}-\gamma}\right) \\
& =\sum_{\beta \succeq \gamma \in \mathbb{N}^{n+1}} \gamma !\left(\begin{array}{l}
\beta \\
\gamma
\end{array}\right)\left(\begin{array}{l}
\alpha^{\prime} \\
\gamma
\end{array}\right) x^{\alpha^{\prime}+\beta^{\prime}-\gamma} \partial^{\alpha+\beta-\gamma} \\
& =x^{\beta^{\prime}} \partial^{\beta} \cdot x^{\alpha^{\prime}} \partial^{\alpha}=\tau\left(x^{\beta} \partial^{\beta^{\prime}}\right) \cdot \tau\left(x^{\alpha} \partial^{\alpha^{\prime}}\right) .
\end{aligned}
$$

Denote

$$
\tilde{D}=t\left(x_{1}^{m_{1}} \partial_{x_{0}}+x_{2}^{m_{2}} \partial_{x_{1}}+\cdots+x_{n-1}^{m_{n-1}} \partial_{x_{n-2}}+x_{n}^{m_{n}} \partial_{x_{n-1}}\right) .
$$

Changing variables

$$
z_{r}=\frac{x_{r}}{x_{n}^{n} \prod_{p=r+1}^{n} m_{p}} \quad \text { for } r \in \overline{0, n-1}
$$

Then

$$
\partial_{z_{r}}=x_{n}^{\prod_{p=r+1}^{n} m_{p}} \partial_{x_{r}} \quad \text { for } r \in \overline{0, n-1} .
$$

Moreover,

$$
\tilde{D}=t\left(\partial_{z_{n-1}}+z_{n-1}^{m_{n-1}} \partial_{z_{n-2}}+\cdots+z_{2}^{m_{2}} \partial_{z_{1}}+z_{1}^{m_{1}} \partial_{z_{0}}\right) .
$$

According to (4.5.15)-(4.3.17), we define $\tilde{\eta}_{n-1}=t$ and

$$
\tilde{\eta}_{r}=\int_{0}^{t}\left(z_{r+1}+\int_{0}^{y_{r+1}}\left(z_{r+2}+\ldots+\int_{0}^{y_{n-2}}\left(z_{n-1}+y_{n-1}\right)^{m_{n-1}} d y_{n-1} \ldots\right)^{m_{r+2}} d y_{r+2}\right)^{m_{r+1}} d y_{r+1}
$$

for $r \in \overline{0, n-2}$. By Lemma 4.5.2,

$$
e^{\tilde{D}}=e^{\tilde{\eta}_{0} \partial_{z_{0}}} e^{\tilde{\eta}_{1} \partial_{z_{1}}} \cdots e^{\tilde{\eta}_{n-1} \partial_{z_{n-1}}}
$$

Note

$$
\begin{aligned}
\eta_{r}^{*}= & x_{n}^{\prod_{p=r+1}^{n} m_{p}} \tilde{\eta}_{r}=\int_{0}^{t}\left(x_{n}^{\prod_{p=r+2}^{n} m_{p}} z_{r+1}+\int_{0}^{y_{r+1}}\left(x_{n}^{\prod_{p=r+2}^{n} m_{p}} z_{r+2}+\right.\right. \\
& \left.\left.\ldots+\int_{0}^{y_{n-2}}\left(x_{n}^{m_{n}} z_{n-1}+x_{n}^{m_{n}} y_{n-1}\right)^{m_{n-1}} d y_{n-1} \ldots\right)^{m_{r+2}} d y_{r+2}\right)^{m_{r+1}} d y_{r+1} \\
= & \int_{0}^{t}\left(x_{r+1}+\int_{0}^{y_{r+1}}\left(x_{r+2}+\ldots+\int_{0}^{y_{n-2}}\left(x_{n-1}\right.\right.\right. \\
& \left.\left.\left.+x_{n}^{m_{n}} y_{n-1}\right)^{m_{n-1}} d y_{n-1} \ldots\right)^{m_{r+2}} d y_{r+2}\right)^{m_{r+1}} d y_{r+1}
\end{aligned}
$$


for $r \in \overline{0, n-2}$ and let $\eta_{n-1}^{*}=t x_{n}^{m_{n}}$. By (4.5.56), we find

$$
e^{\tilde{D}}=e^{\eta_{0}^{*} \partial_{x_{0}}} e^{\eta_{1}^{*} \partial_{x_{1}}} \cdots e^{\eta_{n-1}^{*} \partial_{x_{n-1}}} .
$$

According to Lemma 4.5.5,

$$
\begin{aligned}
& e^{t\left(x_{0} \partial_{x_{1}}^{m_{1}}+x_{1} \partial_{x_{2}}^{m_{2}}+\cdots+x_{n-1} \partial_{x_{n}}^{m_{n}}\right)} \\
= & e^{\tau(\tilde{D})}=\tau\left(e^{\tilde{D}}\right)=\tau\left[e^{\eta_{0}^{*} \partial_{x_{0}}} e^{\eta_{1}^{*} \partial_{x_{1}}} \cdots e^{\eta_{n-1}^{*} \partial_{x_{n-1}}}\right] \\
= & e^{\tau\left(\eta_{n-1}^{*} \partial_{x_{n-1}}\right)} \cdots e^{\tau\left(\eta_{1}^{*} \partial_{x_{1}}\right)} e^{\tau\left(\eta_{0}^{*} \partial_{x_{0}}\right)} \\
= & e^{x_{n-1} \tau\left(\eta_{n-1}^{*}\right)} \cdots e^{\left.x_{1} \tau\left(\eta_{1}^{*}\right)\right)} e^{x_{0} \tau\left(\eta_{0}^{*}\right)} .
\end{aligned}
$$

Denote $\hat{\eta}_{n-1}=\tau\left(\eta_{n-1}^{*}\right)=t \partial_{x_{n}}^{m_{n}}$ and

$$
\begin{aligned}
\hat{\eta}_{r}=\tau\left(\eta_{r}^{*}\right)= & \int_{0}^{t}\left(\partial_{x_{r+1}}+\int_{0}^{y_{r+1}}\left(\partial_{x_{r+2}}+\ldots+\int_{0}^{y_{n-2}}\left(\partial_{x_{n-1}}\right.\right.\right. \\
& \left.\left.\left.+\partial_{x_{n}}^{m_{n}} y_{n-1}\right)^{m_{n-1}} d y_{n-1} \ldots\right)^{m_{r+2}} d y_{r+2}\right)^{m_{r+1}} d y_{r+1}
\end{aligned}
$$

for $r \in \overline{0, n-2}$.

Theorem 4.5.6. We have the following factorization:

$$
e^{\hat{D}}=e^{t\left(\partial_{x_{1}}^{m_{1}}+x_{1} \partial_{x_{2}}^{m_{2}}+\cdots+x_{n-1} \partial_{x_{n}}^{m_{n}}\right)}=e^{x_{n-1} \hat{\eta}_{n-1}} \cdots e^{x_{1} \hat{\eta}_{1}} e^{\hat{\eta}_{0}} .
$$

Next we want to solve the evolution equation

$$
u_{t}=\left(\partial_{x_{1}}^{m_{1}}+x_{1} \partial_{x_{2}}^{m_{2}}+\cdots+x_{n-1} \partial_{x_{n}}^{m_{n}}\right)(u)
$$

subject to the initial condition:

$$
u\left(0, x_{1}, \ldots, x_{n}\right)=f\left(x_{1}, x_{2}, \ldots, x_{n}\right) \quad \text { for } x_{r} \in\left[-a_{r}, a_{r}\right],
$$

where $f$ is a continuous function in $x_{1}, \ldots, x_{n}$. For convenience, we denote

$$
k_{r}^{\dagger}=\frac{k_{r}}{a_{r}}, \quad \vec{k}^{\dagger}=\left(k_{1}^{\dagger}, \ldots, k_{n}^{\dagger}\right) \quad \text { for } \vec{k}=\left(k_{1}, \ldots, k_{n}\right) \in \mathbb{Z}^{n} .
$$

Set

$$
e^{\pi\left(\vec{k}^{\dagger} \cdot \vec{x}\right) i}=e^{\sum_{r=1}^{n} \pi k_{r}^{\dagger} x_{r} i} .
$$

Note that $\hat{\eta}_{r}$ is a polynomial in $t, \partial_{x_{r+1}}, \ldots, \partial_{x_{n}}$. So we denote

$$
\hat{\eta}_{r}=\hat{\eta}_{r}\left(t, \partial_{x_{r+1}}, \ldots, \partial_{x_{n}}\right) .
$$

Observe that

$$
e^{\hat{D}}\left(e^{\pi\left(\vec{k}^{\dagger} \cdot \vec{x}\right) i}\right)=e^{x_{n-1} \eta_{n-1}\left(t, \pi k_{n}^{\dagger} i\right)} \cdots e^{x_{1} \hat{\eta}\left(t, \pi k_{2}^{\dagger} i, \ldots, \pi k_{n}^{\dagger} i\right)} e^{\hat{\eta}\left(t, \pi k_{1}^{\dagger} i, \ldots, \pi k_{n}^{\dagger} i\right)} e^{\pi\left(\vec{k}^{\dagger} \cdot \vec{x}\right) i}
$$


is a solution of $(4.5 .65)$ for any $\vec{k}=\left(k_{1}, \ldots, k_{n}\right) \in \mathbb{Z}^{n}$. Denote the right hand of (4.5.70) as $\phi_{\vec{k}}\left(t, x_{1}, \ldots, x_{n}\right)+\psi_{\vec{k}}\left(t, x_{1}, \ldots, x_{n}\right) i$, where $\phi_{\vec{k}}$ and $\psi_{\vec{k}}$ are real-valued functions. Then

$$
\phi_{\vec{k}}\left(0, x_{1}, \ldots, x_{n}\right)=\cos \pi\left(\vec{k}^{\dagger} \cdot \vec{x}\right), \quad \psi_{\vec{k}}\left(0, x_{1}, \ldots, x_{n}\right)=\sin \pi\left(\vec{k}^{\dagger} \cdot \vec{x}\right) .
$$

We define $0 \prec \vec{k}$ if its first nonzero coordinate is a positive integer.

By Fourier expansion theory, we get:

Theorem 4.5.7. The solution of the equation (4.5.65) subject to (4.5.66) is

$$
u=\sum_{0 \preceq \vec{k} \in \mathbb{Z}^{n}}\left(b_{\vec{k}} \phi_{\vec{k}}\left(t, x_{1}, \ldots, x_{n}\right)+c_{\vec{k}} \psi_{\vec{k}}\left(t, x_{1}, \ldots, x_{n}\right)\right)
$$

with

$$
b_{\vec{k}}=\frac{1}{2^{n-1+\delta_{\vec{k}, \overrightarrow{0}}} a_{1} a_{2} \cdots a_{n}} \int_{-a_{1}}^{a_{1}} \cdots \int_{-a_{n}}^{a_{n}} f\left(x_{1}, \ldots, x_{n}\right) \cos \pi\left(\vec{k}^{\dagger} \cdot \vec{x}\right) d x_{n} \cdots d x_{1}
$$

and

$$
c_{\vec{k}}=\frac{1}{2^{n-1} a_{1} a_{2} \cdots a_{n}} \int_{-a_{1}}^{a_{1}} \ldots \int_{-a_{n}}^{a_{n}} f\left(x_{1}, \ldots, x_{n}\right) \sin \pi\left(\vec{k}^{\dagger} \cdot \vec{x}\right) d x_{n} \cdots d x_{1} .
$$

Example 4.5.1. Consider the case $n=2, m_{1}=m_{2}=2$ and $a_{1}=a_{2}=\pi$. So the problem becomes

$$
u_{t}=u_{x_{1} x_{1}}+x_{1} u_{x_{2} x_{2}}
$$

subject to

$$
u\left(0, x_{1}, x_{2}\right)=f\left(x_{1}, x_{2}\right) \quad \text { for } x_{1}, x_{2} \in[-\pi, \pi]
$$

In this case,

$$
\begin{aligned}
\hat{\eta}_{0}\left(t, \partial_{x_{1}}, \partial_{x_{2}}\right) & =\int_{0}^{t}\left(\partial_{x_{1}}+y_{1} \partial_{x_{2}}^{2}\right)^{2} d y_{1} \\
& =\int_{0}^{t}\left(\partial_{x_{1}}^{2}+2 y_{1} \partial_{x_{1}} \partial_{x_{2}}^{2}+y_{1}^{2} \partial_{x_{2}}^{4}\right) d y_{1} \\
& =t \partial_{x_{1}}^{2}+t^{2} \partial_{x_{1}} \partial_{x_{2}}^{2}+\frac{t^{3} \partial_{x_{2}}^{4}}{3}
\end{aligned}
$$

and $\hat{\eta}_{1}\left(t, \partial_{x_{2}}\right)=t \partial_{x_{2}}^{2}$. Thus

$$
\begin{aligned}
& e^{t x_{1} \partial_{x_{2}}^{2}} e^{t \partial_{x_{1}}^{2}+t^{2} \partial_{x_{1}} \partial_{x_{2}}^{2}+t^{3} \partial_{x_{2}}^{4} / 3}\left(e^{\left(k_{1} x_{1}+k_{2} x_{2}\right) i}\right) \\
= & e^{k_{2}^{4} t^{3} / 3-k_{2}^{2} t x_{1}-k_{1}^{2} t} e^{\left(k_{1} x_{1}+k_{2} x_{2}-k_{1} k_{2}^{2} t^{2}\right) i}
\end{aligned}
$$

Hence

$$
\phi_{\vec{k}}\left(t, x_{1}, x_{2}\right)=e^{k_{2}^{4} t^{3} / 3-k_{2}^{2} t x_{1}-k_{1}^{2} t} \cos \left(k_{1} x_{1}+k_{2} x_{2}-k_{1} k_{2}^{2} t^{2}\right),
$$




$$
\psi_{\vec{k}}\left(t, x_{1}, x_{2}\right)=e^{k_{2}^{4} t^{3} / 3-k_{2}^{2} t x_{1}-k_{1}^{2} t} \sin \left(k_{1} x_{1}+k_{2} x_{2}-k_{1} k_{2}^{2} t^{2}\right) .
$$

The final solution of (4.5.75) and (4.5.76) is

$$
\begin{aligned}
u= & \frac{1}{4 \pi^{2}} \int_{-\pi}^{\pi} \int_{-\pi}^{\pi} f\left(s_{1}, s_{2}\right) d_{s_{1}} d_{s_{2}}+\frac{1}{2 \pi^{2}} \sum_{0 \prec\left(k_{1}, k_{2}\right) \in \mathbb{Z}^{2}} e^{k_{2}^{4} t^{3} / 3-k_{2}^{2} t x_{1}-k_{1}^{2} t} \int_{-\pi}^{\pi} \int_{-\pi}^{\pi} f\left(s_{1}, s_{2}\right) \\
& \times \cos \left[k_{1}\left(x_{1}-s_{1}\right)+k_{2}\left(x_{2}-s_{2}\right)-k_{1} k_{2}^{2} t^{2}\right] d s_{1} d s_{2} .
\end{aligned}
$$

Theorem 4.5.6 gives a way of how to calculate the powers of $\partial_{x_{1}}^{m_{1}}+x_{1} \partial_{x_{2}}^{m_{2}}+\cdots+x_{n-1} \partial_{x_{n}}^{m_{n}}$. Then we can use the powers to solve the equation

$$
\partial_{t}^{m_{0}}(u)=\left(\partial_{x_{1}}^{m_{1}}+x_{1} \partial_{x_{2}}^{m_{2}}+\cdots+x_{n-1} \partial_{x_{n}}^{m_{n}}\right)(u) .
$$

Example 4.5.2. Find the solution of the problem

$$
u_{t t}=u_{x_{1} x_{1}}+x_{1} u_{x_{2} x_{2}}
$$

subject to

$$
u\left(0, x_{1}, x_{2}\right)=f_{1}\left(x_{1}, x_{2}\right), \quad u_{t}\left(0, x_{1}, x_{2}\right)=f_{2}\left(x_{1}, x_{2}\right) \quad \text { for } \quad x_{1}, x_{2} \in[-\pi, \pi] .
$$

Solution. According to the above example,

$$
\left(\partial_{x_{1}}^{2}+x_{1} \partial_{x_{2}}^{2}\right)^{m}=\sum_{n_{r} \in \mathbb{N} ; n_{0}+n_{1}+2 n_{2}+3 n_{3}=m} \frac{m !}{n_{0} ! n_{1} ! n_{2} ! n_{3} ! 3^{n_{3}}} x_{1}^{n_{0}} \partial_{x_{2}}^{2\left(n_{0}+n_{2}+2 n_{3}\right)} \partial_{x_{1}}^{2 n_{1}+n_{2}} .
$$

By Lemma 4.3.1 with $T_{1}=\partial_{t}^{2}, T_{1}^{-}=\int_{(t)}^{2}\left(\right.$ cf. (4.3.31)) and $T_{2}=-\left(\partial_{x_{1}}^{2}+x_{1} \partial_{x_{2}}^{2}\right)$, we have the complex solutions

$$
\begin{aligned}
& \sum_{m=0}^{\infty}\left(-T_{1}^{-} T_{2}\right)^{m}\left(e^{\left(k_{1} x_{1}+k_{2} x_{2}\right) i}\right)=\sum_{m=0}^{\infty} \frac{t^{2 m}\left(\partial_{x_{1}}^{2}+x_{1} \partial_{x_{2}}^{2}\right)^{m}}{(2 m) !}\left(e^{\left(k_{1} x_{1}+k_{2} x_{2}\right) i}\right) \\
= & \sum_{m=0}^{\infty} \sum_{n_{r} \in \mathbb{N} ; n_{0}+n_{1}+2 n_{2}+3 n_{3}=m} \frac{t^{2 m} x_{1}^{n_{0}} \partial_{x_{2}}^{2\left(n_{0}+n_{2}+2 n_{3}\right)} \partial_{x_{1}}^{2 n_{1}+n_{2}}}{(2 m-1) ! ! n_{0} ! n_{1} ! n_{2} ! n_{3} ! 2^{m} 3^{n_{3}}}\left(e^{\left(k_{1} x_{1}+k_{2} x_{2}\right) i}\right) \\
= & \sum_{m=0}^{\infty} \sum_{n_{r} \in \mathbb{N} ; n_{0}+n_{1}+2 n_{2}+3 n_{3}=m} \frac{(-1)^{n_{0}+n_{1}+n_{2}} i^{n_{2}} t^{2 m}}{(2 m-1) ! ! n_{0} ! n_{1} ! n_{2} ! n_{3} ! 2^{m} 3^{n_{3}}} \\
& \times x_{1}^{n_{0}} k_{2}^{2\left(n_{0}+n_{2}+2 n_{3}\right)} k_{1}^{2 n_{1}+n_{2}}\left(e^{\left(k_{1} x_{1}+k_{2} x_{2}\right) i}\right)
\end{aligned}
$$

and

$$
\begin{aligned}
& \sum_{m=0}^{\infty}\left(-T_{1}^{-} T_{2}\right)^{m}\left(t e^{\left(k_{1} x_{1}+k_{2} x_{2}\right) i}\right)=\sum_{m=0}^{\infty} \frac{t^{2 m+1}\left(\partial_{x_{1}}^{2}+x_{1} \partial_{x_{2}}^{2}\right)^{m}}{(2 m+1) !}\left(e^{\left(k_{1} x_{1}+k_{2} x_{2}\right) i}\right) \\
= & \sum_{m=0}^{\infty} \sum_{n_{r} \in \mathbb{N} ; n_{0}+n_{1}+2 n_{2}+3 n_{3}=m} \frac{(-1)^{n_{0}+n_{1}+n_{2}} i^{n_{2}} t^{2 m+1}}{(2 m+1) ! ! n_{0} ! n_{1} ! n_{2} ! n_{3} ! 2^{m} 3^{n_{3}}} \\
& \times x_{1}^{n_{0}} k_{2}^{2\left(n_{0}+n_{2}+2 n_{3}\right)} k_{1}^{2 n_{1}+n_{2}}\left(e^{\left(k_{1} x_{1}+k_{2} x_{2}\right) i}\right)
\end{aligned}
$$


of (4.5.83). Thus we have the following real solutions

$$
\begin{aligned}
& \phi_{k_{1}, k_{2}}\left(t, x_{1}, x_{2}\right)=\sum_{m=0}^{\infty} \sum_{n_{r} \in \mathbb{N} ; n_{0}+n_{1}+4 n_{2}+3 n_{3}=m}(-1)^{n_{0}+n_{1}+n_{2}} k_{1}^{2\left(n_{1}+n_{2}\right)} k_{2}^{2\left(n_{0}+2 n_{2}+2 n_{3}\right)} \\
& \frac{t^{2 m} x_{1}^{n_{0}}}{n_{0} ! n_{1} ! n_{3} ! 2^{m} 3^{n_{3}}}\left[\frac{\cos \left(k_{1} x+k_{2} x\right)}{(2 m-1) ! !\left(2 n_{2}\right) !}+\frac{k_{1} k_{2}^{2} t^{2} \sin \left(k_{1} x+k_{2} x\right)}{4(2 m+3) ! !\left(2 n_{2}+1\right) !}\right] \text {, } \\
& \psi_{k_{1}, k_{2}}\left(t, x_{1}, x_{2}\right)=\sum_{m=0}^{\infty} \sum_{n_{r} \in \mathbb{N} ; n_{0}+n_{1}+4 n_{2}+3 n_{3}=m}(-1)^{n_{0}+n_{1}+n_{2}} k_{1}^{2\left(n_{1}+n_{2}\right)} k_{2}^{2\left(n_{0}+2 n_{2}+2 n_{3}\right)} \\
& \frac{t^{2 m} x_{1}^{n_{0}}}{n_{0} ! n_{1} ! n_{3} ! 2^{m} 3^{n_{3}}}\left[\frac{\sin \left(k_{1} x+k_{2} x\right)}{(2 m-1) ! !\left(2 n_{2}\right) !}-\frac{k_{1} k_{2}^{2} t^{2} \cos \left(k_{1} x+k_{2} x\right)}{4(2 m+3) ! !\left(2 n_{2}+1\right) !}\right] \text {, } \\
& \hat{\phi}_{k_{1}, k_{2}}\left(t, x_{1}, x_{2}\right)=\sum_{m=0}^{\infty} \sum_{n_{r} \in \mathbb{N} ; n_{0}+n_{1}+4 n_{2}+3 n_{3}=m}(-1)^{n_{0}+n_{1}+n_{2}} k_{1}^{2\left(n_{1}+n_{2}\right)} k_{2}^{2\left(n_{0}+2 n_{2}+2 n_{3}\right)} \\
& \frac{t^{2 m+1} x_{1}^{n_{0}}}{n_{0} ! n_{1} ! n_{3} ! 2^{m} 3^{n_{3}}}\left[\frac{\cos \left(k_{1} x+k_{2} x\right)}{(2 m+1) ! !\left(2 n_{2}\right) !}+\frac{k_{1} k_{2}^{2} t^{2} \sin \left(k_{1} x+k_{2} x\right)}{4(2 m+5) ! !\left(2 n_{2}+1\right) !}\right] \text {, } \\
& \hat{\psi}_{k_{1}, k_{2}}\left(t, x_{1}, x_{2}\right)=\sum_{m=0}^{\infty} \sum_{n_{r} \in \mathbb{N} ; n_{0}+n_{1}+4 n_{2}+3 n_{3}=m}(-1)^{n_{0}+n_{1}+n_{2}} k_{1}^{2\left(n_{1}+n_{2}\right)} k_{2}^{2\left(n_{0}+2 n_{2}+2 n_{3}\right)} \\
& \frac{t^{2 m+1} x_{1}^{n_{0}}}{n_{0} ! n_{1} ! n_{3} ! 2^{m} 3^{n_{3}}}\left[\frac{\sin \left(k_{1} x+k_{2} x\right)}{(2 m+1) ! !\left(2 n_{2}\right) !}-\frac{k_{1} k_{2}^{2} t^{2} \cos \left(k_{1} x+k_{2} x\right)}{4(2 m+5) ! !\left(2 n_{2}+1\right) !}\right] \text {. }
\end{aligned}
$$

Moreover,

$$
\begin{gathered}
\phi_{k_{1}, k_{2}}\left(0, x_{1}, x_{2}\right)=\frac{\partial \hat{\phi}_{k_{1}, k_{2}}}{\partial t}\left(0, x_{1}, x_{2}\right)=\cos \left(k_{1} x+k_{2} x\right), \\
\psi_{k_{1}, k_{2}}\left(0, x_{1}, x_{2}\right)=\frac{\partial \hat{\psi}_{k_{1}, k_{2}}}{\partial t}\left(0, x_{1}, x_{2}\right)=\sin \left(k_{1} x+k_{2} x\right), \\
\frac{\partial \phi_{k_{1}, k_{2}}}{\partial t}\left(0, x_{1}, x_{2}\right)=\frac{\partial \psi_{k_{1}, k_{2}}}{\partial t}\left(0, x_{1}, x_{2}\right)=\hat{\phi}_{k_{1}, k_{2}}\left(0, x_{1}, x_{2}\right)=\hat{\psi}_{k_{1}, k_{2}}\left(0, x_{1}, x_{2}\right)=0 .
\end{gathered}
$$

Thus the solution of the problem (4.5.83) and (4.5.84) is

$$
\begin{array}{r}
u=\sum_{0 \preceq\left(k_{1}, k_{2}\right) \in \mathbb{Z}^{2}}\left[a_{k_{1}, k_{2}} \phi_{k_{1}, k_{2}}\left(t, x_{1}, x_{2}\right)+c_{k_{1}, k_{2}} \psi_{k_{1}, k_{2}}\left(t, x_{1}, x_{2}\right)\right. \\
\left.+\hat{a}_{k_{1}, k_{2}} \hat{\phi}_{k_{1}, k_{2}}\left(t, x_{1}, x_{2}\right)+\hat{c}_{k_{1}, k_{2}} \hat{\psi}_{k_{1}, k_{2}}\left(t, x_{1}, x_{2}\right)\right],
\end{array}
$$

where

$$
\begin{gathered}
a_{k_{1}, k_{2}}=\frac{1}{2^{1+\delta_{k_{1}, 0} \delta_{k_{2}, 0} \pi^{2}}} \int_{-\pi}^{\pi} \int_{-\pi}^{\pi} f_{1}\left(s_{1}, s_{2}\right) \cos \left(k_{1} s+k_{2} s\right) d s \\
c_{k_{1}, k_{2}}=\frac{1}{2 \pi^{2}} \int_{-\pi}^{\pi} \int_{-\pi}^{\pi} f_{1}\left(s_{1}, s_{2}\right) \sin \left(k_{1} s+k_{2} s\right) d s
\end{gathered}
$$




$$
\begin{aligned}
\hat{a}_{k_{1}, k_{2}} & =\frac{1}{2^{1+\delta_{k_{1}, 0} \delta_{k_{2}, 0}} \pi^{2}} \int_{-\pi}^{\pi} \int_{-\pi}^{\pi} f_{2}\left(s_{1}, s_{2}\right) \cos \left(k_{1} s+k_{2} s\right) d s \\
\hat{c}_{k_{1}, k_{2}} & =\frac{1}{2 \pi^{2}} \int_{-\pi}^{\pi} \int_{-\pi}^{\pi} f_{2}\left(s_{1}, s_{2}\right) \sin \left(k_{1} s+k_{2} s\right) d s
\end{aligned}
$$

The above results can be generalized as follows. Recall the definition of a tree given in the paragraph of (4.5.6) and (4.5.7). A tree diagram $\mathcal{T}^{d}$ is a tree $\mathcal{T}=(\mathcal{N}, \mathcal{E})$ with a weight map $d: \mathcal{E} \rightarrow \mathbb{N}+1$. A node $\iota_{q}$ of a tree $\mathcal{T}$ is called a tip if there does not exist $q \leq p \leq n$ such that $\left(\iota_{q}, \iota_{p}\right) \in \mathcal{E}$. Set

$$
\Psi=\left\{q \mid \iota_{q} \text { is a tip of } \mathcal{T}\right\} .
$$

Take a tree diagram $\mathcal{T}^{d}$ with $n$ nodes and a set $\Psi^{\dagger}=\left\{m_{q} \mid q \in \Psi\right\}$ of a positive integers. From (4.5.63) and (4.5.64), we have to generalize the operator $\hat{D}$ in (4.5.44) in reverse order and set

$$
D^{\dagger}=t\left(\sum_{\left(\iota_{p}, \iota_{q}\right) \in \mathcal{E}} x_{q} \partial_{x_{p}}^{d\left[\left(\iota_{p}, \iota_{q}\right)\right]}+\sum_{r \in \Psi} \partial_{x_{r}}^{m_{r}}\right) .
$$

Recall the definition of clan around (4.5.36). Given $q \in \overline{2, n}$, we have the clan $\mathcal{C}_{q}=$ $\left\{\iota_{q_{1}}, \iota_{q_{2}}, \ldots, \iota_{q_{r}}\right\}$ of the node $\iota_{q}$ with $1=q_{1}<q_{2}<\cdots<q_{r-1}<q_{r}=q$. If $r=2$, we define $\eta_{q}^{\dagger}=t \partial_{x_{1}}^{d\left[\left(q_{1}, q_{2}\right)\right]}$. When $r>2$, we define

$$
\begin{aligned}
\eta_{q}^{\dagger}= & \int_{0}^{t}\left(\partial_{x_{q_{r-1}}}+\int_{0}^{y_{q_{r-1}}}\left(\partial_{x_{q_{r-2}}}+\ldots+\int_{0}^{y_{3}}\left(\partial_{x_{q_{2}}}\right.\right.\right. \\
& \left.\left.\left.\left.+\partial_{x_{1}}^{\left.d\left[q_{1}, q_{2}\right)\right]} y_{2}\right)^{d\left[\left(q_{2}, q_{3}\right)\right]} d y_{2}\right) \ldots\right)^{d\left[\left(q_{r-2}, q_{r-1}\right)\right]} d y_{r-2}\right)^{d\left[\left(q_{r-1}, q_{r}\right)\right]} d y_{r-1}
\end{aligned}
$$

For $q \in \Psi$, we also define

$$
\begin{aligned}
\eta_{q}^{\boldsymbol{*}}= & \int_{0}^{t}\left(\partial_{x_{q}}+\int_{0}^{y_{q_{r}}}\left(\partial_{x_{q_{r-1}}}+\int_{0}^{y_{q_{r-1}}}\left(\partial_{x_{q_{r-2}}}+\ldots+\int_{0}^{y_{3}}\left(\partial_{x_{q_{2}}}\right.\right.\right.\right. \\
& \left.\left.\left.\left.\left.+\partial_{x_{1}}^{d\left[\left(q_{1}, q_{2}\right)\right]} y_{2}\right)^{d\left[\left(q_{2}, q_{3}\right)\right]} d y_{2}\right) \ldots\right)^{d\left[\left(q_{r-2}, q_{r-1}\right)\right]} d y_{r-2}\right)^{d\left[\left(q_{r-1}, q_{r}\right)\right]} d y_{r-1}\right)^{m_{q}} d y_{q_{r}}
\end{aligned}
$$

By Theorem 4.5.6, we have the following conclusion.

Proposition 4.5.8. The following factorization holds:

$$
e^{D^{\dagger}}=e^{x_{2} \eta_{2}^{\dagger}} e^{x_{3} \eta_{3}^{\dagger}} \cdots e^{x_{n} \eta_{n}^{\dagger}} \prod_{q \in \Psi} e^{\eta_{q}^{*}} .
$$

As Theorem 4.5.7, the above factorization can be used to solve the evolution equation

$$
u_{t}=\left(\sum_{\left(\iota_{p}, \iota_{q}\right) \in \mathcal{E}} x_{q} \partial_{x_{p}}^{\left.d\left[\iota_{p}, \iota_{q}\right)\right]}+\sum_{r \in \Psi} \partial_{x_{r}}^{m_{r}}\right)(u)
$$


subject to $u\left(0, x_{1}, \ldots, x_{n}\right)=f\left(x_{1}, \ldots, x_{n}\right)$.

Since the weight map $d$ is arbitrary and $\Psi^{\dagger}$ can vary, we can do further generalization as follows. Take two sets $\left\{h_{p, q}(x) \mid\left(\iota_{p}, \iota_{q}\right) \in \mathcal{E}\right\}$ and $\left\{h_{q}(x) \mid q \in \Psi\right\}$ of polynomials in $x$. We generalize (4.5.101) to

$$
D^{\dagger}=t\left(\sum_{\left(\iota_{p}, \iota_{q}\right) \in \mathcal{E}} x_{q} h_{p, q}\left(\partial_{x_{p}}\right)+\sum_{r \in \Psi} h_{r}\left(\partial_{x_{r}}\right)\right) .
$$

Given $q \in \overline{2, n}$, we have the clan $\mathcal{C}_{q}=\left\{\iota_{q_{1}}, \iota_{q_{2}}, \ldots, \iota_{q_{r}}\right\}$ of the node $\iota_{q}$ with $1=q_{1}<q_{2}<$ $\cdots<q_{r-1}<q_{r}=q$. If $r=2$, we define $\eta_{q}^{\dagger}=t h_{q_{1}, q_{2}}\left(\partial_{x_{1}}\right)$. When $r>2$, we define

$$
\begin{aligned}
\eta_{q}^{\dagger}= & \int_{0}^{t} h_{q_{r-1}, q_{r}}\left(\partial_{x_{q_{r-1}}}+\int_{0}^{y_{q_{r-1}}} h_{q_{r-2}, q_{r-1}}\left(\partial_{x_{q_{r-2}}}+\ldots\right.\right. \\
& \left.\left.\left.+\int_{0}^{y_{3}} h_{q_{2}, q_{3}}\left(\partial_{x_{q_{2}}}+h_{q_{1}, q_{2}}\left(\partial_{x_{1}}\right) y_{2}\right) d y_{2}\right) \ldots\right) d y_{r-2}\right) d y_{r-1}
\end{aligned}
$$

For $q \in \Psi$, we also define

$$
\begin{aligned}
\eta_{q}^{\mathbf{\alpha}}= & \int_{0}^{t} h_{q}\left(\partial_{x_{q}}+\int_{0}^{y_{q_{r}}} h_{q_{r-1}, q_{r}}\left(\partial_{x_{q_{r-1}}}+\int_{0}^{y_{q_{r-1}}} h_{q_{r-2}, q_{r-1}}\left(\partial_{x_{q_{r-2}}}+\ldots\right.\right.\right. \\
& \left.\left.\left.\left.+\int_{0}^{y_{3}} h_{q_{2}, q_{3}}\left(\partial_{x_{q_{2}}}+h_{q_{1}, q_{2}}\left(\partial_{x_{1}}\right) y_{2}\right) d y_{2}\right) \ldots\right) d y_{r-2}\right) d y_{r-1}\right) d y_{q_{r}} .
\end{aligned}
$$

Then (4.5.104) still holds.

\section{Exercise 4.5}

1. Find the solution of the equation $u_{x x}+x u_{y y}=0$ for $x \in \mathbb{R}$ and $y \in[-\pi, \pi]$ subject to $u(0, y)=f_{1}(y)$ and $u_{x}(0, y)=f_{2}(y)$, where $f_{1}(y)$ and $f_{2}(y)$ are continuous functions on $[-\pi, \pi]$ (cf. Example 4.5.2).

2. Solve the problem $u_{t}=u_{x x}+x u_{y y}+y u_{z z}$ for $t \in \mathbb{R}$ and $x, y, z \in[-\pi, \pi]$ subject to $u(0, x, y, z)=g(x, y, z)$, where $g(x, y, z)$ is a continuous function for $x, y, z \in[-\pi, \pi]$.

3. Use (4.5.104) to solve the problem

$$
u_{t}=\left(y \partial_{x}^{3}+z \partial_{x}^{2}+\partial_{y}^{2}+\partial_{z}^{2}\right)(u)
$$

for $t \in \mathbb{R}$ and $x, y, z \in[-\pi, \pi]$ subject to $u(0, x, y, z)=g(x, y, z)$, where $g(x, y, z)$ is a continuous function for $x, y, z \in[-\pi, \pi]$.

\subsection{Calogero-Sutherland Model}

The Calogero-Sutherland model is an exactly solvable quantum many-body system in one-dimension (cf. [Cf], $[\mathrm{Sg}]$ ), whose Hamiltonian is given by

$$
H_{C S}=\sum_{\iota=1}^{n} \partial_{x_{\iota}}^{2}+K \sum_{1 \leq p<q \leq n} \frac{1}{\sinh ^{2}\left(x_{p}-x_{q}\right)}
$$


where $K$ is a constant. The model was used to study long-range interactions of $n$ particles. Solving the model is equivalent to find eigenfunctions and their corresponding eigenvalues of the Hamiltonian $H_{C S}$ as a differential operator:

$$
H_{C S}\left(f\left(x_{1}, \ldots, x_{n}\right)\right)=\nu f\left(x_{1}, \ldots, x_{n}\right)
$$

with $\nu \in \mathbb{R}$. In other words, the above is the equation of motion for the CalogeroSutherland model.

In this section, we prove that a two-parameter generalization of the Weyl function of type $A$ in representation theory is a solution of the Calogero-Sutherland model. If $n=2$, we find a connection between the Calogero-Sutherland model and the Gauss hypergeometric function. When $n>2$, a new class of multi-variable hypergeometric functions are found based on Etingof's work [Ep]. The results in this section are taken from the author's work [X9]

Change variables

$$
z_{\iota}=e^{2 x_{\iota}} \quad \text { for } \iota \in \overline{1, n} \text {. }
$$

Then

$$
\partial_{x_{\iota}}=2 e^{x_{\iota}} \partial_{z_{\iota}}=2 z_{\iota} \partial_{z_{\iota}} \quad \text { for } \iota \in \overline{1, n}
$$

by the chain rule of taking partial derivatives. Moreover,

$$
\frac{1}{\sinh ^{2}\left(x_{p}-x_{q}\right)}=\frac{4}{\left(e^{x_{p}-x_{q}}-e^{x_{q}-x_{p}}\right)^{2}}=\frac{4}{\left[e^{-x_{p}-x_{q}}\left(e^{2 x_{p}}-e^{2 x_{q}}\right)\right]^{2}}=\frac{4 z_{p} z_{q}}{\left(z_{p}-z_{q}\right)^{2}} .
$$

So the Hamiltonian

$$
H_{C S}=4\left[\sum_{\iota=1}^{n}\left(z_{\iota} \partial_{z_{\iota}}\right)^{2}+K \sum_{1 \leq p<q \leq n} \frac{z_{p} z_{q}}{\left(z_{p}-z_{q}\right)^{2}}\right]
$$

Replacing $\nu$ by $4 \nu$ and $f$ by $\Psi\left(z_{1}, \ldots, z_{n}\right)$, we get the new equation of motion for the Calogero-Sutherland model:

$$
\sum_{\iota=1}^{n}\left(z_{\iota} \partial_{z_{\iota}}\right)^{2}(\Psi)+K\left(\sum_{1 \leq p<q \leq n} \frac{z_{p} z_{q}}{\left(z_{p}-z_{q}\right)^{2}}\right) \Psi=\nu \Psi
$$

First we will introduce some simple but nontrivial solutions.

Let $\left\{f_{p, q}(z) \mid p, q \in \overline{1, n}\right\}$ be a set of one-variable differentiable functions and let $d_{\iota}$ be a one-variable differential operator in $z_{\iota}$ for $\iota \in \overline{1, n}$. It is easy to verified the following lemma: 
Lemma 4.6.1. We have the following equation on differentiation of determinants:

$$
\begin{gathered}
\left(\sum_{\iota=1}^{n} d_{\iota}\right)\left(\left|\begin{array}{cccc}
f_{1,1}\left(z_{1}\right) & f_{1,2}\left(z_{2}\right) & \cdots & f_{1, n}\left(z_{n}\right) \\
f_{2,1}\left(z_{1}\right) & f_{2,2}\left(z_{2}\right) & \cdots & f_{2, n}\left(z_{n}\right) \\
\vdots & \vdots & \vdots & \vdots \\
f_{n, 1}\left(z_{1}\right) & f_{n, 2}\left(z_{2}\right) & \cdots & f_{n, n}\left(z_{n}\right)
\end{array}\right|\right) \\
=\sum_{\iota=1}^{n}\left|\begin{array}{cccc}
f_{1,1}\left(z_{1}\right) & f_{1,2}\left(z_{2}\right) & \cdots & f_{1, n}\left(z_{n}\right) \\
\vdots & \vdots & \vdots & \vdots \\
f_{\iota-1,1}\left(z_{1}\right) & f_{\iota-1,2}\left(z_{2}\right) & \cdots & f_{\iota-1, n}\left(z_{n}\right) \\
d_{1}\left(f_{\iota, 1}\left(z_{1}\right)\right) & d_{2}\left(f_{\iota, 2}\left(z_{2}\right)\right) & \cdots & d_{n}\left(f_{\iota, n}\left(z_{n}\right)\right) \\
f_{\iota+1,1}\left(z_{1}\right) & f_{\iota+1,2}\left(z_{2}\right) & \cdots & f_{\iota+1, n}\left(z_{n}\right) \\
\vdots & \vdots & \vdots & \vdots \\
f_{n, 1}\left(z_{1}\right) & f_{n, 2}\left(z_{2}\right) & \cdots & f_{n, n}\left(z_{n}\right)
\end{array}\right| .
\end{gathered}
$$

Denote the Vandermonde determinant

$$
\mathcal{W}\left(z_{1}, z_{2}, \ldots, z_{n}\right)=\left|\begin{array}{cccc}
1 & 1 & \cdots & 1 \\
z_{n} & z_{n-1} & \cdots & z_{1} \\
z_{n}^{2} & z_{n-1}^{2} & \cdots & z_{1}^{2} \\
\vdots & \vdots & \vdots & \vdots \\
z_{n}^{n-1} & z_{n-1}^{n-1} & \cdots & z_{1}^{n-1}
\end{array}\right|=\prod_{1 \leq p<q \leq n}\left(z_{p}-z_{q}\right) .
$$

According to the last expression,

$$
\begin{aligned}
& \left(\sum_{\iota=1}^{n}\left(z_{\iota} \partial_{z_{\iota}}\right)^{2}\right)(\mathcal{W})=\left(\sum_{r=1}^{n}\left(z_{r} \partial_{z_{r}}\right)^{2}\right)\left(\prod_{1 \leq p<q \leq n}\left(z_{p}-z_{q}\right)\right) \\
= & \sum_{r=1}^{n} z_{r}\left[\sum_{r \neq s \in \overline{1, n}}\left(z_{r} \partial_{z_{r}}\right)^{2}\left(z_{r}-z_{s}\right) \cdot \frac{\mathcal{W}}{z_{r}-z_{s}}\right. \\
& \left.+2 \sum_{1 \leq s_{1}<s_{2} \leq n ; s_{1}, s_{2} \neq r} z_{r} \partial_{z_{r}}\left(z_{s_{1}}-z_{r}\right) \cdot z_{r} \partial_{z_{r}}\left(z_{s_{2}}-z_{r}\right) \cdot \frac{\mathcal{W}}{\left(z_{s_{1}}-z_{r}\right)\left(z_{s_{2}}-z_{r}\right)}\right] \\
= & \sum_{r=1}^{n} z_{r}\left[\sum_{r \neq s \in 1, n} \frac{z_{r} \mathcal{W}}{z_{r}-z_{s}}+2 \sum_{1 \leq s_{1}<s_{2} \leq n ; s_{1}, s_{2} \neq r} \frac{z_{r}^{2} \mathcal{W}}{\left(z_{s_{1}}-z_{r}\right)\left(z_{s_{2}}-z_{r}\right)}\right] \\
= & \sum_{1 \leq r<s \leq n} \frac{\left(z_{r}-z_{s}\right) \mathcal{W}}{z_{r}-z_{s}}+2 \sum_{r=1}^{n} \sum_{1 \leq s_{1}<s_{2} \leq n ; s_{1}, s_{2} \neq r} \frac{z_{r}^{2} \mathcal{W}\left(z_{1}, z_{2}, \ldots, z_{n}\right)}{\left(z_{s_{1}}-z_{r}\right)\left(z_{s_{2}}-z_{r}\right)} \\
= & \left(\frac{n(n-1)}{2}+2 \sum_{r=1}^{n} \sum_{1 \leq s_{1}<s_{2} \leq n ; s_{1}, s_{2} \neq r} \frac{z_{r}^{2}}{\left(z_{s_{1}}-z_{r}\right)\left(z_{s_{2}}-z_{r}\right)}\right) \mathcal{W} .
\end{aligned}
$$

On the other hand, Lemma 4.6.1 implies

$$
\left(\sum_{\iota=1}^{n}\left(z_{\iota} \partial_{z_{\iota}}\right)^{2}\right)(\mathcal{W})=\left(\sum_{\iota=1}^{n-1} \iota^{2}\right) \mathcal{W}=\frac{(n-1) n(2 n-1)}{6} \mathcal{W}
$$


Thus (4.6.10) and (4.6.11) yield

$$
\begin{aligned}
& \sum_{r=1}^{n} \sum_{1 \leq s_{1}<s_{2} \leq n ; s_{1}, s_{2} \neq r} \frac{z_{r}^{2}}{\left(z_{s_{1}}-z_{r}\right)\left(z_{s_{2}}-z_{r}\right)} \\
= & \frac{1}{2}\left[\frac{(n-1) n(2 n-1)}{6}-\frac{n(n-1)}{2}\right] \\
= & \frac{(n-1) n(n-2)}{6}=\left(\begin{array}{l}
n \\
3
\end{array}\right) .
\end{aligned}
$$

Let

$$
\phi_{\mu_{1}, \mu_{2}}\left(z_{1}, \ldots, z_{n}\right)=\left(z_{1} z_{2} \cdots z_{n}\right)^{\mu_{1}} \mathcal{W}^{\mu_{2}}\left(z_{1}, z_{2}, \ldots, z_{n}\right) \quad \text { for } \mu_{1}, \mu_{2} \in \mathbb{R}
$$

where the special case $\phi_{(1-n) / 2,1}$ is the Weyl function of type- $A_{n-1}$ simple Lie algebra. Then

$$
z_{r} \partial_{z_{r}}\left(\phi_{\mu_{1}, \mu_{2}}\right)=\left(\mu_{1}+\mu_{2} \sum_{r \neq s \in \overline{1, n}} \frac{z_{r}}{z_{r}-z_{s}}\right) \phi_{\mu_{1}, \mu_{2}}
$$

for $r \in \overline{1, n}$. Hence

$$
\begin{aligned}
& \sum_{r=1}^{n}\left(z_{r} \partial_{z_{r}}\right)^{2}\left(\phi_{\mu_{1}, \mu_{2}}\right) \\
= & \sum_{r=1}^{n}\left[\mu_{1}^{2}+\sum_{r \neq s \in \overline{1, n}}\left(2 \mu_{1} \mu_{2} \frac{z_{r}}{z_{r}-z_{s}}-\mu_{2} \frac{z_{s} z_{r}}{\left(z_{s}-z_{r}\right)^{2}}+\mu_{2}^{2} \frac{z_{r}^{2}}{\left(z_{s}-z_{r}\right)^{2}}\right)\right. \\
& \left.+2 \mu_{2}^{2} \sum_{1 \leq s_{1}<s_{2} \leq n ; s_{1}, s_{2} \neq r} \frac{z_{r}^{2}}{\left(z_{s_{1}}-z_{r}\right)\left(z_{s_{2}}-z_{r}\right)}\right] \phi_{\mu_{1}, \mu_{2}} \\
= & {\left[n \mu_{1}^{2}+2 \mu_{1} \mu_{2} \sum_{1 \leq r<s \leq n} \frac{z_{r}-z_{s}}{z_{r}-z_{s}}+2 \mu_{2}^{2}\left(\begin{array}{l}
n \\
3
\end{array}\right)-2 \mu_{2} \sum_{1 \leq r<s \leq n} \frac{z_{s} z_{r}}{\left(z_{s}-z_{r}\right)^{2}}\right.} \\
& \left.+\mu_{2}^{2} \sum_{1 \leq r<s \leq n} \frac{z_{r}^{2}+z_{s}^{2}}{\left(z_{s}-z_{r}\right)^{2}}\right] \phi_{\mu_{1}, \mu_{2}} \\
= & {\left[n \mu_{1}^{2}+n(n-1) \mu_{1} \mu_{2}+2 \mu_{2}^{2}\left(\begin{array}{c}
n \\
3
\end{array}\right)-2 \mu_{2} \sum_{1 \leq r<s \leq n} \frac{z_{s} z_{r}}{\left(z_{s}-z_{r}\right)^{2}}\right.} \\
& \left.+\mu_{2}^{2} \sum_{1 \leq r<s \leq n} \frac{z_{r}^{2}+z_{s}^{2}-2 z_{r} z_{s}+2 z_{r} z_{s}}{\left(z_{s}-z_{r}\right)^{2}}\right] \phi_{\mu_{1}, \mu_{2}} \\
= & {\left[n \mu_{1}^{2}+n(n-1)\left(\mu_{1}+\mu_{2} / 2\right) \mu_{2}+2\left(\begin{array}{l}
n \\
3
\end{array}\right) \mu_{2}^{2}\right.} \\
& \left.+2 \mu_{2}\left(\mu_{2}-1\right) \sum_{1 \leq r<s \leq n} \frac{z_{s} z_{r}}{\left(z_{s}-z_{r}\right)^{2}}\right] \phi_{\mu_{1}, \mu_{2}}
\end{aligned}
$$

by (4.6.12) and (4.6.14). Therefore, we have: 
Theorem 6.2. The function $\phi_{\mu_{1}, \mu_{2}}$ satisfies:

$$
\begin{aligned}
& \sum_{r=1}^{n}\left(z_{r} \partial_{z_{r}}\right)^{2}\left(\phi_{\mu_{1}, \mu_{2}}\right)+2 \mu_{2}\left(1-\mu_{2}\right)\left(\sum_{1 \leq l<j \leq n} \frac{z_{l} z_{j}}{\left(z_{l}-z_{j}\right)^{2}}\right) \phi_{\mu_{1}, \mu_{2}} \\
= & {\left[n \mu_{1}^{2}+n(n-1)\left(\mu_{1}+\mu_{2} / 2\right) \mu_{2}+2\left(\begin{array}{c}
n \\
3
\end{array}\right) \mu_{2}^{2}\right] \phi_{\mu_{1}, \mu_{2}}, }
\end{aligned}
$$

equivalently, $\phi_{\mu_{1}, \mu_{2}}\left(e^{2 x_{1}}, \ldots, e^{2 x_{n}}\right)$ is a solution of the Calogero-Sutherland model with $K=$ $2 \mu_{2}\left(1-\mu_{2}\right)$ and the corresponding eigenvalue is $2 n\left[2 \mu_{1}\left(\mu_{1}+(n-1) \mu_{2}\right)+(n-1)(2 n-1) \mu_{2}^{2} / 3\right]$.

The above theorem for generic $\mu_{1}$ and $\mu_{2}$ was proved by us in [X9], and it was known when $\mu_{1}=\mu_{2}$ or $\mu_{1}=0$ before our work [X9]. Next we will explore the connection between the Calogero-Sutherland model and hypergeometric functions.

We first consider the case $n=2$. Now (4.6.16) becomes

$$
\begin{aligned}
& {\left[\left(z_{1} \partial_{z_{1}}\right)^{2}+\left(z_{2} \partial_{z_{2}}\right)^{2}\right]\left(\phi_{\mu_{1}, \mu_{2}}\right)+2 \mu_{2}\left(1-\mu_{2}\right) \frac{z_{1} z_{2}}{\left(z_{1}-z_{2}\right)^{2}} \phi_{\mu_{1}, \mu_{2}} } \\
= & \left(2 \mu_{1}^{2}+2 \mu_{1} \mu_{2}+\mu_{2}^{2}\right) \phi_{\mu_{1}, \mu_{2}} .
\end{aligned}
$$

Let $g(z)$ be a differentiable function. Denote

$$
\xi=\frac{z_{2}}{z_{2}-z_{1}}
$$

Then

$$
z_{1} \partial_{z_{1}}(g(\xi))=-z_{2} \partial_{z_{2}}(g(\xi))=\frac{z_{1} z_{2}}{\left(z_{2}-z_{1}\right)^{2}} g^{\prime}(\xi)
$$

Moreover,

$$
\left(z_{1} \partial_{z_{1}}\right)^{2}(g(\xi))=\left(z_{2} \partial_{z_{2}}\right)^{2}(g(\xi))=\frac{z_{1}^{2} z_{2}^{2}}{\left(z_{1}-z_{2}\right)^{4}} g^{\prime \prime}(\xi)+\frac{z_{1} z_{2}\left(z_{1}+z_{2}\right)}{\left(z_{2}-z_{1}\right)^{3}} g^{\prime}(\xi) .
$$

According to (4.6.14),

$$
\begin{aligned}
& z_{1} \partial_{z_{1}}\left(\phi_{\mu_{1}, \mu_{2}}\right)=\left(\mu_{1}+\mu_{2} \frac{z_{1}}{z_{1}-z_{2}}\right) \phi_{\mu_{1}, \mu_{2}}, \\
& z_{2} \partial_{z_{2}}\left(\phi_{\mu_{1}, \mu_{2}}\right)=\left(\mu_{1}-\mu_{2} \frac{z_{2}}{z_{1}-z_{2}}\right) \phi_{\mu_{1}, \mu_{2}} .
\end{aligned}
$$


By (4.6.19)-(4.6.22), we have

$$
\begin{aligned}
& {\left[\left(z_{1} \partial_{z_{1}}\right)^{2}+\left(z_{2} \partial_{z_{2}}\right)^{2}\right]\left(\phi_{\mu_{1}, \mu_{2}} g(\xi)\right) } \\
= & \phi_{\mu_{1}, \mu_{2}}\left[\left(2 \mu_{2}\left(\mu_{2}-1\right) \frac{z_{1} z_{2}}{\left(z_{1}-z_{2}\right)^{2}}+\left(2 \mu_{1}^{2}+2 \mu_{1} \mu_{2}+\mu_{2}^{2}\right)\right) g(\xi)\right. \\
& +2\left(\mu_{1}+\mu_{2} \frac{z_{1}}{z_{1}-z_{2}}\right) \frac{z_{1} z_{2}}{\left(z_{1}-z_{2}\right)^{2}} g^{\prime}(\xi)-2\left(\mu_{1}-\mu_{2} \frac{z_{2}}{z_{1}-z_{2}}\right) \frac{z_{1} z_{2}}{\left(z_{1}-z_{2}\right)^{2}} g^{\prime}(\xi) \\
& \left.+2 \frac{z_{1}^{2} z_{2}^{2}}{\left(z_{1}-z_{2}\right)^{4}} g^{\prime \prime}(\xi)+2 \frac{z_{1} z_{2}\left(z_{1}+z_{2}\right)}{\left(z_{2}-z_{1}\right)^{3}} g^{\prime}(\xi)\right] \\
= & \phi_{\mu_{1}, \mu_{2}}\left[\left(2 \mu_{2}\left(\mu_{2}-1\right) \frac{z_{1} z_{2}}{\left(z_{1}-z_{2}\right)^{2}}+\left(2 \mu_{1}^{2}+2 \mu_{1} \mu_{2}+\mu_{2}^{2}\right)\right) g(\xi)\right. \\
& \left.+2\left(1-\mu_{2}\right) \frac{z_{1} z_{2}\left(z_{1}+z_{2}\right)}{\left(z_{2}-z_{1}\right)^{3}} g^{\prime}(\xi)+\frac{2 z_{1}^{2} z_{2}^{2}}{\left(z_{1}-z_{2}\right)^{4}} g^{\prime \prime}(\xi)\right] .
\end{aligned}
$$

Observe that

$$
\begin{gathered}
\frac{z_{1}+z_{2}}{z_{2}-z_{1}}=2 \frac{z_{2}}{z_{2}-z_{1}}-1=2 \xi-1, \\
\frac{z_{1} z_{2}}{\left(z_{1}-z_{2}\right)^{2}}=\frac{z_{1} z_{2}}{\left(z_{2}-z_{1}\right)^{2}}=\frac{z_{2}^{2}}{\left(z_{2}-z_{1}\right)^{2}}-\frac{z_{2}}{z_{2}-z_{1}}=\xi(\xi-1) .
\end{gathered}
$$

Thus

$$
\begin{aligned}
& 2\left(1-\mu_{2}\right) \frac{z_{1} z_{2}\left(z_{1}+z_{2}\right)}{\left(z_{2}-z_{1}\right)^{3}} g^{\prime}(\xi)+\frac{2 z_{1}^{2} z_{2}^{2}}{\left(z_{1}-z_{2}\right)^{4}} g^{\prime \prime}(\xi) \\
= & -\frac{2 z_{1} z_{2}}{\left(z_{1}-z_{2}\right)^{2}}\left[\left(1-\mu_{2}\right) \frac{z_{1}+z_{2}}{z_{1}-z_{2}} g^{\prime}(\xi)-\frac{z_{1} z_{2}}{\left(z_{1}-z_{2}\right)^{2}} g^{\prime \prime}(\xi)\right] \\
= & -\frac{2 z_{1} z_{2}}{\left(z_{1}-z_{2}\right)^{2}}\left[\xi(1-\xi) g^{\prime \prime}(\xi)+\left(1-\mu_{2}\right)(1-2 \xi) g^{\prime}(\xi)\right] .
\end{aligned}
$$

Recall the classical Gauss hypergeometric equation

$$
z(1-z) y^{\prime \prime}+[\gamma-(\alpha+\beta+1) z] y^{\prime}-\alpha \beta y=0 .
$$

We take $\gamma=1-\mu_{2}$ and

$$
\alpha+\beta+1=2\left(1-\mu_{2}\right) \Longrightarrow \beta=1-2 \mu_{2}-\alpha,
$$

where $\alpha$ is arbitrary.

Theorem 4.6.3. Let $\alpha, \mu_{1}, \mu_{2} \in \mathbb{R}$. If $g(z)$ is a nonzero function satisfying the following classical Gauss hypergeometric equation

$$
z(1-z) g^{\prime \prime}+\left(1-\mu_{2}\right)(1-2 z) g^{\prime}-\alpha\left(1-\alpha-2 \mu_{2}\right) g=0,
$$

then the function

$$
\psi=\left(z_{1} z_{2}\right)^{\mu_{1}}\left(z_{1}-z_{2}\right)^{\mu_{2}} g\left(\frac{z_{2}}{z_{2}-z_{1}}\right)
$$


satisfies the equation for the Calogero-Sutherland model

$$
\begin{aligned}
& {\left[\left(z_{1} \partial_{z_{1}}\right)^{2}+\left(z_{2} \partial_{z_{2}}\right)^{2}\right](\psi)+2 \mu_{2}\left(1-\mu_{2}\right) \frac{z_{1} z_{2}}{\left(z_{1}-z_{2}\right)^{2}} \psi } \\
= & {\left[2 \mu_{1}^{2}+2 \mu_{1} \mu_{2}+\mu_{2}^{2}+2 \alpha\left(\alpha+2 \mu_{2}-1\right)\right] \psi }
\end{aligned}
$$

with $K=2 \mu_{2}\left(1-\mu_{2}\right)$ and the eigenvalue $\nu=2 \mu_{1}^{2}+2 \mu_{1} \mu_{2}+\mu_{2}^{2}+2 \alpha\left(\alpha+2 \mu_{2}-1\right)$.

Suppose that $\mu_{2}$ is not an integer, then the fundamental solutions of the equation (4.6.29) are ${ }_{2} F_{1}\left(\alpha, 1-\alpha-2 \mu_{2} ; 1-\mu_{2} ; z\right)$ and ${ }_{2} F_{1}\left(\alpha+\mu_{2}, 1-\mu_{2}-\alpha ; 1+\mu_{2} ; z\right) z^{\mu_{2}}$ (cf. $(3.2 .10))$.

Next we consider $n>2$. Let

$$
\Gamma_{A}=\sum_{1 \leq p<q \leq n} \mathbb{N} \epsilon_{q, p}
$$

be the additive semigroup of rank $n(n-1) / 2$ with $\epsilon_{q, p}$ as base elements. For $\alpha=$ $\sum_{1 \leq p<q \leq n} \alpha_{q, p} \epsilon_{q, p} \in \Gamma_{A}$, we denote

$$
\alpha_{1 *}=\alpha_{n}^{*}=0, \quad \alpha_{k *}=\sum_{r=1}^{k-1} \alpha_{k, r}, \quad \alpha_{l}^{*}=\sum_{s=l+1}^{n} \alpha_{s, l}
$$

Given $\vartheta \in \mathbb{C} \backslash\{-\mathbb{N}\}$ and $\tau_{r} \in \mathbb{C}$ with $r \in \overline{1, n}$, we define our $(n(n-1) / 2)$-variable hypergeometric function of type $A$ by

$$
\mathcal{X}_{A}\left(\tau_{1}, . ., \tau_{n} ; \vartheta\right)\left\{z_{j, k}\right\}=\sum_{\beta \in \Gamma_{A}} \frac{\left[\prod_{s=1}^{n-1}\left(\tau_{s}-\beta_{s *}\right)_{\beta_{s}^{*}}\right]\left(\tau_{n}\right)_{\beta_{n *}}}{\beta !(\vartheta)_{\beta_{n *}}} z^{\beta},
$$

where

$$
\beta !=\prod_{1 \leq k<j \leq n} \beta_{j, k} !, \quad z^{\beta}=\prod_{1 \leq k<j \leq n} z_{j, k}^{\beta_{j, k}}
$$

Set

$$
\xi_{r_{2}, r_{1}}^{A}=\prod_{s=r_{1}}^{r_{2}-1} \frac{z_{r_{2}}}{z_{r_{2}}-z_{s}} \quad \text { for } 1 \leq r_{1}<r_{2} \leq n .
$$

Take $\left(\lambda_{1}, \ldots, \lambda_{n}\right) \in \mathbb{C}^{n}$ such that

$$
\lambda_{1}-\lambda_{2}=\cdots=\lambda_{n-2}-\lambda_{n-1}=\mu \text { and } \lambda_{n-1}-\lambda_{n}=\sigma \notin \mathbb{N}
$$

for some constants $\mu$ and $\sigma$. Then we have the following result which was proved by representation theory:

Theorem 4.6.4. The function

$$
\prod_{r=1}^{n} z_{r}^{\lambda_{r}+(n+1) / 2-r} \mathcal{X}_{A}(\mu+1, . ., \mu+1,-\mu ;-\sigma)\left\{\xi_{r_{2}, r_{1}}^{A}\right\}
$$


is a solution of the equation (4.6.7).

Below we want to show that the functions $\mathcal{X}_{A}\left(\tau_{1}, . ., \tau_{n} ; \vartheta\right)\left\{z_{j, k}\right\}$ are indeed natural generalizations of the Gauss hypergeometric function ${ }_{2} F_{1}(\alpha, \beta ; \gamma ; z)$. Note

$$
D=z \frac{d}{d z} \Longrightarrow D^{2}=z^{2} \frac{d^{2}}{d z^{2}}+z \frac{d}{d z}
$$

Then the classical hypergeometric equation (3.2.1) can be rewritten as

$$
(\gamma+D) \frac{d}{d z}(y)=(\alpha+D)(\beta+D)(y) .
$$

Denote

$$
\mathcal{D}_{p *}=\sum_{r=1}^{p-1} z_{p, r} \partial_{z_{p, r}}, \quad \mathcal{D}_{q}^{*}=\sum_{s=q+1}^{n} z_{s, q} \partial_{z_{s, q}} \quad \text { for } p \in \overline{2, n}, q \in \overline{1, n-1} .
$$

The following result was proved by author.

Theorem 4.6.5. We have:

$$
\left(\tau_{r_{2}}-1-\mathcal{D}_{r_{2} *}+\mathcal{D}_{r_{2}}^{*}\right) \partial_{z_{r_{2}, r_{1}}}\left(\mathcal{X}_{A}\right)=\left(\tau_{r_{2}}-1-\mathcal{D}_{r_{2} *}\right)\left(\tau_{r_{1}}-\mathcal{D}_{r_{1} *}+\mathcal{D}_{r_{1}}^{*}\right)\left(\mathcal{X}_{A}\right)
$$

for $1 \leq r_{1}<r_{2} \leq n-1$ and

$$
\left(\vartheta+\mathcal{D}_{n *}\right) \partial_{z_{n, r}}\left(\mathcal{X}_{A}\right)=\left(\tau_{n}+\mathcal{D}_{n *}\right)\left(\tau_{r}-\mathcal{D}_{r *}+\mathcal{D}_{r}^{*}\right)\left(\mathcal{X}_{A}\right)
$$

for $r \in \overline{1, n-1}$.

Recall the differentiation property

$$
\frac{d}{d z}{ }_{2} F_{1}(\alpha, \beta ; \gamma ; z)=\frac{\alpha \beta}{\gamma}{ }_{2} F_{1}(\alpha+1, \beta+1 ; \gamma+1 ; z)
$$

(cf. (3.2.19)). For two positive integers $k_{1}$ and $k_{2}$ such that $k_{1}<k_{2}$, a path from $k_{1}$ to $k_{2}$ is a sequence $\left(m_{0}, m_{1} \ldots, m_{r}\right)$ of positive integers such that

$$
k_{1}=m_{0}<m_{1}<m_{2}<\cdots<m_{r-1}<m_{r}=k_{2} .
$$

One can imagine a path from $k_{1}$ to $k_{2}$ is a way of a super man going from $k_{1}$ th floor to $k_{2}$ th floor through a stairway. Let

$$
\mathcal{P}_{k_{1}}^{k_{2}}=\text { the set of all paths from } k_{1} \text { to } k_{2} .
$$

The path polynomial from $k_{1}$ to $k_{2}$ is defined as

$$
P_{\left[k_{1}, k_{2}\right]}=\sum_{\left(m_{0}, m_{1}, \ldots, m_{r}\right) \in \mathcal{P}_{k_{1}}^{k_{2}}}(-1)^{r} z_{m_{1}, m_{0}} z_{m_{2}, m_{1}} \cdots z_{m_{r-1}, m_{r-2}} z_{m_{r}, m_{r-1}} .
$$


In fact

$$
\left(\begin{array}{ccccc}
1 & 0 & 0 & \cdots & 0 \\
P_{[1,2]} & 1 & 0 & \cdots & 0 \\
P_{[1,3]} & P_{[2,3]} & 1 & \ddots & \vdots \\
\vdots & \vdots & \ddots & \ddots & 0 \\
P_{[1, n]} & P_{[2, n]} & \cdots & P_{[n-1, n]} & 1
\end{array}\right)=\left(\begin{array}{ccccc}
1 & 0 & 0 & \cdots & 0 \\
z_{2,1} & 1 & 0 & \cdots & 0 \\
z_{3,1} & z_{3,2} & 1 & \ddots & \vdots \\
\vdots & \vdots & \ddots & \ddots & 0 \\
z_{n, 1} & z_{n, 2} & \cdots & z_{n, n-1} & 1
\end{array}\right)^{-1}
$$

For convenience, we simply denote

$$
\begin{gathered}
P_{[k, k]}=1 \quad \text { for } 0<k \in \mathbb{N}, \\
\mathcal{X}_{A}=\mathcal{X}_{A}\left(\tau_{1}, . ., \tau_{n} ; \vartheta\right)\left\{z_{j, k}\right\} \\
\mathcal{X}_{A}[l, j]=\mathcal{X}_{A}\left(\tau_{1}, \ldots, \tau_{l}+1, \ldots, \tau_{j}-1, \ldots \tau_{n} ; \vartheta\right)\left\{z_{r_{2}, r_{1}}\right\}
\end{gathered}
$$

obtained from $\mathcal{X}_{A}$ by changing $\tau_{l}$ to $\tau_{l}+1$ and $\tau_{j}$ to $\tau_{j}-1$ for $1 \leq i<j \leq n-1$ and

$$
\mathcal{X}_{A}[k, n]=\mathcal{X}_{A}\left(\tau_{1}, . ., \tau_{k}+1, \ldots, \tau_{n}+1 ; \vartheta+1\right)\left\{z_{r_{2}, r_{1}}\right\}
$$

obtained from $\mathcal{X}_{A}$ by changing $\tau_{k}$ to $\tau_{k}+1, \tau_{n}$ to $\tau_{n}+1$ and $\vartheta$ to $\vartheta+1$ for $k \in \overline{1, n-1}$. The following result was proved by the author.

Theorem 4.6.6. For $1 \leq r_{1}<r_{2} \leq n-1$ and $r \in \overline{1, n-1}$, we have

$$
\begin{aligned}
& \partial_{z_{r_{2}, r_{1}}}\left(\mathcal{X}_{A}\right)=\sum_{s=1}^{r_{1}} \tau_{s} P_{\left[s, r_{1}\right]} \mathcal{X}_{A}\left[s, r_{2}\right], \\
& \partial_{z_{n, r}}\left(\mathcal{X}_{A}\right)=\frac{\tau_{n}}{\vartheta} \sum_{s=1}^{r} \tau_{s} P_{[s, r]} \mathcal{X}_{A}[s, n] .
\end{aligned}
$$

Recall the integral representation

$$
{ }_{2} F_{1}(\alpha, \beta ; \gamma ; z)=\frac{\Gamma(\gamma)}{\Gamma(\beta) \Gamma(\gamma-\beta)} \int_{0}^{1} t^{\beta-1}(1-t)^{\gamma-\beta-1}(1-z t)^{-\alpha} d t
$$

(cf. Theorem 3.2.1). We have the following integral representation:

Theorem 4.6.7. Suppose $\operatorname{Re} \tau_{n}>0$ and $\operatorname{Re}\left(\vartheta-\tau_{n}\right)>0$. We have

$$
\mathcal{X}_{A}=\frac{\Gamma(\vartheta)}{\Gamma\left(\vartheta-\tau_{n}\right) \Gamma\left(\tau_{n}\right)} \int_{0}^{1}\left[\prod_{r=1}^{n-1}\left(\sum_{s=r}^{n-1} P_{[r, s]}+t P_{[r, n]}\right)^{-\tau_{r}}\right] t^{\tau_{n}-1}(1-t)^{\vartheta-\tau_{n}-1} d t
$$

on the region $P_{[r, n]} /\left(\sum_{s=r}^{n-1} P_{[r, s]}\right) \notin(-\infty,-1)$ for $r \in \overline{1, n-1}$.

Heckman and Opdam [HO, Hg1-Hg3, Oe1-Oe5, BO] introduced hypergeometric equations related to root systems and analogous to (4.6.7). They proved the existence of solutions (hypergeometric functions) of their equations. Gel'fand and Graev studied analogues of classical hypergeometric functions (so called GG-functions) by generalizing the differential property of the classical hypergeometric functions (e.g. cf. [GG]). 


\subsection{Maxwell Equations}

The electromagnetic fields in physics are governed by the well-known Maxwell equations (e.g., cf. $[\operatorname{In} 3])$ :

$$
\partial_{t}(\mathbf{E})=\operatorname{curl} \mathbf{B}, \quad \partial_{t}(\mathbf{B})=-\operatorname{curl} \mathbf{E}
$$

with

$$
\operatorname{div} \mathbf{E}=f(x, y, z), \quad \operatorname{div} \mathbf{B}=g(x, y, z),
$$

where the vector function $\mathbf{E}$ stands for the electric field, the vector function $\mathbf{B}$ stands for the magnetic field, the scalar function $f$ is related to the charge density and the scalar function $g$ is related to the magnetic potential. We want to use matrix-differentialoperators and Fourier expansion to solve the Maxwell equations (4.7.1) subject to the following initial condition:

$$
\mathbf{E}(0, x, y, z)=\mathbf{E}_{0}(x, y, z), \quad \mathbf{B}(0, x, y, z)=\mathbf{B}_{0}(x, y, z)
$$

for $x \in\left[-a_{1}, a_{1}\right], y \in\left[-a_{2}, a_{2}\right], z \in\left[-a_{3}, a_{3}\right]$, where $\mathbf{E}_{0}(x, y, z)$ and $\mathbf{B}_{0}(x, y, z)$ are given real vector-valued functions satisfying (4.7.2), and $a_{r}$ are positive real constants. We denote

$$
\mathbf{B}=\left(\begin{array}{c}
B_{1} \\
B_{2} \\
B_{3}
\end{array}\right), \quad \mathbf{E}=\left(\begin{array}{c}
E_{1} \\
E_{2} \\
E_{3}
\end{array}\right)
$$

Then the Maxwell equations becomes

$$
\begin{array}{r}
\partial_{t}(\mathbf{E})=\left(\begin{array}{c}
\partial_{y}\left(B_{3}\right)-\partial_{z}\left(B_{2}\right) \\
\partial_{z}\left(B_{1}\right)-\partial_{x}\left(B_{3}\right) \\
\partial_{x}\left(B_{2}\right)-\partial_{y}\left(B_{1}\right)
\end{array}\right)=\left(\begin{array}{ccc}
0 & -\partial_{z} & \partial_{y} \\
\partial_{z} & 0 & -\partial_{x} \\
-\partial_{y} & \partial_{x} & 0
\end{array}\right) \mathbf{B}, \\
\partial_{t}(\mathbf{B})=-\left(\begin{array}{c}
\partial_{y}\left(E_{3}\right)-\partial_{z}\left(E_{2}\right) \\
\partial_{z}\left(E_{1}\right)-\partial_{x}\left(E_{3}\right) \\
\partial_{x}\left(E_{2}\right)-\partial_{y}\left(E_{1}\right)
\end{array}\right)=-\left(\begin{array}{ccc}
0 & -\partial_{z} & \partial_{y} \\
\partial_{z} & 0 & -\partial_{x} \\
-\partial_{y} & \partial_{x} & 0
\end{array}\right) \mathbf{E} .
\end{array}
$$

Set

$$
\mathbb{D}=\left(\begin{array}{ccc}
0 & -\partial_{z} & \partial_{y} \\
\partial_{z} & 0 & -\partial_{x} \\
-\partial_{y} & \partial_{x} & 0
\end{array}\right)
$$

Then we can combine the two equations in (4.7.1) into one equation:

$$
\partial_{t}\left(\begin{array}{l}
\mathbf{E} \\
\mathbf{B}
\end{array}\right)=\left(\begin{array}{cc}
0 & \mathbb{D} \\
-\mathbb{D} & 0
\end{array}\right)\left(\begin{array}{l}
\mathbf{E} \\
\mathbf{B}
\end{array}\right)
$$

Thus the solution is given by

$$
\left(\begin{array}{l}
\mathbf{E} \\
\mathbf{B}
\end{array}\right)=\left[\exp t\left(\begin{array}{cc}
0 & \mathbb{D} \\
-\mathbb{D} & 0
\end{array}\right)\right]\left(\begin{array}{l}
\mathbf{E}_{0} \\
\mathbf{B}_{0}
\end{array}\right)=\left(\begin{array}{cc}
\cos t \mathbb{D} & \sin t \mathbb{D} \\
-\sin t \mathbb{D} & \cos t \mathbb{D}
\end{array}\right)\left(\begin{array}{l}
\mathbf{E}_{0} \\
\mathbf{B}_{0}
\end{array}\right)
$$


where

$$
\left(\begin{array}{l}
\mathbf{E}_{0} \\
\mathbf{B}_{0}
\end{array}\right)=\left.\left(\begin{array}{l}
\mathbf{E} \\
\mathbf{B}
\end{array}\right)\right|_{t=0}
$$

is a given first-order differentiable field in $x, y, z$ satisfying the constraint (4.7.2).

Now the key point is how to calculate $\cos t \mathbb{D}$ and $\sin t \mathbb{D}$. In order to do this, we consider the $3 \times 3$ skew-symmetric matrix:

$$
A=\left(\begin{array}{rrr}
0 & -a & -b \\
a & 0 & -c \\
b & c & 0
\end{array}\right), \quad 0 \neq a, b, c \in \mathbb{R}
$$

where $\mathbb{R}$ is the field of real numbers. Note that

$$
A^{2}=\left(\begin{array}{rrr}
0 & -a & -b \\
a & 0 & -c \\
b & c & 0
\end{array}\right)\left(\begin{array}{rrr}
0 & -a & -b \\
a & 0 & -c \\
b & c & 0
\end{array}\right)=-\left(\begin{array}{ccc}
a^{2}+b^{2} & b c & -a c \\
b c & a^{2}+c^{2} & a b \\
-a c & a b & b^{2}+c^{2}
\end{array}\right) .
$$

Moreover,

$$
\begin{aligned}
A^{3} & =-\left(\begin{array}{ccc}
a^{2}+b^{2} & b c & -a c \\
b c & a^{2}+c^{2} & a b \\
-a c & a b & b^{2}+c^{2}
\end{array}\right)\left(\begin{array}{rrr}
0 & -a & -b \\
a & 0 & -c \\
b & c & 0
\end{array}\right) \\
& =-\left(a^{2}+b^{2}+c^{2}\right)\left(\begin{array}{rrr}
0 & -a & -b \\
a & 0 & -c \\
b & c & 0
\end{array}\right)=-\left(a^{2}+b^{2}+c^{2}\right) A
\end{aligned}
$$

which implies

$$
A^{2 k+1}=\left[-\left(a^{2}+b^{2}+c^{2}\right)\right]^{k} A, \quad A^{2 k+2}=\left[-\left(a^{2}+b^{2}+c^{2}\right)\right]^{k} A^{2} \quad \text { for } k \in \mathbb{N},
$$

where $\mathbb{N}$ stands for the set of nonnegative integers. Thus

$$
\begin{gathered}
\sin t A=\left(\sum_{k=0}^{\infty} \frac{\left(a^{2}+b^{2}+c^{2}\right)^{k} t^{2 k+1}}{(2 k+1) !}\right) A, \\
\cos t A=I_{3}-\left(\sum_{k=0}^{\infty} \frac{\left(a^{2}+b^{2}+c^{2}\right)^{k} t^{2 k+2}}{(2 k+2) !}\right) A^{2},
\end{gathered}
$$

where $I_{3}$ is the $3 \times 3$ identity matrix.

Denote

$$
\Delta=\partial_{x}^{2}+\partial_{y}^{2}+\partial_{z}^{2}
$$

By (4.7.7), (4.7.15) and (4.7.16), we have:

$$
\sin t \mathbb{D}=\left(\sum_{k=0}^{\infty} \frac{\Delta^{k} t^{2 k+1}}{(2 k+1) !}\right)\left(\begin{array}{ccc}
0 & -\partial_{z} & \partial_{y} \\
\partial_{z} & 0 & -\partial_{x} \\
-\partial_{y} & \partial_{x} & 0
\end{array}\right)
$$


and

$$
\cos t \mathbb{D}=I_{3}+\left(\sum_{k=0}^{\infty} \frac{\Delta^{k} t^{2 k+2}}{(2 k+2) !}\right)\left(\begin{array}{ccc}
\partial_{y}^{2}+\partial_{z}^{2} & -\partial_{x} \partial_{y} & -\partial_{x} \partial_{z} \\
-\partial_{x} \partial_{y} & \partial_{x}^{2}+\partial_{z}^{2} & -\partial_{y} \partial_{z} \\
-\partial_{x} \partial_{z} & -\partial_{y} \partial_{z} & \partial_{x}^{2}+\partial_{y}^{2}
\end{array}\right) .
$$

As operators,

$$
\operatorname{div} \circ \operatorname{curl}=0 .
$$

This shows

$$
\begin{gathered}
\partial_{t}(\operatorname{div} \mathbf{E})=\operatorname{div}\left(\partial_{t} \mathbf{E}\right)=\operatorname{div}(\operatorname{curl} \mathbf{B})=0, \\
\partial_{t}(\operatorname{div} \mathbf{B})=\operatorname{div}\left(\partial_{t} \mathbf{B}\right)=-\operatorname{div}(\operatorname{curl} \mathbf{E})=0 .
\end{gathered}
$$

Thus the constraint (4.7.2) is satisfied if the initial field $\mathbf{E}_{0}$ and $\mathbf{B}_{0}$ satisfy it. Solving (4.7.2), we get

$$
\begin{array}{r}
\mathbf{E}_{0}=\left(\begin{array}{c}
\int_{0}^{x} f(s, y, z) d s-\partial_{y}\left(f_{1}(x, y, z)\right) \\
\partial_{x}\left(f_{1}(x, y, z)\right)-\partial_{z}\left(f_{2}(x, y, z)\right) \\
\partial_{y}\left(f_{2}(x, y, z)\right)
\end{array}\right), \\
\mathbf{B}_{0}=\left(\begin{array}{c}
\int_{0}^{x} g(s, y, z) d s-\partial_{y}\left(g_{1}(x, y, z)\right) \\
\partial_{x}\left(g_{1}(x, y, z)\right)-\partial_{z}\left(g_{2}(x, y, z)\right) \\
\partial_{y}\left(g_{2}(x, y, z)\right)
\end{array}\right),
\end{array}
$$

which imply that $\mathbf{E}_{0}$ is completely determined by two second-order differentiable functions $f_{1}$ and $f_{2}$, and $\mathbf{B}_{0}$ is completely determined by two second-order differentiable functions $g_{1}$ and $g_{2}$. In other words, giving initial fields $\mathbf{E}_{0}$ and $\mathbf{B}_{0}$ is equivalent to giving four second-order differentiable functions $f_{1}, g_{1}, f_{2}, g_{2}$.

For convenience, we denote

$$
k_{r}^{\dagger}=\frac{k_{r}}{a_{r}}, \quad \vec{k}^{\dagger}=\left(k_{1}^{\dagger}, k_{2}^{\dagger}, k_{3}^{\dagger}\right) \quad \text { for } \vec{k}=\left(k_{1}, k_{2}, k_{3}\right) \in \mathbb{Z}^{3} .
$$

Moreover, we write

$$
\vec{x}=\left(\begin{array}{l}
x \\
y \\
z
\end{array}\right)
$$

and

$$
\vec{k}^{\dagger} \cdot \vec{x}=k_{1}^{\dagger} x+k_{2}^{\dagger} y+k_{3}^{\dagger} z
$$

Set

$$
\left|\vec{k}^{\dagger}\right|=\sqrt{\left(k_{1}^{\dagger}\right)^{2}+\left(k_{2}^{\dagger}\right)^{2}+\left(k_{3}^{\dagger}\right)^{2}}
$$

Observe that

$$
-\sum_{s=0}^{\infty} \frac{(-1)^{s} x^{2 s}(\pi t)^{2 s+2}}{(2 s+2) !}=\frac{\cos \pi x t-1}{x^{2}}, \sum_{s=0}^{\infty} \frac{(-1)^{s} x^{2 s}(\pi t)^{2 s+1}}{(2 s+1) !}=\frac{\sin \pi x t}{x} .
$$

Moreover, we treat

$$
\left.\frac{\cos \pi x t-1}{x^{2}}\right|_{x=0}=-\frac{\pi^{2} t^{2}}{2},\left.\quad \frac{\sin \pi x t}{x}\right|_{x=0}=\pi t .
$$


For $\lambda_{1}, \lambda_{2}, \lambda_{3} \in \mathbb{R}$ and $\vec{k} \in \mathbb{Z}^{3}$,

$$
\begin{aligned}
& \cos t \mathbb{D}\left(\begin{array}{c}
\lambda_{1} e^{\pi\left(\vec{k}^{\dagger} \cdot \vec{x}\right) i} \\
\lambda_{2} e^{\pi\left(\vec{k}^{\dagger} \cdot \vec{x}\right) i} \\
\lambda_{3} e^{\pi\left(\vec{k}^{\dagger} \cdot \vec{x}\right) i}
\end{array}\right) \\
= & {\left[I_{3}+\left(\sum_{s=0}^{\infty} \frac{\Delta^{s} t^{2 s+2}}{(2 s+2) !}\right)\left(\begin{array}{ccc}
\partial_{y}^{2}+\partial_{z}^{2} & -\partial_{x} \partial_{y} & -\partial_{x} \partial_{z} \\
-\partial_{x} \partial_{y} & \partial_{x}^{2}+\partial_{z}^{2} & -\partial_{y} \partial_{z} \\
-\partial_{x} \partial_{z} & -\partial_{y} \partial_{z} & \partial_{x}^{2}+\partial_{y}^{2}
\end{array}\right)\right]\left(\begin{array}{c}
\lambda_{1} e^{\pi\left(\vec{k}^{\dagger} \cdot \vec{x}\right) i} \\
\lambda_{2} e^{\pi\left(\vec{k}^{\dagger} \cdot \vec{x}\right) i} \\
\lambda_{3} e^{\pi\left(\vec{k}^{\dagger} \cdot \vec{x}\right) i}
\end{array}\right) } \\
= & \mathbb{K}(\vec{k}, t)\left(\begin{array}{c}
\lambda_{1} e^{\pi\left(\vec{k}^{\dagger} \cdot \vec{x}\right) i} \\
\lambda_{2} e^{\pi\left(\vec{k}^{\dagger} \cdot \vec{x}\right) i} \\
\lambda_{3} e^{\pi\left(\vec{k}^{\dagger} \cdot \vec{x}\right) i}
\end{array}\right)
\end{aligned}
$$

with

$$
\begin{aligned}
& \mathbb{K}(\vec{k}, t)=I_{3}+\frac{\cos \pi t\left|\vec{k}^{\dagger}\right|-1}{\left|\vec{k}^{\dagger}\right|^{2}} \\
& \times\left(\begin{array}{ccc}
\left(k_{2}^{\dagger}\right)^{2}+\left(k_{3}^{\dagger}\right)^{2} & -k_{1}^{\dagger} k_{2}^{\dagger} & -k_{1}^{\dagger} k_{3}^{\dagger} \\
-k_{1}^{\dagger} k_{2}^{\dagger} & \left(k_{1}^{\dagger}\right)^{2}+\left(k_{3}^{\dagger}\right)^{2} & -k_{2}^{\dagger} k_{3}^{\dagger} \\
-k_{1}^{\dagger} k_{3}^{\dagger} & -k_{2}^{\dagger} k_{3}^{\dagger} & \left(k_{1}^{\dagger}\right)^{2}+\left(k_{2}^{\dagger}\right)^{2}
\end{array}\right),
\end{aligned}
$$

and

$$
\begin{aligned}
& \sin t \mathbb{D}\left(\begin{array}{c}
\lambda_{1} e^{\pi\left(\vec{k}^{\dagger} \cdot \vec{x}\right) i} \\
\lambda_{2} e^{\pi\left(\vec{k}^{\dagger} \cdot \vec{x}\right) i} \\
\lambda_{3} e^{\pi\left(\vec{k}^{\dagger} \cdot \vec{x}\right) i}
\end{array}\right) \\
= & \left(\sum_{s=0}^{\infty} \frac{\Delta^{s} t^{2 s+1}}{(2 s+1) !}\right)\left(\begin{array}{ccc}
0 & -\partial_{z} & \partial_{y} \\
\partial_{z} & 0 & -\partial_{x} \\
-\partial_{y} & \partial_{x} & 0
\end{array}\right)\left(\begin{array}{c}
\lambda_{1} e^{\pi\left(\vec{k}^{\dagger} \cdot \vec{x}\right) i} \\
\lambda_{2} e^{\pi\left(\vec{k}^{\dagger} \cdot \vec{x}\right) i} \\
\lambda_{3} e^{\pi\left(\vec{k}^{\dagger} \cdot \vec{x}\right) i}
\end{array}\right) \\
= & i \mathbb{M}(\vec{k}, t)\left(\begin{array}{c}
\lambda_{1} e^{\pi\left(\vec{k}^{\dagger} \cdot \vec{x}\right) i} \\
\lambda_{2} e^{\pi\left(\vec{k}^{\dagger} \cdot \vec{x}\right) i} \\
\lambda_{3} e^{\pi\left(\vec{k}^{\dagger} \cdot \vec{x}\right) i}
\end{array}\right)
\end{aligned}
$$

with

$$
\mathbb{M}(\vec{k}, t)=\left(\begin{array}{ccc}
0 & -\frac{k_{3}^{\dagger} \sin \pi t\left|\vec{k}^{\dagger}\right|}{\left|\vec{k}^{\dagger}\right|} & \frac{k_{2}^{\dagger} \sin \pi t\left|\vec{k}^{\dagger}\right|}{\left|\vec{k}^{\dagger}\right|} \\
\frac{k_{3}^{\dagger} \sin \pi t\left|\vec{k}^{\dagger}\right|}{\left|\vec{k}^{\dagger}\right|} & 0 & -\frac{k_{1}^{\dagger} \sin \pi t\left|\vec{k}^{\dagger}\right|}{\left|\vec{k}^{\dagger}\right|} \\
-\frac{k_{2}^{\dagger} \sin \pi t\left|\vec{k}^{\dagger}\right|}{\left|\vec{k}^{\dagger}\right|} & \frac{k_{1}^{\dagger} \sin \pi t\left|\vec{k}^{\dagger}\right|}{\left|\vec{k}^{\dagger}\right|} & 0
\end{array}\right) .
$$

Thus for $\vec{k} \in \mathbb{Z}^{3}$ and $\lambda_{r} \in \mathbb{R}$ with $r \in \overline{1,6}$, the vector-valued function

$$
\begin{aligned}
& \left(\begin{array}{cc}
\cos t \mathbb{D} & \sin t \mathbb{D} \\
-\sin t \mathbb{D} & \cos t \mathbb{D}
\end{array}\right)\left(\begin{array}{c}
\lambda_{1} e^{\pi\left(\vec{k}^{\dagger} \cdot \vec{x}\right) i} \\
\vdots \\
\lambda_{6} e^{\pi\left(\vec{k}^{\dagger} \cdot \vec{x}\right) i}
\end{array}\right) \\
= & \left(\begin{array}{cc}
\mathbb{K}(\vec{k}, t) & i \mathbb{M}(\vec{k}, t) \\
-i \mathbb{M}(\vec{k}, t) & \mathbb{K}(\vec{k}, t)
\end{array}\right)\left(\begin{array}{c}
\lambda_{1} e^{\pi\left(\vec{k}^{\dagger} \cdot \vec{x}\right) i} \\
\vdots \\
\lambda_{6} e^{\pi\left(\vec{k}^{\dagger} \cdot \vec{x}\right) i}
\end{array}\right)
\end{aligned}
$$


is a complex solution of the equation (4.7.8). Considering the real and imaginary parts of (4.7.35), we get two real solutions of the Maxwell equation (4.7.1):

$$
\begin{gathered}
\mathbf{E}=\mathbb{K}(\vec{k}, t)\left(\begin{array}{c}
\lambda_{1} \cos \pi\left(\vec{k}^{\dagger} \cdot \vec{x}\right) \\
\lambda_{2} \cos \pi\left(\vec{k}^{\dagger} \cdot \vec{x}\right) \\
\lambda_{2} \cos \pi\left(\vec{k}^{\dagger} \cdot \vec{x}\right)
\end{array}\right)-\mathbb{M}(\vec{k}, t)\left(\begin{array}{c}
\lambda_{4} \sin \pi\left(\vec{k}^{\dagger} \cdot \vec{x}\right) \\
\lambda_{5} \sin \pi\left(\vec{k}^{\dagger} \cdot \vec{x}\right) \\
\lambda_{6} \sin \pi\left(\vec{k}^{\dagger} \cdot \vec{x}\right)
\end{array}\right), \\
\mathbf{B}=\mathbb{M}(\vec{k}, t)\left(\begin{array}{c}
\lambda_{1} \sin \pi\left(\vec{k}^{\dagger} \cdot \vec{x}\right) \\
\lambda_{2} \sin \pi\left(\vec{k}^{\dagger} \cdot \vec{x}\right) \\
\lambda_{3} \sin \pi\left(\vec{k}^{\dagger} \cdot \vec{x}\right)
\end{array}\right)+\mathbb{K}(\vec{k}, t)\left(\begin{array}{c}
\lambda_{4} \cos \pi\left(\vec{k}^{\dagger} \cdot \vec{x}\right) \\
\lambda_{5} \cos \pi\left(\vec{k}^{\dagger} \cdot \vec{x}\right) \\
\lambda_{6} \cos \pi\left(\vec{k}^{\dagger} \cdot \vec{x}\right)
\end{array}\right)
\end{gathered}
$$

and

$$
\begin{gathered}
\mathbf{E}=\mathbb{K}(\vec{k}, t)\left(\begin{array}{l}
\mu_{1} \sin \pi\left(\vec{k}^{\dagger} \cdot \vec{x}\right) \\
\mu_{2} \sin \pi\left(\vec{k}^{\dagger} \cdot \vec{x}\right) \\
\mu_{3} \sin \pi\left(\vec{k}^{\dagger} \cdot \vec{x}\right)
\end{array}\right)+\mathbb{M}(\vec{k}, t)\left(\begin{array}{c}
\mu_{4} \cos \pi\left(\vec{k}^{\dagger} \cdot \vec{x}\right) \\
\mu_{5} \cos \pi\left(\vec{k}^{\dagger} \cdot \vec{x}\right) \\
\mu_{6} \cos \pi\left(\vec{k}^{\dagger} \cdot \vec{x}\right)
\end{array}\right), \\
\mathbf{B}=-\mathbb{M}(\vec{k}, t)\left(\begin{array}{c}
\mu_{1} \cos \pi\left(\vec{k}^{\dagger} \cdot \vec{x}\right) \\
\mu_{2} \cos \pi\left(\vec{k}^{\dagger} \cdot \vec{x}\right) \\
\mu_{3} \cos \pi\left(\vec{k}^{\dagger} \cdot \vec{x}\right)
\end{array}\right)+\mathbb{K}(\vec{k}, t)\left(\begin{array}{c}
\mu_{4} \sin \pi\left(\vec{k}^{\dagger} \cdot \vec{x}\right) \\
\mu_{5} \sin \pi\left(\vec{k}^{\dagger} \cdot \vec{x}\right) \\
\mu_{6} \sin \pi\left(\vec{k}^{\dagger} \cdot \vec{x}\right)
\end{array}\right),
\end{gathered}
$$

where $\lambda_{r}, \mu_{r} \in \mathbb{R}$ for $r \in \overline{1,6}$.

Write

$$
\lambda_{r}=b_{r}(\vec{k}), \quad \mu_{r}=c_{r}(\vec{k}) \quad \text { for } r \in \overline{1,6} .
$$

By superposition principle,

$$
\begin{aligned}
\mathbf{E}=\left(\begin{array}{c}
E_{1} \\
E_{2} \\
E_{3}
\end{array}\right)= & \sum_{0 \preceq \vec{k} \in \mathbb{Z}^{3}}\left[\mathbb{K}(\vec{k}, t)\left(\begin{array}{l}
b_{1}(\vec{k}) \cos \pi\left(\vec{k}^{\dagger} \cdot \vec{x}\right)+c_{1}(\vec{k}) \sin \pi\left(\vec{k}^{\dagger} \cdot \vec{x}\right) \\
b_{2}(\vec{k}) \cos \pi\left(\vec{k}^{\dagger} \cdot \vec{x}\right)+c_{2}(\vec{k}) \sin \pi\left(\vec{k}^{\dagger} \cdot \vec{x}\right) \\
b_{3}(\vec{k}) \cos \pi\left(\vec{k}^{\dagger} \cdot \vec{x}\right)+c_{3}(\vec{k}) \sin \pi\left(\vec{k}^{\dagger} \cdot \vec{x}\right)
\end{array}\right)\right. \\
& \left.+\mathbb{M}(\vec{k}, t)\left(\begin{array}{c}
c_{4}(\vec{k}) \cos \pi\left(\vec{k}^{\dagger} \cdot \vec{x}\right)-b_{4}(\vec{k}) \sin \pi\left(\vec{k}^{\dagger} \cdot \vec{x}\right) \\
c_{5}(\vec{k}) \cos \pi\left(\vec{k}^{\dagger} \cdot \vec{x}\right)-b_{5}(\vec{k}) \sin \pi\left(\vec{k}^{\dagger} \cdot \vec{x}\right) \\
c_{6}(\vec{k}) \cos \pi\left(\vec{k}^{\dagger} \cdot \vec{x}\right)-b_{6}(\vec{k}) \sin \pi\left(\vec{k}^{\dagger} \cdot \vec{x}\right)
\end{array}\right)\right]
\end{aligned}
$$

and

$$
\begin{aligned}
\mathbf{B}=\left(\begin{array}{c}
B_{1} \\
B_{2} \\
B_{3}
\end{array}\right)= & \sum_{0 \leq \vec{k} \in \mathbb{Z}^{3}}\left[\mathbb{M}(\vec{k}, t)\left(\begin{array}{c}
b_{1}(\vec{k}) \sin \pi\left(\vec{k}^{\dagger} \cdot \vec{x}\right)-c_{1}(\vec{k}) \cos \pi\left(\vec{k}^{\dagger} \cdot \vec{x}\right) \\
b_{2}(\vec{k}) \sin \pi\left(\vec{k}^{\dagger} \cdot \vec{x}\right)-c_{2}(\vec{k}) \cos \pi\left(\vec{k}^{\dagger} \cdot \vec{x}\right) \\
b_{3}(\vec{k}) \sin \pi\left(\vec{k}^{\dagger} \cdot \vec{x}\right)-c_{3}(\vec{k}) \cos \pi\left(\vec{k}^{\dagger} \cdot \vec{x}\right)
\end{array}\right)\right. \\
& \left.+\mathbb{K}(\vec{k}, t)\left(\begin{array}{c}
b_{4}(\vec{k}) \cos \pi\left(\vec{k}^{\dagger} \cdot \vec{x}\right)+c_{4}(\vec{k}) \sin \pi\left(\vec{k}^{\dagger} \cdot \vec{x}\right) \\
b_{5}(\vec{k}) \cos \pi\left(\vec{k}^{\dagger} \cdot \vec{x}\right)+c_{5}(\vec{k}) \sin \pi\left(\vec{k}^{\dagger} \cdot \vec{x}\right) \\
b_{6}(\vec{k}) \cos \pi\left(\vec{k}^{\dagger} \cdot \vec{x}\right)+c_{6}(\vec{k}) \sin \pi\left(\vec{k}^{\dagger} \cdot \vec{x}\right)
\end{array}\right)\right]
\end{aligned}
$$

is a general solution of the equations in (4.7.1), where $\mathbb{K}(\vec{k}, t)$ is given in (4.7.32) and $\mathbb{M}(\vec{k}, t)$ is given in (4.7.34). 
Note $\mathbb{K}(\vec{k}, 0)=I_{3}$ and $\mathbb{M}(\vec{k}, 0)=0_{3 \times 3}$. So

$$
\mathbf{E}\left(0, x_{1}, x_{2}, x_{3}\right)=\sum_{0 \preceq \vec{k} \in \mathbb{Z}^{3}}\left(\begin{array}{l}
b_{1}(\vec{k}) \cos \pi\left(\vec{k}^{\dagger} \cdot \vec{x}\right)+c_{1}(\vec{k}) \sin \pi\left(\vec{k}^{\dagger} \cdot \vec{x}\right) \\
b_{2}(\vec{k}) \cos \pi\left(\vec{k}^{\dagger} \cdot \vec{x}\right)+c_{2}(\vec{k}) \sin \pi\left(\vec{k}^{\dagger} \cdot \vec{x}\right) \\
b_{3}(\vec{k}) \cos \pi\left(\vec{k}^{\dagger} \cdot \vec{x}\right)+c_{3}(\vec{k}) \sin \pi\left(\vec{k}^{\dagger} \cdot \vec{x}\right)
\end{array}\right)
$$

and

$$
\mathbf{B}\left(0, x_{1}, x_{2}, x_{3}\right)=\sum_{0 \preceq \vec{k} \in \mathbb{Z}^{3}}\left(\begin{array}{l}
b_{4}(\vec{k}) \cos \pi\left(\vec{k}^{\dagger} \cdot \vec{x}\right)+c_{4}(\vec{k}) \sin \pi\left(\vec{k}^{\dagger} \cdot \vec{x}\right) \\
b_{5}(\vec{k}) \cos \pi\left(\vec{k}^{\dagger} \cdot \vec{x}\right)+c_{5}(\vec{k}) \sin \pi\left(\vec{k}^{\dagger} \cdot \vec{x}\right) \\
b_{6}(\vec{k}) \cos \pi\left(\vec{k}^{\dagger} \cdot \vec{x}\right)+c_{6}(\vec{k}) \sin \pi\left(\vec{k}^{\dagger} \cdot \vec{x}\right)
\end{array}\right)
$$

Write

$$
\mathbf{E}_{0}=\left(\begin{array}{c}
h_{1}(x, y, z) \\
h_{2}(x, y, z) \\
h_{3}(x, y, z)
\end{array}\right), \quad \mathbf{B}_{0}=\left(\begin{array}{c}
h_{4}(x, y, z) \\
h_{5}(x, y, z) \\
h_{6}(x, y, z)
\end{array}\right)
$$

which must be of the form (4.7.23) and (4.7.24). By Fourier expansion and the Kovalevskaya Theorem on the existence and uniqueness of the solution of linear partial differential equations, we have:

Theorem 4.7.1. The solution of the initial value problem of the Maxwell equations (4.7.1)-(4.7.3) is given in (4.7.41) and (4.7.42) with

$$
\begin{array}{r}
b_{r}(\vec{k})=\frac{1}{2^{2+\delta_{\vec{k}, \overrightarrow{0}} a_{1} a_{2} a_{3}}} \int_{-a_{1}}^{a_{1}} \int_{-a_{2}}^{a_{2}} \int_{-a_{3}}^{a_{3}} h_{r}(\vec{x}) \cos \pi\left(\vec{k}^{\dagger} \cdot \vec{x}\right) d z d y d x \\
c_{r}(\vec{k})=\frac{1}{4 a_{1} a_{2} a_{3}} \int_{-a_{1}}^{a_{1}} \int_{-a_{2}}^{a_{2}} \int_{-a_{3}}^{a_{3}} h_{r}(\vec{x}) \sin \pi\left(\vec{k}^{\dagger} \cdot \vec{x}\right) d z d y d x .
\end{array}
$$

The above result is due to our work [X10]. Ciattonic, Crosignanic, Di Porto and Yariv [CCDY] found the spatial Kerr solutions as exact solutions of Maxwell equations. Fushchich and Revenko [FR] obtained some exact solutions of the Lorentz-Maxwell equations.

\subsection{Dirac Equation and Acoustic System}

The classical free Dirac equation is:

$$
\left[\sum_{r=0}^{3}\left[\gamma^{r} P_{r}-m\right] \psi=0\right.
$$

with

$$
P_{0}=i \partial_{t}, \quad P_{1}=i \partial_{x}, \quad P_{2}=i \partial_{y}, \quad P_{3}=i \partial_{z}
$$


and the Dirac matrices:

$$
\gamma^{0}=\left(\begin{array}{rr}
I_{2} & 0 \\
0 & -I_{2}
\end{array}\right), \quad \gamma^{r}=\left(\begin{array}{rr}
0 & \sigma_{r} \\
-\sigma_{r} & 0
\end{array}\right), \quad r=1,2,3,
$$

where $m$ is a positive real constant, $I_{2}$ is the $2 \times 2$ identity matrix and

$$
\sigma_{1}=\left(\begin{array}{ll}
0 & 1 \\
1 & 0
\end{array}\right), \quad \sigma_{2}=\left(\begin{array}{rr}
0 & -i \\
i & 0
\end{array}\right), \quad \sigma_{3}=\left(\begin{array}{rr}
1 & 0 \\
0 & -1
\end{array}\right)
$$

are the Pauli matrices. We want to solve the free Dirac equation (4.8.1) subject to the initial condition:

$$
\psi(0, x, y, z)=\psi_{0}(x, y, z) \quad \text { for } x \in\left[-a_{1}, a_{1}\right], y \in\left[-a_{2}, a_{2}\right], z \in\left[-a_{3}, a_{3}\right],
$$

where $\psi_{0}(x, y, z)$ is a given continuous complex vector-valued function.

The Dirac matrices:

$$
\begin{array}{rlr}
\gamma^{0}=\left(\begin{array}{rrrr}
1 & 0 & 0 & 0 \\
0 & 1 & 0 & 0 \\
0 & 0 & -1 & 0 \\
0 & 0 & 0 & -1
\end{array}\right), & \gamma^{1}=\left(\begin{array}{rrrr}
0 & 0 & 0 & 1 \\
0 & 0 & 1 & 0 \\
0 & -1 & 0 & 0 \\
-1 & 0 & 0 & 0
\end{array}\right), \\
\gamma^{2}=\left(\begin{array}{rrrr}
0 & 0 & 0 & -i \\
0 & 0 & i & 0 \\
0 & i & 0 & 0 \\
-i & 0 & 0 & 0
\end{array}\right), & \gamma^{3}=\left(\begin{array}{rrrr}
0 & 0 & 1 & 0 \\
0 & 0 & 0 & -1 \\
-1 & 0 & 0 & 0 \\
0 & 1 & 0 & 0
\end{array}\right) .
\end{array}
$$

Now free Dirac equation is equivalent to: $\partial_{t}(\psi)=\mathbb{D} \psi$ with

$$
\mathbb{D}=\left(\begin{array}{cccc}
m i & 0 & -\partial_{z} & -\partial_{x}+i \partial_{y} \\
0 & m i & -\partial_{x}-i \partial_{y} & \partial_{z} \\
-\partial_{z} & -\partial_{x}+i \partial_{y} & -m i & 0 \\
-\partial_{x}-i \partial_{y} & \partial_{z} & 0 & -m i
\end{array}\right) .
$$

Observe

$$
\mathbb{D}^{2}=\left(\partial_{x}^{2}+\partial_{y}^{2}+\partial_{z}^{2}-m^{2}\right) I_{4},
$$

where $I_{4}$ is the $4 \times 4$ identity matrix. Thus

$$
e^{t \mathbb{D}}=\left(\sum_{s=0}^{\infty} \frac{\left(\partial_{x}^{2}+\partial_{y}^{2}+\partial_{z}^{2}-m^{2}\right)^{s} t^{2 s}}{(2 s) !}\right) I_{4}+\left(\sum_{s=0}^{\infty} \frac{\left(\partial_{x}^{2}+\partial_{y}^{2}+\partial_{z}^{2}-m^{2}\right)^{s} t^{2 s+1}}{(2 s+1) !}\right) \mathbb{D} .
$$

We take the settings (4.7.25)-(4.7.30). Set

$$
\widehat{\mathbb{D}}(\vec{k})=\left(\begin{array}{cccc}
m & 0 & \left.\vec{k}^{\dagger}\right\rangle=\sqrt{\left|\vec{k}^{\dagger}\right|^{2}-m^{2}}, \\
0 & m & k_{1}^{\dagger} i-k_{2}^{\dagger} & k_{1}^{\dagger} i+k_{2}^{\dagger} \\
k_{3}^{\dagger} i & k_{1}^{\dagger} i+k_{2}^{\dagger} i & -m & 0 \\
k_{1}^{\dagger} i-k_{2}^{\dagger} & -k_{3}^{\dagger} i & 0 & -m
\end{array}\right) .
$$


Then

$$
\begin{gathered}
e^{t \mathbb{D}}\left(\begin{array}{c}
a_{1}(\vec{k}) e^{\pi\left(\vec{k}^{\dagger} \cdot \vec{x}\right) i} \\
a_{2}(\vec{k}) e^{\pi\left(\vec{k}^{\dagger} \cdot \vec{x}\right) i} \\
a_{3}(\vec{k}) e^{\pi\left(\vec{k}^{\dagger} \cdot \vec{x}\right) i} \\
a_{4}(\vec{k}) e^{\pi\left(\vec{k}^{\dagger} \cdot \vec{x}\right) i}
\end{array}\right)=\left[\left(\sum_{s=0}^{\infty} \frac{\left(\partial_{x}^{2}+\partial_{y}^{2}+\partial_{z}^{2}-m^{2}\right)^{s} t^{2 s}}{(2 s) !}\right) I_{4}\right. \\
+\left(\sum_{s=0}^{\infty} \frac{\left(\partial_{x}^{2}+\partial_{y}^{2}+\partial_{z}^{2}-m^{2}\right)^{s} t^{2 s+1}}{(2 s+1) !}\right)\left(\begin{array}{c}
a_{1}(\vec{k}) e^{\pi\left(\vec{k}^{\dagger} \cdot \vec{x}\right) i} \\
\mathbb{D}]\left(\begin{array}{c}
a_{2}(\vec{k}) e^{\pi\left(\vec{k}^{\dagger} \cdot \vec{x}\right) i} \\
a_{3}(\vec{k}) e^{\pi\left(\vec{k}^{\dagger} \cdot \vec{x}\right) i} \\
a_{4}(\vec{k}) e^{\pi\left(\vec{k}^{\dagger} \cdot \vec{x}\right) i}
\end{array}\right) \\
=\left[\cos \pi\left\langle\vec{k}^{\dagger}\right\rangle t I_{4}-\frac{\sin \pi\left\langle\vec{k}^{\dagger}\right\rangle t}{\left\langle\vec{k}^{\dagger}\right\rangle} \widehat{\mathbb{D}}(\vec{k})\right.
\end{array}\left(\begin{array}{c}
a_{1}(\vec{k}) e^{\pi\left(\vec{k}^{\dagger} \cdot \vec{x}\right) i} \\
a_{2}(\vec{k}) e^{\pi\left(\vec{k}^{\dagger} \cdot \vec{x}\right) i} \\
a_{3}(\vec{k}) e^{\pi\left(\vec{k}^{\dagger} \cdot \vec{x}\right) i} \\
a_{4}(\vec{k}) e^{\pi\left(\vec{k}^{\dagger} \cdot \vec{x}\right) i}
\end{array}\right)\right.
\end{gathered}
$$

is a solution of the Dirac equation (4.8.1), where $a_{r}(\vec{k})$ with $r \in \overline{1,4}$ are complex constants.

We write

$$
\psi_{0}(x, y, z)=\left(\begin{array}{c}
f_{1}(x, y, z) \\
f_{2}(x, y, z) \\
f_{3}(x, y, z) \\
f_{4}(x, y, z)
\end{array}\right)
$$

and take

$$
a_{r}(\vec{k})=\frac{1}{8 a_{1} a_{2} a_{3}} \int_{-a_{1}}^{a_{1}} \int_{-a_{2}}^{a_{2}} \int_{-a_{3}}^{a_{3}} f_{r}(x, y, z) e^{-\pi\left(\vec{k}^{\dagger} \cdot \vec{x}\right) i} d z d y d x
$$

for $r \in \overline{1,3}$ and $\vec{k} \in \mathbb{Z}^{3}$. By the theory of Fourier expansion,

$$
f_{r}(x, y, z)=\sum_{\vec{k} \in \mathbb{Z}^{3}} a_{r}(\vec{k}) e^{\pi\left(\vec{k}^{\dagger} \cdot \vec{x}\right) i} \text { for } r \in \overline{1,4} .
$$

According to superposition principle and the Kovalevskaya Theorem on the existence and uniqueness of the solution of linear partial differential equations, we obtain:

Theorem 4.8.1. The solution of the initial value problem of the free Dirac equation is:

$$
\psi=\sum_{\vec{k} \in \mathbb{Z}^{3}}\left[\cos \pi\left\langle\vec{k}^{\dagger}\right\rangle t I_{4}-\frac{\sin \pi\left\langle\vec{k}^{\dagger}\right\rangle t}{\left\langle\vec{k}^{\dagger}\right\rangle} \widehat{\mathbb{D}}(\vec{k})\right]\left(\begin{array}{c}
a_{1}(\vec{k}) e^{\pi\left(\vec{k}^{\dagger} \cdot \vec{x}\right) i} \\
a_{2}(\vec{k}) e^{\pi\left(\vec{k}^{\dagger} \cdot \vec{x}\right) i} \\
a_{3}(\vec{k}) e^{\pi\left(\vec{k}^{\dagger} \cdot \vec{x}\right) i} \\
a_{4}(\vec{k}) e^{\pi\left(\vec{k}^{\dagger} \cdot \vec{x}\right) i}
\end{array}\right)
$$

The above result is taken from the author's work [X10]. Ibragimov [In1] studied the invariance of Dirac equations. Fushchich, Shtelen and Spichak [FSS] found a connection between solutions of Dirac and Maxwell equations. Moreover, Hounkonnou and Mendy [HM] obtained some exact solutions of Dirac equation for neutrinos in presence of external 
fields. Furthermore, Inoue [Ia] constructed the fundamental solution for the free Dirac equation by Hamiltonian path-integral method. In addition, Moayedi and Darabi derived the exact solutions of Dirac equation on 2D gravitational background.

The $n$-dimensional generalized acoustic system

$$
\lambda_{t}+\sum_{r=1}^{n} u_{r x_{r}}=0, \quad u_{p t}+\lambda_{x_{p}}=0, \quad p \in \overline{1, n},
$$

comes from the linear approximation of the compressible Euler equations in fluid dynamics. Denote

$$
\vec{u}\left(t, x_{1}, \ldots, x_{n}\right)=\left(\begin{array}{c}
\lambda\left(t, x_{1}, \ldots, x_{n}\right) \\
u_{1}\left(t, x_{1}, \ldots, x_{n}\right) \\
\vdots \\
u_{n}\left(t, x_{1}, \ldots, x_{n}\right)
\end{array}\right), \quad \nabla=\left(\begin{array}{c}
\partial_{x_{1}} \\
\partial_{x_{2}} \\
\vdots \\
\partial_{x_{n}}
\end{array}\right)
$$

Set

$$
\mathbb{A}=\left(\begin{array}{cc}
0 & \nabla^{T} \\
\nabla & 0_{n \times n}
\end{array}\right),
$$

where the up-index " $T$ " denotes the transpose of matrix and $0_{n \times n}$ denotes the $n \times n$ matrix whose all entries are 0 . The system (4.8.18) can be rewritten as

$$
\vec{u}_{t}+\mathbb{A} \vec{u}=0 .
$$

We want to solve (4.8.21) for $t \in \mathbb{R}$ and $x_{r} \in\left[-a_{r}, a_{r}\right]$ with $r \in \overline{1, n}$ subject to

$$
\vec{u}\left(0, x_{1}, \ldots, x_{n}\right)=\left(\begin{array}{c}
\lambda\left(0, x_{1}, \ldots, x_{n}\right) \\
u_{1}\left(0, x_{1}, \ldots, x_{n}\right) \\
\vdots \\
u_{n}\left(0, x_{1}, \ldots, x_{n}\right)
\end{array}\right)=\left(\begin{array}{c}
f_{0}\left(x_{1}, \ldots, x_{n}\right) \\
f_{1}\left(x_{1}, \ldots, x_{n}\right) \\
\vdots \\
f_{n}\left(x_{1}, \ldots, x_{n}\right)
\end{array}\right) .
$$

Recall the Laplace operator

$$
\Delta=\partial_{x_{1}}^{2}+\partial_{x_{2}}^{2}+\cdots+\partial_{x_{n}}^{2}=\nabla^{T} \nabla
$$

We calculate

$$
\mathbb{A}^{2 m+2}=\left(\begin{array}{cc}
\Delta^{m+1} & 0 \\
0 & \Delta^{m} \nabla \nabla^{T}
\end{array}\right), \quad \mathbb{A}^{2 m+1}=\left(\begin{array}{cc}
0 & \Delta^{m} \nabla^{T} \\
\Delta^{m} \nabla & 0_{n \times n}
\end{array}\right) .
$$

Thus

$$
\begin{aligned}
e^{-t \mathbb{A}}= & I_{n+1}+\left(\sum_{m=0}^{\infty} \frac{t^{2 m+2} \Delta^{m}}{(2 m+2) !}\right)\left(\begin{array}{cc}
\Delta & 0 \\
0 & \nabla \nabla^{T}
\end{array}\right) \\
& -\left(\sum_{m=0}^{\infty} \frac{t^{2 m+1} \Delta^{m}}{(2 m+1) !}\right)\left(\begin{array}{cc}
0 & \nabla^{T} \\
\nabla & 0_{n \times n}
\end{array}\right),
\end{aligned}
$$


where $I_{n+1}$ is the $(n+1) \times(n+1)$ identity matrix.

For convenience, we again denote

$$
k_{r}^{\dagger}=\frac{k_{r}}{a_{r}}, \quad \vec{k}^{\dagger}=\left(k_{1}^{\dagger}, \ldots, k_{n}^{\dagger}\right), \quad\left|\vec{k}^{\dagger}\right|=\sqrt{\left(k_{1}^{\dagger}\right)^{2}+\cdots+\left(k_{n}^{\dagger}\right)^{2}}, \quad \vec{k}^{\dagger} \cdot \vec{x}=\sum_{r=1}^{n} k_{r}^{\dagger} x_{r}
$$

for $\vec{k}=\left(k_{1}, \ldots, k_{n}\right) \in \mathbb{Z}^{n}$. Recall the equations in (4.7.25) and take the convention (4.7.30). Let $\mu_{r} \in \mathbb{R}$ with $r \in \overline{0, n}$. Then

$$
\begin{gathered}
e^{-t \mathbb{A}}\left(\begin{array}{c}
\mu_{0} e^{\pi\left(\vec{k}^{\dagger} \cdot \vec{x}\right) i} \\
\mu_{1} e^{\pi\left(\vec{k}^{\dagger} \cdot \vec{x}\right) i} \\
\vdots \\
\mu_{n} e^{\pi\left(\vec{k}^{\dagger} \cdot \vec{x}\right) i}
\end{array}\right)=\left[I_{n+1}+\left(\sum_{m=0}^{\infty} \frac{t^{2 m+2} \Delta^{m}}{(2 m+2) !}\right)\left(\begin{array}{cc}
\Delta & 0 \\
0 & \nabla \nabla^{T}
\end{array}\right)\right. \\
\left.-\left(\sum_{m=0}^{\infty} \frac{t^{2 m+1} \Delta^{m}}{(2 m+1) !}\right)\left(\begin{array}{cc}
0 & \nabla^{T} \\
\nabla & 0_{n \times n}
\end{array}\right)\right]\left(\begin{array}{c}
\mu_{0} e^{\pi\left(\vec{k}^{\dagger} \cdot \vec{x}\right) i} \\
\mu_{1} e^{\pi\left(\vec{k}^{\dagger} \cdot \vec{x}\right) i} \\
\vdots \\
\mu_{n} e^{\pi\left(\vec{k}^{\dagger} \cdot \vec{x}\right) i}
\end{array}\right) \\
=[\mathbb{K}(\vec{k}, t)-i \mathbb{M}(\vec{k}, t)]\left(\begin{array}{c}
\mu_{0} e^{\pi\left(\vec{k}^{\dagger} \cdot \vec{x}\right) i} \\
\mu_{1} e^{\pi\left(\vec{k}^{\dagger} \cdot \vec{x}\right) i} \\
\vdots \\
\mu_{n} e^{\pi\left(\vec{k}^{\dagger} \cdot \vec{x}\right) i}
\end{array}\right)
\end{gathered}
$$

is a complex solution of the equation (4.8.21), where

$$
\mathbb{K}(\vec{k}, t)=\left(\begin{array}{cc}
\cos \pi\left|\vec{k}^{\dagger}\right| t & 0 \\
0 & I_{n}+\left|\vec{k}^{\dagger}\right|^{-2}\left(\cos \pi\left|\vec{k}^{\dagger}\right| t-1\right)\left(\vec{k}^{\dagger}\right)^{T} \vec{k}^{\dagger}
\end{array}\right)
$$

and

$$
\mathbb{M}(\vec{k}, t)=\left|\vec{k}^{\dagger}\right|^{-1} \sin \pi\left|\vec{k}^{\dagger}\right| t\left(\begin{array}{cc}
0 & \vec{k}^{\dagger} \\
\left(\vec{k}^{\dagger}\right)^{T} & 0_{n \times n}
\end{array}\right) .
$$

Considering the real and imaginary parts of (4.8.27), we get two real solutions of the equation (4.8.21):

$$
\vec{u}=\mathbb{K}(\vec{k}, t)\left(\begin{array}{c}
b_{0}(\vec{k}) \cos \pi\left(\vec{k}^{\dagger} \cdot \vec{x}\right) \\
b_{1}(\vec{k}) \cos \pi\left(\vec{k}^{\dagger} \cdot \vec{x}\right) \\
\vdots \\
b_{n}(\vec{k}) \cos \pi\left(\vec{k}^{\dagger} \cdot \vec{x}\right)
\end{array}\right)+\mathbb{M}(\vec{k}, t)\left(\begin{array}{c}
b_{0}(\vec{k}) \sin \pi\left(\vec{k}^{\dagger} \cdot \vec{x}\right) \\
b_{1}(\vec{k}) \sin \pi\left(\vec{k}^{\dagger} \cdot \vec{x}\right) \\
\vdots \\
b_{n}(\vec{k}) \sin \pi\left(\vec{k}^{\dagger} \cdot \vec{x}\right)
\end{array}\right)
$$

and

$$
\vec{u}=\mathbb{K}(\vec{k}, t)\left(\begin{array}{c}
c_{0}(\vec{k}) \sin \pi\left(\vec{k}^{\dagger} \cdot \vec{x}\right) \\
c_{1}(\vec{k}) \sin \pi\left(\vec{k}^{\dagger} \cdot \vec{x}\right) \\
\vdots \\
c_{n}(\vec{k}) \sin \pi\left(\vec{k}^{\dagger} \cdot \vec{x}\right)
\end{array}\right)-\mathbb{M}(\vec{k}, t)\left(\begin{array}{c}
b_{0}(\vec{k}) \cos \pi\left(\vec{k}^{\dagger} \cdot \vec{x}\right) \\
b_{1}(\vec{k}) \cos \pi\left(\vec{k}^{\dagger} \cdot \vec{x}\right) \\
\vdots \\
b_{n}(\vec{k}) \cos \pi\left(\vec{k}^{\dagger} \cdot \vec{x}\right)
\end{array}\right)
$$


We take

$$
\begin{gathered}
b_{r}(\vec{k})=\frac{1}{2^{n-1+\delta_{\vec{k}, 0}} \prod_{r=1}^{n} a_{r}} \int_{-a_{1}}^{a_{1}} \int_{-a_{2}}^{a_{2}} \cdots \int_{-a_{n}}^{a_{n}} f_{r}(\vec{x}) \cos \pi\left(\vec{k}^{\dagger} \cdot \vec{x}\right) d x_{1} d x_{2} \cdots d x_{n}, \\
c_{r}(\vec{k})=\frac{1}{2^{n-1} \prod_{r=1}^{n} a_{r}} \int_{-a_{1}}^{a_{1}} \int_{-a_{2}}^{a_{2}} \cdots \int_{-a_{n}}^{a_{n}} f_{r}(\vec{x}) \sin \pi\left(\vec{k}^{\dagger} \cdot \vec{x}\right) d x_{1} d x_{2} \cdots d x_{n}
\end{gathered}
$$

(cf. (4.8.22)). Then we have the Fourier expansions:

$$
f_{r}\left(x_{1}, \ldots, x_{n}\right)=\sum_{0 \preceq \vec{k} \in \mathbb{Z}^{n}}\left(b_{r}(\vec{k}) \cos \pi\left(\vec{k}^{\dagger} \cdot \vec{x}\right)+c_{r}(\vec{k}) \sin \pi\left(\vec{k}^{\dagger} \cdot \vec{x}\right)\right) .
$$

Note $\mathbb{K}(\vec{k}, 0)=I_{(n+1) \times(n+1)}$ and $\mathbb{M}(\vec{k}, 0)=0_{(n+1) \times(n+1)}$. According to superposition principle and the Kovalevskaya Theorem on the existence and uniqueness of the solution of linear partial differential equations, we obtain:

Theorem 4.8.2. The solution of the $n$-dimensional generalized acoustic system (4.8.18) subject to the initial condition (4.8.22) is

$$
\begin{aligned}
\left(\begin{array}{c}
\lambda \\
u_{1} \\
\vdots \\
u_{n}
\end{array}\right)=\sum_{0 \preceq \vec{k} \in \mathbb{Z}^{n}}\left[\mathbb{K}(\vec{k}, t)\left(\begin{array}{c}
b_{0}(\vec{k}) \cos \pi\left(\vec{k}^{\dagger} \cdot \vec{x}\right)+c_{0}(\vec{k}) \sin \pi\left(\vec{k}^{\dagger} \cdot \vec{x}\right) \\
b_{1}(\vec{k}) \cos \pi\left(\vec{k}^{\dagger} \cdot \vec{x}\right)+c_{1}(\vec{k}) \sin \pi\left(\vec{k}^{\dagger} \cdot \vec{x}\right) \\
\vdots \\
b_{n}(\vec{k}) \cos \pi\left(\vec{k}^{\dagger} \cdot \vec{x}\right)+c_{n}(\vec{k}) \sin \pi\left(\vec{k}^{\dagger} \cdot \vec{x}\right)
\end{array}\right)\right. \\
+\mathbb{M}(\vec{k}, t)\left(\begin{array}{c}
b_{0}(\vec{k}) \sin \pi\left(\vec{k}^{\dagger} \cdot \vec{x}\right)-c_{0}(\vec{k}) \cos \pi\left(\vec{k}^{\dagger} \cdot \vec{x}\right) \\
b_{1}(\vec{k}) \sin \pi\left(\vec{k}^{\dagger} \cdot \vec{x}\right)-c_{1}(\vec{k}) \cos \pi\left(\vec{k}^{\dagger} \cdot \vec{x}\right) \\
\vdots \\
b_{n}(\vec{k}) \sin \pi\left(\vec{k}^{\dagger} \cdot \vec{x}\right)-c_{n}(\vec{k}) \cos \pi\left(\vec{k}^{\dagger} \cdot \vec{x}\right)
\end{array}\right)
\end{aligned}
$$

with $\mathbb{K}(\vec{k}, t)$ given in (4.8.28) and $\mathbb{M}(\vec{k}, t)$ given (4.8.29).

The result in Theorem 4.8.2 was newly obtained. Cao [Cb1] determined all the polynomial solutions of the Navier equation in elasticity and their representation structure. Moreover, he solved the initial-value problem of the Navier equation and the related Lamé equation.

\section{Exercise 4.8}

Solve the Lamé Equations

$$
\vec{u}_{t t}=\left(\kappa \Delta+\nabla \cdot \nabla^{T}\right)(\vec{u})
$$

for $t \in \mathbb{R}$ and $x_{r} \in\left[a_{r},-a_{r}\right]$ with $r \in \overline{1, n}$ subject to

$$
\vec{u}\left(0, x_{1}, \ldots, x_{n}\right)=\vec{g}_{0}\left(x_{1}, \ldots, x_{n}\right), \quad \vec{u}_{t}\left(0, x_{1}, \ldots, x_{n}\right)=\vec{g}_{1}\left(x_{1}, \ldots, x_{n}\right),
$$

where $\kappa$ is a nonzero constant, $a_{r}$ are positive real numbers and $g_{1}, g_{2}$ are continuous functions (cf. [Cb1]). 


\section{Chapter 5}

\section{Nonlinear Scalar Equations}

This chapter deals with nonlinear scalar (one dependent variable) partial differential equations. First we do symmetry analysis on the KdV equation, and obtain the traveling-wave solutions of the KdV equation in terms of the functions $\wp(z), \tan ^{2} z, \operatorname{coth}^{2} z$ and $\operatorname{cn}^{2}(z \mid m)$, respectively. In particular, the soliton solution is obtained by taking $\lim _{m \rightarrow 1}$ of a special case of the last solution. Moreover, we derive the Hirota bilinear presentation of the KdV equation and use it to find the two-soliton solution.

The KP equation can be viewed as an extension of the KdV equation. Any solution of the $\mathrm{KdV}$ equation is obviously a solution of the KP equation. In this chapter, we have done the symmetry analysis on the KP equation and use the symmetry transformations to extend the solutions of the $\mathrm{KdV}$ equation that are independent of $y$ to a more sophisticated solution of the KP equation that depends on $y$. Moreover, we solve the KP equation for solutions that are polynomial in $x$, and obtain many solutions that can not be obtained from the solutions of the KdV equation. Furthermore, we find the Hirota bilinear presentation of the KP equation and obtain the "lump" solution. The above results are well-known (e.g., cf. [AC]) and we reformulate them here just for pedagogic purpose.

Lin, Reisner and Tsien [LRT] (1948) found the equation of transonic gas flows. We derive the symmetry transformations of the equation. Using the stable range of the nonlinear term and generalized power series method, we find a family of singular solutions with seven arbitrary parameter functions in $t$ and a family of analytic solutions with six arbitrary parameter functions in $t$. Khristianovich and Rizhov [KR] (1958) discovered the equations of short waves in connection with the nonlinear reflection of weak shock waves. Khokhlov and Zabolotskaya [KZ] (1969) found an equation for quasi-plane waves in nonlinear acoustics of bounded bundles. The solutions of the above equations similar to those of the LRT equation are derived. Kibel' [Kt] (1954) introduced an equation for geopotential forecast on a middle level. The symmetry transformations and two new families of exact solutions with multiple parameter functions of the equation are derived. 


\subsection{Kortweg and de Vries Equation}

Soliton phenomenon was first observed by J. Scott Russel in 1834 when he was riding on horseback beside the narrow Union Canal near Edinburgh, Scotland. He described his observations as follows:

"I was observing the motion of a boat which was rapidly drawn along a narrow channel by a pair of horse, when the boat suddenly stopped - not so the mass of water in the channel which it had put in motion; it accumulates round the prow of the vessel in a state of violent agitation, then suddenly leaving it behind, rolled forward with great velocity, assuming the form of a large solitary elevation, a rounded, smooth and well-defined heap of water, which continued its course along the channel apparently without change of form or diminution of speed. I followed it on horseback, and overtook it still rolling on at a rate of some eight or nine miles an hour, preserving its original figure some thirty feet long and a foot to a foot and a half in height. Its height gradually diminished, and after a chase of one or two miles I lost it in the windings of the channel. Such, in the month of August 1834, was my first chance interview with that rare and beautiful phenomenon which I called the Wave of Translation... ."

The phenomenon had been theoretically studied by Russel, Airy (1845), Stokes (1847), Boussinesq (1871, 1872) and Rayleigh (1876). Boussinesq's study lead him to discover the $(1+1)$-dimensional Boussinesq equation. There had been an intensive discussion and controversy on whether the inviscid equations of water wave would posses such solitary wave solutions. The problem was finally solved by Kortweg and de Vries (1895). They derived a nonlinear evolution equation governing long one-dimensional, small amplitude, surface gravity waves propagating in a shallow channel of water:

$$
\frac{\partial \eta}{\partial \tau}=\frac{3}{2} \sqrt{\frac{g}{h}} \frac{\partial}{\partial \xi}\left(\frac{1}{2} \eta^{2}+\frac{2}{3} \alpha \eta+\frac{1}{3} \sigma \frac{\partial^{2} \eta}{\partial \xi^{2}}\right), \quad \sigma=\frac{1}{3} h^{2}-\frac{T h}{\rho g},
$$

where $\eta$ is the surface elevation of the wave above the equilibrium level $h, \alpha$ is a small arbitrary constant related to the uniform motion of the liquid, $g$ is the gravitational constant, $T$ is the surface tension and $\rho$ is the density (the term "long" and "small" are meant in comparison to the depth of the channel). By the nondimensional transformation

$$
t=\frac{1}{2} \sqrt{\frac{g}{h \sigma}} \tau, \quad x=-\frac{\xi}{\sqrt{\sigma}}, \quad u=\frac{1}{2} \eta+\frac{1}{3} \alpha,
$$

the equation (5.1.1) becomes

$$
u_{t}+6 u u_{x}+u_{x x x}=0
$$

the standard modern KdV equation.

A transformation is called a symmetry of a partial differential equation if it maps the solution space of the equation to itself. Since the equation (5.1.3) does not contain 
variable coefficients, the translation

$$
T_{a_{1}, a_{2}}(u(t, x))=u\left(t+a_{1}, x+a_{2}\right)
$$

leave (5.1.3) invariant, that is, it changes (5.1.3) to

$$
u_{t}\left(t+a_{1}, x+a_{2}\right)+6 u\left(t+a_{1}, x+a_{2}\right) u_{x}\left(t+a_{1}, x+a_{2}\right)+u_{x x x}\left(t+a_{1}, x+a_{2}\right)=0,
$$

where $a_{1}, a_{2} \in \mathbb{R}$ and the subindices denote the partial derivatives with respect to the original independent variables. Thus it maps a solution of (5.1.3) to another solution of (5.1.3). Equivalently $T_{a_{1}, a_{2}}$ is a symmetry of the KdV equation. Next we want to find dilation (scaling) symmetry. We do the following degree analysis. Suppose that

$$
\operatorname{deg} t=\ell_{1}, \quad \operatorname{deg} x=\ell_{2}, \quad \operatorname{deg} u=\ell_{3} .
$$

We want to make all the terms in (5.1.3) having the same degree in order to find invariant scaling transformation. Note

$$
\operatorname{deg} u_{t}=\ell_{3}-\ell_{1}, \quad \operatorname{deg} u u_{x}=2 \ell_{3}-\ell_{2}, \quad \operatorname{deg} u_{x x x}=\ell_{3}-3 \ell_{2} .
$$

We impose

$$
\ell_{3}-\ell_{1}=2 \ell_{3}-\ell_{2}=\ell_{3}-3 \ell_{2}
$$

Thus

$$
\ell_{1}=3 \ell_{2}, \quad \ell_{3}=-2 \ell_{2} .
$$

Hence the scaling

$$
S_{b}(u(t, x))=b^{2} u\left(b^{3} t, b x\right)
$$

with $0 \neq b \in \mathbb{R}$ keeps (5.1.3) invariant, that is, it changes (5.1.3) to

$$
b^{5}\left[u_{t}\left(b^{3} t, b x\right)+6 u\left(b^{3} t, b x\right) u_{x}\left(b^{3} t, b x\right)+u_{x x x}\left(b^{3} t, b x\right)\right]=0,
$$

where the subindices again denote the partial derivatives with respect to the original independent variables; equivalently,

$$
u_{t}\left(b^{3} t, b x\right)+6 u\left(b^{3} t, b x\right) u_{x}\left(b^{3} t, b x\right)+u_{x x x}\left(b^{3} t, b x\right)=0 .
$$

Thus $S_{b}$ maps a solution of (5.1.3) to another solution of (5.1.3) because (5.1.12) implies (5.1.11). Observe that the transformation $u(t, x) \mapsto u(t, x+c t)$ with $c \in \mathbb{R}$ changes (5.1.3) to

$$
u_{t}(t, x+c t)+c u_{x}(t, x+c t)+6 u(t, x+c t) u_{x}(t, x+c t)+u_{x x x}(t, x+c t)=0,
$$


where the subindices once again denote the partial derivatives with respect to the original independent variables. On the other hand, the transformation $u(t, x) \mapsto u(t, x)-c / 6$ changes (5.1.3) to

$$
u_{t}(t, x)-c u_{x}(t, x)+6 u(t, x) u_{x}(t, x)+u_{x x x}(t, x)=0 .
$$

So (5.1.3) is invariant under the following Galilean boost

$$
G_{c}(u(t, x))=u(t, x+c t)-\frac{c}{6}
$$

with the independent variable $x$ replaced by $x+c t$ and the same meaning of the subindices.

A solution of (5.1.3) is called a traveling-wave solution if it is of the form $u=f(a t+b x)$ with $a, b \in \mathbb{R}$. To find such an interesting solution, we can assume that $u=\xi(x)$ is independent of $t$; otherwise, we replace $u$ by some $G_{c}(u)$ so that the " $t$ " disappears. Under this assumption, (5.1.3) becomes

$$
\xi^{\prime \prime \prime}+6 \xi \xi^{\prime}=0 \sim \xi^{\prime \prime}+3 \xi^{2}=k
$$

If we take $\operatorname{deg} x=1$, we have to take $\operatorname{deg} \xi=-2$ in order to make the two nonzero terms in the first equation in (5.1.16) to have the same degree. This shows that we can try the real function with a pole of order 2 when it is viewed as a complex function. Note $\left(x^{-2}\right)^{\prime \prime}=6 x^{-4}$. Assume $\xi=a x^{-2}$ is a solution of (5.1.16). Then

$$
6 a x^{-4}+2 a^{2} x^{-4}=k \Longrightarrow a=-2 .
$$

So $u=-2 x^{-2}$ is a solution of the KdV equation (5.1.3). Applying $T_{0, a}$ in (5.1.4) and $G_{c}$ in (5.1.15), we get a more general traveling-wave solution

$$
u=-\frac{2}{(x+c t+a)^{2}}-\frac{c}{6} .
$$

Recall the Weierstrass's elliptic function $\wp(z)$ defined in (3.4.9). Moreover, $\wp^{\prime \prime}(z)=$ $6 \wp^{2}(z)-g_{2} / 2$ with the $g_{2}$ given in (3.4.29). In (3.4.9), we take $\omega_{1} \in \mathbb{C}$ such that $\operatorname{Re} \omega_{1}, \operatorname{Im} \omega_{1} \neq 0$ and $\omega_{2}=\overline{\omega_{1}}$. Then $\wp(z)$ is real if $z \in \mathbb{R}$ and $g_{2}$ is a real number. Thus $\xi=-2 \wp(x)$ is a solution of (5.1.16). Applying the transformation in (5.1.4) and (5.1.15), we get the following traveling-wave solution of the $\mathrm{KdV}$ equation (5.1.3):

$$
u=-2 \wp(x+c t+a)-\frac{c}{6}, \quad a, b, c \in \mathbb{R}, b \neq 0 .
$$

Note that for $a \in \mathbb{R}$,

$$
\left(f^{2}(x)+a\right)^{\prime \prime}=\left(f^{2}(x)\right)^{\prime \prime}=2\left[f(x) f^{\prime \prime}(x)+\left(f^{\prime}(x)\right)^{2}\right] .
$$


By (3.5.17),

$$
\begin{aligned}
\tan x \tan ^{\prime \prime} x+\left(\tan ^{\prime} x\right)^{2} & =\tan x\left(2 \tan ^{3} x+2 \tan x\right)+\left(\tan ^{2} x+1\right)^{2} \\
& =3 \tan ^{4} x+4 \tan ^{2} x+1=3\left(\tan ^{2} x+2 / 3\right)^{2}-1 / 3 .
\end{aligned}
$$

Thus $\xi=-2\left(\tan ^{2} x+2 / 3\right)$ is a solution of (5.1.16). Applying the transformations in (5.1.4), (5.1.10) and (5.1.15), we find another traveling-wave solution of the KdV equation (5.1.3):

$$
u=-2 b^{2} \tan ^{2}\left(b x+c b^{3} t+a\right)-\frac{b^{2}(8+c)}{6}, \quad a, b, c \in \mathbb{R}, b \neq 0 .
$$

Taking $c=-8$, we get $u=-b^{2} \tan ^{2}\left(b x-8 b^{3} t+a\right)$. According to (3.5.19),

$\operatorname{coth} x \operatorname{coth}^{\prime \prime} x+\left(\operatorname{coth}^{\prime} x\right)^{2}=\operatorname{coth} x\left(2 \operatorname{coth}^{3} x-2 \operatorname{coth} x\right)+\left(1-\operatorname{coth}^{2} x\right)^{2}$

$$
=3\left(\operatorname{coth}^{2} x-2 / 3\right)^{2}-1 / 3 \text {. }
$$

So we have the following traveling-wave solution of the KdV equation (5.1.3):

$$
u=-2 b^{2} \operatorname{coth}^{2}\left(b x+c b^{3} t+a\right)+\frac{b^{2}(8-c)}{6}, \quad a, b, c \in \mathbb{R}, b \neq 0 .
$$

Taking $c=8$, we get $u=-2 b^{2} \operatorname{coth}^{2}\left(b x+8 b^{3} t+a\right)$.

Next (3.5.10), (3.5.13) and (3.5.14) imply

$$
\begin{aligned}
& \operatorname{sn}(x \mid m) \operatorname{sn}^{\prime \prime}(x \mid m)+\left(\operatorname{sn}^{\prime}(x \mid m)\right)^{2} \\
= & \operatorname{sn}(x \mid m)\left[2 m^{2} \operatorname{sn}^{3}(x \mid m)-\left(m^{2}+1\right) \operatorname{sn}(x \mid m)\right]+\operatorname{cn}^{2}(x \mid m) \mathrm{dn}^{2}(x \mid m) \\
= & 2 m^{2} \operatorname{sn}^{4}(x \mid m)-\left(m^{2}+1\right) \operatorname{sn}^{2}(x \mid m)+\left(1-\operatorname{sn}^{2}(x \mid m)\right)\left(1-m^{2} \operatorname{sn}^{2}(x \mid m)\right) \\
= & 3 m^{2} \operatorname{sn}^{4}(x \mid m)-2\left(m^{2}+1\right) \operatorname{sn}^{2}(x \mid m)+1 \\
= & 3 m^{2}\left(\operatorname{sn}^{2}(x \mid m)-\frac{m^{2}+1}{3 m^{2}}\right)^{2}+\frac{m^{2}-m^{4}-1}{3 m^{2}} .
\end{aligned}
$$

Thus

$$
\xi=-2 m^{2}\left(\operatorname{sn}^{2}(x \mid m)-\frac{2 m^{2}+2}{3 m^{2}}\right)=2 m^{2} \operatorname{cn}^{2}(x \mid m)+\frac{2-4 m^{2}}{3}
$$

is a solution of (5.1.16). Hence we have the following traveling-wave solution of the $\mathrm{KdV}$ equation (5.1.3):

$$
u=2 b^{2} m^{2} \operatorname{cn}^{2}\left(b x+c b^{3} t+a \mid m\right)+\frac{b^{2}\left(4-8 m^{2}-c\right)}{6}, \quad a, b, c \in \mathbb{R}, b \neq 0 .
$$

Taking $c=4-8 m^{2}$, we have $u=2 b^{2} m^{2} \operatorname{cn}^{2}\left(b x+\left(4-8 m^{2}\right) b^{3} t+a \mid m\right)$. Recall $\lim _{m \rightarrow 1} \operatorname{cn}(x \mid m)$ $=\operatorname{sech} x$. Therefore, we have the soliton solution

$$
u=2 b^{2} \operatorname{sech}^{2}\left(b x-4 b^{3} t+a\right),
$$


which describes the phenomenon observed by Russel in 1834 .

There is another obvious solution $u=x / 6 t$ of the $\mathrm{KdV}$ equation (5.1.3). Applying $T_{a_{1}, a_{2}}$ in (5.1.4), we get the following traveling-wave solution of the $\mathrm{KdV}$ equation (5.1.3):

$$
u=\frac{x-a_{2}}{6\left(t-a_{1}\right)}, \quad a_{1}, a_{2} \in \mathbb{R} .
$$

Next we look for the solution of the KdV equation (5.1.3) in the form

$$
u=\rho \partial_{x}^{2} \ln v(t, x)
$$

where $\rho$ is a nonzero constant to be determined when we try to simplify the resulted equation. Then (5.1.3) becomes

$$
\rho \partial_{x}^{2} \partial_{t} \ln v+3 \rho^{2} \partial_{x}\left(\partial_{x}^{2} \ln v\right)^{2}+\rho \partial_{x}^{5} \ln v=0
$$

equivalently,

$$
\partial_{x} \partial_{t} \ln v+3 \rho\left(\partial_{x}^{2} \ln v\right)^{2}+\partial_{x}^{4} \ln v=\nu(t)
$$

for some function $\nu$ in $t$. Note

$$
\begin{gathered}
\partial_{x} \partial_{t} \ln v=\frac{v v_{t x}-v_{t} v_{x}}{v^{2}}, \quad \partial_{x}^{2} \ln v=\frac{v v_{x x}-v_{x}^{2}}{v^{2}} \\
\partial_{x}^{3} \ln v=\frac{v^{2} v_{x x x}-3 v v_{x} v_{x x}+2 v_{x}^{3}}{v^{3}} \\
\partial_{x}^{4} \ln v=\frac{v^{3} v_{x x x x}-4 v^{2} v_{x} v_{x x x}-3 v^{2} v_{x x}^{2}+12 v v_{x}^{2} v_{x x}-6 v_{x}^{4}}{v^{4}} .
\end{gathered}
$$

Since

$$
\left(\partial_{x}^{2} \ln v\right)^{2}=\frac{v^{2} v_{x x}^{2}-2 v v_{x}^{2} v_{x x}+v_{x}^{4}}{v^{4}}
$$

we take $\rho=2$, and (5.1.32) becomes

$$
v v_{t x}-v_{t} v_{x}+v v_{x x x x}-4 v_{x} v_{x x x}+3 v_{x x}^{2}=\nu v^{2}
$$

We assume

$$
v=1+k_{1} e^{a_{1} t+b_{1} x}+k_{2} e^{a_{2} t+b_{2} x}+k_{3} e^{\left(a_{1}+a_{2}\right) t+\left(b_{1}+b_{2}\right) x}, \quad a_{1}, a_{2}, b_{1}, b_{2}, k_{1}, k_{2}, k_{3} \in \mathbb{R}
$$

Then

$$
\begin{gathered}
v_{t}=a_{1} k_{1} e^{a_{1} t+b_{1} x}+a_{2} k_{2} e^{a_{2} t+b_{2} x}+\left(a_{1}+a_{2}\right) k_{3} e^{\left(a_{1}+a_{2}\right) t+\left(b_{1}+b_{2}\right) x} \\
v_{t x}=a_{1} b_{1} k_{1} e^{a_{1} t+b_{1} x}+a_{2} b_{2} k_{2} e^{a_{2} t+b_{2} x}+\left(a_{1}+a_{2}\right)\left(b_{1}+b_{2}\right) k_{3} e^{\left(a_{1}+a_{2}\right) t+\left(b_{1}+b_{2}\right) x} \\
\partial_{x}^{m}(v)=b_{1}^{m} k_{1} e^{a_{1} t+b_{1} x}+b_{2}^{m} k_{2} e^{a_{2} t+b_{2} x}+\left(b_{1}+b_{2}\right)^{m} k_{3} e^{\left(a_{1}+a_{2}\right) t+\left(b_{1}+b_{2}\right) x} .
\end{gathered}
$$


Moreover,

$$
\begin{aligned}
& v v_{t x}-v_{t} v_{x}=v_{t x}+\left(k_{1} e^{a_{1} t+b_{1} x}+k_{2} e^{a_{2} t+b_{2} x}+k_{3} e^{\left(a_{1}+a_{2}\right) t+\left(b_{1}+b_{2}\right) x}\right) \\
& \times\left(a_{1} b_{1} k_{1} e^{a_{1} t+b_{1} x}+a_{2} b_{2} k_{2} e^{a_{2} t+b_{2} x}+\left(a_{1}+a_{2}\right)\left(b_{1}+b_{2}\right) k_{3} e^{\left(a_{1}+a_{2}\right) t+\left(b_{1}+b_{2}\right) x}\right) \\
& -\left(a_{1} k_{1} e^{a_{1} t+b_{1} x}+a_{2} k_{2} e^{a_{2} t+b_{2} x}+\left(a_{1}+a_{2}\right) k_{3} e^{\left(a_{1}+a_{2}\right) t+\left(b_{1}+b_{2}\right) x}\right) \\
& \times\left(b_{1} k_{1} e^{a_{1} t+b_{1} x}+b_{2} k_{2} e^{a_{2} t+b_{2} x}+\left(b_{1}+b_{2}\right) k_{3} e^{\left(a_{1}+a_{2}\right) t+\left(b_{1}+b_{2}\right) x}\right) \\
& =a_{1} b_{1}\left(k_{1} e^{a_{1} t+b_{1} x}+k_{2} k_{3} e^{\left(a_{1}+2 a_{2}\right) t+\left(b_{1}+2 b_{2}\right) x}\right)+\left(k_{2} e^{a_{2} t+b_{2} x}+k_{1} k_{3} e^{\left(2 a_{1}+a_{2}\right) t+\left(2 b_{1}+b_{2}\right) x}\right) \\
& \times a_{2} b_{2}+\left[\left(a_{1}+a_{2}\right)\left(b_{1}+b_{2}\right) k_{3}+k_{1} k_{2}\left(a_{1}-a_{2}\right)\left(b_{1}-b_{2}\right)\right] e^{\left(a_{1}+a_{2}\right) t+\left(b_{1}+b_{2}\right) x}, \\
& v v_{x x x x}-4 v_{x} v_{x x x}+3 v_{x x}^{2}=v_{x x x x}+\left(k_{1} e^{a_{1} t+b_{1} x}+k_{2} e^{a_{2} t+b_{2} x}+k_{3} e^{\left(a_{1}+a_{2}\right) t+\left(b_{1}+b_{2}\right) x}\right) \\
& \times\left(b_{1}^{4} k_{1} e^{a_{1} t+b_{1} x}+b_{2}^{4} k_{2} e^{a_{2} t+b_{2} x}+\left(b_{1}+b_{2}\right)^{4} k_{3} e^{\left(a_{1}+a_{2}\right) t+\left(b_{1}+b_{2}\right) x}\right)-4\left(b_{1} k_{1} e^{a_{1} t+b_{1} x}+b_{2} k_{2}\right. \\
& \left.\times e^{a_{2} t+b_{2} x}+\left(b_{1}+b_{2}\right) k_{3} e^{\left(a_{1}+a_{2}\right) t+\left(b_{1}+b_{2}\right) x}\right)\left(b_{1}^{3} k_{1} e^{a_{1} t+b_{1} x}+\left(b_{1}+b_{2}\right)^{3} k_{3} e^{\left(a_{1}+a_{2}\right) t+\left(b_{1}+b_{2}\right) x}\right. \\
& \left.+b_{2}^{3} k_{2} e^{a_{2} t+b_{2} x}\right)+3\left(b_{1}^{2} k_{1} e^{a_{1} t+b_{1} x}+b_{2}^{2} k_{2} e^{a_{2} t+b_{2} x}+\left(b_{1}+b_{2}\right)^{2} k_{3} e^{\left(a_{1}+a_{2}\right) t+\left(b_{1}+b_{2}\right) x}\right)^{2} \\
& =\left[\left(b_{1}+b_{2}\right)^{4} k_{3}+k_{1} k_{2}\left(b_{1}-b_{2}\right)^{4}\right] e^{\left(a_{1}+a_{2}\right) t+\left(b_{1}+b_{2}\right) x}+k_{2} k_{3} b_{1}^{4} e^{\left(a_{1}+2 a_{2}\right) t+\left(b_{1}+2 b_{2}\right) x} \\
& +k_{1} k_{3} b_{2}^{4} e^{\left(2 a_{1}+a_{2}\right) t+\left(2 b_{1}+b_{2}\right) x}+k_{1} b_{1}^{4} e^{a_{1} t+b_{1} x}+k_{2} b_{2}^{4} e^{a_{2} t+b_{2} x} .
\end{aligned}
$$

Substituting the above expressions into (5.1.37) and taking $\nu \equiv 0$, we find that (5.1.37) is equivalent to

$$
a_{1}=-b_{1}^{3}, \quad a_{2}=-b_{2}^{3}
$$

and

$$
\left(a_{1}+a_{2}\right)\left(b_{1}+b_{2}\right) k_{3}+k_{1} k_{2}\left(a_{1}-a_{2}\right)\left(b_{1}-b_{2}\right)+\left(b_{1}+b_{2}\right)^{4} k_{3}+k_{1} k_{2}\left(b_{1}-b_{2}\right)^{4}=0,
$$

which is equivalent to

$$
3 b_{1} b_{2}\left(b_{1}+b_{2}\right)^{2} k_{3}=3 b_{1} b_{2}\left(b_{1}-b_{2}\right)^{2} k_{1} k_{2} \Longrightarrow k_{3}=\left(\frac{b_{1}-b_{2}}{b_{1}+b_{2}}\right)^{2} k_{1} k_{2}
$$

Hence we have a two-solition solution

$$
u=2 \partial_{x}^{2} \ln \left(1+k_{1} e^{b_{1} x-b_{1}^{3} t}+k_{2} e^{b_{2} x-b_{2}^{3} t}+\left(\frac{b_{1}-b_{2}}{b_{1}+b_{2}}\right)^{2} k_{1} k_{2} e^{\left(b_{1}+b_{2}\right) x-\left(b_{1}^{3}+b_{2}^{3}\right) t}\right)
$$

for the $\mathrm{KdV}$ equation (5.1.3), where $0 \neq b_{1}, b_{2}, k_{1}, k_{2} \in \mathbb{R}$ and $b_{1}+b_{2} \neq 0$. The above two-solition solution was discovered by Hirota (1971) [Hr]. Hirota introduced a bilinear form (now called Hirota bilinear form) as follows. For two functions $f\left(x_{1}, \ldots, x_{n}\right)$ and $g\left(x_{1}, \ldots, x_{n}\right)$, we define the Hirota bilinear form

$$
D_{x_{r_{1}}}^{k_{1}} D_{x_{r_{2}}}^{k_{2}}(f \cdot g)=\sum_{s_{1}=0}^{k_{1}} \sum_{s_{2}=0}^{k_{2}}\left(\begin{array}{l}
k_{1} \\
s_{1}
\end{array}\right)\left(\begin{array}{c}
k_{2} \\
s_{2}
\end{array}\right)(-1)^{s_{1}+s_{2}} \partial_{x_{r_{1}}}^{k_{1}-s_{1}} \partial_{x_{r_{2}}}^{k_{2}-s_{2}}(f) \partial_{x_{r_{1}}}^{s_{1}} \partial_{x_{r_{2}}}^{s_{2}}(g)
$$


for $r_{1}, r_{2} \in \overline{1, n}$ and $k_{1}, k_{2} \in \mathbb{N}$. The reason for the $\mathrm{KdV}$ equation to have the two-soliton solution (5.1.47) is because the equation (5.1.37) with $\nu \equiv 0$ can be written as

$$
D_{t} D_{x}(v \cdot v)+D_{x}^{4}(v \cdot v)=0
$$

which is called the Hirota bilinear form presentation of the KdV equation.

\section{Exercise 5.1}

Find exact solutions of the following one-dimensional Boussinesq equation

$$
u_{t t}+u u_{x x}+\left(u_{x}\right)^{2}+u_{x x x x}=0
$$

(Hint: prove that if $u=f(x)$ is a solution, then so is $\left.f(x+c t)-c^{2}\right)$.

\subsection{Kadomtsev and Petviashvili Equation}

The Kadomtsev and Petviashvili (KP) equation

$$
\left(u_{t}+6 u u_{x}+u_{x x x}\right)_{x}+3 \epsilon u_{y y}=0
$$

with $\epsilon= \pm 1$ is used to describe the evolution of long water waves of small amplitude if they are weakly two-dimensional (cf. $[\mathrm{KP}]$ ). The choice of $\epsilon$ depends on the relevant magnitude of gravity and surface tension. The equation has also been proposed as a model for surface waves and internal waves in straits or channels of varying depth and width.

Let $\alpha(t)$ be a differentiable function. Then the transformation $u(t, x, y) \mapsto u(t, x+\alpha, y)$ changes the KP equation to

$$
\left(u_{t}+\alpha^{\prime} u_{x}+6 u u_{x}+u_{x x x}\right)_{x}+3 \epsilon u_{y y}=0,
$$

where the independent variable $x$ is replaced by $x+\alpha$ and the subindices denote the partial derivatives with respect to the original independent variables. Moreover, the transformation $u \mapsto u-\alpha^{\prime} / 6$ changes the KP equation to

$$
\left(u_{t}-\alpha^{\prime} u_{x}+6 u u_{x}+u_{x x x}\right)_{x}+3 \epsilon u_{y y}=0 .
$$

So the transformation

$$
T_{2, \alpha}(u(t, x, y))=u(t, x+\alpha, y)-\frac{\alpha^{\prime}}{6}
$$

keeps the KP equation invariant with the independent variable $x$ is replaced by $x+\alpha$; equivalently, $T_{2, \alpha}$ maps a solution of the KP equation to another solution of the KP 
equation. Moreover, the transformation $u(t, x, y) \mapsto u(t, x, y+\alpha)$ changes the KP equation to

$$
\left(u_{t}+\alpha^{\prime} u_{y}+6 u u_{x}+u_{x x x}\right)_{x}+3 \epsilon u_{y y}=0
$$

with the independent variable $y$ replaced by $y+\alpha$, and the transformation $u(t, x, y) \mapsto$ $u(t, x+\beta y, y)$ changes the KP equation to

$$
\left(u_{t}+\beta^{\prime} y u_{x}+6 u u_{x}+u_{x x x}\right)_{x}+3 \epsilon u_{y y}+3 \epsilon \beta^{2} u_{x x}+6 \epsilon \beta u_{x y}=0
$$

with the independent variable $x$ replaced by $x+\beta y$; equivalently,

$$
\left(u_{t}+\left(\beta^{\prime} y+3 \epsilon \beta^{2}\right) u_{x}+6 \epsilon \beta u_{y}+6 u u_{x}+u_{x x x}\right)_{x}+3 \epsilon u_{y y}=0 .
$$

Thus the transformation

$$
T_{3, \alpha}(u(t, x, y))=u\left(t, x-\frac{\alpha^{\prime} y}{6 \epsilon}, y+\alpha\right)+\frac{2 \alpha^{\prime \prime} y-\alpha^{\prime 2}}{72 \epsilon}
$$

leaves the KP equation invariant with the independent variable $y$ replaced by $y+\alpha$ and the variable $x$ replaced by $x-\epsilon \alpha^{\prime} y / 6$. Hence $T_{3, \alpha}$ maps a solution of the KP equation to another solution of the KP equation.

From the degree analysis in (5.1.6)-(5.1.9), we can make the KP equation homogeneous if we take $\operatorname{deg} y=2 \operatorname{deg} x=2 \ell_{2}$. Hence the transformation

$$
T_{a, b}(u(t, x, y))=b^{2} u\left(b^{3} t+a, b x, b^{2} y\right)
$$

keeps the KP equation invariant for $a, b \in \mathbb{R}$ and $b \neq 0$. Therefore, the transformation

$$
\mathcal{T}(u(t, x, y))=b^{2} u\left(b^{3} t+a, b\left(x-\epsilon \alpha^{\prime} y / 6+\beta\right), b^{2}(y+\alpha)\right)+\frac{2 \alpha^{\prime \prime} y-\alpha^{\prime 2}}{72 \epsilon}-\frac{\beta^{\prime}}{6}
$$

maps a solution of the KP equation to another solution for any functions $\alpha, \beta$ in $t$ and $a, b \in \mathbb{R}$ with $b \neq 0$.

Note that any solution of the KdV equation is also a solution of the KP equation. Using the above symmetry transformations in (5.2.4) and (5.2.8), we can get more sophisticated solutions of the KP equation from the solutions of the KdV equation in last section: (1)

$$
u=-\frac{2}{(x-\epsilon \alpha y / 6+\beta)^{2}}+\frac{2 \alpha^{\prime} y-\alpha^{2}}{72 \epsilon}-\frac{\beta^{\prime}}{6}
$$

from the solution $u=-2 / x^{2}$ of the KdV equation; (2)

$$
u=-2 \wp(x-\epsilon \alpha y / 6+\beta)+\frac{2 \alpha^{\prime} y-\alpha^{2}}{72 \epsilon}-\frac{\beta^{\prime}}{6}
$$

from the solution $u=-2 \wp(x)$ of the KdV equation; (3)

$$
u=-2 b^{2} \tan ^{2} b(x-\epsilon \alpha y / 6+\beta)+\frac{2 \alpha^{\prime} y-\alpha^{2}}{72 \epsilon}-\frac{8 b^{2}+\beta^{\prime}}{6}
$$


from the solution $u=-2 b^{2}\left(\tan ^{2} b x+2 / 3\right)$ of the KdV equation; (4)

$$
u=-2 b^{2} \operatorname{coth}^{2}(b(x-\epsilon \alpha y / 6+\beta))+\frac{2 \alpha^{\prime} y-\alpha^{2}}{72 \epsilon}+\frac{8 b^{2}-\beta^{\prime}}{6}
$$

from the solution $u=-2 b^{2}\left(\operatorname{coth}^{2} b x-2 / 3\right)$ of the KdV equation; (5)

$$
u=2 m^{2} b^{2} \operatorname{cn}^{2}(b(x-\epsilon \alpha y / 6+\beta) \mid m)+\frac{2 \alpha^{\prime} y-\alpha^{2}}{72 \epsilon}+\frac{\left(4-8 m^{2}\right) b^{2}-\beta^{\prime}}{6}
$$

from the solution $u=2 b^{2} m^{2} \mathrm{cn}^{2}(b x \mid m)+\left(2-4 m^{2}\right) b^{2} / 3$ of the $\mathrm{KdV}$ equation, which becomes a line-soliton solution

$$
u=2 b^{2} \operatorname{sech}^{2}\left(b\left(x-\epsilon c y-\left(3 \epsilon c^{2}+4 b^{2}\right) t+a\right)\right)
$$

when we take $\alpha=6 c, \beta=-\left(3 \epsilon c^{2}+4 b^{2}\right) t+a$ and let $m \rightarrow 1$; $(6)$

$$
u=\frac{x-\epsilon \alpha y / 6+\beta}{6(t+a)}+\frac{2 \alpha^{\prime} y-\alpha^{2}}{72 \epsilon}-\frac{\beta^{\prime}}{6}
$$

from the solution $u=x / 6(t+a)$ of the KdV equation; (7)

$$
\begin{aligned}
u= & 2 \partial_{x}^{2} \ln \left[1+k_{1} e^{b_{1}(x-\epsilon \alpha y / 6+\beta)-b_{1}^{3} t}+k_{2} e^{b_{2}(x-\epsilon \alpha y / 6+\beta)-b_{2}^{3} t}\right. \\
& \left.+\left(\frac{b_{1}-b_{2}}{b_{1}+b_{2}}\right)^{2} k_{1} k_{2} e^{\left(b_{1}+b_{2}\right)(x-\epsilon \alpha y / 6+\beta)-\left(b_{1}^{3}+b_{2}^{3}\right) t}\right]+\frac{2 \alpha^{\prime} y-\alpha^{2}}{72 \epsilon}-\frac{\beta^{\prime}}{6}
\end{aligned}
$$

from the solution (5.1.47) of the KdV equation, which becomes a two-soliton solution

$$
\begin{aligned}
u= & 2 \partial_{x}^{2} \ln \left[1+k_{1} e^{b_{1}\left(x-\epsilon c y-3 \epsilon c^{2} t\right)-b_{1}^{3} t}+k_{2} e^{b_{2}\left(x-\epsilon c y-3 \epsilon c^{2} t\right)-b_{2}^{3} t}\right. \\
& \left.+\left(\frac{b_{1}-b_{2}}{b_{1}+b_{2}}\right)^{2} k_{1} k_{2} e^{\left(b_{1}+b_{2}\right)\left(x-\epsilon c y-3 \epsilon c^{2} t\right)-\left(b_{1}^{3}+b_{2}^{3}\right) t}\right]
\end{aligned}
$$

when we take $\alpha=6 c$ and $\beta=-3 \epsilon c^{2} t$.

Next we assume that

$$
u=h(t, y)+g(t, y) x+f(t, y) x^{2}
$$

is a solution of the KP equation, where $h, g$ and $f$ are functions in $t$ and $x$ to be determined. Then

$$
g_{t}+3 \epsilon h_{y y}+6 g^{2}+12 f h+\left[2 f_{t}+3 \epsilon g_{y y}+36 f g\right] x+3\left(\epsilon f_{y y}+12 f^{2}\right) x^{2}=0,
$$

equivalently,

$$
\begin{gathered}
\epsilon f_{y y}+12 f^{2}=0, \\
2 f_{t}+3 \epsilon g_{y y}+36 f g=0, \\
g_{t}+3 \epsilon h_{y y}+6 g^{2}+12 f h=0 .
\end{gathered}
$$


Recall the Weierstrass's elliptic function $\wp(z)$ defined in (3.4.9). Moreover, $\wp^{\prime \prime}(z)=$ $6 \wp^{2}(z)-g_{2} / 2$ with the $g_{2}$ given in (3.4.29). In (3.4.9), we take $\omega_{1} \in \mathbb{C}$ such that $\operatorname{Re} \omega_{1}, \operatorname{Im} \omega_{1} \neq 0$ and $\omega_{2}=\overline{\omega_{1}}$ for which $g_{2}=0$. Then $\wp(z)$ is real if $z \in \mathbb{R}$. An obvious solution of the system (5.2.22)-(5.2.24) is $f=-\epsilon \wp(y) / 2$ and $g=h=0$. So $u=-\epsilon x^{2} \wp(y) / 2$ is a solution of the KP equation. Applying the transformations in (5.2.4) and (5.2.8), we get a more sophisticated solution

$$
u=-\frac{\epsilon}{2}\left(x-\epsilon \alpha^{\prime} y / 6+\beta\right)^{2} \wp(y+\alpha)+\frac{2 \alpha^{\prime \prime} y-\alpha^{\prime 2}}{72 \epsilon}-\frac{\beta^{\prime}}{6}
$$

for any differentiable functions $\alpha, \beta$ in $t$.

Note that $f=-\epsilon / 2(y-\alpha)^{2}$ is a solution of (5.2.22) for any function $\alpha$ in $t$. Replacing $u$ by $T_{3, \alpha}(u)$ (cf. (5.2.8)), we can assume $\alpha=0$, that is, $f=-\epsilon / 2 y^{2}$. Substituting it into (5.2.23), we get $g_{y y}=6 g / y^{2} \sim y^{2} g_{y y}=6 g$. Assume

$$
g=\sum_{m \in \mathbb{Z}} a_{m}(t) y^{m}
$$

where $a_{m}(t)$ are functions in $t$ to be determined. Then

$$
\sum_{m \in \mathbb{Z}} m(m-1) a_{m} y^{m}=6 \sum_{m \in \mathbb{Z}} a_{m} y^{m} \sim[m(m-1)-6] a_{m}=0, \quad m \in \mathbb{Z} .
$$

Moreover,

$$
[m(m-1)-6] a_{m}=0 \sim(m-3)(m+2) a_{m}=0 .
$$

So $a_{m}=0$ if $m \neq-2,3$. Hence

$$
g=\frac{\beta}{y^{2}}+\gamma y^{3}
$$

where $\beta$ and $\gamma$ are arbitrary functions in $t$.

Recall $u=f x^{2}+g x+h$ and observe

$$
f x^{2}+g x=\frac{-\epsilon x^{2}+2 \beta x}{2 y^{2}}+\gamma y^{3} x=\frac{-\epsilon(x-\epsilon \beta)^{2}+\epsilon \beta^{2}}{2 y^{2}}+\gamma y^{3} x .
$$

Replacing $u$ by $T_{2, \epsilon \beta}(u)$ (cf. (5.2.4)), we can assume $\beta=0$, that is, $g=\gamma y^{3}$. Next (5.2.24) becomes

$$
\gamma^{\prime} y^{3}+3 \epsilon h_{y y}+6 \gamma^{2} y^{6}-\frac{6 \epsilon}{y^{2}} h=0
$$

equivalently,

$$
y^{2} h_{y y}-2 h=-\frac{\epsilon \gamma^{\prime}}{3} y^{5}-2 \epsilon \gamma^{2} y^{8} .
$$

Suppose

$$
h=\sum_{m \in \mathbb{Z}} b_{m}(t) y^{m},
$$


where $b_{m}(t)$ are functions in $t$ to be determined. Substituting it into (5.2.32), we have

$$
\sum_{m \in \mathbb{Z}}[m(m-1)-2] b_{m} y^{m}=-\frac{\epsilon \gamma^{\prime}}{3} y^{5}-2 \epsilon \gamma^{2} y^{8}
$$

Thus

$$
b_{5}=-\frac{\epsilon \gamma^{\prime}}{54}, \quad b_{8}=-\frac{\epsilon \gamma^{2}}{27}, \quad[m(m-1)-2] b_{m}=(m-2)(m+1) b_{m}=0, \quad m \neq 5,8 .
$$

Hence

$$
h=\frac{\vartheta}{y}+\nu y^{2}-\frac{\epsilon \gamma^{\prime}}{54} y^{5}-\frac{\epsilon \gamma^{2}}{27} y^{8},
$$

where $\vartheta$ and $\nu$ are two arbitrary functions in $t$. Therefore, we obtain following solution of the KP equation (5.2.1):

$$
u=-\frac{\epsilon x^{2}}{2 y^{2}}+\gamma x y^{3}+\frac{\vartheta}{y}+\nu y^{2}-\frac{\epsilon \gamma^{\prime}}{54} y^{5}-\frac{\epsilon \gamma^{2}}{27} y^{8}
$$

Applying the transformations in (5.2.4) and (5.2.8), we have:

Theorem 5.2.1. For any functions $\alpha, \beta, \gamma, \vartheta$ and $\nu$ in $t$, the following is a solution of the KP equation (5.2.1):

$$
\begin{aligned}
u= & -\frac{\epsilon\left(x-\alpha^{\prime} y / 6 \epsilon+\beta\right)^{2}}{2(y+\alpha)^{2}}+\gamma\left(x-\alpha^{\prime} y / 6 \epsilon+\beta\right)(y+\alpha)^{3}+\frac{\vartheta}{y+\alpha} \\
& +\nu(y+\alpha)^{2}-\frac{\epsilon \gamma^{\prime}}{54}(y+\alpha)^{5}-\frac{\epsilon \gamma^{2}}{27}(y+\alpha)^{8}+\frac{2 \alpha^{\prime \prime} y-\alpha^{\prime 2}}{72 \epsilon}-\frac{\beta^{\prime}}{6} .
\end{aligned}
$$

Let $f=0$ in (5.2.22). Then (5.2.23) becomes $g_{y y}=0$. So $g=\alpha y+\beta$ for some functions $\alpha$ and $\beta$ in $t$. Now (5.2.24) yields

$$
3 \epsilon h_{y y}+6 \alpha^{2} y^{2}+\left(\alpha^{\prime}+12 \alpha \beta\right) y+6 \beta^{2}+\beta^{\prime}=0
$$

Thus we get the following solution of the KP equation

$$
u=(\alpha y+\beta) x-\frac{\epsilon \alpha^{2}}{6} y^{4}-\frac{\epsilon\left(\alpha^{\prime}+12 \alpha \beta\right)}{18} y^{3}-\frac{\epsilon\left(6 \beta^{2}+\beta^{\prime}\right)}{6} y^{2}+\gamma y+\theta
$$

where $\alpha, \beta, \gamma$ and $\theta$ are arbitrary functions in $t$. Note that the solution (5.2.17) is a special case of the above solution.

Changing variable $u=2 \partial_{x}^{2} \ln v$, we find the following presentation of the KP equation in Hirota bilinear form

$$
D_{t} D_{x}(v \cdot v)+D_{x}^{4}(v \cdot v)+3 \epsilon D_{y}^{2}(v \cdot v)=0
$$


(cf. (5.1.37) and (5.1.49)). Suppose that

$$
v=\left(x+a_{0} t\right)^{2}+b y^{2}+c
$$

is a solution (5.2.41), where all the coefficients are constants to be determined and $b \neq 0$. By (5.2.41),

$$
2\left(a_{0}+3 \epsilon b\right) v-4 a_{0}\left(x+a_{0} t\right)^{2}+12-12 \epsilon b^{2} y^{2}=0,
$$

equivalently,

$$
a_{0}=3 \epsilon b, \quad c=-\frac{\epsilon}{b^{2}}
$$

So

$$
u=2 \partial_{x}^{2} \ln v=2 \partial_{x}^{2} \ln \left((x+3 \epsilon b t)^{2}+b y^{2}-\epsilon / b^{2}\right)
$$

is a solution of the KP equation. Applying the transformations in (5.2.4) and (5.2.8), we obtain the following solution of the KP equation:

$$
u=2 \partial_{x}^{2} \ln \left(\left(x-\epsilon \alpha^{\prime} y / 6+\beta+3 \epsilon b t+a\right)^{2}+b(y+\alpha)^{2}-\epsilon / b^{2}\right)+\frac{2 \alpha^{\prime \prime} y-\alpha^{\prime 2}}{72 \epsilon}-\frac{\beta^{\prime}}{6} .
$$

Taking $\alpha=6 \epsilon t$ and $\beta=-3 \epsilon c^{2} t$, we get the following lump solution of the KP equation:

$$
u=2 \partial_{x}^{2} \ln \left(\left(x-c y+3 \epsilon\left(b-c^{2}\right) t+a\right)^{2}+b(y+6 \epsilon c t)^{2}-\epsilon / b^{2}\right),
$$

where $a, b, c \in \mathbb{R}$ and $b \neq 0$.

Jimbo and Miwa [JM] found the $\tau$-function solutions of the KP equation via the orbit of the vacuum vector for the fermionic representation of the general linear group $G L(\infty)$ and the Boson-Fermion correspondence in quantum field theory. Kupershmidt $[\mathrm{Kb}]$ found geometric-Hamiltonian form for the KP equation. Cao [Cb2] found some algebraic approaches to the exact solutions of the Jimbo-Miwa equation, which is the second equation in the KP hierarchy.

\subsection{Equation of Transonic Gas Flows}

Lin, Reisner and Tsien [LRT] (1948) found the equation

$$
2 u_{t x}+u_{x} u_{x x}-u_{y y}=0
$$

for two-dimensional non-steady motion of a slender body in a compressible fluid, which was later called the "equation of transonic gas flows" (cf. [Me1]).

Mamontov [Me1] (1969) obtained the Lie point symmetries of the above equation and solved the problem of existence of analytic solutions in [Me2] (1972). Sevost'janov [Sg] (1977) found explicit solutions of the equation (5.3.1), describing nonstationary transonic 
flows in plane nozzles. Sukhinin [Sv] (1978) studied the group property and conservation laws of the equation. In this section, we give the stable-range approach to the LRT equation (5.3.1). The results are taken from our work [X8].

First we give an intuitive derivation of the symmetry transformations of the LRT equation due to Mamontov [Me1]. Suppose

$$
\operatorname{deg} u=\ell_{1}, \quad \operatorname{deg} x=\ell_{2} .
$$

To make each nonzero term having the same degree, we have to take

$$
\operatorname{deg} t=2 \ell_{2}-\ell_{1}, \quad \operatorname{deg} y=\frac{3}{2} \ell_{2}-\frac{1}{2} \ell_{1} .
$$

Since the LRT equation (5..3.1) does not contain variable coefficients, it is translation invariant. Thus the transformation

$$
T_{b_{1}, b_{2}}^{(a)}(u(t, x, y))=b_{1}^{2} u\left(b_{1}^{2} b_{2}^{4} t+a, b_{2}^{2} x, b_{1} b_{2}^{3} y\right)
$$

keep the LRT equation invariant for $a, b_{1}, b_{2} \in \mathbb{R}$ such that $b_{1}, b_{2} \neq 0$, with the independent variables $t$ replaced by $b_{1}^{2} b_{2}^{4} t+a, x$ replaced by $b_{2}^{2} x$ and $y$ replaced by $b_{1} b_{2}^{3} y$, where the subindices denote the partial derivatives with respect to the original independent variables. So $T_{b_{1}, b_{2}}^{(a)}$ maps a solutions of the LRT equation to another solution.

Let $\alpha$ be differentiable functions in $t$. Then the transformation $u \mapsto u+\alpha$ keeps (5.3.1) invariant. Moreover, the transformation $u(t, x, y) \mapsto u(t, x+\alpha, y)$ changes the LRT equation to

$$
2 u_{t x}+2 \alpha^{\prime} u_{x x}+u_{x} u_{x x}-u_{y y}=0
$$

with the independent variables $x$ replaced by $x+\alpha$ and the subindices denoting the partial derivatives with respect to the original independent variables. Furthermore, the transformation $u(t, x, y) \mapsto u(t, x, y)-2 \alpha^{\prime} x$ changes the LRT equation to

$$
-4 \alpha^{\prime \prime}+2 u_{t x}-2 \alpha^{\prime} u_{x x}+u_{x} u_{x x}-u_{y y}=0 .
$$

In addition, the transformation $u(t, x, y) \mapsto u(t, x, y)-2 \alpha^{\prime \prime} y^{2}$ changes the LRT equation to

$$
2 u_{t x}+u_{x} u_{x x}-u_{y y}+4 \alpha^{\prime \prime}=0 .
$$

Thus the transformation

$$
T_{2, \alpha}(u(t, x, y))=u(t, x+\alpha, y)-2 \alpha^{\prime} x-2 \alpha^{\prime \prime} y^{2}
$$

keeps the LRT equation invariant with the independent variable $x$ replaced by $x+\alpha$ and the subindices denoting the partial derivatives with respect to the original independent variables; equivalently, $T_{2, \alpha}$ maps a solutions of the LRT equation to another solution. 
Since $u=0$ is a solution, $u=T_{2, \alpha}(0)=-2 \alpha^{\prime} x-2 \alpha^{\prime \prime} y^{2}$ is a nontrivial solution of the LRT equation.

Given a differentiable function $\beta$ in $t$, the transformation $u(t, x, y) \mapsto u(t, x, y+\beta)$ changes the LRT equation to

$$
2 u_{t x}+2 \beta^{\prime} u_{x y}+u_{x} u_{x x}-u_{y y}=0
$$

with the independent variable $y$ replaced by $y+\beta$ and the subindices denoting the partial derivatives with respect to the original independent variables. Moreover, the transformation $u(t, x, y) \mapsto u\left(t, x+\beta^{\prime} y, y\right)$ changes the LRT equation to

$$
2 u_{t x}+2 \beta^{\prime \prime} y u_{x x}+u_{x} u_{x x}-u_{y y}-2 \beta^{\prime} u_{x y}-\beta^{\prime 2} u_{x x}=0
$$

with the independent variable $x$ replaced by $x+\beta^{\prime} y$. Furthermore, the transformation $u(t, x, y) \mapsto u(t, x, y)-2 \beta^{\prime \prime} x y+\beta^{\prime 2} x$ changes the LRT equation to

$$
4 \beta^{\prime} \beta^{\prime \prime}-4{\beta^{\prime \prime}}^{\prime} y+2 u_{t x}-2 \beta^{\prime \prime} y u_{x x}+{\beta^{\prime}}^{2} x u_{x x}+u_{x} u_{x x}-u_{y y}=0
$$

In addition, the transformation $u(t, x, y) \mapsto u(t, x, y)+2 \beta^{\prime} \beta^{\prime \prime} y^{2}-2 \beta^{\prime \prime} y^{3} / 3$ changes the LRT equation to

$$
2 u_{t x}+u_{x} u_{x x}-u_{y y}-4 \beta^{\prime} \beta^{\prime \prime}+4{\beta^{\prime \prime}}^{\prime \prime}=0 .
$$

Therefore, the transformation

$$
T_{3, \beta}(u(t, x, y))=u\left(t, x+\beta^{\prime} y, y+\beta\right)+{\beta^{\prime}}^{2} x+2 \beta^{\prime} \beta^{\prime \prime} y^{2}-2{\beta^{\prime \prime}}^{\prime} y-\frac{2{\beta^{\prime \prime}}^{\prime \prime}}{3} y^{3}
$$

leave the equation (5.3.1) invariant with the independent variables $x$ replaced by $x+\beta^{\prime} y$ and $y$ replaced by $y+\beta$, where the subindices denote the partial derivatives with respect to the original independent variables. In other words, $T_{3, \beta}$ maps a solutions of the LRT equation to another solution. In particular, $u=T_{3, \beta}(0)=\beta^{\prime 2} x+2 \beta^{\prime} \beta^{\prime \prime} y^{2}-2 \beta^{\prime \prime} x y-\frac{2{\beta^{\prime \prime \prime}}^{\prime}}{3} y^{3}$ is a solution of the LRT equation.

In summary, the transformation

$$
\begin{aligned}
T_{b_{1}, b_{2} ; \gamma}^{(a ; \alpha)}(u(t, x, y))= & b_{1}^{2} u\left(b_{1}^{2} b_{2}^{4} t+a, b_{2}^{2}\left(x+\beta^{\prime} y+\alpha\right), b_{1} b_{2}^{3}(y+\beta)\right)+\gamma \\
& +\left({\beta^{\prime}}^{2}-2 \alpha^{\prime}\right) x+2\left(\beta^{\prime}{\beta^{\prime \prime}}^{\prime}-\alpha^{\prime \prime}\right) y^{2}-2 \beta^{\prime \prime} x y-\frac{2 \beta^{\prime \prime}}{3} y^{3}
\end{aligned}
$$

maps a solutions of the LRT equation to another solution.

Note that the maximal finite-dimensional subspace of $\mathbb{R}[x]$ invariant under the transformation $u \mapsto u_{x} u_{x x}$ is $\sum_{r=0}^{3} \mathbb{R} x^{r}$ (stable range). We look for a solution of the form:

$$
u=f(t, y)+g(t, y) x+h(t, y) x^{2}+\xi(t, y) x^{3},
$$


where $f(t, y), g(t, y), h(t, y)$ and $\xi(t, y)$ are suitably-differentiable functions to be determined. Note

$$
\begin{gathered}
u_{x}=g+2 h x+3 \xi x^{2}, \quad u_{x x}=2 h+6 \xi x \\
u_{t x}=g_{t}+2 h_{t} x+3 \xi_{t} x^{2}, \quad u_{y y}=f_{y y}+g_{y y} x+h_{y y} x^{2}+\xi_{y y} x^{3},
\end{gathered}
$$

Now (5.3.1) becomes

$$
2\left(g_{t}+2 h_{t} x+3 \xi_{t} x^{2}\right)+\left(g+2 h x+3 \xi x^{2}\right)(2 h+6 \xi x)-f_{y y}-g_{y y} x-h_{y y} x^{2}-\xi_{y y} x^{3}=0,
$$

which is equivalent to the following system of partial differential equations:

$$
\begin{gathered}
\xi_{y y}=18 \xi^{2}, \\
h_{y y}=6 \xi_{t}+18 \xi h, \\
g_{y y}=4 h_{t}+4 h^{2}+6 \xi g, \\
f_{y y}=2 g_{t}+2 g h .
\end{gathered}
$$

Recall the Weierstrass's elliptic function $\wp(z)$ defined in (3.4.9). Moreover, $\wp^{\prime \prime}(z)=$ $6 \wp^{2}(z)-g_{2} / 2$ with the $g_{2}$ given in (3.4.29). In (3.4.9), we take $\omega_{1} \in \mathbb{C}$ such that $\operatorname{Re} \omega_{1}, \operatorname{Im} \omega_{1} \neq 0$ and $\omega_{2}=\overline{\omega_{1}}$ for which $g_{2}=0$. Then $\wp(z)$ is real if $z \in \mathbb{R}$. An obvious solution of the equation (5.3.19)-(5.3.22) is $\xi=\wp(y) / 3$ and $f=g=h=0$. So $u=x^{3} \wp(y) / 3$ is a solution of the LRT equation (5.3.1). Applying the transformation $T_{1,1 ; \gamma}^{(0 ; \alpha, \beta)}$ in (5.3.14), we get a more sophisticated solution

$$
u=\frac{1}{3}\left(x+\beta^{\prime} y+\alpha\right)^{3} \wp(y+\beta)+\left({\beta^{\prime}}^{2}-2 \alpha^{\prime}\right) x+2\left(\beta^{\prime} \beta^{\prime \prime}-\alpha^{\prime \prime}\right) y^{2}-2 \beta^{\prime \prime} x y-\frac{2 \beta^{\prime \prime \prime}}{3} y^{3}+\gamma
$$

of the LRT equation (5.3.1).

Observe that

$$
\xi=\frac{1}{3 y^{2}}
$$

is a solution of the equation (5.3.19). Substituting (5.3.24) into (5.3.20), we get

$$
h_{y y}=\frac{6}{y^{2}} h .
$$

Write

$$
h(t, y)=\sum_{m \in \mathbb{Z}} \frac{a_{m}(t)}{y^{m}} .
$$

Then (5.3.25) is equivalent to

$$
[m(m+1)-6] a_{m}=0 \sim(m-2)(m+3) a_{m}=0 \quad \text { for } m \in \mathbb{Z}
$$

Thus

$$
h=\frac{\alpha}{y^{2}}+\gamma y^{3}
$$


where $\alpha$ and $\gamma$ are arbitrary differentiable functions in $t$.

Note

$$
\xi x^{3}+h x^{2}=\frac{x^{3}+3 \alpha x^{2}}{3 y^{2}}+\gamma y^{3} x^{2}=\frac{(x+\alpha)^{3}-3 \alpha^{2} x-\alpha^{3}}{3 y^{2}}+\gamma y^{3} x^{2} .
$$

Replacing $u$ by $T_{2,-\alpha}(u)$ (cf. (5.3.8)), we can assume $\alpha=0$, that is, $h=\gamma y^{3}$. Now

$$
h_{t}=\gamma^{\prime} y^{3}, \quad h^{2}=\gamma^{2} y^{6} .
$$

Substituting the above equation into (5.3.21), we have:

$$
g_{y y}-\frac{2}{y^{2}} g=4 \gamma^{\prime} y^{3}+4 \gamma^{2} y^{6}
$$

Write

$$
g(t, y)=\sum_{m \in \mathbb{Z}} b_{m}(t) y^{m} .
$$

Then (5.3.31) is equivalent to

$$
b_{5}=\frac{2 \gamma^{\prime}}{9}, \quad b_{8}=\frac{2 \gamma^{2}}{27}, \quad(m+1)(m-2) a_{m}=0, \quad m \neq 5,8 .
$$

So

$$
g=\frac{\vartheta}{y}+\Im y^{2}+\frac{2 \gamma^{\prime}}{9} y^{5}+\frac{2 \gamma^{2}}{27} y^{8}
$$

where $\vartheta$ and $\Im$ are arbitrary differentiable functions in $t$.

Observe that

$$
\begin{gathered}
g_{t}=\frac{\vartheta^{\prime}}{y}+\Im^{\prime} y^{2}+\frac{2 \gamma^{\prime \prime}}{9} y^{5}+\frac{4 \gamma \gamma^{\prime}}{27} y^{8}, \\
g h=\gamma \vartheta y^{2}+\gamma \Im y^{5}+\frac{2 \gamma \gamma^{\prime}}{9} y^{8}+\frac{2 \gamma^{3}}{27} y^{11} .
\end{gathered}
$$

Hence (5.3.22) becomes

$$
f_{y y}=2\left[\frac{\vartheta^{\prime}}{y}+\left(\Im^{\prime}+\gamma \vartheta\right) y^{2}+\frac{9 \gamma \Im+2 \gamma^{\prime \prime}}{9} y^{5}+\frac{10 \gamma \gamma^{\prime}}{27} y^{8}+\frac{2 \gamma^{3}}{27} y^{11}\right] .
$$

Therefore,

$$
f=2 \vartheta^{\prime} y(\ln y-1)+\frac{\Im^{\prime}+\gamma \vartheta}{6} y^{4}+\frac{9 \gamma \Im+2 \gamma^{\prime \prime}}{189} y^{7}+\frac{2 \gamma \gamma^{\prime}}{243} y^{10}+\frac{\gamma^{3}}{1053} y^{13}+\rho y,
$$

where $\rho$ is any function in $t$.

Theorem 5.3.1. Let $\alpha, \beta, \gamma, \vartheta, \Im, \rho, \mu$ be arbitrary functions in $t$, which are differentiable up to need. We have the following solution of the LRT equation (5.3.1):

$$
\begin{aligned}
u= & \varphi=\frac{x^{3}}{3 y^{2}}+\gamma x^{2} y^{3}+\left(\frac{\vartheta}{y}+\Im y^{2}+\frac{2 \gamma^{\prime}}{9} y^{5}+\frac{2 \gamma^{2}}{27} y^{8}\right) x+2 \vartheta^{\prime} y(\ln y-1) \\
& +\frac{\Im^{\prime}+\gamma \vartheta}{6} y^{4}+\frac{9 \gamma \Im+2 \gamma^{\prime \prime}}{189} y^{7}+\frac{2 \gamma \gamma^{\prime}}{243} y^{10}+\frac{\gamma^{3}}{1053} y^{13}+\rho y .
\end{aligned}
$$


Moreover, $u=T_{1,1 ; \mu}^{(0 ; \alpha,-\beta)}(\varphi)$ is solution of the LRT equation (5.3.1) blowing up on the moving line $y=\beta(t)$.

We remark that the solution $u=T_{1,1 ; \mu}^{(0, \alpha,-\beta)}(\varphi)$ may reflect the phenomenon of abrupt high-speed wind. If we take $\varphi=x^{3} / 3 y^{2}$, then

$$
u=\frac{\left(x+\beta^{\prime} y+\alpha\right)^{3}}{3(y-\beta)^{2}}+\left({\beta^{\prime}}^{2}-2 \alpha^{\prime}\right) x+2\left(\beta^{\prime} \beta^{\prime \prime}-\alpha^{\prime \prime}\right) y^{2}-2 \beta^{\prime \prime} x y-\frac{2 \beta^{\prime \prime}}{3} y^{3}+\mu .
$$

Take the trivial solution $\xi=0$ of (5.3.19), which is the only solution polynomial in $y$. Then (5.3.20) and (5.3.21) become

$$
h_{y y}=0, \quad g_{y y}=4 h_{t}+4 h^{2} .
$$

Replacing $u$ by $T_{3, \alpha}(u)$ for some proper function $\alpha$ in $t$ if necessary (cf. (5.3.13)), we can take $h=\beta y$, where $\beta$ is an arbitrary function in $t$. Hence

$$
g_{y y}=4 \beta^{\prime} y+4 \beta^{2} y^{2} .
$$

So

$$
g=\gamma+\sigma y+\frac{2 \beta^{\prime}}{3} y^{3}+\frac{\beta^{2}}{3} y^{4}
$$

where $\gamma$ and $\sigma$ are arbitrary functions in $t$. Now (5.3.22) yields

$$
f_{y y}=2 \gamma^{\prime}+2\left(\beta \gamma+\sigma^{\prime}\right) y+2 \beta \sigma y^{2}+\frac{4 \beta^{\prime \prime}}{3} y^{3}+\frac{8 \beta \beta^{\prime}}{3} y^{4}+\frac{2}{3} \beta^{3} y^{5} .
$$

Replacing $u$ by some $T_{1,1 ; \alpha}^{(0 ; 0,0)}(u)$ if necessary (cf. (5.3.14)), we have

$$
f=\rho y+\gamma^{\prime} y^{2}+\frac{\beta \gamma+\sigma^{\prime}}{3} y^{3}+\frac{\beta \sigma}{6} y^{4}+\frac{\beta^{\prime \prime}}{15} y^{5}+\frac{4 \beta \beta^{\prime}}{45} y^{6}+\frac{\beta^{3}}{63} y^{7} .
$$

Theorem 5.3.2. The following is a solution of the equation (5.3.1):

$$
\begin{aligned}
u= & \psi=\beta x^{2} y+\left(\gamma+\sigma y+\frac{2 \beta^{\prime}}{3} y^{3}+\frac{\beta^{2}}{3} y^{4}\right) x+\rho y+\gamma^{\prime} y^{2} \\
& +\frac{\beta \gamma+\sigma^{\prime}}{3} y^{3}+\frac{\beta \sigma}{6} y^{4}+\frac{\beta^{\prime \prime}}{15} y^{5}+\frac{4 \beta \beta^{\prime}}{45} y^{6}+\frac{\beta^{3}}{63} y^{7}
\end{aligned}
$$

where $\beta, \gamma, \sigma$ and $\rho$ are arbitrary functions in $t$. Moreover, any solution polynomial in $x$ and $y$ of (5.3.1) must be of the form $u=T_{1,1 ; \vartheta}^{(0 ; 0, \alpha)}(\psi)$, where $\alpha$ and $\vartheta$ are another two arbitrary functions in $t$.

Proof. We only need to prove the last statement. Suppose that $u$ is a solution of (5.3.1) polynomial in $x$ and $y$. By comparing the term with highest degree of $x, u$ must be of the form (5.3.15) and (5.3.19)-(5.3.22) hold. Since $\xi$ is polynomial in $y,(5.3 .19)$ forces $\xi=0$. Then the conclusion follows from the arguments (5.3.41)-(5.3.45). 


\subsection{Short Wave Equation}

Khristianovich and Rizhov [KR] (1958) discovered the equations of short waves

$$
u_{y}-2 v_{t}-2(v-x) v_{x}-2 k v=0, \quad v_{y}+u_{x}=0
$$

in connection with the nonlinear reflection of weak shock waves, where $k$ is a real constant. Bagdoev and Petrosyan [BP] (1985) showed that the modulation equation of a gas-fluid mixture coincides in main orders with the corresponding short-wave equations. Kraenkel, Manna and Merle [KMM] (2000) studied nonlinear short-wave propagation in ferrites and Ermakov [Es] (2006) investigated short-wave interaction in film slicks. By the second equation in (5.4.1), there exist a potential function $w(t, x, y)$ such that $u=w_{y}$ and $v=-w_{x}$. Then the first equation becomes:

$$
2 w_{t x}-2\left(x+w_{x}\right) w_{x x}+w_{y y}+2 k w_{x}=0 .
$$

To solve the short wave equations (5.4.1) is equivalent to solve the equation (5.4.2). The reader may find the other interesting results in literatures such as $[R R D, K p]$. In this section, we want to solve the short wave equation by the stable-range approach. The results come from our work [X13]

The symmetry group and conservation laws of (5.4.2) were first studied by Kucharczyk [Kp] (1965) and later by Khamitova [Kr] (1982). Let $\alpha$ be a differentiable function in $t$. Note that the transformation $w(t, x, y) \mapsto w(t, x+\alpha, y)$ changes the equation (5.4.2) to

$$
2 \alpha^{\prime} w_{x x}+2 w_{t x}-2\left(x+\alpha+w_{x}\right) w_{x x}+w_{y y}+2 k w_{x}=0
$$

with the independent variables $x$ replaced by $x+\alpha$ and the subindices denoting the partial derivatives with respect to the original independent variables. Moreover, the transformation $w(t, x, y) \mapsto w(t, x, y)+\left(\alpha^{\prime}-\alpha\right) x$ changes the equation (5.4.2) to

$$
2\left(\alpha^{\prime \prime}-\alpha^{\prime}\right)+2 w_{t x}-2 \alpha^{\prime} w_{x x}-2\left(x-\alpha+w_{x}\right) w_{x x}+w_{y y}+2 k w_{x}+2 k\left(\alpha^{\prime}-\alpha\right)=0 .
$$

Furthermore, the transformation $w(t, x, y) \mapsto w(t, x, y)+\left(k \alpha+(1-k) \alpha^{\prime}-\alpha^{\prime \prime}\right) y^{2}$ changes the equation (5.4.2) to

$$
2 w_{t x}-2\left(x+w_{x}\right) w_{x x}+w_{y y}+2\left(k \alpha+(1-k) \alpha^{\prime}-\alpha^{\prime \prime}\right)+2 k w_{x}=0 .
$$

Thus the transformation

$$
T_{2, \alpha}(w(t, x, y))=w(t, x+\alpha, y)+\left(\alpha^{\prime}-\alpha\right) x+\left(k \alpha+(1-k) \alpha^{\prime}-\alpha^{\prime \prime}\right) y^{2}
$$

keeps the equation (5.4.2) invariant with the independent variable $x$ replaced by $x+\alpha$, that is, the transformation $T_{2, \alpha}$ maps a solution of (5.4.2) to another solution. In particular, $T_{2, \alpha}(0)=\left(\alpha^{\prime}-\alpha\right) x+\left(k \alpha+(1-k) \alpha^{\prime}-\alpha^{\prime \prime}\right) y^{2}$ is a solution of the equation (5.4.2). 
Given a differentiable function $\beta$ in $t$, the transformation $w(t, x, y) \mapsto w(t, x, y+\beta)$ changes the equation (5.4.2) to

$$
2 \beta^{\prime} w_{x y}+2 w_{t x}-2\left(x+w_{x}\right) w_{x x}+w_{y y}+2 k w_{x}=0
$$

with the independent variable $y$ replaced by $y+\beta$ and the subindices denoting the partial derivatives with respect to the original independent variables. Moreover, the transformation $w(t, x, y) \mapsto w\left(t, x-\beta^{\prime} y, y\right)$ changes the equation (5.4.2) to

$$
-2 \beta^{\prime \prime} y w_{x x}+2 w_{t x}-2\left(x-\beta^{\prime} y+w_{x}\right) w_{x x}+w_{y y}-2 \beta^{\prime} w_{x y}+\beta^{\prime 2} w_{x x}+2 k w_{x}=0
$$

with the independent variable $x$ replaced by $x-\beta^{\prime} y$. Furthermore, the transformation $w(t, x, y) \mapsto w(t, x, y)+{\beta^{\prime 2}}^{2} x / 2+\left(\beta^{\prime}-\beta^{\prime \prime}\right) x y$ changes the equation (5.4.2) to

$$
\begin{aligned}
& 2 \beta^{\prime} \beta^{\prime \prime}+2\left({\beta^{\prime \prime}}^{\prime}-\beta^{\prime \prime}\right) y+2 w_{t x}-2\left(x+{\beta^{\prime}}^{2} / 2+\left(\beta^{\prime}-\beta^{\prime \prime}\right) y+w_{x}\right) w_{x x} \\
& +w_{y y}+2 k w_{x}+k \beta^{\prime 2}+2 k\left(\beta^{\prime}-\beta^{\prime \prime}\right) y=0 .
\end{aligned}
$$

In addition, the transformation

$$
w(t, x, y) \mapsto w(t, x, y)-\left(\beta^{\prime}{\beta^{\prime \prime}}^{\prime}+k{\beta^{\prime}}^{2} / 2\right) y^{2}+\left({\beta^{\prime \prime}}^{\prime \prime}+(k-1) \beta^{\prime \prime}-k \beta^{\prime}\right) y^{3} / 3
$$

changes the equation (5.4.2) to

$$
2 w_{t x}-2\left(x+w_{x}\right) w_{x x}+w_{y y}-\left(2 \beta^{\prime}{\beta^{\prime \prime}}^{\prime}+k{\beta^{\prime}}^{2}\right)+2\left({\beta^{\prime \prime}}^{\prime \prime}+(k-1) \beta^{\prime \prime}-k \beta^{\prime}\right) y+2 k w_{x}=0 .
$$

Therefore, the transformation

$$
\begin{aligned}
T_{3, \beta}(w(t, x, y))= & w\left(t, x-\beta^{\prime} y, y+\beta\right)+\beta^{2} x / 2+\left(\beta^{\prime}-\beta^{\prime \prime}\right) x y \\
& -\left(\beta^{\prime} \beta^{\prime \prime}+k \beta^{\prime 2} / 2\right) y^{2}+\left({\beta^{\prime \prime}}^{\prime \prime}+(k-1) \beta^{\prime \prime}-k \beta^{\prime}\right) y^{3} / 3
\end{aligned}
$$

leaves the equation (5.4.2) invariant with the independent variables $x$ replaced by $x-\beta^{\prime} y$ and $y$ replaced by $y+\beta$, where the subindices denote the partial derivatives with respect to the original independent variables. In other words, $T_{3, \beta}$ maps a solutions of the equation (5.4.2) to another solution. In particular,

$$
u=T_{3, \beta}(0)=\beta^{\prime 2} x / 2+\left(\beta^{\prime}-\beta^{\prime \prime}\right) x y-\left(\beta^{\prime} \beta^{\prime \prime}+k \beta^{\prime 2} / 2\right) y^{2}+\left(\beta^{\prime \prime \prime}+(k-1) \beta^{\prime \prime}-k \beta^{\prime}\right) y^{3} / 3
$$

is a solution of the equation (5.4.2).

To make each term in (5.4.2) having the same degree, we take

$$
\operatorname{deg} w=2 \operatorname{deg} x=4 \operatorname{deg} y, \quad \operatorname{deg} t=0 .
$$

Thus the transformation

$$
T_{a, b}(w(t, x, y))=b^{-4} w\left(t+a, b^{2} x, b y\right)
$$


keeps the equation (5.4.2) invariant with the independent variables $t$ replaced by $t+a$, where $a, b \in \mathbb{R}$ and $b \neq 0$. In summary, the transformation

$$
\begin{aligned}
& T_{a, b ; \gamma}^{(\alpha, \beta)}(w(t, x, y)) \\
= & b^{-4} w\left(t+a, b^{2}\left(x-\beta^{\prime} y+\alpha\right), b(y+\beta)\right)+\gamma+\left(\beta^{\prime}-\beta^{\prime \prime}\right) x y+\left[k \alpha+(1-k) \alpha^{\prime}-\alpha^{\prime \prime}\right. \\
& \left.-\beta^{\prime}{\beta^{\prime \prime}}^{\prime \prime} k{\beta^{\prime}}^{2} / 2\right] y^{2}+\left({\beta^{\prime \prime}}^{\prime \prime}+(k-1) \beta^{\prime \prime}-k \beta^{\prime}\right) y^{3} / 3+\left({\beta^{\prime}}^{2} / 2+\alpha^{\prime}-\alpha\right) x
\end{aligned}
$$

maps a solutions of the equation (5.4.2) to another solution, where $\alpha, \beta, \gamma$ are functions in $t$ and $a, b \in \mathbb{R}$ with $b \neq 0$.

In this section, we study solutions polynomial in $x$ for the short wave equation (5.4.2). By comparing the terms of highest degree in $x$, we find that such a solution must be of the form:

$$
w=f(t, y)+g(t, y) x+h(t, y) x^{2}+\xi(t, y) x^{3},
$$

where $f(t, y), g(t, y), h(t, y)$ and $\xi(t, y)$ are suitably-differentiable functions to be determined. Note

$$
\begin{gathered}
w_{x}=g+2 h x+3 \xi x^{2}, \quad w_{x x}=2 h+6 \xi x, \\
w_{t x}=g_{t}+2 h_{t} x+3 \xi_{t} x^{2}, \quad w_{y y}=f_{y y}+g_{y y} x+h_{y y} x^{2}+\xi_{y y} x^{3},
\end{gathered}
$$

Now (5.4.2) becomes

$$
\begin{aligned}
& 2\left(g_{t}+2 h_{t} x+3 \xi_{t} x^{2}\right)-2\left(g+(2 h+1) x+3 \xi x^{2}\right)(2 h+6 \xi x) \\
& +f_{y y}+g_{y y} x+h_{y y} x^{2}+\xi_{y y} x^{3}+2 k\left(g+2 h x+3 \xi x^{2}\right)=0,
\end{aligned}
$$

which is equivalent to the following systems of partial differential equations:

$$
\begin{gathered}
\xi_{y y}=36 \xi^{2}, \\
h_{y y}=6 \xi(6 h+2-k)-6 \xi_{t}, \\
g_{y y}=8 h^{2}+4(1-k) h+12 \xi g-4 h_{t}, \\
f_{y y}=4 g h-2 g_{t}-2 k g .
\end{gathered}
$$

First we observe that

$$
\xi=\frac{1}{6 y^{2}}
$$

is a solution of the equation (5.4.21). Substituting (5.4.25) into (5.4.22), we get

$$
h_{y y}=\frac{6 h+2-k}{y^{2}} .
$$

Write

$$
h(t, y)=\sum_{m \in \mathbb{Z}} \frac{a_{m}(t)}{y^{m}}
$$


where $a_{m}(t)$ are functions in $t$ to be determined. Then (5.4.26) is equivalent to

$$
a_{0}=\frac{k-2}{6},[m(m+1)-6] a_{m}=(m+3)(m-2) a_{m}=0, \quad m \neq 0 .
$$

Thus

$$
h=\frac{\alpha}{y^{2}}+\frac{k-2}{6}+\gamma y^{3}
$$

where $\alpha$ and $\gamma$ are arbitrary differentiable functions in $t$. Observe

$$
\xi x^{3}+h x^{2}=\frac{x^{3}+6 \alpha x^{2}}{6 y^{2}}+\frac{k-2}{6} x^{2}+\gamma x^{2} y^{3} .
$$

Replacing $w$ by $T_{2,-2 \alpha}(w)$, we can take $\alpha=0$, that is,

$$
h=\frac{k-2}{6}+\gamma y^{3}
$$

Calculate

$$
h^{2}=\frac{(k-2)^{2}}{36}+\frac{(k-2) \gamma}{3} y^{3}+\gamma^{2} y^{6}
$$

Note (5.4.23) becomes

$$
g_{y y}-\frac{2 g}{y^{2}}=\frac{2(k-2)(1-2 k)}{9}-\frac{4\left[(k+1) \gamma+3 \gamma^{\prime}\right]}{3} y^{3}+8 \gamma^{2} y^{6} .
$$

Write

$$
g(t, y)=\sum_{m \in \mathbb{Z}} b_{m}(t) y^{m},
$$

where $b_{m}(t)$ are functions in $t$ to be determined. Now (5.4.33) is equivalent to

$$
\begin{gathered}
(k-2)(1-2 k)=0, \quad b_{5}=-\frac{2(k+1) \gamma}{27}, \quad b_{8}=\frac{4 \gamma^{2}}{27} \\
m(m+3) b_{m+2}=0, \quad m \neq 0,3,6 .
\end{gathered}
$$

Thus $k=1 / 2,2$ and

$$
g=\frac{\vartheta}{y}+\sigma y^{2}-\frac{2(k+1) \gamma+6 \gamma^{\prime}}{27} y^{5}+\frac{4 \gamma^{2}}{27} y^{8},
$$

where $\vartheta$ and $\sigma$ are arbitrary differentiable functions in $t$.

$$
\begin{aligned}
& \text { Note } \\
& \qquad \begin{array}{c}
g_{t}=\frac{\vartheta^{\prime}}{y}+\sigma^{\prime} y^{2}-\frac{2(k+1) \gamma^{\prime}+6 \gamma^{\prime \prime}}{27} y^{5}+\frac{8 \gamma \gamma^{\prime}}{27} y^{8}, \\
g h=\frac{(k-2) \vartheta}{6 y}+\frac{(k-2) \sigma+6 \gamma \vartheta}{6} y^{2}+\frac{81 \gamma \sigma-\left[(k+1) \gamma+3 \gamma^{\prime}\right](k-2)}{81} y^{5} \\
-\frac{2 \gamma\left[(2 k+5) \gamma+9 \gamma^{\prime}\right]}{81} y^{8}+\frac{4 \gamma^{3}}{27} y^{11} .
\end{array}
\end{aligned}
$$


Thus (5.4.24) becomes

$$
\begin{aligned}
f_{y y}= & -\frac{4(k+1) \vartheta+6 \vartheta^{\prime}}{3 y}-\frac{4(k+1) \sigma+6 \sigma^{\prime}-12 \gamma \vartheta}{3} y^{2}-\frac{40 \gamma\left[(k+1) \gamma+3 \gamma^{\prime}\right]}{81} y^{8} \\
& +\frac{8(k+1)^{2} \gamma+12(3 k+2) \gamma^{\prime}-36 \gamma^{\prime \prime}+244 \gamma \sigma}{81} y^{5}+\frac{16 \gamma^{3}}{27} y^{11} .
\end{aligned}
$$

So

$$
\begin{aligned}
f= & \frac{4(k+1) \vartheta+6 \vartheta^{\prime}}{3} y(1-\ln y)-\frac{2(k+1) \sigma+3 \sigma^{\prime}-6 \gamma \vartheta}{18} y^{4}-\frac{4 \gamma\left[(k+1) \gamma+3 \gamma^{\prime}\right]}{729} y^{10} \\
& +\frac{4(k+1)^{2} \gamma+6(3 k+2) \gamma^{\prime}-18 \gamma^{\prime \prime}+122 \gamma \sigma}{1701} y^{7}+\frac{4 \gamma^{3}}{1053} y^{13}+\varsigma y
\end{aligned}
$$

where $\varsigma$ is an arbitrary functions in $t$.

Theorem 5.4.1. Suppose $k=1 / 2,2$. We have the following solution of the equation (5.4.2):

$$
\begin{aligned}
w= & \psi=\frac{x^{3}}{6 y^{2}}+\left(\frac{k-2}{6}+\gamma y^{3}\right) x^{2}+\left(\frac{\vartheta}{y}+\sigma y^{2}-\frac{2(k+1) \gamma+6 \gamma^{\prime}}{27} y^{5}+\frac{4 \gamma^{2}}{27} y^{8}\right) x+\varsigma y \\
& +\frac{4(k+1) \vartheta+6 \vartheta^{\prime}}{3} y(1-\ln y)-\frac{2(k+1) \sigma+3 \sigma^{\prime}-6 \gamma \vartheta}{18} y^{4}-\frac{4 \gamma\left[(k+1) \gamma+3 \gamma^{\prime}\right]}{729} y^{10} \\
& +\frac{4(k+1)^{2} \gamma+6(3 k+2) \gamma^{\prime}-18 \gamma^{\prime \prime}+122 \gamma \sigma}{1701} y^{7}+\frac{4 \gamma^{3}}{1053} y^{13}+\varsigma y,
\end{aligned}
$$

where $\gamma, \vartheta, \sigma$ and $\varsigma$ are arbitrary functions in $t$, whose derivatives appeared in the above exist in a certain open set of $\mathbb{R}$. Moreover, $w=T_{0,1 ; \zeta}^{(\alpha,-\beta)}(\psi)$ is solution of the equation (5.4.2) blowing up on the moving line $y=\beta(t)$.

The simplest case is

$$
\psi=\frac{x^{3}}{6 y^{2}}+\frac{k-2}{6} x^{2} .
$$

So the simplest solution of the equation (5.4.2) blowing up on the moving line $y=\beta(t)$ is

$$
\begin{aligned}
w= & \frac{\left(x+\beta^{\prime} y\right)^{3}}{6(y-\beta)^{2}}+\frac{k-2}{6}\left(x+\beta^{\prime} y\right)^{2}+\frac{\beta^{\prime 2} x}{2}+\left(\beta^{\prime \prime}-\beta^{\prime}\right) x y \\
& -\left(\beta^{\prime} \beta^{\prime \prime}+\frac{k \beta^{\prime 2}}{2}\right) y^{2}-\frac{\beta^{\prime \prime \prime}+(k-1) \beta^{\prime \prime}-k \beta^{\prime}}{3} y^{3} .
\end{aligned}
$$

Take the trivial solution $\xi=0$ of (5.4.21), which is the only solution polynomial in $y$. Then (5.4.22) and (5.4.23) become

$$
h_{y y}=0, \quad g_{y y}=8 h^{2}+4(1-k) h-4 h_{t},
$$

Replacing $u$ by some $T_{3, \beta}(u)$ if necessary (cf.(5.4.13)), we have $h=\gamma y$ for some function $\gamma$ in $t$. Hence

$$
g_{y y}=8 \gamma^{2} y^{2}+4(1-k) \gamma y-4 \gamma^{\prime} y .
$$


So

$$
g=\frac{2 \gamma^{2}}{3} y^{4}+\frac{2\left[(1-k) \gamma-\gamma^{\prime}\right]}{3} y^{3}+\vartheta y+\rho
$$

for some functions $\vartheta$ and $\rho$ in $t$.

Observe

$$
g h=\frac{2 \gamma^{3}}{3} y^{5}+\frac{2 \gamma\left[(1-k) \gamma-\gamma^{\prime}\right]}{3} y^{4}+\gamma \vartheta y^{2}+\gamma \rho y
$$

and

$$
g_{t}=\frac{4 \gamma \gamma^{\prime}}{3} y^{4}+\frac{2\left[(1-k) \gamma^{\prime}-\gamma^{\prime \prime}\right]}{3} y^{3}+\vartheta^{\prime} y+\rho^{\prime}
$$

Now (5.4.24) yields

$$
\begin{aligned}
f_{y y}= & \frac{8 \gamma^{3}}{3} y^{5}+\frac{4 \gamma\left[(2-3 k) \gamma-4 \gamma^{\prime}\right]}{3} y^{4}-\frac{4\left[k(1-k) \gamma-(2 k-1) \gamma^{\prime}-\gamma^{\prime}\right]}{3} y^{3} \\
& +4 \gamma \vartheta y^{2}+\left(4 \gamma \rho-2 k \vartheta-2 \vartheta^{\prime}\right) y-2 k \rho-2 \rho^{\prime}
\end{aligned}
$$

Replacing $u$ by some $T_{0,1 ; \alpha}^{(0 ; 0,0)}(u)$ if necessary (cf. (5.4.16)), we have

$$
\begin{aligned}
f= & \frac{4 \gamma^{3}}{63} y^{7}+\frac{2 \gamma\left[(2-3 k) \gamma-4 \gamma^{\prime}\right]}{45} y^{6}-\frac{k(1-k) \gamma-(2 k-1) \gamma^{\prime}-\gamma^{\prime \prime}}{15} y^{5} \\
& +\frac{\gamma \vartheta}{3} y^{4}+\frac{2 \gamma \rho-k \vartheta-\vartheta^{\prime}}{3} y^{3}-\left(k \rho+\rho^{\prime}\right) y^{2}+\varsigma y
\end{aligned}
$$

for some function $\varsigma$ of $t$.

Theorem 5.4.2. The following is a solution of the equation (5.4.2):

$$
\begin{aligned}
w= & \varphi=\gamma x^{2} y+\left(\frac{2 \gamma^{2}}{3} y^{4}+\frac{2\left[(1-k) \gamma-\gamma^{\prime}\right]}{3} y^{3}+\vartheta y+\rho\right) x \\
& +\frac{4 \gamma^{3}}{63} y^{7}+\frac{2 \gamma\left[(2-3 k) \gamma-4 \gamma^{\prime}\right]}{45} y^{6}-\frac{k(1-k) \gamma-(2 k-1) \gamma^{\prime}-\gamma^{\prime \prime}}{15} y^{5} \\
& +\frac{\gamma \vartheta}{3} y^{4}+\frac{2 \gamma \rho-k \vartheta-\vartheta^{\prime}}{3} y^{3}-\left(k \rho+\rho^{\prime}\right) y^{2}+\varsigma y
\end{aligned}
$$

where $\gamma, \vartheta, \rho$ and $\varsigma$ are arbitrary functions in $t$, whose derivatives exist as they appear. Moreover, any solution polynomial in $x$ and $y$ of (5.4.2) must be of the above form $w=$ $T_{0,1 ; \alpha}^{(0, \beta)}(\varphi)$, where $\alpha$ and $\beta$ are another arbitrary functions in $t$.

\subsection{Khokhlov and Zabolotskaya Equation}

Khokhlov and Zabolotskaya [KZ] (1969) found the equation

$$
2 u_{t x}+\left(u u_{x}\right)_{x}-u_{y y}=0
$$

for quasi-plane waves in nonlinear acoustics of bounded bundles. More specifically, the equation describes the propagation of a diffraction sound beam in a nonlinear medium. 
Kupershmidt [Kb] (1994) constructed a geometric Hamiltonian form for the KhokhlovZabolotskaya equation (5.5.1). Certain group-invariant solutions of (5.1.1) were found by Korsunskii [Ks] (1991), and by Lin and Zhang [LZ] (1995). Sanchez [Sd] (2005) studied long waves in ferromagnetic media via Khokhlov-Zabolotskaya equation. There are the other interesting results on the equation (e.g., cf. [Gj, KS, KiPg, Mo, RN, Ra1, Ra2, Sf, Va]). In this section, we present the stable-range approach to the equation (5.5.1) due to our work $[\mathrm{X} 13]$.

Suppose

$$
\operatorname{deg} u=\ell_{1}, \quad \operatorname{deg} x=\ell_{2} .
$$

To make each nonzero term in (5.5.1) having the same degree, we have to take

$$
\operatorname{deg} t=\ell_{2}-\ell_{1}, \quad \operatorname{deg} y=\ell_{2}-\frac{1}{2} \ell_{1} .
$$

Since the Khokhlov-Zabolotskaya equation (5.5.1) does not contain variable coefficients, it is translation invariant. Thus the transformation

$$
T_{b_{1}, b_{2}}^{(a)}(u(t, x, y))=b_{1}^{2} u\left(b_{1}^{2} b_{2} t+a, b_{2} x, b_{1} b_{2} y\right)
$$

keeps the Khokhlov-Zabolotskaya equation invariant for $a, b_{1}, b_{2} \in \mathbb{R}$ such that $b_{1}, b_{2} \neq 0$, with the independent variables $t$ replaced by $b_{1}^{2} b_{2} t+a, x$ replaced by $b_{2} x$ and $y$ replaced by $b_{1} b_{2} y$, where the subindices denote the partial derivatives with respect to the original independent variables. So $T_{b_{1}, b_{2}}^{(a)}$ maps a solutions of the equation to another solution.

Let $\alpha$ be differentiable functions in $t$. Then the transformation $u(t, x, y) \mapsto u(t, x+\alpha, y)$ changes the Khokhlov-Zabolotskaya equation to

$$
2 \alpha^{\prime} u_{x x}+2 u_{t x}+\left(u u_{x}\right)_{x}-u_{y y}=0 .
$$

with the independent variables $x$ replaced by $x+\alpha$ and the subindices denoting the partial derivatives with respect to the original independent variables. Furthermore, the transformation $u(t, x, y) \mapsto u(t, x, y)-2 \alpha^{\prime}$ changes the Khokhlov-Zabolotskaya equation to

$$
2 u_{t x}-2 \alpha^{\prime} u_{x x}+\left(u u_{x}\right)_{x}-u_{y y}=0 .
$$

Thus the transformation

$$
T_{2, \alpha}(u(t, x, y))=u(t, x+\alpha, y)-2 \alpha^{\prime}
$$

keep the Khokhlov-Zabolotskaya equation invariant with the independent variables $x$ replaced by $x+\alpha$ and the subindices denoting the partial derivatives with respect to the original independent variables; equivalently, $T_{2, \alpha}$ maps a solutions of the KhokhlovZabolotskaya equation to another solution. 
Given a differentiable function $\beta$ in $t$, the transformation $u(t, x, y) \mapsto u(t, x, y+\beta)$ changes the Khokhlov-Zabolotskaya equation to

$$
2 u_{t x}+2 \beta^{\prime} u_{x y}+\left(u u_{x}\right)_{x}-u_{y y}=0
$$

with the independent variable $y$ replaced by $y+\beta$ and the subindices denoting the partial derivatives with respect to the original independent variables. Moreover, the transformation $u(t, x, y) \mapsto u\left(t, x+\beta^{\prime} y, y\right)$ changes the Khokhlov-Zabolotskaya equation to

$$
2 u_{t x}+2 \beta^{\prime \prime} y u_{x x}+\left(u u_{x}\right)_{x}-u_{y y}-2 \beta^{\prime} u_{x y}-\beta^{\prime 2} u_{x x}=0
$$

with the independent variable $x$ replaced by $x+\beta^{\prime} y$. Furthermore, the transformation $u(t, x, y) \mapsto u(t, x, y)-2{\beta^{\prime \prime}}^{\prime}+{\beta^{\prime 2}}^{2}$ changes the Khokhlov-Zabolotskaya equation to

$$
2 u_{t x}-2 \beta^{\prime \prime} y u_{x x}+\beta^{\prime 2} u_{x x}+\left(u u_{x}\right)_{x}-u_{y y}=0 .
$$

Therefore, the transformation

$$
T_{3, \beta}(u(t, x, y))=u\left(t, x+\beta^{\prime} y, y+\beta\right)+{\beta^{\prime 2}}^{2} 2{\beta^{\prime \prime}}^{\prime}
$$

leaves the equation (5.5.1) invariant with the independent variables $x$ replaced by $x+\beta^{\prime} y$ and $y$ replaced by $y+\beta$, where the subindices denote the partial derivatives with respect to the original independent variables. In other words, $T_{3, \beta}$ maps a solutions of the KhokhlovZabolotskaya equation to another solution.

In summary, the transformation

$$
T_{a ; b_{1}, b_{2}}^{(\alpha, \beta)}(u(t, x, y))=b_{1}^{2} u\left(b_{1}^{2} b_{2} t+a, b_{2}\left(x+\beta^{\prime} y+\alpha\right), b_{1} b_{2}(y+\beta)\right)-2 \alpha^{\prime}+\beta^{\prime 2}-2 \beta^{\prime \prime} y
$$

maps a solutions of the Khokhlov-Zabolotskaya equation to another solution.

Comparing the terms with highest degree of $x$, we find that the solution of the equation (5.5.1) polynomial in $x$ must be of the form

$$
u=f(t, y)+g(t, y) x+\xi(t, y) x^{2}
$$

Then

$$
\begin{gathered}
u_{x}=g+2 \xi x, \quad u_{t x}=g_{t}+2 \xi_{t} x, \quad u_{y y}=f_{y y}+g_{y y} x+\xi_{y y} x^{2} \\
\left(u u_{x}\right)_{x}=\partial_{x}\left(f g+\left(g^{2}+2 f \xi\right) x+3 g \xi x^{2}+2 \xi^{2} x^{3}\right)=g^{2}+2 f \xi+6 g \xi x+6 \xi^{2} x^{2} .
\end{gathered}
$$

Substituting them into (5.5.1), we get

$$
2\left(g_{t}+2 \xi_{t} x\right)+g^{2}+2 f \xi+6 g \xi x+6 \xi^{2} x^{2}-f_{y y}-g_{y y} x-\xi_{y y} x^{2}=0,
$$

equivalently,

$$
\xi_{y y}=6 \xi^{2}
$$




$$
\begin{gathered}
g_{y y}-6 g \xi=4 \xi_{t}, \\
f_{y y}-2 f \xi=2 g_{t}+g^{2} .
\end{gathered}
$$

Recall the Weierstrass's elliptic function $\wp(z)$ defined in (3.4.9). Moreover, $\wp^{\prime \prime}(z)=$ $6 \wp^{2}(z)-g_{2} / 2$ with the $g_{2}$ given in (3.4.29). In (3.4.9), we take $\omega_{1} \in \mathbb{C}$ such that $\operatorname{Re} \omega_{1}, \operatorname{Im} \omega_{1} \neq 0$ and $\omega_{2}=\overline{\omega_{1}}$ for which $g_{2}=0$. Then $\wp(z)$ is real if $z \in \mathbb{R}$. An obvious solution of the equation (5.5.17)-(5.5.19) is $\xi=\wp(y)$ and $g=f=0$. Applying $T_{0 ; 1,1}^{(\alpha, \beta)}$, we obtain a more sophisticated solution

$$
u=\left(x+\beta^{\prime} y+\alpha\right)^{2} \wp(y+\beta)-2 \alpha^{\prime}+{\beta^{\prime}}^{2}-2 \beta^{\prime \prime} y .
$$

Observe that $\xi=1 / y^{2}$ is a solution of the equation (5.5.17). Substituting it into (5.5.18), we obtain

$$
g_{y y}-\frac{6 g}{y^{2}}=0 .
$$

Write

$$
g(t, y)=\sum_{m \in \mathbb{Z}} a_{m}(t) y^{m}
$$

Then (5.5.21) becomes

$$
\sum_{m \in \mathbb{Z}}[(m+2)(m+1)-6] a_{m+2}(t) y^{m}=0 \sim(m+4)(m-1) a_{m+2}=0 \text { for } m \in \mathbb{Z}
$$

Hence

$$
g=\frac{\alpha}{y^{2}}+\beta y^{3},
$$

where $\alpha$ and $\beta$ are arbitrary differentiable functions in $t$. Note

$$
x^{2} \xi+x g=\frac{x^{2}+\alpha x}{y^{2}}+\beta x y^{3} .
$$

Replacing $u$ by $T_{2,-\alpha / 2}(u)$, we can take $\alpha=0$. That is, $g=\beta y^{3}$.

We can write (5.5.19) as

$$
f_{y y}-\frac{2}{y^{2}} f=2 \beta^{\prime} y^{3}+\beta^{2} y^{6} .
$$

Suppose

$$
f(t, y)=\sum_{m \in \mathbb{Z}} b_{m}(t) y^{m}
$$

Then (5.5.26) becomes

$$
\sum_{m \in \mathbb{Z}}[(m+2)(m+1)-2] a_{m+2}(t) y^{m}=2 \beta^{\prime} y^{3}+\beta^{2} y^{6},
$$

equivalently,

$$
18 a_{5}=2 \beta^{\prime}, \quad 54 a_{8}=\beta^{2}, \quad(m+3) m a_{m+2}=0, \quad m \neq 3,6 .
$$


Thus

$$
f=\frac{\gamma}{y}+\vartheta y^{2}+\frac{\beta^{\prime}}{9} y^{5}+\frac{\beta^{2}}{54} y^{8}
$$

where $\gamma$ and $\vartheta$ are arbitrary functions in $t$.

Theorem 5.5.1. We have the following solution of the equation:

$$
u=\varphi=\frac{x^{2}}{y^{2}}+\beta x y^{3}+\frac{\gamma}{y}+\vartheta y^{2}+\frac{\beta^{\prime}}{9} y^{5}+\frac{\beta^{2}}{54} y^{8}
$$

where $\beta, \gamma$ and $\vartheta$ are arbitrary functions in $t$. Moreover, $u=T_{0 ; 1,1}^{(\alpha,-\sigma)}(\varphi)$ is solution of the Khokhlov-Zabolotskaya equation (5.5.1) blowing up on the moving line $y=\sigma(t)$.

The simplest solution of the Khokhlov-Zabolotskaya equation (5.5.1) blowing up on the moving line $y=\sigma(t)$ :

$$
u=\frac{\left(x-\sigma^{\prime} y\right)^{2}}{(y-\sigma)^{2}}+{\sigma^{\prime}}^{2}-2{\sigma^{\prime \prime}}^{\prime}
$$

Suppose that $\xi$ is polynomial in $y$, then $\xi=0$ by comparing the terms with highest degree of $y$ in (5.5.17). Then (5.5.18) and (5.5.19) become

$$
g_{y y}=0, \quad f_{y y}=2 g_{t}+g^{2} .
$$

Replacing $u$ by some $T_{3, \alpha}(u)$ (cf. (5.5.11)), we have $g=\beta y$ for some function $\beta$ in $t$. Hence

$$
f_{y y}=2 \beta^{\prime} y+\beta^{2} y^{2}
$$

So

$$
f=\gamma+\sigma y+\frac{\beta^{\prime}}{3} y^{3}+\frac{\beta^{2}}{12} y^{4}
$$

where $\gamma$ and $\sigma$ are arbitrary functions in $t$.

Theorem 5.5.2. The following is a solution of the Khokhlov-Zabolotskaya equation $(5.5 .1)$ :

$$
u=\psi=\beta x y+\gamma+\sigma y+\frac{\beta^{\prime}}{3} y^{3}+\frac{\beta^{2}}{12} y^{4}
$$

where $\beta, \gamma$ and $\sigma$ are arbitrary functions in $t$. Moreover, any solution polynomial in $x$ and $y$ of (5.5.1) must be of the form $u=T_{3, \alpha}(\psi)$.

\subsection{Equation of Geopotential Forecast}

In a book on short term weather forecast, Kibel' [Kt] (1954) used the partial differential equation

$$
\left(H_{x x}+H_{y y}\right)_{t}+H_{x}\left(H_{x x}+H_{y y}\right)_{y}-H_{y}\left(H_{x x}+H_{y y}\right)_{x}=k H_{x}
$$


for geopotential forecast on a middle level in earth sciences, where $k$ is a real constant. Moreover, Kibel' [Kt] found the Gaurvitz solution of the above equation. Syono [Ss] (1958) got another special solution. The other known solutions are related to the physical backgrounds such as configuration of type of narrow gullies and crests, flows of type of isolate whirlwinds, stream flow, springs and drains, hyperbolic points, and cyclone formation. Katkov [Kv1, Kv2] $(1965,1966)$ determined the Lie point symmetries and obtained certain invariant solutions of the above equation. In this section, we give new approaches to the equation (5.6.1).

To make the nonzero terms in (5.6.1) having the same degree, we suppose

$$
\operatorname{deg} x=\operatorname{deg} y=\ell_{1}, \quad \operatorname{deg} H=\ell_{2} .
$$

Then

$$
\ell_{2}-2 \ell_{1}-\operatorname{deg} t=2 \ell_{2}-4 \ell_{1}=\ell_{2}-\ell_{1} \sim \ell_{2}=3 \ell_{1}, \quad \operatorname{deg} t=-\ell_{1} .
$$

Since (5.6.1) dose not contain variable coefficients, it is translation invariant. Thus the transformation

$$
T_{a, b ; c}(H)=c^{-3} H\left(c^{-1} t+a, c x, c y+b\right)
$$

keeps the equation (5.6.1) invariant for $a, b, c \in \mathbb{R}$ and $c \neq 0$ with the independent variables $t$ replaced by $c^{-1} t+a, x$ replaced by $c x$ and $y$ replaced by $c y+b$, where the subindices denote the partial derivatives with respect to the original independent variables. So $T_{a, b ; c}$ maps a solution of the geopotential equation (5.6.1) to another solution.

Let $\alpha$ and $\beta$ be two differentiable functions in $t$. The transformation $H(t, x, y) \mapsto$ $H(t, x+\alpha, y)$ changes the equation (5.6.1) to

$$
\alpha^{\prime}\left(H_{x x}+H_{y y}\right)_{x}+\left(H_{x x}+H_{y y}\right)_{t}+H_{x}\left(H_{x x}+H_{y y}\right)_{y}-H_{y}\left(H_{x x}+H_{y y}\right)_{x}=k H_{x},
$$

with the independent variables $x$ replaced by $x+\alpha$, where the subindices denote the partial derivatives with respect to the original independent variables. Moreover, the transformation $H(t, x, y) \mapsto H(t, x, y)+\alpha^{\prime} y$ changes the equation (5.6.1) to

$$
\left(H_{x x}+H_{y y}\right)_{t}+H_{x}\left(H_{x x}+H_{y y}\right)_{y}-\left(H_{y}+\alpha^{\prime}\right)\left(H_{x x}+H_{y y}\right)_{x}=k H_{x} .
$$

Hence the transformation

$$
T_{\alpha, \beta}(H)=H(t, x+\alpha, y)+\alpha^{\prime} y+\beta
$$

leaves the equation (5.6.1) invariant with the independent variables $x$ replaced by $x+\alpha$, where the subindices denote the partial derivatives with respect to the original independent variables. Thus $T_{\alpha, \beta}$ maps a solution of the geopotential equation (5.6.1) to another solution. 
In summary, the transformation

$$
T_{a, b ; c}^{(\alpha, \beta)}(H(t, x, y))=c^{-3} H\left(c^{-1} t+a, c(x+\alpha), c y+b\right)+\alpha^{\prime} y+\beta
$$

maps a solution of the geopotential equation (5.6.1) to another solution.

Fix two functions $\alpha$ and $\beta$ in $t$. Denote

$$
\varpi=\alpha x+\beta y .
$$

Assume

$$
H=\phi(t, \varpi)+\mu y^{2}+\tau x+\nu y
$$

where $\phi$ is a two-variable function and $\tau, \mu, \nu$ are functions in $t$. Note

$$
\begin{gathered}
H_{x}=\alpha \phi_{\varpi}+\tau, \quad H_{y}=\beta \phi_{\varpi}+2 \mu y+\nu, \quad H_{x x}+H_{y y}=2 \mu+\left(\alpha^{2}+\beta^{2}\right) \phi_{\varpi \varpi} \\
\left(H_{x x}+H_{y y}\right)_{t}=2 \mu^{\prime}+\left(\alpha^{2}+\beta^{2}\right)^{\prime} \phi_{\varpi \varpi}+\left(\alpha^{2}+\beta^{2}\right)\left[\phi_{t \varpi \varpi}+\left(\alpha^{\prime} x+\beta^{\prime} y\right) \phi_{\varpi \varpi \varpi}\right] \\
\left(H_{x x}+H_{y y}\right)_{x}=\left(\alpha^{2}+\beta^{2}\right) \alpha \phi_{\varpi \varpi \varpi}, \quad\left(H_{x x}+H_{y y}\right)_{y}=\left(\alpha^{2}+\beta^{2}\right) \beta \phi_{\varpi \varpi \varpi} .
\end{gathered}
$$

Thus (5.6.1) becomes

$$
\begin{aligned}
& 2 \mu^{\prime}+\left(\alpha^{2}+\beta^{2}\right)^{\prime} \phi_{\varpi \varpi}+\left(\alpha^{2}+\beta^{2}\right) \phi_{t \varpi \varpi}-k\left(\alpha \phi_{\varpi}+\tau\right) \\
& +\left(\alpha^{2}+\beta^{2}\right)\left[\alpha^{\prime} x+\left(\beta^{\prime}-2 \alpha \mu\right) y+\beta \tau-\alpha \nu\right] \phi_{\varpi \varpi \varpi}=0 .
\end{aligned}
$$

In order to solve the above equation, we assume

$$
2 \mu^{\prime}=k \tau, \quad \tau=\alpha \vartheta^{\prime \prime}, \quad \nu=\beta \vartheta^{\prime \prime},
$$

for some function $\vartheta$ in $t$, and

$$
\alpha^{\prime} x+\left(\beta^{\prime}-2 \alpha \mu\right) y=0
$$

Note that (5.6.16) is equivalent to the following system of ordinary differential equations:

$$
\alpha^{\prime}=0, \quad \beta^{\prime}-2 \alpha \mu=0 .
$$

By the first equation and replacing $H$ by $T_{0,0 ; c}(H)$ (cf. (5.6.8)) if necessary, we have $\alpha=1$. So $\tau=\vartheta^{\prime \prime}$ according to the second equation in (5.6.15). Moreover, the first equation in (5.6.15) yields

$$
\mu=\frac{k \vartheta^{\prime}+c_{0}}{2}, \quad c_{0} \in \mathbb{R} .
$$

Hence the second equation in (5.6.17) becomes

$$
\beta^{\prime}-\left(k \vartheta^{\prime}+c_{0}\right)=0 .
$$


Therefore,

$$
\beta=k \vartheta+c_{0} t+d, \quad d \in \mathbb{R} .
$$

According to the third equation in (5.6.15),

$$
\nu=\left(k \vartheta+c_{0} t+d\right) \vartheta^{\prime \prime} .
$$

Now (5.6.14) becomes

$$
\left(\alpha^{2}+\beta^{2}\right)^{\prime} \phi_{\varpi \varpi}+\left(\alpha^{2}+\beta^{2}\right) \phi_{t \varpi \varpi}-k \phi_{\varpi}=0 .
$$

Replacing $H$ by some $T_{0,0 ; 1}^{(0, \varsigma)}(H)$ if necessary, we have:

$$
\left(\alpha^{2}+\beta^{2}\right)^{\prime} \phi_{\varpi}+\left(\alpha^{2}+\beta^{2}\right) \phi_{t \varpi}-k \phi=0 .
$$

The above equation can written as

$$
\left[\left(\alpha^{2}+\beta^{2}\right) \phi_{\varpi}\right]_{t}-k \phi=0 .
$$

So we take the form

$$
\phi=\frac{\hat{\phi}(t, \varpi)}{\alpha^{2}+\beta^{2}}=\frac{\hat{\phi}(t, \varpi)}{1+\left(k \vartheta+c_{0} t+d\right)^{2}} .
$$

Then (5.6.23) becomes

$$
\hat{\phi}_{\varpi t}=\frac{k \hat{\phi}}{1+\left(k \vartheta+c_{0} t+d\right)^{2}} .
$$

We use the separation of variables

$$
\hat{\phi}=\xi(\varpi) \eta(t),
$$

where $\xi$ and $\eta$ are one-variable functions. Then (5.6.26) becomes

$$
\frac{\xi^{\prime}(\varpi)}{k \xi(\varpi)}=\frac{\eta(t)}{\left(1+\left(k \vartheta+c_{0} t+d\right)^{2}\right) \eta^{\prime}(t)},
$$

which must be a constant. To find more solutions, we assume

$$
\frac{\xi^{\prime}(\varpi)}{k \xi(\varpi)}=\frac{\eta(t)}{\left(1+\left(k \vartheta+c_{0} t+d\right)^{2}\right) \eta^{\prime}(t)}=a+b i \neq 0
$$

for some $a, b \in \mathbb{R}$. Thus $\xi^{\prime}=(a+b i) \xi$ and

$$
\eta^{\prime}=\frac{\eta}{(a+b i)\left(1+\left(k \vartheta+c_{0} t+d\right)^{2}\right)}=\frac{(a-b i) \eta}{\left(a^{2}+b^{2}\right)\left(1+\left(k \vartheta+c_{0} t+d\right)^{2}\right)} .
$$

We have

$$
\xi=e^{k(a+b i) \varpi}, \quad \eta=\exp \left(\frac{a-b i}{a^{2}+b^{2}} \int \frac{d t}{1+\left(k \vartheta+c_{0} t+d\right)^{2}}\right),
$$


that is,

$$
\hat{\phi}=e^{k(a+b i) \varpi} \exp \left(\frac{a-b i}{a^{2}+b^{2}} \int \frac{d t}{1+\left(k \vartheta+c_{0} t+d\right)^{2}}\right)
$$

is a complex solution (5.6.26). Since (5.6.26) is a linear equation with real coefficients, the real part

$$
\begin{aligned}
\zeta_{1}= & \exp \left(k a \varpi+\frac{a}{a^{2}+b^{2}} \int \frac{d t}{1+\left(k \vartheta+c_{0} t+d\right)^{2}}\right) \\
& \times \cos \left(k b \varpi-\frac{b}{a^{2}+b^{2}} \int \frac{d t}{1+\left(k \vartheta+c_{0} t+d\right)^{2}}\right)
\end{aligned}
$$

and the imaginary part

$$
\begin{aligned}
\zeta_{2}= & \exp \left(k a \varpi+\frac{a}{a^{2}+b^{2}} \int \frac{d t}{1+\left(k \vartheta+c_{0} t+d\right)^{2}}\right) \\
& \times \sin \left(k b \varpi-\frac{b}{a^{2}+b^{2}} \int \frac{d t}{1+\left(k \vartheta+c_{0} t+d\right)^{2}}\right)
\end{aligned}
$$

are real solutions of (5.6.26). For any $c \in \mathbb{R}$,

$$
\begin{aligned}
\zeta_{1} \sin c+\zeta_{2} \cos c= & \exp \left(k a \varpi+\frac{a}{a^{2}+b^{2}} \int \frac{d t}{1+\left(k \vartheta+c_{0} t+d\right)^{2}}\right) \\
& \times \sin \left(c+k b \varpi-\frac{b}{a^{2}+b^{2}} \int \frac{d t}{1+\left(k \vartheta+c_{0} t+d\right)^{2}}\right)
\end{aligned}
$$

is a solution of (5.6.26) by the additivity of solutions for linear equation. Applying the additivity again, we have more general solution

$$
\begin{aligned}
\hat{\phi}= & \sum_{r=1}^{m} d_{r} \exp \left(k a_{r} \varpi+\frac{a_{r}}{a_{r}^{2}+b_{r}^{2}} \int \frac{d t}{1+\left(k \vartheta+c_{0} t+d\right)^{2}}\right) \\
& \times \sin \left(k b_{r} \varpi+c_{r}-\frac{b_{r}}{a_{r}^{2}+b_{r}^{2}} \int \frac{d t}{1+\left(k \vartheta+c_{0} t+d\right)^{2}}\right),
\end{aligned}
$$

where $a_{r}, b_{r}, c_{r}, d_{r}$ are real constants such that $\left(a_{r}, b_{r}\right) \neq(0,0)$. By (5.6.10), (5.6.18), (5.6.20), (5.6.21) and (5.6.25), we have:

Theorem 5.6.1. Let $\vartheta$ be any function in $t$ and let $a_{r}, b_{r}, c_{r}, d_{r}, c_{0}, d$ for $r=1, \ldots, m$ be real constants such that $(c, d),\left(a_{r}, b_{r}\right) \neq(0,0)$. We have the following solution of the geopotential forecast equation (5.6.1):

$$
\begin{aligned}
H= & \frac{k \vartheta^{\prime}+c_{0}}{2} y^{2}+\vartheta^{\prime \prime}\left[x+\left(k \vartheta+c_{0} t+d\right) y\right]+\frac{1}{1+\left(k \vartheta+c_{0} t+d\right)^{2}} \\
& \times \sum_{r=1}^{m} d_{r} \exp \left(k a_{r}\left[x+\left(k \vartheta+c_{0} t+d\right) y\right]+\frac{a_{r}}{a_{r}^{2}+b_{r}^{2}} \int \frac{d t}{1+\left(k \vartheta+c_{0} t+d\right)^{2}}\right) \\
& \times \sin \left(k b_{r}\left[x+\left(k \vartheta+c_{0} t+d\right) y\right]+c_{r}-\frac{b_{r}}{a_{r}^{2}+b_{r}^{2}} \int \frac{d t}{1+\left(k \vartheta+c_{0} t+d\right)^{2}}\right) .
\end{aligned}
$$


Applying the transformation $T_{0, b ; c}^{(\alpha, \beta)}$ in (5.6.8) to the above solution, we will get a more general solution the geopotential forecast equation (5.6.1).

Next we set

$$
\varpi=x^{2}+y^{2}
$$

Assume

$$
H=\xi(\varpi)-y
$$

where $\xi$ is a one-variable function. Note

$$
\begin{gathered}
H_{x}=2 x \xi^{\prime}, \quad H_{y}=2 y \xi^{\prime}-1, \quad H_{x x}+H_{y y}=4\left(\xi^{\prime}+\varpi \xi^{\prime \prime}\right), \\
\left(H_{x x}+H_{y y}\right)_{x}=8 x\left(2 \xi^{\prime \prime}+\varpi \xi^{\prime \prime \prime}\right), \quad\left(H_{x x}+H_{y y}\right)_{y}=8 y\left(2 \xi^{\prime \prime}+\varpi \xi^{\prime \prime}\right) .
\end{gathered}
$$

Then (5.6.1) is equivalent to:

$$
4\left(2 \xi^{\prime \prime}+\varpi \xi^{\prime \prime \prime}\right)=k \xi^{\prime}
$$

Replacing $H$ by some $T_{0,0 ; 1}^{(0, \varsigma)}(H)$ if necessary, we have:

$$
\xi^{\prime}+\varpi \xi^{\prime \prime}=\frac{k}{4} \xi
$$

To solve the above ordinary differential equation, we assume

$$
\xi=\sum_{s=0}^{\infty} \varpi^{s}\left(a_{s}+b_{s} \ln \varpi\right), \quad a_{s}, b_{s} \in \mathbb{R} .
$$

Observe

$$
\begin{gathered}
\xi^{\prime}=\sum_{s=0}^{\infty} \varpi^{s-1}\left(s a_{s}+b_{s}+s b_{s} \ln \varpi\right) \\
\xi^{\prime \prime}=\sum_{s=0}^{\infty} \varpi^{s-2}\left(s(s-1) a_{s}+(2 s-1) b_{s}+s(s-1) b_{s} \ln \varpi\right) .
\end{gathered}
$$

So (5.6.43) becomes

$$
\sum_{s=0}^{\infty} \varpi^{s-1}\left(s^{2} a_{s}+2 s b_{s}+s^{2} b_{s} \ln \varpi\right)=\frac{k}{4} \sum_{s=0}^{\infty} \varpi^{s}\left(a_{s}+b_{s} \ln \varpi\right),
$$

equivalently,

$$
(s+1)^{2} a_{s+1}+2(s+1) b_{s+1}=\frac{k}{4} a_{s}, \quad(s+1)^{2} b_{s+1}=\frac{k}{4} b_{s} .
$$

Hence

$$
b_{s}=\frac{b_{0} k^{s}}{(s !)^{2} 4^{s}}, \quad a_{s}=\frac{a_{0} k^{s}}{(s !)^{2} 4^{s}}-\frac{2 b_{0} k^{s}}{(s !)^{2} 4^{s}} \sum_{r=1}^{s} \frac{1}{r} \quad \text { for } s>0 .
$$


Thus

$$
\xi=a_{0} \sum_{s=0}^{\infty} \frac{k^{s} \varpi^{s}}{(s !)^{2} 4^{s}}+b_{0}\left[\ln \varpi+\sum_{j=1}^{\infty} \frac{(k \varpi)^{j}}{(j !)^{2} 4^{j}}\left(\ln \varpi-2 \sum_{r=1}^{j} r^{-1}\right)\right] .
$$

Theorem 5.6.2. Let $b$ and $c$ be any real constants. We have the following steady solution of the geopotential forecast equation (5.6.1):

$$
\begin{aligned}
H= & -y+b \sum_{s=0}^{\infty} \frac{k^{s}\left(x^{2}+y^{2}\right)^{s}}{(s !)^{2} 4^{s}}+c\left[\ln \left(x^{2}+y^{2}\right)\right. \\
& \left.+\sum_{j=1}^{\infty} \frac{\left(k\left(x^{2}+y^{2}\right)\right)^{j}}{(j !)^{2} 4^{j}}\left(\ln \left(x^{2}+y^{2}\right)-2 \sum_{r=1}^{j} r^{-1}\right)\right] .
\end{aligned}
$$

Remark 5.6.3. Although the above solution is time independent, we apply $T_{0,0 ; 1}^{(\alpha, \beta)}$ to it and obtain the following time-dependent solution:

$$
\begin{aligned}
H= & \left(\alpha^{\prime}-1\right) y+\beta+b \sum_{s=0}^{\infty} \frac{k^{s}\left((x+\alpha)^{2}+y^{2}\right)^{s}}{(s !)^{2} 4^{s}}+c\left[\ln \left((x+\alpha)^{2}+y^{2}\right)\right. \\
& \left.+\sum_{j=1}^{\infty} \frac{\left(k\left((x+\alpha)^{2}+y^{2}\right)\right)^{j}}{(j !)^{2} 4^{j}}\left(\ln \left((x+\alpha)^{2}+y^{2}\right)-2 \sum_{r=1}^{j} r^{-1}\right)\right],
\end{aligned}
$$

where $\alpha$ and $\beta$ are arbitrary functions in $t$. 


\section{Chapter 6}

\section{Nonlinear Schrödinger and DS Equations}

The two-dimensional cubic nonlinear Schrödinger equation is used to describe the propagation of an intense laser beam through a medium with Kerr nonlinearity. The coupled two-dimensional cubic nonlinear Schrödinger equations are used to describe interaction of electromagnetic waves with different polarizations in nonlinear optics. In this chapter, we solve the above equations by imposing a quadratic condition on the related argument functions and using their symmetry transformations. More complete families of exact solutions of such type are obtained. Many of them are the periodic, quasi-periodic, aperiodic and singular solutions that may have practical significance.

The Davey-Stewartson equations are used to describe the long time evolution of three-dimensional packets of surface waves. Assuming that the argument functions are quadratic in spacial variables, we find in this chapter various exact solutions for the Davey-Stewartson equations.

\subsection{Nonlinear Schrödinger Equation}

The two-dimensional cubic nonlinear Schrödinger equation

$$
i \psi_{t}+\kappa\left(\psi_{x x}+\psi_{y y}\right)+\varepsilon|\psi|^{2} \psi=0
$$

is used to describe the propagation of an intense laser beam through a medium with Kerr nonlinearity, where $t$ is the distance in the direction of propagation, $x$ and $y$ are the transverse spacial coordinates, $\psi$ is a complex valued function in $t, x, y$ standing for electric field amplitude, and $\kappa, \varepsilon$ are nonzero real constants. Akhnediev, Eleonskii and Kulagin [AEK] (1987) found certain exact solutions of (6.1.1) whose real and imaginary parts are linearly dependent over the functions in $t$. Moreover, Gagnon and Winternitz [GW] (1989) found exact solutions of the cubic and quintic nonlinear Schrödinger equation for a cylindrical geometry. Mihalache and Panoin [MP] (1992) used the method of Akhnediev, 
Eleonskii and Kulagin to obtain new solutions which describe the propagation of dark envelope soliton light pulses in optical fibers under the normal group velocity dispersion regime. Furthermore, Saied, EI-Rahman and Ghonamy [SEG] (2003) used various similarity variables to reduce the above equation to certain ordinary differential equations and obtain some exact solutions. However, many of their solutions are equivalent to each other under the action of the known symmetry transformations of the above equation. There are the other interesting results on the equation (6.1.1) (e.g., cf. [AP, Pa, Sy]).

The objective of this section is to give a direct more systematical study on the exact solutions of the nonlinear Schrödinger equation. We solve them by imposing the quadratic condition on the argument functions and using their symmetry transformations. More complete families of explicit exact solutions of this type with multiple parameter functions are obtained. Many of them are the periodic, quasi-periodic, aperiodic and singular solutions that physicists and engineers expect to know. For instance, soliton solutions are sitting in our families. The results are from our work [X14].

To make the nonzero terms in (6.1.1) to have the same degree, we have to take

$$
\operatorname{deg} x=\operatorname{deg} y=-\operatorname{deg} \psi=\frac{1}{2} \operatorname{deg} t .
$$

Moreover, the Laplace operator $\partial_{x}^{2}+\partial_{y}^{2}$ is invariant under rotations and (6.1.1) is translation invariant because it does not contain variable coefficients. Thus the transformation

$$
T_{a ; b ; \theta}^{\left(a_{1}, a_{2}, a_{3}\right)}(\psi)=b e^{a i} \psi\left(b^{2}\left(t+a_{1}\right), b\left(x \cos \theta+y \sin \theta+a_{2}\right), b\left(-x \sin \theta+y \cos \theta+a_{3}\right)\right)
$$

maps a solution of the Schrödinger equation (6.1.1) to another solution, where $a, a_{1}, a_{2}, a_{3}$, $b, \theta \in \mathbb{R}$ and $b \neq 0$.

Fix $a_{1}, a_{2} \in \mathbb{R}$. Note that the transformation $\psi(t, x, y) \mapsto \psi\left(t, x-2 \kappa a_{1} t, y-2 \kappa a_{2} t\right)$ changes the equation (6.1.1) to

$$
-2 \kappa i\left(a_{1} \psi_{x}+a_{2} \psi_{y}\right)+i \psi_{t}+\kappa\left(\psi_{x x}+\psi_{y y}\right)+\varepsilon|\psi|^{2} \psi=0
$$

with the independent variables $x$ replaced by $x-2 \kappa a_{1} t$ and $y$ replaced by $y-2 \kappa a_{2} t$, where the subindices denote the partial derivatives with respect to the original independent variables. Moreover, the transformation $\psi \mapsto e^{\left[\left(a_{1} x+a_{2} y\right)-\kappa\left(a_{1}^{2}+a_{2}^{2}\right) t\right] i} \psi$ changes the equation (6.1.1) to

$$
e^{\left[\left(a_{1} x+a_{2} y\right)-\kappa\left(a_{1}^{2}+a_{2}^{2}\right) t\right] i}\left[i \psi_{t}+2 \kappa i\left(a_{1} \psi_{x}+a_{2} \psi_{y}\right)+\kappa\left(\psi_{x x}+\psi_{y y}\right)+\varepsilon|\psi|^{2} \psi\right]=0 .
$$

Hence the transformation

$$
S_{a_{1}, a_{2}}(\psi(t, x, y))=e^{\left[\left(a_{1} x+a_{2} y\right)-\kappa\left(a_{1}^{2}+a_{2}^{2}\right) t\right] i} \psi\left(t, x-2 \kappa a_{1} t, y-2 \kappa a_{2} t\right)
$$

changes the equation (6.1.1) to

$$
e^{\left(a_{1} x+a_{2} y\right) i}\left[i \psi_{t}+\kappa\left(\psi_{x x}+\psi_{y y}\right)+\varepsilon|\psi|^{2} \psi\right]=0,
$$


equivalently, (6.1.1) holds with the independent variables $x$ replaced by $x-2 \kappa a_{1} t$ and $y$ replaced by $y-2 \kappa a_{2} t$, where the subindices denote the partial derivatives with respect to the original independent variables. Therefore, $S_{a_{1}, a_{2}}$ maps a solution of the Schrödinger equation (6.1.1) to another solution.

Write

$$
\psi=\xi(t, x, y) e^{i \phi(t, x, y)},
$$

where $\xi$ and $\phi$ are real functions in $t, x, y$. Note

$$
\begin{gathered}
\psi_{t}=\left(\xi_{t}+i \xi \phi_{t}\right) e^{i \phi}, \quad \psi_{x}=\left(\xi_{x}+i \xi \phi_{x}\right) e^{i \phi}, \quad \psi_{y}=\left(\xi_{y}+i \xi \phi_{y}\right) e^{i \phi} \\
\psi_{x x}=\left(\xi_{x x}-\xi \phi_{x}^{2}+i\left(2 \xi_{x} \phi_{x}+\xi \phi_{x x}\right)\right) e^{i \phi}, \quad \psi_{y y}=\left(\xi_{y y}-\xi \phi_{y}^{2}+i\left(2 \xi_{y} \phi_{y}+\xi \phi_{y y}\right)\right) e^{i \phi} .
\end{gathered}
$$

So the equation (6.1.1) becomes

$$
\begin{aligned}
& i \xi_{t}-\phi_{t} \xi+\varepsilon \xi^{3}+\kappa\left[\xi_{x x}+\xi_{y y}-\xi\left(\phi_{x}^{2}+\phi_{y}^{2}\right)\right. \\
& \left.+i\left(2 \xi_{x} \phi_{x}+2 \xi_{y} \phi_{y}+\xi\left(\phi_{x x}+\phi_{y y}\right)\right)\right]=0
\end{aligned}
$$

equivalently,

$$
\begin{gathered}
\xi_{t}+\kappa\left(2 \xi_{x} \phi_{x}+2 \xi_{y} \phi_{y}+\xi\left(\phi_{x x}+\phi_{y y}\right)\right)=0, \\
-\xi\left[\phi_{t}+\kappa\left(\phi_{x}^{2}+\phi_{y}^{2}\right)\right]+\kappa\left(\xi_{x x}+\xi_{y y}\right)+\varepsilon \xi^{3}=0 .
\end{gathered}
$$

Note that it is very difficult to solve the above system without pre-assumptions. From the algebraic characteristics of the above system of partial differential equations, it is most affective to assume that $\phi$ is quadratic in $x$ and $y$. After sorting case by case, we only have the following four cases that lead us to exact solutions of (6.1.12) and (6.1.13).

Case 1. $\phi=\beta(t)$ is a function in $t$.

According to (6.1.12), $\xi_{t}=0$. Moreover, (6.1.13) becomes

$$
-\beta^{\prime} \xi+\kappa\left(\xi_{x x}+\xi_{y y}\right)+\varepsilon \xi^{3}=0 .
$$

Replacing $\psi$ by some $T_{a ; 1 ; 0}^{(0,0,0)}(\psi)$, we have

$$
\beta=b t, \quad b \in \mathbb{R} .
$$

Then (6.1.14) becomes

$$
-b \xi+\kappa\left(\xi_{x x}+\xi_{y y}\right)+\varepsilon \xi^{3}=0 .
$$

First we assume $\xi_{y}=0$. The above equation becomes an ordinary differential equation:

$$
-b \xi+\kappa \xi^{\prime \prime}+\varepsilon \xi^{3}=0 .
$$


Recall

$$
\begin{gathered}
\left(\frac{1}{x}\right)^{\prime \prime}=2\left(\frac{1}{x}\right)^{3}, \\
(\tan z)^{\prime \prime}=2\left(\tan ^{3} z+\tan z\right), \quad(\sec z)^{\prime \prime}=2 \sec ^{3} z-\sec z
\end{gathered}
$$

(cf. (3.5.17) and (3.5.18)),

$$
(\operatorname{coth} z)^{\prime \prime}=2\left(\operatorname{coth}^{3} z-\operatorname{coth} z\right), \quad(\operatorname{csch} z)^{\prime \prime}=2 \operatorname{csch}^{3} z+\operatorname{csch} z
$$

(cf. (3.5.19) and (3.5.20)),

$$
\begin{gathered}
\operatorname{sn}^{\prime \prime}(z \mid m)=2 m^{2} \operatorname{sn}^{3}(z \mid m)-\left(m^{2}+1\right) \operatorname{sn}(z \mid m), \\
\mathrm{cn}^{\prime \prime}(z \mid m)=-2 m^{2} \mathrm{cn}^{3}(z \mid m)+\left(2 m^{2}-1\right) \mathrm{cn}(z \mid m), \\
\operatorname{dn}^{\prime \prime}(z \mid m)=-2 \operatorname{dn}^{3}(z \mid m)+\left(2-m^{2}\right) \operatorname{dn}(z \mid m)
\end{gathered}
$$

(cf. (3.5.14)-(3.5.16)).

Substituting $\xi=k f(x)$ to (6.1.17) with $k \in \mathbb{R}$ and $f=1 / x, \tan x, \sec x, \operatorname{coth} x, \operatorname{csch} x$, sn $(x \mid m), \operatorname{cn}(x \mid m), \operatorname{dn}(x \mid m)$, we find the following solutions: if $\kappa \varepsilon<0$,

$$
\begin{array}{cc}
\xi=\frac{1}{x} \sqrt{-\frac{2 \kappa}{\varepsilon}}, \quad b=0 ; & \\
\xi=\sqrt{-\frac{2 \kappa}{\varepsilon}} \tan x, \quad b=2 \kappa ; \\
\xi=\sqrt{-\frac{2 \kappa}{\varepsilon}} \sec x, \quad b=-\kappa ; \\
\xi=\sqrt{-\frac{2 \kappa}{\varepsilon}} \operatorname{coth} x, \quad b=-2 \kappa ; \\
\xi=\sqrt{-\frac{2 \kappa}{\varepsilon}} \operatorname{csch} x, \quad b=\kappa ; \\
\xi=m \sqrt{-\frac{2 \kappa}{\varepsilon}} \operatorname{sn}(x \mid m), \quad b=-\left(1+m^{2}\right) \kappa .
\end{array}
$$

When $\kappa \varepsilon>0$, we get the following solutions:

$$
\begin{array}{cl}
\xi=m \sqrt{\frac{2 \kappa}{\varepsilon}} \operatorname{cn}(x \mid m), & b=\left(2 m^{2}-1\right) \kappa, \\
\xi=\sqrt{\frac{2 \kappa}{\varepsilon}} \operatorname{dn}(x \mid m), & b=\left(2-m^{2}\right) \kappa .
\end{array}
$$

Observe that

$$
\left(\partial_{x}^{2}+\partial_{y}^{2}\right)\left(\sqrt{\frac{1}{x^{2}+y^{2}}}\right)=\left(\sqrt{\frac{1}{x^{2}+y^{2}}}\right)^{3} .
$$


Thus we have solution

$$
\xi=\sqrt{-\frac{\kappa}{\varepsilon\left(x^{2}+y^{2}\right)}}, \quad b=0
$$

if $\kappa \varepsilon<0$.

Theorem 6.1.1. Let $m \in \mathbb{R}$ such that $0<m<1$. The following functions are solutions $\psi$ of the two-dimensional cubic nonlinear cubic nonlinear Schrödinger equation (6.1.1): if $\varepsilon \kappa<0$,

$$
\begin{gathered}
\sqrt{-\frac{2 \kappa}{\varepsilon}} \frac{1}{x}, \sqrt{-\frac{\kappa}{\varepsilon\left(x^{2}+y^{2}\right)}}, e^{2 \kappa t i} \sqrt{-\frac{2 \kappa}{\varepsilon}} \tan x, e^{-\kappa t i} \sqrt{-\frac{2 \kappa}{\varepsilon}} \sec x, \\
e^{-2 \kappa t i} \sqrt{-\frac{2 \kappa}{\varepsilon}} \operatorname{coth} x, e^{\kappa t i} \sqrt{-\frac{2 \kappa}{\varepsilon}} \operatorname{csch} x, m e^{-\left(1+m^{2}\right) \kappa t i} \sqrt{-\frac{2 \kappa}{\varepsilon}} \operatorname{sn}(x \mid m) ;
\end{gathered}
$$

when $\varepsilon \kappa>0$,

$$
m e^{\left(2 m^{2}-1\right) \kappa t i} \sqrt{\frac{2 \kappa}{\varepsilon}} \operatorname{cn}(x \mid m), \quad e^{\left(2-m^{2}\right) \kappa t i} \sqrt{\frac{2 \kappa}{\varepsilon}} \operatorname{dn}(x \mid m) .
$$

Remark 6.1.2. Recall $\lim _{m \rightarrow 1} \mathrm{cn}(x \mid m)=\operatorname{sech} x$. Thus we have the solution

$$
\psi=\lim _{m \rightarrow 1} m e^{\left(2 m^{2}-1\right) \kappa t i} \sqrt{\frac{2 \kappa}{\varepsilon}} \operatorname{cn}(x \mid m)=e^{\kappa t i} \sqrt{\frac{2 \kappa}{\varepsilon}} \operatorname{sech} x .
$$

Applying the transformation $T_{c ; b ; \theta}^{(0, a, 0)}$ (cf. (6.1.3)) and $S_{d, 0}$ (cf. (6.1.6)), we get a soliton solution

$$
\psi=b \sqrt{\frac{2 \kappa}{\varepsilon}} e^{\left(b^{2} \kappa\left(1-d^{2}\right) t+b d(x \cos \theta+y \sin \theta+a)+c\right) i} \operatorname{sech} b(x \cos \theta+y \sin \theta-2 b d \kappa t+a) .
$$

We can also apply the transformations (6.1.3) and (6.1.6) to the other solutions in the above theorem and obtain more general solutions.

Case 2. $\phi=x^{2} / 4 \kappa t+\beta$ for some function $\beta$ of $t$.

In this case, (6.1.12) becomes

$$
\xi_{t}+\frac{x}{t} \xi_{x}+\frac{1}{2 t} \xi=0
$$

Thus

$$
\xi=\frac{1}{\sqrt{t}} \zeta(u, y), \quad u=\frac{x}{t},
$$

for some two-variable function $\zeta$. Now (6.1.13) becomes (6.1.14). Note

$$
\xi_{x x}=t^{-5 / 2} \zeta_{u u}, \quad \xi_{y y}=t^{-1 / 2} \zeta_{y y}, \quad \xi^{3}=t^{-3 / 2} \zeta^{3} .
$$


So (6.1.14) become

$$
-\frac{\beta^{\prime}}{\sqrt{t}} \zeta+\kappa\left(t^{-5 / 2} \zeta_{u u}+t^{-1 / 2} \zeta_{y y}\right)+\varepsilon t^{-3 / 2} \zeta^{3}=0
$$

whose coefficients of $t^{-3 / 2}$ force us to take

$$
\xi=\frac{b}{\sqrt{t}}, \quad b \in \mathbb{R} .
$$

Now (6.1.14) becomes

$$
-\beta^{\prime}+\frac{\varepsilon b^{2}}{t}=0 \Longrightarrow \beta=\varepsilon b^{2} \ln t
$$

because otherwise we can replace $\psi$ by some $T_{a ; 1 ; 0}^{(0,0,0)}(\psi)$.

Case 3. $\phi=x^{2} / 4 \kappa t+y^{2} / 4 \kappa(t-d)+\beta$ for some function $\beta$ in $t$ with $0 \neq d \in \mathbb{R}$.

In this case, (6.1.12) becomes

$$
\xi_{t}+\frac{x}{t} \xi_{x}+\frac{y}{t-d} \xi_{y}+\left(\frac{1}{2 t}+\frac{1}{2(t-d)}\right) \xi=0 .
$$

Hence we have:

$$
\xi=\frac{1}{\sqrt{t(t-d)}} \zeta(u, v), \quad u=\frac{x}{t}, v=\frac{y}{t-d},
$$

for some two-variable function $\zeta$. Again (6.1.13) becomes (6.1.14). Note

$$
\xi_{x x}=t^{-5 / 2}(t-d)^{-1 / 2} \zeta_{u u}, \xi_{y y}=t^{-1 / 2}(t-d)^{-5 / 2} \zeta_{v v}, \xi^{3}=t^{-3 / 2}(t-d)^{-3 / 2} \zeta^{3} .
$$

So (6.1.14) becomes

$$
-\frac{\beta^{\prime}}{\sqrt{t(t-d)}} \zeta+\kappa\left(t^{-5 / 2}(t-d)^{-1 / 2} \zeta_{u u}+t^{-1 / 2}(t-d)^{-5 / 2} \zeta_{v v}\right)+\varepsilon t^{-3 / 2}(t-d)^{-3 / 2} \zeta^{3}=0,
$$

whose coefficients of $t^{-3 / 2}(t-d)^{-3 / 2}$ force us to take

$$
\xi=\frac{b}{\sqrt{t(t-d)}}, \quad b \in \mathbb{R}
$$

Now (6.1.14) becomes

$$
-\beta^{\prime}+\frac{\varepsilon b^{2}}{t(t-d)}=0 \Longrightarrow \beta=\frac{\varepsilon b^{2}}{d} \ln \frac{t-d}{t}
$$

because otherwise we can replace $\psi$ by some $T_{a ; 1 ; 0}^{(0,0,0)}(\psi)$.

Theorem 6.1.3. Let $b, d \in \mathbb{R}$ with $d \neq 0$. The following functions are solutions $\psi$ of the two-dimensional cubic nonlinear cubic nonlinear Schrödinger equation:

$$
b t^{\varepsilon b^{2} i-1 / 2} e^{x^{2} i / 4 \kappa t}, \quad b t^{-\varepsilon b^{2} i / d-1 / 2}(t-d)^{\varepsilon b^{2} i / d-1 / 2} e^{x^{2} i / 4 \kappa t+y^{2} i / 4 \kappa(t-d)} .
$$


Remark 6.1.4. Applying (6.1.3) to the above first solution, we get another solution

$$
\psi=b(t+a)^{a \kappa b^{2} i-1 / 2} \exp \left(\frac{\left(x \cos \theta+y \sin \theta+a_{0}\right)^{2}}{4 \kappa(t+a)}+d\right) i,
$$

for $a, a_{0}, b, d, \theta \in \mathbb{R}$. Moreover, we obtain a more sophisticated solution:

$$
\begin{aligned}
\psi= & b(t+a)^{\kappa b^{2} i-1 / 2} e^{\left(a_{1} x+a_{2} y-\kappa\left(a_{1}^{2}+a_{2}^{2}\right) t+d\right) i} \\
& \times \exp \frac{\left(\left(x-2 \kappa a_{1} t\right) \cos \theta+\left(y-2 \kappa a_{2} t\right) \sin \theta+a_{0}\right)^{2} i}{4 \kappa(t+a)}
\end{aligned}
$$

by applying the transformation (6.1.6) to (6.1.52), where $a_{1}, a_{2} \in \mathbb{R}$.

Case 4. $\phi=\left(x^{2}+y^{2}\right) / 4 \kappa t+\beta$ for some function $\beta$ in $t$.

Under our assumption, (6.1.12) becomes

$$
\xi_{t}+\frac{x}{t} \xi_{x}+\frac{y}{t} \xi_{y}+\frac{1}{t} \xi=0
$$

Thus we have:

$$
\xi=\frac{1}{t} \zeta(u, v), \quad u=\frac{x}{t}, v=\frac{y}{t},
$$

for some two-variable function $\zeta$. Moreover, (6.1.13) becomes

$$
-\beta^{\prime} \zeta+\frac{\kappa}{t^{2}}\left(\zeta_{u u}+\zeta_{v v}\right)+\frac{\varepsilon}{t^{2}} \zeta^{3}=0 .
$$

An obvious solution is

$$
\zeta=d, \quad \beta=-\frac{\varepsilon d^{2}}{t}, \quad d \in \mathbb{R} .
$$

If $\varepsilon \kappa<0$, we have the simple following solutions with $\beta=0$ :

$$
\zeta=\frac{1}{u} \sqrt{-\frac{2 \kappa}{\varepsilon}} \quad \text { or } \quad \sqrt{-\frac{\kappa}{\varepsilon\left(u^{2}+v^{2}\right)}} .
$$

Next we take

$$
\beta^{\prime}=\frac{b}{t^{2}} \Longrightarrow \beta=-\frac{b}{t},
$$

where $b$ is a real constant to be determined. Then (6.1.58) is equivalent to

$$
-b \zeta+\kappa\left(\zeta_{u u}+\zeta_{v v}\right)+\varepsilon \zeta^{3}=0,
$$

which is the equation of the type (6.1.16). By Theorem 6.1.1, we have:

Theorem 6.1.5. Let $m \in \mathbb{R}$ such that $0<m<1$. The following functions are solutions $\psi$ of the two-dimensional cubic nonlinear cubic nonlinear Schrödinger equation (6.1.1): if $\varepsilon \kappa<0$,

$$
\frac{d}{t} e^{\left(x^{2}+y^{2}-4 \kappa \varepsilon d^{2}\right) i / 4 \kappa t} \sqrt{-\frac{2 \kappa}{\varepsilon}}, e^{\left(x^{2}+y^{2}\right) i / 4 \kappa t} \sqrt{-\frac{2 \kappa}{\varepsilon}} \frac{1}{x}, e^{\left(x^{2}+y^{2}\right) i / 4 \kappa t} \sqrt{-\frac{\kappa}{\varepsilon\left(x^{2}+y^{2}\right)}},
$$




$$
\begin{gathered}
\frac{e^{\left(x^{2}+y^{2}-8 \kappa^{2}\right) i / 4 \kappa t}}{t} \sqrt{-\frac{2 \kappa}{\varepsilon}} \tan \frac{x}{t}, \frac{e^{\left(x^{2}+y^{2}+4 \kappa^{2}\right) i / 4 \kappa t}}{t} \sqrt{-\frac{2 \kappa}{\varepsilon}} \sec \frac{x}{t}, \\
\frac{e^{\left(x^{2}+y^{2}+8 \kappa^{2}\right) i / 4 \kappa t}}{t} \sqrt{-\frac{2 \kappa}{\varepsilon}} \operatorname{coth} \frac{x}{t}, \frac{e^{\left(x^{2}+y^{2}-4 \kappa^{2}\right) i / 4 \kappa t}}{t} \sqrt{-\frac{2 \kappa}{\varepsilon}} \operatorname{csch} \frac{x}{t}, \\
\frac{m e^{\left(x^{2}+y^{2}+4\left(1+m^{2}\right) \kappa^{2}\right) i / 4 \kappa t}}{t} \sqrt{-\frac{2 \kappa}{\varepsilon}} \operatorname{sn}\left(\frac{x}{t} \mid m\right) ;
\end{gathered}
$$

when $\varepsilon \kappa>0$,

$$
\begin{gathered}
\frac{m e^{\left(x^{2}+y^{2}+4\left(1-2 m^{2}\right) \kappa^{2}\right) i / 4 \kappa t}}{t} \sqrt{\frac{2 \kappa}{\varepsilon}} \operatorname{cn}\left(\frac{x}{t} \mid m\right), \\
\frac{e^{\left(x^{2}+y^{2}+4\left(m^{2}-2\right) \kappa^{2}\right) i / 4 \kappa t}}{t} \sqrt{\frac{2 \kappa}{\varepsilon}} \operatorname{dn}\left(\frac{x}{t} \mid m\right) .
\end{gathered}
$$

Remark 6.1.6. Recall $\lim _{m \rightarrow 1} \mathrm{cn}(x \mid m)=\operatorname{sech} x$. Thus we have the solution

$$
\psi=\frac{e^{\left(x^{2}+y^{2}-4 \kappa^{2}\right) i / 4 \kappa t}}{t} \sqrt{\frac{2 \kappa}{\varepsilon}} \operatorname{sech} \frac{x}{t} .
$$

Applying the transformations $T_{0 ; b ; \theta}^{\left(a, a_{2}, 0\right)}$ (cf. (6.1.3)) and $S_{a_{1}, 0}$ (cf. (6.1.6)), we get a more general soliton-like solution

$$
\begin{aligned}
\psi= & \sqrt{\frac{2 \kappa}{\varepsilon}} \frac{e^{\left(\left(x-2 a_{1} \kappa t\right)^{2}+y^{2}-4 \kappa^{2} / b^{2}\right) i / 4 \kappa(t-a)+a_{1}\left(x-a_{1} \kappa t\right) i}}{b(t-a)} \\
& \times \operatorname{sech} \frac{\left(x-2 a_{1} \kappa t\right) \cos \theta+y \sin \theta}{b(t-a)} .
\end{aligned}
$$

Of course, applying the general forms of the transformations in (6.1.3) and (6.1.6) to the solutions in the above theorem, we will get more solutions of the Schrödinger equation.

\subsection{Coupled Schrödinger Equations}

The coupled two-dimensional cubic nonlinear Schrödinger equations

$$
\begin{aligned}
& i \psi_{t}+\kappa_{1}\left(\psi_{x x}+\psi_{y y}\right)+\left(\varepsilon_{1}|\psi|^{2}+\epsilon_{1}|\varphi|^{2}\right) \psi=0 \\
& i \varphi_{t}+\kappa_{2}\left(\varphi_{x x}+\varphi_{y y}\right)+\left(\varepsilon_{2}|\psi|^{2}+\epsilon_{2}|\varphi|^{2}\right) \varphi=0
\end{aligned}
$$

are used to describe interaction of electromagnetic waves with different polarizations in nonlinear optics, where $\kappa_{1}, \kappa_{2}, \varepsilon_{1}, \varepsilon_{2}, \epsilon_{1}$ and $\epsilon_{2}$ are real constants. Radhakrishnan and Lakshmanan [RL1] (1995) used Painlevé analysis to find a Hirota bilinearization of the above system of partial differential equations and obtained bright and dark multiple soliton soutions. They [RL2] (1995) also generalized their results to the coupled nonlinear 
Schrödinger equations with higher-order effects. Grébert and Guillot [GG] (1996) construcetd periodic solutions of coupled one-dimensional nonlinear Schrödinger equations with periodic boundary conditions in some resonance situations. Moreover, Hioe and Salter [HS] (2002) found a connections between Lamé functions and solutions of the above coupled equations. In this section, we want to apply the quadratic-argument approach to the coupled nonlinear Schrödinger equations. Results are due to our work [X14].

As (6.1.3), we have the following symmetric transformations of the coupled equations (6.2.1) and (6.2.2):

$$
\begin{aligned}
& T_{a, a_{0} ; ; ; \theta}^{\left(a_{1}, a_{2}, a_{3}\right)}(\psi)=b e^{a i} \psi\left(b^{2}\left(t+a_{1}\right), b\left(x \cos \theta+y \sin \theta+a_{2}\right), b\left(-x \sin \theta+y \cos \theta+a_{3}\right)\right), \\
& T_{a, a_{0} ; ; ; \theta}^{\left(a_{1}, a_{2}, a_{3}\right)}(\varphi)=b e^{a_{0} i} \varphi\left(b^{2}\left(t+a_{1}\right), b\left(x \cos \theta+y \sin \theta+a_{2}\right), b\left(-x \sin \theta+y \cos \theta+a_{3}\right)\right) .
\end{aligned}
$$

Moreover, (6.1.6) implies the following symmetry

$$
\begin{aligned}
& S_{a_{1}, a_{2}}(\psi(t, x, y))=e^{\left[\left(a_{1} x+a_{2} y\right)-\left(a_{1}^{2}+a_{2}^{2}\right) t\right] i / \kappa_{1}} \psi\left(t, x-2 a_{1} t, y-2 a_{2} t\right), \\
& S_{a_{1}, a_{2}}(\varphi(t, x, y))=e^{\left[\left(a_{1} x+a_{2} y\right)-\left(a_{1}^{2}+a_{2}^{2}\right) t\right] i / \kappa_{2}} \varphi\left(t, x-2 a_{1} t, y-2 a_{2} t\right)
\end{aligned}
$$

of the coupled equations. In addition to the above symmetries, we also solve the coupled equations modulo the following symmetry:

$$
\left(\psi, \kappa_{1}, \varepsilon_{1}, \epsilon_{1}\right) \leftrightarrow\left(\varphi, \kappa_{2}, \varepsilon_{2}, \epsilon_{2}\right)
$$

Write

$$
\psi=\xi(t, x, y) e^{i \phi(t, x, y)}, \quad \varphi=\eta(t, x, y) e^{i \mu(t, x, y)}
$$

where $\xi, \phi, \eta$ and $\mu$ are real functions in $t, x, y$. As the arguments in (6.1.8)-(6.1.13), the system (6.2.1) and (6.2.2) is equivalent to the following system for real functions:

$$
\begin{gathered}
\xi_{t}+\kappa_{1}\left(2 \xi_{x} \phi_{x}+2 \xi_{y} \phi_{y}+\xi\left(\phi_{x x}+\phi_{y y}\right)\right)=0, \\
-\xi\left[\phi_{t}+\kappa_{1}\left(\phi_{x}^{2}+\phi_{y}^{2}\right)\right]+\kappa_{1}\left(\xi_{x x}+\xi_{y y}\right)+\left(\varepsilon_{1} \xi^{2}+\epsilon_{1} \eta^{2}\right) \xi=0, \\
\eta_{t}+\kappa_{2}\left(2 \eta_{x} \mu_{x}+2 \eta_{y} \mu_{y}+\eta\left(\mu_{x x}+\mu_{y y}\right)\right)=0, \\
-\eta\left[\mu_{t}+\kappa_{2}\left(\mu_{x}^{2}+\mu_{y}^{2}\right)\right]+\kappa_{2}\left(\eta_{x x}+\eta_{y y}\right)+\left(\varepsilon_{2} \xi^{2}+\epsilon_{2} \eta^{2}\right) \eta=0 .
\end{gathered}
$$

Based on our experience in last section, we will solve the above system according to the following cases. For the convenience, we always assume the conditions on the constants involved in an expression such that it make sense. For instance, when we use $\sqrt{d_{1}-d_{2}}$, we naturally assume $d_{1} \geq d_{2}$.

Case 1. $(\phi, \mu)=(0,0)$ and $\varepsilon_{1} \epsilon_{2}-\varepsilon_{2} \epsilon_{1} \neq 0$. 
In this case, $\xi_{t}=\eta_{t}=0$ by (6.2.9) and (6.2.11). Moreover, (6.2.10) and (6.2.12) become

$$
\kappa_{1}\left(\xi_{x x}+\xi_{y y}\right)+\left(\varepsilon_{1} \xi^{2}+\epsilon_{1} \eta^{2}\right) \xi=0, \quad \kappa_{2}\left(\eta_{x x}+\eta_{y y}\right)+\left(\varepsilon_{2} \xi^{2}+\epsilon_{2} \eta^{2}\right) \eta=0
$$

where $\iota_{1}$ and $\iota_{2}$ are constants to be determined. Assume

$$
\xi=\frac{\iota_{1}}{x}, \quad \eta=\frac{\iota_{2}}{x}
$$

Then (6.2.13) is equivalent to:

$$
\varepsilon_{1} \iota_{1}^{2}+\epsilon_{1} \iota_{2}^{2}+2 \kappa_{1}=0, \quad \varepsilon_{2} \iota_{1}^{2}+\epsilon_{2} \iota_{2}^{2}+2 \kappa_{2}=0
$$

Solving the above linear algebraic equations for $\iota_{1}^{2}$ and $\iota_{2}^{2}$, we have:

$$
\iota_{1}^{2}=\frac{2\left(\epsilon_{1} \kappa_{2}-\epsilon_{2} \kappa_{1}\right)}{\varepsilon_{1} \epsilon_{2}-\varepsilon_{2} \epsilon_{1}}, \quad \iota_{2}^{2}=\frac{2\left(\varepsilon_{2} \kappa_{1}-\varepsilon_{1} \kappa_{2}\right)}{\varepsilon_{1} \epsilon_{2}-\varepsilon_{2} \epsilon_{1}} .
$$

Thus we have the following solution

$$
\xi=\frac{\sigma_{1}}{x} \sqrt{\frac{2\left(\epsilon_{1} \kappa_{2}-\epsilon_{2} \kappa_{1}\right)}{\varepsilon_{1} \epsilon_{2}-\varepsilon_{2} \epsilon_{1}}}, \quad \eta=\frac{\sigma_{2}}{x} \sqrt{\frac{2\left(\varepsilon_{2} \kappa_{1}-\varepsilon_{1} \kappa_{2}\right)}{\varepsilon_{1} \epsilon_{2}-\varepsilon_{2} \epsilon_{1}}}
$$

for $\sigma_{1}, \sigma_{2} \in\{1,-1\}$. Similarly, we have the solution:

$$
\xi=\sigma_{1} \sqrt{\frac{\epsilon_{1} \kappa_{2}-\epsilon_{2} \kappa_{1}}{\left(\varepsilon_{1} \epsilon_{2}-\varepsilon_{2} \epsilon_{1}\right)\left(x^{2}+y^{2}\right)}}, \quad \eta=\sigma_{2} \sqrt{\frac{\varepsilon_{2} \kappa_{1}-\varepsilon_{1} \kappa_{2}}{\left(\varepsilon_{1} \epsilon_{2}-\varepsilon_{2} \epsilon_{1}\right)\left(x^{2}+y^{2}\right)}} .
$$

Case 2. $(\phi, \mu)=\left(k_{1} t, k_{2} t\right)$ with $k_{1}, k_{2} \in \mathbb{R}$.

Again we have $\xi_{t}=\eta_{t}=0$ by (6.2.9) and (6.2.11). Moreover, (6.2.10) and (6.2.12) become

$$
-k_{1} \xi+\kappa_{1}\left(\xi_{x x}+\xi_{y y}\right)+\left(\varepsilon_{1} \xi^{2}+\epsilon_{1} \eta^{2}\right) \xi=0, \quad-k_{2} \eta+\kappa_{2}\left(\eta_{x x}+\eta_{y y}\right)+\left(\varepsilon_{2} \xi^{2}+\epsilon_{2} \eta^{2}\right) \eta=0
$$

First we assume $\varepsilon_{1} \epsilon_{2}-\varepsilon_{2} \epsilon_{1} \neq 0$ and

$$
\xi=\iota_{1} \Im(x), \quad \eta=\iota_{2} \Im(x),
$$

where $\iota_{1}$ and $\iota_{2}$ are constants to be determined. Then (6.2.19) becomes

$$
-k_{1} \Im+\kappa_{1} \Im^{\prime \prime}+\left(\varepsilon_{1} \iota_{1}^{2}+\epsilon_{1} \iota_{2}^{2}\right) \Im^{3}=0, \quad-k_{2} \Im+\kappa_{2} \Im^{\prime \prime}+\left(\varepsilon_{2} \iota_{1}^{2}+\epsilon_{2} \iota_{2}^{2}\right) \Im^{3}=0 .
$$

According to (3.5.17)-(3.5.20), when $\Im=\tan x, \sec x, \operatorname{coth} x$ and $\operatorname{csch} x$, we always have

$$
\varepsilon_{1} \iota_{1}^{2}+\epsilon_{1} \iota_{2}^{2}+2 \kappa_{1}=0, \quad \varepsilon_{2} \iota_{1}^{2}+\epsilon_{2} \iota_{2}^{2}+2 \kappa_{2}=0
$$


Thus for $\sigma_{1}, \sigma_{2} \in\{1,-1\}$, we have the following solutions:

$$
\xi=\sigma_{1} \sqrt{\frac{2\left(\epsilon_{1} \kappa_{2}-\epsilon_{2} \kappa_{1}\right)}{\varepsilon_{1} \epsilon_{2}-\varepsilon_{2} \epsilon_{1}}} \tan x, \quad \eta=\sigma_{2} \sqrt{\frac{2\left(\varepsilon_{2} \kappa_{1}-\varepsilon_{1} \kappa_{2}\right)}{\varepsilon_{1} \epsilon_{2}-\varepsilon_{2} \epsilon_{1}}} \tan x
$$

with $\left(k_{1}, k_{2}\right)=2\left(\kappa_{1}, \kappa_{2}\right)$;

$$
\xi=\sigma_{1} \sqrt{\frac{2\left(\epsilon_{1} \kappa_{2}-\epsilon_{2} \kappa_{1}\right)}{\varepsilon_{1} \epsilon_{2}-\varepsilon_{2} \epsilon_{1}}} \sec x, \quad \eta=\sigma_{2} \sqrt{\frac{2\left(\varepsilon_{2} \kappa_{1}-\varepsilon_{1} \kappa_{2}\right)}{\varepsilon_{1} \epsilon_{2}-\varepsilon_{2} \epsilon_{1}}} \sec x
$$

with $\left(k_{1}, k_{2}\right)=-\left(\kappa_{1}, \kappa_{2}\right)$;

$$
\xi=\sigma_{1} \sqrt{\frac{2\left(\epsilon_{1} \kappa_{2}-\epsilon_{2} \kappa_{1}\right)}{\varepsilon_{1} \epsilon_{2}-\varepsilon_{2} \epsilon_{1}}} \operatorname{coth} x, \quad \eta=\sigma_{2} \sqrt{\frac{2\left(\varepsilon_{2} \kappa_{1}-\varepsilon_{1} \kappa_{2}\right)}{\varepsilon_{1} \epsilon_{2}-\varepsilon_{2} \epsilon_{1}}} \operatorname{coth} x
$$

with $\left(k_{1}, k_{2}\right)=-2\left(\kappa_{1}, \kappa_{2}\right)$;

$$
\xi=\sigma_{1} \sqrt{\frac{2\left(\epsilon_{1} \kappa_{2}-\epsilon_{2} \kappa_{1}\right)}{\varepsilon_{1} \epsilon_{2}-\varepsilon_{2} \epsilon_{1}}} \operatorname{csch} x, \quad \eta=\sigma_{2} \sqrt{\frac{2\left(\varepsilon_{2} \kappa_{1}-\varepsilon_{1} \kappa_{2}\right)}{\varepsilon_{1} \epsilon_{2}-\varepsilon_{2} \epsilon_{1}}} \operatorname{csch} x
$$

with $\left(k_{1}, k_{2}\right)=\left(\kappa_{1}, \kappa_{2}\right)$. Similarly, (3.5.14)-(3.5.16) give us the following solutions:

$$
\xi=m \sigma_{1} \sqrt{\frac{2\left(\epsilon_{1} \kappa_{2}-\epsilon_{2} \kappa_{1}\right)}{\varepsilon_{1} \epsilon_{2}-\varepsilon_{2} \epsilon_{1}}} \operatorname{sn}(x \mid m), \quad \eta=m \sigma_{2} \sqrt{\frac{2\left(\varepsilon_{2} \kappa_{1}-\varepsilon_{1} \kappa_{2}\right)}{\varepsilon_{1} \epsilon_{2}-\varepsilon_{2} \epsilon_{1}}} \operatorname{sn}(x \mid m)
$$

with $\left(k_{1}, k_{2}\right)=-\left(1+m^{2}\right)\left(\kappa_{1}, \kappa_{2}\right)$;

$$
\xi=m \sigma_{1} \sqrt{\frac{2\left(\epsilon_{2} \kappa_{1}-\epsilon_{1} \kappa_{2}\right)}{\varepsilon_{1} \epsilon_{2}-\varepsilon_{2} \epsilon_{1}}} \operatorname{cn}(x \mid m) \quad \eta=m \sigma_{2} \sqrt{\frac{2\left(\varepsilon_{1} \kappa_{2}-\varepsilon_{2} \kappa_{1}\right)}{\varepsilon_{1} \epsilon_{2}-\varepsilon_{2} \epsilon_{1}}} \operatorname{cn}(x \mid m)
$$

with $\left(k_{1}, k_{2}\right)=\left(2 m^{2}-1\right)\left(\kappa_{1}, \kappa_{2}\right)$;

$$
\xi=\sigma_{1} \sqrt{\frac{2\left(\epsilon_{2} \kappa_{1}-\epsilon_{1} \kappa_{2}\right)}{\varepsilon_{1} \epsilon_{2}-\varepsilon_{2} \epsilon_{1}}} \operatorname{dn}(x \mid m), \quad \eta=\sigma_{2} \sqrt{\frac{2\left(\varepsilon_{1} \kappa_{2}-\varepsilon_{2} \kappa_{1}\right)}{\varepsilon_{1} \epsilon_{2}-\varepsilon_{2} \epsilon_{1}}} \operatorname{dn}(x \mid m)
$$

with $\left(k_{1}, k_{2}\right)=\left(2-m^{2}\right)\left(\kappa_{1}, \kappa_{2}\right)$.

If $\left(\varepsilon_{1}, \epsilon_{1}\right)=\varepsilon_{1}\left(1, d^{2}\right)$ and $\left(\varepsilon_{2}, \epsilon_{2}\right)=\varepsilon_{2}\left(1, d^{2}\right)$ with $d \in \mathbb{R}$, then (6.2.19) becomes

$$
-k_{1} \xi+\kappa_{1}\left(\xi_{x x}+\xi_{y y}\right)+\varepsilon_{1}\left(\xi^{2}+d^{2} \eta^{2}\right) \xi=0, \quad-k_{2} \eta+\kappa_{2}\left(\eta_{x x}+\eta_{y y}\right)+\varepsilon_{2}\left(\xi^{2}+d^{2} \eta^{2}\right) \eta=0 .
$$

The sum of squares and $\sin ^{2} x+\cos ^{2} x=1$ motivate us to try

$$
\xi=d \ell \sin x, \quad \eta=\ell \cos x
$$

for any $0 \neq \ell \in \mathbb{R}$. Substitute them into (6.2.30), we have

$$
-k_{1}-\kappa_{1}+d^{2} \ell^{2} \varepsilon_{1}=0,-k_{2}-\kappa_{2}+d^{2} \ell^{2} \varepsilon_{2}=0 .
$$


So

$$
\left(k_{1}, k_{2}\right)=\left(d^{2} \ell^{2} \varepsilon_{1}-\kappa_{1}, d^{2} \ell^{2} \varepsilon_{2}-\kappa_{2}\right) .
$$

When $\left(\varepsilon_{1}, \epsilon_{1}\right)=\varepsilon_{1}\left(1,-d^{2}\right)$ and $\left(\varepsilon_{2}, \epsilon_{2}\right)=\varepsilon_{2}\left(1,-d^{2}\right)$ with $d \in \mathbb{R}$, then (6.2.19) becomes

$$
-k_{1} \xi+\kappa_{1}\left(\xi_{x x}+\xi_{y y}\right)+\varepsilon_{1}\left(\xi^{2}-d^{2} \eta^{2}\right) \xi=0, \quad-k_{2} \eta+\kappa_{2}\left(\eta_{x x}+\eta_{y y}\right)+\varepsilon_{2}\left(\xi^{2}-d^{2} \eta^{2}\right) \eta=0
$$

The difference of squares and $\cosh ^{2} x-\sinh ^{2} x=1$ motivate us to try

$$
\xi=d \ell \cosh x, \quad \eta=\ell \sinh x
$$

for any $0 \neq \ell \in \mathbb{R}$. Substitute them into (6.2.34), we have

$$
-k_{1}+\kappa_{1}+d^{2} \ell^{2} \varepsilon_{1}=0,-k_{2}+\kappa_{2}+d^{2} \ell^{2} \varepsilon_{2}=0
$$

Hence

$$
\left(k_{1}, k_{2}\right)=\left(d^{2} \ell^{2} \varepsilon_{1}+\kappa_{1}, d^{2} \ell^{2} \varepsilon_{2}+\kappa_{2}\right) .
$$

In summary, we have the following theorem.

Theorem 6.2.1. Let $d, \ell, m \in \mathbb{R}$ with $0<m<1$ and let $\sigma_{1}, \sigma_{2} \in\{1,-1\}$. If $a_{1} \epsilon_{2}-\varepsilon_{2} \epsilon_{1} \neq 0$, we have the following solutions of the coupled two-dimensional cubic nonlinear Schrödinger equations (6.2.1) and (6.2.2):

$$
\begin{aligned}
& \psi=\frac{\sigma_{1}}{x} \sqrt{\frac{2\left(\epsilon_{1} \kappa_{2}-\epsilon_{2} \kappa_{1}\right)}{\varepsilon_{1} \epsilon_{2}-\varepsilon_{2} \epsilon_{1}}}, \quad \varphi=\frac{\sigma_{2}}{x} \sqrt{\frac{2\left(\varepsilon_{2} \kappa_{1}-\varepsilon_{1} \kappa_{2}\right)}{\varepsilon_{1} \epsilon_{2}-\varepsilon_{2} \epsilon_{1}}} ; \\
& \psi=\sigma_{1} \sqrt{\frac{\epsilon_{1} \kappa_{2}-\epsilon_{2} \kappa_{1}}{\left(\varepsilon_{1} \epsilon_{2}-\varepsilon_{2} \epsilon_{1}\right)\left(x^{2}+y^{2}\right)}}, \quad \varphi=\sigma_{2} \sqrt{\frac{\varepsilon_{2} \kappa_{1}-\varepsilon_{1} \kappa_{2}}{\left(\varepsilon_{1} \epsilon_{2}-\varepsilon_{2} \epsilon_{1}\right)\left(x^{2}+y^{2}\right)}} ; \\
& \psi=\sigma_{1} \sqrt{\frac{2\left(\epsilon_{1} \kappa_{2}-\epsilon_{2} \kappa_{1}\right)}{\varepsilon_{1} \epsilon_{2}-\varepsilon_{2} \epsilon_{1}}} e^{2 \kappa_{1} t i} \tan x, \quad \varphi=\sigma_{2} \sqrt{\frac{2\left(\varepsilon_{2} \kappa_{1}-\varepsilon_{1} \kappa_{2}\right)}{\varepsilon_{1} \epsilon_{2}-\varepsilon_{2} \epsilon_{1}}} e^{2 \kappa_{2} t i} \tan x \\
& \psi=\sigma_{1} \sqrt{\frac{2\left(\epsilon_{1} \kappa_{2}-\epsilon_{2} \kappa_{1}\right)}{\varepsilon_{1} \epsilon_{2}-\varepsilon_{2} \epsilon_{1}}} e^{-\kappa_{1} t i} \sec x, \quad \varphi=\sigma_{2} \sqrt{\frac{2\left(\varepsilon_{2} \kappa_{1}-\varepsilon_{1} \kappa_{2}\right)}{\varepsilon_{1} \epsilon_{2}-\varepsilon_{2} \epsilon_{1}}} e^{-\kappa_{2} t i} \sec x \\
& \psi=\sigma_{1} \sqrt{\frac{2\left(\epsilon_{1} \kappa_{2}-\epsilon_{2} \kappa_{1}\right)}{\varepsilon_{1} \epsilon_{2}-\varepsilon_{2} \epsilon_{1}}} e^{-2 \kappa_{1} t i} \operatorname{coth} x, \quad \varphi=\sigma_{2} \sqrt{\frac{2\left(\varepsilon_{2} \kappa_{1}-\varepsilon_{1} \kappa_{2}\right)}{\varepsilon_{1} \epsilon_{2}-\varepsilon_{2} \epsilon_{1}}} e^{-2 \kappa_{2} t i} \operatorname{coth} x ; \\
& \psi=\sigma_{1} \sqrt{\frac{2\left(\epsilon_{1} \kappa_{2}-\epsilon_{2} \kappa_{1}\right)}{\varepsilon_{1} \epsilon_{2}-\varepsilon_{2} \epsilon_{1}}} e^{\kappa_{1} t i} \operatorname{csch} x, \quad \varphi=\sigma_{2} \sqrt{\frac{2\left(\varepsilon_{2} \kappa_{1}-\varepsilon_{1} \kappa_{2}\right)}{\varepsilon_{1} \epsilon_{2}-\varepsilon_{2} \epsilon_{1}}} e^{\kappa_{2} t i} \operatorname{csch} x \\
& \psi=m \sigma_{1} \sqrt{\frac{2\left(\epsilon_{1} \kappa_{2}-\epsilon_{2} \kappa_{1}\right)}{\varepsilon_{1} \epsilon_{2}-\varepsilon_{2} \epsilon_{1}}} e^{-\left(1+m^{2}\right) \kappa_{1} t i} \operatorname{sn}(x \mid m), \\
& \varphi=m \sigma_{2} \sqrt{\frac{2\left(\varepsilon_{2} \kappa_{1}-\varepsilon_{1} \kappa_{2}\right)}{\varepsilon_{1} \epsilon_{2}-\varepsilon_{2} \epsilon_{1}}} e^{-\left(1+m^{2}\right) \kappa_{2} t i} \operatorname{sn}(x \mid m)
\end{aligned}
$$




$$
\begin{aligned}
& \psi=m \sigma_{1} \sqrt{\frac{2\left(\epsilon_{2} \kappa_{1}-\epsilon_{1} \kappa_{2}\right)}{\varepsilon_{1} \epsilon_{2}-\varepsilon_{2} \epsilon_{1}}} e^{\left(2 m^{2}-1\right) \kappa_{1} t i} \operatorname{cn}(x \mid m), \\
& \varphi=m \sigma_{2} \sqrt{\frac{2\left(\varepsilon_{1} \kappa_{2}-\varepsilon_{2} \kappa_{1}\right)}{\varepsilon_{1} \epsilon_{2}-\varepsilon_{2} \epsilon_{1}}} e^{\left(2 m^{2}-1\right) \kappa_{2} t i} \operatorname{cn}(x \mid m) \\
& \psi=\sigma_{1} \sqrt{\frac{2\left(\epsilon_{2} \kappa_{1}-\epsilon_{1} \kappa_{2}\right)}{\varepsilon_{1} \epsilon_{2}-\varepsilon_{2} \epsilon_{1}}} e^{\left(2-m^{2}\right) \kappa_{1} t i} \operatorname{dn}(x \mid m), \\
& \varphi=\sigma_{2} \sqrt{\frac{2\left(\varepsilon_{1} \kappa_{2}-\varepsilon_{2} \kappa_{1}\right)}{\varepsilon_{1} \epsilon_{2}-\varepsilon_{2} \epsilon_{1}}} e^{\left(2-m^{2}\right) \kappa_{1} t i} \operatorname{dn}(x \mid m) . \\
& \text { If }\left(\varepsilon_{1}, \epsilon_{1}\right)=\varepsilon_{1}\left(1, d^{2}\right) \text { and }\left(\varepsilon_{2}, \epsilon_{2}\right)=\varepsilon_{2}\left(1, d^{2}\right) \text {, } \\
& \psi=d \ell e^{\left(d^{2} \ell^{2} \varepsilon_{1}-\kappa_{1}\right) t i} \sin x, \quad \varphi=\ell e^{\left(d^{2} \ell^{2} \varepsilon_{2}-\kappa_{2}\right) t i} \cos x .
\end{aligned}
$$

When $\left(\varepsilon_{1}, \epsilon_{1}\right)=\varepsilon_{1}\left(1,-d^{2}\right)$ and $\left(\varepsilon_{2}, \epsilon_{2}\right)=\varepsilon_{2}\left(1,-d^{2}\right)$,

$$
\psi=d \ell e^{\left(d^{2} \ell^{2} \varepsilon_{1}+\kappa_{1}\right) t i} \cosh x, \quad \eta=\ell e^{\left(d^{2} \ell^{2} \varepsilon_{2}+\kappa_{2}\right) t i} \sinh x .
$$

Remark 6.2.2. Applying the symmetric transformations (6.2.3)-(6.2.6) to the above solutions, we can get more sophisticated ones. For instance, by (6.2.38), we get the following traveling-wave solution

$$
\begin{gathered}
\psi=\frac{\sigma_{1} e^{a i+a_{1}\left(x \cos \theta+y \sin \theta+a_{2}-a_{1} t\right) i / \kappa_{1}}}{x \cos \theta+y \sin \theta-2 a_{1} t+a_{2}} \sqrt{\frac{2\left(\epsilon_{1} \kappa_{2}-\epsilon_{2} \kappa_{1}\right)}{\varepsilon_{1} \epsilon_{2}-\varepsilon_{2} \epsilon_{1}}}, \\
\varphi=\frac{\sigma_{2} e^{a_{0} i+a_{1}\left(x \cos \theta+y \sin \theta+a_{2}-a_{1} t\right) i / \kappa_{2}}}{x \cos \theta+y \sin \theta-2 a_{1} t+a_{2}} \sqrt{\frac{2\left(\varepsilon_{2} \kappa_{1}-\varepsilon_{1} \kappa_{2}\right)}{\varepsilon_{1} \epsilon_{2}-\varepsilon_{2} \epsilon_{1}}} .
\end{gathered}
$$

Since $\lim _{m \rightarrow 1} c n(x \mid m)=\operatorname{sech} x,(6.2 .46)$ and (6.2.47) yield the solution

$$
\psi=\sigma_{1} \sqrt{\frac{2\left(\epsilon_{2} \kappa_{1}-\epsilon_{1} \kappa_{2}\right)}{\varepsilon_{1} \epsilon_{2}-\varepsilon_{2} \epsilon_{1}}} e^{\kappa_{1} t i} \operatorname{sech} x, \quad \varphi=\sigma_{2} \sqrt{\frac{2\left(\varepsilon_{1} \kappa_{2}-\varepsilon_{2} \kappa_{1}\right)}{\varepsilon_{1} \epsilon_{2}-\varepsilon_{2} \epsilon_{1}}} e^{\kappa_{2} t i} \operatorname{sech} x .
$$

The symmetric transformations (6.2.3)-(6.2.6) give us the following soliton solution

$$
\begin{aligned}
\psi= & b \sigma_{1} \sqrt{\frac{2\left(\epsilon_{2} \kappa_{1}-\epsilon_{1} \kappa_{2}\right)}{\varepsilon_{1} \epsilon_{2}-\varepsilon_{2} \epsilon_{1}}} e^{\left(b^{2} \kappa_{1} t+a\right) i+a_{1} b\left(x \cos \theta+y \sin \theta+a_{2}-a_{1} b t\right) i / \kappa_{1}} \\
& \times \operatorname{sech} b\left(x \cos \theta+y \sin \theta-2 a_{1} b t+a_{2}\right), \\
\varphi= & b \sigma_{2} \sqrt{\frac{2\left(\varepsilon_{1} \kappa_{2}-\varepsilon_{2} \kappa_{1}\right)}{\varepsilon_{1} \epsilon_{2}-\varepsilon_{2} \epsilon_{1}}} e^{\left(b^{2} \kappa_{2} t+a_{0}\right) i+a_{1} b\left(x \cos \theta+y \sin \theta+a_{2}-a_{1} b t\right) i / \kappa_{2}} \\
& \times \operatorname{sech} b\left(x \cos \theta+y \sin \theta-2 a_{1} b t+a_{2}\right) .
\end{aligned}
$$


If $\left(\varepsilon_{1}, \epsilon_{1}\right)=\varepsilon_{1}\left(1, d^{2}\right)$ and $\left(\varepsilon_{2}, \epsilon_{2}\right)=\varepsilon_{2}\left(1, d^{2}\right),(6.2 .3)-(6.2 .6)$ and (6.2.50) yield the following wave solution

$$
\begin{aligned}
\psi= & b d \ell e^{\left[b^{2}\left(d^{2} \ell^{2} \varepsilon_{1}-\kappa_{1}\right) t+a\right] i+a_{1} b\left(x \cos \theta+y \sin \theta+a_{2}-a_{1} b t\right) i / \kappa_{1}} \\
& \times \sin b\left(x \cos \theta+y \sin \theta-2 a_{1} b t+a_{2}\right), \\
\varphi= & b \ell e^{\left[b^{2}\left(d^{2} \ell^{2} \varepsilon_{2}-\kappa_{2}\right) t+a_{0}\right] i+a_{1} b\left(x \cos \theta+y \sin \theta+a_{2}-a_{1} b t\right) i / \kappa_{2}} \\
& \times \cos b\left(x \cos \theta+y \sin \theta-2 a_{1} b t+a_{2}\right) .
\end{aligned}
$$

Case 3. $\phi=x^{2} / 4 \kappa_{1} t+\beta_{1}$ and $\mu=(x-d)^{2} / 4 \kappa_{2}(t-\ell)+\beta_{2}$ or $\mu=y^{2} / 4 \kappa_{2}(t-\ell)+\beta_{2}$ for some functions $\beta_{1}$ and $\beta_{2}$ in $t$ and real constants $d$ and $\ell$.

First we assume $\mu=(x-d)^{2} / 4 \kappa_{2}(t-\ell)+\beta_{2}$. Then (6.2.9) and (6.2.11) become

$$
\xi_{t}+\frac{x}{t} \xi_{x}+\frac{1}{2 t} \xi=0, \quad \eta_{t}+\frac{x-d}{t-\ell} \eta_{x}+\frac{1}{2(t-\ell)} \eta=0
$$

Thus

$$
\xi=\frac{1}{\sqrt{t}} \hat{\xi}\left(t^{-1} x, y\right), \quad \eta=\frac{1}{\sqrt{t-\ell}} \hat{\eta}\left((t-\ell)^{-1}(x-d), y\right)
$$

for some two-variable functions $\hat{\xi}$ and $\hat{\eta}$. On the other hand, (6.2.10) and (6.2.12) become

$$
\begin{aligned}
& -\beta_{1}^{\prime} \xi+\kappa_{1}\left(\xi_{x x}+\xi_{y y}\right)+\left(\varepsilon_{1} \xi^{2}+\epsilon_{1} \eta^{2}\right) \xi=0 \\
& -\beta_{2}^{\prime} \eta+\kappa_{2}\left(\eta_{x x}+\eta_{y y}\right)+\left(\varepsilon_{2} \xi^{2}+\epsilon_{2} \eta^{2}\right) \eta=0 .
\end{aligned}
$$

As (6.1.40)-(6.1.43), the above two equations force us to take

$$
\xi=\frac{c_{1}}{\sqrt{t}}, \quad \eta=\frac{c_{2}}{\sqrt{t-\ell}}
$$

So (6.2.61) and (6.2.62) are implied by the equations:

$$
\beta_{1}^{\prime}=\frac{c_{1}^{2} \varepsilon_{1}}{t}+\frac{c_{2}^{2} \epsilon_{1}}{t-\ell}, \quad \beta_{2}^{\prime}=\frac{c_{1}^{2} \varepsilon_{2}}{t}+\frac{c_{2}^{2} \epsilon_{2}}{t-\ell} .
$$

For simplicity, we take

$$
\beta_{1}=c_{1}^{2} \varepsilon_{1} \ln t+c_{2}^{2} \epsilon_{1} \ln (t-\ell), \quad \beta_{2}=c_{1}^{2} \varepsilon_{2} \ln t+c_{2}^{2} \epsilon_{2} \ln (t-\ell)
$$

Exact same approach holds for $\mu=y^{2} / 4 \kappa_{2}(t-\ell)+\beta_{2}$.

Theorem 6.2.3. Let $c_{1}, c_{2}, d, \ell \in \mathbb{R}$. We have the following solutions of the coupled two-dimensional cubic nonlinear Schrödinger equations (6.2.1) and (6.2.2):

$$
\psi=c_{1} t^{c_{1}^{2} \varepsilon_{1} i-1 / 2}(t-\ell)^{c_{2}^{2} \epsilon_{1} i} e^{x^{2} i / 2 \kappa_{1} t}, \quad \varphi=c_{2} t^{c_{1}^{2} \varepsilon_{2} i}(t-\ell)^{c_{2}^{2} \epsilon_{2} i-1 / 2} e^{(x-d)^{2} i / 2 \kappa_{2}(t-\ell)}
$$




$$
\psi=c_{1} t^{c_{1}^{2} \varepsilon_{1} i-1 / 2}(t-\ell)^{c_{2}^{2} \epsilon_{1} i} e^{x^{2} i / 2 \kappa_{1} t}, \quad \varphi=c_{2} t^{c_{1}^{2} \varepsilon_{2} i}(t-\ell)^{c_{2}^{2} \epsilon_{2} i-1 / 2} e^{y^{2 i} / 2 \kappa_{2}(t-\ell)} .
$$

Case 4. $\phi=x^{2} / 4 \kappa_{1} t+\beta_{1}$ and $\mu=(x-d)^{2} / 4 \kappa_{2}\left(t-\ell_{1}\right)+y^{2} / 4 \kappa_{2}\left(t-\ell_{2}\right)+\beta_{2}$ for some functions $\beta_{1}$ and $\beta_{2}$ in $t$ and real constants $d, \ell_{1}$ and $\ell_{2}$.

In this case, (6.2.9) and (6.2.11) become

$$
\xi_{t}+\frac{x}{t} \xi_{x}+\frac{1}{2 t} \xi=0, \quad \eta_{t}+\frac{x-d}{t-\ell_{1}} \eta_{x}+\frac{y}{t-\ell_{2}} \eta_{y}+\left(\frac{1}{2\left(t-\ell_{1}\right)}+\frac{1}{2\left(t-\ell_{2}\right)}\right) \xi=0 .
$$

Thus

$$
\xi=\frac{1}{\sqrt{t}} \hat{\xi}\left(t^{-1} x, y\right), \quad \eta=\frac{1}{\sqrt{\left(t-\ell_{1}\right)\left(t-\ell_{2}\right)}} \hat{\eta}\left(\left(t-\ell_{1}\right)^{-1}(x-d),\left(t-\ell_{2}\right)^{-1} y\right)
$$

for some two-variable functions $\hat{\xi}$ and $\hat{\eta}$ by the method of characteristic lines in Section 4.1. Again (6.2.10) and (6.2.12) become (6.2.61) and (6.2.62), respectively. Moreover, they force us to take

$$
\xi=\frac{c_{1}}{\sqrt{t}}, \quad \eta=\frac{c_{2}}{\sqrt{\left(t-\ell_{1}\right)\left(t-\ell_{2}\right)}} .
$$

So (6.2.10) and (6.2.12) are implied by the equations:

$$
\beta_{1}^{\prime}=\frac{c_{1}^{2} \varepsilon_{1}}{t}+\frac{c_{2}^{2} \epsilon_{1}}{\left(t-\ell_{1}\right)\left(t-\ell_{2}\right)}, \quad \beta_{2}^{\prime}=\frac{c_{1}^{2} \varepsilon_{2}}{t}+\frac{c_{2}^{2} \epsilon_{2}}{\left(t-\ell_{1}\right)\left(t-\ell_{2}\right)} .
$$

For simplicity, we get

$$
\beta_{1}=c_{1}^{2} \varepsilon_{1} \ln t+\frac{c_{2}^{2} \epsilon_{1}}{\ell_{2}-\ell_{1}} \ln \frac{t-\ell_{1}}{t-\ell_{2}}, \quad \beta_{2}=\varepsilon_{2} c_{1}^{2} \ln t+\frac{\epsilon_{2} c_{2}^{2}}{\ell_{2}-\ell_{1}} \ln \frac{t-\ell_{1}}{t-\ell_{2}}
$$

if $\ell_{1} \neq \ell_{2}$, and

$$
\beta_{1}=c_{1}^{2} \varepsilon_{1} \ln t-\frac{c_{2}^{2} \epsilon_{1}}{t-\ell_{1}}, \quad \quad \beta_{2}=c_{1}^{2} \varepsilon_{2} \ln t-\frac{c_{2}^{2} \epsilon_{2}}{t-\ell_{1}}
$$

when $\ell_{1}=\ell_{2}$.

Theorem 6.2.4. Let $c_{1}, c_{2}, \ell_{1}, \ell_{2} \in \mathbb{R}$ such that $\ell_{1} \neq \ell_{2}$. We have the following solutions of the coupled two-dimensional cubic nonlinear Schrödinger equations (6.2.1) and (6.2.2):

$$
\begin{aligned}
\psi= & c_{1} t^{c_{1}^{2} \varepsilon_{1} i-1 / 2}\left(t-\ell_{1}\right)^{c_{2}^{2} \epsilon_{1}\left(\ell_{2}-\ell_{1}\right)^{-1} i}\left(t-\ell_{2}\right)^{-c_{2}^{2} \epsilon_{1}\left(\ell_{2}-\ell_{1}\right)^{-1} i} e^{x^{2} i / 4 \kappa_{1} t} \\
\varphi= & c_{2} t^{c_{1}^{2} \varepsilon_{2} i}\left(t-\ell_{1}\right)^{c_{2}^{2} \epsilon_{2}\left(\ell_{2}-\ell_{1}\right)^{-1} i-1 / 2}\left(t-\ell_{2}\right)^{-c_{2}^{2} \epsilon_{2}\left(\ell_{2}-\ell_{1}\right)^{-1} i-1 / 2} \\
& \times \exp \left(\frac{(x-d)^{2} i}{4 \kappa_{2}\left(t-\ell_{1}\right)}+\frac{y^{2} i}{4 \kappa_{2}\left(t-\ell_{1}\right)}\right)
\end{aligned}
$$




$$
\begin{gathered}
\psi=c_{1} t^{c_{1}^{2} \varepsilon_{1} i-1 / 2} \exp \left(\frac{x^{2} i}{4 \kappa_{1} t}-\frac{c_{2}^{2} \epsilon_{1} i}{t-\ell_{1}}\right), \\
\varphi=\frac{c_{2} t^{c_{1}^{2} \varepsilon_{2} i}}{t-\ell_{1}} \exp \frac{\left((x-d)^{2}+y^{2}-4 c_{2}^{2} \kappa_{2} \epsilon_{2}\right) i}{4 \kappa_{2}\left(t-\ell_{1}\right)} .
\end{gathered}
$$

Case 5. For $\ell_{1}, \ell_{2}, \ell, d_{1}, d_{2} \in \mathbb{R}$ and functions $\beta_{1}, \beta_{2}$ in $t$,

$$
\phi=\frac{x^{2}}{4 \kappa_{1} t}+\frac{y^{2}}{4 \kappa_{1}(t-\ell)}+\beta_{1}, \quad \mu=\frac{\left(x-d_{1}\right)^{2}}{4 \kappa_{2}\left(t-\ell_{1}\right)}+\frac{\left(y-d_{2}\right)^{2}}{4 \kappa_{1}\left(t-\ell_{2}\right)}+\beta_{2} .
$$

As the above case, we get

$$
\xi=\frac{c_{1}}{\sqrt{t(t-\ell)}}, \quad \eta=\frac{c_{2}}{\sqrt{\left(t-\ell_{1}\right)\left(t-\ell_{2}\right)}} .
$$

So (6.2.10) and (6.2.12) are implied by the equations:

$$
\beta_{1}^{\prime}=\frac{c_{1}^{2} \varepsilon_{1}}{t(t-\ell)}+\frac{c_{2}^{2} \epsilon_{1}}{\left(t-\ell_{1}\right)\left(t-\ell_{2}\right)}, \quad \beta_{2}^{\prime}=\frac{c_{1}^{2} \varepsilon_{2}}{t}+\frac{c_{2}^{2} \epsilon_{2}}{\left(t-\ell_{1}\right)\left(t-\ell_{2}\right)} .
$$

For simplicity, we have

$$
\beta_{1}=\frac{c_{1}^{2} \varepsilon_{1}}{\ell} \ln \frac{t-\ell}{t}+\frac{c_{2}^{2} \epsilon_{1}}{\ell_{2}-\ell_{1}} \ln \frac{t-\ell_{1}}{t-\ell_{2}}, \quad \beta_{2}=\frac{c_{1}^{2} \varepsilon_{2}}{\ell} \ln \frac{t-\ell}{t}+\frac{c_{2}^{2} \epsilon_{2}}{\ell_{2}-\ell_{1}} \ln \frac{t-\ell_{1}}{t-\ell_{2}}
$$

if $\ell \neq 0$ and $\ell_{1} \neq \ell_{2}$;

$$
\beta_{1}=-\frac{c_{1}^{2} \varepsilon_{1}}{t}+\frac{c_{2}^{2} \epsilon_{1}}{\ell_{2}-\ell_{1}} \ln \frac{t-\ell_{1}}{t-\ell_{2}}, \quad \beta_{2}=-\frac{c_{1}^{2} \varepsilon_{2}}{t}+\frac{c_{2}^{2} \epsilon_{2}}{\ell_{2}-\ell_{1}} \ln \frac{t-\ell_{1}}{t-\ell_{2}}
$$

when $\ell=0$ and $\ell_{1} \neq \ell_{2}$;

$$
\beta_{1}=\frac{c_{1}^{2} \varepsilon_{1}}{t}-\frac{c_{2}^{2} \epsilon_{1}}{t-\ell_{1}}, \quad \beta_{2}=\frac{c_{1}^{2} \varepsilon_{2}}{t} t-\frac{c_{2}^{2} \epsilon_{2}}{t-\ell_{1}}
$$

if $\ell=0$ and $\ell_{1}=\ell_{2}$. Therefore, we obtain:

Theorem 6.2.5. Let $c_{1}, c_{2}, \ell, d_{1}, d_{2}, \ell_{1}, \ell_{2} \in \mathbb{R}$ such that $\ell \neq 0$ and $\ell_{1} \neq \ell_{2}$. We have the following solutions of the coupled two-dimensional cubic nonlinear Schrödinger equations (6.2.1) and (6.2.2):

$$
\begin{gathered}
\psi=\frac{c_{1}}{t} \exp \left(\frac{\left(x^{2}+y^{2}-4 c_{1}^{2} \kappa_{1} \varepsilon_{1}\right) i}{4 \kappa_{1} t}-\frac{c_{2}^{2} \epsilon_{1} i}{t-\ell_{1}}\right) \\
\varphi=\frac{c_{2}}{t-\ell_{1}} \exp \left(\frac{\left(\left(x-d_{1}\right)^{2}+\left(y-d_{2}\right)^{2}-4 c_{2}^{2} \kappa_{2} \epsilon_{2}\right) i}{4 \kappa_{2}\left(t-\ell_{1}\right)}-\frac{c_{1}^{2} \varepsilon_{2} i}{t}\right) \\
\psi=\frac{c_{1}\left(t-\ell_{1}\right)^{c_{2}^{2} \epsilon_{1} i /\left(\ell_{2}-\ell_{1}\right)}\left(t-\ell_{2}\right)^{-c_{2}^{2} \epsilon_{1} i /\left(\ell_{2}-\ell_{1}\right)}}{t} \exp \frac{\left(x^{2}+y^{2}-4 c_{1}^{2} \kappa_{1} \varepsilon_{1}\right) i}{4 \kappa_{1} t}
\end{gathered}
$$




$$
\begin{aligned}
& \varphi= c_{2}\left(t-\ell_{1}\right)^{c_{2}^{2} \epsilon_{2} i /\left(\ell_{2}-\ell_{1}\right)-1 / 2}\left(t-\ell_{2}\right)^{-c_{2}^{2} \epsilon_{2} i /\left(\ell_{2}-\ell_{1}\right)-1 / 2} \\
& \times \exp \left(\frac{\left(x-d_{1}\right)^{2} i}{4 \kappa_{2}\left(t-\ell_{1}\right)}+\frac{\left(y-d_{2}\right)^{2} i}{4 \kappa_{2}\left(t-\ell_{2}\right)}-\frac{c_{1}^{2} \varepsilon_{2} i}{t}\right) ; \\
& \psi= c_{1} t^{-c_{1}^{2} \varepsilon_{1} i / \ell-1 / 2}(t-\ell)^{c_{1}^{2} \varepsilon_{1} i / \ell-1 / 2}\left(t-\ell_{1}\right)^{c_{2}^{2} \epsilon_{1} i /\left(\ell_{2}-\ell_{1}\right)} \\
& \times\left(t-\ell_{2}\right)^{-c_{2}^{2} \epsilon_{1} i /\left(\ell_{2}-\ell_{1}\right)} \exp \left(\frac{x^{2} i}{4 \kappa_{1} t}+\frac{y^{2} i}{4 \kappa_{1}(t-\ell)}\right), \\
& \varphi= c_{2} t^{-c_{1}^{2} \varepsilon_{2} i / \ell}(t-\ell)^{c_{1}^{2} \varepsilon_{2} i / \ell}\left(t-\ell_{1}\right)^{c_{2}^{2} \epsilon_{2} i /\left(\ell_{2}-\ell_{1}\right)-1 / 2} \\
& \times\left(t-\ell_{2}\right)^{-c_{2}^{2} \epsilon_{2} i /\left(\ell_{2}-\ell_{1}\right)-1 / 2} \exp \left(\frac{\left(x-d_{1}\right)^{2} i}{4 \kappa_{2}\left(t-\ell_{1}\right)}+\frac{\left(y-d_{2}\right)^{2} i}{4 \kappa_{2}\left(t-\ell_{2}\right)}\right) .
\end{aligned}
$$

Case 6. For two functions $\beta_{1}, \beta_{2}$ in $t$,

$$
\phi=\frac{x^{2}+y^{2}}{4 \kappa_{1} t}+\beta_{1}, \quad \mu=\frac{x^{2}+y^{2}}{4 \kappa_{2} t}+\beta_{2} .
$$

As Case 4, (6.2.9) and (6.2.11) imply

$$
\xi=\frac{1}{t} \hat{\xi}(u, v), \quad \eta=\frac{1}{t} \hat{\eta}(u, v), \quad u=\frac{x}{t}, v=\frac{y}{t} .
$$

Moreover, (6.2.10) and (6.2.12) become

$$
\begin{aligned}
& -\beta_{1}^{\prime} \hat{\xi}+\frac{\kappa_{1}}{t^{2}}\left(\hat{\xi}_{u u}+\hat{\xi}_{v v}\right)+\frac{1}{t^{2}}\left(\varepsilon_{1} \hat{\xi}^{2}+\epsilon_{1} \hat{\eta}^{2}\right) \hat{\xi}=0, \\
& -\beta_{2}^{\prime} \hat{\eta}+\frac{\kappa_{2}}{t^{2}}\left(\hat{\eta}_{u u}+\hat{\eta}_{v v}\right)+\frac{1}{t^{2}}\left(\varepsilon_{2} \hat{\xi}^{2}+\epsilon_{2} \hat{\eta}^{2}\right) \hat{\eta}=0 .
\end{aligned}
$$

To solve the above system, we assume

$$
\beta_{1}=-\frac{c_{1}}{t}, \quad \beta_{2}=-\frac{c_{2}}{t}, \quad c_{1}, c_{2} \in \mathbb{R} .
$$

Then (6.2.92) and (6.2.93) are equivalent to:

$$
\begin{aligned}
& -c_{1} \hat{\xi}+\kappa_{1}\left(\hat{\xi}_{u u}+\hat{\xi}_{v v}\right)+\left(\varepsilon_{1} \hat{\xi}^{2}+\epsilon_{1} \hat{\eta}^{2}\right) \hat{\xi}=0, \\
& -c_{2} \hat{\eta}+\kappa_{2}\left(\hat{\eta}_{u u}+\hat{\eta}_{v v}\right)+\left(\varepsilon_{2} \hat{\xi}^{2}+\epsilon_{2} \hat{\eta}^{2}\right) \hat{\eta}=0 .
\end{aligned}
$$

For simplicity, we assume $\hat{\xi}$ and $\hat{\eta}$ are independent of $v$. If $\left(\varepsilon_{1}, \epsilon_{1}\right)=\varepsilon_{1}\left(1, d^{2}\right)$ and $\left(\varepsilon_{2}, \epsilon_{2}\right)=\varepsilon_{2}\left(1, d^{2}\right)$ with $d \in \mathbb{R}$, we have the following solution:

$$
\hat{\xi}=d \ell \sin u, \quad \hat{\eta}=\ell \cos u, \quad\left(c_{1}, c_{2}\right)=\left(d^{2} \ell^{2} \varepsilon_{1}-\kappa_{1}, d^{2} \ell^{2} \varepsilon_{2}-\kappa_{2}\right)
$$


for $\ell \in \mathbb{R}$. When $\left(\varepsilon_{1}, \epsilon_{1}\right)=\varepsilon_{1}\left(1,-d^{2}\right)$ and $\left(\varepsilon_{2}, \epsilon_{2}\right)=\varepsilon_{2}\left(1,-d^{2}\right)$ with $d \in \mathbb{R}$, we get the solution:

$$
\hat{\xi}=d \ell \cosh \varpi, \quad \hat{\eta}=\ell \sinh \varpi, \quad\left(c_{1}, c_{2}\right)=\left(d^{2} \ell^{2} \varepsilon_{1}+\kappa_{1}, d^{2} \ell^{2} \varepsilon_{2}+\kappa_{2}\right)
$$

for $\ell \in \mathbb{R}$.

Theorem 6.2.6. For $d, \ell \in \mathbb{R}$, we have the following solutions of the coupled twodimensional cubic nonlinear Schrödinger equations (6.2.1) and (6.2.2):

$$
\begin{aligned}
& \psi=\frac{d \ell \sin (x / t)}{t} \exp \left(\frac{x^{2}+y^{2}}{4 \kappa_{1} t}+\frac{\kappa_{1}-d^{2} \ell^{2} \varepsilon_{1}}{t}\right) i \\
& \varphi=\frac{\ell \cos (x / t)}{t} \exp \left(\frac{x^{2}+y^{2}}{4 \kappa_{2} t}+\frac{\kappa_{2}-d^{2} \ell^{2} \varepsilon_{2}}{t}\right) i
\end{aligned}
$$

if $\left(\varepsilon_{1}, \epsilon_{1}\right)=\varepsilon_{1}\left(1, d^{2}\right)$ and $\left(\varepsilon_{2}, \epsilon_{2}\right)=\varepsilon_{2}\left(1, d^{2}\right)$;

$$
\begin{gathered}
\psi=\frac{d \ell \cosh (x / t)}{t} \exp \left(\frac{x^{2}+y^{2}}{4 \kappa_{1} t}-\frac{\kappa_{1}+d^{2} \ell^{2} \varepsilon_{1}}{t}\right) i \\
\varphi=\frac{\ell \sinh (x / t)}{t} \exp \left(\frac{x^{2}+y^{2}}{4 \kappa_{2} t}-\frac{\kappa_{2}+d^{2} \ell^{2} \varepsilon_{2}}{t}\right) i
\end{gathered}
$$

when $\left(\varepsilon_{1}, \epsilon_{1}\right)=\varepsilon_{1}\left(1,-d^{2}\right)$ and $\left(\varepsilon_{2}, \epsilon_{2}\right)=\varepsilon_{2}\left(1,-d^{2}\right)$.

Remark 6.2.7. Applying the transformation in (6.2.3) and (6.2.4) with $a=a_{0}=$ $a_{2}=a_{3}=0$ to (6.2.99) and (6.2.100), we get a more general wave-like solution:

$$
\begin{gathered}
\psi=\frac{d \ell \sin \left[(x \cos \theta+y \sin \theta) /\left(b\left(t-a_{1}\right)\right)\right]}{b\left(t-a_{1}\right)} \exp \left(\frac{x^{2}+y^{2}}{4 \kappa_{1}\left(t-a_{1}\right)}+\frac{\kappa_{1}-d^{2} \ell^{2} \varepsilon_{1}}{b^{2}\left(t-a_{1}\right)}\right) i \\
\varphi=\frac{\ell \cos \left[(x \cos \theta+y \sin \theta) /\left(b\left(t-a_{1}\right)\right)\right]}{b\left(t-a_{1}\right)} \exp \left(\frac{x^{2}+y^{2}}{4 \kappa_{2}\left(t-a_{1}\right)}+\frac{\kappa_{2}-d^{2} \ell^{2} \varepsilon_{2}}{b^{2}\left(t-a_{1}\right)}\right) i
\end{gathered}
$$

if $\left(\varepsilon_{1}, \epsilon_{1}\right)=\varepsilon_{1}\left(1, d^{2}\right)$ and $\left(\varepsilon_{2}, \epsilon_{2}\right)=\varepsilon_{2}\left(1, d^{2}\right)$, where $a_{1}, b, \theta \in \mathbb{R}$ with $b \neq 0$. We can get more sophisticated wave-like solution if we apply the general forms of the transformations in (6.2.3)-(6.2.6).

Finally, we assume $\varepsilon_{1} \epsilon_{2}-\varepsilon_{2} \epsilon_{1} \neq 0$. Again we assume that $\hat{\xi}$ and $\hat{\eta}$ are independent of $v$. By the arguments in (6.2.19)-(6.2.30), we have:

Theorem 6.2.8. Let $d, \ell, m \in \mathbb{R}$ with $0<m<1$ and let $\sigma_{1}, \sigma_{2} \in\{1,-1\}$. If $\varepsilon_{1} \epsilon_{2}-\varepsilon_{2} \epsilon_{1} \neq 0$, we have the following solutions of the coupled two-dimensional cubic nonlinear Schrödinger equations (6.2.1) and (6.2.2):

$$
\psi=\frac{\sigma_{1}}{x} \sqrt{\frac{2\left(\sigma_{1} \kappa_{2}-\epsilon_{2} \kappa_{1}\right)}{\varepsilon_{1} \epsilon_{2}-\varepsilon_{2} \epsilon_{1}}} \exp \frac{\left(x^{2}+y^{2}\right) i}{4 \kappa_{1} t},
$$




$$
\begin{aligned}
& \varphi=\frac{\sigma_{2}}{x} \sqrt{\frac{2\left(\varepsilon_{2} \kappa_{1}-\varepsilon_{1} \kappa_{2}\right)}{\varepsilon_{1} \epsilon_{2}-\varepsilon_{2} \epsilon_{1}}} \exp \frac{\left(x^{2}+y^{2}\right) i}{4 \kappa_{2} t} ; \\
& \psi=\sigma_{1} \sqrt{\frac{\epsilon_{1} \kappa_{2}-\epsilon_{2} \kappa_{1}}{\left(\varepsilon_{1} \epsilon_{2}-\varepsilon_{2} \epsilon_{1}\right)\left(x^{2}+y^{2}\right)}} \exp \frac{\left(x^{2}+y^{2}\right) i}{4 \kappa_{1} t}, \\
& \varphi=\sigma_{2} \sqrt{\frac{\varepsilon_{2} \kappa_{1}-\varepsilon_{1} \kappa_{2}}{\left(\varepsilon_{1} \epsilon_{2}-\varepsilon_{2} \epsilon_{1}\right)\left(x^{2}+y^{2}\right)}} \exp \frac{\left(x^{2}+y^{2}\right) i}{4 \kappa_{2} t} ; \\
& \psi=\frac{\sigma_{1}}{t} \sqrt{\frac{2\left(\epsilon_{1} \kappa_{2}-\epsilon_{2} \kappa_{1}\right)}{\varepsilon_{1} \epsilon_{2}-\varepsilon_{2} \epsilon_{1}}} \tan \frac{x}{t} \exp \left(\frac{x^{2}+y^{2}}{4 \kappa_{1} t}-\frac{2 \kappa_{1}}{t}\right) i, \\
& \varphi=\frac{\sigma_{2}}{t} \sqrt{\frac{2\left(\varepsilon_{2} \kappa_{1}-\varepsilon_{1} \kappa_{2}\right)}{\varepsilon_{1} \epsilon_{2}-\varepsilon_{2} \epsilon_{1}}} \tan \frac{x}{t} \exp \left(\frac{x^{2}+y^{2}}{4 \kappa_{2} t}-\frac{2 \kappa_{2}}{t}\right) i ; \\
& \psi=\frac{\sigma_{1}}{t} \sqrt{\frac{2\left(\epsilon_{1} \kappa_{2}-\epsilon_{2} \kappa_{1}\right)}{\varepsilon_{1} \epsilon_{2}-\varepsilon_{2} \epsilon_{1}}} \sec \frac{x}{t} \exp \left(\frac{x^{2}+y^{2}}{4 \kappa_{1} t}+\frac{\kappa_{1}}{t}\right) i, \\
& \varphi=\frac{\sigma_{2}}{t} \sqrt{\frac{2\left(\varepsilon_{2} \kappa_{1}-\varepsilon_{1} \kappa_{2}\right)}{\varepsilon_{1} \epsilon_{2}-\varepsilon_{2} \epsilon_{1}}} \sec \frac{x}{t} \exp \left(\frac{x^{2}+y^{2}}{4 \kappa_{2} t}+\frac{\kappa_{2}}{t}\right) i ; \\
& \psi=\frac{\sigma_{1}}{t} \sqrt{\frac{2\left(\epsilon_{1} \kappa_{2}-\epsilon_{2} \kappa_{1}\right)}{\varepsilon_{1} \epsilon_{2}-\varepsilon_{2} \epsilon_{1}}} \operatorname{coth} \frac{x}{t}, \exp \left(\frac{x^{2}+y^{2}}{4 \kappa_{1} t}+\frac{2 \kappa_{1}}{t}\right) i, \\
& \varphi=\frac{\sigma_{2}}{t} \sqrt{\frac{2\left(\varepsilon_{2} \kappa_{1}-\varepsilon_{1} \kappa_{2}\right)}{\varepsilon_{1} \epsilon_{2}-\varepsilon_{2} \epsilon_{1}}} \operatorname{coth} \frac{x}{t} \exp \left(\frac{x^{2}+y^{2}}{4 \kappa_{2} t}+\frac{2 \kappa_{2}}{t}\right) i \\
& \psi=\frac{\sigma_{1}}{t} \sqrt{\frac{2\left(\epsilon_{1} \kappa_{2}-\epsilon_{2} \kappa_{1}\right)}{\varepsilon_{1} \epsilon_{2}-\varepsilon_{2} \epsilon_{1}}} \operatorname{csch} \frac{x}{t} \exp \left(\frac{x^{2}+y^{2}}{4 \kappa_{1} t}-\frac{\kappa_{1}}{t}\right) i, \\
& \varphi=\frac{\sigma_{2}}{t} \sqrt{\frac{2\left(\varepsilon_{2} \kappa_{1}-\varepsilon_{1} \kappa_{2}\right)}{\varepsilon_{1} \epsilon_{2}-\varepsilon_{2} \epsilon_{1}}} \operatorname{csch} \frac{x}{t} \exp \left(\frac{x^{2}+y^{2}}{4 \kappa_{2} t}-\frac{\kappa_{2}}{t}\right) i ; \\
& \psi=\frac{m \sigma_{1}}{t} \sqrt{\frac{2\left(\epsilon_{1} \kappa_{2}-\epsilon_{2} \kappa_{1}\right)}{\varepsilon_{1} \epsilon_{2}-\varepsilon_{2} \epsilon_{1}}} \operatorname{sn}(x / t \mid m) \exp \left(\frac{x^{2}+y^{2}}{4 \kappa_{1} t}+\frac{\left(1+m^{2}\right) \kappa_{1}}{t}\right) i, \\
& \varphi=\frac{m \sigma_{2}}{t} \sqrt{\frac{2\left(\varepsilon_{2} \kappa_{1}-\varepsilon_{1} \kappa_{2}\right)}{\varepsilon_{1} \epsilon_{2}-\varepsilon_{2} \epsilon_{1}}} \operatorname{sn}(x / t \mid m) \exp \left(\frac{x^{2}+y^{2}}{4 \kappa_{2} t}+\frac{\left(1+m^{2}\right) \kappa_{2}}{t}\right) i ; \\
& \psi=\frac{m \sigma_{1}}{t} \sqrt{\frac{2\left(\epsilon_{2} \kappa_{1}-\epsilon_{1} \kappa_{2}\right)}{\varepsilon_{1} \epsilon_{2}-\varepsilon_{2} \epsilon_{1}}} \text { cn }(x / t \mid m) \exp \left(\frac{x^{2}+y^{2}}{4 \kappa_{1} t}+\frac{\left(1-2 m^{2}\right) \kappa_{1}}{t}\right) i, \\
& \varphi=\frac{m \sigma_{2}}{t} \sqrt{\frac{2\left(\varepsilon_{1} \kappa_{2}-\varepsilon_{2} \kappa_{1}\right)}{\varepsilon_{1} \epsilon_{2}-\varepsilon_{2} \epsilon_{1}}} \operatorname{cn}(x / t \mid m) \exp \left(\frac{x^{2}+y^{2}}{4 \kappa_{2} t}+\frac{\left(1-2 m^{2}\right) \kappa_{2}}{t}\right) i \text {; } \\
& \psi=\frac{\sigma_{1}}{t} \sqrt{\frac{2\left(\epsilon_{2} \kappa_{1}-\epsilon_{1} \kappa_{2}\right)}{\varepsilon_{1} \epsilon_{2}-\varepsilon_{2} \epsilon_{1}}} \operatorname{dn}(x / t \mid m) \exp \left(\frac{x^{2}+y^{2}}{4 \kappa_{1} t}+\frac{\left(m^{2}-2\right) \kappa_{1}}{t}\right) i,
\end{aligned}
$$




$$
\varphi=\frac{\sigma_{2}}{t} \sqrt{\frac{2\left(\varepsilon_{1} \kappa_{2}-\varepsilon_{2} \kappa_{1}\right)}{\varepsilon_{1} \epsilon_{2}-\varepsilon_{2} \epsilon_{1}}} \operatorname{dn}(x / t \mid m) \exp \left(\frac{x^{2}+y^{2}}{4 \kappa_{1} t}+\frac{\left(m^{2}-2\right) \kappa_{1}}{t}\right) i .
$$

Remark 6.2.9. Since $\lim _{m \rightarrow 1} c n(x \mid m)=\operatorname{sech} x$, (6.2.119) and (6.2.120) yield the solution

$$
\begin{aligned}
& \psi=\frac{\sigma_{1}}{t} \sqrt{\frac{2\left(\epsilon_{2} \kappa_{1}-\epsilon_{1} \kappa_{2}\right)}{\varepsilon_{1} \epsilon_{2}-\varepsilon_{2} \epsilon_{1}}} \operatorname{sech} \frac{x}{t} \exp \left(\frac{x^{2}+y^{2}}{4 \kappa_{1} t}-\frac{\kappa_{1}}{t}\right) i, \\
& \varphi=\frac{\sigma_{2}}{t} \sqrt{\frac{2\left(\varepsilon_{1} \kappa_{2}-\varepsilon_{2} \kappa_{1}\right)}{\varepsilon_{1} \epsilon_{2}-\varepsilon_{2} \epsilon_{1}}} \operatorname{sech} \frac{x}{t} \exp \left(\frac{x^{2}+y^{2}}{4 \kappa_{2} t}-\frac{\kappa_{2}}{t}\right) i ;
\end{aligned}
$$

Applying the transformation in (6.2.3)-(6.2.4) with $a=a_{0}=a_{2}=a_{3}=0$ and the transformation $S_{c, 0}$ in (6.2.5)-(6.2.6), we get a more general soliton-like solution:

$$
\begin{aligned}
\psi= & \frac{\sigma_{1}}{b^{2}\left(t-a_{1}\right)} \sqrt{\frac{2\left(\epsilon_{2} \kappa_{1}-\epsilon_{1} \kappa_{2}\right)}{\varepsilon_{1} \epsilon_{2}-\varepsilon_{2} \epsilon_{1}}} \operatorname{sech} \frac{(x-2 c t) \cos \theta+y \sin \theta}{b\left(t-a_{1}\right)} \\
& \times \exp \left(\frac{(x-2 c t)^{2}+y^{2}}{4 \kappa_{1}\left(t-a_{1}\right)}-\frac{\kappa_{1}}{b^{2}\left(t-a_{1}\right)}+\frac{c(x-c t)}{\kappa_{1}}+a\right) i, \\
\varphi= & \frac{\sigma_{2}}{b^{2}\left(t-a_{1}\right)} \sqrt{\frac{2\left(\epsilon_{2} \kappa_{1}-\epsilon_{1} \kappa_{2}\right)}{\varepsilon_{1} \epsilon_{2}-\varepsilon_{2} \epsilon_{1}}} \operatorname{sech} \frac{(x-2 c t) \cos \theta+y \sin \theta}{b\left(t-a_{1}\right)} \\
& \quad \times \exp \left(\frac{(x-2 c t)^{2}+y^{2}}{4 \kappa_{2}\left(t-a_{1}\right)}-\frac{\kappa_{2}}{b^{2}\left(t-a_{1}\right)}+\frac{c(x-c t)}{\kappa_{2}}+a_{0}\right) i,
\end{aligned}
$$

where $a, a_{0}, a_{1}, b, c, \theta \in \mathbb{R}$ with $b \neq 0$. We can get more sophisticated soliton-like solution if we apply the general forms of the transformations in (6.2.3)-(6.2.6).

\subsection{Davey and Stewartson Equations}

Davey and Stewartson [DS] (1974) used the method of multiple scales to derive the following system of nonlinear partial differential equations

$$
\begin{gathered}
2 i u_{t}+\epsilon_{1} u_{x x}+u_{y y}-2 \epsilon_{2}|u|^{2} u-2 u v=0, \\
v_{x x}-\epsilon_{1}\left(v_{y y}+2\left(|u|^{2}\right)_{x x}\right)=0
\end{gathered}
$$

that describe the long time evolution of three-dimensional packets of surface waves, where $u$ is a complex-valued function, $v$ is a real valued function and $\epsilon_{1}, \epsilon_{2}= \pm 1$. The equations are called the Davey-Stewartson I equations if $\epsilon_{1}=1$, and the Davey-Stewartson II equations when $\epsilon_{1}=-1$. They were used to study the stability of the uniform Stokes wave train with respect to small disturbance. The soliton solutions of the Davey-Stewartson equations were first studied by Anker and Freeman [AF] (1978). Kirby and Dalrymple 
[KD] (1983) obtained oblique envelope solutions of the equations in intermediate water depth. Omote [Om] (1988) found infinite-dimensional symmetry algebras and an infinite number of conserved quantities for the equations.

Arkadiev, Pogrebkov and Polivanov [APP1] (1989) studied the solutions of the DaveyStewartson II equations whose singularities form closed lines with string-like behavior. They [APP2] (1989) also applied the inverse scattering transform method to the DaveyStewartson II equations. Gilson and Nimmo [GN] (1991) found dromion solutions and Malanyuk [Mt1, Mt2] (1991, 1994) obtained finite-gap solutions of the equations. van de Linden (1992) studied the solutions under a certain boundary condition. Clarkson and Hood $[\mathrm{CH}]$ (1994) obtained certain symmetry reductions of the equations to ordinary differential equations with no intervening steps and provided new exact solutions which are not obtainable by the Lie group approach. Guil and Manas [GM] (1995) found certain solutions of the Davey-Stewartson I equations by deforming dromion. Manas and Santini [MS] (1997) studied a large class of solutions of the Davey-Stewartson II equations by a Wronskian scheme. There are the other interesting works on solutions of the Davey-Stewartson equations (e.g., cf. [Vj]). It is obvious that the some of above solutions are equivalent to each other under the known symmetric transformations. It is time to study solutions of the Davey-Stewartson equations modulo the known symmetric transformations.

In this section, we use the quadratic-argument approach to study exact solutions of the Davey-Stewartson equations modulo the most known symmetry transformations. This is a revision of our earlier preprint [X18].

By (6.1.2), (6.3.1) and (6.3.2), we take

$$
\operatorname{deg} x=\operatorname{deg} y=-\operatorname{deg} u=\frac{1}{2} \operatorname{deg} t=-\frac{1}{2} \operatorname{deg} v
$$

in order to make the nonzero terms in (6.3.1) and (6.3.2) having the same degree. Moreover, the equation (6.3.1) and (6.3.2) are translation invariant because they do not contain variable coefficients. Thus the transformation

$$
T_{a, b}(u(t, x, y))=b u\left(b^{2} t+a, b x, b y\right), \quad T_{a, b}(v(t, x, y))=b^{2} v\left(b^{2} t+a, b x, b y\right)
$$

maps a solution of the Davey-Stewartson equations (6.3.1) and (6.3.2) to another solution, where $a, b \in \mathbb{R}$ and $b \neq 0$. Let $\alpha, \beta$ and $\gamma$ be functions in $t$. The transformation $u(t, x, y) \mapsto$ $u(t, x+\alpha, y+\beta)$ and $v(t, x, y) \mapsto v(t, x+\alpha, y+\beta)$ changes (6.3.1) to

$$
2 i\left(\alpha^{\prime} u_{x}+\beta^{\prime} u_{y}+u_{t}\right)+\epsilon_{1} u_{x x}+u_{y y}-2 \epsilon_{2}|u|^{2} u-2 u v=0
$$

and leaves (6.3.2) invariant, where the independent variables $x$ is replaced by $x+\alpha$, the independent variables $y$ is replaced by $y+\beta$ and the subindices denote the partial derivatives with respect to the original independent variables. Moreover, the transformation 
$u \mapsto e^{-\left(\epsilon_{1} \alpha^{\prime} x+\beta^{\prime} y+\gamma\right) i} u$ and $v \mapsto v$ changes $(6.3 .1)$ to

$$
\begin{aligned}
& 2\left[\left(\left(\epsilon_{1} \alpha^{\prime \prime} x+\beta^{\prime \prime} y\right)+\gamma^{\prime}\right) u+i u_{t}\right]-\left(\epsilon_{1} \alpha^{\prime 2}+{\beta^{\prime}}^{2}\right) u-2 \alpha^{\prime} i u_{x}-2 \beta^{\prime} i u_{y} \\
& +\epsilon_{1} u_{x x}+u_{y y}-2 \epsilon_{2}|u|^{2} u-2 u v=0
\end{aligned}
$$

and leaves (6.3.2) invariant. Furthermore, the transformation

$$
u \mapsto u \text { and } v \mapsto v+\epsilon_{1} \alpha^{\prime \prime} x+{\beta^{\prime \prime}}^{\prime} y-\frac{\epsilon_{1} \alpha^{2}+\beta^{\prime 2}}{2}+\gamma^{\prime}
$$

changes (6.3.1) to

$$
\begin{aligned}
& 2 i u_{t}+\epsilon_{1} u_{x x}+u_{y y}-2 \epsilon_{2}|u|^{2} u-2 u v \\
& +\left[\epsilon_{1} \alpha^{\prime 2}+\beta^{\prime 2}+2 \gamma^{\prime}-2\left(\epsilon_{1} \alpha^{\prime \prime} x+\beta^{\prime \prime} y\right)\right] u=0
\end{aligned}
$$

and keeps (6.3.2) invariant. Thus the transformation

$$
\begin{gathered}
S_{\alpha, \beta, \gamma}(u(t, x, y))=e^{-\left(\epsilon_{1} \alpha^{\prime} x+\beta^{\prime} y+\gamma\right) i} u(t, x+\alpha, y+\beta), \\
S_{\alpha, \beta, \gamma}(v(t, x, y))=v(t, x+\alpha, y+\beta)+\epsilon_{1} \alpha^{\prime \prime} x+\beta^{\prime \prime} y-\frac{\epsilon_{1} \alpha^{\prime 2}+\beta^{\prime 2}}{2}+\gamma^{\prime}
\end{gathered}
$$

maps a solution of the Davey-Stewartson equations (6.3.1) and (6.3.2) to another solution.

Write

$$
u=\xi(t, x, y) e^{i \phi(t, x, y)},
$$

where $\xi$ and $\phi$ are real functions in $t, x, y$. Note

$$
\begin{gathered}
u_{t}=\left(\xi_{t}+i \xi \phi_{t}\right) e^{i \phi}, \quad u_{x}=\left(\xi_{x}+i \xi \phi_{x}\right) e^{i \phi}, \quad u_{y}=\left(\xi_{y}+i \xi \phi_{y}\right) e^{i \phi} \\
u_{x x}=\left(\xi_{x x}-\xi \phi_{x}^{2}+i\left(2 \xi_{x} \phi_{x}+\xi \phi_{x x}\right)\right) e^{i \phi}, \quad u_{y y}=\left(\xi_{y y}-\xi \phi_{y}^{2}+i\left(2 \xi_{y} \phi_{y}+\xi \phi_{y y}\right)\right) e^{i \phi} .
\end{gathered}
$$

Then (6.3.1) is equivalent to

$$
\begin{aligned}
& 2 i \xi_{t}-2 \xi \phi_{t}+\epsilon_{1}\left(\xi_{x x}-\xi \phi_{x}^{2}+i\left(2 \xi_{x} \phi_{x}+\xi \phi_{x x}\right)\right) \\
& +\xi_{y y}-\xi \phi_{y}^{2}+i\left(2 \xi_{y} \phi_{y}+\xi \phi_{y y}\right)-2 \epsilon_{2} \xi^{3}-2 \xi v=0
\end{aligned}
$$

equivalently,

$$
\begin{gathered}
2 \xi_{t}+2\left(\epsilon_{1} \xi_{x} \phi_{x}+\xi_{y} \phi_{y}\right)+\xi\left(\epsilon_{1} \phi_{x x}+\phi_{y y}\right)=0, \\
\xi\left(2 \phi_{t}+\epsilon_{1} \phi_{x}^{2}+\phi_{y}^{2}\right)-\epsilon_{1} \xi_{x x}-\xi_{y y}+2 \epsilon_{2} \xi^{3}+2 \xi v=0 .
\end{gathered}
$$

Moreover, (6.3.2) becomes

$$
v_{x x}-\epsilon_{1}\left(v_{y y}+2\left(\xi^{2}\right)_{x x}\right)=0
$$

Case 1. $\phi=0$. 
In this case, (6.3.15) becomes $\xi_{t}=0$. Moreover, (6.3.16) gives

$$
-\epsilon_{1} \xi_{x x}-\xi_{y y}+2 \epsilon_{2} \xi^{3}+2 \xi v=0 .
$$

Fixing $\ell_{1}, \ell_{2} \in \mathbb{R}$, we denote

$$
\varpi=\ell_{1} x+\ell_{2} y .
$$

Assume $\xi=f(\varpi)$ and $v=g(\varpi)$ for some one-variable functions $f$ and $g$. Then (6.3.17) and (6.3.18) become

$$
\begin{gathered}
\left(\ell_{1}^{2}-\epsilon_{1} \ell_{2}^{2}\right) g^{\prime \prime}-2 \epsilon_{1} \ell_{1}^{2}\left(f^{2}\right)^{\prime \prime}=0, \\
-\left(\epsilon_{1} \ell_{1}^{2}+\ell_{2}^{2}\right) f^{\prime \prime}+2 \epsilon_{2} f^{3}+2 f g=0 .
\end{gathered}
$$

Suppose

$$
\ell_{1}^{2}-\epsilon_{1} \ell_{2}^{2} \neq 0 \text { and } \epsilon_{1} \ell_{1}^{2}+\ell_{2}^{2} \neq 0 \sim \ell_{1}^{4} \neq \ell_{2}^{4} .
$$

Then

$$
g=\frac{2 \ell_{1}^{2} f^{2}}{\epsilon_{1} \ell_{1}^{2}-\ell_{2}^{2}}+c\left(\epsilon_{1} \ell_{1}^{2}+\ell_{2}^{2}\right)
$$

is a solution of (6.3.20) with $c \in \mathbb{R}$.

Substituting (6.3.23) into (6.3.21), we get

$$
-\left(\epsilon_{1} \ell_{1}^{2}+\ell_{2}^{2}\right) f^{\prime \prime}+2 \frac{\left(2+\epsilon_{1} \epsilon_{2}\right) \ell_{1}^{2}-\epsilon_{2} \ell_{2}^{2}}{\epsilon_{1} \ell_{1}^{2}-\ell_{2}^{2}} f^{3}+2 c\left(\epsilon_{1} \ell_{1}^{2}+\ell_{2}^{2}\right) f=0,
$$

equivalently,

$$
f^{\prime \prime}+2 \frac{\left(2+\epsilon_{1} \epsilon_{2}\right) \ell_{1}^{2}-\epsilon_{2} \ell_{2}^{2}}{\ell_{2}^{4}-\ell_{1}^{4}} f^{3}-2 c f=0 .
$$

If

$$
\epsilon_{2}=1 \text { and } \ell_{2}= \pm \sqrt{2+\epsilon_{1}} \ell_{1},
$$

then (6.3.25) becomes $f^{\prime \prime}=2 c f$. Assuming $c=2 c_{1}^{2}$ with $c_{1} \in \mathbb{R}$, we have the solution

$$
f=a_{1} e^{2 c_{1} \varpi}+a_{2} e^{-2 c_{1} \varpi} \text { and } g=-f^{2}+8 c_{1}^{2} \ell_{1}^{2} .
$$

Letting $c=-2 c_{1}^{2}$ with $c_{1} \in \mathbb{R}$, we obtain another solution

$$
f=a_{1} \sin 2 c_{1} \varpi \text { and } g=-f^{2}-8 c_{1}^{2} \ell_{1}^{2} .
$$

Since $\varpi=\ell_{1} x+\ell_{2} y=\ell_{1}\left(x \pm \sqrt{2+\epsilon_{1}} y\right)$, we can take $2 c_{1} \ell_{1}=1$ if we replace $u$ by $T_{0,\left(2 c_{1} \ell_{1}\right)^{-1}}(u)$ and $v$ by $T_{0,\left(2 c_{1} \ell_{1}\right)^{-1}}(v)$. Thus have

$$
\begin{gathered}
f=a_{1} e^{x \pm \sqrt{2+\epsilon_{1}} y}+a_{2} e^{-x \mp \sqrt{2+\epsilon_{1}} y} \text { and } g=-f^{2}+2 ; \\
f=a_{1} \sin \left(x \pm \sqrt{2+\epsilon_{1}} y\right) \text { and } g=-f^{2}-2 .
\end{gathered}
$$

Next we assume

$$
\left(2+\epsilon_{1} \epsilon_{2}\right) \ell_{1}^{2}-\epsilon_{2} \ell_{2}^{2} \neq 0 .
$$


Recall (6.1.18)-(6.1.23). Substituting $\xi=f=k \varphi(x)$ to (6.3.25) with $k \in \mathbb{R}$ and $\varphi=$ $1 / x, \tan x, \sec x, \operatorname{coth} x, \operatorname{csch} x, \operatorname{sn}(x \mid m), \operatorname{cn}(x \mid m), \operatorname{dn}(x \mid m)$, we find the following solutions:

$$
\begin{aligned}
& f=\frac{1}{\varpi} \sqrt{\frac{\ell_{1}^{4}-\ell_{2}^{4}}{\left(2+\epsilon_{1} \epsilon_{2}\right) \ell_{1}^{2}-\epsilon_{2} \ell_{2}^{2}}}, \quad g=\frac{2 \ell_{1}^{2} f^{2}}{\epsilon_{1} \ell_{1}^{2}-\ell_{2}^{2}} ; \\
& f=\sqrt{\frac{\ell_{1}^{4}-\ell_{2}^{4}}{\left(2+\epsilon_{1} \epsilon_{2}\right) \ell_{1}^{2}-\epsilon_{2} \ell_{2}^{2}}} \tan \varpi, \quad g=\frac{2 \ell_{1}^{2} f^{2}}{\epsilon_{1} \ell_{1}^{2}-\ell_{2}^{2}}+\epsilon_{1} \ell_{1}^{2}+\ell_{2}^{2} ; \\
& f=\sqrt{\frac{\ell_{1}^{4}-\ell_{2}^{4}}{\left(2+\epsilon_{1} \epsilon_{2}\right) \ell_{1}^{2}-\epsilon_{2} \ell_{2}^{2}}} \sec \varpi, \quad g=\frac{2 \ell_{1}^{2} f^{2}}{\epsilon_{1} \ell_{1}^{2}-\ell_{2}^{2}}-\frac{\epsilon_{1} \ell_{1}^{2}+\ell_{2}^{2}}{2} \text {; } \\
& f=\sqrt{\frac{\ell_{1}^{4}-\ell_{2}^{4}}{\left(2+\epsilon_{1} \epsilon_{2}\right) \ell_{1}^{2}-\epsilon_{2} \ell_{2}^{2}}} \operatorname{coth} \varpi, \quad g=\frac{2 \ell_{1}^{2} f^{2}}{\epsilon_{1} \ell_{1}^{2}-\ell_{2}^{2}}-\epsilon_{1} \ell_{1}^{2}-\ell_{2}^{2} ; \\
& f=\sqrt{\frac{\ell_{1}^{4}-\ell_{2}^{4}}{\left(2+\epsilon_{1} \epsilon_{2}\right) \ell_{1}^{2}-\epsilon_{2} \ell_{2}^{2}}} \operatorname{csch} \varpi, \quad g=\frac{2 \ell_{1}^{2} f^{2}}{\epsilon_{1} \ell_{1}^{2}-\ell_{2}^{2}}+\frac{\epsilon_{1} \ell_{1}^{2}+\ell_{2}^{2}}{2} ; \\
& f=m \sqrt{\frac{\ell_{1}^{4}-\ell_{2}^{4}}{\left(2+\epsilon_{1} \epsilon_{2}\right) \ell_{1}^{2}-\epsilon_{2} \ell_{2}^{2}}} \operatorname{sn}(\varpi \mid m), \quad g=\frac{2 \ell_{1}^{2} f^{2}}{\epsilon_{1} \ell_{1}^{2}-\ell_{2}^{2}}-\frac{\left(m^{2}+1\right)\left(\epsilon_{1} \ell_{1}^{2}+\ell_{2}^{2}\right)}{2} \text {; } \\
& f=m \sqrt{\frac{\ell_{2}^{4}-\ell_{1}^{4}}{\left(2+\epsilon_{1} \epsilon_{2}\right) \ell_{1}^{2}-\epsilon_{2} \ell_{2}^{2}}} \text { cn }(\varpi \mid m), \quad g=\frac{2 \ell_{1}^{2} f^{2}}{\epsilon_{1} \ell_{1}^{2}-\ell_{2}^{2}}+\frac{\left(2 m^{2}-1\right)\left(\epsilon_{1} \ell_{1}^{2}+\ell_{2}^{2}\right)}{2} \text {; } \\
& f=\sqrt{\frac{\ell_{2}^{4}-\ell_{1}^{4}}{\left(2+\epsilon_{1} \epsilon_{2}\right) \ell_{1}^{2}-\epsilon_{2} \ell_{2}^{2}}} \operatorname{dn}(\varpi \mid m), \quad g=\frac{2 \ell_{1}^{2} f^{2}}{\epsilon_{1} \ell_{1}^{2}-\ell_{2}^{2}}+\frac{\left(2-m^{2}\right)\left(\epsilon_{1} \ell_{1}^{2}+\ell_{2}^{2}\right)}{2} .
\end{aligned}
$$

In summary, we have:

Theorem 6.3.1. If $\epsilon_{2}=1$, we have the following solutions of the Davey-Stewartson equations (6.3.1) and (6.3.2): for $a_{1}, a_{2} \in \mathbb{R}$ and $a_{1} \neq 0$,

$$
\begin{gathered}
u=a_{1} e^{x \pm \sqrt{2+\epsilon_{1}} y}+a_{2} e^{-x \mp \sqrt{2+\epsilon_{1}} y} \text { and } v=-u^{2}+2 \\
u=a_{1} \sin \left(x \pm \sqrt{2+\epsilon_{1}} y\right) \text { and } v=-u^{2}-2 .
\end{gathered}
$$

Let $\ell_{1}, \ell_{2} \in \mathbb{R}$ such that

$$
\ell_{1}^{4} \neq \ell_{2}^{4} \text { and }\left(2+\epsilon_{1} \epsilon_{2}\right) \ell_{1}^{2} \neq \epsilon_{2} \ell_{2}^{2}
$$

Then we the following solutions of the Davey-Stewartson equations (6.3.1) and (6.3.2):

$$
\begin{gathered}
u=\frac{1}{\ell_{1} x+\ell_{2} y} \sqrt{\frac{\ell_{1}^{4}-\ell_{2}^{4}}{\left(2+\epsilon_{1} \epsilon_{2}\right) \ell_{1}^{2}-\epsilon_{2} \ell_{2}^{2}}}, \quad v=\frac{2 \ell_{1}^{2} u^{2}}{\epsilon_{1} \ell_{1}^{2}-\ell_{2}^{2}} ; \\
u=\sqrt{\frac{\ell_{1}^{4}-\ell_{2}^{4}}{\left(2+\epsilon_{1} \epsilon_{2}\right) \ell_{1}^{2}-\epsilon_{2} \ell_{2}^{2}}} \tan \left(\ell_{1} x+\ell_{2} y\right), \quad v=\frac{2 \ell_{1}^{2} u^{2}}{\epsilon_{1} \ell_{1}^{2}-\ell_{2}^{2}}+\epsilon_{1} \ell_{1}^{2}+\ell_{2}^{2} ;
\end{gathered}
$$




$$
\begin{aligned}
& u=\sqrt{\frac{\ell_{1}^{4}-\ell_{2}^{4}}{\left(2+\epsilon_{1} \epsilon_{2}\right) \ell_{1}^{2}-\epsilon_{2} \ell_{2}^{2}}} \sec \left(\ell_{1} x+\ell_{2} y\right), \quad v=\frac{2 \ell_{1}^{2} u^{2}}{\epsilon_{1} \ell_{1}^{2}-\ell_{2}^{2}}-\frac{\epsilon_{1} \ell_{1}^{2}+\ell_{2}^{2}}{2} \\
& u=\sqrt{\frac{\ell_{1}^{4}-\ell_{2}^{4}}{\left(2+\epsilon_{1} \epsilon_{2}\right) \ell_{1}^{2}-\epsilon_{2} \ell_{2}^{2}}} \operatorname{coth}\left(\ell_{1} x+\ell_{2} y\right), \quad v=\frac{2 \ell_{1}^{2} u^{2}}{\epsilon_{1} \ell_{1}^{2}-\ell_{2}^{2}}-\epsilon_{1} \ell_{1}^{2}-\ell_{2}^{2} \\
& u=\sqrt{\frac{\ell_{1}^{4}-\ell_{2}^{4}}{\left(2+\epsilon_{1} \epsilon_{2}\right) \ell_{1}^{2}-\epsilon_{2} \ell_{2}^{2}}} \operatorname{csch}\left(\ell_{1} x+\ell_{2} y\right), \quad v=\frac{2 \ell_{1}^{2} u^{2}}{\epsilon_{1} \ell_{1}^{2}-\ell_{2}^{2}}+\frac{\epsilon_{1} \ell_{1}^{2}+\ell_{2}^{2}}{2} \text {; } \\
& u=m \sqrt{\frac{\ell_{1}^{4}-\ell_{2}^{4}}{\left(2+\epsilon_{1} \epsilon_{2}\right) \ell_{1}^{2}-\epsilon_{2} \ell_{2}^{2}}} \operatorname{sn}\left(\ell_{1} x+\ell_{2} y \mid m\right) \\
& v=\frac{2 \ell_{1}^{2} u^{2}}{\epsilon_{1} \ell_{1}^{2}-\ell_{2}^{2}}-\frac{\left(m^{2}+1\right)\left(\epsilon_{1} \ell_{1}^{2}+\ell_{2}^{2}\right)}{2} \\
& u=m \sqrt{\frac{\ell_{2}^{4}-\ell_{1}^{4}}{\left(2+\epsilon_{1} \epsilon_{2}\right) \ell_{1}^{2}-\epsilon_{2} \ell_{2}^{2}}} \operatorname{cn}\left(\ell_{1} x+\ell_{2} y \mid m\right), \\
& v=\frac{2 \ell_{1}^{2} u^{2}}{\epsilon_{1} \ell_{1}^{2}-\ell_{2}^{2}}+\frac{\left(2 m^{2}-1\right)\left(\epsilon_{1} \ell_{1}^{2}+\ell_{2}^{2}\right)}{2} ; \\
& u=\sqrt{\frac{\ell_{2}^{4}-\ell_{1}^{4}}{\left(2+\epsilon_{1} \epsilon_{2}\right) \ell_{1}^{2}-\epsilon_{2} \ell_{2}^{2}}} \operatorname{dn}\left(\ell_{1} x+\ell_{2} y \mid m\right), v=\frac{2 \ell_{1}^{2} u^{2}}{\epsilon_{1} \ell_{1}^{2}-\ell_{2}^{2}}+\frac{\left(2-m^{2}\right)\left(\epsilon_{1} \ell_{1}^{2}+\ell_{2}^{2}\right)}{2} .
\end{aligned}
$$

Remark 6.3.2. Since $\lim _{m \rightarrow 1} \operatorname{dn}(x \mid m)=\operatorname{sech} x$, (6.3.52) yields the solution

$$
u=\sqrt{\frac{\ell_{2}^{4}-\ell_{1}^{4}}{\left(2+\epsilon_{1} \epsilon_{2}\right) \ell_{1}^{2}-\epsilon_{2} \ell_{2}^{2}}} \operatorname{sech}\left(\ell_{1} x+\ell_{2} y\right), v=\frac{2 \ell_{1}^{2} u^{2}}{\epsilon_{1} \ell_{1}^{2}-\ell_{2}^{2}}+\frac{\epsilon_{1} \ell_{1}^{2}+\ell_{2}^{2}}{2} .
$$

Applying $S_{\alpha, \beta, \gamma}$ in (6.3.9) and (6.3.10), we get a more general solution

$$
\begin{aligned}
u= & \sqrt{\frac{\ell_{2}^{4}-\ell_{1}^{4}}{\left(2+\epsilon_{1} \epsilon_{2}\right) \ell_{1}^{2}-\epsilon_{2} \ell_{2}^{2}}} e^{-\left(\epsilon_{1} \alpha^{\prime} x+\beta^{\prime} y+\gamma\right) i} \operatorname{sech}\left(\ell_{1}(x+\alpha)+\ell_{2}(y+\beta)\right), \\
v= & \frac{2 \ell_{1}^{2}\left(\epsilon_{1} \ell_{1}^{2}+\ell_{2}^{2}\right)}{\epsilon_{2} \ell_{2}^{2}-\left(2+\epsilon_{1} \epsilon_{2}\right) \ell_{1}^{2}} \operatorname{sech}^{2}\left(\ell_{1}(x+\alpha)+\ell_{2}(y+\beta)\right) \\
& +\frac{\epsilon_{1} \ell_{1}^{2}+\ell_{2}^{2}}{2}+\epsilon_{1} \alpha^{\prime \prime} x+\beta^{\prime \prime} y-\frac{\epsilon_{1}\left(\alpha^{\prime}\right)^{2}+\left(\beta^{\prime}\right)^{2}}{2}+\gamma^{\prime}
\end{aligned}
$$

where $\alpha, \beta$ and $\gamma$ are arbitrary functions of $t$. Taking $\alpha=a_{1} t, \beta=a_{2} t$ and $\gamma=$ $\left(\epsilon_{1} a_{1}^{2}+a_{2}^{2}\right) t / 2$, we have a solition solution

$$
u=\sqrt{\frac{\ell_{2}^{4}-\ell_{1}^{4}}{\left(2+\epsilon_{1} \epsilon_{2}\right) \ell_{1}^{2}-\epsilon_{2} \ell_{2}^{2}}} e^{-\left(\epsilon_{1} a_{1} x+a_{2} y+\left(\epsilon_{1} a_{1}^{2}+a_{2}^{2}\right) t / 2\right) i} \operatorname{sech}\left(\ell_{1} x+\ell_{2} y+\left(a_{1} \ell_{1}+a_{2} \ell_{2}\right) t\right),
$$




$$
v=\frac{2 \ell_{1}^{2}\left(\epsilon_{1} \ell_{1}^{2}+\ell_{2}^{2}\right)}{\epsilon_{2} \ell_{2}^{2}-\left(2+\epsilon_{1} \epsilon_{2}\right) \ell_{1}^{2}} \operatorname{sech}^{2}\left(\ell_{1} x+\ell_{2} y+\left(a_{1} \ell_{1}+a_{2} \ell_{2}\right) t\right)+\frac{\epsilon_{1} \ell_{1}^{2}+\ell_{2}^{2}}{2},
$$

where $a_{1}, a_{2} \in \mathbb{R}$.

Case 2. $\phi=\epsilon_{1} x^{2} / 2 t$ or $y^{2} / 2 t$.

Suppose $\phi=\epsilon_{1} x^{2} / 2 t$. Then (6.3.15) and (6.3.16) become

$$
\begin{gathered}
\xi_{t}+\frac{x}{t} \xi_{x}+\frac{1}{2 t} \xi=0 \\
-\epsilon_{1} \xi_{x x}-\xi_{y y}+2 \epsilon_{2} \xi^{3}+2 \xi v=0 .
\end{gathered}
$$

By (6.1.40)-(6.1.43), we have

$$
\xi=\frac{a}{\sqrt{t}}, \quad v=-\frac{\epsilon_{2} a^{2}}{t}, \quad a \in \mathbb{R},
$$

which satisfies (6.3.17). Moreover, (6.3.60) also holds when $\phi=y^{2} / 2 t$.

Case 3. $\phi=\epsilon_{1} x^{2} / 2 t+y^{2} / 2(t-d)$ with $0 \neq d \in \mathbb{R}$.

In this case, (6.1.45) and (6.3.59) hold. By (6.1.46)-(6.1.49),

$$
\xi=\frac{a}{\sqrt{t(t-d)}}, \quad v=-\frac{\epsilon_{2} a^{2}}{t(t-d)}, \quad a \in \mathbb{R} .
$$

In summary, we have:

Theorem 6.3.3. For $a, d \in \mathbb{R}$ with $d \neq 0$, we have the following solutions of the Davey-Stewartson equations (6.3.1) and (6.3.2):

$$
\begin{gathered}
u=\frac{a e^{\epsilon_{1} x^{2} i / 2 t}}{\sqrt{t}}, \quad v=-\frac{\epsilon_{2} a^{2}}{t} ; \\
u=\frac{a e^{y^{2} i / 2 t}}{\sqrt{t}}, \quad v=-\frac{\epsilon_{2} a^{2}}{t} ; \\
u=\frac{a e^{\left(\epsilon_{1} x^{2} / 2 t+y^{2} / 2(t-d)\right) i}}{\sqrt{t(t-d)}}, \quad v=-\frac{\epsilon_{2} a^{2}}{t(t-d)} .
\end{gathered}
$$

Case 4. $\phi=\left(\epsilon_{1} x^{2}+y^{2}\right) / 2 t$.

In this case, (6.3.15) becomes (6.1.54). So

$$
\xi=\frac{1}{t} \zeta(z, s), \quad z=\frac{x}{t}, s=\frac{y}{t},
$$


for some two-variable function $\zeta$ by (6.1.55). Moreover, (6.3.16) becomes

$$
-\frac{\epsilon_{1} \zeta_{z z}+\zeta_{s s}}{t^{2}}+2 \frac{\epsilon_{2} \zeta^{3}}{t^{2}}+2 \zeta v=0 .
$$

Assume

$$
v=\frac{\eta(z, s)}{t^{2}}
$$

for some two-variable functions $\eta$. Then (6.3.66) becomes

$$
-\epsilon_{1} \zeta_{z z}-\zeta_{s s}+2 \epsilon_{2} \zeta^{3}+2 \zeta \eta=0
$$

and (6.3.17) becomes

$$
\eta_{z z}-\epsilon_{1}\left(\eta_{s s}+2\left(\zeta^{2}\right)_{z z}\right)=0 .
$$

By the arguments in (6.3.17)-(6.3.39), we obtain:

Theorem 6.3.4. If $\epsilon_{2}=1$, we have the following solutions of the Davey-Stewartson equations (6.3.1) and (6.3.2): for $a_{1}, a_{2} \in \mathbb{R}$ and $a_{1} \neq 0$,

$$
\begin{gathered}
u=\frac{e^{\left(\epsilon_{1} x^{2}+y^{2}\right) i / 2 t}}{t}\left(a_{1} e^{\left(x \pm \sqrt{2+\epsilon_{1}} y\right) / t}+a_{2} e^{\left(-x \mp \sqrt{2+\epsilon_{1}} y\right) / t}\right), \\
v=\frac{1}{t^{2}}\left[2-\left(a_{1} e^{\left(x \pm \sqrt{2+\epsilon_{1}} y\right) / t}+a_{2} e^{\left(-x \mp \sqrt{2+\epsilon_{1}} y\right) / t}\right)^{2}\right] ; \\
u=\frac{a_{1} e^{\left(\epsilon_{1} x^{2}+y^{2}\right) i / 2 t}}{t} \sin \frac{x \pm \sqrt{2+\epsilon_{1}} y}{t}, \quad v=-\frac{1}{t^{2}}\left(2+a_{1}^{2} \sin ^{2} \frac{x \pm \sqrt{2+\epsilon_{1}} y}{t}\right) ;
\end{gathered}
$$

Let $\ell_{1}, \ell_{2} \in \mathbb{R}$ such that

$$
\ell_{1}^{4} \neq \ell_{2}^{4} \text { and }\left(2+\epsilon_{1} \epsilon_{2}\right) \ell_{1}^{2} \neq \epsilon_{2} \ell_{2}^{2} .
$$

Then we the following solutions of the Davey-Stewartson equations (6.3.1) and (6.3.2):

$$
\begin{aligned}
& u=\frac{e^{\left(\epsilon_{1} x^{2}+y^{2}\right) i / 2 t}}{\ell_{1} x+\ell_{2} y} \sqrt{\frac{\ell_{1}^{4}-\ell_{2}^{4}}{\left(2+\epsilon_{1} \epsilon_{2}\right) \ell_{1}^{2}-\epsilon_{2} \ell_{2}^{2}}}, \quad v=\frac{2 \ell_{1}^{2}\left(\epsilon_{1} \ell_{1}^{2}+\ell_{2}^{2}\right)}{\left(\left(2+\epsilon_{1} \epsilon_{2}\right) \ell_{1}^{2}-\epsilon_{2} \ell_{2}^{2}\right)\left(\ell_{1} x+\ell_{2} y\right)^{2}} ; \\
& u=\sqrt{\frac{\ell_{1}^{4}-\ell_{2}^{4}}{\left(2+\epsilon_{1} \epsilon_{2}\right) \ell_{1}^{2}-\epsilon_{2} \ell_{2}^{2}}} \frac{e^{\left(\epsilon_{1} x^{2}+y^{2}\right) i / 2 t}}{t} \tan \frac{\ell_{1} x+\ell_{2} y}{t}, \\
& v=\frac{2 \ell_{1}^{2}\left(\epsilon_{1} \ell_{1}^{2}+\ell_{2}^{2}\right)}{\left(\left(2+\epsilon_{1} \epsilon_{2}\right) \ell_{1}^{2}-\epsilon_{2} \ell_{2}^{2}\right) t^{2}} \tan ^{2} \frac{\ell_{1} x+\ell_{2} y}{t}+\frac{\epsilon_{1} \ell_{1}^{2}+\ell_{2}^{2}}{t^{2}} ; \\
& u=\sqrt{\frac{\ell_{1}^{4}-\ell_{2}^{4}}{\left(2+\epsilon_{1} \epsilon_{2}\right) \ell_{1}^{2}-\epsilon_{2} \ell_{2}^{2}}} \frac{e^{\left(\epsilon_{1} x^{2}+y^{2}\right) i / 2 t}}{t} \sec \frac{\ell_{1} x+\ell_{2} y}{t}, \\
& v=\frac{2 \ell_{1}^{2}\left(\epsilon_{1} \ell_{1}^{2}+\ell_{2}^{2}\right)}{\left(\left(2+\epsilon_{1} \epsilon_{2}\right) \ell_{1}^{2}-\epsilon_{2} \ell_{2}^{2}\right) t^{2}} \sec ^{2} \frac{\ell_{1} x+\ell_{2} y}{t}-\frac{\epsilon_{1} \ell_{1}^{2}+\ell_{2}^{2}}{2 t^{2}} ; \\
& u=\sqrt{\frac{\ell_{1}^{4}-\ell_{2}^{4}}{\left(2+\epsilon_{1} \epsilon_{2}\right) \ell_{1}^{2}-\epsilon_{2} \ell_{2}^{2}}} \frac{e^{\left(\epsilon_{1} x^{2}+y^{2}\right) i / 2 t}}{t} \operatorname{coth} \frac{\ell_{1} x+\ell_{2} y}{t},
\end{aligned}
$$




$$
\begin{aligned}
& v=\frac{2 \ell_{1}^{2}\left(\epsilon_{1} \ell_{1}^{2}+\ell_{2}^{2}\right)}{\left(\left(2+\epsilon_{1} \epsilon_{2}\right) \ell_{1}^{2}-\epsilon_{2} \ell_{2}^{2}\right) t^{2}} \operatorname{coth}^{2} \frac{\ell_{1} x+\ell_{2} y}{t}-\frac{\epsilon_{1} \ell_{1}^{2}+\ell_{2}^{2}}{t^{2}} \\
& u=\sqrt{\frac{\ell_{1}^{4}-\ell_{2}^{4}}{\left(2+\epsilon_{1} \epsilon_{2}\right) \ell_{1}^{2}-\epsilon_{2} \ell_{2}^{2}}} \frac{e^{\left(\epsilon_{1} x^{2}+y^{2}\right) i / 2 t}}{t} \operatorname{csch} \frac{\ell_{1} x+\ell_{2} y}{t}, \\
& v=\frac{2 \ell_{1}^{2}\left(\epsilon_{1} \ell_{1}^{2}+\ell_{2}^{2}\right)}{\left(\left(2+\epsilon_{1} \epsilon_{2}\right) \ell_{1}^{2}-\epsilon_{2} \ell_{2}^{2}\right) t^{2}} \operatorname{csch}^{2} \frac{\ell_{1} x+\ell_{2} y}{t}+\frac{\epsilon_{1} \ell_{1}^{2}+\ell_{2}^{2}}{2 t^{2}} \\
& u=m \sqrt{\frac{\ell_{1}^{4}-\ell_{2}^{4}}{\left(2+\epsilon_{1} \epsilon_{2}\right) \ell_{1}^{2}-\epsilon_{2} \ell_{2}^{2}}} \frac{e^{\left(\epsilon_{1} x^{2}+y^{2}\right) i / 2 t}}{t} \mathrm{sn}\left(\frac{\ell_{1} x+\ell_{2} y}{t} \mid m\right), \\
& v=\frac{2 \ell_{1}^{2}\left(\epsilon_{1} \ell_{1}^{2}+\ell_{2}^{2}\right)}{\left(\left(2+\epsilon_{1} \epsilon_{2}\right) \ell_{1}^{2}-\epsilon_{2} \ell_{2}^{2}\right) t^{2}} \operatorname{sn}^{2}\left(\frac{\ell_{1} x+\ell_{2} y}{t} \mid m\right)-\frac{\left(m^{2}+1\right)\left(\epsilon_{1} \ell_{1}^{2}+\ell_{2}^{2}\right)}{2 t^{2}} \text {; } \\
& u=m \sqrt{\frac{\ell_{2}^{4}-\ell_{1}^{4}}{\left(2+\epsilon_{1} \epsilon_{2}\right) \ell_{1}^{2}-\epsilon_{2} \ell_{2}^{2}}} \frac{e^{\left(\epsilon_{1} x^{2}+y^{2}\right) i / 2 t}}{t} \mathrm{cn}\left(\frac{\ell_{1} x+\ell_{2} y}{t} \mid m\right), \\
& v=-\frac{2 \ell_{1}^{2}\left(\epsilon_{1} \ell_{1}^{2}+\ell_{2}^{2}\right)}{\left(\left(2+\epsilon_{1} \epsilon_{2}\right) \ell_{1}^{2}-\epsilon_{2} \ell_{2}^{2}\right) t^{2}} \mathrm{cn}^{2}\left(\frac{\ell_{1} x+\ell_{2} y}{t} \mid m\right)+\frac{\left(2 m^{2}-1\right)\left(\epsilon_{1} \ell_{1}^{2}+\ell_{2}^{2}\right)}{2 t^{2}} \text {; } \\
& u=\sqrt{\frac{\ell_{2}^{4}-\ell_{1}^{4}}{\left(2+\epsilon_{1} \epsilon_{2}\right) \ell_{1}^{2}-\epsilon_{2} \ell_{2}^{2}}} \frac{e^{\left(\epsilon_{1} x^{2}+y^{2}\right) i / 2 t}}{t} \mathrm{dn}\left(\frac{\ell_{1} x+\ell_{2} y}{t} \mid m\right), \\
& v=-\frac{2 \ell_{1}^{2}\left(\epsilon_{1} \ell_{1}^{2}+\ell_{2}^{2}\right)}{\left(\left(2+\epsilon_{1} \epsilon_{2}\right) \ell_{1}^{2}-\epsilon_{2} \ell_{2}^{2}\right) t^{2}} \operatorname{dn}^{2}\left(\frac{\ell_{1} x+\ell_{2} y}{t} \mid m\right)+\frac{\left(2-m^{2}\right)\left(\epsilon_{1} \ell_{1}^{2}+\ell_{2}^{2}\right)}{2 t^{2}} \text {. }
\end{aligned}
$$

Remark 6.3.5. Since $\lim _{m \rightarrow 1} \operatorname{dn}(x \mid m)=\operatorname{sech} x$, (6.3.87) and (6.3.88) yield the solution

$$
\begin{gathered}
u=\sqrt{\frac{\ell_{2}^{4}-\ell_{1}^{4}}{\left(2+\epsilon_{1} \epsilon_{2}\right) \ell_{1}^{2}-\epsilon_{2} \ell_{2}^{2}}} \frac{e^{\left(\epsilon_{1} x^{2}+y^{2}\right) i / 2 t}}{t} \operatorname{sech} \frac{\ell_{1} x+\ell_{2} y}{t}, \\
v=-\frac{2 \ell_{1}^{2}\left(\epsilon_{1} \ell_{1}^{2}+\ell_{2}^{2}\right)}{\left(\left(2+\epsilon_{1} \epsilon_{2}\right) \ell_{1}^{2}-\epsilon_{2} \ell_{2}^{2}\right) t^{2}} \operatorname{sech}^{2} \frac{\ell_{1} x+\ell_{2} y}{t}+\frac{\epsilon_{1} \ell_{1}^{2}+\ell_{2}^{2}}{2 t^{2}} .
\end{gathered}
$$

Applying $S_{a_{1} t, a_{2} t,\left(\epsilon_{1} a_{1}^{2}+a_{2}^{2}\right) t / 2}$ in (6.3.9) and (6.3.10), we get a solition-like solution

$$
\begin{aligned}
& u= \sqrt{\frac{\ell_{2}^{4}-\ell_{1}^{4}}{\left(2+\epsilon_{1} \epsilon_{2}\right) \ell_{1}^{2}-\epsilon_{2} \ell_{2}^{2}}} \frac{e^{\left(\epsilon_{1} x^{2}+y^{2}\right) i / 2 t-\left(\epsilon_{1} a_{1} x+a_{2} y+\left(\epsilon_{1} a_{1}^{2}+a_{2}^{2}\right) t / 2\right) i}}{t} \\
& \times \operatorname{sech} \frac{\ell_{1} x+\ell_{2} y+\left(a_{1} \ell_{1}+a_{2} \ell_{2}\right) t}{t}, \\
& v=-\frac{2 \ell_{1}^{2}\left(\epsilon_{1} \ell_{1}^{2}+\ell_{2}^{2}\right)}{\left(\left(2+\epsilon_{1} \epsilon_{2}\right) \ell_{1}^{2}-\epsilon_{2} \ell_{2}^{2}\right) t^{2}} \operatorname{sech}^{2} \frac{\ell_{1} x+\ell_{2} y+\left(a_{1} \ell_{1}+a_{2} \ell_{2}\right) t}{t}+\frac{\epsilon_{1} \ell_{1}^{2}+\ell_{2}^{2}}{2 t^{2}} .
\end{aligned}
$$




\section{Chapter 7}

\section{Dynamic Convection in a Sea}

The rotation of the earth influences both the atmospheric and oceanic flows. In fact, the fast rotation and small aspect ratio are two main characteristics of the large scale atmospheric and oceanic flows. The small aspect ratio characteristic leads to the primitive equations, and the fast rotation leads to the quasi-geostropic equations (e.g., cf. [GC, LTW1, LTW2, Pj]). A main objective in climate dynamics and in geophysical fluid dynamics is to understand and predict the periodic, quasi-periodic, aperiodic, and fully turbulent characteristics of the large scale atmospheric and oceanic flows (e.g., cf. [HMW, Le]). The general model of atmospheric and oceanic flows is very complicated. In this chapter, we study a simplified model of dynamic convection in a sea due to Ovsiannikov (1967) (e.g., cf. Page 203 in [In3]).

In Section 7.1, we present the equations for dynamic convection in a sea and the symmetry analysis on them. In Section 7.2, we use a new variable of moving line to solve the equations. An approach of using the product of cylindrical invariant function with $z$ is introduced in Section 7.3. In Section 7.4, we reduce the three-dimensional (spacial) equations into a two-dimensional problem and then solve it with three different ansatzes (assumptions). This chapter is a revision of our earlier preprint [X17].

\subsection{Equations and Symmetries}

The following equations

$$
\begin{gathered}
u_{x}+v_{y}+w_{z}=0, \quad \rho=p_{z}, \\
\rho_{t}+u \rho_{x}+v \rho_{y}+w \rho_{z}=0, \\
u_{t}+u u_{x}+v u_{y}+w u_{z}+v=-\frac{1}{\rho} p_{x}, \\
v_{t}+u v_{x}+v v_{y}+w v_{z}-u=-\frac{1}{\rho} p_{y}
\end{gathered}
$$

are used to describe the dynamic convection of a sea in geophysics, where $u, v$ and $w$ are components of velocity vector of relative motion of fluid in Cartesian coordinates $(x, y, z)$, 
$\rho=\rho(x, y, z, t)$ is the density of fluid and $p$ is the pressure (e.g., cf. Page 203 in [In3]). Ovsiannikov determined the Lie point symmetries of the above equations and found two very special solutions.

Let us first do degree analysis. Denote

$$
\operatorname{deg} u=\ell, \quad \operatorname{deg} x=\ell_{1}, \quad \operatorname{deg} y=\ell_{2}, \quad \operatorname{deg} x=\ell_{3} .
$$

To make the nonzero terms in (7.1.1)-(7.1.4) to have the same degree, we have to take

$$
\begin{gathered}
\operatorname{deg} u_{x}=\operatorname{deg} v_{y} \Longrightarrow \operatorname{deg} v=\ell+\ell_{2}-\ell_{1}, \\
\operatorname{deg} u_{x}=\operatorname{deg} w_{z} \Longrightarrow \operatorname{deg} w=\ell+\ell_{3}-\ell_{1}, \\
\operatorname{deg} u_{t}=\operatorname{deg} u u_{x} \Longrightarrow \operatorname{deg} t=\ell_{1}-\ell, \\
\operatorname{deg} u_{t}=\operatorname{deg} v \sim 2 \ell-\ell_{1}=\ell+\ell_{2}-\ell_{1} \Longrightarrow \ell_{2}=\ell, \\
\operatorname{deg} v_{t}=\operatorname{deg} u \sim 2 \ell+\ell_{2}-2 \ell_{1}=\ell \Longrightarrow \ell_{1}=\ell, \\
\operatorname{deg} \rho=\operatorname{deg} p_{z} \Longrightarrow \operatorname{deg} \rho=\operatorname{deg} p-\ell_{3}, \\
\operatorname{deg} u=\operatorname{deg} \frac{1}{\rho} p_{y} \sim \ell=\operatorname{deg} p-\operatorname{deg} \rho-\ell_{2} \Longrightarrow \ell=\ell_{3}-\ell_{2} \Longrightarrow \ell_{3}=2 \ell .
\end{gathered}
$$

In summary,

$$
\begin{gathered}
\operatorname{deg} u=\operatorname{deg} v=\operatorname{deg} x=\operatorname{deg} y=\ell, \\
\operatorname{deg} w=\operatorname{deg} z=2 \ell=\operatorname{deg} p-\operatorname{deg} \rho, \quad \operatorname{deg} t=0 .
\end{gathered}
$$

Moreover, the equations (7.1.1)-(7.1.4) are translation invariant because they do not contain variable coefficients. Thus the transformation

$$
\begin{gathered}
T_{a ; b_{1}, b_{2}}(u(t, x, y, z))=b_{1}^{-1} u\left(t+a, b_{1} x, b_{1} y, b_{1}^{2} z\right), \\
T_{a ; b_{1}, b_{2}}(v(t, x, y, z))=b_{1}^{-1} v\left(t+a, b_{1} x, b_{1} y, b_{1}^{2} z\right), \\
T_{a ; b_{1}, b_{2}}(w(t, x, y, z))=b_{1}^{-2} w\left(t+a, b_{1} x, b_{1} y, b_{1}^{2} z\right), \\
T_{a ; b_{1}, b_{2}}(\rho(t, x, y, z))=b_{2} \rho\left(t+a, b_{1} x, b_{1} y, b_{1}^{2} z\right), \\
T_{a ; b_{1}, b_{2}}(p(t, x, y, z))=b_{1}^{-2} b_{2} p\left(t+a, b_{1} x, b_{1} y, b_{1}^{2} z\right)
\end{gathered}
$$

is a symmetry of the equations (7.1.1)-(7.1.4).

Let $\alpha$ be a function in $t$. Note that the transformation

$$
F(t, x, y, z) \mapsto F(t, x+\alpha, y, z) \text { with } F=u, v, w, p, \rho
$$

leaves (7.1.1) invariant and changes (7.1.2)-(7.1.4) to:

$$
\alpha^{\prime} \rho_{x}+\rho_{t}+u \rho_{x}+v \rho_{y}+w \rho_{z}=0
$$




$$
\begin{aligned}
\alpha^{\prime} u_{x}+u_{t}+u u_{x}+v u_{y}+w u_{z}+v & =-\frac{1}{\rho} p_{x}, \\
\alpha^{\prime} v_{x}+v_{t}+u v_{x}+v v_{y}+w v_{z}-u & =-\frac{1}{\rho} p_{y},
\end{aligned}
$$

where the independent variable $x$ is replaced by $x+\alpha$ and the partial derivatives are with respect to the original variables. Thus the transformation

$$
F(t, x, y, z) \mapsto F(t, x+\alpha, y, z)-\delta_{u, F} \alpha^{\prime} \text { with } F=u, v, w, p, \rho
$$

leaves (7.1.1) and (7.1.2) invariant, and changes (7.1.3) and (7.1.4) to

$$
\begin{gathered}
-\alpha^{\prime \prime}+u_{t}+u u_{x}+v u_{y}+w u_{z}+v=-\frac{1}{\rho} p_{x} \\
v_{t}+u v_{x}+v v_{y}+w v_{z}-u+\alpha^{\prime}=-\frac{1}{\rho} p_{y} .
\end{gathered}
$$

On the other hand, the transformation

$$
F(t, x, y, z) \mapsto F\left(t, x, y, z+\alpha^{\prime \prime} x-\alpha^{\prime} y\right) \text { with } F=u, v, w, p, \rho
$$

leaves the second equation in (7.1.1) invariant and changes the first equation in (7.1.1), and (7.1.2)-(7.1.4) to:

$$
\begin{gathered}
\alpha^{\prime \prime} u_{z}+u_{x}-\alpha^{\prime} v_{z}+v_{y}+w_{z}=0, \\
\left(\alpha^{\prime \prime} x-\alpha^{\prime \prime} y\right) \rho_{z}+\rho_{t}+\alpha^{\prime \prime} u \rho_{z}+u \rho_{x}-\alpha^{\prime} v \rho_{z}+v \rho_{y}+w \rho_{z}=0, \\
\left(\alpha^{\prime \prime \prime} x-\alpha^{\prime \prime} y\right) u_{z}+u_{t}+\alpha^{\prime \prime} u u_{z}+u u_{x}-\alpha^{\prime} v u_{z}+v u_{y}+w u_{z}+v=-\frac{1}{\rho} p_{x}-\alpha^{\prime \prime}, \\
\left(\alpha^{\prime \prime \prime} x-\alpha^{\prime \prime} y\right) v_{z}+v_{t}+\alpha^{\prime \prime} u v_{z}+u v_{x}-\alpha^{\prime} v v_{z}+v v_{y}+w v_{z}-u=-\frac{1}{\rho} p_{y}+\alpha^{\prime} .
\end{gathered}
$$

Thus we have the following symmetry transformation of (7.1.1)-(7.1.4):

$$
S_{1, \alpha}(F(t, x, y, z))=F\left(t, x+\alpha, y, z+\alpha^{\prime \prime} x-\alpha^{\prime} y\right)-\delta_{u, F} \alpha^{\prime} \text { with } F=u, v, p, \rho
$$

and

$$
S_{1, \alpha}(w(t, x, y, z))=w\left(t, x+\alpha, y, z+\alpha^{\prime \prime} x-\alpha^{\prime} y\right)-\alpha^{\prime \prime} u+\alpha^{\prime} v-\alpha^{\prime \prime} x+\alpha^{\prime \prime} y .
$$

Similarly, we have the symmetry transformation of (7.1.1)-(7.1.4):

$$
S_{2, \alpha}(F(t, x, y, z))=F\left(t, x, y+\alpha, z+\alpha^{\prime} x+\alpha^{\prime \prime} y\right)-\delta_{v, F} \alpha^{\prime} \text { with } F=u, v, p, \rho
$$

and

$$
S_{2, \alpha}(w(t, x, y, z))=w\left(t, x+\alpha, y, z+\alpha^{\prime} x+\alpha^{\prime \prime} y\right)-\alpha^{\prime} u-\alpha^{\prime \prime} v-\alpha^{\prime \prime} x-\alpha^{\prime \prime} y .
$$

Let $\beta$ be another function in $t$. We have the following symmetry transformation of (7.1.1)(7.1.4):

$$
S_{\alpha, \beta}(F(t, x, y, z))=F(t, x, y, z+\alpha)-\delta_{w, F} \alpha^{\prime}+\delta_{p, F} \beta \text { with } F=u, v, w, p, \rho .
$$

The above transformations transform one solution of the equations (7.1.1)-(7.1.4) into another solution. Applying the above transformations to any solution found in this chapter will yield another solution with four extra parameter functions. 


\subsection{Moving-Line Approach}

Let $\alpha$ and $\beta$ be given functions in $t$. Denote the variable of moving line

$$
\varpi=\alpha^{\prime} x+\beta^{\prime} y+z
$$

Suppose that $f, g, h$ are functions in $t, x, y, z$ that are linear in $x, y, z$ such that

$$
f_{x}+g_{y}+h_{z}=0
$$

We assume

$$
\begin{gathered}
u=\phi(t, \varpi)+f, \quad v=\psi(t, \varpi)+g, \\
w=h-\alpha^{\prime} \phi(t, \varpi)-\beta^{\prime} \psi(t, \varpi), \quad p=\zeta(t, \varpi),
\end{gathered}
$$

where $\phi, \psi, \zeta$ are two-variable functions to be determined. Note that the first equation in (7.1.1) naturally holds and $\rho=p_{z}=\zeta_{\varpi}$ by the second equation in (7.1.1). Moreover, (7.1.2)-(7.1.4) become

$$
\begin{gathered}
\zeta_{\varpi t}+\zeta_{\varpi \varpi}\left(\alpha^{\prime \prime} x+\beta^{\prime \prime} y+\alpha^{\prime} f+\beta^{\prime} g+h\right)=0, \\
f_{t}+g+f f_{x}+g f_{y}+h f_{z}+\alpha^{\prime}+\phi_{t}+\left(f_{x}-\alpha^{\prime} f_{z}\right) \phi+\left(f_{y}-\beta^{\prime} f_{z}+1\right) \psi \\
+\phi_{\varpi}\left(\alpha^{\prime \prime} x+\beta^{\prime \prime} y+\alpha^{\prime} f+\beta^{\prime} g+h\right)=0, \\
g_{t}-f+f g_{x}+g g_{y}+h g_{z}+\beta^{\prime}+\psi_{t}+\left(g_{x}-\alpha^{\prime} g_{z}-1\right) \phi+\left(g_{y}-\beta^{\prime} g_{z}\right) \psi \\
+\psi_{\varpi}\left(\alpha^{\prime \prime} x+\beta^{\prime \prime} y+\alpha^{\prime} f+\beta^{\prime} g+h\right)=0 .
\end{gathered}
$$

In order to solve the above system of partial differential equations, we assume

$$
\alpha^{\prime \prime} x+\beta^{\prime \prime} y+\alpha^{\prime} f+\beta^{\prime} g+h=-\gamma^{\prime} \varpi=-\gamma^{\prime}\left(\alpha^{\prime} x+\beta^{\prime} y+z\right)
$$

for some function $\gamma$ in $t$, and

$$
\begin{aligned}
& f_{t}+g+f f_{x}+g f_{y}+h f_{z}+\alpha^{\prime}=0, \\
& g_{t}-f+f g_{x}+g g_{y}+h g_{z}+\beta^{\prime}=0 .
\end{aligned}
$$

Then (7.2.5)-(7.2.7) become

$$
\begin{gathered}
\zeta_{\varpi t}-\gamma^{\prime} \varpi \zeta_{\varpi \varpi}=0, \\
\phi_{t}+\left(f_{x}-\alpha^{\prime} f_{z}\right) \phi+\left(f_{y}-\beta^{\prime} f_{z}+1\right) \psi-\gamma^{\prime} \varpi \phi_{\varpi}=0, \\
\psi_{t}+\left(g_{x}-\alpha^{\prime} g_{z}-1\right) \phi+\left(g_{y}-\beta^{\prime} g_{z}\right) \psi-\gamma^{\prime} \varpi \psi_{\varpi}=0 .
\end{gathered}
$$

According to (7.2.8),

$$
h=-\alpha^{\prime \prime} x-\beta^{\prime \prime} y-\alpha^{\prime} f-\beta^{\prime} g-\gamma^{\prime} \varpi .
$$


Substituting the above equation into (7.2.9) and (7.2.10), we have:

$$
\begin{aligned}
& f_{t}+f\left(f_{x}-\alpha^{\prime} f_{z}\right)+g\left(f_{y}-\beta^{\prime} f_{z}+1\right)-f_{z}\left(\alpha^{\prime \prime} x+\beta^{\prime \prime} y+\gamma^{\prime} \varpi\right)+\alpha^{\prime}=0, \\
& g_{t}+f\left(g_{x}-\alpha^{\prime} g_{z}-1\right)+g\left(g_{y}-\beta^{\prime} g_{z}\right)-g_{z}\left(\alpha^{\prime \prime} x+\beta^{\prime \prime} y+\gamma^{\prime} \varpi\right)+\beta^{\prime}=0 .
\end{aligned}
$$

Our linearity assumption implies that

$$
A=\left(\begin{array}{cc}
f_{x}-\alpha^{\prime} f_{z} & f_{y}-\beta^{\prime} f_{z}+1 \\
g_{x}-\alpha^{\prime} g_{z}-1 & g_{y}-\beta^{\prime} g_{z}
\end{array}\right)
$$

is a matrix function in $t$. In order to solve the system (7.2.12) and (7.2.13), and the system (7.2.15) and (7.2.16), we need the commutativity of $A$ with $d A / d t$. For simplicity, we assume

$$
f_{y}-\beta^{\prime} f_{z}+1=g_{x}-\alpha^{\prime} g_{z}-1=0 .
$$

So

$$
f_{y}=\beta^{\prime} f_{z}-1, \quad g_{x}=\alpha^{\prime} g_{z}+1 .
$$

Moreover, (7.2.15) and (7.2.16) become

$$
\begin{aligned}
& f_{t}+f\left(f_{x}-\alpha^{\prime} f_{z}\right)-f_{z}\left(\alpha^{\prime \prime} x+\beta^{\prime \prime} y+\gamma^{\prime} \varpi\right)+\alpha^{\prime}=0, \\
& g_{t}+g\left(g_{y}-\beta^{\prime} g_{z}\right)-g_{z}\left(\alpha^{\prime \prime} x+\beta^{\prime \prime} y+\gamma^{\prime} \varpi\right)+\beta^{\prime}=0 .
\end{aligned}
$$

Write

$$
\begin{gathered}
f=\alpha_{1} x+\left(\beta^{\prime} \alpha_{2}-1\right) y+\alpha_{2} z+\alpha_{3}, \\
g=\left(\alpha^{\prime} \beta_{2}+1\right) x+\beta_{1} y+\beta_{2} z+\beta_{3}
\end{gathered}
$$

by our linearity assumption and (7.2.19), where $\alpha_{l}$ and $\beta_{j}$ are functions in $t$.

Now (7.2.20) is equivalent to the following system of ordinary differential equations:

$$
\begin{gathered}
\alpha_{1}^{\prime}+\alpha_{1}\left(\alpha_{1}-\alpha^{\prime} \alpha_{2}\right)-\alpha_{2}\left(\alpha^{\prime \prime}+\gamma^{\prime} \alpha^{\prime}\right)=0, \\
\left(\beta^{\prime} \alpha_{2}\right)^{\prime}+\left(\beta^{\prime} \alpha_{2}-1\right)\left(\alpha_{1}-\alpha^{\prime} \alpha_{2}\right)-\alpha_{2}\left(\beta^{\prime \prime}+\gamma^{\prime} \beta^{\prime}\right)=0, \\
\alpha_{2}^{\prime}+\alpha_{2}\left(\alpha_{1}-\alpha^{\prime} \alpha_{2}-\gamma^{\prime}\right)=0, \\
\alpha_{3}^{\prime}+\alpha_{3}\left(\alpha_{1}-\alpha^{\prime} \alpha_{2}\right)+\alpha^{\prime}=0 .
\end{gathered}
$$

Observe that (7.2.25) $-\beta^{\prime} \times(7.2 .26)$ becomes

$$
-\alpha_{1}+\alpha^{\prime} \alpha_{2}=0 .
$$

So (7.2.26) becomes

$$
\alpha_{2}^{\prime}-\gamma^{\prime} \alpha_{2}=0 \Longrightarrow \alpha_{2}=b_{1} e^{\gamma}, \quad b_{1} \in \mathbb{R} .
$$


According to (7.2.28),

$$
\alpha_{1}=b_{1} \alpha^{\prime} e^{\gamma}
$$

With the data (7.2.29) and (7.2.30), (7.2.24) naturally holds. By (7.2.27), we take

$$
\alpha_{3}=-\alpha
$$

Note that (7.2.21) is equivalent to the following system of ordinary differential equations:

$$
\begin{gathered}
\alpha^{\prime} \beta_{2}^{\prime}+\left(\alpha^{\prime} \beta_{2}+1\right)\left(\beta_{1}-\beta^{\prime} \beta_{2}\right)-\alpha^{\prime} \beta_{2} \gamma^{\prime}=0, \\
\beta_{1}^{\prime}+\beta_{1}\left(\beta_{1}-\beta^{\prime} \beta_{2}\right)-\beta_{2}\left(\beta^{\prime \prime}+\beta^{\prime} \gamma^{\prime}\right)=0 \\
\beta_{2}^{\prime}+\beta_{2}\left(\beta_{1}-\beta^{\prime} \beta_{2}-\gamma^{\prime}\right)=0 \\
\beta_{3}^{\prime}+\beta_{3}\left(\beta_{1}-\beta^{\prime} \beta_{2}\right)+\beta^{\prime}=0 .
\end{gathered}
$$

Similarly, we have:

$$
\beta_{1}=b_{2} \beta^{\prime} e^{\gamma}, \quad \beta_{2}=b_{2} e^{\gamma}, \quad \beta_{3}=\beta
$$

with $b_{2} \in \mathbb{R}$. Moreover, (7.2.2) gives $\gamma^{\prime}=0$ by (7.2.14), (7.2.28) and (7.2.36). We take $\gamma=0$. Therefore, $\phi=\Im(\varpi)$ and $\psi=\iota(\varpi)$ by (7.2.12) and (7.2.13) for some one-variable functions $\Im$ and $\iota$. Furthermore, we take $\zeta=\sigma(\varpi)$ by (7.2.11) for another one-variable function $\sigma$. In summary, we have:

Theorem 7.2.1. Let $\alpha, \beta$ be functions in $t$ and let $b_{1}, b_{2} \in \mathbb{R}$. Suppose that $\Im, \iota$ and $\sigma$ are arbitrary one-variable functions. The following is a solution of the equations (7.1.1)-(7.1.4) of dynamic convection in a sea:

$$
\begin{gathered}
u=b_{1} \alpha^{\prime} x+\left(b_{1} \beta^{\prime}-1\right) y+b_{1} z-\alpha+\Im\left(\alpha^{\prime} x+\beta^{\prime} y+z\right), \\
v=\left(b_{2} \alpha^{\prime}+1\right) x+b_{2} \beta^{\prime} y+b_{2} z+\beta+\iota\left(\alpha^{\prime} x+\beta^{\prime} y+z\right), \\
w=-\left(\alpha^{\prime \prime}+b_{1} \alpha^{\prime 2}+\left(b_{2} \alpha^{\prime}+1\right) \beta^{\prime}\right) x-\left(\beta^{\prime \prime}+\alpha^{\prime}\left(b_{1} \beta^{\prime}-1\right)+b_{2} \beta^{\prime 2}\right) y-\left(b_{1} \alpha^{\prime}+b_{2} \beta^{\prime}\right) z \\
+\alpha \alpha^{\prime}-\beta \beta^{\prime}-\alpha^{\prime} \Im\left(\alpha^{\prime} x+\beta^{\prime} y+z\right)-\beta^{\prime} \iota\left(\alpha^{\prime} x+\beta^{\prime} y+z\right), \\
p=\sigma\left(\alpha^{\prime} x+\beta^{\prime} y+z\right), \quad \rho=\sigma^{\prime}\left(\alpha^{\prime} x+\beta^{\prime} y+z\right) .
\end{gathered}
$$




\subsection{Approach of Cylindrical Product}

Let $\sigma$ be a fixed one-variable function and set the variable of cylindrical product:

$$
\varpi=z \sigma\left(x^{2}+y^{2}\right)
$$

Suppose that $f$ and $g$ are functions in $t, x, z$ that are linear homogeneous in $x, y$ and

$$
h=\frac{\gamma}{\sigma}-z\left(f_{x}+g_{y}\right)
$$

where $\gamma$ is a function in $t$. Assume

$$
u=f+y \psi(t, \varpi), \quad v=g-x \psi(t, \varpi), \quad w=h, \quad p=\phi(t, \varpi)
$$

where $\psi$ and $\phi$ are two-variable functions. Note

$$
\begin{gathered}
u_{t}=f_{t}+y \psi_{t}, \quad u_{x}=f_{x}+2 x y z \sigma^{\prime} \psi_{\varpi}, \\
u_{y}=f_{y}+\psi+2 y^{2} z \sigma^{\prime} \psi_{\varpi}, \quad u_{z}=f_{z}+y \sigma \psi_{\varpi}, \\
v_{t}=g_{t}-x \psi_{t}, \quad v_{x}=g_{x}-\psi-2 x^{2} z \sigma^{\prime} \psi_{\varpi}, \\
v_{y}=g_{y}-2 x y z \sigma^{\prime} \psi_{\varpi}, \quad v_{z}=g_{z}-x \sigma \psi_{\varpi} .
\end{gathered}
$$

Hence (7.1.3) becomes

$$
\begin{aligned}
& u_{t}+u u_{x}+v u_{y}+w u_{z}+v=f_{t}+y \psi_{t}+(f+y \psi)\left(f_{x}+2 x y z \sigma^{\prime} \psi_{\varpi}\right) \\
& +(g-x \psi)\left(f_{y}+1+\psi+2 y^{2} z \sigma^{\prime} \psi_{\varpi}\right)+y \sigma h \psi_{\varpi} \\
= & f_{t}+f f_{x}+g\left(1+f_{y}\right)+x\left(g_{x}-f_{y}-1\right) \psi-x \psi^{2} \\
& +y\left[\psi_{t}+\left(f_{x}+g_{y}\right) \psi+\left(2(x f+y g) \sigma^{\prime} z+h \sigma\right) \psi_{\varpi}\right]=-\frac{2 x z \sigma^{\prime}}{\sigma}
\end{aligned}
$$

and (7.1.4) gives

$$
\begin{aligned}
& v_{t}+u v_{x}+v v_{y}+w v_{z}-u=g_{t}-x \psi_{t}+(f+y \psi)\left(g_{x}-1-\psi-2 x^{2} z \sigma^{\prime} \psi_{\varpi}\right) \\
& +(g-x \psi)\left(g_{y}-2 x y z \sigma^{\prime} \psi_{\varpi}\right)-x \sigma h \psi_{\varpi} \\
= & g_{t}+f\left(g_{x}-1\right)+g g_{y}-y\left(1+f_{y}-g_{x}\right) \psi-y \psi^{2} \\
& -x\left[\psi_{t}+\left(f_{x}+g_{y}\right) \psi+\left(2(x f+y g) \sigma^{\prime} z+h \sigma\right) \psi_{\varpi}\right]=-\frac{2 y z \sigma^{\prime}}{\sigma} .
\end{aligned}
$$

In order to solve the above system of differential equations, we assume

$$
f=\alpha^{\prime} x-\frac{y}{2}, \quad g=\frac{x}{2}+\alpha^{\prime} y, \quad \sigma\left(x^{2}+y^{2}\right)=\frac{1}{x^{2}+y^{2}}
$$

for some function $\alpha$ in $t$. According to (7.3.2),

$$
h=\frac{\gamma}{\sigma}-2 \alpha^{\prime} z
$$


Now (7.3.8) becomes

$$
\left(\alpha^{\prime \prime}+\alpha^{\prime 2}+4^{-1}-\psi^{2}\right) x+y\left[\psi_{t}+2 \alpha^{\prime} \psi+\left(\gamma-4 \alpha^{\prime} \varpi\right) \psi_{\varpi}\right]=2 x \varpi
$$

and (7.3.9) yields

$$
\left(\alpha^{\prime \prime}+\alpha^{\prime 2}+4^{-1}-\psi^{2}\right) y-x\left[\psi_{t}+2 \alpha^{\prime} \psi+\left(\gamma-4 \alpha^{\prime} \varpi\right) \psi_{\varpi}\right]=2 y \varpi .
$$

The above system is equivalent to

$$
\begin{gathered}
\alpha^{\prime \prime}+\alpha^{\prime 2}+4^{-1}-\psi^{2}=2 \varpi, \\
\psi_{t}+2 \alpha^{\prime} \psi+\left(\gamma-4 \alpha^{\prime} \varpi\right) \psi_{\varpi}=0 .
\end{gathered}
$$

By (7.3.14), we take

$$
\psi=\sqrt{\alpha^{\prime \prime}+\alpha^{2}+4^{-1}-2 \varpi},
$$

due to the skew-symmetry of $(u, x)$ and $(v, y)$. Substituting (7.3.16) into (7.3.15), we get

$$
\alpha^{\prime \prime \prime}+2 \alpha^{\prime} \alpha^{\prime \prime}+4 \alpha^{\prime}\left(\alpha^{\prime \prime}+\alpha^{\prime 2}+4^{-1}-2 \varpi\right)-2\left(\gamma-4 \alpha^{\prime} \varpi\right)=0,
$$

equivalently,

$$
\gamma=2 \alpha^{\prime 3}+3 \alpha^{\prime} \alpha^{\prime \prime}+\frac{\alpha^{\prime \prime \prime}+\alpha^{\prime}}{2}
$$

According to the second equation in (7.1.1), we have $\rho=\sigma \phi_{\varpi}$. Note

$$
\begin{gathered}
\rho_{t}=\sigma \phi_{\varpi t}, \quad \rho_{x}=2 x \sigma^{\prime}\left(\phi_{\varpi}+\varpi \phi_{\varpi \varpi}\right), \\
\rho_{y}=2 y \sigma^{\prime}\left(\phi_{\varpi}+\varpi \phi_{\varpi \varpi}\right), \quad \rho_{z}=\sigma^{2} \phi_{\varpi \varpi} .
\end{gathered}
$$

So (7.1.2) becomes

$$
\phi_{\varpi t}-2 \alpha^{\prime} \phi_{\varpi}+\left(\gamma-4 \alpha^{\prime} \varpi\right) \phi_{\varpi \varpi}=0 .
$$

Modulo some $S_{0, \beta}$ in (7.1.36), the above equation is equivalent to:

$$
\phi_{t}+2 \alpha^{\prime} \phi+\left(\gamma-4 \alpha^{\prime} \varpi\right) \phi_{\varpi}=0
$$

Set

$$
\tilde{\psi}=e^{2 \alpha} \psi, \quad \tilde{\phi}=e^{2 \alpha} \phi
$$

Then (7.3.15) and (7.3.22) are equivalent to the equations:

$$
\tilde{\psi}_{t}+\left(\gamma-4 \alpha^{\prime} \varpi\right) \tilde{\psi}_{\varpi}=0, \quad \tilde{\phi}_{t}+\left(\gamma-4 \alpha^{\prime} \varpi\right) \tilde{\phi}_{\varpi}=0,
$$

respectively. So we have the solution

$$
\tilde{\phi}=\Im(\tilde{\psi}) \Longrightarrow \phi=e^{-2 \alpha} \Im\left(e^{2 \alpha} \sqrt{\alpha^{\prime \prime}+\alpha^{\prime 2}+4^{-1}-2 \varpi}\right)
$$


for some one-variable function $\Im$. Thus we have:

Theorem 7.3.1. Let $\alpha$ be any function in $t$ and let $\Im$ be arbitrary one-variable function. The following is a solution of the equations (7.1.1)-(7.1.4) of dynamic convection in a sea:

$$
\begin{gathered}
u=\alpha^{\prime} x-\frac{y}{2}+y \sqrt{\alpha^{\prime \prime}+\alpha^{\prime 2}+\frac{1}{4}-\frac{2 z}{x^{2}+y^{2}}}, \\
v=\alpha^{\prime} y+\frac{x}{2}-x \sqrt{\alpha^{\prime \prime}+\alpha^{\prime 2}+\frac{1}{4}-\frac{2 z}{x^{2}+y^{2}}}, \\
w=\left(2 \alpha^{\prime 3}+3 \alpha^{\prime} \alpha^{\prime \prime}+\frac{\alpha^{\prime \prime}+\alpha^{\prime}}{2}\right)\left(x^{2}+y^{2}\right)-2 \alpha^{\prime} z, \\
p=e^{-2 \alpha} \Im\left(e^{2 \alpha} \sqrt{\alpha^{\prime \prime}+\alpha^{\prime 2}+\frac{1}{4}-\frac{2 z}{x^{2}+y^{2}}}\right), \\
\rho=-\frac{\Im^{\prime}\left(e^{2 \alpha} \sqrt{\alpha^{\prime \prime}+\alpha^{\prime 2}+\frac{1}{4}-\frac{2 z}{x^{2}+y^{2}}}\right)}{\left(x^{2}+y^{2}\right) \sqrt{\alpha^{\prime}+\alpha^{2}+\frac{1}{4}-\frac{2 z}{x^{2}+y^{2}}}} .
\end{gathered}
$$

Remark 7.3.2. Let $\beta_{1}, \beta_{2}, \beta_{3}$ and $\gamma$ be functions in $t$. Applying $S_{1, \beta_{1}}$ in (7.1.32)(7.1.33), $S_{2, \beta_{2}}$ in (7.1.34)-(7.1.35) and $S_{\beta_{3}, \gamma}$ in (7.1.36) to the above solution, we get a more general solution:

$$
\begin{aligned}
& u=\left(y+\beta_{2}\right) \sqrt{\alpha^{\prime \prime}+\alpha^{\prime 2}+\frac{1}{4}-\frac{2\left(z+\left(\left(\beta_{1}^{\prime \prime}+\beta_{2}^{\prime}\right) x+\left(\beta_{2}^{\prime \prime}-\beta_{1}^{\prime}\right) y+\beta_{3}\right)\right.}{\left(x+\beta_{1}\right)^{2}+\left(y+\beta_{2}\right)^{2}}} \\
& +\alpha^{\prime}\left(x+\beta_{1}\right)-\frac{y+\beta_{2}}{2}-\beta_{1}^{\prime}, \\
& v=-\left(x+\beta_{2}\right) \sqrt{\alpha^{\prime \prime}+\alpha^{\prime 2}+\frac{1}{4}-\frac{2\left(z+\left(\left(\beta_{1}^{\prime \prime}+\beta_{2}^{\prime}\right) x+\left(\beta_{2}^{\prime \prime}-\beta_{1}^{\prime}\right) y+\beta_{3}\right)\right.}{\left(x+\beta_{1}\right)^{2}+\left(y+\beta_{2}\right)^{2}}} \\
& +\alpha^{\prime}\left(y+\beta_{2}\right)+\frac{x+\beta_{1}}{2}-\beta_{2}^{\prime}, \\
& w=\left(2 \alpha^{\prime 3}+3 \alpha^{\prime} \alpha^{\prime \prime}+\frac{\alpha^{\prime \prime}+\alpha^{\prime}}{2}\right)\left(\left(x+\beta_{1}\right)^{2}+\left(y+\beta_{2}\right)^{2}\right) \\
& -2 \alpha^{\prime}\left(z+\left(\beta_{1}^{\prime \prime}+\beta_{2}^{\prime}\right) x+\left(\beta_{2}^{\prime \prime}-\beta_{1}^{\prime}\right) y+\beta_{3}\right)-\beta_{3}^{\prime} \\
& -\left(\beta_{1}^{\prime \prime}+\beta_{2}^{\prime}\right) u+\left(\beta_{1}^{\prime}-\beta_{2}^{\prime \prime}\right) v-\left(\beta_{1}^{\prime \prime}+\beta_{2}^{\prime \prime}\right) x+\left(\beta_{1}^{\prime \prime}-\beta_{2}^{\prime \prime \prime}\right) y, \\
& p=e^{-2 \alpha} \Im\left(e^{2 \alpha} \sqrt{\alpha^{\prime \prime}+\alpha^{\prime 2}+\frac{1}{4}-\frac{2\left(z+\left(\left(\beta_{1}^{\prime \prime}+\beta_{2}^{\prime}\right) x+\left(\beta_{2}^{\prime \prime}-\beta_{1}^{\prime}\right) y+\beta_{3}\right)\right.}{\left(x+\beta_{1}\right)^{2}+\left(y+\beta_{2}\right)^{2}}}\right)+\gamma, \\
& \Im^{\prime}\left(e^{2 \alpha} \sqrt{\alpha^{\prime \prime}+\alpha^{\prime 2}+\frac{1}{4}-\frac{2\left(z+\left(\left(\beta_{1}^{\prime \prime}+\beta_{2}^{\prime}\right) x+\left(\beta_{2}^{\prime \prime}-\beta_{1}^{\prime}\right) y+\beta_{3}\right)\right.}{\left(x+\beta_{1}\right)^{2}+\left(y+\beta_{2}\right)^{2}}}\right) \\
& \rho=-\frac{\left(\left(x+\beta_{1}\right)^{2}+\left(y+\beta_{2}\right)^{2}\right] \sqrt{\alpha^{\prime \prime}+\alpha^{\prime 2}+\frac{1}{4}-\frac{2\left(z+\left(\left(\beta_{1}^{\prime \prime}+\beta_{2}^{\prime}\right) x+\left(\beta_{2}^{\prime \prime}-\beta_{1}^{\prime}\right) y+\beta_{3}\right)\right.}{\left(x+\beta_{1}\right)^{2}+\left(y+\beta_{2}\right)^{2}}}}{\text {. }} \text {. }
\end{aligned}
$$




\subsection{Dimensional Reduction}

Suppose that $u, v, \zeta$ and $\eta$ are functions in $t, x, y$. Assume

$$
w=\zeta-\left(u_{x}+v_{y}\right) z, \quad p=z+\eta, \quad \rho=1 .
$$

Then the equations (7.1.1)-(7.1.4) are equivalent to the following two-dimensional problem:

$$
\begin{aligned}
& u_{t}+u u_{x}+v u_{y}+v=-\eta_{x} \\
& v_{t}+u v_{x}+v v_{y}-u=-\eta_{y} .
\end{aligned}
$$

The compatibility $\eta_{x y}=\eta_{y x}$ gives

$$
\left(u_{y}-v_{x}\right)_{t}+u\left(u_{y}-v_{x}\right)_{x}+v\left(u_{y}-v_{x}\right)_{y}+\left(u_{x}+v_{y}\right)\left(u_{y}-v_{x}+1\right)=0 .
$$

Let $\vartheta$ be a function in $t, x, y$ that is harmonic in $x$ and $y$, i.e.

$$
\vartheta_{x x}+\vartheta_{y y}=0
$$

We assume

$$
u=\vartheta_{x x}, \quad v=\vartheta_{x y} .
$$

Then (7.4.4) naturally holds. Indeed,

$$
\begin{aligned}
& u_{t}+u u_{x}+v u_{y}+v=\left(\vartheta_{x t}+2^{-1}\left(\vartheta_{x x}^{2}+\vartheta_{x y}^{2}\right)+\vartheta_{y}\right)_{x}, \\
& v_{t}+u v_{x}+v v_{y}-u=\left(\vartheta_{x t}+2^{-1}\left(\vartheta_{x x}^{2}+\vartheta_{x y}^{2}\right)+\vartheta_{y}\right)_{y} .
\end{aligned}
$$

By (7.4.2) and (7.4.3), we take

$$
\eta=-\vartheta_{x t}-\vartheta_{y}-\frac{1}{2}\left(\vartheta_{x x}^{2}+\vartheta_{x y}^{2}\right)
$$

Hence we have the following easy result:

Proposition 7.4.1. Let $\vartheta$ and $\zeta$ be functions in $t, x, y$ such that (7.4.5) holds. The following is a solution of the equations (7.1.1)-(7.1.4) of dynamic convection in a sea:

$$
\begin{gathered}
u=\vartheta_{x x}, \quad v=\vartheta_{x y}, \quad w=\zeta, \\
\rho=1, \quad p=z-\vartheta_{x t}-\vartheta_{y}-\frac{1}{2}\left(\vartheta_{x x}^{2}+\vartheta_{x y}^{2}\right) .
\end{gathered}
$$

The above approach is the well-known rotation-free approach. We are more interested in the approaches that the rotation may not be zero. Let $f$ and $g$ be functions in $t, x, y$ that are linear in $x, y$. Denote

$$
\varpi=x^{2}+y^{2}
$$


Consider

$$
u=f+y \phi(t, \varpi), \quad v=g-x \phi(t, \varpi),
$$

where $\phi$ is a two-variable function to be determined. Then

$$
\begin{aligned}
& u_{x}=f_{x}+2 x y \phi_{\varpi}, \quad u_{y}=f_{y}+\phi+2 y^{2} \phi_{\varpi}, \\
& v_{x}=g_{x}-\phi-2 x^{2} \phi_{\varpi}, \quad u_{y}=g_{y}-2 x y \phi_{\varpi} .
\end{aligned}
$$

Thus

$$
u_{x}+v_{y}=f_{x}+g_{y}, \quad u_{y}-v_{x}=f_{y}-g_{x}+2(\varpi \phi)_{\varpi} .
$$

For simplicity, we assume

$$
f=-\frac{\alpha^{\prime} x}{2 \alpha}-\frac{y}{2}, \quad g=\frac{x}{2}-\frac{\alpha^{\prime} y}{2 \alpha}
$$

for some functions $\alpha$ and $\beta$ in $t$. Then (7.4.4) becomes

$$
(\varpi \phi)_{\varpi t}-\frac{\alpha^{\prime}}{\alpha} \varpi(\varpi \phi)_{\varpi \varpi}-\frac{\alpha^{\prime}}{\alpha}(\varpi \phi)_{\varpi}=0 .
$$

Hence

$$
\phi=\frac{\gamma+\Im(\alpha \varpi)}{\varpi}
$$

for some function $\gamma$ in $t$ and one-variable function $\Im$.

Now (7.4.12), (7.4.17) and (7.4.19) imply

$$
\begin{gathered}
u=-\frac{\alpha^{\prime} x}{2 \alpha}-\frac{y}{2}+\frac{(\gamma+\Im(\alpha \varpi)) y}{\varpi}, \\
v=\frac{x}{2}-\frac{\alpha^{\prime} y}{2 \alpha}-\frac{(\gamma+\Im(\alpha \varpi)) x}{\varpi} .
\end{gathered}
$$

By (7.4.13) and (7.4.17), we calculate

$$
\begin{aligned}
& u_{t}+u u_{x}+v u_{y}+v \\
= & f_{t}+y \phi_{t}+(f+y \phi)\left(f_{x}+2 x y \phi_{\varpi}\right)+(g-x \phi)\left(f_{y}+\phi+2 y^{2} \phi_{\varpi}\right)+g-x \phi \\
= & f_{t}+f f_{x}+g\left(f_{y}+1\right)+y \phi_{t}+\left(f_{x} y-f_{y} x+g-x\right) \phi+2(f x+g y) y \phi_{\varpi}-x \phi^{2} \\
= & \left.\left(\frac{3 \alpha^{\prime 2}-2 \alpha \alpha^{\prime \prime}}{4 \alpha^{2}}+\frac{1}{4}\right) x-x \phi^{2}+y\left(\phi_{t}-\frac{\alpha^{\prime}}{\alpha}(\varpi \phi)_{\varpi}\right)\right), \\
& v_{t}+u v_{x}+v v_{y}-u \\
= & g_{t}-x \phi_{t}+(f+y \phi)\left(g_{x}-\phi-2 x^{2} \phi_{\varpi}\right)+(g-x \phi)\left(g_{y}-2 x y \phi_{\varpi}\right)-f-y \phi \\
= & g_{t}+f\left(g_{x}-1\right)+g g_{y}-x \phi_{t}+\left(g_{x} y-g_{y} x-f-y\right) \phi-2(f x+g y) x \phi_{\varpi}-y \phi^{2} \\
= & \left.\left(\frac{3 \alpha^{\prime 2}-2 \alpha \alpha^{\prime \prime}}{4 \alpha^{2}}+\frac{1}{4}\right) y-x \phi^{2}-x\left(\phi_{t}-\frac{\alpha^{\prime}}{\alpha}(\varpi \phi)_{\varpi}\right)\right) .
\end{aligned}
$$


On the other hand, (7.4.19) says that

$$
\phi_{t}-\frac{\alpha^{\prime}}{\alpha}(\varpi \phi)_{\varpi}=\frac{\gamma^{\prime}+\alpha^{\prime} \varpi \Im^{\prime}(\alpha \varpi)}{\varpi}-\alpha^{\prime} \varsigma^{\prime}(\alpha \varpi)=\frac{\gamma^{\prime}}{\varpi}
$$

Thus (7.4.2) and (7.4.3) yield

$$
\begin{aligned}
& \left(\frac{3 \alpha^{\prime 2}-2 \alpha \alpha^{\prime \prime}}{4 \alpha^{2}}+\frac{1}{4}\right) x+\frac{\gamma^{\prime} y}{\varpi}-x \phi^{2}=-\eta_{x}, \\
& \left(\frac{3 \alpha^{\prime 2}-2 \alpha \alpha^{\prime \prime}}{4 \alpha^{2}}+\frac{1}{4}\right) y-\frac{\gamma^{\prime} x}{\varpi}-y \phi^{2}=-\eta_{y}
\end{aligned}
$$

by (7.4.22) and (7.4.23). Hence

$$
\eta=\frac{1}{2} \int \frac{(\gamma+\Im(\alpha \varpi))^{2} d \varpi}{\varpi^{2}}-\frac{1}{2}\left(\frac{3 \alpha^{2}-2 \alpha \alpha^{\prime \prime}}{4 \alpha^{2}}+\frac{1}{4}\right) \varpi+\gamma^{\prime} \arctan \frac{y}{x}
$$

Theorem 7.4.2. Let $\alpha, \gamma$ be any functions in $t$. Suppose that $\Im$ is an arbitrary one-variable function and $\zeta$ is any function in $t, x, y$. The following is a solution of the equations (7.1.1)-(7.1.4) of dynamic convection in a sea:

$$
\begin{gathered}
u=-\frac{\alpha^{\prime} x}{2 \alpha}-\frac{y}{2}+\frac{\left(\gamma+\Im\left(\left(x^{2}+y^{2}\right) \alpha\right)\right) y}{x^{2}+y^{2}}, \\
v=\frac{x}{2}-\frac{\alpha^{\prime} y}{2 \alpha}-\frac{\left(\gamma+\Im\left(\left(x^{2}+y^{2}\right) \alpha\right)\right) x}{x^{2}+y^{2}}, \\
w=\frac{\alpha^{\prime}}{\alpha} z+\zeta, \quad \rho=1, \\
p=z+\frac{1}{2} \int \frac{(\gamma+\Im(\alpha \varpi))^{2} d \varpi}{\varpi^{2}}-\frac{\left(3 \alpha^{\prime 2}-2 \alpha \alpha^{\prime \prime}\right) \alpha^{-2}+1}{8}\left(x^{2}+y^{2}\right)+\gamma^{\prime} \arctan \frac{y}{x} \\
\text { with } \varpi=x^{2}+y^{2} .
\end{gathered}
$$

Next we assume

$$
u=\varepsilon(t, x), \quad v=\phi(t, x)+\psi(t, x) y,
$$

where $\varepsilon, \phi$ and $\psi$ are functions in $t, x$ to be determined. Substituting (7.4.32) into (7.4.4), we get

$$
\phi_{t x}+\psi_{t x} y+\varepsilon\left(\phi_{x x}+\psi_{x x} y\right)+(\phi+\psi y) \psi_{x}+\left(\varepsilon_{x}+\psi\right)\left(\phi_{x}+\psi_{x} y-1\right)=0,
$$

equivalently,

$$
\begin{gathered}
\left(\phi_{t}+\varepsilon \phi_{x}+\phi \psi-\varepsilon\right)_{x}-\psi=0, \\
\left(\psi_{t}+\varepsilon \psi_{x}+\psi^{2}\right)_{x}=0
\end{gathered}
$$


For simplicity, we take

$$
\psi=-\alpha^{\prime}
$$

a function in $t$.

Denote

$$
\phi=\hat{\phi}+x .
$$

Then (7.4.34) becomes

$$
\left(\hat{\phi}_{t}+\varepsilon \hat{\phi}_{x}-\alpha^{\prime} \hat{\phi}\right)_{x}=0 .
$$

To solve the above equation, we assume

$$
\varepsilon=\frac{\beta}{\hat{\phi}_{x}}-\frac{\vartheta_{t}(t, x)}{\vartheta_{x}(t, x)}
$$

for some functions $\beta$ in $t$, and $\vartheta$ in $t$ and $x$. We have the following solution of (7.4.38):

$$
\hat{\phi}=e^{\alpha} \Im(\vartheta) \Longrightarrow \phi=e^{\alpha} \Im(\vartheta)+x \Longrightarrow v=e^{\alpha} \Im(\vartheta)+x-\alpha^{\prime} y
$$

for another one-variable function $\Im$. Moreover,

$$
\varepsilon=\frac{\beta e^{-\alpha}}{\vartheta_{x} \Im^{\prime}(\vartheta)}-\frac{\vartheta_{t}}{\vartheta_{x}} .
$$

Note

$$
\begin{aligned}
& u_{t}+u u_{x}+v u_{y}+v=\frac{\left(\beta e^{-\alpha}\right)^{\prime}}{\vartheta_{x} \Im^{\prime}(\vartheta)}-\frac{\beta e^{-\alpha}\left(\vartheta_{x t} \Im^{\prime}(\vartheta)+\vartheta_{t} \vartheta_{x} \Im^{\prime \prime}(\vartheta)\right)}{\left(\vartheta_{x} \Im^{\prime}(\vartheta)\right)^{2}}-\frac{\vartheta_{t t} \vartheta_{x}-\vartheta_{t} \vartheta_{x t}}{\vartheta_{x}^{2}} \\
& +\left(\frac{\beta e^{-\alpha}}{\vartheta_{x} \Im^{\prime}(\vartheta)}-\frac{\vartheta_{t}}{\vartheta_{x}}\right)\left(\frac{\beta e^{-\alpha}}{\vartheta_{x} \Im^{\prime}(\vartheta)}-\frac{\vartheta_{t}}{\vartheta_{x}}\right)_{x}+e^{\alpha} \Im(\vartheta)+x-\alpha^{\prime} y
\end{aligned}
$$

By (7.4.36), (7.4.40) and (7.4.41),

$$
\begin{aligned}
& \phi_{t}+\varepsilon \phi_{x}+\psi \phi-\varepsilon \\
= & e^{\alpha}\left(\alpha^{\prime} \Im(\vartheta)+\vartheta_{t} \Im^{\prime}(\vartheta)\right)+\left(\frac{\beta e^{-\alpha}}{\vartheta_{x} \Im^{\prime}(\vartheta)}-\frac{\vartheta_{t}}{\vartheta_{x}}\right) e^{\alpha} \vartheta_{x} \Im^{\prime}(\vartheta)-\alpha^{\prime}\left(e^{\alpha} \Im(\vartheta)+x\right) \\
= & \beta-\alpha^{\prime} x .
\end{aligned}
$$

Thus (7.4.32) (7.4.36) and (7.4.43) yield

$$
\begin{aligned}
v_{t}+u v_{x}+v v_{y}-u & =\phi_{t}+\psi_{t} y+\varepsilon\left(\phi_{x}+\psi_{x} y-1\right)+(\phi+\psi y) \psi \\
& =\phi_{t}+\varepsilon \phi_{x}+\psi \phi-\varepsilon+\left(\psi_{t}+\varepsilon \psi_{x}+\psi^{2}\right) y \\
& =\beta-\alpha^{\prime} x+\left(-\alpha^{\prime \prime}+\alpha^{2}\right) y .
\end{aligned}
$$

According to (7.4.2) and (7.4.3),

$$
\begin{aligned}
\eta= & \int\left(\frac{\beta e^{-\alpha}\left(\vartheta_{x t} \Im^{\prime}(\vartheta)+\vartheta_{t} \vartheta_{x} \Im^{\prime \prime}(\vartheta)\right)}{\left(\vartheta_{x} \Im^{\prime}(\vartheta)\right)^{2}}+\frac{\vartheta_{t t} \vartheta_{x}-\vartheta_{t} \vartheta_{x t}}{\vartheta_{x}^{2}}-\frac{\left(\beta e^{-\alpha}\right)^{\prime}}{\vartheta_{x} \Im^{\prime}(\vartheta)}-e^{\alpha} \Im(\vartheta)\right) d x \\
& +\alpha^{\prime} x y-\beta y+\frac{\left(\alpha^{\prime \prime}-\alpha^{\prime 2}\right) y^{2}-x^{2}}{2}-\frac{1}{2}\left(\frac{\beta e^{-\alpha}}{\vartheta_{x} \Im^{\prime}(\vartheta)}-\frac{\vartheta_{t}}{\vartheta_{x}}\right)^{2} .
\end{aligned}
$$


Theorem 7.4.3. Let $\alpha, \beta$ be functions in $t$ and let $\Im$ be a one-variable function. Suppose that $\vartheta$ is a function in $t, x$, and $\zeta$ is function in $t, x, y$. The following is a solution of the equations (7.1.1)-(7.1.4) of dynamic convection in a sea:

$$
\begin{gathered}
u=\frac{\beta e^{-\alpha}}{\vartheta_{x} \Im^{\prime}(\vartheta)}-\frac{\vartheta_{t}}{\vartheta_{x}}, \quad v=e^{\alpha} \Im(\vartheta)+x-\alpha^{\prime} y \\
w=\left(\alpha^{\prime}+\frac{\beta e^{-\alpha}\left(\vartheta_{x x} \Im^{\prime}(\vartheta)+\vartheta_{x}^{2} \Im^{\prime \prime}(\vartheta)\right)}{\left(\vartheta_{x} \Im^{\prime}(\vartheta)\right)^{2}}+\frac{\vartheta_{x t} \vartheta_{x}-\vartheta_{t} \vartheta_{x x}}{\vartheta_{x}^{2}}\right) z+\zeta, \quad \rho=1, \\
p=z+\int\left(\frac{\beta e^{-\alpha}\left(\vartheta_{x t} \Im^{\prime}(\vartheta)+\vartheta_{t} \vartheta_{x} \Im^{\prime \prime}(\vartheta)\right)}{\left(\vartheta_{x} \Im^{\prime}(\vartheta)\right)^{2}}+\frac{\vartheta_{t t} \vartheta_{x}-\vartheta_{t} \vartheta_{x t}}{\vartheta_{x}^{2}}-\frac{\left(\beta e^{-\alpha}\right)^{\prime}}{\vartheta_{x} \Im^{\prime}(\vartheta)}-e^{\alpha} \Im(\vartheta)\right) d x \\
+\alpha^{\prime} x y-\beta y+\frac{\left(\alpha^{\prime \prime}-\alpha^{\prime 2}\right) y^{2}-x^{2}}{2}-\frac{1}{2}\left(\frac{\beta e^{-\alpha}}{\vartheta_{x} \Im^{\prime}(\vartheta)}-\frac{\vartheta_{t}}{\vartheta_{x}}\right)^{2}
\end{gathered}
$$

Finally, we suppose that $\alpha, \beta$ are functions in $t$ and $f, g$ are functions in $t, x, y$ that are linear homogeneous in $x$ and $y$. Denote $\varpi=\alpha x+\beta y$. Assume

$$
u=f+\beta \phi(t, \varpi), \quad v=g-\alpha \phi(t, \varpi) .
$$

Then

$$
u_{y}-v_{x}=f_{y}-g_{x}+\left(\alpha^{2}+\beta^{2}\right) \phi_{\varpi}, \quad u_{x}+v_{y}=f_{x}+g_{y} .
$$

Now (7.4.4) becomes

$$
\begin{aligned}
& f_{y t}-g_{x t}+\left(\alpha^{2}+\beta^{2}\right)^{\prime} \phi_{\varpi}+\left(\alpha^{2}+\beta^{2}\right)\left(\phi_{\varpi t}+\left(\alpha^{\prime} x+\beta^{\prime} y+\alpha f+\beta g\right) \phi_{\varpi \varpi}\right) \\
& +\left(f_{x}+g_{y}\right)\left(f_{y}-g_{x}+1+\left(\alpha^{2}+\beta^{2}\right) \phi_{\varpi}\right)=0 .
\end{aligned}
$$

In order to solve the above equation, we assume

$$
\begin{gathered}
g_{x}=\varphi, \quad f_{y}=\varphi-1, \\
\alpha^{\prime} x+\beta^{\prime} y+\alpha f+\beta g=0
\end{gathered}
$$

for some function $\varphi$ in $t$. The equation (7.4.52) is equivalent to:

$$
\begin{aligned}
\alpha^{\prime}+\alpha f_{x}+\varphi \beta & =0 \Longrightarrow f_{x}=-\frac{\alpha^{\prime}+\varphi \beta}{\alpha} \\
\beta^{\prime}+\beta g_{y}+\alpha(\varphi-1) & =0 \Longrightarrow g_{y}=-\frac{\beta^{\prime}+\alpha(\varphi-1)}{\beta} .
\end{aligned}
$$

Thus

$$
f=-\frac{\alpha^{\prime}+\varphi \beta}{\alpha} x+(\varphi-1) y, \quad g=\varphi x-\frac{\beta^{\prime}+\alpha(\varphi-1)}{\beta} y .
$$


Now (7.4.51) becomes

$$
\phi_{\varpi t}-\left(\frac{\alpha^{\prime}+\varphi \beta}{\alpha}+\frac{\beta^{\prime}+\alpha(\varphi-1)}{\beta}-\frac{\left(\alpha^{2}+\beta^{2}\right)^{\prime}}{\alpha^{2}+\beta^{2}}\right) \phi_{\varpi}=0 .
$$

Thus we have the following solution

$$
\phi=\frac{\alpha \beta}{\alpha^{2}+\beta^{2}} e^{\int\left(\alpha \beta^{-1}(\varphi-1)+\alpha^{-1} \beta \varphi\right) d t} \Im^{\prime}(\varpi),
$$

where $\Im$ is an arbitrary one-variable function. Note that (7.4.56) and (7.4.58) give

$$
\begin{aligned}
& u_{t}+u u_{x}+v u_{y}+v \\
= & f_{t}+\beta^{\prime} \phi+\beta \phi_{t}+\left(\alpha^{\prime} x+\beta^{\prime} y\right) \beta \phi_{\varpi}+(f+\beta \phi)\left(f_{x}+\alpha \beta \phi_{\varpi}\right) \\
& +(g-\alpha \phi)\left(f_{y}+1+\beta^{2} \phi_{\varpi}\right) \\
= & f_{t}+f f_{x}+g\left(f_{y}+1\right)+\left(\beta^{\prime}+\beta f_{x}-\alpha\left(f_{y}+1\right)\right) \phi+\beta \phi_{t} \\
& +\left(\alpha^{\prime} x+\beta^{\prime} y+\alpha f+\beta g\right) \beta \phi_{\varpi} \\
= & \frac{\alpha^{2} \beta}{\alpha^{2}+\beta^{2}}\left(\frac{2\left(\alpha \beta^{\prime}-\alpha^{\prime} \beta\right)}{\alpha^{2}+b^{2}}-1\right) e^{\int\left(\alpha \beta^{-1}(\varphi-1)+\alpha^{-1} \beta \varphi\right) d t \Im^{\prime}(\varpi)} \\
& +\left(\frac{2 \alpha^{\prime 2}+(\varphi \beta)^{2}+3 \alpha^{\prime} \beta \varphi-\alpha(\varphi \beta)^{\prime}-\alpha \alpha^{\prime \prime}}{\alpha^{2}}+\varphi^{2}\right) x \\
& +\left(\varphi^{\prime}-\frac{(\varphi-1)\left(\alpha^{\prime}+\varphi \beta\right)}{\alpha}-\frac{\varphi\left(\beta^{\prime}+\alpha(\varphi-1)\right)}{\beta}\right) y, \\
& v_{t}+u v_{x}+v v_{y}-u \\
= & g_{t}-\alpha^{\prime} \phi-\alpha \phi_{t}-\left(\alpha^{\prime} x+\beta^{\prime} y\right) \alpha \phi_{\varpi}+(f+\beta \phi)\left(g_{x}-1-2 \alpha^{2} \phi_{\varpi}\right) \\
& +(g-\alpha \phi)\left(g_{y}-2 \alpha \beta \phi_{\varpi}\right) \\
= & g_{t}+f\left(g_{x}-1\right)+g g_{y}-\left(\alpha^{\prime}+\alpha g_{y}-\beta\left(g_{x}-1\right)\right) \phi-\alpha \phi_{t} \\
& -\left(\alpha^{\prime} x+\beta^{\prime} y+\alpha f+\beta g\right) \alpha \phi_{\varpi} \\
= & \frac{\alpha \beta^{2}}{\alpha^{2}+\beta^{2}}\left(\frac{2\left(\alpha \beta^{\prime}-\alpha^{\prime} \beta\right)}{\alpha^{2}+b^{2}}-1\right) e^{\int\left(\alpha \beta^{-1}(\varphi-1)+\alpha^{-1} \beta \varphi\right) d t} \Im^{\prime}(\varpi)+\left[(\varphi-1)^{2}\right. \\
& \left.-\frac{\beta((\varphi-1) \alpha)^{\prime}+\beta \beta^{\prime \prime}-2 \beta^{\prime 2}-((\varphi-1) \alpha)^{2}-3 \alpha \beta^{\prime}(\varphi-1)}{\beta^{2}}\right] y \\
& +\left(\varphi^{\prime}-\frac{(\varphi-1)\left(\alpha^{\prime}+\varphi \beta\right)}{\alpha}-\frac{\varphi\left(\beta^{\prime}+\alpha(\varphi-1)\right)}{\beta}\right) x . \\
B y & 4.2)
\end{aligned}
$$

By (7.4.2) and (7.4.3),

$$
\begin{aligned}
\eta= & \frac{y^{2}}{2}\left(\frac{\beta((\varphi-1) \alpha)^{\prime}+\beta \beta^{\prime \prime}-2{\beta^{\prime}}^{2}-((\varphi-1) \alpha)^{2}-3 \alpha \beta^{\prime}(\varphi-1)}{\beta^{2}}-(\varphi-1)^{2}\right) \\
& -\frac{x^{2}}{2}\left(\frac{2 \alpha^{\prime 2}+(\varphi \beta)^{2}+3 \alpha^{\prime} \beta \varphi-\alpha(\varphi \beta)^{\prime}-\alpha \alpha^{\prime \prime}}{\alpha^{2}}+\varphi^{2}\right) \\
& +\left[\frac{(\varphi-1)\left(\alpha^{\prime}+\varphi \beta\right)}{\alpha}-\varphi^{\prime}+\frac{\varphi\left(\beta^{\prime}+\alpha(\varphi-1)\right)}{\beta}\right] x y \\
& +\frac{\alpha \beta}{\alpha^{2}+\beta^{2}}\left(1-\frac{2\left(\alpha \beta^{\prime}-\alpha^{\prime} \beta\right)}{\alpha^{2}+b^{2}}\right) e^{\int\left(\alpha \beta^{-1}(\varphi-1)+\alpha^{-1} \beta \varphi\right) d t} \Im(\varpi) .
\end{aligned}
$$


Theorem 7.4.4. Let $\alpha, \beta, \varphi$ be functions in $t$ and let $\Im$ be a one-variable function. Suppose that $\zeta$ is a function in $t, x, y$. The following is a solution of the equations (7.1.1)(7.1.4) of dynamic convection in a sea:

$$
\begin{aligned}
& u=(\varphi-1) y-\frac{\left(\alpha^{\prime}+\varphi \beta\right) x}{\alpha}+\frac{\alpha \beta^{2}}{\alpha^{2}+\beta^{2}} e^{\int\left(\alpha \beta^{-1}(\varphi-1)+\alpha^{-1} \beta \varphi\right) d t} \Im^{\prime}(\alpha x+\beta y), \\
& v=\varphi x-\frac{\left(\beta^{\prime}+(\varphi-1) \alpha\right) y}{\beta}-\frac{\alpha^{2} \beta}{\alpha^{2}+\beta^{2}} e^{\int\left(\alpha \beta^{-1}(\varphi-1)+\alpha^{-1} \beta \varphi\right) d t} \Im^{\prime}(\alpha x+\beta y), \\
& w=\left(\frac{\alpha^{\prime}+\varphi \beta}{\alpha}+\frac{\beta^{\prime}+(\varphi-1) \alpha}{\beta}\right) z+\zeta, \quad \rho=1 \\
& p=z+\frac{y^{2}}{2}\left(\frac{\beta((\varphi-1) \alpha)^{\prime}+\beta \beta^{\prime \prime}-2 \beta^{\prime 2}-((\varphi-1) \alpha)^{2}-3 \alpha \beta^{\prime}(\varphi-1)}{\beta^{2}}-(\varphi-1)^{2}\right) \\
& -\frac{x^{2}}{2}\left(\frac{2 \alpha^{\prime 2}+(\varphi \beta)^{2}+3 \alpha^{\prime} \beta \varphi-\alpha(\varphi \beta)^{\prime}-\alpha \alpha^{\prime \prime}}{\alpha^{2}}+\varphi^{2}\right) \\
& +x y\left[\frac{(\varphi-1)\left(\alpha^{\prime}+\varphi \beta\right)}{\alpha}-\varphi^{\prime}+\frac{\varphi\left(\beta^{\prime}+\alpha(\varphi-1)\right)}{\beta}\right] \\
& +\frac{\alpha \beta}{\alpha^{2}+\beta^{2}}\left(1-\frac{2\left(\alpha \beta^{\prime}-\alpha^{\prime} \beta\right)}{\alpha^{2}+b^{2}}\right) e^{\int\left(\alpha \beta^{-1}(\varphi-1)+\alpha^{-1} \beta \varphi\right) d t} \Im(\alpha x+\beta y) .
\end{aligned}
$$




\section{Chapter 8}

\section{Boussinesq Equations in Geophysics}

Boussinesq systems of nonlinear partial differential equations are fundamental equations in geophysical fluid dynamics. In this chapter, we use asymmetric ideas and moving frames to solve the two-dimensional Boussinesq equations with partial viscosity terms and the three-dimensional stratified rotating Boussinesq equations. We obtain new families of explicit exact solutions with multiple parameter functions. Many of them are the periodic, quasi-periodic, aperiodic solutions that may have practical significance. By Fourier expansion and some of our solutions, one can obtain discontinuous solutions. In addition, the symmetries of these equations are used to simplify our arguments.

In Section 8.1, we solve the two-dimensional Boussinesq equations and obtain four families of explicit exact solutions. In Section 8.2, we give the symmetry analysis on the three-dimensional stratified rotating Boussinesq equations. In Section 8.3, we find the solutions of the three-dimensional equations that are linear in $x$ and $y$. In Section 8.4, we obtain two families of explicit exact solutions under certain conditions on the variable z. In Section 8.5, we obtain a family of explicit exact solutions of the three-dimensional equations that are independent of $x$. The status can be changed by applying symmetry transformations. This chapter is a revision of our preprint [X16].

\subsection{Two-Dimensional Equations}

The Boussinesq system for the incompressible fluid in $\mathbb{R}^{2}$ is

$$
\begin{gathered}
u_{t}+u u_{x}+v u_{y}-\nu \Delta u=-p_{x}, \quad v_{t}+u v_{x}+v v_{y}-\nu \Delta v-\theta=-p_{y}, \\
\theta_{t}+u \theta_{x}+v \theta_{y}-\kappa \Delta \theta=0, \quad u_{x}+v_{y}=0,
\end{gathered}
$$

where $(u, v)$ is the velocity vector field, $p$ is the scalar pressure, $\theta$ is the scalar temperature, $\nu \geq 0$ is the viscosity and $\kappa \geq 0$ is the thermal diffusivity. The above system is a simple model in atmospheric sciences (e.g., cf. [Ma]). Chae [Cd] proved the global regularity, and Hou and $\mathrm{Li}[\mathrm{HL}]$ obtained the well-posedness of the above system. 
Let us do the degree analysis. Note that $\Delta=\partial_{x}^{2}+\partial_{y}^{2}$ in this case. To make the nonzero terms to have the same degree, we have to take

$$
\begin{gathered}
\operatorname{deg} x=\operatorname{deg} y=\ell \text { and } \operatorname{deg} u u_{x}=\operatorname{deg} u_{x x} \Longrightarrow \operatorname{deg} u=-\ell, \\
\operatorname{deg} v v_{y}=\operatorname{deg} v_{y y} \Longrightarrow \operatorname{deg} v=-\ell, \quad \operatorname{deg} u_{t}=\operatorname{deg} u_{x x} \Longrightarrow \operatorname{deg} t=2 \ell, \\
\operatorname{deg} p_{x}=\operatorname{deg} u u_{x} \Longrightarrow \operatorname{deg} p=-2 \ell, \quad \operatorname{deg} \theta=\operatorname{deg} v_{t}=-3 \ell
\end{gathered}
$$

Moreover, (8.1.1) and (8.1.2) are translation invariant because they do not contain variable coefficients. Thus the transformation

$$
\begin{aligned}
& T_{a, b}(u(t, x, y))=b u\left(b^{2} t+a, b x, b y\right), T_{a, b}(v(t, x, y))=b v\left(b^{2} t+a, b x, b y\right), \\
& T_{a, b}(p(t, x, y))=b^{2}\left(b^{2} t+a, b x, b y\right), \quad T_{a, b}(\theta(t, x, y))=b^{3} \theta\left(b^{2} t+a, b x, b y\right)
\end{aligned}
$$

is a symmetry of the equations (8.1.1) and (8.1.2), where $a, b \in \mathbb{R}$ with $b \neq 0$. By the arguments in (7.1.20)-(7.1.24), we have the following symmetry of the equations (8.1.1) and (8.1.2):

$$
\begin{gathered}
S_{\alpha, \beta ; \gamma}(u(t, x, y))=u(t, x+\alpha, y+\beta)-\alpha^{\prime}, \quad S_{\alpha, \beta ; \gamma}(\theta(t, x, y))=\theta(t, x+\alpha, y+\beta), \\
S_{\alpha, \beta ; \gamma}(v(t, x, y))=v(t, x+\alpha, y+\beta)-\beta^{\prime} \\
S_{\alpha, \beta ; \gamma}(p(t, x, y))=p(t, x+\alpha, y+\beta)+\alpha^{\prime \prime} x+\beta^{\prime \prime} y+\gamma
\end{gathered}
$$

where $\alpha, \beta$ and $\gamma$ are arbitrary functions in $t$.

According to the second equation in (8.1.2), we take the potential form:

$$
u=\xi_{y}, \quad v=-\xi_{x}
$$

for some functions $\xi$ in $t, x, y$. Then the two-dimensional Boussinesq equations become

$$
\begin{gathered}
\xi_{y t}+\xi_{y} \xi_{x y}-\xi_{x} \xi_{y y}-\nu \Delta \xi_{y}=-p_{x}, \quad \xi_{x t}+\xi_{y} \xi_{x x}-\xi_{x} \xi_{x y}-\nu \Delta \xi_{x}+\theta=p_{y}, \\
\theta_{t}+\xi_{y} \theta_{x}-\xi_{x} \theta_{y}-\kappa \Delta \theta=0 .
\end{gathered}
$$

By our assumption $p_{x y}=p_{y x}$, the compatible condition of the equations in (8.1.12) is

$$
(\Delta \xi)_{t}+\xi_{y}(\Delta \xi)_{x}-\xi_{x}(\Delta \xi)_{y}-\nu \Delta^{2} \xi+\theta_{x}=0
$$

Now we first solve the system (8.1.13) and (8.1.14). To do this, we impose some asymmetric conditions.

Firs we assume

$$
\theta=\varepsilon(t, y), \quad \xi=\phi(t, y)+x \psi(t, y)
$$


for some functions $\varepsilon, \phi$ and $\psi$ in $t, y$. Then (8.1.13) becomes

$$
\varepsilon_{t}-\psi \varepsilon_{y}-\kappa \varepsilon_{y y}=0
$$

Moreover, (8.1.14) becomes

$$
\phi_{y y t}+x \psi_{y y t}+\left(\phi_{y}+x \psi_{y}\right) \psi_{y y}-\psi\left(\phi_{y y y}+x \psi_{y y y}\right)-\nu\left(\phi_{y y y y}+x \psi_{y y y y}\right)=0
$$

equivalently,

$$
\begin{aligned}
& \phi_{y y t}+\phi_{y} \psi_{y y}-\psi \phi_{y y y}-\nu \phi_{y y y y}=0 \\
& \psi_{y y t}+\psi_{y} \psi_{y y}-\psi \psi_{y y y}-\nu \psi_{y y y y}=0 .
\end{aligned}
$$

The above two equations are equivalent to:

$$
\begin{gathered}
\phi_{y t}+\phi_{y} \psi_{y}-\psi \phi_{y y}-\nu \phi_{y y y}=\alpha_{1}, \\
\psi_{y t}+\psi_{y}^{2}-\psi \psi_{y y}-\nu \psi_{y y y}=\alpha_{2}
\end{gathered}
$$

for some functions $\alpha_{1}$ and $\alpha_{2}$ in $t$ to be determined.

Observe that

$$
\psi=6 \nu y^{-1}
$$

is a solution of $(8.1 .21)$ with $\alpha_{2}=0$. In order to solve (8.1.20), we assume

$$
\phi=\sum_{m=1}^{\infty} \gamma_{m} y^{m}
$$

where $\gamma_{m}$ are functions in $t$ to be determined. Now (8.1.20) becomes

$$
\sum_{m=1}^{\infty}\left[m \gamma_{m}^{\prime}-\nu(m+2)(m+3)(m+4) \gamma_{m+2}\right] y^{m-1}-6 \nu \gamma_{1} y^{-2}-18 \nu \gamma_{2} y^{-1}=\alpha_{1}
$$

equivalently,

$$
\begin{gathered}
\gamma_{1}=\gamma_{2}=0, \quad \alpha_{1}=-60 \nu \gamma_{3}, \\
m \gamma_{m}^{\prime}-\nu(m+2)(m+3)(m+4) \gamma_{m+2}=0, \quad m>1 .
\end{gathered}
$$

Thus

$$
\begin{gathered}
\gamma_{2 m+2}=\frac{2 m \gamma_{2 m}^{\prime}}{\nu(2 m+2)(2 m+3)(2 m+4)}=0, \quad m \geq 1, \\
\gamma_{2 m+3}=\frac{(2 m+1) \gamma_{2 m+1}^{\prime}}{\nu(2 m+3)(2 m+4)(2 m+5)}=\frac{360 \gamma_{3}^{(m)}}{\nu^{m}(2 m+3)(2 m+5) !}, \quad m \geq 1 .
\end{gathered}
$$

For simplicity, we redenote $\alpha=\gamma_{3}$. Then

$$
\phi=360 \sum_{m=0}^{\infty} \frac{\alpha^{(m)} y^{2 m+3}}{\nu^{m}(2 m+3)(2 m+5) !} .
$$


To solve (8.1.16), we also assume

$$
\varepsilon=\sum_{n=0}^{\infty} \beta_{n} y^{n}
$$

where $\beta_{n}$ are functions in $t$ to be determined. Then (8.1.16) becomes

$$
6 \nu \beta_{1} y^{-1}+\sum_{n=0}^{\infty}\left[\beta_{n}^{\prime}-(n+2)(6 \nu+(n+1) \kappa) \beta_{n+2}\right] y^{n}=0,
$$

that is, $\beta_{1}=0$ and

$$
\beta_{n}^{\prime}-(n+2)(6 \nu+(n+1) \kappa) \beta_{n+2}=0, \quad n \geq 0 .
$$

Hence

$$
\theta=\beta+\sum_{n=1}^{\infty} \frac{\beta^{(n)} y^{2 n}}{2^{n} n ! \prod_{r=1}^{n}(6 \nu+(2 r-1) \kappa)},
$$

where $\beta$ is an arbitrary function in $t$. Moreover, (8.1.11), (8.1.20), (8.1.21) and (8.1.25) lead to

$$
\begin{aligned}
& u_{t}+u u_{x}+v u_{y}-\nu \Delta u \\
= & \phi_{y t}+x \psi_{y t}+\left(\phi_{y}+x \psi_{y}\right) \psi_{y}-\psi\left(\phi_{y y}+x \psi_{y y}\right)-\nu\left(\phi_{y y y}+x \psi_{y y y}\right) \\
= & \phi_{y t}+\phi_{y}^{2}-\psi \phi_{y y}-\nu \phi_{y y y}+\left(\psi_{y t}+\psi_{y} \psi_{y}-\psi \psi_{y y}-\nu \psi_{y y y}\right) x \\
= & \alpha_{2} x+\alpha_{1}=-60 \nu \alpha .
\end{aligned}
$$

Furthermore, (8.1.22) and (8.1.33) give

$$
\begin{aligned}
& v_{t}+u v_{x}+v v_{y}-\nu \Delta(v)-\theta=-\psi_{t}+\psi \psi_{y}+\nu \psi_{y y}-\theta \\
= & -24 \nu^{2} y^{-3}-\beta-\sum_{n=1}^{\infty} \frac{\beta^{(n)} y^{2 n}}{2^{n} n ! \prod_{r=1}^{n}(6 \nu+(2 r-1) \kappa)} .
\end{aligned}
$$

By (8.1.15), (8.1.22) and (8.1.29),

$$
\xi=6 \nu x y^{-1}+360 \sum_{m=0}^{\infty} \frac{\alpha^{(m)} y^{2 m+3}}{\nu^{m}(2 m+3)(2 m+5) !} .
$$

According (8.1.1) and (8.1.11), we have:

Theorem 8.1.1. The following is a solution of the two-dimensional Boussinesq equations (8.1.1)-(8.1.2):

$$
\begin{gathered}
u=360 \sum_{m=0}^{\infty} \frac{\alpha^{(m)} y^{2 m+2}}{\nu^{m}(2 n+5) !}-6 \nu x y^{-2}, \quad v=-6 \nu y^{-1}, \\
p=60 \nu \alpha x+12 \nu^{2} y^{-2}+\beta y+\sum_{n=1}^{\infty} \frac{\beta^{(n)} y^{2 n+1}}{2^{n} n !(2 n+1) \prod_{r=1}^{n}(6 \nu+(2 r-1) \kappa)}
\end{gathered}
$$


and $\theta$ is given in (8.1.33), where $\alpha$ and $\beta$ are arbitrary functions in $t$.

Remark 8.1.2. Let $\gamma, \gamma_{1}, \gamma_{2}$ be arbitrary functions in $t$. Applying the symmetry transformation $S_{\gamma_{1}, \gamma_{2} ; \gamma}$ in (8.1.8)-(8.1.10) to the above solution, we get a more general solution of the two-dimensional Boussinesq equations (8.1.1)-(8.1.2):

$$
\begin{gathered}
u=360 \sum_{m=0}^{\infty} \frac{\alpha^{(m)}\left(y+\gamma_{2}\right)^{2 m+2}}{\nu^{m}(2 n+5) !}-6 \nu\left(x+\gamma_{1}\right)\left(y+\gamma_{2}\right)^{-2}-\gamma_{1}^{\prime}, \\
v=-6 \nu\left(y+\gamma_{2}\right)^{-1}-\gamma_{2}^{\prime}, \\
\theta=\beta+\sum_{n=1}^{\infty} \frac{\beta^{(n)}\left(y+\gamma_{2}\right)^{2 n}}{2^{n} n ! \prod_{r=1}^{n}(6 \nu+(2 r-1) \kappa)}, \\
p=60 \nu \alpha\left(x+\gamma_{1}\right)+12 \nu^{2}\left(y+\gamma_{2}\right)^{-2}+\beta\left(y+\gamma_{2}\right)+\gamma_{1}^{\prime \prime} x+\gamma_{2}^{\prime \prime} y+\gamma \\
\beta^{(n)}\left(y+\gamma_{2}\right)^{2 n+1} \\
+\sum_{n=1}^{\infty} \frac{2^{n} n !(2 n+1) \prod_{r=1}^{n}(6 \nu+(2 r-1) \kappa)}{}
\end{gathered}
$$

Let $c$ be a fixed real constant and let $\gamma$ be a fixed function in $t$. We define

$$
\begin{array}{cc}
\zeta_{1}(y)=\frac{e^{\gamma y}-c e^{-\gamma y}}{2}, & \eta_{1}(y)=\frac{e^{\gamma y}+c e^{-\gamma y}}{2} \\
\zeta_{0}(y)=\sin \gamma y, & \eta_{0}(y)=\cos \gamma y .
\end{array}
$$

Then

$$
\begin{gathered}
\eta_{r}^{2}(y)+(-1)^{r} \zeta_{r}^{2}(y)=c^{r} \\
\partial_{y}\left(\zeta_{r}(y)\right)=\gamma \eta_{r}(y), \quad \partial_{y}\left(\eta_{r}(y)\right)=-(-1)^{r} \gamma \zeta_{r}(y)
\end{gathered}
$$

and

$$
\partial_{y}\left(\zeta_{r}(y)\right)=\gamma^{\prime} y \eta_{r}(y), \quad \partial_{t}\left(\eta_{r}(y)\right)=-(-1)^{r} \gamma^{\prime} y \zeta_{r}(y)
$$

where we treat $0^{0}=1$ when $c=r=0$.

First we assume

$$
\psi=\beta_{1} y+\beta_{2} \zeta_{r}(y)
$$

for some functions $\beta_{1}$ and $\beta_{2}$ in $t$, where $r=0,1$. Then (8.1.21) becomes

$$
\begin{aligned}
& \beta_{1}^{\prime}+\left(\beta_{2} \gamma\right)^{\prime} \eta_{r}-(-1)^{r} \beta_{2} \gamma \gamma^{\prime} y \zeta_{r}+\left(\beta_{1}+\beta_{2} \gamma \eta_{r}\right)^{2} \\
& +(-1)^{r} \beta_{2} \gamma^{2}\left(\beta_{1} y+\beta_{2} \zeta_{r}\right) \zeta_{r}+(-1)^{r} \nu \beta_{2} \gamma^{3} \eta_{r} \\
= & \beta_{1}^{\prime}+c^{r} \beta_{2}^{2} \gamma^{2}+\beta_{1}^{2}+\left[\left(\beta_{2} \gamma\right)^{\prime}+(-1)^{r} \nu \beta_{2} \gamma^{3}+2 \beta_{1} \beta_{2} \gamma\right] \eta_{r} \\
& +(-1)^{r} \beta_{2} \gamma\left(\beta_{1} \gamma-\gamma^{\prime}\right) y \zeta_{r}=\alpha_{2},
\end{aligned}
$$


which is implied by the following equations:

$$
\begin{gathered}
\beta_{1}^{\prime}+c^{r} \beta_{2}^{2} \gamma^{2}+\beta_{1}^{2}=\alpha_{2}, \quad \beta_{1} \gamma-\gamma^{\prime}=0 \\
\left(\beta_{2} \gamma\right)^{\prime}+(-1)^{r} \nu \beta_{2} \gamma^{3}+2 \beta_{1} \beta_{2} \gamma=0 .
\end{gathered}
$$

For convenience, we assume

$$
\gamma=\sqrt{\alpha^{\prime}}
$$

for some increasing function $\alpha$ in $t$. Thus we have

$$
\beta_{1}=\frac{\gamma^{\prime}}{\gamma}=\frac{\alpha^{\prime \prime}}{2 \alpha^{\prime}}
$$

by the second equation in (8.1.50). Now (8.1.51) becomes

$$
\left(\beta_{2} \gamma\right)^{\prime}+\left((-1)^{r} \nu \alpha^{\prime}+\frac{\alpha^{\prime \prime}}{\alpha^{\prime}}\right) \beta_{2} \gamma=0
$$

Hence

$$
\beta_{2} \gamma=\frac{b_{1} e^{-(-1)^{r} \nu \alpha}}{\alpha^{\prime}} \Longrightarrow \beta_{2}=\frac{b_{1} e^{-(-1)^{r} \nu \alpha}}{\sqrt{\left(\alpha^{\prime}\right)^{3}}}, \quad b_{1} \in \mathbb{R} .
$$

To solve (8.1.20), we assume

$$
\phi=\beta_{3} \eta_{r}(y)
$$

for some function $\beta_{3}$. Now (8.1.20) becomes

$$
\begin{aligned}
& -(-1)^{r}\left[\left(\beta_{3} \gamma\right)^{\prime} \zeta_{r}+\beta_{3} \gamma \gamma^{\prime} y \eta_{r}+\beta_{3} \gamma \zeta_{r}\left(\beta_{1}+\beta_{2} \gamma \eta_{r}\right)-\beta_{3} \gamma^{2}\left(\beta_{1} y+\beta_{2} \zeta\right) \eta_{r}\right]-\nu \beta_{3} \gamma^{3} \zeta \\
= & -\left[(-1)^{r}\left(\left(\beta_{3} \gamma\right)^{\prime}+\beta_{1} \beta_{3} \gamma\right)+\nu \beta_{3} \gamma^{3}\right] \zeta_{r}(y)=\alpha_{1}
\end{aligned}
$$

by $(8.1 .46),(8.1 .47)$ and the second equation in (8.1.50), equivalently, $\alpha_{1}=0$ and

$$
(-1)^{r}\left(\left(\beta_{3} \gamma\right)^{\prime}+\beta_{1} \beta_{3} \gamma\right)+\nu \beta_{3} \gamma^{3}=0
$$

According to (8.1.52) and (8.1.53),

$$
\left(\beta_{3} \gamma\right)^{\prime}+\left(\frac{\alpha^{\prime \prime}}{2 \alpha^{\prime}}+(-1)^{r} \alpha^{\prime}\right) \beta_{3} \gamma=0
$$

Thus

$$
\beta_{3} \gamma=\frac{b_{2} e^{-(-1)^{r} \nu \alpha}}{\sqrt{\alpha^{\prime}}} \Longrightarrow \beta_{3}=\frac{b_{2} e^{-(-1)^{r} \nu \alpha}}{\alpha^{\prime}},
$$

where $b_{2}$ is a real constant.

In order to solve (8.1.16), we assume

$$
\varepsilon=b e^{\gamma_{1} \eta_{r}(y)}
$$


where $b$ is a real constant and $\gamma_{1}$ is a function in $t$. Then (8.1.16) changes to

$$
b\left(\gamma_{1}^{\prime} \eta_{r}-(-1)^{r} \gamma_{1} \gamma^{\prime} y \zeta_{r}\right)+(-1)^{r} b \gamma_{1} \gamma\left(\beta_{1} y+\beta_{2} \zeta_{r}\right) \zeta_{r}-b \kappa \gamma_{1} \gamma^{2}\left(-(-1)^{r} \eta_{r}+\gamma_{1} \zeta_{r}^{2}\right)=0
$$

which is implied by

$$
\gamma_{1}^{\prime}+(-1)^{r} \kappa \gamma^{2} \gamma_{1}=0, \quad(-1)^{r} \beta_{2}-\kappa \gamma \gamma_{1}=0
$$

Then the first equation and (8.1.52) imply

$$
\gamma_{1}=b_{3} e^{-(-1)^{r} \kappa \alpha}
$$

for some constant $b_{3}$. By the second equations in (8.1.63) and (8.1.55), we have:

$$
(-1)^{r} \frac{b_{1} e^{-(-1)^{r} \nu \alpha}}{\sqrt{\left(\alpha^{\prime}\right)^{3}}}=b_{3} \kappa \sqrt{\alpha^{\prime}} e^{-(-1)^{r} \kappa \alpha} .
$$

For convenience, we take

$$
b_{1}=(-1)^{r} \kappa b_{3} .
$$

Then (8.1.65) is implied by

$$
\alpha^{\prime} e^{(-1)^{r}(\nu-\kappa) \alpha / 2}=1
$$

If $\nu=\kappa,(8.1 .65)$ is implied by $\alpha=t$. When $\nu \neq \kappa,(8.1 .65)$ becomes

$$
\left(\frac{2 e^{(-1)^{r}(\nu-\kappa) \alpha / 2}}{\nu-\kappa}\right)^{\prime}=(-1)^{r}
$$

Thus

$$
\alpha=\frac{2(-1)^{r}}{\nu-\kappa} \ln \left[(-1)^{r}(\nu-\kappa) t / 2+c_{0}\right], \quad c_{0} \in \mathbb{R} .
$$

Suppose $\nu=\kappa$. Then $\gamma=\sqrt{\alpha^{\prime}}=1$ and $\beta_{1}=0$. By (8.1.48), (8.1.55), (8.1.56) and (8.1.60),

$$
\phi=b_{2} e^{-(-1)^{r} \nu t} \eta_{r}(y), \quad \psi=(-1)^{r} b_{3} \nu e^{-(-1)^{r} \nu t} \zeta_{r}(y)
$$

Moreover, (8.1.15), (8.1.61) and (8.1.64) yield

$$
\theta=b \exp \left(b_{3} e^{-(-1)^{r} \nu t} \eta_{r}(y)\right)
$$

Furthermore, (8.1.15) and (8.1.66) give

$$
\xi=b_{2} e^{-(-1)^{r} \nu t} \eta_{r}(y)+(-1)^{r} b_{3} \nu e^{-(-1)^{r} \nu t} x \zeta_{r}(y) .
$$

According to (8.1.11),

$$
\begin{gathered}
u=\xi_{y}=(-1)^{r}\left[-b_{2} e^{-(-1)^{r} \nu t} \zeta_{r}(y)+b_{3} \nu e^{-(-1)^{r} \nu t} x \eta_{r}(y)\right] \\
v=-\xi_{x}=-(-1)^{r} b_{3} \nu e^{-(-1)^{r} \nu t} \zeta_{r}(y) .
\end{gathered}
$$


Note

$$
\begin{gathered}
u_{t}+u u_{x}+v u_{y}-\nu \Delta u=b_{3}^{2} \nu^{2} c^{r} e^{-(-1)^{r} 2 \nu t} x, \\
v_{t}+u v_{x}+v v_{y}-\nu \Delta v-\theta=v v_{y}-b \exp \left(b_{3} e^{-(-1)^{r} \nu t} \eta_{r}(y)\right) .
\end{gathered}
$$

By (8.1.1), we have

$$
p=b \int \exp \left(b_{3} e^{-(-1)^{r} \nu t} \eta_{r}(y)\right) d y-\frac{1}{2} b_{3}^{2} \nu^{2} e^{-(-1)^{r} 2 \nu t}\left(c^{r} x^{2}+\zeta_{r}^{2}(y)\right) .
$$

Theorem 8.1.3. Suppose $\kappa=\nu$. For $b, b_{2}, b_{3}, c \in \mathbb{R}$, we have the following solutions of the two-dimensional Boussinesq equations (8.1.1)-(8.1.2): (1)

$$
\begin{gathered}
u=\frac{e^{\nu t}}{2}\left[b_{2}\left(e^{y}-c e^{-y}\right)-b_{3} \nu x\left(e^{y}+c e^{-y}\right)\right], \\
v=\frac{1}{2} b_{3} \nu e^{\nu t}\left(e^{y}-c e^{-y}\right) \\
\theta=b \exp \left(b_{3} e^{\nu t}\left(e^{y}+c e^{-y}\right) / 2\right)
\end{gathered}
$$

and

$$
p=b \int \exp \left(b_{3} e^{\nu t}\left(e^{y}+c e^{-y}\right) / 2\right) d y-\frac{1}{2} b_{3}^{2} \nu^{2} e^{2 \nu t}\left(c x^{2}+\left(e^{y}-c e^{-y}\right)^{2} / 4\right)
$$

$$
\begin{gathered}
u=e^{-\nu t}\left[-b_{2} \sin y+b_{3} \nu x \cos y\right], \quad v=-b_{3} \nu e^{-\nu t} \sin y \\
\theta=b \exp \left(b_{3} e^{-\nu t} \cos y\right)
\end{gathered}
$$

and

$$
p=b \int \exp \left(b_{3} e^{-\nu t} \cos y\right) d y-\frac{1}{2} b_{3}^{2} \nu^{2} e^{-2 \nu t}\left(x^{2}+\cos ^{2} y\right)
$$

Applying the symmetry transformations in (8.1.6)-(8.1.10) to the above solutions, we can get more general solutions the two-dimensional Boussinesq equations (8.1.1)-(8.1.2).

Consider the case $\nu \neq \kappa$. Then

$$
\gamma=\sqrt{\alpha^{\prime}}=\frac{1}{\sqrt{(-1)^{r}(\nu-\kappa) t / 2+c_{0}}}
$$

by (8.1.69). Moreover,

$$
\beta_{1}=\frac{\gamma^{\prime}}{\gamma}=\frac{(-1)^{r}(\kappa-\nu)}{4\left[(-1)^{r}(\nu-\kappa) t / 2+c_{0}\right]}
$$

by (8.1.53),

$$
\beta_{2}=\frac{b_{1} e^{-(-1)^{r} \nu \alpha}}{\sqrt{\left(\alpha^{\prime}\right)^{3}}}=(-1)^{r} b_{3} \kappa\left[(-1)^{r}(\nu-\kappa) t / 2+c_{0}\right]^{2 \nu /(\kappa-\nu)+3 / 2}
$$


according to (8.1.55), (8.1.66) and (8.1.69),

$$
\beta_{3}=\frac{b_{2} e^{-(-1)^{r} \nu \alpha}}{\alpha^{\prime}}=b_{2}\left[(-1)^{r}(\nu-\kappa) t / 2+c_{0}\right]^{2 \nu /(\kappa-\nu)+1}
$$

by $(8.1 .60)$, and

$$
\gamma_{1}=b_{3} e^{-(-1)^{r} \kappa \alpha}=b_{3}\left[(-1)^{r}(\nu-\kappa) t / 2+c_{0}\right]^{2 \kappa /(\kappa-\nu)}
$$

by (8.1.64). Thus (8.1.56) and (8.1.88) yield

$$
\phi=b_{2}\left[(-1)^{r}(\nu-\kappa) t / 2+c_{0}\right]^{2 \nu /(\kappa-\nu)+1} \eta_{r}(y) .
$$

Furthermore,

$$
\psi=\frac{(-1)^{r}(\kappa-\nu) y}{4\left[(-1)^{r}(\nu-\kappa) t / 2+c_{0}\right]}+(-1)^{r} b_{3} \kappa\left[(-1)^{r}(\nu-\kappa) t / 2+c_{0}\right]^{2 \nu /(\kappa-\nu)+3 / 2} \zeta_{r}(y)
$$

by (8.1.48), (8.1.86) and (8.1.87).

According to (8.1.15), (8.1.61) and (8.1.89),

$$
\theta=b \exp \left(b_{3}\left[(-1)^{r}(\nu-\kappa) t / 2+c_{0}\right]^{2 \kappa /(\kappa-\nu)} \eta_{r}(y)\right)
$$

By (8.1.15),

$$
\begin{aligned}
\xi= & \frac{(-1)^{r}(\kappa-\nu) x y}{4\left[(-1)^{r}(\nu-\kappa) t / 2+c_{0}\right]}+(-1)^{r} b_{3} \kappa\left[(-1)^{r}(\nu-\kappa) t / 2+c_{0}\right]^{2 \nu /(\kappa-\nu)+3 / 2} x \zeta_{r}(y) \\
& +b_{2}\left[(-1)^{r}(\nu-\kappa) t / 2+c_{0}\right]^{2 \nu /(\kappa-\nu)+1} \eta_{r}(y) .
\end{aligned}
$$

Then (8.1.11) and (8.1.93) say that

$$
\begin{aligned}
u= & \frac{(-1)^{r}(\kappa-\nu) x}{4\left[(-1)^{r}(\nu-\kappa) t / 2+c_{0}\right]}+(-1)^{r} b_{3} \kappa\left[(-1)^{r}(\nu-\kappa) t / 2+c_{0}\right]^{2 \nu /(\kappa-\nu)+1} x \eta_{r}(y) \\
& -(-1)^{r} b_{2}\left[(-1)^{r}(\nu-\kappa) t / 2+c_{0}\right]^{2 \nu /(\kappa-\nu)+1 / 2} \zeta_{r}(y), \\
v= & \frac{(-1)^{r}(\nu-\kappa) y}{4\left[(-1)^{r}(\nu-\kappa) t / 2+c_{0}\right]}-(-1)^{r} b_{3} \kappa\left[(-1)^{r}(\nu-\kappa) t / 2+c_{0}\right]^{2 \nu /(\kappa-\nu)+3 / 2} \zeta_{r}(y),
\end{aligned}
$$

By (8.1.20) with $\alpha_{1}=0$ and (8.1.21) with $\alpha_{2}$ given in (8.1.49), we have

$$
\begin{aligned}
& u_{t}+u u_{x}+v u_{y}-\nu \Delta u \\
= & \phi_{y t}+x \psi_{y t}+\left(\phi_{y}+x \psi_{y}\right) \psi_{y}-\psi\left(\phi_{y y}+x \psi_{y y}\right)-\nu\left(\phi_{y y y}+x \psi_{y y y}\right) \\
= & \phi_{y t}+\phi_{y}^{2}-\psi \phi_{y y}-\nu \phi_{y y y}+\left(\psi_{y t}+\psi_{y} \psi_{y}-\psi \psi_{y y}-\nu \psi_{y y y}\right) x \\
= & \left(\beta_{1}^{\prime}+c^{r} \beta_{2}^{2} \gamma^{2}+\beta_{1}^{2}\right) x=b_{3}^{2} c^{r} \kappa^{2}\left[(-1)^{r}(\nu-\kappa) t / 2+c_{0}\right]^{4 \nu /(\kappa-\nu)+2} x \\
& +\frac{3(\nu-\kappa)^{2} x}{16\left[(-1)^{r}(\nu-\kappa) t / 2+c_{0}\right]^{2}} .
\end{aligned}
$$


Moreover, (8.1.48), the second equation in (8.1.50) and (8.1.85)-(8.1.87) yield

$$
\begin{aligned}
& v_{t}+u v_{x}+v v_{y}-\nu \Delta(v)-\theta=-\psi_{t}+\psi \psi_{y}+\nu \psi_{y y}-\theta \\
= & -\left(\beta_{1}^{\prime} y+\beta_{2}^{\prime} \zeta_{r}+\beta_{2} \gamma^{\prime} y \eta_{r}\right)+\left(\beta_{1} y+\beta_{2} \zeta_{r}\right)\left(\beta_{1}+\beta_{2} \gamma \eta_{r}\right)-(-1)^{r} \nu \beta_{2} \gamma^{2} \zeta_{r}-\theta \\
= & \left(\beta_{1}^{2}-\beta_{1}^{\prime}\right) y+\left(\beta_{1} \beta_{2}-\beta_{2}^{\prime}-(-1)^{r} \nu \beta_{2} \gamma^{2}\right) \zeta_{r}+\beta_{2}\left(\beta_{1} \gamma-\gamma^{\prime}\right) y \eta_{r}+\frac{\beta_{2}^{2}}{2} \partial_{y}\left(\zeta_{r}^{2}\right)-\theta \\
= & \frac{3(\nu-\kappa)^{2} y}{16\left[(-1)^{r}(\nu-\kappa) t / 2+c_{0}\right]^{2}}-b e^{b_{3}\left[(-1)^{r}(\nu-\kappa) t / 2+c_{0}\right]^{2 \kappa /(\kappa-\nu)} \eta_{r}(y)} \\
& +b_{3} \kappa(\kappa-\nu)\left[(-1)^{r}(\nu-\kappa) t / 2+c_{0}\right]^{2 \nu /(\kappa-\nu)+1 / 2} \zeta_{r}(y) \\
& +\frac{b_{3}^{2}}{2} \kappa^{2}\left[(-1)^{r}(\nu-\kappa) t / 2+c_{0}\right]^{4 \nu /(\kappa-\nu)+3} \partial_{y} \zeta_{r}^{2}(y) .
\end{aligned}
$$

According to (8.1.11), we have

$$
\begin{aligned}
p= & b \int e^{b_{3}\left[(-1)^{r}(\nu-\kappa) t / 2+c_{0}\right]^{2 \kappa /(\kappa-\nu)} \eta_{r}(y)} d y-\frac{b_{3}^{2}}{2} c^{r} \kappa^{2}\left[(-1)^{r}(\nu-\kappa) t / 2+c_{0}\right]^{4 \nu /(\kappa-\nu)+2} x^{2} \\
& -\frac{3(\nu-\kappa)^{2}\left(x^{2}+y^{2}\right)}{32\left[(-1)^{r}(\nu-\kappa) t / 2+c_{0}\right]^{2}}-\frac{b_{3}^{2}}{2} \kappa^{2}\left[(-1)^{r}(\nu-\kappa) t / 2+c_{0}\right]^{4 \nu /(\kappa-\nu)+3} \zeta_{r}^{2}(y) \\
& +(-1)^{r} b_{3} \kappa(\kappa-\nu)\left[(-1)^{r}(\nu-\kappa) t / 2+c_{0}\right]^{2 \nu /(\kappa-\nu)+1} \eta_{r}(y) .
\end{aligned}
$$

Theorem 8.1.4. Suppose $\kappa \neq \nu$. For $b, b_{2}, b_{3}, c, c_{0} \in \mathbb{R}$, we have the following solutions of the two-dimensional Boussinesq equations (8.1.1)-(8.1.2): (1)

$$
\begin{aligned}
u= & -\frac{b_{3}}{2} \kappa\left[(\kappa-\nu) t / 2+c_{0}\right]^{2 \nu /(\kappa-\nu)+1} x\left(e^{y / \sqrt{(\kappa-\nu) t / 2+c_{0}}}+c e^{y / \sqrt{(\kappa-\nu) t / 2+c_{0}}}\right) \\
& +\frac{b_{2}}{2}\left[(\kappa-\nu) t / 2+c_{0}\right]^{2 \nu /(\kappa-\nu)+1 / 2}\left(e^{y / \sqrt{(\kappa-\nu) t / 2+c_{0}}}-c e^{y / \sqrt{(\kappa-\nu) t / 2+c_{0}}}\right) \\
& +\frac{(\nu-\kappa) x}{4\left[(\kappa-\nu) t / 2+c_{0}\right]}, \\
v= & \frac{b_{3}}{2} \kappa\left[(\kappa-\nu) t / 2+c_{0}\right]^{2 \nu /(\kappa-\nu)+3 / 2} x\left(e^{y / \sqrt{(\kappa-\nu) t / 2+c_{0}}}-c e^{y / \sqrt{(\kappa-\nu) t / 2+c_{0}}}\right) \\
& +\frac{(\kappa-\nu) y}{4\left[(\kappa-\nu) t / 2+c_{0}\right]}, \\
\theta= & b \exp \left(2^{-1} b_{3}\left[(\kappa-\nu) t / 2+c_{0}\right]^{2 \kappa /(\kappa-\nu)}\left(e^{y / \sqrt{(\kappa-\nu) t / 2+c_{0}}}+c e^{y / \sqrt{(\kappa-\nu) t / 2+c_{0}}}\right)\right)
\end{aligned}
$$

and

$$
\begin{aligned}
p= & b \int \exp \left(2^{-1} b_{3}\left[(\kappa-\nu) t / 2+c_{0}\right]^{2 \kappa /(\kappa-\nu)}\left(e^{y / \sqrt{(\kappa-\nu) t / 2+c_{0}}}+c e^{y / \sqrt{(\kappa-\nu) t / 2+c_{0}}}\right)\right) d y \\
& -\frac{b_{3}^{2}}{8} \kappa^{2}\left[(\kappa-\nu) t / 2+c_{0}\right]^{4 \nu /(\kappa-\nu)+3}\left(e^{y / \sqrt{(\kappa-\nu) t / 2+c_{0}}}-c e^{y / \sqrt{(\kappa-\nu) t / 2+c_{0}}}\right) \\
& -\frac{b_{3}}{2} \kappa(\kappa-\nu)\left[(\kappa-\nu) t / 2+c_{0}\right]^{2 \nu /(\kappa-\nu)+1}\left(e^{y / \sqrt{(\kappa-\nu) t / 2+c_{0}}}+c e^{y / \sqrt{(\kappa-\nu) t / 2+c_{0}}}\right) \\
& -\frac{b_{3}^{2}}{2} c \kappa^{2}\left[(\kappa-\nu) t / 2+c_{0}\right]^{4 \nu /(\kappa-\nu)+2} x^{2}-\frac{3(\nu-\kappa)^{2}\left(x^{2}+y^{2}\right)}{32\left[(\kappa-\nu) t / 2+c_{0}\right]^{2}}
\end{aligned}
$$




$$
\begin{aligned}
u= & b_{3} \kappa\left[(\nu-\kappa) t / 2+c_{0}\right]^{2 \nu /(\kappa-\nu)+1} x \cos \frac{y}{\sqrt{(\nu-\kappa) t / 2+c_{0}}}+\frac{(\kappa-\nu) x}{4\left[(\nu-\kappa) t / 2+c_{0}\right]} \\
& -b_{2}\left[(\nu-\kappa) t / 2+c_{0}\right]^{2 \nu /(\kappa-\nu)+1 / 2} \sin \frac{y}{\sqrt{(\nu-\kappa) t / 2+c_{0}}}, \\
v= & \frac{(\nu-\kappa) y}{4\left[(\nu-\kappa) t / 2+c_{0}\right]}-b_{3} \kappa\left[(\nu-\kappa) t / 2+c_{0}\right]^{2 \nu /(\kappa-\nu)+3 / 2} \sin \frac{y}{\sqrt{(\nu-\kappa) t / 2+c_{0}}}, \\
& \theta=b \exp \left(b_{3}\left[(\nu-\kappa) t / 2+c_{0}\right]^{2 \kappa /(\kappa-\nu)} \cos \frac{y}{\sqrt{(\nu-\kappa) t / 2+c_{0}}}\right) \\
p= & \quad \int \exp \left(b_{3}\left[(\nu-\kappa) t / 2+c_{0}\right]^{2 \kappa /(\kappa-\nu)} \cos \frac{y}{\sqrt{(\nu-\kappa) t / 2+c_{0}}}\right) d y \\
& -\frac{b_{3}^{2}}{2} \kappa^{2}\left[(\nu-\kappa) t / 2+c_{0}\right]^{4 \nu /(\kappa-\nu)+2} x^{2}-\frac{3(\nu-\kappa)^{2}\left(x^{2}+y^{2}\right)}{32\left[(\nu-\kappa) t / 2+c_{0}\right]^{2}} \\
& -\frac{b_{3}^{2}}{2} \kappa^{2}\left[(\nu-\kappa) t / 2+c_{0}\right]^{4 \nu /(\kappa-\nu)+3} \sin ^{2} \frac{y}{\sqrt{(\nu-\kappa) t / 2+c_{0}}} \\
& +b_{3} \kappa(\kappa-\nu)\left[(\nu-\kappa) t / 2+c_{0}\right]^{2 \nu /(\kappa-\nu)+1} \cos \frac{y}{\sqrt{(\nu-\kappa) t / 2+c_{0}}} .
\end{aligned}
$$

Applying the symmetry transformations in (8.1.6)-(8.1.10) to the above solutions, we can get more general solutions the two-dimensional Boussinesq equations (8.1.1)-(8.1.2).

Let $\gamma$ be a function in $t$. Denote the moving frame

$$
\mathcal{X}=x \cos \gamma+y \sin \gamma, \quad \mathcal{Y}=y \cos \gamma-x \sin \gamma
$$

Then

$$
\partial_{t}(\mathcal{X})=\gamma^{\prime} \mathcal{Y}, \quad \partial_{t}(\mathcal{Y})=-\gamma^{\prime} \mathcal{X}
$$

By the chain rule of taking partial derivatives,

$$
\partial_{x}=\cos \gamma \partial_{\mathcal{X}}-\sin \gamma \partial_{\mathcal{Y}}, \quad \partial_{y}=\sin \gamma \partial_{\mathcal{X}}+\cos \gamma \partial_{\mathcal{Y}}
$$

Solving the above system, we get

$$
\partial_{\mathcal{X}}=\cos \gamma \partial_{x}+\sin \gamma \partial_{y}, \quad \partial_{\mathcal{Y}}=-\sin \gamma \partial_{x}+\cos \gamma \partial_{y}
$$

Moreover, (8.1.107) and (8.1.110) imply

$$
\partial_{\mathcal{X}}(\mathcal{Y})=0, \quad \partial_{\mathcal{Y}}(\mathcal{X})=0
$$

In particular,

$$
\Delta=\partial_{x}^{2}+\partial_{y}^{2}=\partial_{\mathcal{X}}^{2}+\partial_{\mathcal{Y}}^{2}, \quad x^{2}+y^{2}=\mathcal{X}^{2}+\mathcal{Y}^{2}
$$


We assume

$$
\xi=\phi(t, \mathcal{X})-\frac{\gamma^{\prime}}{2}\left(x^{2}+y^{2}\right), \quad \theta=\psi(t, \mathcal{X})
$$

where $\phi$ and $\psi$ are functions in $t, \mathcal{X}$. Note

$$
\xi_{y} \partial_{x}-\xi_{x} \partial_{y}=\left(\mathcal{X}-\phi_{\mathcal{X}}\right) \partial_{\mathcal{Y}}-\gamma^{\prime} \mathcal{Y} \partial_{\mathcal{X}}
$$

Then (8.1.13) becomes

$$
\psi_{t}-\kappa \psi_{\mathcal{X X}}=0
$$

and (8.1.14) becomes

$$
-2 \gamma^{\prime \prime}+\phi_{t \mathcal{X} \mathcal{X}}-\nu \phi_{\mathcal{X} \mathcal{X} \mathcal{X}}+\psi_{\mathcal{X}} \cos \gamma=0
$$

by (8.1.111) and (8.1.114). Modulo the transformation in (8.1.8)-(8.1.11), the above equation is equivalent to

$$
-2 \gamma^{\prime \prime} \mathcal{X}+\phi_{t \mathcal{X}}-\nu \phi_{\mathcal{X X X}}+\psi \cos \gamma=0
$$

Note that (8.1.115) is a heat conduction equation. Assume $\nu=\kappa$. We take its solution

$$
\psi=\sum_{r=1}^{m} a_{r} d_{r} e^{a_{r}^{2} \kappa t \cos 2 b_{r} t+a_{r} \mathcal{X} \cos b_{r}} \sin \left(a_{r}^{2} \kappa t \sin 2 b_{r}+a_{r} \mathcal{X} \sin b_{r}+b_{r}+c_{r}\right),
$$

where $a_{r}, b_{r}, c_{r}, d_{r} \in \mathbb{R}$ with $\left(a_{r}, b_{r}\right) \neq(0,0)$ and $d_{r} \neq 0$. Then

$$
\psi=\partial_{\mathcal{X}}\left[\sum_{r=1}^{m} d_{r} e^{a_{r}^{2} \kappa t \cos 2 b_{r} t+a_{r} \mathcal{X} \cos b_{r}} \sin \left(a_{r}^{2} \kappa t \sin 2 b_{r}+a_{r} \mathcal{X} \sin b_{r}+c_{r}\right)\right] .
$$

Moreover, (8.1.117) is implied by the following equation:

$$
\begin{aligned}
& 2 \nu \gamma^{\prime}-\gamma^{\prime \prime} \mathcal{X}^{2}+\phi_{t}-\nu \phi_{\mathcal{X} \mathcal{X}}+\left[\sum_{r=1}^{m} d_{r} e^{a_{r}^{2} \kappa t \cos 2 b_{r}+a_{r} \mathcal{X} \cos b_{r}}\right. \\
& \left.\times \sin \left(a_{r}^{2} \kappa t \sin 2 b_{r}+a_{r} \mathcal{X} \sin b_{r}+c_{r}\right)\right] \cos \gamma=0
\end{aligned}
$$

by (8.1.119). Thus we have the following solution of (8.1.117):

$$
\begin{aligned}
\phi= & -\left[\sum_{r=1}^{r} d_{r} e^{a_{r}^{2} \kappa t \cos 2 b_{r}+a_{r} \mathcal{X} \cos b_{r}} \sin \left(a_{r}^{2} \kappa t \sin 2 b_{r}+a_{r} \mathcal{X} \sin b_{r}+c_{r}\right)\right] \int \cos \gamma d t \\
& +\gamma^{\prime} \mathcal{X}^{2}+\sum_{s=1}^{n} \hat{d}_{s} e^{\hat{a}_{s}^{2} \kappa t \cos 2 \hat{b}_{s}+\hat{a}_{s} \mathcal{X} \cos \hat{b}_{s}} \sin \left(\hat{a}_{s}^{2} \kappa t \sin 2 \hat{b}_{s}+\hat{a}_{s} \mathcal{X} \sin \hat{b}_{s}+\hat{c}_{s}\right),
\end{aligned}
$$

where $\hat{a}_{s}, \hat{b}_{s}, \hat{c}_{s}, \hat{d}_{s}$ are real numbers.

Suppose $\nu \neq \kappa$. To make (8.1.117) solvable, we choose the following solution of (8.1.115):

$$
\psi=\sum_{r=1}^{m} a_{r} d_{r} e^{a_{r}^{2} \kappa t+a_{r} \mathcal{X}}
$$


Now (8.1.117) is implied by the following equation:

$$
2 \nu \gamma^{\prime}-\gamma^{\prime \prime} \mathcal{X}^{2}+\phi_{t}-\nu \phi_{\mathcal{X} X}+\sum_{r=1}^{m} d_{r} e^{a_{r}^{2} \kappa t+a_{r} \mathcal{X}} \cos \gamma=0
$$

We obtain the following solution of (8.1.117):

$$
\begin{aligned}
\phi= & \gamma^{\prime} \mathcal{X}^{2}+\sum_{s=1}^{n} \hat{d}_{s} e^{\hat{a}_{s}^{2} \kappa t \cos 2 \hat{b}_{s}+\hat{a}_{s} \mathcal{X} \cos \hat{b}_{s}} \sin \left(\hat{a}_{s}^{2} \kappa t \sin 2 \hat{b}_{s}+\hat{a}_{s} \mathcal{X} \sin \hat{b}_{s}+\hat{c}_{s}\right) \\
& -\sum_{r=1}^{m} d_{r} e^{a_{r}^{2} \nu t+a_{r} \mathcal{X}} \int e^{a_{r}^{2}(\kappa-\nu) t} \cos \gamma d t
\end{aligned}
$$

Note

$$
u=\phi_{\mathcal{X}} \sin \gamma-\gamma^{\prime} y, \quad v=\gamma^{\prime} x-\phi_{\mathcal{X}} \cos \gamma
$$

Moreover,

$$
u \partial_{x}+v \partial_{y}=-\phi_{\mathcal{X}} \partial_{\mathcal{Y}}+\gamma^{\prime}\left(x \partial_{y}-y \partial_{x}\right)
$$

By (8.1.117) and (8.1.126), we find

$$
\begin{aligned}
& u_{t}+u u_{x}+v u_{y}-\nu \Delta u \\
& =\gamma^{\prime} \phi_{\mathcal{X}} \cos \gamma+\phi_{\mathcal{X} t} \sin \gamma+\gamma^{\prime} \mathcal{Y} \phi_{\mathcal{X} \mathcal{X}} \sin \gamma-\gamma^{\prime \prime} y+\gamma^{\prime} \phi_{\mathcal{X}} \partial_{\mathcal{Y}}(y) \\
& +\gamma^{\prime}\left(x \partial_{y}-y \partial_{x}\right)\left(\phi_{\mathcal{X}}\right) \sin \gamma-\gamma^{\prime 2} x-\nu \phi_{\mathcal{X X X}} \sin \gamma \\
& =\left(\phi_{\mathcal{X} t}-\nu \phi_{\mathcal{X X X}}\right) \sin \gamma+2 \gamma^{\prime} \phi_{\mathcal{X}} \cos \gamma-\gamma^{\prime 2} x-\gamma^{\prime \prime} y \\
& =\left(2 \gamma^{\prime \prime} \mathcal{X}-\psi \cos \gamma\right) \sin \gamma+2 \gamma^{\prime} \phi_{\mathcal{X}} \cos \gamma-\gamma^{\prime 2} x-\gamma^{\prime \prime} y \text {, } \\
& =\gamma^{\prime \prime}(x \sin 2 \gamma-y \cos 2 \gamma)+\left(2 \gamma^{\prime} \phi_{\mathcal{X}}-\psi \sin \gamma\right) \cos \gamma-\gamma^{\prime 2} x \text {, } \\
& v_{t}+u v_{x}+v v_{y}-\nu \Delta v-\theta \\
& =\gamma^{\prime} \phi_{\mathcal{X}} \sin \gamma-\phi_{\mathcal{X} t} \cos \gamma-\gamma^{\prime} \mathcal{Y} \phi_{\mathcal{X X}} \cos \gamma+\gamma^{\prime \prime} x-\gamma^{\prime} \phi_{\mathcal{X}} \partial_{\mathcal{Y}}(x) \\
& -\gamma^{\prime}\left(x \partial_{y}-y \partial_{x}\right)\left(\phi_{\mathcal{X}}\right) \cos \gamma-\gamma^{\prime 2} y+\nu \phi_{\mathcal{X} X \mathcal{X}} \cos \gamma-\psi \\
& =\left(\nu \phi_{\mathcal{X} X \mathcal{X}}-\phi_{\mathcal{X} t}\right) \cos \gamma+2 \gamma^{\prime} \phi_{\mathcal{X}} \sin \gamma-\gamma^{\prime 2} y+\gamma^{\prime \prime} x-\psi \\
& =\left(\psi \cos \gamma-2 \gamma^{\prime \prime} \mathcal{X}\right) \cos \gamma+2 \gamma^{\prime} \phi_{\mathcal{X}} \sin \gamma-\gamma^{\prime 2} y+\gamma^{\prime \prime} x-\psi \\
& =-\gamma^{\prime \prime}(x \cos 2 \gamma+y \sin 2 \gamma)+\left(2 \gamma^{\prime} \phi \mathcal{X}-\psi \sin \gamma\right) \sin \gamma-\gamma^{\prime 2} y \text {. }
\end{aligned}
$$

According to (8.1.1),

$$
p=\frac{\gamma^{\prime 2}-\gamma^{\prime \prime} \sin 2 \gamma}{2} x^{2}+\frac{\gamma^{\prime 2}+\gamma^{\prime \prime} \sin 2 \gamma}{2} y^{2}+\gamma^{\prime \prime} x y \cos 2 \gamma+\int \psi d \mathcal{X} \sin \gamma-2 \gamma^{\prime} \phi
$$

Theorem 8.1.5. Let $\gamma$ be any function in $t$ and denote $\mathcal{X}=x \cos \gamma+y \sin \gamma$. Take

$$
\left\{a_{r}, b_{r}, c_{r}, d_{r}, \hat{a}_{s}, \hat{b}_{s}, \hat{c}_{s}, \hat{d}_{s} \mid r=1, \ldots, m ; s=1, \ldots, n\right\} \subset \mathbb{R}
$$


If $\nu=\kappa$, we have the following solutions of the two-dimensional Boussinesq equations (8.1.1)-(8.1.2):

$$
\begin{aligned}
u= & \left\{\sum_{s=1}^{n} \hat{a}_{s} \hat{d}_{s} e^{\hat{a}_{s}^{2} \kappa t \cos 2 \hat{b}_{s}+\hat{a}_{s} \mathcal{X} \cos \hat{b}_{s}} \sin \left(\hat{a}_{s}^{2} \kappa t \sin 2 \hat{b}_{s}+\hat{a}_{s} \mathcal{X} \sin \hat{b}_{s}+\hat{b}_{s}+\hat{c}_{s}\right)\right. \\
& -\left[\sum_{r=1}^{m} a_{r} d_{r} e^{a_{r}^{2} \kappa t \cos 2 b_{r}+a_{r} \mathcal{X} \cos b_{r}} \sin \left(a_{r}^{2} \kappa t \sin 2 b_{r}+b_{r}+a_{r} \mathcal{X} \sin b_{r}+c_{r}\right)\right] \\
& \left.\times \int \cos \gamma d t+2 \gamma^{\prime} \mathcal{X}\right\} \sin \gamma-\gamma^{\prime} y, \\
v= & -\left\{\sum_{s=1}^{n} \hat{a}_{s} \hat{d}_{s} e^{\hat{a}_{s}^{2} \kappa t \cos 2 \hat{b}_{s}+\hat{a}_{s} \mathcal{X} \cos \hat{b}_{s}} \sin \left(\hat{a}_{s}^{2} \kappa t \sin 2 \hat{b}_{s}+\hat{a}_{s} \mathcal{X} \sin \hat{b}_{s}+\hat{b}_{s}+\hat{c}_{s}\right)\right. \\
& -\left[\sum_{r=1}^{m} a_{r} d_{r} e^{a_{r}^{2} \kappa t \cos 2 b_{r}+a_{r} \mathcal{X} \cos b_{r}} \sin \left(a_{r}^{2} \kappa t \sin 2 b_{r}+a_{r} \mathcal{X} \sin b_{r}+b_{r}+c_{r}\right)\right] \\
& \left.\times \int \cos \gamma d t+2 \gamma^{\prime} \mathcal{X}\right\} \cos \gamma+\gamma^{\prime} x,
\end{aligned}
$$

$\theta=\psi$ in (8.1.118), and

$$
\begin{aligned}
p= & \left(\sin \gamma+2 \gamma^{\prime} \int \cos \gamma\right)\left[\sum_{r=1}^{m} d_{r} e^{a_{r}^{2} \kappa t \cos 2 b_{r}+a_{r} \mathcal{X} \cos b_{r}} \sin \left(a_{r}^{2} \kappa t \sin 2 b_{r}+a_{r} \mathcal{X} \sin b_{r}+c_{r}\right)\right] \\
& +\frac{{\gamma^{\prime}}^{2}-2{\gamma^{\prime \prime}}^{\prime} \sin 2 \gamma}{2} x^{2}+\frac{\gamma^{\prime 2}+\gamma^{\prime \prime} \sin 2 \gamma}{2} y^{2}+\gamma^{\prime \prime} x y \cos 2 \gamma-\gamma^{\prime 2} \mathcal{X}^{2} \\
& -2 \gamma^{\prime} \sum_{s=1}^{n} \hat{d}_{s} e^{\hat{a}_{s}^{2} \kappa t \cos 2 \hat{b}_{s}+\hat{a}_{s} \mathcal{X} \cos \hat{b}_{s}} \sin \left(\hat{a}_{s}^{2} \kappa t \sin 2 \hat{b}_{s}+\hat{a}_{s} \mathcal{X} \sin \hat{b}_{s}+\hat{c}_{s}\right)
\end{aligned}
$$

When $\nu \neq \kappa$, we have the following solutions of the two-dimensional Boussinesq equations (8.1.1)-(8.1.2):

$$
\begin{aligned}
u= & \left\{\sum_{s=1}^{n} \hat{a}_{s} \hat{d}_{s} e^{\hat{a}_{s}^{2} \kappa t \cos 2 \hat{b}_{s}+\hat{a}_{s} \mathcal{X} \cos \hat{b}_{s}} \sin \left(\hat{a}_{s}^{2} \kappa t \sin 2 \hat{b}_{s}+\hat{a}_{s} \mathcal{X} \sin \hat{b}_{s}+\hat{b}_{s}+\hat{c}_{s}\right)\right. \\
& \left.+2 \gamma^{\prime} \mathcal{X}-\sum_{r=1}^{m} a_{r} d_{r} e^{a_{r}^{2} \nu t+a_{r} \mathcal{X}} \int e^{a_{r}^{2}(\kappa-\nu) t} \cos \gamma d t\right\} \sin \gamma-\gamma^{\prime} y, \\
v= & -\left\{\sum_{s=1}^{n} \hat{a}_{s} \hat{d}_{s} e^{\hat{a}_{s}^{2} \kappa t \cos 2 \hat{b}_{s}+\hat{a}_{s} \mathcal{X} \cos \hat{b}_{s}} \sin \left(\hat{a}_{s}^{2} \kappa t \sin 2 \hat{b}_{s}+\hat{a}_{s} \mathcal{X} \sin \hat{b}_{s}+\hat{b}_{s}+\hat{c}_{s}\right)\right. \\
& \left.+2 \gamma^{\prime} \mathcal{X}-\sum_{r=1}^{m} a_{r} d_{r} e^{a_{r}^{2} \nu t+a_{r} \mathcal{X}} \int e^{a_{r}^{2}(\kappa-\nu) t} \cos \gamma d t\right\} \cos \gamma+\gamma^{\prime} x,
\end{aligned}
$$


$\theta=\psi$ in (8.1.122), and

$$
\begin{aligned}
p= & \frac{\gamma^{\prime 2}-\gamma^{\prime \prime} \sin 2 \gamma}{2} x^{2}+\frac{\gamma^{\prime 2}+\gamma^{\prime \prime} \sin 2 \gamma}{2} y^{2}+\gamma^{\prime \prime} x y \cos 2 \gamma-2 \gamma^{\prime 2} \mathcal{X}^{2} \\
& -2 \gamma^{\prime} \sum_{s=1}^{n} \hat{d}_{s} e^{\hat{a}_{s}^{2} \kappa t \cos 2 \hat{b}_{s}+\hat{a}_{s} \mathcal{X} \cos \hat{b}_{s}} \sin \left(\hat{a}_{s}^{2} \kappa t \sin 2 \hat{b}_{s}+\hat{a}_{s} \mathcal{X} \sin \hat{b}_{s}+\hat{c}_{s}\right) \\
& +\sum_{r=1}^{m} d_{r} e^{a_{r}^{2} \nu t+a_{r} \mathcal{X}}\left(2 \gamma^{\prime} \int e^{a_{r}^{2}(\kappa-\nu) t} \cos \gamma d t+\sin \gamma\right) .
\end{aligned}
$$

Remark 8.1.6. By Fourier expansion, we can use the above solution to obtain the one depending on two piecewise continuous functions of $\mathcal{X}$. Applying the symmetry transformations in (8.1.6)-(8.1.10) to the above solution, we can get more general solutions of the two-dimensional Boussinesq equations (8.1.1)-(8.1.2).

\subsection{Three-Dimensional Equations and Symmetry}

Another slightly simplified version of the system of primitive equations in geophysics is the three-dimensional stratified rotating Boussinesq system (e.g., cf. [LTW], [Pj]):

$$
\begin{gathered}
u_{t}+u u_{x}+v u_{y}+w u_{z}-\frac{1}{R_{0}} v=\sigma\left(\Delta u-p_{x}\right), \\
v_{t}+u v_{x}+v v_{y}+w v_{z}+\frac{1}{R_{0}} u=\sigma\left(\Delta v-p_{y}\right), \\
w_{t}+u w_{x}+v w_{y}+w w_{z}-\sigma R T=\sigma\left(\Delta w-p_{z}\right), \\
T_{t}+u T_{x}+v T_{y}+w T_{z}=\Delta T+w, \\
u_{x}+v_{y}+w_{z}=0,
\end{gathered}
$$

where $(u, v, w)$ is the velocity vector filed, $T$ is the temperature function, $p$ is the pressure function, $\sigma$ is the Prandtle number, $R$ is the thermal Rayleigh number and $R_{0}$ is the Rossby number. Moreover, the vector $\left(1 / R_{0}\right)(-v, u, 0)$ represents the Coriolis force and the term $w$ in (8.2.4) is derived using stratification. So the above equations are the extensions of Navier-Stokes equations by adding the Coriolis force and the stratified temperature equation. Due to the Coriolis force, the two-dimensional system (8.1.1) and (8.1.2) is not a special case of the above three-dimensional system. Hsia, Ma and Wang [HMW] studied the bifurcation and periodic solutions of the above system (8.2.1)-(8.2.5).

After the degree analysis, we find that the three-dimensional stratified rotating Boussinesq system is not dilation invariant. It is translation invariant. Let $\alpha$ be a function in $t$. The transformation

$$
F(t, x, y, z) \mapsto F(t, x+\alpha, y, z)-\delta_{u, F} \alpha^{\prime} \quad \text { for } F=u, v, w, T, p
$$


leaves (8.2.3)-(8.2.5) invariant and changes (8.2.1) and (8.2.2) to

$$
-\alpha^{\prime \prime}+u_{t}+u u_{x}+v u_{y}+w u_{z}-\frac{1}{R_{0}} v=\sigma\left(\Delta u-p_{x}\right)
$$

and

$$
v_{t}+u v_{x}+v v_{y}+w v_{z}+\frac{1}{R_{0}} u-\frac{\alpha^{\prime}}{R_{0}}=\sigma\left(\Delta v-p_{y}\right),
$$

where the independent variable $x$ is replaced by $x+\alpha$ and the partial derivatives are with respect to the original variables. Thus the transformation

$$
S_{1, \alpha}(F(t, x, y, z))=F(t, x+\alpha, y, z)-\delta_{u, F} \alpha^{\prime}+\delta_{p, F} \sigma^{-1}\left(\alpha^{\prime \prime} x+\alpha^{\prime} y / R_{0}\right)
$$

for $F=u, v, w, T, p$, is a symmetry of the system (8.2.1)-(8.2.5). Similarly, we have the following symmetry of the system (8.2.1)-(8.2.5):

$$
S_{2, \alpha}(F(t, x, y, z))=F(t, x, y+\alpha, z)-\delta_{v, F} \alpha^{\prime}+\delta_{p, F} \sigma^{-1}\left(\alpha^{\prime \prime} y-\alpha^{\prime} x / R_{0}\right)
$$

for $F=u, v, w, T, p$.

Note that the transformation

$$
F(t, x, y, z) \mapsto F(t, x, y, z+\alpha)-\delta_{w, F} \alpha^{\prime} \quad \text { for } F=u, v, w, T, p
$$

leaves (8.2.1), (8.2.2) and (8.2.5) invariant, and changes (8.2.3) and (8.2.4) to

$$
-\alpha^{\prime \prime}+w_{t}+u w_{x}+v w_{y}+w w_{z}-\sigma R T=\sigma\left(\Delta w-p_{z}\right)
$$

and

$$
T_{t}+u T_{x}+v T_{y}+w T_{z}=\Delta T+w-\alpha^{\prime}
$$

where the independent variable $x$ is replaced by $x+\alpha$ and the partial derivatives are with respect to the original variables. Hence the transformation

$$
S_{3, \alpha}(F(t, x, y, z))=F(t, x, y, z+\alpha)-\delta_{w, F} \alpha^{\prime}+\delta_{p, F}\left(\sigma^{-1} \alpha^{\prime \prime}-\alpha / R\right) z-\delta_{T, F} \alpha
$$

for $F=u, v, w, T, p$, is a symmetry of the system (8.2.1)-(8.2.5) . Obviously, the transformation

$$
S_{4, \alpha}(F(t, x, y, z))=F(t, x, y, z)+\delta_{p, F} \alpha^{\prime}
$$

for $F=u, v, w, T, p$, is a symmetry of the system.

For convenience of computation, we denote

$$
\begin{gathered}
\Phi_{1}=u_{t}+u u_{x}+v u_{y}+w u_{z}-\frac{1}{R_{0}} v-\sigma\left(u_{x x}+u_{y y}+u_{z z}\right), \\
\Phi_{2}=v_{t}+u v_{x}+v v_{y}+w v_{z}+\frac{1}{R_{0}} u-\sigma\left(v_{x x}+v_{y y}+v_{z z}\right),
\end{gathered}
$$




$$
\Phi_{3}=w_{t}+u w_{x}+v w_{y}+w w_{z}-\sigma R T-\sigma\left(w_{x x}+w_{y y}+w_{z z}\right) .
$$

Then the equations (8.2.1)-(8.2.3) become

$$
\Phi_{1}+\sigma p_{x}=0, \quad \Phi_{2}+\sigma p_{y}=0, \quad \Phi_{3}+\sigma p_{z}=0 .
$$

Our strategy is to solve the following compatibility conditions:

$$
\partial_{y}\left(\Phi_{1}\right)=\partial_{x}\left(\Phi_{2}\right), \quad \partial_{z}\left(\Phi_{1}\right)=\partial_{x}\left(\Phi_{3}\right), \quad \partial_{z}\left(\Phi_{2}\right)=\partial_{y}\left(\Phi_{3}\right) .
$$

\subsection{Asymmetric Approach I}

Starting from this section, we use asymmetric methods to solve the stratified rotating Boussinesq equations (8.2.1)-(8.2.5).

First we assume

$$
\begin{gathered}
u=\phi_{z}(t, z) x+\varsigma(t, z) y+\mu(t, z), \quad v=\tau(t, z) x+\psi_{z}(t, z) y+\varepsilon(t, z), \\
w=-\phi(t, z)-\psi(t, z), \quad T=\vartheta(t, z)+z,
\end{gathered}
$$

where $\phi, \vartheta, \varsigma, \mu, \tau$, and $\varepsilon$ are functions of $t, z$ to be determined. Then

$$
\begin{aligned}
\Phi_{1}= & \phi_{t z} x+\varsigma_{t} y+\mu_{t}+\phi_{z}\left(\phi_{z} x+\varsigma y+\mu\right)+\left(\varsigma-1 / R_{0}\right)\left(\tau x+\psi_{z} y+\varepsilon\right) \\
& -(\phi+\psi)\left(\phi_{z z} x+\varsigma_{z} y+\mu_{z}\right)-\sigma\left(\phi_{z z z} x+\varsigma_{z z} y+\mu_{z z}\right) \\
= & {\left[\phi_{t z}+\phi_{z}^{2}+\tau\left(\varsigma-1 / R_{0}\right)-\phi_{z z}(\phi+\psi)-\sigma \phi_{z z z}\right] x } \\
& +\left[\varsigma_{t}+\varsigma \phi_{z}+\psi_{z}\left(\varsigma-1 / R_{0}\right)-\varsigma_{z}(\phi+\psi)-\sigma \varsigma_{z z}\right] y \\
& +\mu_{t}+\mu \phi_{z}+\left(\varsigma-1 / R_{0}\right) \varepsilon-\mu_{z}(\phi+\psi)-\sigma \mu_{z z}, \\
\Phi_{2}= & \tau_{t} x+\psi_{t z} y+\varepsilon_{t}+\psi_{z}\left(\tau x+\psi_{z} y+\varepsilon\right)+\left(\tau+1 / R_{0}\right)\left(\phi_{z} x+\varsigma y+\mu\right) \\
& -(\phi+\psi)\left(\tau_{z} x+\psi_{z z} y+\varepsilon_{z}\right)-\sigma\left(\tau_{z z} x+\psi_{z z z} y+\varepsilon_{z z}\right) \\
= & {\left[\psi_{t z}+\psi_{z}^{2}+\varsigma\left(\tau+1 / R_{0}\right)-(\phi+\psi) \psi_{z z}-\sigma \psi_{z z z}\right] y } \\
& +\left[\tau_{t}+\tau \psi_{z}+\left(\tau+1 / R_{0}\right) \phi_{z}-(\phi+\psi) \tau_{z}-\sigma \tau_{z z}\right] x \\
& +\varepsilon_{t}+\varepsilon \psi_{z}+\left(\tau+1 / R_{0}\right) \mu-(\phi+\psi) \varepsilon_{z}-\sigma \varepsilon_{z z}, \\
& \Phi_{3}=-\phi_{t}-\psi_{t}+(\phi+\psi)\left(\phi_{z}+\psi_{z}\right)-\sigma R(\vartheta+z)+\sigma\left(\phi_{z z}+\psi_{z z}\right) .
\end{aligned}
$$

Thus (8.2.20) is equivalent to the following system of partial differential equations:

$$
\begin{gathered}
\phi_{t z}+\phi_{z}^{2}+\tau\left(\varsigma-1 / R_{0}\right)-\phi_{z z}(\phi+\psi)-\sigma \phi_{z z z}=\alpha_{1}, \\
\varsigma_{t}+\varsigma \phi_{z}+\psi_{z}\left(\varsigma-1 / R_{0}\right)-\varsigma_{z}(\phi+\psi)-\sigma \varsigma_{z z}=\alpha, \\
\mu_{t}+\mu \phi_{z}+\left(\varsigma-1 / R_{0}\right) \varepsilon-\mu_{z}(\phi+\psi)-\sigma \mu_{z z}=\alpha_{2},
\end{gathered}
$$




$$
\begin{gathered}
\psi_{t z}+\psi_{z}^{2}+\varsigma\left(\tau+1 / R_{0}\right)-(\phi+\psi) \psi_{z z}-\sigma \psi_{z z z}=\beta_{1}, \\
\tau_{t}+\tau \psi_{z}+\left(\tau+1 / R_{0}\right) \phi_{z}-(\phi+\psi) \tau_{z}-\sigma \tau_{z z}=\alpha \\
\varepsilon_{t}+\varepsilon \psi_{z}+\left(\tau+1 / R_{0}\right) \mu-(\phi+\psi) \varepsilon_{z}-\sigma \varepsilon_{z z}=\beta_{2}
\end{gathered}
$$

for some $\alpha, \alpha_{1}, \alpha_{2}, \beta_{1}, \beta_{2}$ are functions in $t$.

Let $0 \neq b$ and $c$ be fixed real constants. We define

$$
\begin{aligned}
\zeta_{1}(z)=\frac{e^{b z}-c e^{-b z}}{2}, & \eta_{1}(z)=\frac{e^{b z}+c e^{-b z}}{2}, \\
\zeta_{0}(z)=\sin b z, & \eta_{0}(z)=\cos b z .
\end{aligned}
$$

Then

$$
\eta_{r}^{2}(z)+(-1)^{r} \zeta_{r}^{2}(z)=c^{r}
$$

We assume

$$
\begin{gathered}
\phi=b^{-1} \gamma_{1} \zeta_{r}(z), \quad \psi=b^{-1}\left(\gamma_{2} \zeta_{r}(z)+\gamma_{3} \eta_{r}(z)\right), \\
\varsigma=\gamma_{4}\left(\gamma_{2} \eta_{r}(z)-(-1)^{r} \gamma_{3} \zeta_{r}(z)\right), \quad \tau=\gamma_{5} \gamma_{1} \eta_{r}(z), \quad \gamma_{4} \gamma_{5}=1,
\end{gathered}
$$

where $\gamma_{j}$ are functions in $t$ to be determined. Moreover, (8.3.6) becomes

$$
\left(\gamma_{1}^{\prime}+(-1)^{r} b^{2} \sigma \gamma_{1}-\gamma_{1} \gamma_{5} / R_{0}\right) \eta_{r}(z)+\left(\gamma_{1}+\gamma_{2}\right) \gamma_{1} c^{r}=\alpha_{1}
$$

which is implied by

$$
\begin{gathered}
\alpha_{1}=\left(\gamma_{1}+\gamma_{2}\right) \gamma_{1} c^{r} \\
\gamma_{1}^{\prime}+(-1)^{r} b^{2} \sigma \gamma_{1}-\gamma_{1} \gamma_{5} / R_{0}=0 .
\end{gathered}
$$

On the other hand, (8.3.10) becomes

$$
\left[\left(\gamma_{1} \gamma_{5}\right)^{\prime}+\gamma_{1} / R_{0}+(-1)^{r} b^{2} \sigma \gamma_{1} \gamma_{5}\right] \eta_{r}+\gamma_{1} \gamma_{5}\left(\gamma_{1}+\gamma_{2}\right) c^{r}=\alpha
$$

which gives

$$
\begin{gathered}
\alpha=\gamma_{1} \gamma_{5}\left(\gamma_{1}+\gamma_{2}\right) c^{r} \\
\left(\gamma_{1} \gamma_{5}\right)^{\prime}+(-1)^{r} b^{2} \sigma \gamma_{1} \gamma_{5}+\gamma_{1} / R_{0}=0
\end{gathered}
$$

Solving (8.3.19) and (8.3.22) for $\gamma_{1}$ and $\gamma_{1} \gamma_{5}$, we get

$$
\gamma_{1}=b_{1} e^{-(-1)^{r} b^{2} \sigma t} \sin \frac{t}{R_{0}}, \quad \gamma_{1} \gamma_{5}=b_{1} e^{-(-1)^{r} b^{2} \sigma t} \cos \frac{t}{R_{0}},
$$

where $b_{1}$ is a real constant. In particular, we take

$$
\gamma_{5}=\cot \frac{t}{R_{0}}
$$


Observe that (8.3.7) becomes

$$
\begin{aligned}
& {\left[\left(\gamma_{2} \gamma_{4}\right)^{\prime}+(-1)^{r} b^{2} \sigma \gamma_{2} \gamma_{4}-\gamma_{2} / R_{0}\right] \eta_{r}+\gamma_{4}\left(\gamma_{1} \gamma_{2}+\gamma_{2}^{2}+(-1)^{r} \gamma_{3}^{2}\right) c^{r}} \\
& -(-1)^{r}\left[\left(\gamma_{3} \gamma_{4}\right)^{\prime}+(-1)^{r} b^{2} \sigma \gamma_{2} \gamma_{4}-\gamma_{3} / R_{0}\right] \zeta_{r}=\alpha
\end{aligned}
$$

and (8.3.9) becomes

$$
\begin{aligned}
& {\left[\gamma_{2}^{\prime}+(-1)^{r} b^{2} \sigma \gamma_{2}+\gamma_{2} \gamma_{4} / R_{0}\right] \eta_{r}+\left(\gamma_{1} \gamma_{2}+\gamma_{2}^{2}+(-1)^{r} \gamma_{3}^{2}\right) c^{r}} \\
& -(-1)^{r}\left[\gamma_{3}^{\prime}+(-1)^{r} b^{2} \sigma \gamma_{3}+\gamma_{3} \gamma_{4} / R_{0}\right] \zeta_{r}=\beta_{1},
\end{aligned}
$$

equivalently,

$$
\begin{gathered}
\alpha=\gamma_{4}\left(\gamma_{1} \gamma_{2}+\gamma_{2}^{2}+(-1)^{r} \gamma_{3}^{2}\right) c^{r}, \\
\beta_{1}=\left(\gamma_{1} \gamma_{2}+\gamma_{2}^{2}+(-1)^{r} \gamma_{3}^{2}\right) c^{r}, \\
\left(\gamma_{2} \gamma_{4}\right)^{\prime}+(-1)^{r} b^{2} \sigma \gamma_{2} \gamma_{4}-\gamma_{2} / R_{0}=0, \\
\gamma_{2}^{\prime}+(-1)^{r} b^{2} \sigma \gamma_{2}+\gamma_{2} \gamma_{4} / R_{0}=0, \\
\left(\gamma_{3} \gamma_{4}\right)^{\prime}+(-1)^{r} b^{2} \sigma \gamma_{2} \gamma_{4}-\gamma_{3} / R_{0}=0, \\
\gamma_{3}^{\prime}+(-1)^{r} b^{2} \sigma \gamma_{3}+\gamma_{3} \gamma_{4} / R_{0}=0 .
\end{gathered}
$$

Solving (8.3.29)-(8.3.32) under the assumption $\gamma_{4} \gamma_{5}=1$, we obtain

$$
\begin{array}{ll}
\gamma_{2} \gamma_{4}=b_{2} e^{-(-1)^{r} b^{2} \sigma t} \sin \frac{t}{R_{0}}, & \gamma_{2}=b_{2} e^{-(-1)^{r} b^{2} \sigma t} \cos \frac{t}{R_{0}}, \\
\gamma_{3} \gamma_{4}=b_{3} e^{-(-1)^{r} b^{2} \sigma t} \sin \frac{t}{R_{0}}, & \gamma_{3}=b_{3} e^{-(-1)^{r} b^{2} \sigma t} \cos \frac{t}{R_{0}} .
\end{array}
$$

In particular, we have:

$$
\gamma_{4}=\tan \frac{t}{R_{0}} .
$$

According to (8.3.21) and (8.3.27),

$$
\gamma_{1} \gamma_{5}\left(\gamma_{1}+\gamma_{2}\right) c^{r}=\gamma_{4}\left(\gamma_{1} \gamma_{2}+\gamma_{2}^{2}+(-1)^{r} \gamma_{3}^{2}\right) c^{r} .
$$

Multiplying $\gamma_{4}$ to the above equation and dividing by $c^{r}$, we have

$$
\gamma_{1}\left(\gamma_{1}+\gamma_{2}\right)=\gamma_{1} \gamma_{4}\left(\gamma_{2} \gamma_{4}\right)+\left(\gamma_{2} \gamma_{4}\right)^{2}+(-1)^{r}\left(\gamma_{3} \gamma_{4}\right)^{2} .
$$

By (8.3.23) and (8.3.33)-(8.3.35), the above equation is equivalent to

$$
b_{1}^{2} \sin ^{2} \frac{t}{R_{0}}+\frac{b_{1} b_{2}}{2} \sin \frac{2 t}{R_{0}}=b_{1} b_{2} \tan \frac{t}{R_{0}} \sin ^{2} \frac{t}{R_{0}}+\left(b_{2}^{2}+(-1)^{r} b_{3}^{2}\right) \sin ^{2} \frac{t}{R_{0}},
$$

which can be rewritten as

$$
-b_{1} b_{2} \cos \frac{2 t}{R_{0}} \tan \frac{t}{R_{0}}+\left(b_{2}^{2}-b_{1}^{2}+(-1)^{r} b_{3}^{2}\right) \sin \frac{2 t}{R_{0}}=0 .
$$


Thus

$$
b_{1} b_{2}=0, \quad b_{2}^{2}-b_{1}^{2}+(-1)^{r} b_{3}^{2}=0
$$

So

$$
r=0, \quad b_{2}=0, \quad b_{1}=b_{3}
$$

or

$$
r=1, \quad b_{1}=0, \quad b_{2}=b_{3} .
$$

Assume $r=0$ and $b_{1} \neq 0$. Then

$$
\begin{array}{r}
\phi=b^{-1} b_{1} e^{-b^{2} \sigma t} \sin b z \sin \frac{t}{R_{0}}, \quad \psi=b^{-1} b_{1} e^{-b^{2} \sigma t} \cos b z \cos \frac{t}{R_{0}}, \\
\varsigma=-b_{1} e^{-b^{2} \sigma t} \sin b z \sin \frac{t}{R_{0}}, \quad \tau=b_{1} e^{-b^{2} \sigma t} \cos b z \cos \frac{t}{R_{0}} .
\end{array}
$$

Moreover, we take $\mu=\varepsilon=\vartheta=0$. So (8.2.4), (8.3.8) and (8.3.11) naturally hold. Observe

$$
\Phi_{1}=\gamma_{1}^{2}\left(x+\gamma_{5} y\right)=b_{1}^{2} e^{-2 b^{2} \sigma t}\left(x \sin \frac{t}{R_{0}}+y \cos \frac{t}{R_{0}}\right) \sin \frac{t}{R_{0}}
$$

by (8.3.3), (8.3.6)-(8.3.8), (8.3.18) and (8.3.21). Similarly

$$
\Phi_{2}=b_{1}^{2} e^{-2 b^{2} \sigma t}\left(x \sin \frac{t}{R_{0}}+y \cos \frac{t}{R_{0}}\right) \cos \frac{t}{R_{0}} .
$$

According to (8.3.5),

$$
\Phi_{3}=\left[b^{-1} R_{0}^{-1} b_{1} e^{-b^{2} \sigma t}-b^{-1} b_{1}^{2} e^{-2 b^{2} \sigma t} \cos \left(b z-\frac{t}{R_{0}}\right)\right] \sin \left(b z-\frac{t}{R_{0}}\right)-R \sigma z .
$$

By (8.2.19), we have

$$
\begin{aligned}
p= & \frac{R z^{2}}{2}+\frac{b_{1} e^{-b^{2} \sigma t}}{b^{2} \sigma R_{0}} \cos \left(b z-\frac{t}{R_{0}}\right)-\frac{b_{1}^{2} e^{-2 b^{2} \sigma t}}{2 \sigma b^{2}} \cos ^{2}\left(b z-\frac{t}{R_{0}}\right) \\
& -\frac{b_{1}^{2} e^{-2 b^{2} \sigma t}}{2 \sigma}\left(y^{2} \cos ^{2} \frac{t}{R_{0}}+x^{2} \sin ^{2} \frac{t}{R_{0}}+x y \sin \frac{2 t}{R_{0}}\right) .
\end{aligned}
$$

Suppose $r=1$ and $b_{2} \neq 0$. Then

$$
\phi=\tau=\mu=\varepsilon=\vartheta=0, \quad \psi=b^{-1} b_{2} e^{b z+b^{2} \sigma t} \cos \frac{t}{R_{0}}, \quad \varsigma=b_{2} e^{b z+b^{2} \sigma t} \sin \frac{t}{R_{0}} .
$$

Moreover,

$$
\Phi_{1}=\Phi_{2}=0, \quad \Phi_{3}=b^{-1} b_{2} R_{0}^{-1} e^{b z+b^{2} \sigma t} \sin \frac{t}{R_{0}}+b^{-1} b_{2}^{2} e^{2\left(b z+b^{2} \sigma t\right)} \cos ^{2} \frac{t}{R_{0}}-R \sigma z .
$$

According to (8.2.19),

$$
p=\frac{R z^{2}}{2}-\frac{b_{2} e^{b z+b^{2} \sigma t}}{b^{2} \sigma R_{0}} \sin \frac{t}{R_{0}}-\frac{b_{2}^{2} e^{2\left(b z+b^{2} \sigma t\right)}}{2 b^{2} \sigma} \cos ^{2} \frac{t}{R_{0}} .
$$


by (8.3.1) and (8.3.2), we get:

Theorem 8.3.1. Let $b, b_{1}, b_{2} \in \mathbb{R}$ with $b \neq 0$. We have the following solutions of the three-dimensional stratified rotating Boussinesq equations (8.2.1)-(8.2.5): (1)

$$
\begin{gathered}
u=b_{1} e^{-b^{2} \sigma t}(x \cos b z-y \sin b z) \sin \frac{t}{R_{0}}, \quad v=b_{1} e^{-b^{2} \sigma t}(x \cos b z-y \sin b z) \cos \frac{t}{R_{0}}, \\
w=-b^{-1} b_{1} e^{-b^{2} \sigma t} \cos \left(b z-\frac{t}{R_{0}}\right), \quad T=z
\end{gathered}
$$

and $p$ is given in (8.3.48); (2)

$$
\begin{gathered}
u=b_{2} e^{b z+b^{2} \sigma t} y \sin \frac{t}{R_{0}}, \quad v=b_{2} e^{b z+b^{2} \sigma t} y \cos \frac{t}{R_{0}}, \\
w=-b^{-1} b_{2} e^{b z+b^{2} \sigma t} \cos \frac{t}{R_{0}} \quad T=z
\end{gathered}
$$

and $p$ is given in (8.3.51).

Next we assume $\phi=\varsigma=\psi=\tau=0$. Then

$$
\mu_{t}-\frac{1}{R_{0}} \varepsilon-\sigma \mu_{z z}=\alpha_{2}, \quad \varepsilon_{t}+\frac{1}{R_{0}} \nu-\sigma \varepsilon_{z z}=\beta_{2}, \quad \vartheta_{t}-\vartheta_{z z}=0 .
$$

Solving them, we get:

Theorem 8.3.2. Let $a_{s}, b_{s}, c_{s}, d_{s}, \hat{a}_{r}, \hat{b}_{r}, \hat{c}_{r}, \hat{d}_{r}, \tilde{a}_{j}, \tilde{b}_{j}, \tilde{c}_{j}, \tilde{d}_{j}$ be real numbers. We have the following solutions of the three-dimensional stratified rotating Boussinesq equations (8.2.1)-(8.2.5):

$$
\begin{aligned}
u= & \cos \frac{t}{R_{0}} \sum_{s=1}^{m} d_{s} e^{a_{s}^{2} \sigma t \cos 2 b_{s}+a_{s} z \cos b_{s}} \sin \left(a_{s}^{2} \sigma t \sin 2 b_{s}+a_{s} z \sin b_{s}+c_{s}\right) \\
& +\sin \frac{t}{R_{0}} \sum_{r=1}^{n} \hat{d}_{r} e^{\hat{a}_{r}^{2} \sigma t \cos 2 \hat{b}_{r}+a_{r} z \cos \hat{b}_{r}} \sin \left(\hat{a}_{r}^{2} \sigma t \sin 2 \hat{b}_{r}+\hat{a}_{r} z \sin \hat{b}_{r}+\hat{c}_{r}\right), \\
v= & -\sin \frac{t}{R_{0}} \sum_{s=1}^{m} d_{s} e^{a_{s}^{2} \sigma t \cos 2 b_{s}+a_{s} z \cos b_{s}} \sin \left(a_{s}^{2} \sigma t \sin 2 b_{s}+a_{s} z \sin b_{s}+c_{s}\right) \\
& +\cos \frac{t}{R_{0}} \sum_{r=1}^{n} \hat{d}_{r} e^{\hat{a}_{r}^{2} \sigma t \cos 2 \hat{b}_{r}+a_{r} z \cos \hat{b}_{r}} \sin \left(\hat{a}_{r}^{2} \sigma t \sin 2 \hat{b}_{r}+\hat{a}_{r} z \sin \hat{b}_{r}+\hat{c}_{r}\right), \\
w= & 0, \quad T=z+\sum_{j=1}^{k} \tilde{a}_{j} \tilde{d}_{j} e^{\tilde{a}_{j}^{2} t \cos 2 \tilde{b}_{j}+\tilde{a}_{j} z \cos \tilde{b}_{j}} \sin \left(\tilde{a}_{j}^{2} t \sin 2 \tilde{b}_{j}+\tilde{a}_{j} z \sin \tilde{b}_{j}+\tilde{b}_{j}+\tilde{c}_{j}\right), \\
& p=\frac{R z^{2}}{2}+R \sum_{j=1}^{k} \tilde{d}_{j} e^{\tilde{a}_{j}^{2} t \cos 2 \tilde{b}_{j}+\tilde{a}_{j} z \cos \tilde{b}_{j}} \sin \left(\tilde{a}_{j}^{2} t \sin 2 \tilde{b}_{j}+\tilde{a}_{j} z \sin \tilde{b}_{j}+\tilde{c}_{j}\right) .
\end{aligned}
$$

Remark 8.3.3. By Fourier expansion, we can use the above solution to obtain the one depending on three arbitrary piecewise continuous functions of $z$. 


\subsection{Asymmetric Approach II}

In this section, we solve the stratified rotating Boussinesq equations (8.2.1)-(8.2.5) under the assumption

$$
u_{z}=v_{z}=w_{z z}=T_{z z}=0
$$

Let $\gamma$ be a function in $t$ and we use the moving frame in (8.1.107). Assume

$$
\begin{aligned}
u=f(t, \mathcal{X}) \sin \gamma-\gamma^{\prime} y, & v=-f(t, \mathcal{X}) \cos \gamma+\gamma^{\prime} x, \\
w=\phi(t, \mathcal{X}), & T=\psi(t, \mathcal{X})+z,
\end{aligned}
$$

for some functions $f, \phi$ and $\psi$ in $t$ and $\mathcal{X}$.

Using (8.1.108)-(8.1.112) and (8.2.16)-(8.2.18), we get

$$
u \partial_{x}+v \partial_{y}=-f \partial_{\mathcal{Y}}+\gamma^{\prime}\left(x \partial_{y}-y \partial_{x}\right)
$$

and

$$
\begin{gathered}
\Phi_{1}=-\left(\gamma^{\prime 2}+\gamma^{\prime} / R_{0}\right) x-\gamma^{\prime \prime} y+f_{t} \sin \gamma+\left(2 \gamma^{\prime}+1 / R_{0}\right) f \cos \gamma-\sigma f_{\mathcal{X} \mathcal{X}} \sin \gamma \\
\Phi_{2}=-\left(\gamma^{\prime 2}+\gamma^{\prime} / R_{0}\right) y+\gamma^{\prime \prime} x-f_{t} \cos \gamma+\left(2 \gamma^{\prime}+1 / R_{0}\right) f \sin \gamma+\sigma f_{\mathcal{X} \mathcal{X}} \cos \gamma \\
\Phi_{3}=\phi_{t}-\sigma \phi_{\mathcal{X} \mathcal{X}}-\sigma R(\psi+z) .
\end{gathered}
$$

By (8.2.20), we have

$$
\begin{gathered}
-2 \gamma^{\prime \prime}+f_{\mathcal{X} t}-\sigma f_{\mathcal{X} \mathcal{X} \mathcal{X}}=0, \\
\phi_{t}-\sigma \phi_{\mathcal{X X}}-\sigma R \psi=0
\end{gathered}
$$

Moreover, (8.2.4) becomes

$$
\psi_{t}-\psi_{\mathcal{X X}}=0
$$

Solving (8.4.8), we have:

$$
f=2 \gamma^{\prime} \mathcal{X}+\sum_{j=1}^{m} a_{j} d_{j} e^{a_{j}^{2} \kappa t \cos 2 b_{j}+a_{j} \mathcal{X} \cos b_{j}} \sin \left(a_{j}^{2} \kappa t \sin 2 b_{j}+a_{j} \mathcal{X} \sin b_{j}+b_{j}+c_{j}\right)
$$

where $a_{j}, b_{j}, c_{j}, d_{j}$ are arbitrary real numbers. Moreover, (8.4.9) and (8.4.10) yield

$$
\begin{gathered}
\phi=\sum_{r=1}^{n} \hat{d}_{r} e^{\hat{a}_{r}^{2} t \cos 2 \hat{b}_{r}+\hat{a}_{r} \mathcal{X} \cos \hat{b}_{r}} \sin \left(\hat{a}_{r}^{2} t \sin 2 \hat{b}_{r}+\hat{a}_{r} \mathcal{X} \sin \hat{b}_{r}+\hat{c}_{r}\right)+\sigma R t \psi \\
\psi=\sum_{s=1}^{k} \tilde{d}_{s} e^{\tilde{a}_{s}^{2} t \cos 2 \tilde{b}_{s}+\tilde{a}_{s} \mathcal{X} \cos \tilde{b}_{s}} \sin \left(\tilde{a}_{s}^{2} t \sin 2 \tilde{b}_{s}+\tilde{a}_{s} \mathcal{X} \sin \tilde{b}_{s}+\tilde{c}_{s}\right)
\end{gathered}
$$


if $\sigma=1$, and

$$
\begin{aligned}
\phi= & \sum_{r=1}^{n} \hat{d}_{r} e^{\hat{a}_{r}^{2} \sigma t \cos 2 \hat{b}_{r}+\hat{a}_{r} \mathcal{X} \cos \hat{b}_{r}} \sin \left(\hat{a}_{r}^{2} \sigma t \sin 2 \hat{b}_{r}+\hat{a}_{r} \mathcal{X} \sin \hat{b}_{r}+\hat{c}_{r}\right) \\
& +\frac{\sigma R}{1-\sigma} \sum_{s=1}^{k} \tilde{d}_{s} e^{2} \tilde{a}_{s}^{2 t \cos 2 \tilde{b}_{s}+\tilde{a}_{s} \mathcal{X} \cos \tilde{b}_{s}} \sin \left(\tilde{a}_{s}^{2} t \sin 2 \tilde{b}_{s}+\tilde{a}_{s} \mathcal{X} \sin \tilde{b}_{s}+\tilde{c}_{s}\right) \\
& \psi \sum_{s=1}^{k} \tilde{a}_{s}^{2} \tilde{d}_{s} e^{\tilde{a}_{s}^{2} t \cos 2 \tilde{b}_{s}+\tilde{a}_{s} \mathcal{X} \cos \tilde{b}_{s}} \sin \left(\tilde{a}_{s}^{2} t \sin 2 \tilde{b}_{s}+\tilde{a}_{s} \mathcal{X} \sin \tilde{b}_{s}+2 \tilde{b}_{s}+\tilde{c}_{s}\right)
\end{aligned}
$$

when $\sigma \neq 1$, where $\hat{a}_{r}, \hat{b}_{r}, \hat{c}_{r}, \hat{d}_{r}, \tilde{a}_{s}, \tilde{b}_{s}, \tilde{c}_{s}, \tilde{d}_{s}$ are arbitrary real numbers.

Now

$$
\begin{gathered}
\Phi_{1}=\left(\gamma^{\prime \prime} \sin 2 \gamma-\gamma^{\prime 2}-\gamma^{\prime} / R_{0}\right) x-\gamma^{\prime \prime} y \cos 2 \gamma+\left(2 \gamma^{\prime}+1 / R_{0}\right) f \cos \gamma, \\
\Phi_{2}=-\left(\gamma^{\prime \prime} \sin 2 \gamma+\gamma^{\prime 2}+\gamma^{\prime} / R_{0}\right) y-\gamma^{\prime \prime} x \cos 2 \gamma+\left(2 \gamma^{\prime}+1 / R_{0}\right) f \sin \gamma
\end{gathered}
$$

and $\Phi_{3}=-\sigma R z$. Thanks to (8.2.19), we have

$$
\begin{aligned}
p= & -\frac{2 \gamma^{\prime}+1 / R_{0}}{\sigma}\left[\gamma^{\prime} \mathcal{X}^{2}+\sum_{j=1}^{m} d_{j} e^{a_{j}^{2} \kappa t \cos 2 b_{j}+a_{j} \mathcal{X} \cos b_{j}} \sin \left(a_{j}^{2} \kappa t \sin 2 b_{j}+a_{j} \mathcal{X} \sin b_{j}+c_{j}\right)\right] \\
& +\frac{R}{2} z^{2}+\frac{\left(\gamma^{\prime 2}+\gamma^{\prime} / R_{0}\right)\left(x^{2}+y^{2}\right)+\gamma^{\prime \prime}\left(y^{2}-x^{2}\right) \sin 2 \gamma}{2 \sigma}+\frac{\gamma^{\prime \prime}}{\sigma} x y \cos 2 \gamma
\end{aligned}
$$

Theorem 8.4.1. Let $a_{j}, b_{j}, c_{j}, d_{j}, \hat{a}_{r}, \hat{b}_{r}, \hat{c}_{r}, \hat{d}_{r}, \tilde{a}_{s}, \tilde{b}_{s}, \tilde{c}_{s}, \tilde{d}_{s}$ be real numbers and let $\gamma$ be any function in $t$. Denote $\mathcal{X}=x \cos \gamma+y \sin \gamma$. We have the following solutions of the three-dimensional stratified rotating Boussinesq equations (8.2.1)-(8.2.5):

$$
\begin{aligned}
u= & {\left[\sum_{j=1}^{m} a_{j} d_{j} e^{a_{j}^{2} \kappa t \cos 2 b_{j}+a_{j} \mathcal{X} \cos b_{j}} \sin \left(a_{j}^{2} \kappa t \sin 2 b_{j}+a_{j} \mathcal{X} \sin b_{j}+b_{j}+c_{j}\right)\right.} \\
& \left.+2 \gamma^{\prime} \mathcal{X}\right] \sin \gamma-\gamma^{\prime} y, \\
v= & {\left[-\sum_{j=1}^{m} a_{j} d_{j} e^{a_{j}^{2} \kappa t \cos 2 b_{j}+a_{j} \mathcal{X} \cos b_{j}} \sin \left(a_{j}^{2} \kappa t \sin 2 b_{j}+a_{j} \mathcal{X} \sin b_{j}+b_{j}+c_{j}\right)\right.} \\
& \left.+2 \gamma^{\prime} \mathcal{X}\right] \cos \gamma+\gamma^{\prime} x,
\end{aligned}
$$

$p$ is given in (8.4.18);

$$
\begin{aligned}
w= & \sum_{r=1}^{n} \hat{d}_{r} e^{\hat{a}_{r}^{2} t \cos 2 \hat{b}_{r}+\hat{a}_{r} \mathcal{X} \cos \hat{b}_{r}} \sin \left(\hat{a}_{r}^{2} t \sin 2 \hat{b}_{r}+\hat{a}_{r} \mathcal{X} \sin \hat{b}_{r}+\hat{c}_{r}\right) \\
& +\sigma R t \sum_{s=1}^{k} \tilde{d}_{s} e^{\tilde{a}_{s}^{2} t \cos 2 \tilde{b}_{s}+\tilde{a}_{s} \mathcal{X} \cos \tilde{b}_{s}} \sin \left(\tilde{a}_{s}^{2} t \sin 2 \tilde{b}_{s}+\tilde{a}_{s} \mathcal{X} \sin \tilde{b}_{s}+\tilde{c}_{s}\right),
\end{aligned}
$$




$$
T=z+\sum_{s=1}^{k} \tilde{d}_{s} e^{\tilde{a}_{s}^{2} t \cos 2 \tilde{b}_{s}+\tilde{a}_{s} \mathcal{X} \cos \tilde{b}_{s}} \sin \left(\tilde{a}_{s}^{2} t \sin 2 \tilde{b}_{s}+\tilde{a}_{s} \mathcal{X} \sin \tilde{b}_{s}+\tilde{c}_{s}\right)
$$

if $\sigma=1$, and

$$
\begin{aligned}
w= & \sum_{r=1}^{n} \hat{d}_{r} e^{\hat{a}_{r}^{2} \sigma t \cos 2 \hat{b}_{r}+\hat{a}_{r} \mathcal{X} \cos \hat{b}_{r}} \sin \left(\hat{a}_{r}^{2} \sigma t \sin 2 \hat{b}_{r}+\hat{a}_{r} \mathcal{X} \sin \hat{b}_{r}+\hat{c}_{r}\right) \\
& +\frac{\sigma R}{1-\sigma} \sum_{s=1}^{k} \tilde{d}_{s} e^{\tilde{a}_{s}^{2} t \cos 2 \tilde{b}_{s}+\tilde{a}_{s} \mathcal{X} \cos \tilde{b}_{s}} \sin \left(\tilde{a}_{s}^{2} t \sin 2 \tilde{b}_{s}+\tilde{a}_{s} \mathcal{X} \sin \tilde{b}_{s}+\tilde{c}_{s}\right) \\
T & =z+\sum_{s=1}^{k} \tilde{a}_{s}^{2} \tilde{d}_{s} e^{\tilde{a}_{s}^{2} t \cos 2 \tilde{b}_{s}+\tilde{a}_{s} \mathcal{X} \cos \tilde{b}_{s}} \sin \left(\tilde{a}_{s}^{2} t \sin 2 \tilde{b}_{s}+\tilde{a}_{s} \mathcal{X} \sin \tilde{b}_{s}+2 \tilde{b}_{s}+\tilde{c}_{s}\right)
\end{aligned}
$$

when $\sigma \neq 1$.

Remark 8.4.2. By Fourier expansion, we can use the above solution to obtain the one depending on three arbitrary piecewise continuous functions of $\mathcal{X}$.

Next we set

$$
\varpi=x^{2}+y^{2}
$$

We assume

$$
\begin{array}{ll}
u=y \phi(t, \varpi), & v=-x \phi(t, \varpi), \\
w=\psi(t, \varpi), & T=\vartheta(t, \varpi)+z
\end{array}
$$

where $\phi, \psi$ and $\vartheta$ are functions in $t, \varpi$. Note that (8.2.16)-(8.2.18) give

$$
\begin{gathered}
\Phi_{1}=y \phi_{t}+\frac{x}{R_{0}} \phi-x \phi^{2}-4 \sigma y(\varpi \phi)_{\varpi \varpi}, \\
\Phi_{2}=-x \phi_{t}+\frac{y}{R_{0}} \phi-y \phi^{2}+4 \sigma x(\varpi \phi)_{\varpi \varpi}, \\
\Phi_{3}=\psi_{t}-\sigma R(\vartheta+z)-4 \sigma\left(\varpi \psi_{\varpi}\right)_{\varpi} .
\end{gathered}
$$

According to (8.2.20),

$$
\begin{gathered}
{\left[\varpi\left(\phi_{t}-4 \sigma(\varpi \phi)_{\varpi \varpi}\right)\right]_{\varpi}=0,} \\
\partial_{x}\left[\psi_{t}-\sigma R \vartheta-4 \sigma\left(\varpi \psi_{\varpi}\right)_{\varpi}\right]=\partial_{y}\left[\psi_{t}-\sigma R \vartheta-4 \sigma\left(\varpi \psi_{\varpi}\right)_{\varpi}\right]=0, \\
\phi_{t}-4 \sigma(\varpi \phi)_{\varpi \varpi}=\frac{\alpha^{\prime}}{\varpi}, \\
\psi_{t}-\sigma R \vartheta-4 \sigma\left(\varpi \psi_{\varpi}\right)_{\varpi}=\beta^{\prime}
\end{gathered}
$$

for some functions $\alpha$ and $\beta$ in $t$.

Write

$$
\phi=\sum_{j=-1}^{\infty} \alpha_{j} \varpi^{j}
$$


where $\alpha_{j}$ are functions in $t$ to be determined. Then (8.4.33) becomes

$$
\sum_{j=-1}^{\infty}\left(\alpha_{j}^{\prime}-4 \sigma(j+2)(j+1) \alpha_{j+1}\right) \varpi^{j}=\frac{\alpha^{\prime}}{\varpi}
$$

equivalently,

$$
\alpha_{-1}^{\prime}=\alpha^{\prime}, 4 \sigma(j+2)(j+1) \alpha_{j+1}=\alpha_{j}^{\prime} \text { for } j \geq 0 .
$$

We take $\alpha_{-1}=\alpha$ and redenote $\alpha_{0}=\gamma$. The above second equation implies

$$
\alpha_{j}=\frac{\gamma^{(j)}}{j !(j+1) !(4 \sigma)^{j}} \quad \text { for } j \geq 0 .
$$

So

$$
\phi=\frac{\alpha}{\varpi}+\sum_{j=0}^{\infty} \frac{\gamma^{(j)} \varpi^{j}}{j !(j+1) !(4 \sigma)^{j}} .
$$

Observe that (8.2.4) becomes

$$
\vartheta_{t}-4\left(\varpi \vartheta_{\varpi}\right)_{\varpi}=0
$$

by (8.4.25)-(8.4.27). The arguments in the above show

$$
\vartheta=\sum_{r=0}^{\infty} \frac{\gamma_{1}^{(r)} \varpi^{r}}{r !(r+1) !(4 \sigma)^{r}},
$$

where $\gamma_{1}$ is an arbitrary function in $t$. Substituting (8.4.41) into (8.4.34), we get

$$
\psi_{t}-4 \sigma\left(\varpi \psi_{\varpi}\right)_{\varpi}=\beta^{\prime}+4 \sigma R \sum_{r=0}^{\infty} \frac{\gamma_{1}^{(r)} \varpi^{r}}{r !(r+1) !(4 \sigma)^{r}} .
$$

Write

$$
\psi=\sum_{r=1}^{\infty} \beta_{r} \varpi^{r},
$$

where $\beta_{r}$ are functions in $t$ to be determined. Then (8.4.42) becomes

$$
\sum_{r=0}^{\infty}\left(\beta_{r}^{\prime}-4 \sigma(r+2)(r+1) \beta_{r+1}\right) \varpi^{r}=\beta^{\prime}+4 \sigma R \sum_{r=0}^{\infty} \frac{\gamma_{1}^{(r)} \varpi^{r}}{r !(r+1) !(4 \sigma)^{r}},
$$

equivalently,

$$
\begin{gathered}
8 \sigma \beta_{1}=\beta_{0}^{\prime}-\beta^{\prime}-4 \sigma R \gamma, \\
\beta_{r+1}=\frac{\beta_{r}^{\prime}}{4 \sigma(r+2)(r+1)}-\frac{R \gamma_{1}^{(r)}}{(r+2) !(r+1) !(4 \sigma)^{r}} \quad \text { for } r \geq 1 .
\end{gathered}
$$

Thus

$$
\beta_{r}=\frac{\beta_{0}^{(r)}-\beta^{(r)}}{r !(r+1) !(4 \sigma)^{r}}-\frac{R \gamma_{1}^{(r-1)}}{(r+1) !(r-1) !(4 \sigma)^{r-1}} \quad \text { for } r \geq 1 .
$$


So

$$
\psi=\beta_{0}+\sum_{r=1}^{\infty} \frac{\left(\beta_{0}^{(r)}-\beta^{(r)}-4 \sigma R \gamma_{1}^{(r-1)}\right) \varpi^{r}}{r !(r+1) !(4 \sigma)^{r}}
$$

Now (8.4.28), (8.4.29) and (8.4.33) give

$$
\begin{gathered}
\Phi_{1}=\frac{\alpha^{\prime} y}{\varpi}+\frac{x}{R_{0}} \phi-x \phi^{2}, \\
\Phi_{2}=-\frac{\alpha^{\prime} x}{\varpi}+\frac{y}{R_{0}} \phi-y \phi^{2} .
\end{gathered}
$$

Moreover,

$$
\Phi_{3}=\beta^{\prime}-\sigma R z
$$

by (8.4.30) and (8.4.34). Thanks to (8.2.19), we have

$$
\begin{aligned}
p= & \frac{R z^{2}}{2}+\frac{\alpha^{\prime}}{\sigma} \arctan \frac{y}{x}-\frac{\beta^{\prime}}{\sigma} z-\frac{\alpha \ln \left(x^{2}+y^{2}\right)}{2 \sigma R_{0}}-\frac{1}{\sigma R_{0}} \sum_{j=0}^{\infty} \frac{\gamma^{(j)}\left(x^{2}+y^{2}\right)^{j+1}}{[(j+1) !]^{2}(4 \sigma)^{j}} \\
& -\frac{\alpha^{2}}{2 \sigma\left(x^{2}+y^{2}\right)}-\frac{\alpha \gamma \ln \left(x^{2}+y^{2}\right)}{\sigma}+\frac{\alpha}{\sigma} \sum_{j=1}^{\infty} \frac{\gamma^{(j)}\left(x^{2}+y^{2}\right)^{j}}{j j !(j+1) !(4 \sigma)^{j}} \\
& +\frac{1}{2 \sigma} \sum_{j_{1}, j_{2}=0}^{\infty} \frac{\gamma^{\left(j_{1}\right)} \gamma^{\left(j_{2}\right)}\left(x^{2}+y^{2}\right)^{j_{1}+j_{2}+1}}{\left(j_{1}+j_{2}+1\right) j_{1} ! j_{2} !\left(j_{1}+1\right) !\left(j_{2}+1\right) !(4 \sigma)^{j_{1}+j_{2}}} .
\end{aligned}
$$

By (8.4.25)-(8.4.27), (8.4.39), (8.4.41), and (8.4.48), we have:

Theorem 8.4.3 Let $\alpha, \beta, \beta_{0}, \gamma, \gamma_{1}$ be any functions in $t$. We have the following solutions of the three-dimensional stratified rotating Boussinesq equations (8.2.1)-(8.2.5):

$$
\begin{gathered}
u=\frac{\alpha y}{x^{2}+y^{2}}+y \sum_{j=0}^{\infty} \frac{\gamma^{(j)}\left(x^{2}+y^{2}\right)^{j}}{j !(j+1) !(4 \sigma)^{j}} \\
v=-\frac{\alpha x}{x^{2}+y^{2}}-x \sum_{j=0}^{\infty} \frac{\gamma^{(j)}\left(x^{2}+y^{2}\right)^{j}}{j !(j+1) !(4 \sigma)^{j}} \\
w=\beta_{0}+\sum_{r=1}^{\infty} \frac{\left(\beta_{0}^{(r)}-\beta^{(r)}-4 \sigma R \gamma_{1}^{(r-1)}\right)\left(x^{2}+y^{2}\right)^{r}}{r !(r+1) !(4 \sigma)^{r}} \\
T=z+\sum_{r=0}^{\infty} \frac{\gamma_{1}^{(r)}\left(x^{2}+y^{2}\right)^{r}}{r !(r+1) !(4 \sigma)^{r}}
\end{gathered}
$$

and $p$ is given in (8.4.52). 


\subsection{Asymmetric Approach III}

In this section, we solve (8.2.1)-(8.2.5) under the assumption $v_{x}=w_{x}=T_{x}=0$.

Let $c$ be a real constant. Set

$$
\varpi=y \cos c+z \sin c .
$$

Suppose

$$
\begin{gathered}
u=f(t, \varpi), \quad v=\phi(t, \varpi) \sin c, \\
w=-\phi(t, \varpi) \cos c, \quad T=\psi(t, \varpi)+z,
\end{gathered}
$$

where $f, \phi$ and $\psi$ are functions in $t$ and $\varpi$. According to (8.2.16)-(8.2.18),

$$
\begin{gathered}
\Phi_{1}=f_{t}-\sigma f_{\varpi \varpi}-\frac{\sin c}{R_{0}} \phi, \\
\Phi_{2}=\left(\phi_{t}-\sigma \phi_{\varpi \varpi}\right) \sin c+\frac{1}{R_{0}} f, \\
\Phi_{3}=\left(\sigma \phi_{\varpi \varpi}-\phi_{t}\right) \cos c-\sigma R(\psi+z) .
\end{gathered}
$$

By (8.2.20),

$$
\begin{gathered}
f_{\varpi t}-\sigma f_{\varpi \varpi \varpi}-\frac{\sin c}{R_{0}} \phi_{\varpi}=0, \\
\left(\phi_{t}-\sigma \phi_{\varpi \varpi}\right)_{\varpi}+\frac{\sin c}{R_{0}} f_{\varpi}+\sigma R \psi_{\varpi} \cos c=0 .
\end{gathered}
$$

For simplicity, we take

$$
\begin{gathered}
f_{t}-\sigma f_{\varpi \varpi}-\frac{\sin c}{R_{0}} \phi=0, \\
\phi_{t}-\sigma \phi_{\varpi \varpi}+\frac{\sin c}{R_{0}} f+\sigma R \psi \cos c=0 .
\end{gathered}
$$

Denote

$$
\left(\begin{array}{l}
\hat{f} \\
\hat{\phi}
\end{array}\right)=\left(\begin{array}{cc}
\cos \frac{t \sin c}{R_{0}} & -\sin \frac{t \sin c}{R_{0}} \\
\sin \frac{t \sin c}{R_{0}} & \cos \frac{t \sin c}{R_{0}}
\end{array}\right)\left(\begin{array}{l}
f \\
\phi
\end{array}\right) .
$$

Then (8.5.9) and (8.5.10) become

$$
\begin{aligned}
& \hat{f}_{t}-\sigma \hat{f}_{\varpi \varpi}-\sigma R \psi \cos c \sin \frac{t \sin c}{R_{0}}=0, \\
& \hat{\phi}_{t}-\sigma \hat{\phi}_{\varpi \varpi}+\sigma R \psi \cos c \cos \frac{t \sin c}{R_{0}}=0 .
\end{aligned}
$$

On the other hand, (8.2.4) becomes

$$
\psi_{t}-\psi_{\varpi \varpi}=0 .
$$

Assume $\sigma=1$. We have the following solution:

$$
\psi=\sum_{j=1}^{m} a_{j} d_{j} e^{a_{j}^{2} t \cos 2 b_{j}+a_{j} \varpi \cos b_{j}} \sin \left(a_{j}^{2} t \sin 2 b_{j}+a_{j} \varpi \sin b_{j}+b_{j}+c_{j}\right),
$$




$$
\begin{aligned}
\hat{f}=- & R R_{0} \cot c \cos \frac{t \sin c}{R_{0}} \sum_{j=1}^{m} a_{j} d_{j} e^{a_{j}^{2} t \cos 2 b_{j}+a_{j} \varpi \cos b_{j}} \sin \left(a_{j}^{2} t \sin 2 b_{j}+a_{j} \varpi \sin b_{j}+b_{j}+c_{j}\right) \\
& +\sum_{r=1}^{n} \hat{a}_{r} \hat{d}_{r} e^{\hat{a}_{r}^{2} t \cos 2 \hat{b}_{r}+\hat{a}_{r} \varpi \cos \hat{b}_{r}} \sin \left(\hat{a}_{r}^{2} t \sin 2 \hat{b}_{r}+\hat{a}_{r} \varpi \sin \hat{b}_{r}+\hat{b}_{r}+\hat{c}_{r}\right), \\
\hat{\phi}=- & R R_{0} \cot c \sin \frac{t \sin c}{R_{0}} \sum_{j=1}^{m} a_{j} d_{j} e^{a_{j}^{2} t \cos 2 b_{j}+a_{j} \varpi \cos b_{j}} \sin \left(a_{j}^{2} t \sin 2 b_{j}+a_{j} \varpi \sin b_{j}+b_{j}+c_{j}\right) \\
& +\sum_{s=1}^{k} \tilde{a}_{s} \tilde{d}_{s} e^{\tilde{a}_{s}^{2} t \cos 2 \tilde{b}_{s}+\tilde{a}_{s} \varpi \cos \tilde{b}_{s}} \sin \left(\tilde{a}_{s}^{2} t \sin 2 \tilde{b}_{s}+\tilde{a}_{s} \varpi \sin \tilde{b}_{s}+\tilde{b}_{s}+\tilde{c}_{s}\right),
\end{aligned}
$$

where $a_{j}, b_{j}, c_{j}, \hat{a}_{r}, \hat{b}_{r}, \hat{c}_{r}, \hat{d}_{r}, \tilde{a}_{s}, \tilde{b}_{s}, \tilde{c}_{s}, \tilde{d}_{s}$ are arbitrary real numbers. According to (8.5.11),

$$
\begin{aligned}
f= & -R R_{0} \cot c \cos \frac{2 t \sin c}{R_{0}} \sum_{j=1}^{m} a_{j} d_{j} e^{a_{j}^{2} t \cos 2 b_{j}+a_{j} \varpi \cos b_{j}} \sin \left(a_{j}^{2} t \sin 2 b_{j}+a_{j} \varpi \sin b_{j}+b_{j}+c_{j}\right) \\
& +\cos \frac{t \sin c}{R_{0}} \sum_{r=1}^{n} \hat{a}_{r} \hat{d}_{r} e^{\hat{a}_{r}^{2} t \cos 2 \hat{b}_{r}+\hat{a}_{r} \varpi \cos \hat{b}_{r}} \sin \left(\hat{a}_{r}^{2} t \sin 2 \hat{b}_{r}+\hat{a}_{r} \varpi \sin \hat{b}_{r}+\hat{b}_{r}+\hat{c}_{r}\right) \\
& +\sin \frac{t \sin c}{R_{0}} \sum_{s=1}^{k} \tilde{a}_{s} \tilde{d}_{s} e^{\tilde{a}_{s}^{2} t \cos 2 \tilde{b}_{s}+\tilde{a}_{s} \varpi \cos \tilde{b}_{s}} \sin \left(\tilde{a}_{s}^{2} t \sin 2 \tilde{b}_{s}+\tilde{a}_{s} \varpi \sin \tilde{b}_{s}+\tilde{b}_{s}+\tilde{c}_{s}\right), \\
& \phi=-\sin \frac{t \sin c}{R_{0}} \sum_{r=1}^{n} \hat{a}_{r} \hat{d}_{r} e^{\hat{a}_{r}^{2} t \cos 2 \hat{b}_{r}+\hat{a}_{r} \varpi \cos \hat{b}_{r}} \sin \left(\hat{a}_{r}^{2} t \sin 2 \hat{b}_{r}+\hat{a}_{r} \varpi \sin \hat{b}_{r}+\hat{b}_{r}+\hat{c}_{r}\right) \\
& +\cos \frac{t \sin c}{R_{0}} \sum_{s=1}^{k} \tilde{a}_{s} \tilde{d}_{s} e^{\tilde{a}_{s}^{2} t \cos 2 \tilde{b}_{s}+\tilde{a}_{s} \varpi \cos \tilde{b}_{s}} \sin \left(\tilde{a}_{s}^{2} t \sin 2 \tilde{b}_{s}+\tilde{a}_{s} \varpi \sin \tilde{b}_{s}+\tilde{b}_{s}+\tilde{c}_{s}\right) .
\end{aligned}
$$

Suppose $\sigma \neq 1$. We take the following solution of (8.5.14):

$$
\psi=\sum_{j=1}^{m} a_{j} d_{j} e^{a_{j}^{2} t+a_{j} \varpi},
$$

where $a_{j}, d_{j}$ are real constants. Substituting

$$
\hat{f}=\alpha_{j} e^{a_{j}^{2} t+a_{j} \varpi}, \quad \hat{\phi}=\beta_{j} e^{a_{j}^{2} t+a_{j} \varpi}, \quad \psi=a_{j} d_{j} e^{a_{j}^{2} t+a_{j} \varpi}
$$

into (8.5.12) and (8.5.13), we get

$$
\begin{aligned}
& \alpha_{j}^{\prime}+a_{j}^{2}(1-\sigma) \alpha_{j}-\sigma R a_{j} d_{j} \cos c \sin \frac{t \sin c}{R_{0}}=0 \\
& \beta_{j}^{\prime}+a_{j}^{2}(1-\sigma) \beta_{j}+\sigma R a_{j} d_{j} \cos c \cos \frac{t \sin c}{R_{0}}=0 .
\end{aligned}
$$


We have the solutions

$$
\begin{gathered}
\alpha_{j}=\sigma R a_{j} d_{j} \cos c \frac{a_{j}^{2}(1-\sigma) \sin \frac{t \sin c}{R_{0}}-R_{0}^{-1} \sin c \cos \frac{t \sin c}{R_{0}}}{a_{j}^{4}(1-\sigma)^{2}+R_{0}^{-2} \sin ^{2} c}, \\
\beta_{j}=-\sigma R a_{j} d_{j} \cos c \frac{a_{j}^{2}(1-\sigma) \cos \frac{t \sin c}{R_{0}}+R_{0}^{-1} \sin c \sin \frac{t \sin c}{R_{0}}}{a_{j}^{4}(1-\sigma)^{2}+R_{0}^{-2} \sin ^{2} c} .
\end{gathered}
$$

Thus we have the following solutions of (8.5.12) and (8.5.13):

$$
\begin{aligned}
\hat{f}= & \sigma R \sum_{j=1}^{m} a_{j} d_{j} e^{a_{j}^{2} t+a_{j} \varpi} \frac{\cos c\left[a_{j}^{2}(1-\sigma) \sin \frac{t \sin c}{R_{0}}-R_{0}^{-1} \sin c \cos \frac{t \sin c}{R_{0}}\right]}{a_{j}^{4}(1-\sigma)^{2}+R_{0}^{-2} \sin ^{2} c} \\
& +\sum_{r=1}^{n} \hat{a}_{r} \hat{d}_{r} e^{\hat{a}_{r}^{2} \sigma t \cos 2 \hat{b}_{r}+\hat{a}_{r} \varpi \cos \hat{b}_{r}} \sin \left(\hat{a}_{r}^{2} \sigma t \sin 2 \hat{b}_{r}+\hat{a}_{r} \varpi \sin \hat{b}_{r}+\hat{b}_{r}+\hat{c}_{r}\right), \\
\hat{\phi}= & \sigma R \sum_{j=1}^{m} a_{j} d_{j} e^{a_{j}^{2} t+a_{j} \varpi} \frac{\cos c\left[a_{j}^{2}(\sigma-1) \cos \frac{t \sin c}{R_{0}}-R_{0}^{-1} \sin c \sin \frac{t \sin c}{R_{0}}\right]}{a_{j}^{4}(1-\sigma)^{2}+R_{0}^{-2} \sin ^{2} c} \\
& +\sum_{s=1}^{k} \tilde{a}_{s} \tilde{d}_{s} e^{\tilde{e}_{s}^{2} \sigma t \cos 2 \tilde{b}_{s}+\tilde{a}_{s} \varpi \cos \tilde{b}_{s}} \sin \left(\tilde{a}_{s}^{2} \sigma t \sin 2 \tilde{b}_{s}+\tilde{a}_{s} \varpi \sin \tilde{b}_{s}+\tilde{b}_{s}+\tilde{c}_{s}\right),
\end{aligned}
$$

where $\hat{a}_{r}, \hat{b}_{r}, \hat{c}_{r}, \hat{d}_{r}, \tilde{a}_{s}, \tilde{b}_{s}, \tilde{c}_{s}, \tilde{d}_{s}$ are arbitrary real numbers.

According to (8.5.11),

$$
\begin{aligned}
f= & \cos \frac{t \sin c}{R_{0}} \sum_{r=1}^{n} \hat{a}_{r} \hat{d}_{r} e^{\hat{a}_{r}^{2} \sigma t \cos 2 \hat{b}_{r}+\hat{a}_{r} \varpi \cos \hat{b}_{r}} \sin \left(\hat{a}_{r}^{2} \sigma t \sin 2 \hat{b}_{r}+\hat{a}_{r} \varpi \sin \hat{b}_{r}+\hat{b}_{r}+\hat{c}_{r}\right) \\
& +\sin \frac{t \sin c}{R_{0}} \sum_{s=1}^{k} \tilde{a}_{s} \tilde{d}_{s} e^{\tilde{a}_{s}^{2} \sigma t \cos 2 \tilde{b}_{s}+\tilde{a}_{s} \varpi \cos \tilde{s}_{s}} \sin \left(\tilde{a}_{s}^{2} \sigma t \sin 2 \tilde{b}_{s}+\tilde{a}_{s} \varpi \sin \tilde{b}_{s}+\tilde{b}_{s}+\tilde{c}_{s}\right) \\
& -\sigma R \sum_{j=1}^{m} \frac{a_{j} d_{j} e^{a_{j}^{2} t+a_{j} \varpi} \sin 2 c}{2 R_{0}\left(a_{j}^{4}(1-\sigma)^{2}+R_{0}^{-2} \sin ^{2} c\right)} \\
\phi= & -\sin \frac{t \sin c}{R_{0}} \sum_{r=1}^{n} \hat{a}_{r} \hat{d}_{r} e^{\hat{a}_{r}^{2} \sigma t \cos 2 \hat{b}_{r}+\hat{a}_{r} \varpi \cos \hat{b}_{r}} \sin \left(\hat{a}_{r}^{2} \sigma t \sin 2 \hat{b}_{r}+\hat{a}_{r} \varpi \sin \hat{b}_{r}+\hat{b}_{r}+\hat{c}_{r}\right) \\
& +\cos \frac{t \sin c}{R_{0}} \sum_{s=1}^{k} \tilde{a}_{s} \tilde{d}_{s} e^{\tilde{a}_{s}^{2} \sigma t \cos 2 \tilde{b}_{s}+\tilde{a}_{s} \varpi \cos \tilde{s}_{s}} \sin \left(\tilde{a}_{s}^{2} \sigma t \sin 2 \tilde{b}_{s}+\tilde{a}_{s} \varpi \sin \tilde{b}_{s}+\tilde{b}_{s}+\tilde{c}_{s}\right) \\
& +\sigma R \sum_{j=1}^{m} \frac{a_{j}^{3} d_{j}(\sigma-1) e^{a_{j}^{2} t+a_{j} \varpi} \cos c}{a_{j}^{4}(1-\sigma)^{2}+R_{0}^{-2} \sin ^{2} c} .
\end{aligned}
$$

By (8.5.4)-(8.5.6), (8.5.9) and (8.5.10), $\Phi_{1}=0$,

$$
\Phi_{2}=\left(\frac{\cos c}{R_{0}} f-\sigma R \psi \sin c\right) \cos c,
$$




$$
\Phi_{3}=\left(\frac{\cos c}{R_{0}} f-\sigma R \psi \sin c\right) \sin c-\sigma R z
$$

Thanks to (8.2.19),

$$
\begin{aligned}
p= & \frac{R \cos ^{2} c}{\sin c} \cos \frac{2 t \sin c}{R_{0}} \sum_{j=1}^{m} d_{j} e^{a_{j}^{2} t \cos 2 b_{j}+a_{j} \varpi \cos b_{j}} \sin \left(a_{j}^{2} t \sin 2 b_{j}+a_{j} \varpi \sin b_{j}+c_{j}\right) \\
& -\frac{\cos c}{R_{0}} \cos \frac{t \sin c}{R_{0}} \sum_{r=1}^{n} \hat{d}_{r} e^{\hat{a}_{r}^{2} t \cos 2 \hat{b}_{r}+\hat{a}_{r} \varpi \cos \hat{b}_{r}} \sin \left(\hat{a}_{r}^{2} t \sin 2 \hat{b}_{r}+\hat{a}_{r} \varpi \sin \hat{b}_{r}+\hat{c}_{r}\right) \\
& -\frac{\cos c}{R_{0}} \sin \frac{t \sin c}{R_{0}} \sum_{s=1}^{k} \tilde{d}_{s} e^{\tilde{a}_{s}^{2} t \cos 2 \tilde{b}_{s}+\tilde{a}_{s} \varpi \cos \tilde{b}_{s}} \sin \left(\tilde{a}_{s}^{2} t \sin 2 \tilde{b}_{s}+\tilde{a}_{s} \varpi \sin \tilde{b}_{s}+\tilde{c}_{s}\right) \\
& +R \sin c \sum_{j=1}^{m} d_{j} e^{a_{j}^{2} t \cos 2 b_{j}+a_{j} \varpi \cos b_{j}} \sin \left(a_{j}^{2} t \sin 2 b_{j}+a_{j} \varpi \sin b_{j}+c_{j}\right)+\frac{R}{2} z^{2}
\end{aligned}
$$

if $\sigma=1$, and

$$
\begin{aligned}
p= & -\frac{\cos c}{\sigma R_{0}} \cos \frac{t \sin c}{R_{0}} \sum_{r=1}^{n} \hat{d}_{r} e^{\hat{a}_{r}^{2} \sigma t \cos 2 \hat{b}_{r}+\hat{a}_{r} \varpi \cos \hat{b}_{r}} \sin \left(\hat{a}_{r}^{2} \sigma t \sin 2 \hat{b}_{r}+\hat{a}_{r} \varpi \sin \hat{b}_{r}+\hat{c}_{r}\right) \\
& -\frac{\cos c}{\sigma R_{0}} \sin \frac{t \sin c}{R_{0}} \sum_{s=1}^{k} \tilde{d}_{s} e^{\tilde{a}_{s}^{2} \sigma t \cos 2 \tilde{b}_{s}+\tilde{a}_{s} \varpi \cos \tilde{b}_{s}} \sin \left(\tilde{a}_{s}^{2} \sigma t \sin 2 \tilde{b}_{s}+\tilde{a}_{s} \varpi \sin \tilde{b}_{s}+\tilde{c}_{s}\right) \\
& +\sum_{j=1}^{m} \frac{d_{j} R e^{a_{j}^{2} t+a_{j} \varpi} \sin 2 c \cos c}{2 R_{0}^{2}\left(a_{j}^{4}(1-\sigma)^{2}+R_{0}^{-2} \sin ^{2} c\right)}+R \sin c \sum_{j=1}^{m} d_{j} e^{a_{j}^{2} t+a_{j} \varpi}+\frac{R}{2} z^{2}
\end{aligned}
$$

when $\sigma \neq 1$.

In summary, we have:

Theorem 8.5.1. Let $a_{j}, b_{j}, c_{j}, \hat{a}_{r}, \hat{b}_{r}, \hat{c}_{r}, \hat{d}_{r}, \tilde{a}_{s}, \tilde{b}_{s}, \tilde{c}_{s}, \tilde{d}_{s}, c$ be arbitrary real numbers. Denote $\varpi=y \cos x+z \sin c$. We have the following solutions of the three-dimensional stratified rotating Boussinesq equations (8.2.1)-(8.2.5):

$$
u=f, \quad v=\phi \sin c, \quad w=-\phi \cos c, \quad T=\psi+z,
$$

where (1) $\sigma=1, f$ is given in (8.5.18), $\phi$ is given in (8.5.19), $\psi$ is given in (8.5.15) and $p$ is given in (8.5.32); (2) $\sigma \neq 1, f$ is given in (8.5.28), $\phi$ is given in (8.5.29), $\psi$ is given in (8.5.20) and $p$ is given in (8.5.33).

Remark 8.5.2. By Fourier expansion, we can use the above solution to obtain the one depending on three arbitrary piecewise continuous functions of $\varpi$. 


\section{Chapter 9}

\section{Navier-Stokes Equations}

In this chapter, we introduce a method of imposing asymmetric conditions on the velocity vector with respect to independent spacial variables and a method of moving frame for solving the three dimensional Navier-Stokes equations. Seven families of non-steady rotating asymmetric solutions with various parameters are obtained. In particular, one family of solutions blow up on a moving plane, which may be used to study abrupt highspeed rotating flows. Using Fourier expansion and two families of our solutions, one can obtain discontinuous solutions that may be useful in study of shock waves. Another family of solutions are partially cylindrical invariant, containing two parameter functions in $t$, which may be used to describe incompressible fluid in a nozzle. Most of our solutions are globally analytic with respect to spacial variables. The results are due to our work [X12]. Cao [Cb3] applied our approaches to the magnetohydrodynamic equations of incompressible viscous fluids with finite electrical conductivity, which describe the motion of viscous electrically conducting fluids in a magnetic field.

\subsection{Background and Symmetry}

The most fundamental differential equations in the motion of incompressible viscous fluid are the Navier-Stokes equations:

$$
\begin{aligned}
u_{t}+u u_{x}+v u_{y}+w u_{z}+\frac{1}{\rho} p_{x} & =\nu\left(u_{x x}+u_{y y}+u_{z z}\right), \\
v_{t}+u v_{x}+v v_{y}+w v_{z}+\frac{1}{\rho} p_{y} & =\nu\left(v_{x x}+v_{y y}+v_{z z}\right), \\
w_{t}+u w_{x}+v w_{y}+w w_{z}+\frac{1}{\rho} p_{z} & =\nu\left(w_{x x}+w_{y y}+w_{z z}\right), \\
u_{x}+v_{y}+w_{z} & =0,
\end{aligned}
$$

where $(u, v, w)$ stands for the velocity vector of the fluid, $p$ stands for the pressure of the fluid, $\rho$ is the density constant and $\nu$ is the coefficient constant of the kinematic viscosity. 
Assuming nullity of certain components of the tensor of momentum flow density, Landau [Ll] (1944) found an exact solution of the Navier-Stokes equations (9.1.1)-(9.1.4), which describes axially symmetrical jet discharging from a thin pipe into unbounded space. Moreover, Kapitanskii [Kl] (1978) found certain cylindrical invariant solutions of the equations and Yakimov [Y] (1984) obtained exact solutions with a singularity of the type of a vortex filament situated on a half line. Furthermore, Gryn [Gv] (1991) obtained certain exact solution describing flows between porous walls in the presence of injection and suction at identical rates. Brutyan and Karapivskii [BK] (1992) got exact solutions describing the evolution of a vortex structure in a generalized shear flow. Leipnik [Lr] (1996) obtained exact solutions by recursive series of diffusive quotients. In addition, Polyanin [Pa] (2001) used the method of generalized separation of variables to find certain exact solutions and Vyskrebtsov [Vv] (2001) studied self-similar solutions for an axisymmetric flow of a viscous incompressible flow. There also are other works on exact solutions on the Navier-Stokes equations (e.g., cf. [Bv, Pv, Sh1, Sh2]).

A $3 \times 3$ real matrix $A$ is called orthogonal if $A^{T} A=A A^{T}=I_{3}$, where the up-index " $T$ " denotes the transpose of matrix. To show that the Navier-Stokes are invariant under the orthogonal transformation, we need to rewrite the Navier-Stokes equations in terms of matrices and column vectors (which are also viewed as special matrices). Denote

$$
\begin{gathered}
\vec{u}=\left(\begin{array}{c}
u \\
v \\
w
\end{array}\right), \quad \vec{x}=\left(\begin{array}{c}
x \\
y \\
z
\end{array}\right)=\left(\begin{array}{c}
x_{1} \\
x_{2} \\
x_{3}
\end{array}\right), \\
\nabla=\left(\begin{array}{c}
\partial_{x} \\
\partial_{y} \\
\partial_{z}
\end{array}\right), \quad \Delta=\nabla^{T} \nabla=\partial_{x}^{2}+\partial_{y}^{2}+\partial_{z}^{2} .
\end{gathered}
$$

Note $\vec{u}^{T} \nabla=u \partial_{x}+v \partial_{y}+w \partial_{w}$. Then (9.1.1)-(9.1.3) become

$$
\vec{u}_{t}+\left(\vec{u}^{T} \nabla\right)(\vec{u})+\frac{1}{\rho} \nabla(p)=\nu \Delta(\vec{u})
$$

and (9.1.4) changes to

$$
\nabla^{T} \vec{u}=0
$$

For a $3 \times 3$ orthogonal matrix $A=\left(a_{r, s}\right)_{3 \times 3}$, we define

$$
T_{A}\left(\vec{u}\left(t, \vec{x}^{T}\right)\right)=A \vec{u}\left(t, \vec{x}^{T} A\right), \quad T_{A}\left(p\left(t, \vec{x}^{T}\right)\right)=p\left(t, \vec{x}^{T} A\right) .
$$

Note that for any function $f(t, \vec{x})$ in $t, x, y, z$,

$$
\nabla\left(f\left(t, \vec{x}^{T} A\right)\right)=A\left(\begin{array}{c}
f_{x}\left(t, \vec{x}^{T} A\right) \\
f_{y}\left(t, \vec{x}^{T} A\right) \\
f_{z}\left(t, \vec{x}^{T} A\right)
\end{array}\right)=A \nabla(f)\left(t, \vec{x}^{T} A\right),
$$


equivalently,

$$
\begin{gathered}
\partial_{x_{r}}\left(f\left(t, \vec{x}^{T} A\right)\right)=\sum_{s=1}^{s} a_{r, s} f_{x_{s}}\left(t, \vec{x}^{T} A\right) \quad \text { for } r \in \overline{1,3} . \\
\Delta\left(f\left(t, \vec{x}^{T} A\right)\right) \\
=\left(\nabla^{T} \nabla\right)\left(f\left(t, \vec{x}^{T} A\right)\right)=\nabla^{T}\left[\nabla\left(f\left(t, \vec{x}^{T} A\right)\right)\right]=\nabla^{T}\left[(A \nabla(f))\left(t, \vec{x}^{T} A\right)\right] \\
=\left[\nabla^{T} A^{T}(A \nabla(f))\right]\left(t, \vec{x}^{T} A\right)=\left[\nabla^{T} \nabla(f)\right]\left(t, \vec{x}^{T} A\right)=\Delta(f)\left(t, \vec{x}^{T} A\right) .
\end{gathered}
$$

Now

$$
\begin{aligned}
& \left.\partial_{t}\left(T_{A}(\vec{u})\right)+\left(T_{A}(\vec{u})\right)^{T} \nabla\left(T_{A}(\vec{u})\right)\right)+\frac{1}{\rho} \nabla\left(T_{A}(p)\right) \\
= & \partial_{t}\left(A \vec{u}\left(t, \vec{x}^{T} A\right)\right)+\vec{u}^{T}\left(t, \vec{x}^{T} A\right) A^{T} \nabla\left(A \vec{u}\left(t, \vec{x}^{T} A\right)\right)+\frac{1}{\rho} \nabla\left(p\left(t, \vec{x}^{T} A\right)\right) \\
= & A \vec{u}_{t}\left(t, \vec{x}^{T} A\right)+\left[\left(\vec{u}^{T}\left(t, \vec{x}^{T} A\right) A^{T} A \nabla\right)(A \vec{u})\right]\left(t, \vec{x}^{T} A\right)+\frac{1}{\rho} A \nabla(p)\left(t, \vec{x}^{T} A\right) \\
= & \left.A \vec{u}_{t}\left(t, \vec{x}^{T} A\right)+\left[\left(\vec{u}^{T}\left(t, \vec{x}^{T} A\right) \nabla\right)(A \vec{u})\right]\left(t, \vec{x}^{T} A\right)\right)+\frac{1}{\rho} A \nabla(p)\left(t, \vec{x}^{T} A\right) \\
= & A \vec{u}_{t}\left(t, \vec{x}^{T} A\right)+A\left(\vec{u}^{T}\left(t, \vec{x}^{T} A\right) \nabla\right)(\vec{u})\left(t, \vec{x}^{T} A\right)+\frac{1}{\rho} A \nabla(p)\left(t, \vec{x}^{T} A\right) \\
= & A\left[\vec{u}_{t}\left(t, \vec{x}^{T} A\right)+\left(\vec{u}^{T}\left(t, \vec{x}^{T} A\right) \nabla\right)(\vec{u})\left(t, \vec{x}^{T} A\right)+\frac{1}{\rho} \nabla(p)\left(t, \vec{x}^{T} A\right)\right], \\
& \nu \Delta\left(\left(T_{A}(\vec{u})\right)\right)=\nu \Delta\left(A \vec{u}\left(t, \vec{x}^{T} A\right)\right)=\nu A \Delta\left(\vec{u}\left(t, \vec{x}^{T} A\right)=A\left[\nu \Delta(\vec{u})\left(t, \vec{x}^{T} A\right)\right)\right]
\end{aligned}
$$

by $(9.1 .12)$, and

$$
\begin{aligned}
\nabla^{T}\left(T_{A}(\vec{u})\right) & =\nabla^{T}\left(A \vec{u}\left(t, \vec{x}^{T} A\right)\right)=A \nabla^{T}\left(\vec{u}\left(t, \vec{x}^{T} A\right)\right) \\
& =A A^{T}\left(\nabla^{T} \vec{u}\right)\left(t, \vec{x}^{T} A\right)=\left(\nabla^{T} \vec{u}\right)\left(t, \vec{x}^{T} A\right) .
\end{aligned}
$$

If $[\vec{u}(t, x, y, z), p(t, x, y, z)]$ is a solution of the Navier-Stokes equations (9.1.1)-(9.1.4), then

$$
\vec{u}_{t}\left(t, \vec{x}^{T} A\right)+\left(\vec{u}^{T}\left(t, \vec{x}^{T} A\right) \nabla\right)(\vec{u})\left(t, \vec{x}^{T} A\right)+\frac{1}{\rho} \nabla(p)\left(t, \vec{x}^{T} A\right)=\nu \Delta(\vec{u})\left(t, \vec{x}^{T} A\right)
$$

and

$$
\left(\nabla^{T} \vec{u}\right)\left(t, \vec{x}^{T} A\right)=0
$$

Thus

$$
\left.\partial_{t}\left(T_{A}(\vec{u})\right)+\left(T_{A}(\vec{u})\right)^{T} \nabla\left(T_{A}(\vec{u})\right)\right)+\frac{1}{\rho} \nabla\left(T_{A}(p)\right)=\nu \Delta\left(\left(T_{A}(\vec{u})\right)\right)
$$

by (9.1.13) and (9.1.14). Moreover, (9.1.15) implies

$$
\nabla^{T}\left(T_{A}(\vec{u})\right)=0 .
$$


Therefore, $\left[T_{A}(\vec{u}), T_{A}(p)\right]$ is also a solution of the the Navier-Stokes equations (9.1.1)(9.1.4), that is, $T_{A}$ is a symmetry of the equations.

Let us do the degree analysis. Due to the term $\Delta(u)$ in (9.1.1), we assume

$$
\operatorname{deg} x=\operatorname{deg} y=\operatorname{deg} z=\ell_{1} .
$$

Moreover, to make the nonzero terms in (9.1.4) to have the same degree, we have to take

$$
\operatorname{deg} u=\operatorname{deg} v=\operatorname{deg} w=\ell_{2} .
$$

Note that in (9.1.1),

$$
\operatorname{deg} u_{t}=\operatorname{deg} u u_{x}=\operatorname{deg} p_{x}=\operatorname{deg} \Delta(u)
$$

Thus

$$
\operatorname{deg} t=2 \ell_{1}=-\operatorname{deg} p, \quad \ell_{2}=-\ell_{1} .
$$

Moreover, the Navier-Stokes equations are translation invariant because they do not contain variable coefficients. Hence the transformation

$$
\begin{aligned}
& T_{a, b}(\vec{u}(t, x, y, z))=b \vec{u}\left(b^{2} t+a, b x, b y, b z\right), \\
& T_{a, b}(p(t, x, y, z))=b^{2} p\left(b^{2} t+a, b x, b y, b z\right)
\end{aligned}
$$

keeps the Navier-Stokes equations invariant for $a, b \in \mathbb{R}$ with $b \neq 0$, that is, $T_{a, b}$ maps a solution of (9.1.1)-(9.1.4) to another solution.

Let $\alpha$ be a function in $t$. Note that the transformation

$$
\vec{u}(t, x, y, z) \mapsto \vec{u}(t, x+\alpha, y, z), \quad p(t, x, y, z) \mapsto p(t, x+\alpha, y, z)
$$

changes the equation (9.1.7) to

$$
\vec{u}_{t}^{T}+\alpha^{\prime} \vec{u}_{x}^{T}+\vec{u}^{T}(\nabla(u), \nabla(v), \nabla(w))+\frac{1}{\rho} \nabla^{T}(p)=\nu \Delta\left(\vec{u}^{T}\right)
$$

and keeps (9.1.4) invariant, where the independent variable $x$ is replaced by $x+\alpha$ and the partial derivatives are with respect to the original variables. On the other hand, the transformation

$$
\vec{u}^{T}(t, x, y, z) \mapsto \vec{u}^{T}(t, x, y, z)-\left(\alpha^{\prime}, 0,0\right), \quad p(t, x, y, z) \mapsto p(t, x, y, z)+\rho \alpha^{\prime \prime} x
$$

changes the equation (9.1.7) to

$$
\vec{u}_{t}^{T}+\vec{u}^{T}(\nabla(u), \nabla(v), \nabla(w))-\alpha^{\prime} \vec{u}_{x}^{T}+\frac{1}{\rho} \nabla^{T}(p)=\nu \Delta\left(\vec{u}^{T}\right)
$$

by (9.1.1)-(9.1.3) and keeps (9.1.4) invariant. Thus the transformation

$$
T_{1, \alpha}\left(\vec{u}^{T}(t, x, y, z)\right)=\vec{u}^{T}(t, x+\alpha, y, z)-\left(\alpha^{\prime}, 0,0\right)
$$




$$
T_{1, \alpha}(p(t, x, y, z))=p(t, x+\alpha, y, z)+\rho \alpha^{\prime \prime} x
$$

is a symmetry of the Navier-Stokes equations. Symmetrically, we have that the transformation

$$
\begin{gathered}
T_{\alpha_{1}, \alpha_{2}, \alpha_{3} ; \beta}\left(\vec{u}^{T}(t, x, y, z)\right)=\vec{u}^{T}\left(t, x+\alpha_{1}, y+\alpha_{2}, z+\alpha_{3}\right)-\left(\alpha_{1}^{\prime}, \alpha_{2}^{\prime}, \alpha_{3}^{\prime}\right), \\
T_{\alpha_{1}, \alpha_{2}, \alpha_{3} ; \beta}(p(t, x, y, z))=p\left(t, x+\alpha_{1}, y+\alpha_{2}, z+\alpha_{3}\right)+\rho\left(\alpha_{1}^{\prime \prime} x+\alpha_{2}^{\prime \prime} y+\alpha_{3}^{\prime \prime} z\right)+\beta
\end{gathered}
$$

is a symmetry of the Navier-Stokes equations for any functions $\alpha_{1}, \alpha_{2}, \alpha_{3}$ and $\beta$ in $t$.

\subsection{Asymmetric Approaches}

In this section, we will solve the incompressible Navier-Stokes equations (9.1.1)-(9.1.4) by imposing asymmetric assumptions on $u, v$ and $w$.

For convenience of computation, we denote

$$
\begin{gathered}
\Phi_{1}=u_{t}+u u_{x}+v u_{y}+w u_{z}-\nu\left(u_{x x}+u_{y y}+u_{z z}\right), \\
\Phi_{2}=v_{t}+u v_{x}+v v_{y}+w v_{z}-\nu\left(v_{x x}+v_{y y}+v_{z z}\right), \\
\Phi_{3}=w_{t}+u w_{x}+v w_{y}+w w_{z}-\nu\left(w_{x x}+w_{y y}+w_{z z}\right) .
\end{gathered}
$$

Then the Navier-Stokes equations become

$$
\Phi_{1}+\frac{1}{\rho} p_{x}=0, \quad \Phi_{2}+\frac{1}{\rho} p_{y}=0, \quad \Phi_{3}+\frac{1}{\rho} p_{z}=0
$$

and $u_{x}+v_{y}+w_{z}=0$. Our strategy is first to solve the following compatibility conditions:

$$
\partial_{y}\left(\Phi_{1}\right)=\partial_{x}\left(\Phi_{2}\right), \quad \partial_{z}\left(\Phi_{1}\right)=\partial_{x}\left(\Phi_{3}\right), \quad \partial_{z}\left(\Phi_{2}\right)=\partial_{y}\left(\Phi_{3}\right)
$$

and then find $p$ via (9.2.4).

Let us first look for simplest non-steady solutions of the Navier-Stokes equations (indeed, the corresponding Euler equations) that are not rotation free. This will help the reader to better understand our later approaches. Assume

$$
u=\gamma_{1} x-\alpha_{1} y-\alpha_{2} z, \quad v=\alpha_{1} x+\gamma_{2} y-\alpha_{3} z, \quad w=\alpha_{2} x+\alpha_{3} y+\gamma_{3} z,
$$

where $\alpha_{j}$ and $\gamma_{j}$ are functions in $t$ such that $\gamma_{1}+\gamma_{2}+\gamma_{3}=0$. Then

$$
\begin{aligned}
& \Phi_{1}=\left(\gamma_{1}^{\prime}+\gamma_{1}^{2}-\alpha_{1}^{2}-\alpha_{2}^{2}\right) x-\left(\alpha_{1}^{\prime}-\alpha_{1} \gamma_{3}+\alpha_{2} \alpha_{3}\right) y+\left(\alpha_{1} \alpha_{3}-\alpha_{2}^{\prime}+\alpha_{2} \gamma_{2}\right) z, \\
& \Phi_{2}=\left(\alpha_{1}^{\prime}-\alpha_{1} \gamma_{3}-\alpha_{2} \alpha_{3}\right) x+\left(\gamma_{2}^{\prime}+\gamma_{2}^{2}-\alpha_{1}^{2}-\alpha_{3}^{2}\right) y-\left(\alpha_{3}^{\prime}+\alpha_{1} \alpha_{2}-\alpha_{3} \gamma_{1}\right) z, \\
& \Phi_{3}=\left(\alpha_{2}^{\prime}+\alpha_{1} \alpha_{3}-\alpha_{2} \gamma_{2}\right) x+\left(\alpha_{3}^{\prime}-\alpha_{1} \alpha_{2}-\alpha_{3} \gamma_{1}\right) y+\left(\gamma_{3}^{\prime}+\gamma_{3}^{2}-\alpha_{2}^{2}-\alpha_{3}^{2}\right) z .
\end{aligned}
$$


Furthermore,

Note

$$
\begin{aligned}
& \partial_{y}\left(\Phi_{1}\right)=\partial_{x}\left(\Phi_{2}\right) \Longrightarrow \gamma_{3}=\frac{\alpha_{1}^{\prime}}{\alpha_{1}}, \\
& \partial_{z}\left(\Phi_{1}\right)=\partial_{x}\left(\Phi_{3}\right) \Longrightarrow \gamma_{2}=\frac{\alpha_{2}^{\prime}}{\alpha_{2}}, \\
& \partial_{z}\left(\Phi_{2}\right)=\partial_{y}\left(\Phi_{3}\right) \Longrightarrow \gamma_{1}=\frac{\alpha_{3}^{\prime}}{\alpha_{3}} .
\end{aligned}
$$

$$
\gamma_{1}+\gamma_{2}+\gamma_{3}=0 \sim \frac{\alpha_{1}^{\prime}}{\alpha_{1}}+\frac{\alpha_{2}^{\prime}}{\alpha_{2}}+\frac{\alpha_{3}^{\prime}}{\alpha_{3}}=0 \sim \alpha_{1} \alpha_{2} \alpha_{3}=c
$$

for some real constant. Moreover,

$$
\begin{gathered}
\Phi_{1}=\left(\alpha_{3}^{\prime \prime} \alpha_{3}^{-1}-\alpha_{1}^{2}-\alpha_{2}^{2}\right) x-\alpha_{2} \alpha_{3} y+\alpha_{1} \alpha_{3} z \\
\Phi_{2}=-\alpha_{2} \alpha_{3} x+\left(\alpha_{2}^{\prime \prime} \alpha_{2}^{-1}-\alpha_{1}^{2}-\alpha_{3}^{2}\right) y-\alpha_{1} \alpha_{2} z \\
\Phi_{3}=\alpha_{1} \alpha_{3} x-\alpha_{1} \alpha_{2} y+\left(\alpha_{1}^{\prime \prime} \alpha_{1}^{-1}-\alpha_{2}^{2}-\alpha_{3}^{2}\right) z
\end{gathered}
$$

By (9.2.4),

$$
\begin{aligned}
p= & \frac{\rho}{2}\left[\left(\alpha_{1}^{2}+\alpha_{2}^{2}-\alpha_{3}^{\prime \prime} \alpha_{3}^{-1}\right) x^{2}+\left(\alpha_{1}^{2}+\alpha_{3}^{2}-\alpha_{2}^{\prime \prime} \alpha_{2}^{-1}\right) y^{2}+\left(\alpha_{2}^{2}+\alpha_{3}^{2}-\alpha_{1}^{\prime \prime} \alpha_{1}^{-1}\right) z^{2}\right] \\
& +\rho\left(\alpha_{2} \alpha_{3} x y-\alpha_{1} \alpha_{3} x z+\alpha_{1} \alpha_{2} y z\right),
\end{aligned}
$$

after replacing $p$ by some $T_{0,0,0 ; \beta}(p)$ if necessary (cf. (9.1.32) and (9.1.33)).

Proposition 9.2.1. Let $\alpha_{1}, \alpha_{2}$ and $\alpha_{3}$ be functions in $t$ such that $\alpha_{1} \alpha_{2} \alpha_{3}=c$ for some real constant $c$. Then we have the following solution of the Navier-Stokes equations (9.1.1)-(9.1.4):

$$
u=\frac{\alpha_{3}{ }^{\prime}}{\alpha_{3}} x-\alpha_{1} y-\alpha_{2} z, \quad v=\alpha_{1} x+\frac{\alpha_{2}{ }^{\prime}}{\alpha_{2}} y-\alpha_{3} z, \quad w=\alpha_{2} x+\alpha_{3} y+\frac{\alpha_{1}{ }^{\prime}}{\alpha_{1}} z
$$

and $p$ is given in (9.2.17).

Next we assume

$$
v=-\frac{\beta^{\prime \prime}}{2 \beta^{\prime}} y, \quad w=\psi(t, z),
$$

where $\beta$ is a function in $t, \psi$ is a function of $t, z$ and $v$ is so written just for computational convenience by our earlier experience. According to (9.1.4),

$$
u=f(t, y, z)+\left(\frac{\beta^{\prime \prime}}{2 \beta^{\prime}}-\psi_{z}\right) x
$$

for some function $f$ of $t, y, z$. Then

$$
\begin{aligned}
\Phi_{1}= & f_{t}+f\left(\frac{\beta^{\prime \prime}}{2 \beta^{\prime}}-\psi_{z}\right)-\frac{\beta^{\prime \prime}}{2 \beta^{\prime}} y f_{y}+\psi f_{z}-\nu\left(f_{y y}+f_{z z}\right) \\
& +\left[\left(\frac{\beta^{\prime \prime}}{2 \beta^{\prime}}-\psi_{z}\right)^{2}+\frac{\beta^{\prime}{\beta^{\prime \prime}}^{\prime \prime}-\beta^{\prime 2}}{2 \beta^{\prime 2}}-\psi_{z t}-\psi \psi_{z z}+\nu \psi_{z z z}\right] x,
\end{aligned}
$$




$$
\Phi_{2}=\frac{\left(3{\beta^{\prime \prime}}^{2}-2 \beta^{\prime}{\beta^{\prime \prime}}^{\prime \prime}\right) y}{4{\beta^{\prime}}^{2}}, \quad \Phi_{3}=\psi_{t}+\psi \psi_{z}-\nu \psi_{z z} .
$$

Thus (9.2.5) is equivalent to the following equations:

$$
\begin{gathered}
\mathcal{T}\left[f_{t}+f\left(\frac{\beta^{\prime \prime}}{2 \beta^{\prime}}-\psi_{z}\right)-\frac{\beta^{\prime \prime}}{2 \beta^{\prime}} y f_{y}+\psi f_{z}-\nu\left(f_{y y}+f_{z z}\right)\right]=0, \\
\mathcal{T}\left[\psi_{z}^{2}-\frac{\beta^{\prime \prime}}{\beta^{\prime}} \psi_{z}-\psi_{z t}-\psi \psi_{z z}+\nu \psi_{z z z}\right]=0
\end{gathered}
$$

with $\mathcal{T}=\partial_{y}, \partial_{z}$. The above two equations are equivalent to

$$
\begin{gathered}
f_{t}+f\left(\frac{\beta^{\prime \prime}}{2 \beta^{\prime}}-\psi_{z}\right)-\frac{\beta^{\prime \prime}}{2 \beta^{\prime}} y f_{y}+\psi f_{z}-\nu\left(f_{y y}+f_{z z}\right)=\tau_{1}, \\
\psi_{z}^{2}-\frac{\beta^{\prime \prime}}{\beta^{\prime}} \psi_{z}-\psi_{z t}-\psi \psi_{z z}+\nu \psi_{z z z}=\tau_{2}
\end{gathered}
$$

for some functions $\tau_{1}$ and $\tau_{2}$ in $t$.

We solve (9.2.26) first. The idea is to linearize it. Note that

$$
\psi=e^{\nu \gamma \pm \sqrt{\gamma^{\prime}} z}, e^{-\nu \gamma} \sin \sqrt{\gamma^{\prime}} z, e^{-\gamma} \cos \sqrt{\gamma^{\prime}} z
$$

can simplify the expression

$$
-\psi_{z t}+\nu \psi_{z z z}
$$

for any increasing function $\gamma$ in $t$ such that $\gamma^{\prime} \not \equiv 0$. The nonlinear term $\psi_{z}^{2}-\psi \psi_{z z}$ hints us to use

$$
\begin{array}{ll}
\xi_{0}=e^{\nu \gamma}\left(\epsilon_{1} e^{\sqrt{\gamma^{\prime}} z}-\epsilon_{2} e^{-\sqrt{\gamma^{\prime}} z}\right), & \xi_{1}=e^{-\nu \gamma} \sin \left(\sqrt{\gamma^{\prime}} z\right), \\
\zeta_{0}=e^{\nu \gamma}\left(\epsilon_{1} e^{\sqrt{\gamma^{\prime}} z}+\epsilon_{2} e^{-\sqrt{\gamma^{\prime}} z}\right), & \zeta_{1}=e^{-\nu \gamma} \cos \left(\sqrt{\gamma^{\prime}} z\right),
\end{array}
$$

where $\epsilon_{1}, \epsilon_{2} \in \mathbb{R}$. In fact,

$$
\zeta_{0}^{2}-\xi_{0}^{2}=4 \epsilon_{1} \epsilon_{2} e^{2 \nu \gamma}, \quad \xi_{1}^{2}+\zeta_{1}^{2}=e^{-2 \nu \gamma} .
$$

Assume

$$
\psi=\lambda \xi_{r}+\mu z,
$$

where $r=0,1$ and $\lambda, \mu$ are functions in $t$ to be determined. We calculate

$$
\begin{gathered}
\psi_{z}=\lambda \sqrt{\gamma^{\prime}} \zeta_{r}+\mu, \quad \psi_{z z}=(-1)^{r} \lambda \gamma^{\prime} \xi_{r}, \quad \psi_{z z z}=(-1)^{r} \lambda \gamma^{\prime 3 / 2} \zeta_{r}, \\
\psi_{t z}=(-1)^{r} \lambda \sqrt{\gamma^{\prime}}\left(\nu \gamma^{\prime} \zeta_{r}+\gamma^{\prime \prime} z \xi_{r} / 2 \sqrt{\gamma^{\prime}}\right)+\left(\lambda^{\prime} \sqrt{\gamma^{\prime}}+\lambda \gamma^{\prime \prime} / 2 \sqrt{\gamma^{\prime}}\right) \zeta_{r} .
\end{gathered}
$$

Substituting (9.2.33) and (9.2.34) into (9.2.26), we find

$$
\begin{aligned}
& \lambda^{2} \gamma^{\prime}\left(\zeta_{r}^{2}-(-1)^{r} \xi_{r}^{2}\right)+2 \lambda \mu \sqrt{\gamma^{\prime}} \zeta_{r}+\mu^{2}-(-1)^{r} \lambda \mu \gamma^{\prime} z \xi_{r}-\beta^{\prime \prime} \lambda \sqrt{\gamma^{\prime}} \zeta_{r} / \beta^{\prime}-\beta^{\prime \prime} \mu / \beta^{\prime} \\
& -(-1)^{r} \lambda \gamma^{\prime \prime} z \xi_{r} / 2-\left(\lambda^{\prime} \sqrt{\gamma^{\prime}}+\lambda \gamma^{\prime \prime} / 2 \sqrt{\gamma^{\prime}}\right) \zeta_{r}=\tau_{2},
\end{aligned}
$$


equivalently

$$
\lambda^{2} \gamma^{\prime}\left(\zeta_{r}^{2}-(-1)^{r} \xi_{r}^{2}\right)+\mu^{2}-\beta^{\prime \prime} \mu / \beta^{\prime}=\tau_{2}
$$

by the terms that are independent of spacial variables,

$$
-(-1)^{r} \lambda \mu \gamma^{\prime}-(-1)^{r} \lambda \gamma^{\prime \prime} / 2=0
$$

by the coefficients of $z \xi_{r}$ and

$$
2 \lambda \mu \sqrt{\gamma^{\prime}}-\beta^{\prime \prime} \lambda \sqrt{\gamma^{\prime}} / \beta^{\prime}-\left(\lambda^{\prime} \sqrt{\gamma^{\prime}}+\lambda \gamma^{\prime \prime} / 2 \sqrt{\gamma^{\prime}}\right)=0
$$

by the coefficients of $\zeta_{r}$. According to (9.2.37),

$$
\mu=-\frac{\gamma^{\prime \prime}}{2 \gamma^{\prime}}
$$

Substituting it into (9.2.38), we get

$$
-\beta^{\prime \prime} \lambda \sqrt{\gamma^{\prime}} / \beta^{\prime}-\lambda^{\prime} \sqrt{\gamma^{\prime}}-3 \lambda \gamma^{\prime \prime} / 2 \sqrt{\gamma^{\prime}}=0 \Longrightarrow \lambda=\frac{1}{\beta^{\prime} \sqrt{\gamma^{\prime 3}}} .
$$

So

$$
\psi=\frac{\xi_{r}}{\beta^{\prime} \sqrt{\gamma^{\prime 3}}}-\frac{\gamma^{\prime \prime} z}{2 \gamma^{\prime}}
$$

and

$$
\tau_{2}=\frac{4 \epsilon_{1} \epsilon_{2} e^{2 \nu \gamma} \delta_{r, 0}+e^{-2 \nu \gamma} \delta_{r, 1}}{\left(\beta^{\prime} \gamma^{\prime}\right)^{2}}+\frac{\gamma^{\prime \prime 2}}{4 \gamma^{\prime 2}}+\frac{{\beta^{\prime \prime}}^{\prime \prime}}{2 \beta^{\prime} \gamma^{\prime}}
$$

by (9.2.36). According to (9.2.21), (9.2.25) and (9.2.26),

$$
\Phi_{1}=\tau_{1}+\left[\frac{2 \beta^{\prime} \beta^{\prime \prime \prime}-\beta^{\prime \prime 2}}{4{\beta^{\prime}}^{2}}+\frac{4 \epsilon_{1} \epsilon_{2} e^{2 \nu \gamma} \delta_{r, 0}+e^{-2 \nu \gamma} \delta_{r, 1}}{\left(\beta^{\prime} \gamma^{\prime}\right)^{2}}+\frac{{\gamma^{\prime \prime}}^{2}}{4{\gamma^{\prime}}^{2}}+\frac{\beta^{\prime \prime} \gamma^{\prime \prime}}{2 \beta^{\prime} \gamma^{\prime}}\right] x
$$

Substituting (9.2.41) into (9.2.25), we find

$$
f_{t}+\left(\frac{\beta^{\prime \prime}}{2 \beta^{\prime}}+\frac{\gamma^{\prime \prime}}{2 \gamma^{\prime}}\right) f+\frac{\xi_{r} f_{z}-\sqrt{\gamma^{\prime}} \zeta_{r} f}{\beta^{\prime} \sqrt{\gamma^{\prime 3}}}-\frac{\beta^{\prime \prime}}{2 \beta^{\prime}} y f_{y}-\frac{\gamma^{\prime \prime}}{2 \gamma^{\prime}} z f_{z}-\nu\left(f_{y y}+f_{z z}\right)=\tau_{1} .
$$

We assume

$$
f=\frac{g(t, \varpi) \zeta_{r}}{\sqrt{\beta^{\prime} \gamma^{\prime}}}, \quad \varpi=\sqrt{\beta^{\prime}} y,
$$

where $g(t, \varpi)$ is a two-variable function to be determined. We calculate

$$
\begin{gathered}
f_{t}=\frac{g_{t} \zeta_{r}}{\sqrt{\beta^{\prime} \gamma^{\prime}}}-\left(\frac{\beta^{\prime \prime}}{2 \beta^{\prime}}+\frac{\gamma^{\prime \prime}}{2 \gamma^{\prime}}\right) f+(-1)^{r} \frac{\gamma^{\prime \prime} z g \xi_{r}}{2 \gamma^{\prime} \sqrt{\beta^{\prime}}}+\frac{(-1)^{r} \nu \gamma^{\prime} g \zeta_{r}}{\sqrt{\beta^{\prime} \gamma^{\prime}}}+\frac{\beta^{\prime \prime} y g_{\varpi} \zeta_{r}}{2 \beta^{\prime} \sqrt{\gamma^{\prime}}}, \\
f_{y}=\frac{g_{\varpi} \zeta_{r}}{\sqrt{\gamma^{\prime}}}, \quad f_{y y}=\frac{\sqrt{\beta^{\prime}} g_{\varpi \varpi} \zeta_{r}}{\sqrt{\gamma^{\prime}}},
\end{gathered}
$$




$$
f_{z}=\frac{(-1)^{r} g \xi_{r}}{\sqrt{\beta^{\prime}}}, \quad f_{z z}=\frac{(-1)^{r} \sqrt{\gamma^{\prime}} g \zeta_{r}}{\sqrt{\beta^{\prime}}} .
$$

Substituting (9.2.46)-(9.2.48) into (9.2.44), we get

$$
\frac{g_{t} \zeta_{r}}{\sqrt{\beta^{\prime} \gamma^{\prime}}}+\frac{\left((-1)^{r} \xi_{r}^{2}-\zeta_{r}^{2}\right) g}{\sqrt{\left(\beta^{\prime} \gamma^{\prime}\right)^{3}}}-\frac{\nu \sqrt{\beta^{\prime}} g_{\varpi \varpi} \zeta_{r}}{\sqrt{\gamma^{\prime}}}=\tau_{1} .
$$

Case 1. $g=a \in \mathbb{R}$.

In this case

$$
f=\frac{a \zeta_{r}}{\sqrt{\beta^{\prime} \gamma^{\prime}}}, \quad \tau_{1}=\frac{\left((-1)^{r} \xi_{r}^{2}-\zeta_{r}^{2}\right) g}{\sqrt{\left(\beta^{\prime} \gamma^{\prime}\right)^{3}}}=-\frac{a\left(4 \epsilon_{1} \epsilon_{2} e^{2 \nu \gamma} \delta_{r, 0}+e^{-2 \nu \gamma} \delta_{r, 1}\right)}{\sqrt{\left(\beta^{\prime} \gamma^{\prime}\right)^{3}}}
$$

by $(9.2 .49)$

Case 2. $r=0=\epsilon_{2}$ and $\epsilon_{1}=1$.

In this case, $\tau_{1}=0$ and

$$
g_{t}-\nu \beta^{\prime} g_{\varpi \varpi}=0
$$

by (6.2.49). So

$$
g=e^{\nu\left((a+c i)^{2} \beta\right)+(a+c i) \varpi}
$$

is a complex solution of (9.2.51) for any $a, c \in \mathbb{R}$. Thus we have real solutions

$$
e^{\nu\left(a^{2}-c^{2}\right) \beta+a \varpi} \sin (2 a c \nu \beta+c \varpi), \quad e^{\nu\left(a^{2}-c^{2}\right) \beta+a \varpi} \cos (2 a c \nu \beta+c \varpi) .
$$

In particular, any linear combination

$$
\begin{aligned}
& e^{\nu\left(a^{2}-c^{2}\right) \beta+a \varpi}\left(C_{1} \sin (2 a c \nu \beta+c \varpi)+C_{2} \cos (2 a c \nu \beta+c \varpi)\right) \\
= & b e^{\nu\left(a^{2}-c^{2}\right) \beta+a \varpi} \sin (2 a c \nu \beta+c \varpi+\theta)
\end{aligned}
$$

of them is a solution of (9.2.51), where $C_{1}, C_{2} \in \mathbb{R}$ and $b=\sqrt{C_{1}^{2}+C_{2}^{2}}, C_{1} / b=\cos \theta$. By superposition principle, we have more general solution:

$$
g=\sum_{s=1}^{n} b_{s} e^{\nu\left(a_{s}^{2}-c_{s}^{2}\right) \beta+a_{s} \varpi} \sin \left(2 a_{s} c_{s} \nu \beta+c_{s} \varpi+\theta_{s}\right)
$$

for $a_{s}, b_{s}, c_{s}, \theta_{s} \in \mathbb{R}$ such that $b_{s} \neq 0,\left(a_{s}, c_{s}\right) \neq(0,0)$. Recall $\varpi=\sqrt{\beta^{\prime}} y$. Thanks to (9.2.45),

$$
f=\frac{\zeta_{r}}{\sqrt{\beta^{\prime} \gamma^{\prime}}} \sum_{s=1}^{n} b_{s} e^{\nu\left(a_{s}^{2}-c_{s}^{2}\right) \beta+a_{s} \sqrt{\beta^{\prime} y}} \sin \left(2 a_{s} c_{s} \nu \beta+c_{s} \sqrt{\beta^{\prime}} y+\theta_{s}\right) .
$$


Next we calculate the pressure $p$ via (9.2.4). First we assume $g=a$ and $r=1$. In this case,

$$
\psi=\frac{e^{-\nu \gamma} \sin \left(\sqrt{\gamma^{\prime}} z\right)}{\beta^{\prime} \sqrt{\gamma^{\prime 3}}}-\frac{\gamma^{\prime \prime} z}{2 \gamma^{\prime}}
$$

Denote

$$
\hat{\psi}=-\frac{e^{-\nu \gamma} \cos \left(\sqrt{\gamma^{\prime}} z\right)}{\beta^{\prime} \gamma^{\prime}}-\frac{\gamma^{\prime \prime} z^{2}}{4 \gamma^{\prime}} .
$$

Then $\hat{\psi}_{z}=\psi$. According to (9.2.4), (9.2.22), (9.2.43) and (9.2.50),

$$
\begin{aligned}
p= & \rho\left(\nu \psi_{z}-\frac{\psi^{2}}{2}-\hat{\psi}_{t}+\frac{e^{-2 \nu \gamma} x}{\sqrt{\left(\beta^{\prime} \gamma^{\prime}\right)^{3}}}-\frac{\left(3 \beta^{\prime \prime 2}-2 \beta^{\prime} \beta^{\prime \prime}\right) y^{2}}{8 \beta^{\prime 2}}\right) \\
& -\frac{\rho x^{2}}{2}\left[\frac{2 \beta^{\prime}{\beta^{\prime \prime}}^{\prime \prime}-{\beta^{\prime \prime}}^{2}}{4 \beta^{\prime 2}}+\frac{e^{-2 \nu \gamma}}{\left(\beta^{\prime} \gamma^{\prime}\right)^{2}}+\frac{{\gamma^{\prime \prime}}^{2}}{4{\gamma^{\prime}}^{2}}+\frac{\left.{\beta^{\prime \prime} \gamma^{\prime \prime}}_{2 \beta^{\prime} \gamma^{\prime}}\right] .}{}\right.
\end{aligned}
$$

Consider the case $g=a$ and $r=0$. We have

$$
\psi=\frac{e^{\nu \gamma}\left(\epsilon_{1} e^{\sqrt{\gamma^{\prime}} z}-\epsilon_{2} e^{-\sqrt{\gamma^{\prime}} z}\right)}{\beta^{\prime} \sqrt{\gamma^{\prime 3}}}-\frac{\gamma^{\prime \prime} z}{2 \gamma^{\prime}} .
$$

Denote

$$
\hat{\psi}=\frac{e^{\nu \gamma}\left(\epsilon_{1} e^{\sqrt{\gamma^{\prime}} z}+\epsilon_{2} e^{-\sqrt{\gamma^{\prime}} z}\right)}{\beta^{\prime} \gamma^{\prime}}-\frac{\gamma^{\prime \prime} z^{2}}{4 \gamma^{\prime}} .
$$

According to (9.2.4), (9.2.22), (9.2.43) and (9.2.56),

$$
\begin{aligned}
p= & \rho\left(\nu \psi_{z}-\frac{\psi^{2}}{2}-\hat{\psi}_{t}+\frac{4 \epsilon_{1} \epsilon_{2} e^{2 \nu \gamma} x}{\sqrt{\left(\beta^{\prime} \gamma^{\prime}\right)^{3}}}-\frac{\left(3{\beta^{\prime \prime}}^{2}-2 \beta^{\prime} \beta^{\prime \prime}\right) y^{2}}{8 \beta^{\prime 2}}\right) \\
& -\frac{\rho x^{2}}{2}\left[\frac{2 \beta^{\prime}{\beta^{\prime \prime}}^{\prime \prime}-{\beta^{\prime \prime}}^{2}}{4{\beta^{\prime}}^{2}}+\frac{4 \epsilon_{1} \epsilon_{2} e^{2 \nu \gamma}}{\left(\beta^{\prime} \gamma^{\prime}\right)^{2}}+\frac{{\gamma^{\prime \prime}}^{2}}{4{\gamma^{\prime}}^{2}}+\frac{\left.{\beta^{\prime \prime} \gamma^{\prime \prime}}_{2 \beta^{\prime} \gamma^{\prime}}\right] .}{}\right.
\end{aligned}
$$

Suppose $r=0=\epsilon_{2}$ and $\epsilon_{1}=1$. Then the pressure is the corresponding special case of $(9.2 .62)$ :

$$
\begin{aligned}
p= & \rho\left(\nu \psi_{z}-\frac{\psi^{2}}{2}-\hat{\psi}_{t}-\frac{\left(3{\beta^{\prime \prime}}^{2}-2 \beta^{\prime}{\beta^{\prime \prime}}^{\prime \prime}\right) y^{2}}{8 \beta^{\prime 2}}\right) \\
& -\frac{\rho x^{2}}{2}\left[\frac{2 \beta^{\prime}{\beta^{\prime \prime \prime}}^{\prime \prime}-{\beta^{\prime \prime}}^{2}}{4{\beta^{\prime}}^{2}}+\frac{{\gamma^{\prime \prime}}^{2}}{4{\gamma^{\prime}}^{2}}+\frac{\left.{\beta^{\prime \prime} \gamma^{\prime \prime}}_{2 \beta^{\prime} \gamma^{\prime}}\right]}{}\right.
\end{aligned}
$$

with

$$
\psi=\frac{e^{\nu \gamma} e^{\sqrt{\gamma^{\prime}} z}}{\beta^{\prime} \sqrt{\gamma^{\prime 3}}}-\frac{\gamma^{\prime \prime} z}{2 \gamma^{\prime}}, \quad \hat{\psi}=\frac{e^{\nu \gamma} e^{\sqrt{\gamma^{\prime}} z}}{\beta^{\prime} \gamma^{\prime}}-\frac{\gamma^{\prime \prime} z^{2}}{4 \gamma^{\prime}} .
$$

Theorem 9.2.2. Let $\alpha, \beta$ and $\gamma$ be any functions in $t$. For any $0 \neq a, \epsilon_{1}, \epsilon_{2} \in \mathbb{R}$, we have the following solutions of the Navier Stokes equations (9.1.1)-(9.1.4):

$$
u=\frac{a e^{-\nu \gamma} \cos \left(\sqrt{\gamma^{\prime}} z\right)}{\sqrt{\beta^{\prime} \gamma^{\prime}}}+\left(\frac{\beta^{\prime \prime}}{2 \beta^{\prime}}+\frac{\gamma^{\prime \prime}}{2 \gamma^{\prime}}-\frac{e^{-\nu \gamma} \cos \left(\sqrt{\gamma^{\prime}} z\right)}{\beta^{\prime} \gamma}\right) x,
$$




$$
v=-\frac{\beta^{\prime \prime}}{2 \beta^{\prime}} y, \quad w=\frac{e^{-\nu \gamma} \sin \left(\sqrt{\gamma^{\prime}} z\right)}{\beta^{\prime} \sqrt{\gamma^{\prime 3}}}-\frac{\gamma^{\prime \prime} z}{2 \gamma^{\prime}},
$$

and $p$ is given in (9.2.59);

$$
\begin{gathered}
u=\frac{a e^{\nu \gamma}\left(\epsilon_{1} e^{\sqrt{\gamma^{\prime}} z}+\epsilon_{2} e^{-\sqrt{\gamma^{\prime}} z}\right)}{\sqrt{\beta^{\prime} \gamma^{\prime}}}+\left(\frac{\beta^{\prime \prime}}{2 \beta^{\prime}}+\frac{\gamma^{\prime \prime}}{2 \gamma^{\prime}}-\frac{e^{\nu \gamma}\left(\epsilon_{1} e^{\sqrt{\gamma^{\prime}} z}+\epsilon_{2} e^{-\sqrt{\gamma^{\prime}} z}\right)}{\beta^{\prime} \gamma}\right) x, \\
v=-\frac{\beta^{\prime \prime}}{2 \beta^{\prime}} y, \quad w=\frac{e^{\nu \gamma}\left(\epsilon_{1} e^{\sqrt{\gamma^{\prime}} z}-\epsilon_{2} e^{-\sqrt{\gamma^{\prime}} z}\right)}{\beta^{\prime} \sqrt{\gamma^{\prime 3}}}-\frac{\gamma^{\prime \prime} z}{2 \gamma^{\prime}},
\end{gathered}
$$

and $p$ is given in (9.2.62).

For $a_{s}, b_{s}, c_{s}, \theta_{s} \in \mathbb{R}$ with $s \in \overline{1, n}$ such that $b_{s} \neq 0,\left(a_{s}, c_{s}\right) \neq(0,0)$, we have the following solutions of the Navier Stokes equations (9.1.1)-(9.1.4):

$$
\begin{gathered}
u=\frac{e^{\nu \gamma+\sqrt{\gamma^{\prime}} z}}{\sqrt{\beta^{\prime} \gamma^{\prime}}} \sum_{s=1}^{n} b_{s} e^{\nu\left(a_{s}^{2}-c_{s}^{2}\right) \beta+a_{s} \sqrt{\beta^{\prime} y}} \sin \left(2 a_{s} c_{s} \nu \beta+c_{s} \sqrt{\beta^{\prime}} y+\theta_{s}\right) \\
+\left(\frac{\beta^{\prime \prime}}{2 \beta^{\prime}}+\frac{\gamma^{\prime \prime}}{2 \gamma^{\prime}}-\frac{e^{\nu \gamma} e^{\sqrt{\gamma^{\prime}} z}}{\beta^{\prime} \gamma}\right) x, \\
v=-\frac{\beta^{\prime \prime}}{2 \beta^{\prime}} y, \quad w=\frac{e^{\nu \gamma+\sqrt{\gamma^{\prime}} z}}{\beta^{\prime} \sqrt{\gamma^{\prime 3}}}-\frac{\gamma^{\prime \prime} z}{2 \gamma^{\prime}}
\end{gathered}
$$

and $p$ is given in (9.2.63).

Remark 9.2.3. We can use Fourier expansion to solve the system (9.2.51) for $g\left(t, \sqrt{\beta^{\prime}} y\right)$ with given $g\left(0, \sqrt{\beta^{\prime}(0)} y\right)$. In this way, we can obtain discontinuous solutions of the Navier-Stokes equations (9.1.1)-(9.1.4), which may be useful in studying shock waves.

For $\theta \in \mathbb{R}$, we denote the rotation

$$
A=\left(\begin{array}{ccc}
1 & 0 & 0 \\
0 & \cos \theta & \sin \theta \\
0 & -\sin \theta & \cos \theta
\end{array}\right), \quad A^{T}=\left(\begin{array}{ccc}
1 & 0 & 0 \\
0 & \cos \theta & -\sin \theta \\
0 & \sin \theta & \cos \theta
\end{array}\right) .
$$

Applying $T_{A}$ in (9.1.9) to the above first solution, we get

$$
\begin{aligned}
& u=\frac{a e^{-\nu \gamma} \cos \left(\sqrt{\gamma^{\prime}}(y \sin \theta+z \cos \theta)\right)}{\sqrt{\beta^{\prime} \gamma^{\prime}}} \\
& +\left(\frac{\beta^{\prime \prime}}{2 \beta^{\prime}}+\frac{\gamma^{\prime \prime}}{2 \gamma^{\prime}}-\frac{\left.e^{-\nu \gamma} \cos \left(\sqrt{\gamma^{\prime}}(y \sin \theta+z \cos \theta)\right)\right)}{\beta^{\prime} \gamma}\right) x, \\
& v=\left(\frac{e^{-\nu \gamma} \sin \left(\sqrt{\gamma^{\prime}}(y \sin \theta+z \cos \theta)\right)}{\beta^{\prime} \sqrt{{\gamma^{\prime}}^{3}}}-\frac{\gamma^{\prime \prime}(y \sin \theta+z \cos \theta)}{2 \gamma^{\prime}}\right) \sin \theta \\
& -\frac{\beta^{\prime \prime}}{2 \beta^{\prime}}(y \cos \theta-z \sin \theta) \cos \theta,
\end{aligned}
$$




$$
\begin{aligned}
w= & \left(\frac{e^{-\nu \gamma} \sin \left(\sqrt{\gamma^{\prime}}(y \sin \theta+z \cos \theta)\right)}{\beta^{\prime} \sqrt{\gamma^{\prime 3}}}-\frac{\gamma^{\prime \prime}(y \sin \theta+z \cos \theta)}{2 \gamma^{\prime}}\right) \cos \theta \\
& +\frac{\beta^{\prime \prime}}{2 \beta^{\prime}}(y \cos \theta-z \sin \theta) \sin \theta
\end{aligned}
$$

and

$$
\begin{aligned}
p= & \rho\left[\nu \psi_{z}(t, y \sin \theta+z \cos \theta)-\frac{\psi^{2}(t, y \sin \theta+z \cos \theta)}{2}-\hat{\psi}_{t}(t, y \sin \theta+z \cos \theta)\right. \\
& \left.+\frac{e^{-2 \nu \gamma} x}{\sqrt{\left(\beta^{\prime} \gamma^{\prime}\right)^{3}}}-\frac{\left(3{\beta^{\prime \prime}}^{2}-2 \beta^{\prime} \beta^{\prime \prime \prime}\right)(y \cos \theta-z \sin \theta)^{2}}{8{\beta^{\prime}}^{2}}\right] \\
& -\frac{\rho x^{2}}{2}\left[\frac{2 \beta^{\prime}{\beta^{\prime \prime}}^{\prime \prime}-{\beta^{\prime \prime}}^{2}}{4{\beta^{\prime 2}}^{2}}+\frac{e^{-2 \nu \gamma}}{\left(\beta^{\prime} \gamma^{\prime}\right)^{2}}+\frac{{\gamma^{\prime \prime}}^{2}}{4{\gamma^{\prime}}^{2}}+\frac{{\beta^{\prime \prime}}^{\prime \prime}}{2 \beta^{\prime} \gamma^{\prime}}\right]
\end{aligned}
$$

Set

$$
\varpi=x^{2}+y^{2}
$$

Consider

$$
u=y \phi(t, \varpi), \quad v=-x \phi(t, \varpi), \quad w=\psi(t, \varpi),
$$

where $\phi$ and $\psi$ are functions in $t, \varpi$. Then (9.2.1)-(9.2.3) give

$$
\begin{gathered}
\Phi_{1}=y \phi_{t}-x \phi^{2}-4 y \nu(\varpi \phi)_{\varpi \varpi}, \\
\Phi_{2}=-x \phi_{t}-y \phi^{2}+4 x \nu(\varpi \phi)_{\varpi \varpi}, \\
\Phi_{3}=\psi_{t}-4 \nu\left(\psi_{\varpi}+\varpi \psi_{\varpi \varpi}\right) .
\end{gathered}
$$

Note that $\partial_{y}\left(\Phi_{1}\right)=\partial_{x}\left(\Phi_{2}\right)$ becomes

$$
(\varpi \phi)_{\varpi t}-4 \nu\left((\varpi \phi)_{\varpi \varpi}+\varpi(\varpi \phi)_{\varpi \varpi \varpi}\right)=0 .
$$

Set

$$
\hat{\phi}=(\varpi \phi)_{\varpi}
$$

Then (9.2.81) becomes

$$
\hat{\phi}_{t}-4 \nu\left(\hat{\phi}_{\varpi}+\varpi \hat{\phi}_{\varpi \varpi}\right)=0
$$

Suppose that

$$
\hat{\phi}=\sum_{m=0}^{\infty} a_{m}(t) \varpi^{m},
$$

where $a_{m}(t)$ are functions in $t$ to be determined. Then (9.2.83) becomes

$$
\sum_{m=0}^{\infty} a_{m}^{\prime} \varpi^{m}=4 \nu \sum_{m=0}^{\infty} m^{2} a_{m} \varpi^{m-1}
$$


equivalently,

$$
a_{m}=\frac{a_{0}^{(m)}}{(4 \nu)^{m}(m !)^{2}} \quad \text { for } m \in \mathbb{N} .
$$

Write $\alpha(t)=a_{0}(t)$. We have

$$
\hat{\phi}=\sum_{m=0}^{\infty} \frac{\alpha^{(m)} \varpi^{m}}{(4 \nu)^{m}(m !)^{2}} .
$$

By (9.2.82), we get

$$
\phi=\beta \varpi^{-1}+\sum_{m=0}^{\infty} \frac{\alpha^{(m)} \varpi^{m}}{(4 \nu)^{m} m !(m+1) !}
$$

for a function $\beta$ in $t$.

Note

$$
\begin{gathered}
\phi_{t}=\beta^{\prime} \varpi^{-1}+\sum_{m=0}^{\infty} \frac{\alpha^{(m+1)} \varpi^{m}}{(4 \nu)^{m} m !(m+1) !}, \\
4 \nu(\varpi \phi)_{\varpi \varpi}=4 \nu \hat{\phi}_{\varpi}=\sum_{m=1}^{\infty} \frac{\alpha^{(m)} \varpi^{m-1}}{(4 \nu)^{m-1}(m-1) ! m !} .
\end{gathered}
$$

Thus

$$
\phi_{t}-4 \nu(\varpi \phi)_{\varpi \varpi}=\beta^{\prime} \varpi^{-1} .
$$

Therefore,

$$
\Phi_{1}=\frac{\beta^{\prime} y}{x^{2}+y^{2}}-x \phi^{2}
$$

and

$$
\Phi_{2}=-\frac{\beta^{\prime} x}{x^{2}+y^{2}}-y \phi^{2} .
$$

On the other hand, Equations $\partial_{z}\left(\Phi_{1}\right)=\partial_{x}\left(\Phi_{3}\right)$ and $\partial_{z}\left(\Phi_{2}\right)=\partial_{y}\left(\Phi_{3}\right)$ are implied by the following differential equation:

$$
\psi_{t}-4 \nu\left(\psi_{\varpi}+\varpi \psi_{\varpi \varpi}\right)=0
$$

(cf. (9.2.80)). Similarly, we have the solution:

$$
\psi=\sum_{n=0}^{\infty} \frac{\gamma^{(n)} \varpi^{n}}{(4 \nu)^{n}(n !)^{2}},
$$

where $\gamma$ is a smooth function in $t$. With this $\psi, \Phi_{3}=0$. By (9.2.4), (9.2.76), (9.2.77), (9.2.88), (9.2.92), (9.2.93) and (9.2.95), we obtain:

Theorem 9.2.4. Let $\alpha, \gamma$ be any smooth functions in $t$ and let $\beta$ be any differentiable function in $t$. We have the following solution of the Navier-Stokes equations (9.1.1)(9.1.4):

$$
u=\frac{\beta y}{x^{2}+y^{2}}+y \sum_{m=0}^{\infty} \frac{\alpha^{(m)}\left(x^{2}+y^{2}\right)^{m}}{(4 \nu)^{m} m !(m+1) !},
$$




$$
\begin{gathered}
v=-\frac{\beta x}{x^{2}+y^{2}}-x \sum_{m=0}^{\infty} \frac{\alpha^{(m)}\left(x^{2}+y^{2}\right)^{m}}{(4 \nu)^{m} m !(m+1) !}, \\
w=\sum_{n=0}^{\infty} \frac{\gamma^{(n)}\left(x^{2}+y^{2}\right)^{n}}{(4 \nu)^{n}(n !)^{2}} \\
p=\rho \beta^{\prime} \arctan \frac{y}{x}+\rho \sum_{m, n=0}^{\infty} \frac{\alpha^{(m)} \alpha^{(n)}\left(x^{2}+y^{2}\right)^{m+n+1}}{2(m+n+1) m !(m+1) ! n !(n+1) !(4 \nu)^{m+n}} .
\end{gathered}
$$

Remark 9.2.5. When $\alpha$ and $\gamma$ are polynomials in $t$, the summations in the above theorem are finite. Let $\gamma_{1}, \gamma_{2}, \gamma_{3}$ and $\vartheta$ be functions in $t$. For $\theta \in \mathbb{R}$, we the matrices in (9.2.71). Recall the transformations in (9.1.9) and (9.1.32)-(9.1.33). Applying $T_{A} T_{\gamma_{1}, \gamma_{2}, \gamma_{3} ; \vartheta}$ to the above solution, we get the following solution of the Navier-Stokes equations with six parameter functions in $t$ :

$$
\begin{aligned}
& u=\left(y \cos \theta-z \sin \theta+\gamma_{2}\right) \sum_{m=0}^{\infty} \frac{\alpha^{(m)}\left[\left(x+\gamma_{1}\right)^{2}+\left(y \cos \theta-z \sin \theta+\gamma_{2}\right)^{2}\right]^{m}}{(4 \nu)^{m} m !(m+1) !} \\
& +\frac{\beta\left(y \cos \theta-z \sin \theta+\gamma_{2}\right)}{\left(x+\gamma_{1}\right)^{2}+\left(y \cos \theta-z \sin \theta+\gamma_{2}\right)^{2}}-\gamma_{1}^{\prime}, \\
& v=-\left[\sum_{m=0}^{\infty} \frac{\alpha^{(m)}\left[\left(x+\gamma_{1}\right)^{2}+\left(y \cos \theta-z \sin \theta+\gamma_{2}\right)^{2}\right]^{m}}{(4 \nu)^{m} m !(m+1) !}\right. \\
& \left.+\frac{\beta x}{\left(x+\gamma_{1}\right)^{2}+\left(y \cos \theta-z \sin \theta+\gamma_{2}\right)^{2}}-\gamma_{2}^{\prime}\right] \cos \theta-\gamma_{2}^{\prime} \\
& +\sin \theta \sum_{n=0}^{\infty} \frac{\gamma^{(n)}\left[\left(x+\gamma_{1}\right)^{2}+\left(y \cos \theta-z \sin \theta+\gamma_{2}\right)^{2}\right]^{n}}{(4 \nu)^{n}(n !)^{2}}, \\
& w=\left[\sum_{m=0}^{\infty} \frac{\alpha^{(m)}\left[\left(x+\gamma_{1}\right)^{2}+\left(y \cos \theta-z \sin \theta+\gamma_{2}\right)^{2}\right]^{m}}{(4 \nu)^{m} m !(m+1) !}\right. \\
& \left.+\frac{\beta x}{\left(x+\gamma_{1}\right)^{2}+\left(y \cos \theta-z \sin \theta+\gamma_{2}\right)^{2}}-\gamma_{2}^{\prime}\right] \sin \theta-\gamma_{3}^{\prime} \\
& +\cos \theta \sum_{n=0}^{\infty} \frac{\gamma^{(n)}\left[\left(x+\gamma_{1}\right)^{2}+\left(y \cos \theta-z \sin \theta+\gamma_{2}\right)^{2}\right]^{n}}{(4 \nu)^{n}(n !)^{2}}, \\
& p=\rho \sum_{m, n=0}^{\infty} \frac{\alpha^{(m)} \alpha^{(n)}\left[\left(x+\gamma_{1}\right)^{2}+\left(y \cos \theta-z \sin \theta+\gamma_{2}\right)^{2}\right]^{m+n+1}}{2(m+n+1) m !(m+1) ! n !(n+1) !(4 \nu)^{m+n}} \\
& +\rho \beta^{\prime} \arctan \frac{y \cos \theta-z \sin \theta+\gamma_{2}}{x}+\rho\left(\gamma_{1}^{\prime \prime} x+\gamma_{2}^{\prime \prime} y+\gamma_{3}^{\prime \prime} z\right)+\vartheta .
\end{aligned}
$$




\subsection{Moving-Frame Approach I}

Let $\alpha, \beta$ be given differentiable functions in $t$. Denote

$$
\Upsilon=\left(\begin{array}{ccc}
\cos \alpha & \sin \alpha \cos \beta & \sin \alpha \sin \beta \\
-\sin \alpha & \cos \alpha \cos \beta & \cos \alpha \sin \beta \\
0 & -\sin \beta & \cos \beta
\end{array}\right)
$$

and

$$
Q=\left(\begin{array}{ccc}
0 & \alpha^{\prime} & \beta^{\prime} \sin \alpha \\
-\alpha^{\prime} & 0 & \beta^{\prime} \cos \alpha \\
-\beta^{\prime} \sin \alpha & -\beta^{\prime} \cos \alpha & 0
\end{array}\right)
$$

Then

$$
\Upsilon^{-1}=\Upsilon^{T}=\left(\begin{array}{ccc}
\cos \alpha & -\sin \alpha & 0 \\
\sin \alpha \cos \beta & \cos \alpha \cos \beta & -\sin \beta \\
\sin \alpha \sin \beta & \cos \alpha \sin \beta & \cos \beta
\end{array}\right)
$$

and

$$
\frac{d}{d t}(\Upsilon)=Q \Upsilon
$$

Define the moving frames:

$$
\overrightarrow{\mathcal{U}}=\left(\begin{array}{c}
\mathcal{U} \\
\mathcal{V} \\
\mathcal{W}
\end{array}\right)=\Upsilon\left(\begin{array}{c}
u(t, x, y, z) \\
v(t, x, y, z) \\
w(t, x, y, z)
\end{array}\right), \quad \overrightarrow{\mathcal{X}}=\left(\begin{array}{c}
\mathcal{X} \\
\mathcal{Y} \\
\mathcal{Z}
\end{array}\right)=\Upsilon\left(\begin{array}{l}
x \\
y \\
z
\end{array}\right) .
$$

Set

$$
\widetilde{\nabla}^{T}=\left(\partial_{\mathcal{X}}, \partial_{\mathcal{Y}}, \partial_{\mathcal{Z}}\right)
$$

Then

$$
\nabla=\Upsilon^{T} \widetilde{\nabla}
$$

Thus

$$
\Delta=\partial_{x}^{2}+\partial_{y}^{2}+\partial_{z}^{2}=\nabla^{T} \nabla=\left(\widetilde{\nabla}^{T} \Upsilon\right)\left(\Upsilon^{T} \widetilde{\nabla}\right)=\widetilde{\nabla}^{T} \widetilde{\nabla}=\partial_{\mathcal{X}}^{2}+\partial_{\mathcal{Y}}^{2}+\partial_{\mathcal{Z}}^{2}
$$

Recall the notion in (9.1.5). The equation (9.3.7) yields

$$
u_{x}+v_{y}+w_{z}=\nabla^{T} \vec{u}=\left(\widetilde{\nabla}^{T} \Upsilon\right)\left(\Upsilon^{-1} \overrightarrow{\mathcal{U}}\right)=\widetilde{\nabla}^{T} \overrightarrow{\mathcal{U}}=\mathcal{U}_{\mathcal{X}}+\mathcal{V}_{\mathcal{Y}}+\mathcal{W}_{\mathcal{Z}}
$$

and

$$
\vec{u}^{T} \nabla=\left(\Upsilon^{T} \overrightarrow{\mathcal{U}}\right)^{T}\left(\Upsilon^{T} \widetilde{\nabla}\right)=\overrightarrow{\mathcal{U}}^{T} \Upsilon \Upsilon^{T} \widetilde{\nabla}=\overrightarrow{\mathcal{U}}^{T} \widetilde{\nabla}
$$

According to (9.3.3) and (9.3.5),

$$
\overrightarrow{\mathcal{U}}=\Upsilon \vec{u}\left(t, \vec{x}^{T}\right)=\Upsilon \vec{u}\left(t, \overrightarrow{\mathcal{X}}^{T} \Upsilon\right), \quad p(t, \vec{x})=p\left(t, \overrightarrow{\mathcal{X}}^{T} \Upsilon\right)
$$

By (9.3.3) and (9.3.4), we get

$$
\partial_{t}(\overrightarrow{\mathcal{X}})=\frac{d}{d t}(\Upsilon) \vec{x}=Q \Upsilon \vec{x}=Q \overrightarrow{\mathcal{X}}
$$




$$
\partial_{t}(\overrightarrow{\mathcal{U}})=\frac{d}{d t}(\Upsilon) \vec{u}+\Upsilon \vec{u}_{t}=Q \Upsilon \vec{u}+\Upsilon \vec{u}_{t}=Q \overrightarrow{\mathcal{U}}+\Upsilon \vec{u}_{t} .
$$

On the other hand,

$$
\partial_{t}(\overrightarrow{\mathcal{U}})=\overrightarrow{\mathcal{U}}_{t}+\left(\partial_{t}\left(\mathcal{X}^{T}\right) \widetilde{\nabla}\right)(\overrightarrow{\mathcal{U}})=\overrightarrow{\mathcal{U}}_{t}+\left(\mathcal{X}^{T} Q^{T} \widetilde{\nabla}\right)(\overrightarrow{\mathcal{U}})
$$

Thus

$$
\Upsilon \vec{u}_{t}=\overrightarrow{\mathcal{U}}_{t}+\left(\mathcal{X}^{T} Q^{T} \widetilde{\nabla}\right)(\overrightarrow{\mathcal{U}})-Q \overrightarrow{\mathcal{U}}
$$

Multiplying $\Upsilon$ to (9.1.7) from the left side, we get

$$
\Upsilon \vec{u}_{t}+\left(\vec{u}^{T} \nabla\right)(\Upsilon \vec{u})+\frac{1}{\rho} \Upsilon \nabla(p)=\nu \Delta(\Upsilon \vec{u}),
$$

which is equivalent to

$$
\overrightarrow{\mathcal{U}}_{t}+\left(\mathcal{X}^{T} Q^{T} \widetilde{\nabla}\right)(\overrightarrow{\mathcal{U}})-Q \overrightarrow{\mathcal{U}}+\left(\overrightarrow{\mathcal{U}}^{T} \widetilde{\nabla}\right)(\overrightarrow{\mathcal{U}})+\frac{1}{\rho} \widetilde{\nabla}(p)=\nu \Delta(\overrightarrow{\mathcal{U}})
$$

by (9.3.7)-(9.3.9) and (9.3.15). Moreover, (9.1.8), (9.3.5) and (9.3.7) imply

$$
\left(\widetilde{\nabla}^{T} \Upsilon\right)\left(\Upsilon^{-1} \overrightarrow{\mathcal{U}}\right)=0 \sim \widetilde{\nabla}^{T} \overrightarrow{\mathcal{U}}=0
$$

Next we want to find the analogue of (9.2.4). According to (9.3.2), (9.3.8) and (9.3.17), we denote

$$
\begin{aligned}
& R_{1}=\mathcal{U}_{t}+\alpha^{\prime}\left(\mathcal{Y} \mathcal{U}_{\mathcal{X}}-\mathcal{X} \mathcal{U}_{\mathcal{Y}}-\mathcal{V}\right)+\beta^{\prime}\left(\mathcal{Z} \mathcal{U}_{\mathcal{X}}-\mathcal{X} \mathcal{U}_{\mathcal{Z}}-\mathcal{W}\right) \sin \alpha \\
& +\beta^{\prime}\left(\mathcal{Z} \mathcal{U}_{\mathcal{Y}}-\mathcal{Y} \mathcal{U}_{\mathcal{Z}}\right) \cos \alpha+\mathcal{U} \mathcal{U}_{\mathcal{X}}+\mathcal{V} \mathcal{U}_{\mathcal{Y}}+\mathcal{W} \mathcal{U}_{\mathcal{Z}}-\nu \Delta(\mathcal{U}) \\
& R_{2}=\mathcal{V}_{t}+\alpha^{\prime}\left(\mathcal{Y} \mathcal{V}_{\mathcal{X}}-\mathcal{X} \mathcal{V}_{\mathcal{Y}}+\mathcal{U}\right)+\beta^{\prime}\left(\mathcal{Z} \mathcal{V}_{\mathcal{X}}-\mathcal{X} \mathcal{V}_{\mathcal{Z}}\right) \sin \alpha \\
& +\beta^{\prime}\left(\mathcal{Z} \mathcal{V}_{\mathcal{Y}}-\mathcal{Y} \mathcal{V}_{\mathcal{Z}}-\mathcal{W}\right) \cos \alpha+\mathcal{U} \mathcal{V}_{\mathcal{X}}+\mathcal{V} \mathcal{V}_{\mathcal{Y}}+\mathcal{W} \mathcal{V}_{\mathcal{Z}}-\nu \Delta(\mathcal{V}) \\
& R_{3}=\mathcal{W}_{t}+\alpha^{\prime}\left(\mathcal{Y} \mathcal{W}_{\mathcal{X}}-\mathcal{X} \mathcal{W}_{\mathcal{Y}}\right)+\beta^{\prime}\left(\mathcal{Z} \mathcal{W}_{\mathcal{X}}-\mathcal{X} \mathcal{W}_{\mathcal{Z}}+\mathcal{U}\right) \sin \alpha \\
& +\beta^{\prime}\left(\mathcal{Z} \mathcal{W}_{\mathcal{Y}}-\mathcal{Y} \mathcal{W}_{\mathcal{Z}}+\mathcal{V}\right) \cos \alpha+\mathcal{U} \mathcal{W}_{\mathcal{X}}+\mathcal{V} \mathcal{W}_{\mathcal{Y}}+\mathcal{W} \mathcal{W}_{\mathcal{Z}}-\nu \Delta(\mathcal{W})
\end{aligned}
$$

Then the Navier-Stokes equations (9.1.1)-(9.1.4) become

$$
\begin{gathered}
R_{1}+\frac{1}{\rho} p_{\mathcal{X}}=0, \quad R_{2}+\frac{1}{\rho} p_{\mathcal{Y}}=0, \quad R_{3}+\frac{1}{\rho} p_{\mathcal{Z}}=0 \\
\mathcal{U}_{\mathcal{X}}+\mathcal{V}_{\mathcal{Y}}+\mathcal{W}_{\mathcal{Z}}=0
\end{gathered}
$$

by (9.3.17) and (9.3.18). Instead of solving the equations in (9.3.21), we will first solve the following compatibility equations:

$$
\partial_{\mathcal{Y}}\left(R_{1}\right)=\partial_{\mathcal{X}}\left(R_{2}\right), \quad \partial_{\mathcal{Z}}\left(R_{1}\right)=\partial_{\mathcal{X}}\left(R_{3}\right), \quad \partial_{\mathcal{Z}}\left(R_{2}\right)=\partial_{\mathcal{Y}}\left(R_{3}\right)
$$


for $\mathcal{U}, \mathcal{V}, \mathcal{W}$, and then find $p$ from the equations via (9.3.22).

Let $f, g, h$ be functions of $t, \mathcal{X}, \mathcal{Y}, \mathcal{Z}$ that are linear in $\mathcal{X}, \mathcal{Y}, \mathcal{Z}$ and $f_{\mathcal{X}}+g_{\mathcal{Y}}+h_{\mathcal{Z}}=0$. Assume

$$
\mathcal{U}=f+6 \nu \mathcal{X}^{-1}, \quad \mathcal{V}=g+6 \nu \mathcal{Y} \mathcal{X}^{-2}, \quad \mathcal{W}=h .
$$

Then (9.3.19)-(9.3.21) become

$$
\begin{aligned}
R_{1}= & f_{t}+f f_{\mathcal{X}}+f_{\mathcal{Y}} g+f_{\mathcal{Z}} h+6 \nu f_{\mathcal{X}} \mathcal{X}^{-1}+\alpha^{\prime}\left(\mathcal{Y} f_{\mathcal{X}}-\mathcal{X} f_{\mathcal{Y}}-g\right) \\
& +\beta^{\prime}\left(\mathcal{Z} f_{\mathcal{X}}-\mathcal{X} f_{\mathcal{Z}}-h\right) \sin \alpha+\beta^{\prime}\left(\mathcal{Z} f_{\mathcal{Y}}-\mathcal{Y} f_{\mathcal{Z}}\right) \cos \alpha \\
& -6 \nu\left(f-\mathcal{Y} f_{\mathcal{Y}}+2 \alpha^{\prime} \mathcal{Y}+\beta^{\prime} \mathcal{Z} \sin \alpha\right) \mathcal{X}^{-2}-48 \nu^{2} \mathcal{X}^{-3}, \\
R_{2}= & g_{t}+f g_{\mathcal{X}}+g g_{\mathcal{Y}}+g_{\mathcal{Z}} h+\alpha^{\prime}\left(\mathcal{Y}_{g_{\mathcal{X}}}-\mathcal{X} g_{\mathcal{Y}}+f\right)+\beta^{\prime}\left(\mathcal{Z} g_{\mathcal{X}}-\mathcal{X} g_{\mathcal{Z}}\right) \sin \alpha \\
& -6 \nu g_{\mathcal{X}} \mathcal{X}^{-1}+6 \nu\left(g+\beta^{\prime} \mathcal{Z} \cos \alpha+\mathcal{Y} g_{\mathcal{Y}}\right) \mathcal{X}^{-2} \\
+ & \beta^{\prime}\left(\mathcal{Z} g_{\mathcal{Y}}-g_{\mathcal{Z}} \mathcal{Y}-h\right) \cos \alpha-12 \nu \mathcal{Y}\left(f+\alpha^{\prime} \mathcal{Y}+\beta^{\prime} \mathcal{Z} \sin \alpha\right) \mathcal{X}^{-3}, \\
R_{3}= & h_{t}+f h_{\mathcal{X}}+g h_{\mathcal{Y}}+h h_{\mathcal{Z}}+\alpha^{\prime}\left(\mathcal{Y} h_{\mathcal{X}}-\mathcal{X} h_{\mathcal{Y}}\right) \\
& +\beta^{\prime}\left(\mathcal{Z} h_{\mathcal{X}}-\mathcal{X} h_{\mathcal{Z}}+f\right) \sin \alpha+\beta^{\prime}\left(\mathcal{Z} h_{\mathcal{Y}}-\mathcal{Y} h_{\mathcal{Z}}+g\right) \cos \alpha \\
& +6 \nu\left(h_{x}+\beta^{\prime} \sin \alpha\right) \mathcal{X}^{-1}+6 \nu\left(h_{\mathcal{Y}}+\beta^{\prime} \cos \alpha\right) \mathcal{Y} \mathcal{X}^{-2} .
\end{aligned}
$$

By the coefficients of $\mathcal{X}^{-4}$ in $\partial_{\mathcal{Y}}\left(R_{1}\right)=\partial_{\mathcal{X}}\left(R_{2}\right)$, we take

$$
f=\gamma \mathcal{X}-\alpha^{\prime} \mathcal{Y}-\beta^{\prime} \mathcal{Z} \sin \alpha,
$$

where $\gamma$ is a functions in $t$. Moreover, the coefficients of $\mathcal{X}^{-3}$ and the coefficients of $\mathcal{X}^{-2}$ in $\partial_{\mathcal{Y}}\left(R_{1}\right)=\partial_{\mathcal{X}}\left(R_{2}\right)$ imply

$$
g=\alpha^{\prime} \mathcal{X}+\gamma \mathcal{Y}-\beta^{\prime} \mathcal{Z} \cos \alpha .
$$

Furthermore, $\partial_{\mathcal{Y}}\left(R_{1}\right)=\partial_{\mathcal{X}}\left(R_{2}\right)$ does not contain $\mathcal{X}^{-1}$.

According to the coefficients of $\mathcal{X}^{-2}$ in $\partial_{\mathcal{Z}}\left(R_{2}\right)=\partial_{\mathcal{Y}}\left(R_{3}\right)$, we find $h_{\mathcal{Y}}=-\beta^{\prime} \cos \alpha$. Moreover, the coefficients of $\mathcal{X}^{-2}$ in $\partial_{\mathcal{Z}}\left(R_{1}\right)=\partial_{\mathcal{X}}\left(R_{3}\right)$ force $h_{x}=-\beta^{\prime} \sin \alpha$. The condition $f_{\mathcal{X}}+g_{\mathcal{Y}}+h_{\mathcal{Z}}=0$ implies $h_{\mathcal{Z}}=-2 \gamma$. For simplicity, we take

$$
h=-\left(\beta^{\prime} \mathcal{X} \sin \alpha+\beta^{\prime} \mathcal{Y} \cos \alpha+2 \gamma \mathcal{Z}\right) .
$$

With the above $f, g$ and $h$, we have:

$$
\begin{aligned}
R_{1}= & \left(\gamma^{\prime}+\gamma^{2}-\alpha^{\prime 2}+3{\beta^{\prime}}^{2} \sin ^{2} \alpha\right) \mathcal{X}+12 \nu \alpha^{\prime} \mathcal{Y} \mathcal{X}^{-2}-48 \nu^{2} \mathcal{X}^{-3} \\
& +\left(3 \beta^{\prime 2} \sin \alpha \cos \alpha-\alpha^{\prime \prime}-2 \alpha^{\prime} \gamma\right) \mathcal{Y}+\left(4 \beta^{\prime} \gamma-\beta^{\prime \prime}\right) \mathcal{Z} \sin \alpha, \\
R_{2}= & \left(\gamma^{\prime}+\gamma^{2}-\alpha^{\prime 2}+3{\beta^{\prime}}^{2} \cos ^{2} \alpha\right) \mathcal{Y}-12 \nu \alpha^{\prime} \mathcal{X}^{-1} \\
& +\left(\alpha^{\prime \prime}+2 \alpha^{\prime} \gamma+3{\beta^{\prime}}^{2} \sin \alpha \cos \alpha\right) \mathcal{X}+\left(4 \beta^{\prime} \gamma-\beta^{\prime \prime}\right) \mathcal{Z} \cos \alpha,
\end{aligned}
$$




$$
R_{3}=\left(4 \gamma^{2}-2 \gamma^{\prime}-\beta^{\prime 2}\right) \mathcal{Z}+\left(4 \beta^{\prime} \gamma-\beta^{\prime \prime}\right)(\mathcal{X} \sin \alpha+\mathcal{Y} \cos \alpha)
$$

Thanks to (9.3.32)-(9.3.34), (9.3.24) is now equivalent to

$$
-\alpha^{\prime \prime}-2 \alpha^{\prime} \gamma=\alpha^{\prime \prime}+2 \alpha^{\prime} \gamma \Longrightarrow \gamma=-\frac{\alpha^{\prime \prime}}{2 \alpha^{\prime}}
$$

Thus

$$
\begin{gathered}
\mathcal{U}=-\frac{\alpha^{\prime \prime}}{2 \alpha^{\prime}} \mathcal{X}-\alpha^{\prime} \mathcal{Y}-\beta^{\prime} \mathcal{Z} \sin \alpha+6 \nu \mathcal{X}^{-1} \\
\mathcal{V}=\alpha^{\prime} \mathcal{X}-\frac{\alpha^{\prime \prime}}{2 \alpha^{\prime}} \mathcal{Y}-\beta^{\prime} \mathcal{Z} \cos \alpha+6 \nu \mathcal{Y} \mathcal{X}^{-2} \\
\mathcal{W}=\frac{\alpha^{\prime \prime}}{\alpha^{\prime}} \mathcal{Z}-\beta^{\prime} \mathcal{X} \sin \alpha-\beta^{\prime} \mathcal{Y} \cos \alpha
\end{gathered}
$$

by (9.3.25), (9.3.29)-(9.3.31) and (9.3.35). Moreover, (9.3.24) and (9.3.32)-(9.3.34) imply

$$
\begin{aligned}
p= & \rho\left\{\frac{\left(2 \alpha^{\prime} \alpha^{\prime \prime \prime}+4 \alpha^{4}-3 \alpha^{\prime \prime 2}\right)\left(\mathcal{X}^{2}+\mathcal{Y}^{2}\right)}{8 \alpha^{\prime 2}}+\left(\beta^{\prime \prime}+2 \alpha^{\prime \prime} \beta^{\prime} / \alpha^{\prime}\right) \mathcal{Z}(\mathcal{X} \sin \alpha+\mathcal{Y} \cos \alpha)\right. \\
& \left.-\frac{3 \beta^{\prime 2}(\mathcal{X} \sin \alpha+\mathcal{Y} \cos \alpha)^{2}}{2}+12 \nu\left(3 \nu \mathcal{X}^{-2}-\alpha^{\prime} \mathcal{Y} \mathcal{X}^{-1}\right)+\frac{\left(\alpha^{\prime} \beta^{\prime 2}-\alpha^{\prime \prime \prime}\right) \mathcal{Z}^{2}}{2 \alpha^{\prime}}\right\}
\end{aligned}
$$

Note $\vec{u}=\Upsilon^{-1} \overrightarrow{\mathcal{U}}$ by (9.3.5). Thus (9.3.3) yields

$$
\begin{aligned}
u= & \left(\frac{\alpha^{\prime \prime}}{2 \alpha^{\prime}}-6 \nu \mathcal{Y} \mathcal{X}^{-2}\right)(\mathcal{Y} \sin \alpha-\mathcal{X} \cos \alpha)-\alpha^{\prime}(\mathcal{X} \sin \alpha+\mathcal{Y} \cos \alpha) \\
v= & \left(6 \nu \mathcal{Y} \mathcal{X}^{-2}-\frac{\alpha^{\prime \prime}}{2 \alpha^{\prime}}\right)(\mathcal{X} \sin \alpha+\mathcal{Y} \cos \alpha) \cos \beta+\alpha^{\prime}(\mathcal{X} \cos \alpha-\mathcal{Y} \sin \alpha) \cos \beta \\
& -\beta^{\prime} \mathcal{Z} \cos \beta+\left(\beta^{\prime} \mathcal{X} \sin \alpha+\beta^{\prime} \mathcal{Y} \cos \alpha-\frac{\alpha^{\prime \prime}}{\alpha^{\prime}} \mathcal{Z}\right) \sin \beta \\
w= & \left(6 \nu \mathcal{Y} \mathcal{X}^{-2}-\frac{\alpha^{\prime \prime}}{2 \alpha^{\prime}}\right)(\mathcal{X} \sin \alpha+\mathcal{Y} \cos \alpha) \sin \beta+\alpha^{\prime}(\mathcal{X} \cos \alpha-\mathcal{Y} \sin \alpha) \sin \beta \\
& -\beta^{\prime} \mathcal{Z} \sin \beta+\left(\frac{\alpha^{\prime \prime}}{\alpha^{\prime}} \mathcal{Z}-\beta^{\prime} \mathcal{X} \sin \alpha-\beta^{\prime} \mathcal{Y} \cos \alpha\right) \cos \beta
\end{aligned}
$$

According to (9.3.5), $\overrightarrow{\mathcal{X}}=\Upsilon \vec{u}$. So (9.3.1) gives

$$
\begin{gathered}
\mathcal{Y} \sin \alpha-\mathcal{X} \cos \alpha=-x, \quad \mathcal{X} \sin \alpha+\mathcal{Y} \cos \alpha=y \cos \beta+z \sin \beta, \\
\mathcal{X}^{2}+\mathcal{Y}^{2}=x^{2}+(y \cos \beta+z \sin \beta)^{2} .
\end{gathered}
$$

Therefore, we have the following theorem:

Theorem 9.3.1. Let $\alpha$ and $\beta$ be functions in $t$ with $\alpha^{\prime} \neq 0$. We have the following solution of the Navier-Stokes equations (9.1.1)-(9.1.4):

$$
u=\frac{6 \nu x[(y \cos \beta+z \sin \beta) \cos \alpha-x \sin \alpha]}{[(y \cos \beta+z \sin \beta) \sin \alpha+x \cos \alpha]^{2}}-\frac{\alpha^{\prime \prime} x}{2 \alpha^{\prime}}-\alpha^{\prime}(y \cos \beta+z \sin \beta),
$$




$$
\begin{aligned}
v= & \frac{6 \nu[(y \cos \beta+z \sin \beta) \cos \alpha-x \sin \alpha](y \cos \beta+z \sin \beta) \cos \beta}{[(y \cos \beta+z \sin \beta) \sin \alpha+x \cos \alpha]^{2}}+\alpha^{\prime} x \cos \beta \\
& +\left[\beta^{\prime} \sin 2 \beta+\left(\alpha^{\prime \prime} / 2 \alpha^{\prime}\right)\left(\sin ^{2} \beta-\cos 2 \beta\right)\right] y-\left[\beta^{\prime} \cos 2 \beta+\left(3 \alpha^{\prime \prime} / 4 \alpha^{\prime}\right) \sin 2 \beta\right] z, \\
w= & \frac{6 \nu[(y \cos \beta+z \sin \beta) \cos \alpha-x \sin \alpha](y \cos \beta+z \sin \beta) \sin \beta}{[(y \cos \beta+z \sin \beta) \sin \alpha+x \cos \alpha]^{2}}+\alpha^{\prime} x \sin \beta \\
& -\left[\beta^{\prime} \cos 2 \beta+\left(3 \alpha^{\prime \prime} / 4 \alpha^{\prime}\right) \sin 2 \beta\right] y+\left[\left(\alpha^{\prime \prime} / 2 \alpha^{\prime}\right)\left(\cos ^{2} \beta+\cos 2 \beta\right)-\beta^{\prime} \sin 2 \beta\right] z
\end{aligned}
$$

and

$$
\begin{aligned}
p= & \rho\left\{\frac{12 \nu\left[6 \nu+\alpha^{\prime}\left[\left(x^{2}-(y \cos \beta+z \sin \beta)^{2}\right) \sin 2 \alpha-2 x(y \cos \beta+z \sin \beta) \cos 2 \alpha\right]\right]}{2[(y \cos \beta+z \sin \beta) \sin \alpha+x \cos \alpha]^{2}}\right. \\
& +\frac{\left(2 \alpha^{\prime}{\alpha^{\prime \prime}}^{\prime \prime}+4 \alpha^{\prime 4}-3{\alpha^{\prime \prime}}^{2}\right)\left[x^{2}+(y \cos \beta+z \sin \beta)^{2}\right]}{8{\alpha^{\prime}}^{2}} \\
& +\left(\beta^{\prime \prime} / 2+\alpha^{\prime \prime} \beta^{\prime} / \alpha^{\prime}\right)\left[\left(z^{2}-y^{2}\right) \sin 2 \beta+2 y z \cos 2 \beta\right] \\
& \left.-\frac{3 \beta^{\prime 2}(y \cos \beta+z \sin \beta)^{2}}{2}+\frac{\left(\alpha^{\prime} \beta^{\prime 2}-\alpha^{\prime \prime \prime}\right)(z \cos \beta-y \sin \beta)^{2}}{2 \alpha^{\prime}}\right\} .
\end{aligned}
$$

The above solution blows up on the following rotating plane:

$$
\left\{(x, y, z) \in \mathbb{R}^{3} \mid(y \cos \beta+z \sin \beta) \sin \alpha+x \cos \alpha=0\right\} .
$$

Applying the symmetry transformation in (9.1.32) and (9.1.33) to the above solution, we can get a solutions with six parameter functions and blowing up on a more general moving plane. Next let $f$ be a function in $t, \mathcal{Y}, \mathcal{Z}$ such that $\partial_{\mathcal{Y}}^{2}(f)=\partial_{\mathcal{Z}}^{2}(f)=0$, and let $\phi, \psi$ be functions in $t, \mathcal{X}$. Suppose that $\gamma$ is a function in $t$. Assume

$$
\mathcal{U}=f-2 \gamma^{\prime} \mathcal{X}, \quad \mathcal{V}=\phi+\gamma^{\prime} \mathcal{Y}, \quad \mathcal{W}=\psi+\gamma^{\prime} \mathcal{Z}
$$

Then

$$
\begin{aligned}
& R_{1}= f_{t}-2 \gamma^{\prime \prime} \mathcal{X}-\alpha^{\prime}\left(3 \gamma^{\prime} \mathcal{Y}+\mathcal{X} f_{\mathcal{Y}}+\phi\right)-\beta^{\prime}\left(3 \gamma^{\prime} \mathcal{Z}+\mathcal{X} f_{\mathcal{Z}}+\psi\right) \sin \alpha \\
&+\beta^{\prime}\left(\mathcal{Z} f_{\mathcal{Y}}-\mathcal{Y} f_{\mathcal{Z}}\right) \cos \alpha-2 \gamma^{\prime}\left(f-2 \gamma^{\prime} \mathcal{X}\right)+f_{\mathcal{Y}}\left(\phi+\gamma^{\prime} \mathcal{Y}\right)+f_{\mathcal{Z}}\left(\psi+\gamma^{\prime} \mathcal{Z}\right) \\
& R_{2}=\phi_{t}+\gamma^{\prime \prime} \mathcal{Y}+\alpha^{\prime}\left(\mathcal{Y} \phi_{\mathcal{X}}-3 \gamma^{\prime} \mathcal{X}+f\right)+\beta^{\prime} \mathcal{Z} \phi_{\mathcal{X}} \sin \alpha-\beta^{\prime} \psi \cos \alpha \\
& \quad+\left(f-2 \gamma^{\prime} \mathcal{X}\right) \phi_{\mathcal{X}}+\gamma^{\prime} \phi+\gamma^{\prime 2} \mathcal{Y}-\nu \phi_{\mathcal{X} \mathcal{X}} \\
& R_{3}=\psi_{t}+\gamma^{\prime \prime} \mathcal{Z}+\alpha^{\prime} \mathcal{Y} \psi_{\mathcal{X}}+\beta^{\prime}\left(\mathcal{Z} \psi_{\mathcal{X}}-3 \gamma^{\prime} \mathcal{X}+f\right) \sin \alpha-\nu \psi_{\mathcal{X X}} \\
& \quad+\beta^{\prime} \phi \cos \alpha+\left(f-2 \gamma^{\prime} \mathcal{X}\right) \psi_{\mathcal{X}}+\gamma^{\prime}\left(\psi+\gamma^{\prime} \mathcal{Z}\right) .
\end{aligned}
$$

Now (9.3.24) becomes

$$
\begin{aligned}
& \phi_{t \mathcal{X}}+\left(\alpha^{\prime} \mathcal{Y}+\beta^{\prime} \mathcal{Z} \sin \alpha+f\right) \phi_{\mathcal{X X}}-\beta^{\prime} \psi_{\mathcal{X}} \cos \alpha-2 \gamma^{\prime}\left(\mathcal{X}_{\mathcal{X}}\right)_{\mathcal{X}} \\
& +\gamma^{\prime} \phi_{\mathcal{X}}-\nu \phi_{\mathcal{X X X}}=f_{t \mathcal{Y}}-\beta^{\prime} f_{\mathcal{Z}} \cos \alpha-\gamma^{\prime} f_{\mathcal{Y}}
\end{aligned}
$$




$$
\begin{aligned}
& \psi_{t \mathcal{X}}+\left(\alpha^{\prime} \mathcal{Y}+\beta^{\prime} \mathcal{Z} \sin \alpha+f\right) \psi_{\mathcal{X X}}-\nu \psi_{\mathcal{X X} \mathcal{X}}+\beta^{\prime} \phi_{\mathcal{X}} \cos \alpha \\
& -2\left(\gamma^{\prime} \mathcal{X} \psi_{\mathcal{X}}\right)_{\mathcal{X}}+\gamma^{\prime} \psi_{\mathcal{X}}=f_{t \mathcal{Z}}+\beta^{\prime} f_{\mathcal{Y}} \cos \alpha-\gamma^{\prime} f_{\mathcal{Z}}, \\
& \alpha^{\prime} f_{\mathcal{Z}}+\left(\beta^{\prime} \sin \alpha+f_{\mathcal{Z}}\right) \phi_{\mathcal{X}}=\left(\alpha^{\prime}+f_{\mathcal{Y}}\right) \psi_{\mathcal{X}}+\beta^{\prime} f_{\mathcal{Y}} \sin \alpha .
\end{aligned}
$$

By (9.3.54) and (9.3.55), we take

$$
f=-\alpha^{\prime} \mathcal{Y}-\beta^{\prime} \mathcal{Z} \sin \alpha
$$

Note that (9.3.56) is implied by (9.3.57). Integrating (9.3.54) and (9.3.55), we obtain

$$
\begin{gathered}
\phi_{t}-2 \gamma^{\prime} \mathcal{X} \phi_{\mathcal{X}}+\gamma^{\prime} \phi-\nu \phi_{\mathcal{X X}}-\beta^{\prime} \psi \cos \alpha=\left[\beta^{\prime 2} \sin \alpha \cos \alpha+\alpha^{\prime} \gamma^{\prime}-\alpha^{\prime \prime}\right] \mathcal{X}+\beta_{1} \\
\psi_{t}-2 \gamma^{\prime} \mathcal{X} \psi_{\mathcal{X}}+\gamma^{\prime} \psi-\nu \psi_{\mathcal{X} \mathcal{X}}+\beta^{\prime} \phi \cos \alpha \\
=-\left[\left(\beta^{\prime} \sin \alpha\right)^{\prime}+\alpha^{\prime} \beta^{\prime} \cos \alpha-\gamma^{\prime} \beta^{\prime} \sin \alpha\right] \mathcal{X}+\beta_{2},
\end{gathered}
$$

where $\beta_{1}$ and $\beta_{2}$ are arbitrary functions in $t$. To solve the above problem, we write

$$
\beta^{\prime}=\frac{\varphi^{\prime}}{\cos \alpha}, \quad \gamma=\frac{1}{4} \ln \mu^{\prime}
$$

and set

$$
\begin{gathered}
\left(\begin{array}{c}
\hat{\phi} \\
\hat{\psi}
\end{array}\right)=\sqrt[4]{\mu^{\prime}}\left(\begin{array}{cc}
\cos \varphi & -\sin \varphi \\
\sin \varphi & \cos \varphi
\end{array}\right)\left(\begin{array}{c}
\phi \\
\psi
\end{array}\right) \\
\left(\begin{array}{c}
\gamma_{1} \\
\gamma_{2}
\end{array}\right)=\int \frac{1}{\sqrt[4]{\mu^{\prime}}}\left(\begin{array}{cc}
\cos \varphi & -\sin \varphi \\
\sin \varphi & \cos \varphi
\end{array}\right)\left(\begin{array}{c}
\varphi^{\prime 2} \tan \alpha+\frac{\alpha^{\prime} \mu^{\prime \prime}}{4 \mu^{\prime}}-\alpha^{\prime \prime} \\
-\left(\varphi^{\prime} \tan \alpha\right)^{\prime}-\alpha^{\prime} \varphi^{\prime}+\frac{\mu^{\prime \prime} \varphi^{\prime}}{4 \mu^{\prime}} \tan \alpha
\end{array}\right) d t
\end{gathered}
$$

Then (9.3.58) and (9.3.59) are equivalent to:

$$
\begin{aligned}
& \hat{\phi}_{t}-\frac{\mu^{\prime \prime}}{2 \mu^{\prime}} \mathcal{X} \hat{\phi}_{\mathcal{X}}-\nu \hat{\phi}_{\mathcal{X} \mathcal{X}}=\gamma_{1}^{\prime} \sqrt{\mu^{\prime}} \mathcal{X}+\varphi_{1}^{\prime}, \\
& \hat{\psi}_{t}-\frac{\mu^{\prime \prime}}{2 \mu^{\prime}} \mathcal{X} \hat{\psi}_{\mathcal{X}}-\nu \hat{\psi}_{\mathcal{X} \mathcal{X}}=\gamma_{2}^{\prime} \sqrt{\mu^{\prime}} \mathcal{X}+\varphi_{2}^{\prime},
\end{aligned}
$$

where $\varphi_{1}$ and $\varphi_{2}$ are arbitrary functions in $t$. Note the first two terms in the above equations motivate us to write

$$
\hat{\phi}=\tilde{\phi}(t, \varpi)+\gamma_{1} \varpi+\varphi_{1}, \quad \hat{\psi}=\tilde{\psi}(t, \varpi)+\gamma_{2} \varpi+\varphi_{2}, \quad \varpi=\sqrt{\mu^{\prime}} \mathcal{X}
$$

Then the above equations become equations:

$$
\tilde{\phi}_{t}-\nu \mu^{\prime} \tilde{\phi}_{\varpi \varpi}=0, \quad \tilde{\psi}_{t}-\nu \mu^{\prime} \tilde{\psi}_{\varpi \varpi}=0
$$


Thus we have the following solution:

$$
\begin{aligned}
& \tilde{\phi}=\sum_{r=1}^{m} a_{r} d_{r} e^{a_{r}^{2} \nu \mu \cos 2 b_{r}+a_{r} \varpi \cos b_{r}} \sin \left(a_{r}^{2} \nu \mu \sin 2 b_{r}+a_{r} \varpi \sin b_{r}+b_{r}+c_{r}\right), \\
& \tilde{\psi}=\sum_{s=1}^{n} \hat{a}_{s} \hat{d}_{s} e^{\hat{a}_{s}^{2} \nu \mu \cos 2 \hat{b}_{s}+\hat{a}_{s} \varpi \cos \hat{b}_{s}} \sin \left(\hat{a}_{s}^{2} \nu \mu \sin 2 \hat{b}_{s}+\hat{a}_{s} \varpi \sin \hat{b}_{s}+\hat{b}_{s}+\hat{c}_{s}\right),
\end{aligned}
$$

where $a_{r}, \hat{a}_{s}, b_{r}, \hat{b}_{s}, c_{r}, \hat{c}_{s}, d_{r}$ and $\hat{d}_{s}$ are real constants. Therefore,

$$
\begin{aligned}
\hat{\phi}= & \sum_{r=1}^{m} a_{r} d_{r} e^{a_{r}^{2} \nu \mu \cos 2 b_{r}+a_{r} \sqrt{\mu^{\prime}} \mathcal{X} \cos b_{r}} \sin \left(a_{r}^{2} \nu \mu \sin 2 b_{r}+a_{r} \sqrt{\mu^{\prime}} \mathcal{X} \sin b_{r}+b_{r}+c_{r}\right) \\
& +\gamma_{1} \sqrt{\mu^{\prime}} \mathcal{X}+\varphi_{1}, \\
\hat{\psi}= & \sum_{s=1}^{n} \hat{a}_{s} \hat{d}_{s} e^{\hat{a}_{s}^{2} \nu \mu \cos 2 \hat{b}_{s}+\hat{a}_{s} \sqrt{\mu^{\prime}} \mathcal{X} \cos \hat{b}_{s}} \sin \left(\hat{a}_{s}^{2} \nu \mu \sin 2 \hat{b}_{s}+\hat{a}_{s} \sqrt{\mu^{\prime}} \mathcal{X} \sin \hat{b}_{s}+\hat{b}_{s}+\hat{c}_{s}\right) \\
& +\gamma_{2} \sqrt{\mu^{\prime}} \mathcal{X}+\varphi_{2} .
\end{aligned}
$$

According to (9.3.61), we have

$$
\begin{aligned}
& \phi=\frac{\cos \varphi}{\sqrt[4]{\mu^{\prime}}} \sum_{r=1}^{m} a_{r} d_{r} e^{a_{r}^{2} \nu \mu \cos 2 b_{r}+a_{r} \sqrt{\mu^{\prime}} \mathcal{X} \cos b_{r}} \sin \left(a_{r}^{2} \nu \mu \sin 2 b_{r}+a_{r} \sqrt{\mu^{\prime}} \mathcal{X} \sin b_{r}+b_{r}+c_{r}\right) \\
& +\frac{\sin \varphi}{\sqrt[4]{\mu^{\prime}}} \sum_{s=1}^{n} \hat{a}_{s} \hat{d}_{s} e^{\hat{a}_{s}^{2} \nu \mu \cos 2 \hat{b}_{s}+\hat{a}_{s} \sqrt{\mu^{\prime}} \mathcal{X} \cos \hat{b}_{s}} \sin \left(\hat{a}_{s}^{2} \nu \mu \sin 2 \hat{b}_{s}+\hat{a}_{s} \sqrt{\mu^{\prime}} \mathcal{X} \sin \hat{b}_{s}+\hat{b}_{s}+\hat{c}_{s}\right) \\
& +\sqrt[4]{\mu^{\prime}}\left(\gamma_{1} \cos \varphi+\gamma_{2} \sin \varphi\right) \mathcal{X}+\sigma_{1} \\
& \psi=-\frac{\sin \varphi}{\sqrt[4]{\mu^{\prime}}} \sum_{r=1}^{m} a_{r} d_{r} e^{a_{r}^{2} \nu \mu \cos 2 b_{r}+a_{r} \sqrt{\mu^{\prime}} \mathcal{X} \cos b_{r}} \sin \left(a_{r}^{2} \nu \mu \sin 2 b_{r}+a_{r} \sqrt{\mu^{\prime}} \mathcal{X} \sin b_{r}+b_{r}+c_{r}\right) \\
& +\frac{\cos \varphi}{\sqrt[4]{\mu^{\prime}}} \sum_{s=1}^{n} \hat{a}_{s} \hat{d}_{s} e^{\hat{a}_{s}^{2} \nu \mu \cos 2 \hat{b}_{s}+\hat{a}_{s} \sqrt{\mu^{\prime}} \mathcal{X} \cos \hat{b}_{s}} \sin \left(\hat{a}_{s}^{2} \nu \mu \sin 2 \hat{b}_{s}+\hat{a}_{s} \sqrt{\mu^{\prime}} \mathcal{X} \sin \hat{b}_{s}+\hat{b}_{s}+\hat{c}_{s}\right) \\
& +\sqrt[4]{\mu^{\prime}}\left(\gamma_{2} \cos \varphi-\gamma_{1} \sin \varphi\right) \mathcal{X}+\sigma_{2}
\end{aligned}
$$

where $\sigma_{1}$ and $\sigma_{2}$ are arbitrary functions in $t$. By (9.3.50), (9.3.57), (9.3.60), (9.3.71) and $(9.3 .72)$

$$
\begin{gathered}
\mathcal{U}=-\alpha^{\prime} \mathcal{Y}-\varphi^{\prime} \mathcal{Z} \tan \alpha-\frac{\mu^{\prime \prime} \mathcal{X}}{2 \mu^{\prime}} \\
\mathcal{V}=\frac{\cos \varphi}{\sqrt[4]{\mu^{\prime}}} \sum_{r=1}^{m} a_{r} d_{r} e^{a_{r}^{2} \nu \mu \cos 2 b_{r}+a_{r} \sqrt{\mu^{\prime}} \mathcal{X} \cos b_{r}} \sin \left(a_{r}^{2} \nu \mu \sin 2 b_{r}+a_{r} \sqrt{\mu^{\prime}} \mathcal{X} \sin b_{r}+b_{r}+c_{r}\right) \\
+\frac{\sin \varphi}{\sqrt[4]{\mu^{\prime}}} \sum_{s=1}^{n} \hat{a}_{s} \hat{d}_{s} e^{\hat{a}_{s}^{2} \nu \mu \cos 2 \hat{b}_{s}+\hat{a}_{s} \sqrt{\mu^{\prime}} \mathcal{X} \cos \hat{b}_{s}} \sin \left(\hat{a}_{s}^{2} \nu \mu \sin 2 \hat{b}_{s}+\hat{a}_{s} \sqrt{\mu^{\prime}} \mathcal{X} \sin \hat{b}_{s}+\hat{b}_{s}+\hat{c}_{s}\right) \\
+\sqrt[4]{\mu^{\prime}}\left(\gamma_{1} \cos \varphi+\gamma_{2} \sin \varphi\right) \mathcal{X}+\frac{\mu^{\prime \prime} \mathcal{Y}}{4 \mu^{\prime}}+\sigma_{1},
\end{gathered}
$$




$$
\begin{aligned}
\mathcal{W}= & -\frac{\sin \varphi}{\sqrt[4]{\mu^{\prime}}} \sum_{r=1}^{m} a_{r} d_{r} e^{a_{r}^{2} \nu \mu \cos 2 b_{r}+a_{r} \sqrt{\mu^{\prime}} \mathcal{X} \cos b_{r}} \sin \left(a_{r}^{2} \nu \mu \sin 2 b_{r}+a_{r} \sqrt{\mu^{\prime}} \mathcal{X} \sin b_{r}+b_{r}+c_{r}\right) \\
& +\frac{\cos \varphi}{\sqrt[4]{\mu^{\prime}}} \sum_{s=1}^{n} \hat{a}_{s} \hat{d}_{s} e^{\hat{a}_{s}^{2} \nu \mu \cos 2 \hat{b}_{s}+\hat{a}_{s} \sqrt{\mu^{\prime}} \mathcal{X} \cos \hat{b}_{s}} \sin \left(\hat{a}_{s}^{2} \nu \mu \sin 2 \hat{b}_{s}+\hat{a}_{s} \sqrt{\mu^{\prime}} \mathcal{X} \sin \hat{b}_{s}+\hat{b}_{s}+\hat{c}_{s}\right) \\
& +\sqrt[4]{\mu^{\prime}}\left(\gamma_{2} \cos \varphi-\gamma_{1} \sin \varphi\right) \mathcal{X}++\frac{\mu^{\prime \prime} \mathcal{Z}}{4 \mu^{\prime}}+\sigma_{2} .
\end{aligned}
$$

To find the pressure $p$, we recalculate

$$
\begin{gathered}
R_{1}=\left(\varphi^{\prime 2} \mathcal{Y}-2 \varphi^{\prime} \psi\right) \tan \alpha-2 \alpha^{\prime} \phi-\alpha^{\prime \prime} \mathcal{Y}-\left(\varphi^{\prime \prime} \tan \alpha+\alpha^{\prime} \varphi^{\prime}\left(1+\sec ^{2} \alpha\right)\right) \mathcal{Z} \\
-\frac{\mu^{\prime \prime}\left(\alpha^{\prime} \mathcal{Y}+\varphi^{\prime} \mathcal{Z} \tan \alpha\right)}{2 \mu^{\prime}}+\left(\alpha^{\prime 2}+\varphi^{\prime 2} \tan ^{2} \alpha\right) \mathcal{X}+\frac{\left(3 \mu^{\prime \prime 2}-2 \mu^{\prime} \mu^{\prime \prime}\right) \mathcal{X}}{4 \mu^{\prime 2}} \\
R_{2}=\frac{\left(4 \mu^{\prime} \mu^{\prime \prime \prime}-3 \mu^{\prime \prime 2}\right) \mathcal{Y}}{16 \mu^{\prime 2}}-\alpha^{\prime 2} \mathcal{Y}+\sigma_{1}^{\prime}+\left(\varphi^{\prime} \mathcal{X}-\alpha^{\prime} \mathcal{Z}\right) \varphi^{\prime} \tan \alpha-\frac{\left(\alpha^{\prime} \mu^{\prime \prime}+2 \alpha^{\prime \prime} \mu^{\prime}\right) \mathcal{X}}{2 \mu^{\prime}} \\
R_{3}=\frac{\left(4 \mu^{\prime \prime}-3 \mu^{\prime 2}\right) \mathcal{Z}}{16 \mu^{\prime 2}}-\frac{\mu^{\prime \prime} \mathcal{X}+2 \alpha^{\prime} \mu^{\prime} \mathcal{Y}}{2 \mu^{\prime}} \varphi^{\prime} \tan \alpha+\sigma_{2}^{\prime} \\
-\varphi^{\prime 2} \mathcal{Z} \tan ^{2} \alpha-\left(\varphi^{\prime \prime} \tan \alpha+\alpha^{\prime} \varphi^{\prime}\left(1+\sec ^{2} \alpha\right)\right) \mathcal{X}
\end{gathered}
$$

by (9.3.51)-(9.3.53), (9.3.57)-(9.3.60), (9.3.71) and (9..72). Thanks to (9.3.22), we have

$$
\begin{aligned}
& p=\rho\left\{\left(\alpha^{\prime \prime} \mathcal{Y}+\left(\varphi^{\prime \prime} \tan \alpha+\alpha^{\prime} \varphi^{\prime}\left(1+\sec ^{2} \alpha\right)\right) \mathcal{Z}\right) \mathcal{X}+\frac{\mu^{\prime \prime}\left(\alpha^{\prime} \mathcal{Y}+\varphi^{\prime} \mathcal{Z} \tan \alpha\right) \mathcal{X}}{2 \mu^{\prime}}\right. \\
& -\sigma_{1}^{\prime} \mathcal{Y}-\sigma_{2}^{\prime} \mathcal{Z}+\frac{\mathcal{X}^{2}}{2}\left(\frac{\left(2 \mu^{\prime} \mu^{\prime \prime \prime}-3 \mu^{\prime \prime 2}\right)}{4 \mu^{\prime 2}}-\alpha^{\prime 2}-\varphi^{\prime 2} \tan ^{2} \varphi\right)+\frac{\alpha^{\prime 2} \mathcal{Y}^{2}+\gamma^{\prime 2} \mathcal{Z}^{2} \tan ^{2} \alpha}{2} \\
& +\frac{\left(3 \mu^{\prime \prime 2}-4 \mu^{\prime} \mu^{\prime \prime}\right)\left(\mathcal{Y}^{2}+\mathcal{Z}^{2}\right)}{32 \mu^{\prime 2}}+\left(\alpha^{\prime} \mathcal{Z}-\varphi^{\prime} \mathcal{X}\right) \varphi^{\prime} \mathcal{Y} \tan \alpha+2 \frac{\alpha^{\prime} \cos \varphi-\varphi^{\prime} \sin \varphi \tan \alpha}{\sqrt[4]{\mu^{\prime 3}}} \\
& \times \sum_{r=1}^{m} d_{r} e^{a_{r}^{2} \nu \mu \cos 2 b_{r}+a_{r} \sqrt{\mu^{\prime}} \mathcal{X} \cos b_{r}} \sin \left(a_{r}^{2} \nu \mu \sin 2 b_{r}+a_{r} \sqrt{\mu^{\prime}} \mathcal{X} \sin b_{r}+c_{r}\right) \\
& +2\left(\alpha^{\prime} \sigma_{1}+\varphi^{\prime} \sigma_{2} \tan \alpha\right) \mathcal{X}+2 \frac{\alpha^{\prime} \sin \varphi+\varphi^{\prime} \cos \varphi \tan \alpha}{\sqrt[4]{\mu^{\prime 3}}} \\
& \times \sum_{s=1}^{n} \hat{d}_{s} e^{\hat{a}_{s}^{2} \nu \mu \cos 2 \hat{b}_{s}+\hat{a}_{s} \sqrt{\mu^{\prime}} \mathcal{X} \cos \hat{b}_{s}} \sin \left(\hat{a}_{s}^{2} \nu \mu \sin 2 \hat{b}_{s}+\hat{a}_{s} \sqrt{\mu^{\prime}} \mathcal{X} \sin \hat{b}_{s}+\hat{c}_{s}\right) \\
& \left.+\sqrt[4]{\mu^{\prime}}\left[\gamma_{1}\left(\alpha^{\prime} \cos \varphi-\varphi^{\prime} \sin \varphi \tan \alpha\right)+\gamma_{2}\left(\alpha^{\prime} \sin \varphi+\varphi^{\prime} \cos \varphi \tan \alpha\right)\right] \mathcal{X}^{2} .\right\}
\end{aligned}
$$

By (9.3.3), (9.3.5) and (9.3.73)-(9.3.75), we get:

Theorem 9.3.2. Let $\alpha, \varphi, \mu, \sigma_{1}, \sigma_{2}$ be functions in $t$ with $\mu^{\prime}>0$. Take real constants $\left\{r, \hat{a}_{s}, b_{r}, \hat{b}_{s}, c_{r}, \hat{c}_{s}, d_{r}, \hat{d}_{s} \mid i=1, \ldots, m ; s=1, \ldots, n\right\}$. Denote $\beta=\int \varphi^{\prime} \sec \alpha d t$ and define 
$\gamma_{1}, \gamma_{2}$ by (9.3.62). Take the notations $\mathcal{X}, \mathcal{Y}, \mathcal{Z}$ given in (9.3.1) and (9.3.5). We have the following solution of the Navier-Stokes equations (9.1.1)-(9.1.4):

$$
\begin{aligned}
& u=-\left(\frac{\mu^{\prime \prime} \mathcal{X}}{2 \mu^{\prime}}+\alpha^{\prime} \mathcal{Y}+\varphi^{\prime} \mathcal{Z} \tan \alpha\right) \cos \alpha-\left[\frac{\cos \varphi}{\sqrt[4]{\mu^{\prime}}} \sum_{r=1}^{m} a_{r} d_{r} e^{a_{r}^{2} \nu \mu \cos 2 b_{r}+a_{r} \sqrt{\mu^{\prime}} \mathcal{X} \cos b_{r}}\right. \\
& \times \sin \left(a_{r}^{2} \nu \mu \sin 2 b_{r}+a_{r} \sqrt{\mu^{\prime}} \mathcal{X} \sin b_{r}+b_{r}+c_{r}\right)+\frac{\sin \varphi}{\sqrt[4]{\mu^{\prime}}} \sum_{s=1}^{n} \hat{a}_{s} \hat{d}_{s} e^{\hat{a}_{s}^{2} \nu \mu \cos 2 \hat{b}_{s}+\hat{a}_{s} \sqrt{\mu^{\prime}} \mathcal{X} \cos \hat{b}_{s}} \\
& \times \sin \left(\hat{a}_{s}^{2} \nu \mu \sin 2 \hat{b}_{s}+\hat{a}_{s} \sqrt{\mu^{\prime}} \mathcal{X} \sin \hat{b}_{s}+\hat{b}_{s}+\hat{c}_{s}\right) \\
& \left.+\sqrt[4]{\mu^{\prime}}\left(\gamma_{1} \cos \varphi+\gamma_{2} \sin \varphi\right) \mathcal{X}+\frac{\mu^{\prime \prime} \mathcal{Y}}{4 \mu^{\prime}}+\sigma_{1}\right] \sin \alpha, \\
& v=\left(\frac{\mu^{\prime \prime} \mathcal{X}}{2 \mu^{\prime}}-\alpha^{\prime} \mathcal{Y}-\varphi^{\prime} \mathcal{Z} \tan \alpha\right) \sin \alpha \cos \beta+\frac{\mu^{\prime \prime}(\mathcal{Y} \cos \alpha \cos \beta-\mathcal{Z} \sin \beta)}{4 \mu^{\prime}} \\
& +\frac{\cos \varphi \cos \alpha \cos \beta+\sin \varphi \sin \beta}{\sqrt[4]{\mu^{\prime}}} \sum_{r=1}^{m} a_{r} d_{r} e^{a_{r}^{2} \nu \mu \cos 2 b_{r}+a_{r} \sqrt{\mu^{\prime}} \mathcal{X} \cos b_{r}} \\
& \times \sin \left(a_{r}^{2} \nu \mu \sin 2 b_{r}+a_{r} \sqrt{\mu^{\prime}} \mathcal{X} \sin b_{r}+b_{r}+c_{r}\right)+\frac{\sin \varphi \cos \alpha \cos \beta-\cos \varphi \sin \beta}{\sqrt[4]{\mu^{\prime}}} \\
& \times \sum_{s=1}^{n} \hat{a}_{s} \hat{d}_{s} e^{\hat{a}_{s}^{2} \nu \mu \cos 2 \hat{b}_{s}+\hat{a}_{s} \sqrt{\mu^{\prime}} \mathcal{X} \cos \hat{b}_{s}} \sin \left(\hat{a}_{s}^{2} \nu \mu \sin 2 \hat{b}_{s}+\hat{a}_{s} \sqrt{\mu^{\prime}} \mathcal{X} \sin \hat{b}_{s}+\hat{b}_{s}+\hat{c}_{s}\right) \\
& +\sqrt[4]{\mu^{\prime}}\left[\gamma_{1}(\cos \varphi \cos \alpha \cos \beta+\sin \varphi \sin \beta)+\gamma_{2}(\sin \varphi \cos \alpha \cos \beta-\cos \varphi \sin \beta)\right] \mathcal{X} \\
& +\sigma_{1} \cos \alpha \cos \beta-\sigma_{2} \sin \beta \text {, } \\
& w=\left(\frac{\mu^{\prime \prime} \mathcal{X}}{2 \mu^{\prime}}-\alpha^{\prime} \mathcal{Y}-\varphi^{\prime} \mathcal{Z} \tan \alpha\right) \sin \alpha \sin \beta+\frac{\mu^{\prime \prime}(\mathcal{Y} \cos \alpha \sin \beta+\mathcal{Z} \cos \beta)}{4 \mu^{\prime}} \\
& +\frac{\cos \varphi \cos \alpha \sin \beta-\sin \varphi \cos \beta}{\sqrt[4]{\mu^{\prime}}} \sum_{r=1}^{m} a_{r} d_{r} e^{a_{r}^{2} \nu \mu \cos 2 b_{r}+a_{r} \sqrt{\mu^{\prime}} \mathcal{X} \cos b_{r}} \\
& \times \sin \left(a_{r}^{2} \nu \mu \sin 2 b_{r}+a_{r} \sqrt{\mu^{\prime}} \mathcal{X} \sin b_{r}+b_{r}+c_{r}\right)+\frac{\sin \varphi \cos \alpha \sin \beta+\cos \varphi \cos \beta}{\sqrt[4]{\mu^{\prime}}}
\end{aligned}
$$

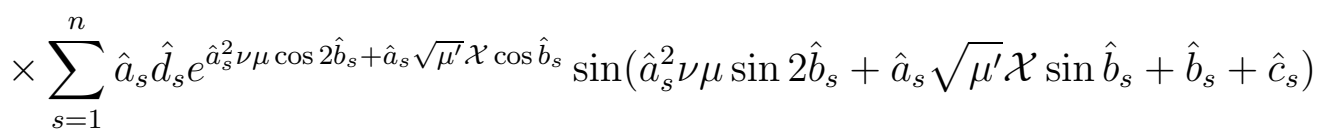

$$
\begin{aligned}
& +\sqrt[4]{\mu^{\prime}}\left[\gamma_{1}(\cos \varphi \cos \alpha \sin \beta-\sin \varphi \cos \beta)+\gamma_{2}(\sin \varphi \cos \alpha \sin \beta+\cos \varphi \cos \beta)\right] \mathcal{X} \\
& +\sigma_{1} \cos \alpha \sin \beta+\sigma_{2} \cos \beta \text {, }
\end{aligned}
$$

and $p$ is given in (9.3.79).

Remark 9.3.3. We can use Fourier expansion to solve the system (9.3.66) for $\tilde{\phi}\left(t, \sqrt{\mu^{\prime}} \mathcal{X}\right)$ and $\tilde{\psi}\left(t, \sqrt{\mu^{\prime}} \mathcal{X}\right)$ with given $\tilde{\phi}\left(0, \sqrt{\mu^{\prime}(0)} \mathcal{X}\right)$ and $\tilde{\psi}\left(0, \sqrt{\mu^{\prime}(0)} \mathcal{X}\right)$. In this way, we can obtain discontinuous solutions of the Navier-Stokes equations (9.1.1)-(9.1.4), which may be useful in studying shock waves. 


\subsection{Moving-Frame Approach II}

Motivated from the first solution in Theorem 9.2.2, we will solve the equations (9.3.17) and (9.3.18) by sin, cos, sinh and cosh functions.

First we rewrite (9.3.19)-(9.3.21):

$$
\begin{aligned}
& R_{1}=\mathcal{U}_{t}+\left(\alpha^{\prime} \mathcal{Y}+\beta^{\prime} \mathcal{Z} \sin \alpha+\mathcal{U}\right) \mathcal{U}_{\mathcal{X}}+\left(\mathcal{V}-\alpha^{\prime} \mathcal{X}+\beta^{\prime} \mathcal{Z} \cos \alpha\right) \mathcal{U}_{\mathcal{Y}} \\
& +\left(\mathcal{W}-\beta^{\prime}(\mathcal{X} \sin \alpha+\mathcal{Y} \cos \alpha)\right) \mathcal{U}_{\mathcal{Z}}-\alpha^{\prime} \mathcal{V}-\beta^{\prime} \mathcal{W} \sin \alpha-\nu \Delta(\mathcal{U}) \\
& R_{2}=\mathcal{V}_{t}+\left(\alpha^{\prime} \mathcal{Y}+\beta^{\prime} \mathcal{Z} \sin \alpha+\mathcal{U}\right) \mathcal{V}_{\mathcal{X}}+\left(\mathcal{V}-\alpha^{\prime} \mathcal{X}+\beta^{\prime} \mathcal{Z} \cos \alpha\right) \mathcal{V}_{\mathcal{Y}} \\
& +\left(\mathcal{W}-\beta^{\prime}(\mathcal{X} \sin \alpha+\mathcal{Y} \cos \alpha)\right) \mathcal{V}_{\mathcal{Z}}+\alpha^{\prime} \mathcal{U}-\beta^{\prime} \mathcal{W} \cos \alpha-\nu \Delta(\mathcal{V}) \\
& R_{3}=\mathcal{W}_{t}+\left(\alpha^{\prime} \mathcal{Y}+\beta^{\prime} \mathcal{Z} \sin \alpha+\mathcal{U}\right) \mathcal{W}_{\mathcal{X}}+\left(\mathcal{V}-\alpha^{\prime} \mathcal{X}+\beta^{\prime} \mathcal{Z} \cos \alpha\right) \mathcal{W}_{\mathcal{Y}} \\
& +\left(\mathcal{W}-\beta^{\prime}(\mathcal{X} \sin \alpha+\mathcal{Y} \cos \alpha)\right) \mathcal{W}_{\mathcal{Z}}+\beta^{\prime}(\mathcal{U} \sin \alpha+\mathcal{V} \cos \alpha)-\nu \Delta(\mathcal{W})
\end{aligned}
$$

Let $\alpha_{1}, \beta_{1}, \gamma$ be functions in $t$. Set

$$
\begin{gathered}
\xi_{0}=\sinh \left(\alpha_{1} \mathcal{Y}+\beta_{1} \mathcal{Z}\right), \quad \zeta_{0}=\cosh \left(\alpha_{1} \mathcal{Y}+\beta_{1} \mathcal{Z}\right), \quad \phi_{0}=\sinh \gamma \mathcal{X}, \\
\psi_{0}=\cosh \gamma \mathcal{X}, \quad \xi_{1}=\sin \left(\alpha_{1} \mathcal{Y}+\beta_{1} \mathcal{Z}\right), \quad \zeta_{1}=\cos \left(\alpha_{1} \mathcal{Y}+\beta_{1} \mathcal{Z}\right), \\
\phi_{1}=\sin \gamma \mathcal{X}, \quad \psi_{1}=\cos \gamma \mathcal{X}, \quad \Delta_{1}=\partial_{\mathcal{Y}}^{2}+\partial_{\mathcal{Z}}^{2} .
\end{gathered}
$$

Suppose that $f$ and $h$ are functions in $t, \mathcal{Y}, \mathcal{Z}$. Moreover, $\sigma$ and $\tau$ are functions in $t$. According to (9.3.29)-(9.3.31), we assume

$$
\begin{gathered}
\mathcal{U}=-\alpha^{\prime} \mathcal{Y}-\beta^{\prime} \mathcal{Z} \sin \alpha-\left(f_{\mathcal{Y}}+h_{\mathcal{Z}}\right) \mathcal{X}-\left(\alpha_{1} \sigma+\beta_{1} \tau\right) \zeta_{r} \phi_{s} \\
\mathcal{V}=\alpha^{\prime} \mathcal{X}-\beta^{\prime} \mathcal{Z} \cos \alpha+f+\sigma \gamma \xi_{r} \psi_{s}, \quad \mathcal{W}=\beta^{\prime}(\mathcal{X} \sin \alpha+\mathcal{Y} \cos \alpha)+h+\tau \gamma \xi_{r} \psi_{s}
\end{gathered}
$$

By (9.4.1)-(9.4.3), we have

$$
\begin{aligned}
& R_{1}=-\left(\alpha_{1} \sigma+\beta_{1} \tau\right)^{\prime} \zeta_{r} \phi_{s}-\left(\alpha_{1} \sigma+\beta_{1} \tau\right)\left[(-1)^{r}\left(\alpha_{1}^{\prime} \mathcal{Y}+\beta_{1}^{\prime} \mathcal{Z}\right) \xi_{r} \phi_{s}+\gamma^{\prime} \mathcal{X} \zeta_{r} \psi_{s}\right] \\
& -\left(f_{\mathcal{Y} t}+h_{\mathcal{Z} t}\right) \mathcal{X}+\left(\left(f_{\mathcal{Y}}+h_{\mathcal{Z}}\right) \mathcal{X}+\left(\alpha_{1} \sigma+\beta_{1} \tau\right) \zeta_{r} \phi_{s}\right)\left(f_{\mathcal{Y}}+h_{\mathcal{Z}}+\gamma\left(\alpha_{1} \sigma+\beta_{1} \tau\right) \zeta_{r} \psi_{s}\right) \\
& -\alpha^{\prime}\left(f+\alpha^{\prime} \mathcal{X}-\beta^{\prime} \mathcal{Z} \cos \alpha+\gamma \sigma \xi_{r} \psi_{s}\right)-\beta^{\prime}\left(\beta^{\prime}(\mathcal{X} \sin \alpha+\mathcal{Y} \cos \alpha)+h+\gamma \tau \xi_{r} \psi_{s}\right) \sin \alpha \\
& -\left(f+\gamma \sigma \xi_{r} \psi_{s}\right)\left(\alpha^{\prime}+\left(f_{\mathcal{Y Y}}+h_{\mathcal{Y Z}}\right) \mathcal{X}+(-1)^{r} \alpha_{1}\left(\alpha_{1} \sigma+\beta_{1} \tau\right) \xi_{r} \phi_{s}\right)-\left(h+\gamma \tau \xi_{r} \psi_{s}\right) \\
& \times\left(\beta^{\prime} \sin \alpha+\left(f_{\mathcal{Y Z}}+h_{\mathcal{Z Z}}\right) \mathcal{X}+(-1)^{r} \beta_{1}\left(\alpha_{1} \sigma+\beta_{1} \tau\right) \xi_{r} \phi_{s}\right)+\nu\left\{\Delta_{1}\left(f_{\mathcal{Y}}+h_{\mathcal{Z}}\right) \mathcal{X}\right. \\
& \left.+\left(\alpha_{1} \sigma+\beta_{1} \tau\right)\left[(-1)^{r}\left(\alpha_{1}^{2}+\beta_{1}^{2}\right)+(-1)^{s} \gamma^{2}\right] \zeta_{r} \phi_{s}\right\}-\alpha^{\prime \prime} \mathcal{Y}-\left(\beta^{\prime} \sin \alpha\right)^{\prime} \mathcal{Z} \\
& =\left\{\left(\gamma\left(f_{\mathcal{Y}}+h_{\mathcal{Z}}\right)-\gamma^{\prime}\right) \mathcal{X} \zeta_{r} \psi_{s}-(-1)^{r}\left(\alpha_{1}^{\prime} \mathcal{Y}+\beta_{1}^{\prime} \mathcal{Z}+\alpha_{1} f+\beta_{1} h\right) \xi_{r} \phi_{s}\right\}\left(\alpha_{1} \sigma+\beta_{1} \tau\right) \\
& +\left\{\left(\alpha_{1} \sigma+\beta_{1} \tau\right)\left[(-1)^{s} \nu \gamma^{2}+(-1)^{r} \nu\left(\alpha_{1}^{2}+\beta_{1}^{2}\right)+f_{\mathcal{Y}}+h_{\mathcal{Z}}\right]-\left(\alpha_{1} \sigma+\tau \beta_{1}\right)^{\prime}\right\} \zeta_{r} \phi_{s} \\
& -\gamma\left\{2\left(\sigma \alpha^{\prime}+\tau \beta^{\prime} \sin \alpha\right)+\left[\sigma\left(f_{\mathcal{Y Y}}+h_{\mathcal{Y Z}}\right)+\tau\left(f_{\mathcal{Y Z}}+h_{\mathcal{Z Z}}\right)\right] \mathcal{X}\right\} \xi_{r} \psi_{s}-\left(f_{\mathcal{Y} t}+h_{\mathcal{Z} t}\right) \mathcal{X} \\
& +\left(f_{\mathcal{Y}}+h_{\mathcal{Z}}\right)^{2} \mathcal{X}-f\left(\alpha^{\prime}+\left(f_{\mathcal{Y Y}}+h_{\mathcal{Y Z}}\right) \mathcal{X}\right)-h\left(\beta^{\prime} \sin \alpha+\left(f_{\mathcal{Y Z}}+h_{\mathcal{Z Z}}\right) \mathcal{X}\right) \\
& -\alpha^{\prime}\left(f+\alpha^{\prime} \mathcal{X}-\beta^{\prime} \mathcal{Z} \cos \alpha\right)-\beta^{\prime}\left(\beta^{\prime}(\mathcal{X} \sin \alpha+\mathcal{Y} \cos \alpha)+h\right) \sin \alpha-\alpha^{\prime \prime} \mathcal{Y} \\
& -\left(\beta^{\prime} \sin \alpha\right)^{\prime} \mathcal{Z}+\nu \Delta_{1}\left(f_{\mathcal{Y}}+h_{\mathcal{Z}}\right) \mathcal{X}+\gamma\left(\alpha_{1} \sigma+\beta_{1} \tau\right)^{2} \phi_{s} \psi_{s}
\end{aligned}
$$




$$
\begin{aligned}
& R_{2}=\alpha^{\prime \prime} \mathcal{X}-\left(\beta^{\prime} \cos \alpha\right)^{\prime} \mathcal{Z}+f_{t}+(\gamma \sigma)^{\prime} \xi_{r} \psi_{s}+\gamma \sigma\left(\left(\alpha_{1}^{\prime} \mathcal{Y}+\beta_{1}^{\prime} \mathcal{Z}\right) \zeta_{r} \psi_{s}+(-1)^{s} \gamma^{\prime} \mathcal{X} \xi_{r} \phi_{s}\right) \\
& -\alpha^{\prime}\left[\alpha^{\prime} \mathcal{Y}+\beta^{\prime} \mathcal{Z} \sin \alpha+\left(f_{\mathcal{Y}}+h_{\mathcal{Z}}\right) \mathcal{X}+\left(\alpha_{1} \sigma+\beta_{1} \tau\right) \zeta_{r} \phi_{s}\right]-\beta^{\prime}\left[\beta^{\prime}(\mathcal{X} \sin \alpha+\mathcal{Y} \cos \alpha)\right. \\
& \left.+h+\gamma \tau \xi_{r} \psi_{s}\right] \cos \alpha-\left[\left(f_{\mathcal{Y}}+h_{\mathcal{Z}}\right) \mathcal{X}+\left(\alpha_{1} \sigma+\beta_{1} \tau\right) \zeta_{r} \phi_{s}\right]\left(\alpha^{\prime}+(-1)^{s} \gamma^{2} \sigma \xi_{r} \phi_{s}\right) \\
& +\left(f+\gamma \sigma \xi_{r} \psi_{s}\right)\left(f_{\mathcal{Y}}+\alpha_{1} \gamma \sigma \zeta_{r} \psi_{s}\right)+\left(h+\gamma \tau \xi_{r} \psi_{s}\right)\left(f_{\mathcal{Z}}-\beta^{\prime} \cos \alpha+\beta_{1} \gamma \sigma \zeta_{r} \psi_{s}\right) \\
& -\nu\left[\Delta_{1}(f)+\gamma \sigma\left((-1)^{r}\left(\alpha_{1}^{2}+\beta_{1}^{2}\right)+(-1)^{s} \gamma^{2}\right)\right] \xi_{r} \psi_{s} \\
& =\alpha^{\prime \prime} \mathcal{X}+f_{t}+\gamma \sigma\left(\alpha_{1}^{\prime} \mathcal{Y}+\beta_{1}^{\prime} \mathcal{Z}+\alpha_{1} f+\beta_{1} h\right) \zeta_{r} \psi_{s}+(-1)^{s} \gamma \sigma\left(\gamma^{\prime}-\gamma\left(f_{\mathcal{Y}}+h_{\mathcal{Z}}\right)\right) \mathcal{X} \xi_{r} \phi_{s} \\
& +\left\{\gamma\left[\sigma f_{\mathcal{Y}}+\tau f_{\mathcal{Z}}-\nu \sigma\left[(-1)^{r}\left(\alpha_{1}^{2}+\beta_{1}^{2}\right)+(-1)^{s} \gamma^{2}\right]-2 \tau \beta^{\prime} \cos \alpha\right]+(\gamma \sigma)^{\prime}\right\} \xi_{r} \psi_{s} \\
& -2 \alpha^{\prime}\left(\alpha_{1} \sigma+\beta_{1} \tau\right) \zeta_{r} \phi_{s}-\left(\beta^{\prime} \cos \alpha\right)^{\prime} \mathcal{Z}-\alpha^{\prime}\left[\alpha^{\prime} \mathcal{Y}+\beta^{\prime} \mathcal{Z} \sin \alpha+2\left(f_{\mathcal{Y}}+h_{\mathcal{Z}}\right) \mathcal{X}\right] \\
& -\beta^{\prime}\left[\beta^{\prime}(\mathcal{X} \sin \alpha+\mathcal{Y} \cos \alpha)+h\right] \cos \alpha+f f_{\mathcal{Y}}+h\left(f_{\mathcal{Z}}-\beta^{\prime} \cos \alpha\right)-\nu \Delta_{1}(f) \\
& +\gamma^{2} \sigma\left(\alpha_{1} \sigma+\beta_{1} \tau\right) \xi_{r} \zeta_{r} \\
& R_{3}=\left(\beta^{\prime} \sin \alpha\right)^{\prime} \mathcal{X}+\left(\beta^{\prime} \cos \alpha\right)^{\prime} \mathcal{Y}+h_{t}+(\tau \gamma)^{\prime} \xi_{r} \psi_{s}+\tau \gamma\left[\left(\alpha_{1}^{\prime} \mathcal{Y}+\beta_{1}^{\prime} \mathcal{Z}\right) \zeta_{r} \psi_{s}\right. \\
& \left.+(-1)^{s} \gamma^{\prime} \mathcal{X} \xi_{r} \phi_{s}\right]-\left[\left(f_{\mathcal{Y}}+h_{\mathcal{Z}}\right) \mathcal{X}+\left(\alpha_{1} \sigma+\beta_{1} \tau\right) \zeta_{r} \phi_{s}\right]\left(\beta^{\prime} \sin \alpha+(-1)^{s} \tau \gamma^{2} \xi_{r} \phi_{s}\right) \\
& +\left(f+\sigma \gamma \xi_{r} \psi_{s}\right)\left(\beta^{\prime} \cos \alpha+h_{\mathcal{Y}}+\alpha_{1} \tau \gamma \zeta_{r} \psi_{s}\right)+\left(h+\tau \gamma \xi_{r} \psi_{s}\right)\left(h_{\mathcal{Z}}+\beta_{1} \tau \gamma \zeta_{r} \psi_{s}\right) \\
& -\beta^{\prime}\left(\alpha^{\prime} \mathcal{Y}+\beta^{\prime} \mathcal{Z} \sin \alpha+\left(f_{\mathcal{Y}}+h_{\mathcal{Z}}\right) \mathcal{X}+\left(\alpha_{1} \sigma+\beta_{1} \tau\right) \zeta_{r} \phi_{s}\right) \sin \alpha+\beta^{\prime}\left(\alpha^{\prime} \mathcal{X}-\beta^{\prime} \mathcal{Z} \cos \alpha\right. \\
& \left.+f+\sigma \gamma \xi_{r} \psi_{s}\right) \cos \alpha-\nu\left[\Delta_{1}(h)+\gamma \tau\left((-1)^{r}\left(\alpha_{1}^{2}+\beta_{1}^{2}\right)+(-1)^{s} \gamma^{2}\right)\right] \xi_{r} \psi_{s} \\
& =\gamma \tau\left(\alpha_{1}^{\prime} \mathcal{Y}+\beta_{1}^{\prime} \mathcal{Z}+\alpha_{1} f+\beta_{1} h\right) \zeta_{r} \psi_{s}+\left\{(\tau \gamma)^{\prime}-\nu \gamma \tau\left[(-1)^{r}\left(\alpha_{1}^{2}+\beta_{1}^{2}\right)+(-1)^{s} \gamma^{2}\right]\right. \\
& \left.+\gamma\left(2 \beta^{\prime} \sigma \cos \alpha+\sigma h_{\mathcal{Y}}+\tau h_{\mathcal{Z}}\right)\right\} \xi_{r} \psi_{s}-2 \beta^{\prime}\left(\alpha_{1} \sigma+\beta_{1} \tau\right) \zeta_{r} \phi_{s} \sin \alpha+(-1)^{s} \gamma \tau\left(\gamma^{\prime}\right. \\
& \left.-\gamma\left(f_{\mathcal{Y}}+h_{\mathcal{Z}}\right)\right) \mathcal{X} \xi_{r} \phi_{s}+\left(\beta^{\prime} \sin \alpha\right)^{\prime} \mathcal{X}+\left(\beta^{\prime} \cos \alpha\right)^{\prime} \mathcal{Y}+h_{t}-\beta^{\prime}\left(f_{\mathcal{Y}}+h_{\mathcal{Z}}\right) \mathcal{X} \sin \alpha \\
& +f\left(\beta^{\prime} \cos \alpha+h_{\mathcal{Y}}\right)+h h_{\mathcal{Z}}-\beta^{\prime}\left(\alpha^{\prime} \mathcal{Y}+\beta^{\prime} \mathcal{Z} \sin \alpha+\left(f_{\mathcal{Y}}+h_{\mathcal{Z}}\right) \mathcal{X}\right) \sin \alpha+\beta^{\prime}\left(\alpha^{\prime} \mathcal{X}\right. \\
& \left.-\beta^{\prime} \mathcal{Z} \cos \alpha+f\right) \cos \alpha-\nu \Delta_{1}(h)+\gamma^{2} \tau\left(\alpha_{1} \sigma+\beta_{1} \tau\right) \xi_{r} \zeta_{r} .
\end{aligned}
$$

By the coefficients of $\xi_{r} \psi_{s}$ in the equation $\partial_{\mathcal{Y}}\left(R_{1}\right)=\partial_{\mathcal{X}}\left(R_{2}\right)$, we have

$$
\gamma^{2} \sigma=(-1)^{r+s+1} \alpha_{1}\left(\alpha_{1} \sigma+\beta_{1} \tau\right), \quad\left[\sigma\left(f_{\mathcal{Y Y}}+h_{\mathcal{Y Z}}\right)+\tau\left(f_{\mathcal{Y Z}}+h_{\mathcal{Z Z Z}}\right)\right]_{\mathcal{Y}}=0 .
$$

Moreover, the coefficients of $\zeta_{r} \phi_{s}$ in the equation $\partial_{\mathcal{Y}}\left(R_{1}\right)=\partial_{\mathcal{X}}\left(R_{2}\right)$ suggest

$$
\left(f_{\mathcal{Y}}+h_{\mathcal{Z}}\right)_{\mathcal{Y}}=0
$$

which implies the second equation in (9.4.12). According the coefficients of $\xi_{r} \phi_{s}$ in the equation $\partial_{\mathcal{Y}}\left(R_{1}\right)=\partial_{\mathcal{X}}\left(R_{2}\right)$, we get

$$
\sigma \beta_{1} h_{\mathcal{Y}}=\tau \alpha_{1}\left(f_{\mathcal{Z}}-2 \beta^{\prime} \cos \alpha\right) .
$$

Furthermore, the coefficients of $\zeta_{r} \psi_{s}$ in the equation $\partial_{\mathcal{Y}}\left(R_{1}\right)=\partial_{\mathcal{X}}\left(R_{2}\right)$ yield

$$
\alpha_{1} \beta^{\prime} \sin \alpha=\alpha^{\prime} \beta_{1}
$$


Symmetrically, we have (9.4.15),

$$
\gamma^{2} \tau=(-1)^{r+s+1} \beta_{1}\left(\alpha_{1} \sigma+\beta_{1} \tau\right), \quad\left(f_{\mathcal{Y}}+h_{\mathcal{Z}}\right)_{\mathcal{Z}}=0
$$

and

$$
\tau \alpha_{1} f_{\mathcal{Z}}=\sigma \beta_{1}\left(h_{\mathcal{Y}}+2 \beta^{\prime} \cos \alpha\right)
$$

(cf. (9.4.7) and (9.4.8)). By the first equation in (9.4.12) and (9.4.16), we have

$$
\sigma \beta_{1}=\tau \alpha_{1}
$$

Then (9.4.14) is implied by (9.4.17) and (9.4.18). Note that the equations of the coefficients $\xi_{r} \psi_{s}, \zeta_{r} \psi_{s}, \xi_{r} \phi_{s}$ and $\zeta_{r} \phi_{s}$ in $\partial_{\mathcal{Z}}\left(R_{2}\right)=\partial_{\mathcal{Y}}\left(R_{3}\right)$ are implied by (9.4.15), (9.4.17) and (9.4.18).

According to (9.4.13) and the second equation in (9.4.16),

$$
f_{\mathcal{Y}}+h_{\mathcal{Z}}=\gamma_{1}
$$

a function in $t$. Under the conditions in (9.4.15), the first equation in (9.4.16), and (9.4.17)-(9.4.19), $\partial_{\mathcal{Y}}\left(R_{1}\right)=\partial_{\mathcal{X}}\left(R_{2}\right)$ becomes

$$
\alpha^{\prime} h_{\mathcal{Z}}-\beta^{\prime} h_{\mathcal{Y}} \sin \alpha=\alpha^{\prime \prime}
$$

$\partial_{\mathcal{Z}}\left(R_{1}\right)=\partial_{\mathcal{X}}\left(R_{3}\right)$ is equivalent to

$$
\beta^{\prime} h_{\mathcal{Z}} \sin \alpha+\alpha^{\prime} h_{y}=\beta^{\prime} \gamma_{1} \sin \alpha-\left(\beta^{\prime} \sin \alpha\right)^{\prime}-2 \alpha^{\prime} \beta^{\prime} \cos \alpha
$$

and $\partial_{\mathcal{Z}}\left(R_{2}\right)=\partial_{\mathcal{Y}}\left(R_{3}\right)$ says

$$
\left(f f_{\mathcal{Y}}+h f_{\mathcal{Z}}\right)_{\mathcal{Z}}=\left(f h_{\mathcal{Y}}+h h_{\mathcal{Z}}\right)_{\mathcal{Y}}+2 \beta^{\prime} \gamma_{1} \cos \alpha
$$

By (9.4.17) and (9.4.19)-(9.4.21), we assume that $f_{\mathcal{Y}}, f_{\mathcal{Z}}, h_{\mathcal{Y}}$ and $h_{\mathcal{Z}}$ are functions in $t$. Then (9.4.22) can be written as

$$
\left(f_{\mathcal{Y}}+h_{\mathcal{Z}}\right) f_{\mathcal{Z}}=\left(f_{\mathcal{Y}}+h_{\mathcal{Z}}\right) h_{\mathcal{Y}}+2 \beta^{\prime} \gamma_{1} \cos \alpha
$$

which is implied by (9.4.17) and (9.4.19). Solving (9.4.20) and (9.4.21), we get

$$
\begin{gathered}
h_{\mathcal{Y}}=\frac{\alpha^{\prime} \beta^{\prime} \gamma_{1} \sin \alpha-\left(\alpha^{\prime} \beta^{\prime} \sin \alpha\right)^{\prime}-2 \alpha^{\prime 2} \beta^{\prime} \cos \alpha}{\alpha^{\prime 2}+{\beta^{\prime}}^{2} \sin ^{2} \alpha} \\
h_{\mathcal{Z}}=\frac{\alpha^{\prime} \alpha^{\prime \prime}+{\beta^{\prime 2}}^{2} \gamma_{1} \sin ^{2} \alpha-\left(\beta^{\prime} \sin \alpha\right)\left(\beta^{\prime} \sin \alpha\right)^{\prime}-\alpha^{\prime} \beta^{\prime 2} \sin 2 \alpha}{\alpha^{\prime 2}+{\beta^{\prime}}^{2} \sin ^{2} \alpha} .
\end{gathered}
$$

Moreover,

$$
f_{\mathcal{Y}}=\frac{\gamma_{1} \alpha^{\prime 2}-\alpha^{\prime} \alpha^{\prime \prime}+\left(\beta^{\prime} \sin \alpha\right)\left(\beta^{\prime} \sin \alpha\right)^{\prime}+\alpha^{\prime} \beta^{\prime 2} \sin 2 \alpha}{\alpha^{\prime 2}+\beta^{\prime 2} \sin ^{2} \alpha}
$$


by (9.4.19) and (9.4.25), and

$$
f_{\mathcal{Z}}=\frac{\alpha^{\prime} \beta^{\prime} \gamma_{1} \sin \alpha-\left(\alpha^{\prime} \beta^{\prime} \sin \alpha\right)^{\prime}+2{\beta^{\prime 2}}^{2} \sin ^{2} \alpha \cos \alpha}{\alpha^{\prime 2}+\beta^{\prime 2} \sin ^{2} \alpha}
$$

by (9.4.17) and (9.4.24). With the above data, we take

$$
f=f_{\mathcal{Y}} \mathcal{Y}+f_{\mathcal{Z}} \mathcal{Z}, \quad h=h_{\mathcal{Y}} \mathcal{Y}+h_{\mathcal{Z}} \mathcal{Z} .
$$

Furthermore, (9.4.18) and the first equation in (9.4.16) yield $r+s+1 \in 2 \mathbb{Z}$,

$$
\begin{gathered}
\alpha_{1}=\varphi \alpha^{\prime}, \quad \gamma= \pm \varphi \sqrt{\alpha^{\prime 2}+\beta^{\prime 2} \sin ^{2} \alpha}, \\
\beta_{1}=\varphi \beta^{\prime} \sin \alpha, \quad \sigma=\mu \alpha^{\prime}, \quad \tau=\mu \beta^{\prime} \sin \alpha .
\end{gathered}
$$

In particular, $\alpha, \beta, \gamma_{1}, \varphi$ and $\mu$ are arbitrary functions in $t$. Thanks to (9.3.22) and (9.4.9)(9.4.11), the pressure

$$
\begin{aligned}
& p=\rho\left\{\gamma \mu \varphi^{-1}\left[\left(\gamma^{\prime}-\gamma \gamma_{1}\right) \mathcal{X} \zeta_{r} \phi_{s}-\left(\left(\varphi \alpha^{\prime}\right)^{\prime} \mathcal{Y}+\left(\varphi \beta^{\prime} \sin \alpha\right)^{\prime} \mathcal{Z}+\varphi\left(\alpha^{\prime} f+\beta^{\prime} h \sin \alpha\right)\right) \xi_{r} \psi_{s}\right]\right. \\
& +(-1)^{s} \varphi^{-1}\left[(\gamma \mu)^{\prime}-\gamma \mu \varphi^{\prime} \varphi^{-1}\right] \zeta_{r} \psi_{s}+2 \mu\left(\alpha^{\prime 2}+\beta^{\prime 2} \sin ^{2} \alpha\right) \xi_{r} \phi_{s}+2\left(\alpha^{\prime} f+\beta^{\prime} h \sin \alpha\right) \mathcal{X} \\
& +\frac{\alpha^{\prime 2}+\beta^{\prime 2} \sin ^{2} \alpha+\gamma_{1}^{\prime}-\gamma_{1}^{2}}{2} \mathcal{X}^{2}+\left[\left(\beta^{\prime} \sin \alpha\right)^{\prime}-\alpha^{\prime} \beta^{\prime} \cos \alpha\right] \mathcal{X} \mathcal{Z}-\frac{1}{2} \gamma^{4} \mu^{2} \varphi^{-2}\left(\phi_{s}^{2}+\xi_{r}^{2}\right) \\
& +\left(\frac{\beta^{\prime 2}}{2} \sin 2 \alpha+\alpha^{\prime \prime}\right) \mathcal{X} \mathcal{Y}+\left[\left(\beta^{\prime} \cos \alpha\right)^{\prime}+\alpha^{\prime} \beta^{\prime} \sin \alpha-f_{\mathcal{Z} t}-f_{\mathcal{Y}} f_{\mathcal{Z}}-h_{\mathcal{Y}} h_{\mathcal{Z}}\right] \mathcal{Y} \mathcal{Z} \\
& \left.+\frac{\beta^{\prime 2}-h_{\mathcal{Z} t}-f_{\mathcal{Z}}^{2}-h_{\mathcal{Z}}^{2}}{2} \mathcal{Z}^{2}+\frac{\alpha^{\prime 2}+\beta^{\prime 2} \cos \alpha-f_{\mathcal{Y} t}-f_{\mathcal{Y}}^{2}-h_{\mathcal{Y}}^{2}}{2} \mathcal{Y}^{2}\right\}
\end{aligned}
$$

By (9.3.3) and (9.3.5), we have the following theorem:

Theorem 9.4.1. Let $\alpha, \beta, \gamma_{1}, \varphi$ and $\mu$ be arbitrary functions in $t$ such that $\varphi \neq 0$ and $\alpha^{\prime 2}+\beta^{\prime 2} \sin ^{2} \alpha \neq 0$. The notations $\mathcal{X}, \mathcal{Y}$ and $\mathcal{Z}$ are defined in (9.3.5) via (9.3.1), and $\alpha_{1}, \beta_{1}$ and $\gamma$ are given in (9.4.29) and (9.4.30). Moreover, $f_{\mathcal{Y}}, f_{\mathcal{Z}}, h_{\mathcal{Y}}, h_{\mathcal{Z}}$ and $f, h$ are given in (9.4.24)-(9.4.28). We have the following solution of the Navier-Stokes equations (9.1.1)-(9.1.4): (1)

$$
\begin{aligned}
u= & -\alpha^{\prime}(\mathcal{X} \sin \alpha+\mathcal{Y} \cos \alpha)-\left(f+\mu \alpha^{\prime} \gamma \sinh \left(\alpha_{1} \mathcal{Y}+\beta_{1} \mathcal{Z}\right) \cos \gamma \mathcal{X}\right) \sin \alpha \\
& -\left(\gamma_{1} \mathcal{X}+\varphi \mu\left(\alpha^{\prime 2}+\beta^{\prime 2} \sin ^{2} \alpha\right) \cosh \left(\alpha_{1} \mathcal{Y}+\beta_{1} \mathcal{Z}\right) \sin \gamma \mathcal{X}\right) \cos \alpha \\
v= & \left(f \cos \alpha-\beta^{\prime} \mathcal{Z}\right) \cos \beta-\left(\alpha^{\prime} \sin \alpha \cos \beta+\beta^{\prime} \cos \alpha \sin \beta\right) \mathcal{Y} \\
& -\left(\gamma_{1} \mathcal{X}+\varphi \mu\left(\alpha^{\prime 2}+\beta^{\prime 2} \sin ^{2} \alpha\right) \cosh \left(\alpha_{1} \mathcal{Y}+\beta_{1} \mathcal{Z}\right) \sin \gamma \mathcal{X}\right) \sin \alpha \cos \beta-h \sin \beta \\
& +\left(\alpha^{\prime} \cos \alpha \cos \beta-\beta^{\prime} \sin \alpha \sin \beta\right)\left(\mathcal{X}+\gamma \mu \sinh \left(\alpha_{1} \mathcal{Y}+\beta_{1} \mathcal{Z}\right) \cos \gamma \mathcal{X}\right) \\
w= & \left(\beta^{\prime} \cos \alpha \cos \beta-\alpha^{\prime} \sin \alpha \sin \beta\right) \mathcal{Y}+\left(f \cos \alpha-\beta^{\prime} \mathcal{Z}\right) \sin \beta \\
& -\left(\gamma_{1} \mathcal{X}+\varphi \mu\left(\alpha^{\prime 2}+\beta^{\prime 2} \sin ^{2} \alpha\right) \cosh \left(\alpha_{1} \mathcal{Y}+\beta_{1} \mathcal{Z}\right) \sin \gamma \mathcal{X}\right) \sin \alpha \sin \beta+h \cos \beta \\
& +\left(\alpha^{\prime} \cos \alpha \sin \beta+\beta^{\prime} \sin \alpha \cos \beta\right)\left(\mathcal{X}+\gamma \mu \sinh \left(\alpha_{1} \mathcal{Y}+\beta_{1} \mathcal{Z}\right) \cos \gamma \mathcal{X}\right)
\end{aligned}
$$




$$
\begin{aligned}
& p=\rho\left\{\gamma \mu \varphi ^ { - 1 } \left[\left(\gamma^{\prime}-\gamma \gamma_{1}\right) \mathcal{X} \cosh \left(\alpha_{1} \mathcal{Y}+\beta_{1} \mathcal{Z}\right) \sin \gamma \mathcal{X}-\left(\left(\varphi \alpha^{\prime}\right)^{\prime} \mathcal{Y}+\left(\varphi \beta^{\prime} \sin \alpha\right)^{\prime} \mathcal{Z}\right.\right.\right. \\
& \left.\left.+\varphi\left(\alpha^{\prime} f+\beta^{\prime} h \sin \alpha\right)\right) \sinh \left(\alpha_{1} \mathcal{Y}+\beta_{1} \mathcal{Z}\right) \cos \gamma \mathcal{X}\right]-\varphi^{-1}\left[(\gamma \mu)^{\prime}-\gamma \mu \varphi^{\prime} \varphi^{-1}\right] \\
& \times \cosh \left(\alpha_{1} \mathcal{Y}+\beta_{1} \mathcal{Z}\right) \cos \gamma \mathcal{X}+2 \mu\left(\alpha^{\prime 2}+\beta^{\prime 2} \sin ^{2} \alpha\right) \sinh \left(\alpha_{1} \mathcal{Y}+\beta_{1} \mathcal{Z}\right) \sin \gamma \mathcal{X} \\
& +2\left(\alpha^{\prime} f+\beta^{\prime} h \sin \alpha\right) \mathcal{X}+\left[\left(\beta^{\prime} \cos \alpha\right)^{\prime}+\alpha^{\prime} \beta^{\prime} \sin \alpha-f_{\mathcal{Z} t}-f_{\mathcal{Y}} f_{\mathcal{Z}}-h_{\mathcal{Y}} h_{\mathcal{Z}}\right] \mathcal{Y} \mathcal{Z} \\
& +\frac{\alpha^{\prime 2}+\beta^{\prime 2} \sin ^{2} \alpha+\gamma_{1}^{\prime}-\gamma_{1}^{2} \mathcal{X}^{2}+\left[\left(\beta^{\prime} \sin \alpha\right)^{\prime}-\alpha^{\prime} \beta^{\prime} \cos \alpha\right] \mathcal{X} \mathcal{Z}}{2} \\
& +\left(\frac{\beta^{\prime 2}}{2} \sin 2 \alpha+\alpha^{\prime \prime}\right) \mathcal{X} \mathcal{Y}-\frac{1}{2} \gamma^{4} \mu^{2} \varphi^{-2}\left(\sin ^{2} \gamma \mathcal{X}+\sinh ^{2}\left(\alpha_{1} \mathcal{Y}+\beta_{1} \mathcal{Z}\right)\right) \\
& +\frac{\beta^{\prime 2}-h_{\mathcal{Z} t}-f_{\mathcal{Z}}^{2}-h_{\mathcal{Z}}^{2}}{2} \mathcal{Z}^{2}+\frac{\left.\alpha^{\prime 2}+\beta^{\prime 2} \cos \alpha-f_{\mathcal{Y} t}-f_{\mathcal{Y}}^{2}-h_{\mathcal{Y}}^{2} \mathcal{Y}^{2}\right\}}{2}
\end{aligned}
$$

$u=-\alpha^{\prime}(\mathcal{X} \sin \alpha+\mathcal{Y} \cos \alpha)-\left(f+\mu \alpha^{\prime} \gamma \sin \left(\alpha_{1} \mathcal{Y}+\beta_{1} \mathcal{Z}\right) \cosh \gamma \mathcal{X}\right) \sin \alpha$ $-\left(\gamma_{1} \mathcal{X}+\varphi \mu\left(\alpha^{\prime 2}+{\beta^{\prime}}^{2} \sin ^{2} \alpha\right) \cos \left(\alpha_{1} \mathcal{Y}+\beta_{1} \mathcal{Z}\right) \sinh \gamma \mathcal{X}\right) \cos \alpha$

$v=\left(f \cos \alpha-\beta^{\prime} \mathcal{Z}\right) \cos \beta-\left(\alpha^{\prime} \sin \alpha \cos \beta+\beta^{\prime} \cos \alpha \sin \beta\right) \mathcal{Y}$ $-\left(\gamma_{1} \mathcal{X}+\varphi \mu\left(\alpha^{\prime 2}+{\beta^{\prime}}^{2} \sin ^{2} \alpha\right) \cos \left(\alpha_{1} \mathcal{Y}+\beta_{1} \mathcal{Z}\right) \sinh \gamma \mathcal{X}\right) \sin \alpha \cos \beta-h \sin \beta$ $+\left(\alpha^{\prime} \cos \alpha \cos \beta-\beta^{\prime} \sin \alpha \sin \beta\right)\left(\mathcal{X}+\gamma \mu \sin \left(\alpha_{1} \mathcal{Y}+\beta_{1} \mathcal{Z}\right) \cosh \gamma \mathcal{X}\right)$,

$w=\left(\beta^{\prime} \cos \alpha \cos \beta-\alpha^{\prime} \sin \alpha \sin \beta\right) \mathcal{Y}+\left(f \cos \alpha-\beta^{\prime} \mathcal{Z}\right) \sin \beta$ $-\left(\gamma_{1} \mathcal{X}+\varphi \mu\left(\alpha^{\prime 2}+{\beta^{\prime}}^{2} \sin ^{2} \alpha\right) \cos \left(\alpha_{1} \mathcal{Y}+\beta_{1} \mathcal{Z}\right) \sinh \gamma \mathcal{X}\right) \sin \alpha \sin \beta+h \cos \beta$ $+\left(\alpha^{\prime} \cos \alpha \sin \beta+\beta^{\prime} \sin \alpha \cos \beta\right)\left(\mathcal{X}+\gamma \mu \sin \left(\alpha_{1} \mathcal{Y}+\beta_{1} \mathcal{Z}\right) \cosh \gamma \mathcal{X}\right)$

$$
\begin{aligned}
& p=\rho\left\{\gamma \mu \varphi ^ { - 1 } \left[\left(\gamma^{\prime}-\gamma \gamma_{1}\right) \mathcal{X} \cos \left(\alpha_{1} \mathcal{Y}+\beta_{1} \mathcal{Z}\right) \sinh \gamma \mathcal{X}-\left(\left(\varphi \alpha^{\prime}\right)^{\prime} \mathcal{Y}+\left(\varphi \beta^{\prime} \sin \alpha\right)^{\prime} \mathcal{Z}\right.\right.\right. \\
& \left.\left.+\varphi\left(\alpha^{\prime} f+\beta^{\prime} h \sin \alpha\right)\right) \sin \left(\alpha_{1} \mathcal{Y}+\beta_{1} \mathcal{Z}\right) \cosh \gamma \mathcal{X}\right]+\varphi^{-1}\left[(\gamma \mu)^{\prime}-\gamma \mu \varphi^{\prime} \varphi^{-1}\right] \\
& \times \cos \left(\alpha_{1} \mathcal{Y}+\beta_{1} \mathcal{Z}\right) \cosh \gamma \mathcal{X}+2 \mu\left(\alpha^{\prime 2}+\beta^{\prime 2} \sin ^{2} \alpha\right) \sin \left(\alpha_{1} \mathcal{Y}+\beta_{1} \mathcal{Z}\right) \sinh \gamma \mathcal{X} \\
& +2\left(\alpha^{\prime} f+\beta^{\prime} h \sin \alpha\right) \mathcal{X}+\left[\left(\beta^{\prime} \cos \alpha\right)^{\prime}+\alpha^{\prime} \beta^{\prime} \sin \alpha-f_{\mathcal{Z} t}-f_{\mathcal{Y}} f_{\mathcal{Z}}-h_{\mathcal{Y}} h_{\mathcal{Z}}\right] \mathcal{Y} \mathcal{Z} \\
& +\frac{\alpha^{\prime 2}+\beta^{\prime 2} \sin ^{2} \alpha+\gamma_{1}^{\prime}-\gamma_{1}^{2}}{2} \mathcal{X}^{2}+\left[\left(\beta^{\prime} \sin \alpha\right)^{\prime}-\alpha^{\prime} \beta^{\prime} \cos \alpha\right] \mathcal{X} \mathcal{Z} \\
& +\left(\frac{\beta^{\prime 2}}{2} \sin 2 \alpha+\alpha^{\prime \prime}\right) \mathcal{X} \mathcal{Y}-\frac{1}{2} \gamma^{4} \mu^{2} \varphi^{-2}\left(\sinh ^{2} \gamma \mathcal{X}+\sin ^{2}\left(\alpha_{1} \mathcal{Y}+\beta_{1} \mathcal{Z}\right)\right) \\
& \left.+\frac{\beta^{\prime 2}-h_{\mathcal{Z} t}-f_{\mathcal{Z}}^{2}-h_{\mathcal{Z}}^{2}}{2} \mathcal{Z}^{2}+\frac{\alpha^{\prime 2}+\beta^{\prime 2} \cos \alpha-f_{\mathcal{Y} t}-f_{\mathcal{Y}}^{2}-h_{\mathcal{Y}}^{2}}{2} \mathcal{Y}^{2}\right\}
\end{aligned}
$$

Let $\gamma_{1}, \gamma_{2}$ be functions in $t$ and let $a, b, c$ be real numbers. Denote

$$
\phi_{0}=e^{\gamma_{1} \mathcal{Y}+\gamma_{2} \mathcal{Z}}-a e^{-\gamma_{1} \mathcal{Y}-\gamma_{2} \mathcal{Z}}, \quad \phi_{1}=\sin \left(\gamma_{1} \mathcal{Y}+\gamma_{2} \mathcal{Z}\right)
$$




$$
\begin{gathered}
\psi_{0}=e^{\gamma_{1} \mathcal{Y}+\gamma_{2} \mathcal{Z}}+a e^{-\gamma_{1} \mathcal{Y}-\gamma_{2} \mathcal{Z}}, \quad \psi_{1}=\cos \left(\gamma_{1} \mathcal{Y}+\gamma_{2} \mathcal{Z}\right), \\
\xi_{0}=b e^{\gamma_{1} \mathcal{Y}+\gamma_{2} \mathcal{Z}}-c e^{-\gamma_{1} \mathcal{Y}-\gamma_{2} \mathcal{Z}}, \quad \xi_{1}=c \sin \left(\gamma_{1} \mathcal{Y}+\gamma_{2} \mathcal{Z}+b\right), \\
\zeta_{0}=b e^{\gamma_{1} \mathcal{Y}+\gamma_{2} \mathcal{Z}}+c e^{-\gamma_{1} \mathcal{Y}-\gamma_{2} \mathcal{Z}}, \quad \zeta_{1}=c \cos \left(\gamma_{1} \mathcal{Y}+\gamma_{2} \mathcal{Z}+b\right) .
\end{gathered}
$$

Suppose that $\sigma, \tau$ are functions in $t$ and $f, k, h$ are functions in $t, \mathcal{X}, \mathcal{Y}, \mathcal{Z}$ such that $h$ and $g$ are linear in $\mathcal{X}, Y, \mathcal{Z}$ and

$$
f_{\mathcal{X}}+k_{\mathcal{Y}}+h_{\mathcal{Z}}=0 .
$$

Motivated from the above solution, we consider the solution of the form:

$$
\begin{gathered}
\mathcal{U}=-\alpha^{\prime} \mathcal{Y}-\beta^{\prime} \mathcal{Z} \sin \alpha+f-\left(\gamma_{1}^{2}+\gamma_{2}^{2}\right)\left(\tau \zeta_{r} \mathcal{X}+\sigma \psi_{r} \mathcal{X}^{2}\right) \\
\mathcal{V}=\alpha^{\prime} \mathcal{X}-\beta^{\prime} \mathcal{Z} \cos \alpha+k+\gamma_{1}\left(\tau \xi_{r}+2 \sigma \phi_{r} \mathcal{X}\right) \\
\mathcal{W}=\beta^{\prime}(\mathcal{X} \sin \alpha+\mathcal{Y} \cos \alpha)+h+\gamma_{2}\left(\tau \xi_{r}+2 \sigma \phi_{r} \mathcal{X}\right)
\end{gathered}
$$

For convenience of computation, we denote

$$
\gamma=\gamma_{1}^{2}+\gamma_{2}^{2}, \quad f^{*}=f-f_{x} \mathcal{X} \quad \Delta_{1}=\partial_{\mathcal{Y}}^{2}+\partial_{\mathcal{Z}}^{2} .
$$

Now (9.4.1) becomes

$$
\begin{aligned}
& R_{1}=-\alpha^{\prime \prime} \mathcal{Y}-\left(\beta^{\prime} \sin \alpha\right)^{\prime} \mathcal{Z}+f_{t}-(-1)^{r} \gamma\left(\gamma_{1}^{\prime} \mathcal{Y}+\gamma_{2}^{\prime} \mathcal{Z}\right)\left(\tau \xi_{r} \mathcal{X}+\sigma \phi_{r} \mathcal{X}^{2}\right) \\
& +\left((-1)^{r} \nu \gamma^{2} \tau-(\gamma \tau)^{\prime}\right) \zeta_{r} \mathcal{X}+\left(f-\gamma\left(\tau \zeta_{r} \mathcal{X}+\sigma \psi_{r} \mathcal{X}^{2}\right)\right)\left(f_{\mathcal{X}}-\gamma\left(\tau \zeta_{r}+2 \sigma \psi_{r} \mathcal{X}\right)\right) \\
& +\left(k+\gamma_{1}\left(\tau \xi_{r}+2 \sigma \phi_{r} \mathcal{X}\right)\right)\left[f_{\mathcal{Y}}-2 \alpha^{\prime}-(-1)^{r} \gamma \gamma_{1}\left(\tau \xi_{r} \mathcal{X}+\sigma \phi_{r} \mathcal{X}^{2}\right)\right]-\nu \Delta_{1}(f) \\
& +\left(h+\gamma_{2}\left(\tau \xi_{r}+2 \sigma \phi_{r} \mathcal{X}\right)\right)\left[f_{\mathcal{Z}}-2 \beta^{\prime} \sin \alpha-(-1)^{r} \gamma \gamma_{2}\left(\tau \xi_{r} \mathcal{X}+\sigma \phi_{r} \mathcal{X}^{2}\right)\right]+2 \nu \gamma \sigma \psi_{r} \\
& -\alpha^{\prime}\left(\alpha^{\prime} \mathcal{X}-\beta^{\prime} \mathcal{Z} \cos \alpha\right)-\beta^{\prime 2}(\mathcal{X} \sin \alpha+\mathcal{Y} \cos \alpha) \sin \alpha+\left((-1)^{r} \nu \gamma^{2} \sigma-(\gamma \sigma)^{\prime}\right) \psi_{r} \mathcal{X}^{2} \\
& =-\left(\alpha^{\prime 2}+\beta^{\prime 2} \sin ^{2} \alpha\right) \mathcal{X}-\left(\alpha^{\prime \prime}+2^{-1}{\beta^{\prime}}^{2} \sin 2 \alpha\right) \mathcal{Y}+\left(\alpha^{\prime} \beta^{\prime} \cos \alpha-\left(\beta^{\prime} \sin \alpha\right)^{\prime}\right) \mathcal{Z} \\
& +\gamma^{2}\left[\tau^{2}\left(4 b \delta_{0, r}+c \delta_{1, r}\right) c \mathcal{X}+3 \sigma \tau\left(2 \delta_{0, r}(a b+c)+\delta_{1, r} c \cos b\right) \mathcal{X}^{2}+2 \sigma^{2}\left(4 a \delta_{0, r}+\delta_{1, r}\right) \mathcal{X}^{3}\right] \\
& -(-1)^{r} \gamma\left(\gamma_{1}^{\prime} \mathcal{Y}+\gamma_{2}^{\prime} \mathcal{Z}+k \gamma_{1}+h \gamma_{2}\right)\left(\tau \xi_{r} \mathcal{X}+\sigma \phi_{r} \mathcal{X}^{2}\right)+f f_{\mathcal{X}}+k\left(f_{\mathcal{Y}}-2 \alpha^{\prime}\right) \\
& +h\left(f_{\mathcal{Z}}-2 \beta^{\prime} \sin \alpha\right)+\left((-1)^{r} \nu \gamma^{2} \sigma-(\gamma \sigma)^{\prime}-3 \gamma \sigma f_{\mathcal{X}}\right) \psi_{r} \mathcal{X}^{2}+\nu\left(2 \gamma \sigma \psi_{r}-\Delta_{1}(f)\right) \\
& -\gamma \tau f^{*} \zeta_{r}-\left[\left((\gamma \tau)^{\prime}+2 \gamma \tau f_{\mathcal{X}}-(-1)^{r} \nu \gamma^{2} \tau\right) \zeta_{r}+2 \gamma \sigma f^{*} \psi_{r}\right] \mathcal{X}+f_{t} \\
& +\left(\gamma_{1}\left(f_{\mathcal{Y}}-2 \alpha^{\prime}\right)+\gamma_{2}\left(f_{\mathcal{Z}}-2 \beta^{\prime} \sin \alpha\right)\right)\left(\tau \xi_{r}+2 \sigma \phi_{r} \mathcal{X}\right)
\end{aligned}
$$

To solve (9.3.24), we assume

$$
\gamma_{1}^{\prime} \mathcal{Y}+\gamma_{2}^{\prime} \mathcal{Z}+k \gamma_{1}+h \gamma_{2}=0
$$

and

$$
(-1)^{r} \nu \gamma^{2} \sigma-(\gamma \sigma)^{\prime}-3 \gamma \sigma f_{\mathcal{X}}=0,
$$


Moreover, (9.4.2) and (9.4.3) become

$$
\begin{aligned}
& R_{2}=\alpha^{\prime \prime} \mathcal{X}-\left(\beta^{\prime} \cos \alpha\right)^{\prime} \mathcal{Z}+\left(\left(\gamma_{1} \tau\right)^{\prime}-(-1)^{r} \nu \gamma \gamma_{1} \tau\right) \xi_{r}+2\left(\left(\gamma_{1} \sigma\right)^{\prime}-(-1)^{r} \nu \gamma \gamma_{1} \sigma\right) \phi_{r} \mathcal{X} \\
& +k_{t}+\left(\gamma_{1}^{\prime} \mathcal{Y}+\gamma_{2}^{\prime} \mathcal{Z}\right) \gamma_{1}\left(\tau \zeta_{r}+2 \sigma \psi_{r} \mathcal{X}\right)+\left(f-\gamma\left(\tau \zeta_{r} \mathcal{X}+\sigma \psi_{r} \mathcal{X}^{2}\right)\right)\left(2 \alpha^{\prime}+k_{\mathcal{X}}+2 \gamma_{1} \sigma \phi_{r}\right) \\
& +\left(k+\gamma_{1}\left(\tau \xi_{r}+2 \sigma \phi_{r} \mathcal{X}\right)\right)\left(k_{\mathcal{Y}}+\gamma_{1}^{2}\left(\tau \zeta_{r}+2 \sigma \psi_{r} \mathcal{X}\right)\right)-\beta^{\prime 2}(\mathcal{X} \sin \alpha+\mathcal{Y} \cos \alpha) \cos \alpha \\
& -\alpha^{\prime}\left(\alpha^{\prime} \mathcal{Y}+\beta^{\prime} \mathcal{Z} \sin \alpha\right)+\left(h+\gamma_{2}\left(\tau \xi_{r}+2 \sigma \phi_{r} \mathcal{X}\right)\right)\left(k_{\mathcal{Z}}-2 \beta^{\prime} \cos \alpha+\gamma_{1} \gamma_{2}\left(\tau \zeta_{r}+2 \sigma \psi_{r} \mathcal{X}\right)\right) \\
& =\left(\alpha^{\prime \prime}-2^{-1}{\beta^{\prime}}^{2} \sin 2 \alpha+f_{\mathcal{X}}\left(2 \alpha^{\prime}+k_{\mathcal{X}}\right)\right) \mathcal{X}-\left(\alpha^{\prime 2}+{\beta^{\prime 2}}^{2} \cos ^{2} \alpha\right) \mathcal{Y}+k_{t}+k k_{\mathcal{Y}} \\
& +\left[\tau\left(\gamma_{1} k_{\mathcal{Y}}+\gamma_{2}\left(k_{\mathcal{Z}}-2 \beta^{\prime} \cos \alpha\right)\right)+\left(\gamma_{1} \tau\right)^{\prime}-(-1)^{r} \nu \gamma \gamma_{1} \tau\right] \xi_{r}-\left(\left(\beta^{\prime} \cos \alpha\right)^{\prime}+\alpha^{\prime} \beta^{\prime} \sin \alpha\right) \mathcal{Z} \\
& +\gamma \sigma\left(2 \sigma \gamma_{1} \phi_{r}-2 \alpha^{\prime}-k_{\mathcal{X}}\right) \psi_{r} \mathcal{X}^{2}+f^{*}\left(2 \alpha^{\prime}+k_{\mathcal{X}}+2 \gamma_{1} \sigma \phi_{r}\right)+\gamma \gamma_{1} \tau^{2} \xi_{r} \zeta_{r} \\
& +h\left(k_{\mathcal{Z}}-2 \beta^{\prime} \cos \alpha\right)+\left\{2 \gamma \gamma_{1} \sigma \tau \xi_{r} \psi_{r}+2\left[\left(\gamma_{1} \sigma\right)^{\prime}-\sigma \gamma_{1}\left(h_{\mathcal{Z}}+(-1)^{r} \nu \gamma\right)\right.\right. \\
& \left.\left.\left.+\gamma_{2} \sigma\left(k_{\mathcal{Z}}-2 \beta^{\prime} \cos \alpha\right)\right)\right] \phi_{r}-\gamma \tau\left(2 \alpha^{\prime}+k_{\mathcal{X}}\right) \zeta_{r}\right\} \mathcal{X} \\
& R_{3}=\left(\beta^{\prime} \sin \alpha\right)^{\prime} \mathcal{X}+\left(\beta^{\prime} \cos \alpha\right)^{\prime} \mathcal{Y}+\left(\gamma_{2} \tau\right)^{\prime} \xi_{r}+2\left(\gamma_{2} \sigma\right)^{\prime} \phi_{r} \mathcal{X}-(-1)^{r} \nu \gamma_{2} \gamma\left(\tau \xi_{r}+2 \sigma \phi_{r} \mathcal{X}\right) \\
& +\left(\gamma_{1}^{\prime} \mathcal{Y}+\gamma_{2}^{\prime} \mathcal{Z}\right) \gamma_{2}\left(\tau \zeta_{r}+2 \sigma \psi_{r} \mathcal{X}\right)+\left(f-\gamma\left(\tau \zeta_{r} \mathcal{X}+\sigma \psi_{r} \mathcal{X}^{2}\right)\right)\left(2 \beta^{\prime} \sin \alpha+h_{\mathcal{X}}+2 \gamma_{2} \sigma \phi_{r}\right) \\
& +\left(k+\gamma_{1}\left(\tau \xi_{r}+2 \sigma \phi_{r} \mathcal{X}\right)\right)\left(2 \beta^{\prime} \cos \alpha+h_{\mathcal{Y}}+\gamma_{1} \gamma_{2}\left(\tau \zeta_{r}+2 \sigma \psi_{r} \mathcal{X}\right)-\beta^{\prime 2} \mathcal{Z}+h_{t}\right. \\
& +\alpha^{\prime} \beta^{\prime}(\mathcal{X} \cos \alpha-\mathcal{Y} \sin \alpha)+\left(h+\gamma_{2}\left(\tau \xi_{r}+2 \sigma \phi_{r} \mathcal{X}\right)\right)\left(h_{\mathcal{Z}}+\gamma_{2}^{2}\left(\tau \zeta_{r}+2 \sigma \psi_{r} \mathcal{X}\right)\right) \\
& =\left[\left(\beta^{\prime} \sin \alpha\right)^{\prime}+\alpha^{\prime} \beta^{\prime} \cos \alpha+f_{\mathcal{X}}\left(2 \beta^{\prime} \sin \alpha+h_{\mathcal{X}}\right)\right] \mathcal{X}+\left[\left(\beta^{\prime} \cos \alpha\right)^{\prime}-\alpha^{\prime} \beta^{\prime} \sin \alpha\right] \mathcal{Y} \\
& +\left[\left(\gamma_{2} \tau\right)^{\prime}+\left(\gamma_{1}\left(2 \beta^{\prime} \cos \alpha+h_{\mathcal{Y}}\right)+\gamma_{2} h_{\mathcal{Z}}-(-1)^{r} \nu \gamma \gamma_{2}\right) \tau\right] \xi_{r}+\left\{2 \gamma \gamma_{2} \tau \sigma \xi_{r} \psi_{r}+2\left[\left(\gamma_{2} \sigma\right)^{\prime}\right.\right. \\
& \left.\left.-\gamma_{2} \sigma\left(k_{\mathcal{Y}}+(-1)^{r} \nu \gamma\right)+\gamma_{1} \sigma\left(2 \beta^{\prime} \cos \alpha+h_{\mathcal{Y}}\right)\right] \phi_{r}-\gamma \tau\left(2 \beta^{\prime} \sin \alpha+h_{\mathcal{X}}\right) \zeta_{r}\right\} \mathcal{X} \\
& +f^{*}\left(2 \beta^{\prime} \sin \alpha+h_{\mathcal{X}}+2 \gamma_{2} \sigma \phi_{r}\right)+k\left(2 \beta^{\prime} \cos \alpha+h_{\mathcal{Y}}\right)+h_{t}+h h_{\mathcal{Z}}+\gamma \gamma_{2} \tau^{2} \xi_{r} \zeta_{r} \\
& +\gamma \sigma\left(2 \gamma_{2} \sigma \phi_{r}-2 \beta^{\prime} \sin \alpha-h_{\mathcal{X}}\right) \psi_{r} \mathcal{X}^{2}-\beta^{\prime 2} \mathcal{Z}
\end{aligned}
$$

by $(9.4 .50)$.

Thanks to the coefficients of $\mathcal{X}^{2}$ in $\partial_{\mathcal{Z}}\left(R_{2}\right)=\partial_{\mathcal{Y}}\left(R_{3}\right)$, we have:

$$
\gamma_{2}\left(2 \alpha^{\prime}+k_{\mathcal{X}}\right)=\gamma_{1}\left(2 \beta^{\prime} \sin \alpha+h_{\mathcal{X}}\right)
$$

According to (9.4.50),

$$
k_{\mathcal{X}} \gamma_{1}+h_{\mathcal{X}} \gamma_{2}=0, \quad \gamma_{1}^{\prime}+\gamma_{1} k_{\mathcal{Y}}+\gamma_{2} h_{\mathcal{Y}}=0, \quad \gamma_{2}^{\prime}+\gamma_{1} k_{\mathcal{Z}}+\gamma_{2} h_{\mathcal{Z}}=0
$$

Solving (9.4.54) and the first equation in (9.4.55), we obtain

$$
k_{\mathcal{X}}=2 \gamma^{-1} \gamma_{2}\left(\beta^{\prime} \gamma_{1} \sin \alpha-\alpha^{\prime} \gamma_{2}\right), \quad h_{\mathcal{X}}=-2 \gamma^{-1} \gamma_{1}\left(\beta^{\prime} \gamma_{1} \sin \alpha-\alpha^{\prime} \gamma_{2}\right)
$$

Moreover, the coefficients of $\mathcal{X}$ in $\partial_{\mathcal{Z}}\left(R_{2}\right)=\partial_{\mathcal{Y}}\left(R_{3}\right)$ give

$$
\gamma_{1}^{\prime} \gamma_{2}-\gamma_{1} \gamma_{2}^{\prime}+\gamma_{1} \gamma_{2}\left(k_{\mathcal{Y}}-h_{\mathcal{Z}}\right)+\gamma_{2}^{2} k_{\mathcal{Z}}-\gamma_{1}^{2} h_{\mathcal{Y}}-2 \gamma \beta^{\prime} \cos \alpha=0
$$


by (9.4.50). According to (9.4.55), the above equation can be rewritten as

$$
k_{\mathcal{Z}}-h_{\mathcal{Y}}=2 \beta^{\prime} \cos \alpha .
$$

Furthermore, (9.4.54) and the coefficients of $\mathcal{X}^{0}$ in $\partial_{\mathcal{Z}}\left(R_{2}\right)=\partial_{\mathcal{Y}}\left(R_{3}\right)$ show that $f$ is a function of $t$ and $\gamma_{1} \mathcal{Y}+\gamma_{2} \mathcal{Z}$ by the method of characteristics in Section 4.1. According to the coefficients of $\mathcal{X}$ in $\partial_{\mathcal{Y}}\left(R_{1}\right)=\partial_{\mathcal{X}}\left(R_{2}\right)$ and $\partial_{\mathcal{Z}}\left(R_{1}\right)=\partial_{\mathcal{X}}\left(R_{3}\right)$, we take

$$
f^{*}=\varphi \vartheta_{r}+\sigma \tilde{\varpi} \phi_{r}+\alpha_{1},
$$

where $\varphi$ and $\alpha_{1}$ are functions in $t$, and

$$
\tilde{\varpi}=\gamma_{1} \mathcal{Y}+\gamma_{2} \mathcal{Z}, \quad \vartheta_{0}=b_{1} e^{\tilde{\varpi}}-c_{1} e^{-\tilde{\varpi}}, \quad \vartheta_{1}=c_{1} \sin \left(\tilde{\varpi}+b_{1}\right)
$$

for $b_{1}, c_{1} \in \mathbb{R}$.

Note

$$
2 \sigma\left(\gamma_{1} f_{\mathcal{Y}}+\gamma_{2} f_{\mathcal{Z}}\right) \phi_{r}=2 \gamma \sigma f_{\tilde{\varpi}}^{*} \phi_{r}
$$

Denote

$$
\hat{\vartheta}_{0}=b_{1} e^{\tilde{\varpi}}+c_{1} e^{-\tilde{\varpi}}, \quad \hat{\vartheta}_{1}=c_{1} \cos \left(\tilde{\varpi}+b_{1}\right) .
$$

Then

$$
f_{\tilde{\varpi}}^{*}=\varphi \hat{\vartheta}_{r}+\sigma\left(\phi_{r}+\tilde{\varpi} \psi_{r}\right), \quad f_{\tilde{\varpi} \tilde{\varpi}}^{*}=(-1)^{r}\left(\varphi \vartheta_{r}+\sigma \tilde{\varpi} \phi_{r}\right)+2 \sigma \psi_{r} .
$$

Moreover.

$$
\begin{aligned}
& \partial_{\mathcal{Y}}\left(2 \gamma \sigma f_{\tilde{\varpi}}^{*} \phi_{r}-2 \gamma \sigma f^{*} \psi_{r}\right) \\
= & 2 \gamma \gamma_{1} \sigma\left[f_{\tilde{\varpi} \tilde{\varpi}}^{*} \phi_{r}+f_{\tilde{\varpi}}^{*} \psi_{r}-\left(f_{\tilde{\varpi}}^{*} \psi_{r}+(-1)^{r} f^{*} \phi_{r}\right)\right] \\
= & 2 \gamma \gamma_{1} \sigma\left[\left((-1)^{r}\left(\varphi \vartheta_{r}+\sigma \tilde{\varpi} \phi_{r}\right)+2 \sigma \psi_{r}\right) \phi_{r}-(-1)^{r}\left(\varphi \vartheta_{r}+\sigma \tilde{\varpi} \phi_{r}+\alpha_{1}\right) \phi_{r}\right] \\
= & 4 \gamma \gamma_{1} \sigma^{2} \phi_{r} \psi_{r}-(-1)^{r} 2 \alpha_{1} \gamma \gamma_{1} \sigma \phi_{r} .
\end{aligned}
$$

Similarly,

$$
\partial_{\mathcal{Z}}\left(2 \gamma \sigma f_{\widetilde{\varpi}}^{*} \phi_{r}-2 \gamma \sigma f^{*} \psi_{r}\right)=4 \gamma \gamma_{2} \sigma^{2} \phi_{r} \psi_{r}-(-1)^{r} 2 \alpha_{1} \gamma \gamma_{2} \sigma \phi_{r} .
$$

Now the coefficients of $\mathcal{X}$ in $\partial_{\mathcal{Y}}\left(R_{1}\right)=\partial_{\mathcal{X}}\left(R_{2}\right)$ give

$$
\begin{aligned}
& -(-1)^{r} \gamma_{1}\left[\left((\gamma \tau)^{\prime}+2 \gamma \tau f_{\mathcal{X}}-(-1)^{r} \nu \gamma^{2} \tau\right) \xi_{r}+2 \alpha_{1} \gamma \sigma \phi_{r}\right] \\
& -4 \gamma_{1} \sigma\left(\gamma_{1} \alpha^{\prime}+\gamma_{2} \beta^{\prime} \sin \alpha\right) \psi_{r}=-2 \gamma \sigma\left(2 \alpha^{\prime}+k_{\mathcal{X}}\right) \psi_{r}
\end{aligned}
$$

by (9.4.49), (9.4.52) and (9.4.64). According to (9.4.49), (9.4.53) and (9.4.65), the coefficients of $\mathcal{X}$ in $\partial_{\mathcal{X}}\left(R_{1}\right)=\partial_{\mathcal{X}}\left(R_{3}\right)$ imply

$$
\begin{aligned}
& -(-1)^{r} \gamma_{2}\left[\left((\gamma \tau)^{\prime}+2 \gamma \tau f_{\mathcal{X}}-(-1)^{r} \nu \gamma^{2} \tau\right) \xi_{r}+2 \alpha_{1} \gamma \sigma \phi_{r}\right] \\
& -4 \gamma_{2} \sigma\left(\gamma_{1} \alpha^{\prime}+\gamma_{2} \beta^{\prime} \sin \alpha\right) \psi_{r}=-2 \gamma \sigma\left(2 \beta^{\prime} \sin \alpha+h_{\mathcal{X}}\right) \psi_{r} .
\end{aligned}
$$


Observe that (9.4.56) yields

$$
\begin{gathered}
\gamma\left(2 \alpha^{\prime}+k_{\mathcal{X}}\right)=2 \alpha^{\prime} \gamma+2 \gamma_{2}\left(\beta^{\prime} \gamma_{1} \sin \alpha-\alpha^{\prime} \gamma_{2}\right)=2 \gamma_{1}\left(\gamma_{1} \alpha^{\prime}+\gamma_{2} \beta^{\prime} \sin \alpha\right) \\
\gamma\left(2 \beta^{\prime} \sin \alpha+h_{\mathcal{X}}\right)=2 \beta^{\prime} \gamma \sin \alpha-2 \gamma_{1}\left(\beta^{\prime} \gamma_{1} \sin \alpha-\alpha^{\prime} \gamma_{2}\right)=2 \gamma_{2}\left(\gamma_{2} \beta^{\prime} \sin \alpha+\gamma_{1} \alpha^{\prime}\right)
\end{gathered}
$$

Thus (9.4.66) and (9.4.67) are implied by

$$
\left((\gamma \tau)^{\prime}+2 \gamma \tau f_{\mathcal{X}}-(-1)^{r} \nu \gamma^{2} \tau\right) \xi_{r}+2 \alpha_{1} \gamma \sigma \phi_{r}=0
$$

As (9.4.64) and (9.4.65), Expressions (9.4.40)-(9.4.43) and (9.4.59)-(9.4.62) give

$$
\begin{aligned}
& \gamma \tau \partial_{\mathcal{Y}}\left(f_{\tilde{\varpi}}^{*} \xi_{r}-f^{*} \zeta_{r}\right)=\gamma \gamma_{1} \tau\left(2 \sigma \xi_{r} \psi_{r}-(-1)^{r} \alpha_{1} \xi_{r}+\hat{c}_{r} \sigma\right) \\
& \gamma \tau \partial_{\mathcal{Z}}\left(f_{\tilde{\varpi}}^{*} \xi_{r}-f^{*} \zeta_{r}\right)=\gamma \gamma_{2} \tau\left(2 \sigma \xi_{r} \psi_{r}-(-1)^{r} \alpha_{1} \xi_{r}+\hat{c}_{r} \sigma\right)
\end{aligned}
$$

where

$$
\hat{c}_{0}=\xi_{0} \psi_{0}-\zeta_{0} \phi_{0}=2(a b-c), \quad \hat{c}_{1}=\xi_{1} \psi_{1}-z e t a_{1} \phi_{1}=c \sin b
$$

Moreover,

$$
k f_{\mathcal{Y}}+h f_{\mathcal{Z}}=\left(\gamma_{1} k+\gamma_{2} h\right) f_{\tilde{\varpi}}^{*}=-\left(\gamma_{1}^{\prime} \mathcal{Y}+\gamma_{2}^{\prime} \mathcal{Z}\right) f_{\tilde{\varpi}}^{*}=-\partial_{t}(\tilde{\varpi}) f_{\tilde{\varpi}}^{*}
$$

by (6.4.55). On the other hand,

$$
\partial_{t}\left(f^{*}\right)=f_{t}^{*}+\partial_{t}(\tilde{\varpi}) f_{\tilde{\varpi}}^{*}
$$

Thus the coefficients of $\mathcal{X}^{0}$ in $\partial_{\mathcal{Y}}\left(R_{1}\right)=\partial_{\mathcal{X}}\left(R_{2}\right)$ give

$$
\begin{aligned}
& {\left.\left[\left(f_{\mathcal{X}}-(-1)^{r} \gamma \nu\right) \varphi+\varphi^{\prime}\right) \vartheta_{r}+\left(\left(f_{\mathcal{X}}-(-1)^{r} \nu \gamma\right) \sigma+\sigma^{\prime}\right) \tilde{\varpi} \phi_{r}-\alpha_{1} \gamma \tau \zeta_{r}\right] \mathcal{Y} } \\
= & 2 \alpha^{\prime \prime}-\left(2 \alpha^{\prime}+k_{\mathcal{X}}\right) h_{\mathcal{Z}}+k_{\mathcal{X} t}+\left(h_{\mathcal{X}}+2 \beta^{\prime} \sin \alpha\right) h_{\mathcal{Y}}-\hat{c}_{r} \gamma_{1} \gamma \sigma \tau \\
+ & 2\left[\left(\gamma_{1} \sigma\right)^{\prime}-\gamma_{1} \sigma\left(h_{\mathcal{Z}}+(-1)^{r} \nu \gamma\right)+\gamma_{2} \sigma h_{\mathcal{Y}}\right] \phi_{r}
\end{aligned}
$$

and the coefficients of $\mathcal{X}^{0}$ in $\partial_{\mathcal{X}}\left(R_{1}\right)=\partial_{\mathcal{X}}\left(R_{3}\right)$ yield

$$
\begin{aligned}
& {\left.\left[\left(f_{\mathcal{X}}-(-1)^{r} \gamma \nu\right) \varphi+\varphi^{\prime}\right) \vartheta_{r}+\left(\left(f_{\mathcal{X}}-(-1)^{r} \nu \gamma\right) \sigma+\sigma^{\prime}\right) \tilde{\varpi} \phi_{r}-\alpha_{1} \gamma \tau \zeta_{r}\right]_{\mathcal{Z}} } \\
= & 2\left(\beta^{\prime} \sin \alpha\right)^{\prime}+h_{\mathcal{X} t}-\left(h_{\mathcal{X}}+2 \beta^{\prime} \sin \alpha\right) k_{\mathcal{Y}}+\left(k_{\mathcal{X}}+2 \alpha^{\prime}\right) k_{\mathcal{Z}}-\hat{c}_{r} \gamma_{2} \gamma \sigma \tau \\
+ & 2\left[\left(\gamma_{2} \sigma\right)^{\prime}-\gamma_{2} \sigma\left(k_{\mathcal{Y}}+(-1)^{r} \nu \gamma\right)+\gamma_{1} \sigma k_{\mathcal{Z}}\right] \phi_{r}
\end{aligned}
$$

by (9.4.44), (9.4.49), (9.4.52), (9.4.53), (9.4.58), (9.4.68), (9.4.69) and (9.4.71)-(9.4.74). Thus we have:

$$
2 \alpha^{\prime \prime}-\left(2 \alpha^{\prime}+k_{\mathcal{X}}\right) h_{\mathcal{Z}}+k_{\mathcal{X} t}+\left(h_{\mathcal{X}}+2 \beta^{\prime} \sin \alpha\right) h_{\mathcal{Y}}-\hat{c}_{r} \gamma_{1} \gamma \sigma \tau=0
$$


and

$$
2\left(\beta^{\prime} \sin \alpha\right)^{\prime}+h_{\mathcal{X} t}-\left(h_{\mathcal{X}}+2 \beta^{\prime} \sin \alpha\right) k_{\mathcal{Y}}+\left(k_{\mathcal{X}}+2 \alpha^{\prime}\right) k_{\mathcal{Z}}-\hat{c}_{r} \gamma_{2} \gamma \sigma \tau=0 .
$$

For simplicity, we only consider two special cases a follows.

Case 1. $\vartheta_{r}=\zeta_{r}, \sigma=0, \gamma_{1}=\alpha^{\prime} \mu$ and $\gamma_{2}=\beta^{\prime} \mu \sin \alpha$, where $\mu$ is a function in $t$.

In this case,

$$
k_{\mathcal{X}}=h_{\mathcal{X}}=0
$$

by (9.4.56). Moreover, (9.4.78) and (9.4.79) becomes

$$
\alpha^{\prime} h_{\mathcal{Z}}-\beta^{\prime} \sin \alpha h_{\mathcal{Y}}=\alpha^{\prime \prime}, \quad \alpha^{\prime} k_{\mathcal{Z}}-\beta^{\prime} \sin \alpha k_{\mathcal{Y}}=-\left(\beta^{\prime} \sin \alpha\right)^{\prime} .
$$

Furthermore, (9.4.55) becomes

$$
\begin{gathered}
\alpha^{\prime} k_{\mathcal{Y}}+\beta^{\prime} \sin \alpha h_{\mathcal{Y}}=-\alpha^{\prime \prime}-\alpha^{\prime} \frac{\mu^{\prime}}{\mu}, \\
\alpha^{\prime} k_{\mathcal{Z}}+\beta^{\prime} \sin \alpha h_{\mathcal{Z}}=-\left(\beta^{\prime} \sin \alpha\right)^{\prime}-\frac{\beta^{\prime} \mu^{\prime}}{\mu} \sin \alpha .
\end{gathered}
$$

Adding (9.4.82) to the first equation in (9.4.81), we get

$$
\alpha^{\prime}\left(k_{\mathcal{Y}}+h_{\mathcal{Z}}\right)=-\alpha^{\prime} \frac{\mu^{\prime}}{\mu} \sim \alpha^{\prime} f_{\mathcal{X}}=\alpha^{\prime} \frac{\mu^{\prime}}{\mu} \Longrightarrow f_{\mathcal{X}}=\frac{\mu^{\prime}}{\mu}
$$

by (9.4.44). Note

$$
h_{\mathcal{Z}}=-f_{\mathcal{X}}-h_{\mathcal{Y}}=-\frac{\mu^{\prime}}{\mu}-k_{\mathcal{Y}} .
$$

Substituting (9.4.85) into the first equation (9.4.81), we have

$$
h_{\mathcal{y}}=-\frac{\mu\left(\alpha^{\prime} k_{y}+\alpha^{\prime \prime}\right)+\alpha^{\prime} \mu^{\prime}}{\beta^{\prime} \mu \sin \alpha} .
$$

In addition, the second equation in (9.4.81) yields

$$
k_{\mathcal{Z}}=\frac{\beta^{\prime} \sin \alpha k_{y}-\left(\beta^{\prime} \sin \alpha\right)^{\prime}}{\alpha^{\prime}} .
$$

Note that (9.4.85)-(9.4.87) satisfy (9.4.82) and (9.4.83).

According to (9.4.58),

$$
\frac{\beta^{\prime} \sin \alpha k_{y}-\left(\beta^{\prime} \sin \alpha\right)^{\prime}}{\alpha^{\prime}}+\frac{\mu\left(\alpha^{\prime} k_{y}+\alpha^{\prime \prime}\right)+\alpha^{\prime} \mu^{\prime}}{\beta^{\prime} \mu \sin \alpha}=2 \beta^{\prime} \cos \alpha .
$$

Thus

$$
\mu\left(\alpha^{\prime 2}+\beta^{\prime 2} \sin ^{2} \alpha\right) k_{\mathcal{Y}}-\mu\left(\beta^{\prime} \sin \alpha\right)^{\prime} \beta^{\prime} \sin \alpha+\mu \alpha^{\prime} \alpha^{\prime \prime}+\alpha^{\prime 2} \mu^{\prime}=\alpha^{\prime} \beta^{\prime 2} \mu \sin 2 \alpha
$$




$$
\Longrightarrow k_{\mathcal{Y}}=\frac{\mu\left[\alpha^{\prime}\left(\beta^{\prime 2} \sin 2 \alpha-\alpha^{\prime \prime}\right)+\left(\beta^{\prime} \sin \alpha\right)^{\prime} \beta^{\prime} \sin \alpha\right]-\alpha^{\prime 2} \mu^{\prime}}{\mu\left(\alpha^{\prime 2}+\beta^{\prime 2} \sin ^{2} \alpha\right)} .
$$

By (9.4.87),

$$
k_{\mathcal{Z}}=\frac{\left[\mu\left({\beta^{\prime 2}}^{2} \sin 2 \alpha-\alpha^{\prime \prime}\right)-\alpha^{\prime} \mu^{\prime}\right] \beta^{\prime} \sin \alpha-\mu \alpha^{\prime}\left(\beta^{\prime} \sin \alpha\right)^{\prime}}{\mu\left(\alpha^{\prime 2}+\beta^{\prime 2} \sin ^{2} \alpha\right)} .
$$

Moreover,

$$
h_{\mathcal{Y}}=k_{\mathcal{Z}}-2 \beta^{\prime} \cos \alpha=-\frac{\beta^{\prime}\left[\left(\mu \alpha^{\prime \prime}+\alpha^{\prime} \mu^{\prime}\right) \sin \alpha+2 \mu \alpha^{\prime 2} \cos \alpha\right]+\mu \alpha^{\prime}\left(\beta^{\prime} \sin \alpha\right)^{\prime}}{\mu\left(\alpha^{\prime 2}+{\beta^{\prime}}^{2} \sin ^{2} \alpha\right)} .
$$

In addition, (9.4.85) gives

$$
h_{\mathcal{Z}}=-\frac{\alpha^{\prime}\left(\beta^{\prime 2} \sin 2 \alpha-\alpha^{\prime \prime}\right)+\left(\beta^{\prime} \sin \alpha\right)^{\prime} \beta^{\prime} \sin \alpha+\beta^{\prime 2} \sin ^{2} \alpha}{\alpha^{\prime 2}+\beta^{\prime 2} \sin ^{2} \alpha} .
$$

In particular, $k=k_{\mathcal{Y}} \mathcal{Y}+k_{\mathcal{Z}} \mathcal{Z}$ and $h=h_{\mathcal{Y}} \mathcal{Y}+h_{\mathcal{Z}} \mathcal{Z}$ are determined by (9.4.90)-(9.4.93).

Now (9.4.70) is equivalent to

$$
(\gamma \tau)^{\prime}+2 \gamma \tau f_{\mathcal{X}}-(-1)^{r} \nu \gamma^{2} \tau=0
$$

According to (9.4.84), the above equation can be written as

$$
(\gamma \tau)^{\prime}+\frac{2 \mu^{\prime}}{\mu}(\gamma \tau)-(-1)^{r} \nu \gamma(\gamma \tau)=0
$$

So

$$
\gamma \tau=\frac{1}{\mu^{2}} \exp \left((-1)^{r} \nu \int \gamma d t\right)=\frac{1}{\mu^{2}} \exp \left[(-1)^{r} \nu \int \mu^{2}\left(\alpha^{\prime 2}+\beta^{\prime 2} \sin ^{2} \alpha\right) d t\right]
$$

Hence

$$
\tau=\frac{\exp \left[(-1)^{r} \nu \int \mu^{2}\left(\alpha^{\prime 2}+\beta^{\prime 2} \sin ^{2} \alpha\right) d t\right]}{\mu^{4}\left(\alpha^{\prime 2}+{\beta^{\prime}}^{2} \sin ^{2} \alpha\right)} .
$$

Note that (9.4.76) and (9.4.77) are implied by

$$
\begin{gathered}
\left(f_{\mathcal{X}}-(-1)^{r} \gamma \nu\right) \varphi+\varphi^{\prime}-\alpha_{1} \gamma \tau=0 \\
\Longrightarrow \alpha_{1}=\frac{\left[\mu \mu^{\prime}-(-1)^{r} \mu^{4}\left(\alpha^{\prime 2}+\beta^{\prime 2} \sin ^{2} \alpha\right)\right] \varphi+\mu^{2} \varphi^{\prime}}{\exp \left[(-1)^{r} \nu \int \mu^{2}\left(\alpha^{\prime 2}+\beta^{\prime 2} \sin ^{2} \alpha\right) d t\right]} .
\end{gathered}
$$

It can be verified that the equation for the coefficients of $\mathcal{X}^{0}$ in $\partial_{\mathcal{Z}}\left(R_{2}\right)=\partial_{\mathcal{Y}}\left(R_{3}\right)$ is implied by (9.4.55), (9.4.58) and the assumption that $\sigma=0, \gamma_{1}=\alpha^{\prime} \mu$ and $\gamma_{2}=\beta^{\prime} \mu \sin \alpha$.

According to (9.4.45)-(9.4.47), (9.4.59), (9.4.90)-(9.4.93), (9.4.97) and (9.4.99), we have

$$
\begin{aligned}
\mathcal{U}= & \frac{\mu^{\prime}}{\mu} \mathcal{X}-\alpha^{\prime} \mathcal{Y}-\beta^{\prime} \mathcal{Z} \sin \alpha+\left[\varphi-\mu^{-2} \exp \left[(-1)^{r} \nu \int \mu^{2}\left(\alpha^{\prime 2}+\beta^{\prime 2} \sin ^{2} \alpha\right) d t\right]\right] \zeta_{r} \\
& +\frac{\left[\mu \mu^{\prime}-(-1)^{r} \mu^{4}\left(\alpha^{\prime 2}+{\beta^{\prime}}^{2} \sin ^{2} \alpha\right)\right] \varphi+\mu^{2} \varphi^{\prime}}{\exp \left[(-1)^{r} \nu \int \mu^{2}\left(\alpha^{\prime 2}+{\beta^{\prime}}^{2} \sin ^{2} \alpha\right) d t\right]},
\end{aligned}
$$




$$
\begin{gathered}
\mathcal{V}=\alpha^{\prime} \mathcal{X}-\beta^{\prime} \mathcal{Z} \cos \alpha+k+\frac{\alpha^{\prime} \xi_{r} \exp \left[(-1)^{r} \nu \int \mu^{2}\left(\alpha^{\prime 2}+\beta^{\prime 2} \sin ^{2} \alpha\right) d t\right]}{\mu^{3}\left(\alpha^{\prime 2}+{\beta^{\prime}}^{2} \sin ^{2} \alpha\right)} \\
\mathcal{W}=\beta^{\prime}(\mathcal{X} \sin \alpha+\mathcal{Y} \cos \alpha)+h+\frac{\beta^{\prime} \xi_{r} \sin \alpha \exp \left[(-1)^{r} \nu \int \mu^{2}\left(\alpha^{\prime 2}+\beta^{\prime 2} \sin ^{2} \alpha\right) d t\right]}{\mu^{3}\left(\alpha^{\prime 2}+\beta^{\prime 2} \sin ^{2} \alpha\right)} .
\end{gathered}
$$

Observe that $f^{*}=\varphi \zeta_{r}+\alpha_{1}$ and so

$$
f_{\tilde{\varpi}}^{*} \xi_{r}-f^{*} \zeta_{r}=\varphi\left((-1)^{r} \xi_{r}^{2}-\zeta_{r}^{2}\right)-\alpha_{1} \zeta_{r}=-\left(4 b \delta_{0, r}+c \delta_{1, r}\right) c \varphi-\alpha_{1} \zeta_{r} .
$$

Hence

$$
\begin{aligned}
R_{1}= & \left(\mu^{\prime} / \mu-\alpha^{\prime 2}-{\beta^{\prime}}^{2} \sin ^{2} \alpha\right) \mathcal{X}+\left(\alpha^{\prime \prime}+2 \alpha^{\prime} \mu^{\prime} / \mu-2^{-1} \beta^{\prime 2} \sin 2 \alpha\right) \mathcal{Y} \\
& +\left(\alpha^{\prime} \beta^{\prime} \cos \alpha+\left(\beta^{\prime} \sin \alpha\right)^{\prime}+2 \beta^{\prime} \mu^{\prime} \sin \alpha / \mu\right) \mathcal{Z} \\
& +\gamma \tau\left(4 b \delta_{0, r}+c \delta_{1, r}\right) c(\gamma \tau \mathcal{X}-\varphi)-2 \gamma \tau \xi_{r} / \mu
\end{aligned}
$$

by (9.4.49), (9.4.55), (9.4.74), (9.4.75) and (9.4.103). Moreover, (9.4.52), (9.4.53), (9.4.55) and (9.4.58) yield

$$
\begin{aligned}
R_{2}= & \left(\alpha^{\prime \prime}-2^{-1}{\beta^{\prime}}^{2} \sin 2 \alpha+2 \alpha^{\prime} \mu^{\prime} / \mu\right) \mathcal{X}+\left(k_{\mathcal{Y} t}-\alpha^{\prime 2}-{\beta^{\prime}}^{2} \cos ^{2} \alpha\right) \mathcal{Y}+ \\
& +\gamma_{1}\left(\tau^{\prime}-(-1)^{r} \nu \gamma \tau\right) \xi_{r}+\left(\left(k_{\mathcal{Z}}-\beta^{\prime} \cos \alpha\right)^{\prime}-\alpha^{\prime} \beta^{\prime} \sin \alpha\right) \mathcal{Z} \\
& +2 \alpha^{\prime} f^{*}+\gamma \gamma_{1} \tau^{2} \xi_{r} \zeta_{r}-2 \alpha^{\prime} \gamma \tau \zeta_{r} \mathcal{X}+k k_{\mathcal{Y}}+h h_{\mathcal{Y}} \\
R_{3}= & {\left[\left(\beta^{\prime} \sin \alpha\right)^{\prime}+\alpha^{\prime} \beta^{\prime} \cos \alpha+2 \beta^{\prime} \mu^{\prime} \sin \alpha / \mu\right] \mathcal{X}+\left[\left(h_{\mathcal{Y}}+\beta^{\prime} \cos \alpha\right)^{\prime}-\alpha^{\prime} \beta^{\prime} \sin \alpha\right] \mathcal{Y} } \\
& +\gamma_{2}\left(\tau^{\prime}-(-1)^{r} \nu \gamma \tau\right) \xi_{r}-2 \beta^{\prime} \gamma \tau \sin \alpha \zeta_{r} \mathcal{X}+\left(h_{\mathcal{Z} t}-\beta^{\prime 2}\right) \mathcal{Z} \\
& +2 \beta^{\prime} \sin \alpha f^{*}+k k_{\mathcal{Z}}+h h_{\mathcal{Z}}+\gamma \gamma_{2} \tau^{2} \xi_{r} \zeta_{r} .
\end{aligned}
$$

By (9.3.3), (9.3.5), (9.3.22), (9.4.100)-(9.4.102) and (9.4.104)-(9.4.106), we have the following theorem:

Theorem 9.4.2. Let $\alpha, \beta, \varphi, \mu$ be arbitrary functions in $\operatorname{such}$ that $\mu\left(\alpha^{\prime 2}+\beta^{\prime 2} \sin ^{2} \alpha\right) \neq$ 0 , and let $b, c$ be arbitrary real constants. Define the moving frame $\mathcal{X}, \mathcal{Y}$ and $\mathcal{Z}$ by (9.3.1) and (9.3.5), and

$$
\begin{array}{ll}
\xi_{0}=b e^{\mu\left(\alpha^{\prime} \mathcal{Y}+\beta^{\prime} \mathcal{Z} \sin \alpha\right)}-c e^{-\mu\left(\alpha^{\prime} \mathcal{Y}+\beta^{\prime} \mathcal{Z} \sin \alpha\right)}, & \xi_{1}=c \sin \left[\mu\left(\alpha^{\prime} \mathcal{Y}+\beta^{\prime} \mathcal{Z} \sin \alpha\right)+b\right] \\
\zeta_{0}=b e^{\mu\left(\alpha^{\prime} \mathcal{Y}+\beta^{\prime} \mathcal{Z} \sin \alpha\right)}+c e^{-\mu\left(\alpha^{\prime} \mathcal{Y}+\beta^{\prime} \mathcal{Z} \sin \alpha\right)}, & \zeta_{1}=c \cos \left[\mu\left(\alpha^{\prime} \mathcal{Y}+\beta^{\prime} \mathcal{Z} \sin \alpha\right)+b\right]
\end{array}
$$

Moreover, $k=k_{\mathcal{Y}} \mathcal{Y}+k_{\mathcal{Z}} \mathcal{Z}$ and $h=h_{\mathcal{Y}} \mathcal{Y}+h_{\mathcal{Z}} \mathcal{Z}$ are defined by (9.4.90)-(9.4.93). For 
$r=0,1$, we have the following solution of the Navier-Stokes equations (9.1.1)-(9.1.4):

$$
\begin{aligned}
& u=\left(\frac{\mu^{\prime}}{\mu} \mathcal{X}-\alpha^{\prime} \mathcal{Y}+\left[\varphi-\mu^{-2} \exp \left[(-1)^{r} \nu \int \mu^{2}\left(\alpha^{\prime 2}+\beta^{\prime 2} \sin ^{2} \alpha\right) d t\right]\right] \zeta_{r}\right) \cos \alpha \\
& -\left[\alpha^{\prime} \mathcal{X}+k+\frac{\alpha^{\prime} \xi_{r} \exp \left[(-1)^{r} \nu \int \mu^{2}\left(\alpha^{\prime 2}+\beta^{\prime 2} \sin ^{2} \alpha\right) d t\right]}{\mu^{3}\left(\alpha^{\prime 2}+\beta^{\prime 2} \sin ^{2} \alpha\right)}\right] \sin \alpha \\
& +\frac{\left[\mu \mu^{\prime}-(-1)^{r} \mu^{4}\left(\alpha^{\prime 2}+{\beta^{\prime}}^{2} \sin ^{2} \alpha\right)\right] \varphi+\mu^{2} \varphi^{\prime}}{\exp \left[(-1)^{r} \nu \int \mu^{2}\left(\alpha^{\prime 2}+\beta^{\prime 2} \sin ^{2} \alpha\right) d t\right]} \cos \alpha, \\
& v=\left(\frac{\mu^{\prime}}{\mu} \mathcal{X}-\alpha^{\prime} \mathcal{Y}+\left[\varphi-\mu^{-2} \exp \left[(-1)^{r} \nu \int \mu^{2}\left(\alpha^{\prime 2}+\beta^{\prime 2} \sin ^{2} \alpha\right) d t\right]\right] \zeta_{r}\right) \sin \alpha \cos \beta \\
& +\left[\left(\alpha^{\prime} \mathcal{X}+k\right) \cos \alpha-\beta^{\prime} \mathcal{Z}\right] \cos \beta-\left[\beta^{\prime}(\mathcal{X} \sin \alpha+\mathcal{Y} \cos \alpha)+h\right] \sin \beta \\
& +\frac{\left(\alpha^{\prime} \cos \alpha \cos \beta-\beta^{\prime} \sin \alpha \sin \beta\right) \xi_{r} \exp \left[(-1)^{r} \nu \int \mu^{2}\left(\alpha^{\prime 2}+{\beta^{\prime}}^{2} \sin ^{2} \alpha\right) d t\right]}{\mu^{3}\left(\alpha^{\prime 2}+\beta^{\prime 2} \sin ^{2} \alpha\right)} \\
& +\frac{\left[\mu \mu^{\prime}-(-1)^{r} \mu^{4}\left(\alpha^{\prime 2}+{\beta^{\prime}}^{2} \sin ^{2} \alpha\right)\right] \varphi+\mu^{2} \varphi^{\prime}}{\exp \left[(-1)^{r} \nu \int \mu^{2}\left(\alpha^{\prime 2}+\beta^{\prime 2} \sin ^{2} \alpha\right) d t\right]} \sin \alpha \cos \beta, \\
& w=\left(\frac{\mu^{\prime}}{\mu} \mathcal{X}-\alpha^{\prime} \mathcal{Y}+\left[\varphi-\mu^{-2} \exp \left[(-1)^{r} \nu \int \mu^{2}\left(\alpha^{\prime 2}+{\beta^{\prime 2}}^{2} \sin ^{2} \alpha\right) d t\right]\right] \zeta_{r}\right) \sin \alpha \sin \beta \\
& +\left[\left(\alpha^{\prime} \mathcal{X}+k\right) \cos \alpha-\beta^{\prime} \mathcal{Z}\right] \sin \beta+\left[\beta^{\prime}(\mathcal{X} \sin \alpha+\mathcal{Y} \cos \alpha)+h\right] \cos \beta \\
& +\frac{\left(\alpha^{\prime} \cos \alpha \sin \beta+\beta^{\prime} \sin \alpha \cos \beta\right) \xi_{r} \exp \left[(-1)^{r} \nu \int \mu^{2}\left(\alpha^{\prime 2}+{\beta^{\prime 2}}^{2} \sin ^{2} \alpha\right) d t\right]}{\mu^{3}\left(\alpha^{\prime 2}+\beta^{\prime 2} \sin ^{2} \alpha\right)} \\
& +\frac{\left[\mu \mu^{\prime}-(-1)^{r} \mu^{4}\left(\alpha^{\prime 2}+\beta^{\prime 2} \sin ^{2} \alpha\right)\right] \varphi+\mu^{2} \varphi^{\prime}}{\exp \left[(-1)^{r} \nu \int \mu^{2}\left(\alpha^{\prime 2}+{\beta^{\prime}}^{2} \sin ^{2} \alpha\right) d t\right]} \sin \alpha \sin \beta, \\
& p=\rho\left\{\left(\alpha^{\prime 2}+{\beta^{\prime}}^{2} \sin ^{2} \alpha-\mu^{\prime} / \mu\right) \mathcal{X}^{2} / 2+\left(2^{-1}{\beta^{\prime}}^{2} \sin 2 \alpha-\alpha^{\prime \prime}-2 \alpha^{\prime} \mu^{\prime} / \mu\right) \mathcal{X Y}\right. \\
& -\left(\alpha^{\prime} \beta^{\prime} \cos \alpha+\left(\beta^{\prime} \sin \alpha\right)^{\prime}+2 \beta^{\prime} \mu^{\prime} \sin \alpha / \mu\right) \mathcal{X} \mathcal{Z}-2 \gamma \tau \xi_{r} \mathcal{X} / \mu \\
& +2^{-1}\left[\left(\alpha^{\prime 2}+\beta^{\prime 2} \cos ^{2} \alpha-k_{\mathcal{Y} t}\right) \mathcal{Y}^{2}+\left({\beta^{\prime}}^{2}-h_{\mathcal{Z} t}\right) \mathcal{Z}^{2}-k^{2}-h^{2}\right] \\
& +\left(4 b \delta_{0, r}+c \delta_{1, r}\right) c \mu^{-2} \exp \left[(-1)^{r} \nu \int \mu^{2}\left(\alpha^{\prime 2}+{\beta^{\prime}}^{2} \sin ^{2} \alpha\right) d t\right] \\
& \times\left(\varphi-2^{-1} \mu^{-2} \exp \left[(-1)^{r} \nu \int \mu^{2}\left(\alpha^{\prime 2}+\beta^{\prime 2} \sin ^{2} \alpha\right) d t\right] \mathcal{X}\right) \\
& +\left(\alpha^{\prime} \beta^{\prime} \sin \alpha-\left(k_{\mathcal{Z}}-\beta^{\prime} \cos \alpha\right)^{\prime}\right) \mathcal{Y} \mathcal{Z}-\frac{\xi^{2} \exp \left[(-1)^{r} 2 \nu \int \mu^{2}\left(\alpha^{\prime 2}+\beta^{\prime 2} \sin ^{2} \alpha\right) d t\right]}{2 \mu^{8}\left(\alpha^{\prime 2}+\beta^{\prime 2} \sin ^{2} \alpha\right)} \\
& -2 \mu^{-1} \varphi \xi_{r}-\frac{2\left(\alpha^{\prime} \mathcal{Y}+\beta^{\prime} \sin \alpha \mathcal{Z}\right)\left[\mu \mu^{\prime}-(-1)^{r} \mu^{4}\left(\alpha^{\prime 2}+\beta^{\prime 2} \sin ^{2} \alpha\right)\right] \varphi+\mu^{2} \varphi^{\prime}}{\exp \left[(-1)^{r} \nu \int \mu^{2}\left(\alpha^{\prime 2}+{\beta^{\prime}}^{2} \sin ^{2} \alpha\right) d t\right]} \\
& -(-1)^{r} \frac{\zeta_{r}\left[\mu^{4}\left(\alpha^{\prime 2}+{\beta^{\prime}}^{2} \sin ^{2} \alpha\right)\right]^{\prime} \exp \left[(-1)^{r} \nu \int \mu^{2}\left(\alpha^{\prime 2}+{\beta^{\prime}}^{2} \sin ^{2} \alpha\right) d t\right]}{\mu^{4}\left(\alpha^{\prime 2}+\beta^{\prime 2} \sin ^{2} \alpha\right)} .
\end{aligned}
$$


Case 2. $\gamma_{2}=\alpha_{1}=\tau=0$ and $\gamma_{1} \neq 0$.

Under the assumption, (9.4.70) naturally holds. According to (9.4.55) and (9.4.58),

$$
k_{\mathcal{Y}}=-\frac{\gamma_{1}^{\prime}}{\gamma_{1}}, \quad k_{\mathcal{X}}=k_{\mathcal{Z}}=0, \quad h_{\mathcal{Y}}=-2 \beta^{\prime} \cos \alpha .
$$

Note $\gamma=\gamma_{1}^{2}$. Moreover, (9.4.56) says

$$
h_{\mathcal{X}}=-2 \beta^{\prime} \sin \alpha .
$$

Furthermore, (9.4.78) becomes

$$
2 \alpha^{\prime \prime}-2 \alpha^{\prime} h_{\mathcal{Z}}=0 \Longrightarrow h_{Z}=\frac{\alpha^{\prime \prime}}{\alpha^{\prime}}
$$

and (9.4.79) is satisfied naturally. Equation (9.4.44) yields

$$
f_{\mathcal{X}}=\frac{\gamma_{1}^{\prime}}{\gamma_{1}}-\frac{\alpha^{\prime \prime}}{\alpha^{\prime}} .
$$

Now (9.4.76) and (9.4.77) are equivalent to

$$
\begin{gathered}
\frac{\varphi^{\prime}}{\varphi}=\frac{\sigma^{\prime}}{\sigma}=(-1)^{r} \gamma \nu-f_{\mathcal{X}}=(-1)^{r} \nu \gamma_{1}^{2}-\frac{\gamma_{1}^{\prime}}{\gamma_{1}}+\frac{\alpha^{\prime \prime}}{\alpha^{\prime}}, \\
\frac{\left(\gamma_{1} \sigma\right)^{\prime}}{\left(\gamma_{1} \sigma\right)}=h_{\mathcal{Z}}+(-1)^{r} \nu \gamma=(-1)^{r} \nu \gamma_{1}^{2}+\frac{\alpha^{\prime \prime}}{\alpha^{\prime}} .
\end{gathered}
$$

According to (9.4.117),

$$
\varphi=b_{2} \alpha^{\prime} \gamma_{1}^{-1} e^{(-1)^{r} \nu \int \gamma_{1}^{2} d t}, \quad \sigma=b_{3} \alpha^{\prime} \gamma_{1}^{-1} e^{(-1)^{r} \nu \int \gamma_{1}^{2} d t}
$$

with $b_{2}, b_{3} \in \mathbb{R}$. Moreover, (9.4.118) is satisfied by the above $\sigma$.

Next

$$
\gamma \sigma=b_{2} \alpha^{\prime} \gamma_{1} e^{(-1)^{r} \nu \int \gamma_{1}^{2} d t} \Longrightarrow \frac{(\gamma \sigma)^{\prime}}{(\gamma \sigma)}=(-1)^{r} \nu \gamma_{1}^{2}+\frac{\alpha^{\prime \prime}}{\alpha^{\prime}}+\frac{\gamma_{1}^{\prime}}{\gamma} .
$$

On the other hand, (9.4.51) implies

$$
\frac{(\gamma \sigma)^{\prime}}{(\gamma \sigma)}=(-1)^{r} \nu \gamma-3 f_{\mathcal{X}}=(-1)^{r} \nu \gamma_{1}^{2}-\frac{3 \gamma_{1}^{\prime}}{\gamma_{1}}+\frac{3 \alpha^{\prime \prime}}{\alpha^{\prime}} .
$$

So

$$
\frac{\alpha^{\prime \prime}}{\alpha^{\prime}}=2 \frac{\gamma_{1}^{\prime}}{\gamma} \Longrightarrow \gamma_{1}=c_{2} \sqrt{\alpha^{\prime}}, \quad 0 \neq c_{2} \in \mathbb{R}
$$

Thus

$$
\varphi=b_{2} c_{2}^{-1} \sqrt{\alpha^{\prime}} e^{(-1)^{r} c_{2}^{2} \nu \alpha}, \quad \sigma=b_{3} c_{2}^{-1} \sqrt{\alpha^{\prime}} e^{(-1)^{r} c_{2}^{2} \nu \alpha}, \quad f_{\mathcal{X}}=k_{\mathcal{Y}}=-\frac{\alpha^{\prime \prime}}{2 \alpha^{\prime}} .
$$


Observe

$$
\begin{aligned}
& \gamma_{1} f_{\mathcal{Y}} \phi_{r}-\gamma f^{*} \psi_{r} \\
= & \gamma\left[\left(\varphi \hat{\vartheta}_{r}+\sigma \phi_{r}+\sigma \tilde{\varpi} \psi_{r}\right) \phi_{r}-\left(\varphi \vartheta_{r}+\sigma \tilde{\varpi} \phi_{r}\right) \psi_{r}\right] \\
= & \gamma\left[\varphi\left(\hat{\vartheta}_{r} \phi_{r}-\vartheta_{r} \psi_{r}\right)+\sigma \phi_{r} \xi_{r}\right] \\
= & {\left[2\left(c-a b_{1}\right) \delta_{r, 0}-c_{1} \sin b_{1} \delta_{r, 1}\right] \gamma \varphi+\gamma \sigma \phi_{r}^{2} }
\end{aligned}
$$

by (9.4.60) and (9.4.62). According to (9.4.49), (9.4.52), (9.4.53), (9.4.113) and (9.4.114), we have

$$
\begin{aligned}
R_{1}= & {\left[f_{\mathcal{X} t}-\alpha^{\prime 2}-\beta^{\prime 2} \sin ^{2} \alpha+2 \gamma \varphi \sigma\left[2\left(c-a b_{1}\right) \delta_{r, 0}-c_{1} \sin b_{1} \delta_{r, 1}\right]\right] \mathcal{X} } \\
& -\left(\alpha^{\prime \prime}+2^{-1} \beta^{\prime 2} \sin 2 \alpha\right) \mathcal{Y}+\left(\alpha^{\prime} \beta^{\prime} \cos \alpha-\left(\beta^{\prime} \sin \alpha\right)^{\prime}\right) \mathcal{Z}+2 \gamma^{2} \sigma^{2}\left(4 a \delta_{0, r}+\delta_{1, r}\right) \mathcal{X}^{3} \\
& -2 \alpha^{\prime} k-2 \beta^{\prime} h \sin \alpha+2 \sigma\left(\gamma \sigma \phi_{r}-2 \gamma_{1} \alpha^{\prime}\right) \phi_{r} \mathcal{X} \\
R_{2}= & \left(\alpha^{\prime \prime}-2^{-1} \beta^{\prime 2} \sin 2 \alpha+2 \alpha^{\prime} f_{\mathcal{X}}\right) \mathcal{X}-\left(\alpha^{\prime 2}+\beta^{\prime 2} \cos ^{2} \alpha\right) \mathcal{Y}+k_{t}+k k_{\mathcal{Y}} \\
& -\left(\left(\beta^{\prime} \cos \alpha\right)^{\prime}+\alpha^{\prime} \beta^{\prime} \sin \alpha\right) \mathcal{Z}-2 \beta^{\prime} h \cos \alpha \\
& +\gamma \sigma\left(2 \sigma \gamma_{1} \phi_{r}-2 \alpha^{\prime}\right) \psi_{r} \mathcal{X}^{2}+f^{*}\left(2 \alpha^{\prime}+2 \gamma_{1} \sigma \phi_{r}\right), \\
R_{3}=[ & \left.\left(\beta^{\prime} \sin \alpha\right)^{\prime}+\alpha^{\prime} \beta^{\prime} \cos \alpha\right] \mathcal{X}+\left[\left(\beta^{\prime} \cos \alpha\right)^{\prime}-\alpha^{\prime} \beta^{\prime} \sin \alpha\right] \mathcal{Y}+h_{t}+h h_{\mathcal{Z}}-\beta^{\prime 2} \mathcal{Z} .
\end{aligned}
$$

In particular, (9.3.24) holds by (9.4.113), (9.4.114) and (9.4.123).

Expressions (9.4.45)-(9.4.47) become

$$
\begin{gathered}
\mathcal{U}=-\frac{\alpha^{\prime \prime}}{2 \alpha^{\prime}} \mathcal{X}-\alpha^{\prime} \mathcal{Y}-\beta^{\prime} \mathcal{Z} \sin \alpha+\left[b_{2} c_{2}^{-1} \sqrt{\alpha^{\prime}} \vartheta_{r}+b_{3} \alpha^{\prime}\left(\mathcal{Y} \phi_{r}-c_{2} \sqrt{\alpha^{\prime}} \psi_{r} \mathcal{X}^{2}\right)\right] e^{(-1)^{r} c_{2}^{2} \nu \alpha} \\
\mathcal{V}=\alpha^{\prime} \mathcal{X}-\frac{\alpha^{\prime \prime}}{2 \alpha^{\prime}} \mathcal{Y}-\beta^{\prime} \mathcal{Z} \cos \alpha+2 b_{3} c_{2}^{2} \alpha^{\prime 2} e^{(-1)^{r} c_{2}^{2} \nu \alpha} \phi_{r} \mathcal{X} \\
\mathcal{W}=-\beta^{\prime}(\mathcal{X} \sin \alpha+\mathcal{Y} \cos \alpha)+\frac{\alpha^{\prime \prime}}{\alpha^{\prime}} \mathcal{Z}
\end{gathered}
$$

By (9.3.3), (9.3.5), (9.3.22), (9.4.113), (9.4.114), (9.4.123) and (9.4.125)-(9.4.130), we have the following theorem:

Theorem 9.4.3. Let $\alpha, \beta$ be arbitrary functions in $t$ and let $a, b_{1}, b_{2}, c_{2}$ be real constants. Define the moving frame $\mathcal{X}, \mathcal{Y}$ and $\mathcal{Z}$ by (9.3.1) and (9.3.5), and

$$
\begin{gathered}
\phi_{0}=e^{c_{2} \sqrt{\alpha^{\prime}} \mathcal{Y}}-a e^{-c_{2} \sqrt{\alpha^{\prime}} \mathcal{Y}}, \quad \phi_{1}=\sin \left(c_{2} \sqrt{\alpha^{\prime}} \mathcal{Y}\right), \quad \psi_{0}=e^{c_{2} \sqrt{\alpha^{\prime}} \mathcal{Y}}+a e^{-c_{2} \sqrt{\alpha^{\prime}} \mathcal{Y}}, \\
\psi_{1}=\cos \left(c_{2} \sqrt{\alpha^{\prime}} \mathcal{Y}\right), \quad \vartheta_{0}=b_{1} e^{c_{2} \sqrt{\alpha^{\prime}} \mathcal{Y}}-c_{1} e^{-c_{2} \sqrt{\alpha^{\prime}} \mathcal{Y}}, \quad \vartheta_{1}=c_{1} \sin \left(c_{2} \sqrt{\alpha^{\prime}} \mathcal{Y}+b_{1}\right) .
\end{gathered}
$$

For $r=0,1$, we have the following solution of the Navier-Stokes equations (9.1.1)-(9.1.4):

$$
\begin{aligned}
u= & {\left[-\alpha^{\prime \prime} \mathcal{X} /\left(2 \alpha^{\prime}\right)-\alpha^{\prime} \mathcal{Y}+\left[b_{2} c_{2}^{-1} \sqrt{\alpha^{\prime}} \vartheta_{r}+b_{3} \alpha^{\prime}\left(\mathcal{Y} \phi_{r}-c_{2} \sqrt{\alpha^{\prime}} \psi_{r} \mathcal{X}^{2}\right)\right] e^{(-1)^{r} c_{2}^{2} \nu \alpha}\right] \cos \alpha } \\
& -\left[\alpha^{\prime} \mathcal{X}-\alpha^{\prime \prime} \mathcal{Y} /\left(2 \alpha^{\prime}\right)+2 b_{3} c_{2}^{2} \alpha^{\prime 2} e^{(-1)^{r} c_{2}^{2} \nu \alpha} \phi_{r} \mathcal{X}\right] \sin \alpha,
\end{aligned}
$$




$$
\begin{aligned}
v= & {\left[-\alpha^{\prime \prime} \mathcal{X} /\left(2 \alpha^{\prime}\right)-\alpha^{\prime} \mathcal{Y}+\left[b_{2} c_{2}^{-1} \sqrt{\alpha^{\prime}} \vartheta_{r}+b_{3} \alpha^{\prime}\left(\mathcal{Y} \phi_{r}-c_{2} \sqrt{\alpha^{\prime}} \psi_{r} \mathcal{X}^{2}\right)\right] e^{(-1)^{r} c_{2}^{2} \nu \alpha}\right] \sin \alpha \cos \beta } \\
& +\left[\left[\alpha^{\prime} \mathcal{X}-\alpha^{\prime \prime} \mathcal{Y} /\left(2 \alpha^{\prime}\right)+2 b_{3} c_{2}^{2} \alpha^{\prime 2} e^{(-1)^{r} c_{2}^{2} \nu \alpha} \phi_{r} \mathcal{X}\right] \cos \alpha-\beta^{\prime} \mathcal{Z}\right] \cos \beta \\
& +\left[\beta^{\prime}(\mathcal{X} \sin \alpha+\mathcal{Y} \cos \alpha)-\alpha^{\prime \prime} \mathcal{Z} / \alpha^{\prime}\right] \sin \beta
\end{aligned}
$$

$$
\begin{aligned}
w= & {\left[-\alpha^{\prime \prime} \mathcal{X} /\left(2 \alpha^{\prime}\right)-\alpha^{\prime} \mathcal{Y}+\left[b_{2} c_{2}^{-1} \sqrt{\alpha^{\prime}} \vartheta_{r}+b_{3} \alpha^{\prime}\left(\mathcal{Y} \phi_{r}-c_{2} \sqrt{\alpha^{\prime}} \psi_{r} \mathcal{X}^{2}\right)\right] e^{(-1)^{r} c_{2}^{2} \nu \alpha}\right] \sin \alpha \sin \beta } \\
& +\left[\left[\alpha^{\prime} \mathcal{X}-\alpha^{\prime \prime} \mathcal{Y} /\left(2 \alpha^{\prime}\right)+2 b_{3} c_{2}^{2} \alpha^{\prime 2} e^{(-1)^{r} c_{2}^{2} \nu \alpha} \phi_{r} \mathcal{X}\right] \cos \alpha-\beta^{\prime} \mathcal{Z}\right] \sin \beta \\
& -\left[\beta^{\prime}(\mathcal{X} \sin \alpha+\mathcal{Y} \cos \alpha)-\alpha^{\prime \prime} \mathcal{Z} / \alpha^{\prime}\right] \cos \beta
\end{aligned}
$$

$$
\begin{aligned}
& p=\frac{\rho}{2}\left\{\left[\frac{\alpha^{\prime} \alpha^{\prime \prime \prime}-\alpha^{\prime \prime 2}}{2 \alpha^{\prime 2}}+\alpha^{\prime 2}-3{\beta^{\prime}}^{2} \sin ^{2} \alpha-2 b_{2} b_{3}\left[2\left(c-a b_{1}\right) \delta_{r, 0}-c_{1} \sin b_{1} \delta_{r, 1}\right]\right.\right. \\
& \left.\times \alpha^{\prime 2} e^{(-1)^{r} 2 c_{2}^{2} \nu \alpha}\right] \mathcal{X}^{2}-3 \beta^{\prime 2} \mathcal{X} \mathcal{Y} \sin 2 \alpha-\left(b_{3} c_{2}\right)^{2}\left(4 a \delta_{0, r}+\delta_{1, r}\right) \alpha^{\prime 3} e^{(-1)^{r} 2 c_{2}^{2} \nu \alpha} \mathcal{X}^{4} \\
& +2 \frac{\left(\alpha^{\prime} \beta^{\prime \prime}+2 \alpha^{\prime \prime} \beta^{\prime}\right) \sin \alpha}{\alpha^{\prime}} \mathcal{X} \mathcal{Z}+2 b_{3} c_{2}^{-1} \alpha^{\prime 2} e^{(-1)^{r} c_{2}^{2} \nu \alpha}\left(2-b_{3} c_{2} e^{(-1)^{r} c_{2}^{2} \nu \alpha} \phi_{r}\right) \phi_{r} \mathcal{X}^{2} \\
& -4 c_{2}^{-1} \sqrt{\alpha^{\prime 3}} e^{(-1)^{r} c_{2}^{2} \nu \alpha} \int\left(b_{2} \vartheta_{r}+b_{3} c_{2} \sqrt{\alpha^{\prime}} \mathcal{Y} \phi_{r}\right)\left(1+b_{3} e^{(-1)^{r} c_{2}^{2} \nu \alpha} \phi_{r}\right) d \mathcal{Y} \\
& +2 \frac{\left(\beta^{\prime \prime}-2 \alpha^{\prime \prime} \beta^{\prime}\right) \cos \alpha}{\alpha^{\prime}} \mathcal{Y} \mathcal{Z}+\frac{\left(\alpha^{\prime 2} \beta^{\prime 2}-\alpha^{\prime} \alpha^{\prime \prime \prime}+\alpha^{\prime 2}\right) \mathcal{Z}^{2}}{\alpha^{\prime 2}} \\
& \left.+\left[\frac{2 \alpha^{\prime} \alpha^{\prime \prime \prime}-3{\alpha^{\prime \prime}}^{2}}{4 \alpha^{\prime 2}}+\alpha^{\prime 2}-3{\beta^{\prime 2}}^{2} \cos ^{2} \alpha\right] \mathcal{Y}^{2}\right\}
\end{aligned}
$$




\section{Bibliography}

[AMH] M. B. Abdel-Maleck and M. M. Helal, Similarity solutions for magneto-forcedunsteady free convective laminar boundary-layer flow, J. Comp. Appl. Math. 218 (2008), 202-214.

[AC] M. J. Ablowitz and P. A. Clarkson, Solitons, Nonlinear Evolution Equations and Inverse Scattering, London Math. Soc Lect. Notes 149, Cambridge University Press, 1991.

[AAR] G. Andrews, R. Askey and R. Roy, Special Functions, Cambridge University Press, 1999.

[AEK] N. Akhmediev, V. Eleonskii and N. Kulagin, First-order exact solutions of the nonlinear Schrödinger equation, Teoret. Mat. Fiz. 72 (1987), 183-196.

[Ay] Y. Almog, Thin boundary layer of chiral smectics, Calc. Var. 33 (2008), 299-328.

[AF] D. Anker and N. C. Freeman, On the soliton solutions of the Davey-Stewartson equation for long waves, Proc. Roy. Soc. London Ser. A 360 (1978), 529-540.

[APP1] V. A. Arkadiev, A. K. Pogrebkov and M. C. Polivanov, Closed string solution of the Davey-Stewartson equation, Inverse Problem 5 (1989), L1-L6.

[APP2] V. A. Arkadiev, A. K. Pogrebkov and M. C. Polivanov, Inverse sacttering transform method and soliton solution for the Davey-Stewartson II equation, Phys. D 36 (1989), 189-197.

[AP] A. Azzollini and A. Pomponio, Ground state solutions for the nonlinear SchrödingerMaxwell equations, J. Math. Anal. Appl. 345 (2008), 90-108.

[BP] A. G. Bagdoev and L. G. Petrosyan, Justification of the applicability of short wave equations in obtaining an equation for modulation of a gas-fluid mixture, Izv. Akad. Nauk Armyan. SSR Ser. Mekh. 38 (1985), no. 4, 58-66.

[BG1] J. Barros-Neto and I. M. Gel'fand, Fundamental solutions for the Tricomi operator, Duke Math. J. 98 (1999), 465-483. 
[BG2] J. Barros-Neto and I. M. Gel'fand, Fundamental solutions for the Tricomi operator II, Duke Math. J. 111 (2002), 561-584.

[BO] R. Beerends and E. Opdam, Certain hypergeometric series related to the root system BC, Trans. Amer. Math. Soc. 119 (1993), 581-609.

[Bj] J. Boussinesq, Essai sur la theéries des eaux courants; Mém. Acad. Sci. Inst. Nat. France; 23 (1) (1877), 1-680.

[BK] M. A. Brutyan and P. L. Krapivsky, Exact solutions of Navier-Stokes equations describing the evolution of a vortex structure in generalized shear flow, Comput. Math. Phys. 32 (1992), 270-272.

[Bv] V. O. Bytev, Invariant solutions of the Navier-Stokes equations, Zhumal Prikladnoi Mekhaniki i Tekhnicheskoi Fiziki 6 (1972), 56.

[Cf] F. Calogero, Solution of the one-dimensional $n$-body problem with quadratic and /or inversely quadratic pair potentials, J. Math. Phys. 12 (1971), 419-432.

[Cb1] B. Cao, Solutions of Navier Equations and Their Representation Structure, Adv. Appl. Math. 43 (2009), 331-374.

[Cb2] B. Cao, Solutions of Jimbo-Miwa equation and Konopelchenko-Dubrovsky equations, Acta Appl. Math. 112 (2010), 181-203.

[Cb3] B. Cao, Asymmetric and moving-frame approaches to MHD equations, Acta Math. Sinica 28 (2012), no. 1, 1-36.

[Cd] D. Chae, Global regularity for the 2D Boussinesq equations with partial viscosity, Adv. Math. 203 (2006), 497-513.

[CLMV] J. Cheng, S. Liao, R. N. Mohapatra and Vajravelu, Series solutions of nano boundary layer flows by means of the homotopy analysis method, J. Math. Anal. Apll. 343 (2008), 233-145.

[CCDY] A. Ciattonic, B. Crosignanic, P. Di Porto and A. Yariv, Perfect optical solutions: spatial Kerr solutions as exact solutions of Maxwell's equations, J. Opt. Soc. Amer. B Opt. Phys. 22 (2005), no. 7, 1384-1394.

[CH] P. A. Clarkson and S. Hood, New symmetry reductions and exact solutions of the the Davey-Stewartson system. I. Reductions to ordinary differential equations, J. Math. Phys. 35 (1994), 255-283. 
[DS] A. Davey and K. Stewartson, On three-dimensional packets of surface waves, Proc. Roy. Soc. London Ser. A 338 (1974), 101-110.

[Ep] P. Etingof, Quantum integrable systems and representations of Lie algebras, J. Math. Phys. 36 (1995), no. 6,2637-2651.

[EFS] I. V. Egorov, A. V. Fedorov and V. G. Soudakov, Receptivity of a hypersonic boundary layer over a flat plate with a porous coating, J. Fluid Mech. 601 (2008), 165-187.

[Es] S. Ermakov, Short wave/long wave interaction and amplification of decimeter-scale wind waves in film slicks, Geophysical Research Absstracts 8 (2006), 00469.

[FR] V. I. Fushchich and I. V. Revenko, Exact solutions of the Lorentz-Maxwell equations, Dokl. Akad. Nauk Ukrain. SSR 85 (1989), no. 6, 28-31.

[FSS] V. I. Fushchich, V. M. Shtelen and S. V. Spichak, On the connection between solutions of Dirac and Maxwell equations, dual Poincaré invariance and superalgebras of invariance and solutions of nonlinear Dirac equations, J. Phys. A24 (1991), no. 8, 1683-1698.

[GW] L. Gagnon and P. Winternitz, Exact solutions of the cubic and quintic nonlinear Schrödinger equation for a cylindrical geometry, Phys. Rev. A 22 (1989), 296

[GS] V. Galaktionov and S. Svirshchevskii, Exact Solutions and Invariant Subspaces of Nonlinear Partial Differential Equations in Mechanics and Physics, Chapman \& Hall/CRC, Boca Raton, FL, 2007.

[GM] C. Gardner and G. Morikawa, Similarity in the asymptotic behaviour of collision free hydrodynamic waves and water waves, Courant Inst. Math. Sci. Res. Rep. NYO9082, New York University, New York, 1960.

[GGKM1] C. Gardner, J. Greene, M. Kruskal and R. Miura, Method for solving the Korteweg and de Vries equation, Phys. Rev. Lett. 19 (1967), 1095-1097.

[GGKM2] C. Gardner, J. Greene, M. Kruskal and R. Miura, Korteweg-de Vries equation and generalizations VI. Methods for exact solution, Commun. Pure Appl. Math. 27, (1974), 97-133.

[GG] I. M. Gel'fand and M. I. Graev, GG-functions and their relation to general hypergeometric functions, Russian Math. Surveys 52(1997), no. 4, 639-684. 
[GDi1] I. M. Gel'fand and L. A. Dikii, Asymptotic behaviour of the resolvent of SturmLiouville equations and the algebra of the Korteweg-de Vries equations, Russian Math. Surveys 30:5 (1975), 77-113.

[GDi2] I. M. Gel'fand and L. A. Dikii, A Lie algebra structure in a formal variational Calculation, Func. Anal. Appl. 10 (1976), 16-22.

[GDo1] I. M. Gel'fand and I. Ya. Dorfman, Hamiltonian operators and algebraic structures related to them, Funkts. Anal. Prilozhen 13 (1979), 13-30.

[GDo2] I. M. Gel'fand and I. Ya. Dorfman, Schouten brackets and Hamiltonian operators, Funkts. Anal. Prilozhen 14 (1980), 71-74.

[GDo3] I. M. Gel'fand and I. Ya. Dorfman, Hamiltonian operators and infinitedimensional Lie algebras, Funkts. Anal. Prilozhen 14 (1981), 23-40.

[GC] M. Gill and S. Childress, Topics in Geophysical Fluid Dynamics, Atmospheric Dynamics, Dynamo Theory, and Climate Dynamics, Springer-verlag, New York, 1987.

[GN] C. R. Gilson and J. J. C. Nimmo, A direct method for dromion solutions of the Davey-Stewartson equations and their asymptotic properties, Proc. Roy. Soc. London Ser. A 435 (1991) , 339-357.

[Gj] J. Gibbons, The Khokhlov-Zabolotskaya equation and the inverse scattering problem of classical mechanics, Dynamical Problems in Soliton Systems (Kyoto, 1984), 36-41, Springer, Berlin, 1985.

[GG] B. Grébert and J. Guillot, Periodic solutions of coupled nonlinear Schrödinger equations in nonlinear optics: the resonant case, Appl. Math. Lett. 9 (1996), 65-68.

[Gv] V. I. Gryn, Exact solutions of Navier-Stokes equations, J. Appl. Math. Mech. 55 (1991), 301-309.

[GM] F. Guil and M. Manas, Deformation of the dromion and solutions of the DaveyStewartson I equation, Phys. Lett. A 209 (1995), 39-47.

[Hg1] G. Heckman, Root systems and hypergeometric functions II, Compositio. Math. 64 (1987), 353-373.

[Hg2] G. Heckman, Heck algebras and hypergeometric functions, Invent. Math. 100 (1990), 403-417.

[Hg3] G. Heckman, An elementary approach to the hypergeometric shift operators of Opdam, Invent. Math. 103 (1990), 341-350. 
[HO] G. Heckman and E. Opdam, Root systems and hypergeometric functions I, Compositio. Math. 64 (1987), 329-352.

[HS] F. Hioe and T. Salter, Special set and solutions of coupled nonlinear Schrödinger equations, J. Phys. A: Math. Gen. 35 (2002), no. 42, 8913-8928.

[Hr] R. Hirota, Exact solutions of the Korteweg-de Vries equation for multiple collisions of solitons, Phys. Rev. Lett. 27 (1971), 1192-1194.

[HM] M. N. Hounkonnou and J. E. B. Mendy, Exact solutions of Dirac equation for neutrinos in presence of external fields, J. Math. Phys. 40 (1999), no. 9, 4240-4254.

[HMW] C. Hsia, T. Ma and S. Wang, Stratified rotating Boussinesq equations in geophysical fluid dynamics: dynamic bifurcation and periodic solutions, J. Math. Phys. 48 (2007), no. 6, 06560.

[HL] T. Hou and C. Li, Global well-posedness of the viscous Boussinesq equations, Discrete Contin. Dyn. Syst. 12 (2005), 1-12.

[In1] N. H. Ibragimov, On invariance of Dirac equations, Dokl. Akad. Nauk S.S.S.R. 185 (1969), 1226-1228.

[In2] N. H. Ibragimov, Lie Group Analysis of Differential Equations, Volume 1, CRC Handbook, CRC Press, 1995.

[In3] N. H. Ibragimov, Lie Group Analysis of Differential Equations, Volume 2, CRC Handbook, CRC Press, 1995.

[Ik] K. Ilin, Viscous boundary layers in flows through a domain with permeable boundary, Euro. J. Mech. B/Fluids 27 (2008), 514-538.

[Ia] A. Inoue, On construction of the fundamental solution for the free Dirac equation by Hamiltonian path-integral method. The classical counterpart of Zitterbewegung. Japanese J. Math. 24 (1998), 297-334.

[INP1] A. Ishak, R. Naar and I. Pop, MHD boudary-layer flow of a micropolar fluid past a wedge with constant wall heat flux, Commun. Nonl. Sci. Num. Sim. 14 (2009), 108-118.

[INP2] A. Ishak, R. Naar and I. Pop, Dual solutions in mixed convection boudary layer flow of micropolar fluids, Commun. Nonl. Sci. Num. Sim. 14 (2009), 1324-1333.

[JM] M. Jimbo and T. Miwa, Solitons and infinite dimensional Lie algebras, Publ. RIMS. Kyoto Univ. 19 (1983), 943-1001. 
[Kv] V. Kac, Infinite Dimensional Lie Algebras, 3rd Edition, Cambridge University Press, 1990.

[KP] B. B. Kadomitsev and V. I. Petviashvili, On the stability of solitary waves waekly dispersive media, Sov. Phys. Dokl. 15 (1970), 539-541.

[Kv1] V. I. Katkov, One class of exact solutions of the geopotential forecast equation, Izvestiya Akad. Nauk S.S.S.R., Fizica Atmosferi i Okcana 1 (1965), 1088.

[Kv2] V. I. Katkov, Exact solutions of the geopotential forecast equation, Izvestiya Akad. Nauk S.S.S.R., Fizica Atmosferi i Okcana 2 (1966), 1193.

[Kl] L. B. Kapitanskii, Group analysis of Navier-Stokes equations and Euler equations with rotational symmetry and new exact solutions of these equations, Dokl. Akad. Nauk S.S.S.R. 243 (1978), 901.

[Kr] R. S. Khamitova, Group structure and a basis of conservation laws, Teor. Mat. Fiz 52 (1982), no. 2, 244

[KZ] R. V. Khokhlov and E. A. Zabolotskaya, Quasi-plane waves in nonlinear acoustics of bounded bundles, Akust. Zh. 15 (1969), no. 1, 40

[KR] S. A. Khristianovich and O. S. Razhov, On nonlinear reflection of weak shock waves, Prikl. Mat. Tekh. 22 (1958), no. 5, 586

[Kt] T. W. Kibel', Introduction to Hydrodynamical Methods of Short Term Weather Forecast, Gosteoretizdat, Moscow, 1954.

[KD] J. T. Kirby and R. A. Dalrymple, Oblique envelope solutions of the DaveyStewartson equations in intermediate water depth, Phys. Fluids 26 (1983), 2916-2918.

[KS] A. Kocdryavtsev and V. Sapozhnikov, Symmetries of the generalized KhokhlovZabolotskaya equation, Acous. Phys. 4 (1998), 541-546.

[KiPg] I. Kostin and G. Panasenko, Khokhlov-Zabolotskaya-Kuzentsov-type equation: Nonlinear acoustics in heterogeneous media, SIMA J. Math. 40 (2008), 699-715.

[Ks] S. V. Korsunskii, Self-similar solutions of two-dimensional equations of KhokhlovZabolotskaya type, Mat. Fiz. Nelinein. Mekh. 16 (1991), 81-87.

[KMM] R. Kraenkel, M. Manna and V. Merle, Nonlinear short-wave propagation in ferrites, Phys. Rev. E 61 (2000), 976-979. 
[KMGZ] M. Kruskal, R. Miura, C. Gardner and N. Zabusky, Korteweg-de Vries equation and generalizations $\mathrm{V}$. Uniqueness and nonexistence of polynomial conservation laws, J. Math. Phys. 11 (1970), 952-960.

[Kp] P. Kucharczyk, Group properties of the "short waves" equations in gas dynamics, Bull. Acad. Polon. Sci., Ser. Sci. Techn. XIII (1965), no. 5, 469

[Kb] B. A. Kupershmidt, Geometric-Hamiltonian forms for the Kadomtsev and Petviashvili and Khokhlov-Zabolotskaya equations, Geometry in Partial Differential Equations, 155-172, World Scientific Publishing, River Edge, NJ, 1994.

[Ll] L. Landau, A new exact solutions of Navier-Stokes equations, C. R. (Doklady) Acad. Sci. URSS (N. S.) 43 (1944), 286-288.

[Lr] R. B. Leipnik, Exact solutions of Navier-Stokes equations by recursive series of diffusive equations, C. R. Math. Rep. Acad. Sci. Canada 18 (1996), 211-216.

[Ls] S.-J. Liao, A general approach to get series solution of non-similarity boundary-layer flows, Commun. Nonl. Sci. Num. Sim. 14 (2009), 2144-2159.

[Lie] S. Lie, Begrüdung einer Invariantentheorie der Berührungstransformationen, Math. Ann. 8 (1874), 215-288.

[LRT] C. C. Lin, E. Reissner and H. S. Tsien, On two-dimensional non-steady motion of a slender body in a compressible fluid, J. Math. Phys. 27 (1948), no. 3, 220.

[LZ] J. Lin and J. Zhang, Similarity reductions for the Khokhlov-Zabolotskaya equation, Comm. Theoret. Phys. 24 (1995), no. 1, 69-74.

[LTW1] J. Lions, R. Teman and S. Wang, New formulations of the primitive equations of the atmosphere and applications, Nonlinearity 5 (1992), 237-288.

[LTW2] J. Lions, R. Teman and S. Wang, On the equations of large-scale ocean, Nonlinearity 5 (1992), 1007-1053.

[Llg] L. G. Loitsyanskii, Laminar Boundary Layer, Fizmatgiz, Moscow, 1962.

[Le] E. N. Lorenz, Deterministic nonperiodic flow, J. Atmos. Sci. 20 (1963), 130-141.

[Mf] F. Magri, A simple model of the integrable Hamiltonian equation, J. Math. Phys. 19 (1978), 1156-1162.

[Ma] A. Majda, Introduction to PDEs and Waves for the Atmosphere and Ocean, Courant Lecture Note in Mathematics, Vol. 9, AMS and CIMS, 2003. 
[Mt1] T. M. Malanyuk, Finite-gap solutions of the Davey-Stewartson 2 equations, Russian Math. Surveys 46 (1991), 193-194.

[Mt2] T. M. Malanyuk, Finite-gap solutions of the Davey-Stewartson 1 equations, Nonlinear Sci. 4 (1994), 1-21.

[MS] M. Manas and P. Santini, Solutions of the Davey-Stewartson equation with arbitrary rational localization and nontrivial interaction, Phsy. Lett. A 227 (1997), 325-334.

[Me1] E. V. Mamontov, On the theory of nonstationary transonic flows, Dokl. Acad. Nauk SSSR 185 (1969), no. 3, 538

[Me2] E. V. Mamontov, Analytic perturbations in a nonstationary transonic stream, Dinamika Splosn. Sredy Vyp. 10 (1972), 217-222.

[MGK] R. Miura, C. Gardner and M. Kruskal, Korteweg-de Vries equation and generalizations II. Existence of conservation laws and constants of motion, J. Math. Phys. 9 (1968), 1204-1209.

[MP] D. Mihalache and N. Panoin, Exact solutions of nonlinear Schrödinger equation for positive group velocity dispersion, J. Math. Phys. 33 (1992), no. 6, 2323-2328.

[MD] S. K. Moayedi and F. Darabi, Exact solutions of Dirac equation on 2D gravitational background, Phys. Lett A322 (2004), no. 3-4, 173-178.

[Mo] O. Morozov, Cartan's structure theory of symmetry pseudo-groups for the Khokhlov-Zabolotskaya equation, Acta Appl. Math. 101 (2008), 231-241.

[NMM] R. Naz, F. M. Mahomed and D. P. Mason, Symmetry solutions of a third-order ordinary differential equation which arises from Prandtl boundary layer equations, $J$. Nonl. Math. Phys. 15 (2008), Supplement 1, 179-191.

[OP] M. Olslanetsky and A. Perelomov, Completely integrable Hamiltonian systems connected with semisimple Lie algebras, Invent. Math. 37 (1976), 93-108.

[Op] P. Olver, Applications of Lie Groups to Differential Equations, Second Edition, Springer-Verlag New York, Inc., 1991.

[Om] M. Omote, Infinite-dimensional symmetry algebras and an infinite number of conserved quantities of the (2+1)-dimensional Davey-Stewartson equation, J. Math. Phys. 29 (1988), 2599-2603.

[Oe1] E. Opdam, Root systems and hypergeometric functions III, Compositio. Math. 67 (1988), 21-49. 
[Oe2] E. Opdam, Root systems and hypergeometric functions IV, Compositio. Math. 67 (1988), 191-209.

[Oe3] E. Opdam, Some applications of hypergeometric shift operators, Invent. Math. 98 (1989), 1-18.

[Oe4] E. Opdam, An analogue of the Gauss summation formula for hypergeometric functions related to root systems, Math. Z. 212 (1993), 313-336.

[Oe5] E. Opdam, Cuspital hypergeometric functions, Methods Appl. Anal. 6 (1999), 6780 .

[Pa] A. Pankov, On decay of solutions to nonlinear Schrödinger equation, Proc. Amer. Math. Soc. 136 (2008), no. 7, 2565-2570.

[Pj] J. Pedlosky, Geophsical Fluid Dynamics, 2rd Edition, Springer-verlag, New York, 1987.

[Pa] A. D. Polyanin, Exact solutions of the Navier-Stokes equations with generalized separation of variables, Dokl. Phys. 46 (2001), 726-731.

[PZ] A. D. Polyanin and V. F. Zaitsev, Handbook of Exact Solutions of Ordinary Differential Equations, Second Edition, Chapman\&Hall/CRC, 2003

[Pv] V. V. Pukhnachev, Invariant solutions of Navier-Stokes equations describing motions with free boundary, Dokl. Akad. Nauk S.S.S.R. 202 (1972), 302.

[RL1] R. Radhakrishnan and M. Lakshmanan, Bright and dark soliton solutions to coupled nonlinear Schrödinger equations, J. Phys. A: Math. Gen. 28 (1995), no. 9, 26832692.

[RL2] R. Radhakrishnan and M. Lakshmanan, Exact soliton solutions to coupled nonlinear Schrödinger equations with higher-order effects, Phys. Rev. E(3) 54 (1995), no. 3, 2949-2955.

[RN] C. Roy and M. Nasker, Towards the conservation laws and Lie symmetries for the Khokhlov-Zabolotskaya equation in three dimensions, J. Phys. A 19 (1986), no. 10, 1775-1781.

[RRD] S. Roy, C. Roy and M. De, Loop algebra of Lie symmetries for a short-wave equation, Internat. J. Theoret. Phys. 27 (1988), no. 1, 47-55.

[Ra1] A. Rozanova, The Khokhlov-Zabolotskaya-Kuznetsov equation, Math. Acad. Sci. Paris 344 (2007), 337-342. 
[Ra2] A. Rozanova Qualitative analysis of the Khokhlov-Zabolotskaya equation, Math. Models Mathods Appl. Sci. 18 (2008), 781-812.

[RV] A. I. Ruban and K. N. Vonatsos, Discontinuous solutions of the boundary-layer equations, J. Fluid Mech. 614 (2008), 407-424.

[RS] O. S. Ryzhov and G. M. Shefter, On unsteady gas flows in Laval nozzles, Soviet Physics. Dokl. 4 (1959), 939-942.

[SEG] E. Saied, R. EI-Rahman and M. Ghonamy, On the exact solution of (2+1)dimensional cubic nonlinear Schrödinger (NLS) equation", J. Phy. A: Math. Gen. 36 (2003), 6751-6770.

[Sd] D. Sanchez, Long waves in ferromagnetic media, Khokhlov-Zabolotskaya equation, J. Diff. Equ. 210 (2005), 263-289.

[Sy] Y. Sato, Sign-changing multi-peak solutions for nonlinear Schrödinger equation with critical frequency, Comm. Pure Appl. Anal. 7 (2008), no. 4, 883-903.

[Sf] F. Schwarz, Symmetries of the Khokhlov-Zabolotskaya equation, J. Phys. A 20 (1987), no. 6, 1613.

[Sg] G. D. Sevost'janov, An equation for nonstationary transonic flows of an ideal gas, Izv. Acad. Nauk SSSR Meh. Zidk. Gaza, 1977, no.1, 105-109.

[Sh1] H.-C. Shen, The theory of functions of a complex variable under Dirac-Pauli representation and its application in fluid dynamics I, Appl. Math. Mech. (English Ed.) 7 (1986), 391-411.

[Sh2] H.-C. Shen, Exact solutions of Navier-Stokes equations - the theory of functions of a complex variable under Dirac-Pauli representation and its application in fluid dynamics II, Appl. Math. Mech. (English Ed.) 7 (1986), 557-562.

[Sv] S. V. Sukhinin, Group property and conservation laws of the equation of transonic motion of gas, Dinamika Splosh. Sredi 36 (1978), 130.

[Sb] B. Sutherland, Exact results for a quantum many-body problem in one-dimension, Phys. Rev. A 5 (1972), 1372-1376.

[Ss] S. Syono, Various simple solutions of the barotropic vorticity equation, in Vortex, Collected papers of the numerical weather prediction group in Tokyo (1958), 3.

[T] J.-C. Tsai, Similarity solutions for boundary layer flows with prescribed surface temperature, Appl. Math. Lett. 21 (2008), 67-73. 
[Vj] J. van der Linden, Solutions of the Davey-Stewartson equation with boundary condition, Phys. Lett. A 182 (1992), 155-189.

[Va] A. M. Vinogradov and E. M. Vorob'ev, Application of symmetries for finding of exact solutions of Khokhlov-Zabolotskaya equation, Akust. Zh. 22 (1976), no. 1, 22

[Vv] V. G. Vyskrebtsov, New exact solutions of Navier-Stokes equations for axisymmetric self-similar fluid flows, J. Math. Sci. (New York) 104 (2001), 1456-1463.

[WG] Z. Wang and D. Guo, Special functions, World Scientific, Singapore, 1998.

[X1] X. Xu, Hamiltonian superoperators, J. Phys. A 28 (1995), 1681-1698.

[X2] X. Xu, Hamiltonian operators and associative algebras with a derivation, Lett. Math. Phys. 33 (1995), 1-6.

[X3] X. Xu, Differential invariants of classical groups, Duke Math. J. 94 (1998), 543-572.

[X4] X. Xu, Variational calculus of supervariables and related algebraic structures, $J$. Algebra 223 (2000), 386-437.

[X5] X. Xu, Equivalence of conformal superalgebras to Hamiltonian superoperators, Algebra Colloq. 9 (2001), 63-92.

[X6] X. Xu, Poisson and Hamiltonian superpairs over polarized associative algebras, $J$. Phys. A 34 (2001), 4241-4265.

[X7] X. Xu, Tree diagram Lie algebras of differential operators and evolution partial differential equations, J. Lie Theory 16 2006, no.4, 691-718.

[X8] X. Xu, Stable-range approach to the equation of nonstationary transonic gas flows, Quart. Appl. Math. 65 (2007), 529-547.

[X9] X. Xu, Path hypergeometric functions, J. Algebra and Its Applications. 6 (2007), $595-653$.

[X10] X. Xu, Matrix-differential-operator approach to the Maxwell equations and the Dirac equation, Acta Appl. Math. 102 (2008), 237-247.

[X11] X. Xu, Flag partial differential equations and representations of Lie algebras, Acta Appl. Math. 102 (2008), 249-280.

[X12] X. Xu, Asymmetric and moving-frame approaches Navier-Stokes equations, Quart. Appl. Math. 67 (2009), 163-193. 
[X13] X. Xu, Stable-Range approach to short wave and Khokhlov-Zabolotskaya equations, Acta Appl. Math. 106 (2009), 433-454.

[X14] X. Xu, Quadratic-Argument approach to nonlinear Schrödinger equation and coupled ones, Acta Appl. Math. 110 (2010), 749-769.

[X15] X. Xu, New algebraic approaches to classical boundary layer problems, Acta Math. Sinica (English Series) 27 (2011), 1023-1070.

[X16] X. Xu, Asymmetric and moving-frame approaches to the 2D and 3D Boussinesq equations, arXiv:0806.4910v1[physics.flu-dyn].

[X17] X. Xu, Multiple parameter function approaches to the equation of dynamic convection in a sea, arXiv:0812.1831v1[math-ph].

[X18] X. Xu, Quadratic-Argument approach to the Davey-Stewartson equations, arXiv:0812.1833v1[math-ph].

[Y] A. Yu. Yakimov, Exact solutions of Navier-Stokes equations in the presence of a vortex singularity on a ray, Dokl. Acad. Nauk SSSR 276 (1984), 79-82.

[ZC] W. Zhai and D.-Y. Chen, Rational solutions of the general nonlinear Schrödinger equation with derivative, Phys. Lett. A 372 (2008), no. 23, 4217-4221.

[Z] D. Zwillinger, Handbook of Differential Equations, Third Edition, Academic Press, 1998.

$\operatorname{xxx}$ 